Bianca Gusenbauer

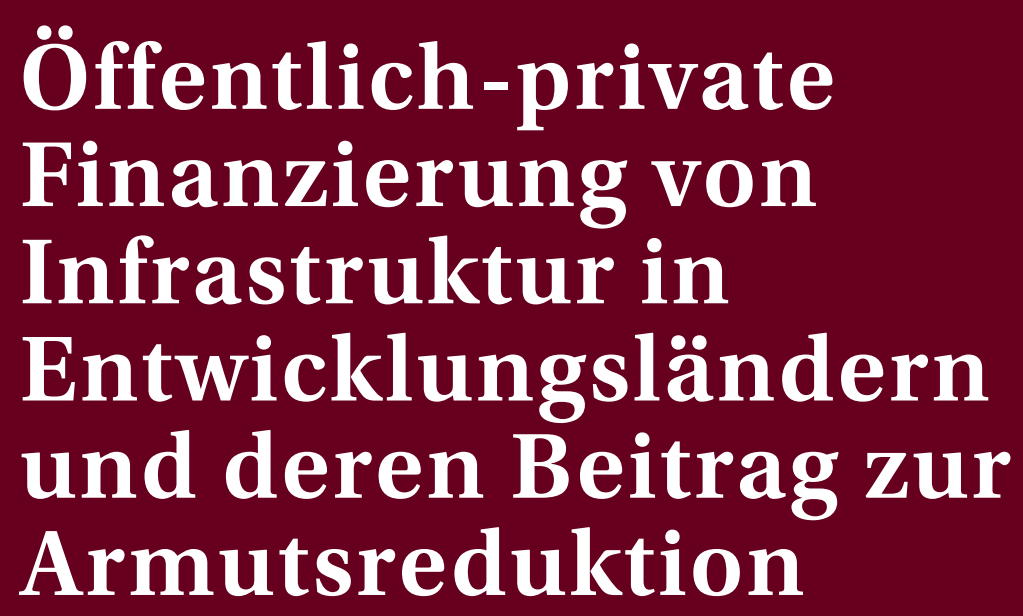

Fallstudien in Vietnam und auf den Philippinen 


\section{Öffentlich-private Finanzierung von Infrastruktur in Entwicklungsländern und deren Beitrag zur Armutsreduktion}

Die Dissertation ist an der Schnittstelle von Betriebwirtschaftslehre und Entwicklungsökonomie angesiedelt. Basierend auf einem Multimethodenansatz werden jeweils zwei Fallstudien in Vietnam und auf den Philippinen aus beiden Perspektiven dargestellt. Der entwicklungsökonomische Teil der Arbeit umfasst die Darstellung von Good Governance und Global Governance. Anschließend werden die Auswirkungen von ausländischen Direktinvestitionen und von Energie- und Straßeninfrastruktur auf Wirtschaftswachstum und Armutsreduktion diskutiert. Ein weiterer Schwerpunkt liegt auf der Darstellung von Public Private Partnerships. Keine Fallstudie liefert einen direkten langfristigen Beitrag zur Armutsreduktion, aber sie tragen zum Wirtschaftswachstum bei. Zusammenfassend kann festgehalten werden, dass die positiven Auswirkungen von privat finanzierter Infrastruktur auf die Armutsreduktion überschätzt werden.

Bianca Gusenbauer absolvierte ihre Studien der Handelswissenschaften und der Betriebswirtschaft an der Wirtschaftsuniversität Wien. Von 2004 bis 2008 war sie als Wissenschaftliche Mitarbeiterin und Doktoratsstudentin am Institut für Betriebswirtschaftslehre des Außenhandels tätig und promovierte im November 2008. Ihr Forschungsinteresse liegt in den Bereichen Infrastrukturfinanzierung, Armutsreduktion und Wechselkursmanagement. Ihre Forschungsaufenthalte führten sie u.a. an die UCLA, an die Wirtschaftsuniversität Prag, nach Vietnam und auf die Philippinen. Seit Mitte 2008 ist die Autorin im Finanzbereich in der Infrastrukturfinanzierung tätig. 
Öffentlich-private Finanzierung von Infrastruktur in Entwicklungsländern und deren Beitrag zur Armutsreduktion 


\section{Forschungsergebnisse der Wirtschaftsuniversität Wien}

\section{Band 29}

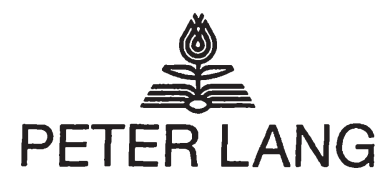

Frankfurt am Main · Berlin · Bern - Bruxelles - New York · Oxford · Wien Bianca Gusenbauer - 978-3-631-75445-0 
Bianca Gusenbauer

\section{Öffentlich-private Finanzierung von Infrastruktur in Entwicklungsländern und deren Beitrag zur Armutsreduktion}

Fallstudien in Vietnam und auf den Philippinen

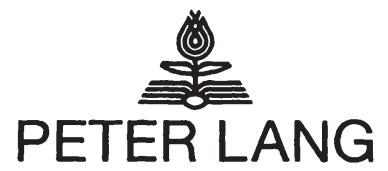

Internationaler Verlag der Wissenschaften 
Bibliografische Information der Deutschen Nationalbibliothek Die Deutsche Nationalbibliothek verzeichnet diese Publikation in der Deutschen Nationalbibliografie; detaillierte bibliografische Daten sind im Internet über <http://www.d-nb.de> abrufbar.

Open Access: The online version of this publication is published on www.peterlang.com and www.econstor.eu under the international Creative Commons License CC-BY 4.0. Learn more on how you can use and share this work: http://creativecommons.org/licenses/ by/4.0.

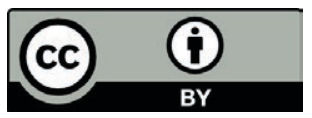

This book is available Open Access thanks to the kind support of ZBW - Leibniz-Informationszentrum Wirtschaft.

Gefördert durch die Wirtschaftsuniversität Wien.

\author{
Umschlaggestaltung: \\ Atelier Platen, nach einem Entwurf \\ der Werbeagentur Publique. \\ Universitätslogo der Wirtschaftsuniversität Wien: \\ Abdruck mit freundlicher Genehmigung \\ der Wirtschaftsuniversität Wien. \\ Gedruckt auf alterungsbeständigem, \\ säurefreiem Papier. \\ ISSN 1613-3056 \\ ISBN 978-3-631-58319-7 \\ ISBN 978-3-631-75445-0 (eBook) \\ (C) Peter Lang $\mathrm{GmbH}$ \\ Internationaler Verlag der Wissenschaften \\ Frankfurt am Main 2009 \\ Alle Rechte vorbehalten.
}

Das Werk einschließlich aller seiner Teile ist urheberrechtlich geschützt. Jede Verwertung außerhalb der engen Grenzen des Urheberrechtsgesetzes ist ohne Zustimmung des Verlages unzulässig und strafbar. Das gilt insbesondere für Vervielfältigungen, Übersetzungen, Mikroverfilmungen und die Einspeicherung und Verarbeitung in elektronischen Systemen.

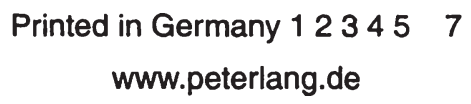




\section{Abstract:}

Die Dissertation ist an der Schnittstelle von Betriebswirtschaftslehre und Entwicklungsökonomie angesiedelt und unternimmt den Versuch, Fragen über die Auswirkungen der Privatisierungs- und Deregulierungsreformen im Infrastruktursektor in beiden Disziplinen zu beantworten. Basierenden auf einem Multimethodenansatz werden jeweils zwei Fallstudien in Vietnam und auf den Philippinen aus beiden Perspektiven dargestellt.

Der entwicklungsökonomische Teil der Arbeit widmet sich zuerst den Veränderungen bei den Unterstützungsansätzen und konzentriert sich dabei auf die Darstellung von Good Governance und Global Governance. Daran anschließend werden die Auswirkungen von ausländischen Direktinvestitionen und von Energie- und Straßeninfrastruktur auf Wirtschaftswachstum und Armutsreduktion diskutiert.

Aus betriebswirtschaftlicher Perspektive trat durch die Privatisierungen und Deregulierungen vor allem der Begriff der Public Private Partnerships in den Diskussionsmittelpunkt. Daher wird Partnerschaft für die Arbeit definiert und darauf aufbauend werden die verschiedenen Formen der Zusammenarbeit zwischen privatem und öffentlichem Sektor gezeigt. Bei der Finanzierung von privaten Infrastrukturinvestitionen in Entwicklungsländern nehmen Internationale Finanzinstitutionen eine wichtige Unterstützungsrolle ein und deren Angebot bzw. innovative Ansätze für die Unterstützung des privaten Sektors werden in der Arbeit dargestellt.

Alle Projekte weisen einen wesentlichen Anteil an institutioneller Unterstützung und unterschiedlichen Instrumenten auf. Keines der Projekte liefert einen direkten langfristigen Beitrag zur Armutsreduktion, aber sie tragen zum Wirtschaftswachstum bei. Zusammenfassend kann festgehalten werden, dass die positiven Auswirkungen von privat finanzierter Infrastruktur auf die Armutsreduktion überschätzt werden. 
Bianca Gusenbauer - 978-3-631-75445-0

Downloaded from PubFactory at 01/11/2019 04:47:32AM

via free access 


\section{Inhaltsverzeichnis}

Abbildungsverzeichnis $\quad 13$

Tabellenverzeichnis $\quad 15$

Abkürzungsverzeichnis $\quad 17$

$1 \quad$ Einleitung $\quad 21$

1.1 Positionierung der Arbeit 21

$\begin{array}{lll}1.2 & \text { Zielsetzung } & 22\end{array}$

$\begin{array}{lll}1.3 & \text { Methodik } & 23\end{array}$

$\begin{array}{lll}1.4 & \text { Aufbau der Arbeit } & 28\end{array}$

$2 \quad$ Aspekte von Governance in der Entwicklungspolitik 33

2.1 Einführung $\quad 33$

2.1.1 Definition 34

2.1.2 Entwicklungen des Governance-Konzeptes 36

2.2 Good Governance 38

2.2.1 Einführung und Definition 38

2.2.2 Historisch-theoretische Darstellung von Good Governance im Lichte der entwicklungstheoretischen Ansätze 40

2.2.2.1 Von den Anfängen bis zum Washington-Consensus 40

2.2.2.2 Post-Washington-Consensus bis zu den Millennium
Development Goals

$\begin{array}{lll}\text { 2.2.2.3 Zusammenfassung } & 47\end{array}$

2.2.3 Kritik an Good Governance $\quad 48$

2.2.4 New Public Management und Good Governance 51

2.2.4.1 Privatisierung 52

2.2.4.2 Dezentralisierung $\quad 54$

2.3 Global Governance $\quad 55$

2.3.1 Einführung und Definition 56

2.3.2 Historisch-theoretische Darstellung von Global Governance im Lichte der entwicklungstheoretischen Ansätze $\quad 58$

$\begin{array}{ll}\text { 2.3.3 Kritik an Global Governance } & 60\end{array}$

2.4 Zusammenfassung $\quad 62$

3 Private Infrastrukturinvestitionen in Entwicklungsländern 65

$\begin{array}{lll}3.1 & \text { Begriffsabgrenzung } & 65\end{array}$

3.1.1 Definition von Ausländischer Direktinvestition 65

$\begin{array}{lll}3.1 .2 & \text { Definition von Infrastruktur } & 67\end{array}$

3.1.3 Definition von Armut $\quad 70$

3.2 Auswirkungen von ADI auf Entwicklungsländer $\quad \mathbf{7 4}$

$\begin{array}{lll}3.2 .1 \quad \text { Einführung } & 75\end{array}$

$\begin{array}{lll}\text { 3.2.2 ADI und Spillover-Effekte } & 76\end{array}$ 
3.2.3 ADI und Wirtschaftswachstum

3.3 Auswirkungen von Infrastruktur auf Armutsreduktion $\quad 83$

$\begin{array}{lll}\text { 3.3.1 Einführung } & 83\end{array}$

3.3.2 Infrastruktur und Armutsreduktion 85

3.3.2.1 Indirekte Auswirkung von Infrastruktur auf Armutsreduktion 85

3.3.2.2 Direkte Auswirkung von Infrastruktur auf Armutsreduktion $\quad 86$

3.3.2.3 Konzeptioneller Rahmen für die Darstellung der Auswirkungen von Infrastruktur auf Armutsreduktion $\quad 87$

$\begin{array}{lll}\text { 3.3.3 Energieinfrastruktur und Armutsreduktion } & 89\end{array}$

3.3.4 Transportinfrastruktur und Armutsreduktion 91

$\begin{array}{lll}3.4 & \text { Zusammenfassung } & 93\end{array}$

4 Öffentlich-private Bereitstellung von Infrastruktur 97

$\begin{array}{lll}4.1 & \text { Einführung } & 98\end{array}$

4.1.1 Definition von Partnerschaft in einem kooperativen Modell 98

$\begin{array}{ll}\text { 4.1.2 Definition von Public Private Partnerships } & 100\end{array}$

4.1.3 Ziele und Interessen der Partner 103

$\begin{array}{lll}\text { 4.1.4 Risiken und Problembereiche } & 106\end{array}$

4.2 PPI in der Entwicklungspolitik 110

4.2.1 Auswirkungen der entwicklungspolitischen Veränderungen in der Infrastrukturfinanzierung $\quad 110$

4.2.2 Die Rolle der ODA in der Infrastrukturfinanzierung 112

4.2.3 Die Rolle des privaten Sektors in der Infrastrukturfinanzierung 113

$\begin{array}{lll}\text { 4.2.3.1 PPI-Ströme nach Sektoren } & 113\end{array}$

$\begin{array}{lll}\text { 4.2.3.2 PPI-Ströme nach Regionen } & 114\end{array}$

4.2.3.3 Zusammenfassung der PPI-Entwicklung in den

4.2.4 Eine neue Rollenverteilung in der Infrastrukturfinanzierung 117

4.3 Länderrisiken bei Infrastrukturinvestitionen in

$\begin{array}{lll}\text { 4.3.1 Einführung } & 119\end{array}$

$\begin{array}{ll}\text { 4.3.2 Wirtschaftliche Risiken } & 121\end{array}$

$\begin{array}{ll}\text { 4.3.2.1 Absatz- und Zulieferrisiken } & 121\end{array}$

$\begin{array}{ll}\text { 4.3.2.2 Delkredererisiko } & 122\end{array}$

4.3.3 Politisches Risiken 122

\begin{tabular}{ll} 
4.3.3.1 Politische Risiken i.e.S. & 122 \\
\hline
\end{tabular}

4.3.3.2 KT-ZM Risiko 123

$\begin{array}{lll}\text { 4.3.4 Wechselkursrisiken } & 124\end{array}$

$\begin{array}{ll}\text { 4.3.4.1 Währungsumrechnungsrisiko } & 125\end{array}$

$\begin{array}{ll}\text { 4.3.4.2 Währungsumwechslungsrisiko } & 125\end{array}$

4.3.4.3 Ökonomisches Wechselkursrisiko 126

4.4 Öffentlich-private Infrastrukturmodelle $\quad 126$

$\begin{array}{ll}4.4 .1 \quad \text { Einführung } & 126\end{array}$

$\begin{array}{ll}\text { 4.4.2 Service- und Management Modelle } & 128\end{array}$ 
$\begin{array}{lll}\text { 4.4.2.1 Serviceverträge } & 128\end{array}$

$\begin{array}{ll}\text { 4.4.2.2 Managementverträge } & 129\end{array}$

4.4.3 Transfer of Operation Rights (TOR) - Modelle 130

4.4.3.1 Leasingmodelle 131

$\begin{array}{lll}\text { 4.4.3.2 Konzessionsmodell } & 134\end{array}$

4.4.4 Public Private Partnership - Modelle 136

$\begin{array}{lll}\text { 4.4.5 } & \text { Betreibermodelle } & 138\end{array}$

4.5 Zusammenfassung 140

5 Die Unterstützung von privaten Infrastrukturinvestitionen durch internationale und nationale Finanzinstitutionen $\quad 144$

$\begin{array}{lll}5.1 & \text { Einführung } & 145\end{array}$

5.1.1 Rolle der Projektfinanzierung im Infrastruktursektor 145

5.1.2 Mindestkriterien für private Infrastrukturinvestitionen 146

5.1.3 Die Kofinanzierungsrolle der IFIs bei privaten Investitionen $\quad 147$

5.2 Finanzierungs- und Absicherungsinstrumente von internationalen und nationalen Finanzinstitutionen in der Infrastrukturfinanzierung

$\begin{array}{lll}5.2 .1 & \text { Einführung } & 148\end{array}$

$\begin{array}{ll}\text { 5.2.2 Multilaterale Institutionen } & 149\end{array}$

$\begin{array}{lll}5.2 .2 .1 & \text { IFC } & 150\end{array}$

$\begin{array}{lll}5.2 .2 .2 & \text { MIGA } & 153\end{array}$

5.2.2.3 IBRD und IDA 154

5.2.2.4 Fonds- und Programmbeteiligungen der Weltbankgruppe 155

$\begin{array}{ll}\text { 5.2.2.5 Asian Development Bank } & 158\end{array}$

5.2.3 Europäische Institutionen 163

5.2.3.1 Europäische Investitionsbank (EIB) 163

5.2.3.2 Europäische Kommission 164

5.2.4 Österreichische Beteiligung an Internationalen Finanzinstitutionen 165

5.2.5 Österreichische Institutionen 165

$\begin{array}{ll}\text { 5.2.5.1 Austrian Development Agency } & 166\end{array}$

$\begin{array}{lll}\text { 5.2.5.2 Austria Wirtschaftsservice (AWS) } & 168\end{array}$

5.2.5.3 Oesterreichische Kontrollbank (OeKB) 169

5.2.6 Zusammenfassung der Instrumente für PPI 170

5.3 Detailvorstellung und kritische Würdigung einzelner

$\begin{array}{lll}\text { 5.3.1 Garantien } & 172\end{array}$

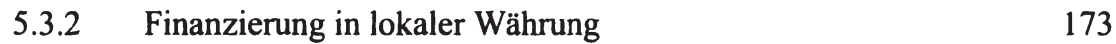

$\begin{array}{lll}\text { 5.3.3 Output-based Aid } & 177\end{array}$

$\begin{array}{lll}\text { 5.3.4 Sub-souveräne Finanzierung } & 179\end{array}$

$\begin{array}{lll}5.4 & \text { Zusammenfassung } & 183\end{array}$ 
$\begin{array}{lll}6 & \text { Fallstudien } & 187\end{array}$

$\begin{array}{lll}\text { 6.1 Einführung } & 187\end{array}$

6.1.1 Kriterien für die Auswahl der Fallstudien 188

6.1.1.1 Region $\quad 188$

6.1.1.2 Sektor 188

6.1.1.3 Art der PPI 190

6.1.1.4 Aktualität der Fallstudien $\quad 191$

6.1.1.5 Zugang zu den Daten 191

6.1.1.6 Ergebnis des Auswahlprozesses $\quad 191$

6.1.2 Aufbau der Fallstudien 192

6.2 Vietnam 193

6.2.1 Einführung 193

6.2.1.1 Eckdaten 193

6.2.1.2 Wirtschaftliche Rahmenbedingungen 194

$\begin{array}{lll}\text { 6.2.2 Phu My 2.2 } & 197\end{array}$

6.2.2.1 Eckdaten 197

6.2.2.2 Entstehung 198

6.2.2.3 Finanzierungs- und Absicherungsinstrumente 198

6.2.2.4 Vertragsstruktur 203

$\begin{array}{lll}\text { 6.2.3 Phu My } 3 & 206\end{array}$

6.2.3.1 Eckdaten 206

$\begin{array}{ll}\text { 6.2.3.2 Entstehung } & 208\end{array}$

6.2.3.3 Finanzierungs- und Absicherungsinstrumente 208

$\begin{array}{ll}\text { 6.2.3.4 Vertragsstruktur } & 212\end{array}$

6.2.4 Zusammenfassende Gegenüberstellung von Phu My 2.2
und Phu My 3

6.2.5 Auswirkungen von Phu My 2.2 und Phu My 3 auf die
Armutsreduktion

$\begin{array}{lll}\text { 6.3 Philippinen } & 224\end{array}$

6.3.1 Einführung 224

$\begin{array}{ll}\text { 6.3.1.1 Eckdaten } & 224\end{array}$

6.3.1.2 Wirtschaftliche Rahmenbedingungen 225

6.3.2 Kraftwerk Steag State Power Inc. Mindanao 228

$\begin{array}{ll}\text { 6.3.2.1 Eckdaten } & 228\end{array}$

6.3.2.2 Entstehung 228

6.3.2.3 Finanzierungs- und Absicherungsinstrumente 229

6.3.2.4 Vertragsstruktur 233

6.3.2.5 Zusammenfassende Gegenüberstellung von SPI 237

6.3.2.6 Auswirkungen des Kraftwerkes Steag State Power Inc.
Mindanao auf die Armutsreduktion

6.3.3 Northern Luzon Expressway (NLEX) 243

6.3.3.1 Eckdaten 243

6.3.3.2 Entstehung 244

6.3.3.3 Finanzierungs- und Absicherungsinstrumente 245 
6.3.3.5 Zusammenfassende Gegenüberstellung von NLEX 254

6.3.3.6 Auswirkungen der NLEX auf die Armutsreduktion 259

6.4 Zusammenfassung 262

7 Conclusio $\quad 271$

7.1 Resümee 271

\begin{tabular}{ll}
7.2 & Fazit und Ausblick \\
\hline
\end{tabular}

Anhang $\quad 279$

Literaturverzeichnis $\quad 285$ 
Bianca Gusenbauer - 978-3-631-75445-0

Downloaded from PubFactory at 01/11/2019 04:47:32AM

via free access 


\section{Abbildungsverzeichnis}

Abbildung 1: $\quad$ Positionierung des Themas 22

Abbildung 2: Forschungsfragen $\quad 23$

Abbildung 3: Methodische Vorgehensweise 26

Abbildung 4: Zirkuläres Modell der Datenanalyse 29

Abbildung 5: Aufbau der Arbeit 31

Abbildung 6: Kapitelübersicht Aspekte von Governance in der Entwicklungspolitik 33

Abbildung 7: Übersicht der Governance-Konzepte $\quad 37$

Abbildung 8: Millennium Development Goals 46

Abbildung 9: Entwicklungstheoretische Einordnung von

Good Governance $\quad 48$

Abbildung 10: Kapitelübersicht Private Investitionen in die Infrastruktur in Entwicklungsländern

Abbildung 11: Einordnung der ausländischen Direktinvestitionen 66

Abbildung 12: Der multidimensionale Armutsrahmen der OECD 72

Abbildung 13: Auswirkungen von Infrastruktur auf Wachstum und Armutsreduktion

Abbildung 14: Mögliche positive Auswirkungen von Energieinfrastruktur auf die Armen 90

Abbildung 15: Mögliche positive Auswirkungen von Transportinfrastruktur auf die Armen 92

Abbildung 16: Kapitelübersicht Öffentlich-private Infrastrukturmodelle 97

Abbildung 17: Ziele und Interessen der Akteure in einer PPP 107

Abbildung 18: Vereinfachte Darstellung der Annahmen des

Washington-Consensus bei der Privatisierung von Infrastruktur

Abbildung 19: Anteil der bilateralen Entwicklungshilfe für Infrastruktur 113

Abbildung 20: Einteilung der Länderrisiken 120

Abbildung 21: Kontinuum Staat - Privat 127

Abbildung 22: Leistungsbeziehungen in Serviceverträgen 129

Abbildung 23: Leistungsbeziehungen bei Managementverträgen 130

Abbildung 24: Leistungsbeziehungen in einem Leasingmodell, Staat als Leasinggeber

Abbildung 25: Leistungsbeziehungen im Leasingmodell, Staat als Leasingnehmer

Abbildung 26: Leistungsbeziehungen im Konzessionsmodell 135

Abbildung 27: Leistungsbeziehungen im PPP-Modell 137

Abbildung 28: Leistungsbeziehungen im BOT-Modell 139

Abbildung 29: Kapitelübersicht Unterstützung von privaten Infrastrukturinvestitionen durch internationale und nationale Finanzinstitutionen 
Abbildung 30: Organisationen der Weltbankgruppe $\quad 150$

Abbildung 31: Instrumente der IFC 151

Abbildung 32: Instrumente der ADB 159

Abbildung 33: Ablauf des Complementary Financing Schemes 160

Abbildung 34: Ablauf einer Political Risk Guarantee 161

Abbildung 35: Ablauf einer Partial Risk Guarantee 162

Abbildung 36: Österreichische Beteiligungen an Internationalen

Finanzinstitutionen 166

Abbildung 37: Cross-Currency Swap der ADB 176

Abbildung 38: Gestaltung der Übergangssubvention 178

Abbildung 39: Indirekter Kredit durch den souveränen Staat an die dezentrale Regierungseinheit 182

Abbildung 40: Direkter Kredit an die sub-souveräne Regierungseinheit 182

Abbildung 41: Kapitelübersicht Fallstudien $\quad 187$

Abbildung 42: Energieproduktion in Vietnam 196

Abbildung 43: Finanzierungs- und Absicherungsstruktur von Phu My 2.2202

Abbildung 44: Projektbeteiligte bei Phu My 2.2 204

Abbildung 45: Finanzierungs- und Absicherungsstruktur von Phu My 3211

Abbildung 46: Projektbeteiligte bei Phu My $3 \quad 213$

Abbildung 47: Auswirkungen von Phu My 2.2 und Phu My 3 auf

Wachstum und Armutsreduktion 223

Abbildung 48: Finanzierungs- und Absicherungsstruktur von

SPI Mindanao 232

Abbildung 49: Projektbeteiligte bei Steag State Power Inc. Kraftwerk Mindanao

Abbildung 50: Auswirkungen des SPI Kraftwerkes auf Wachstum und Armutsreduktion

Abbildung 51: Ursprüngliche Finanzierungs- und Absicherungsstruktur von NLEX

Abbildung 52: Restrukturierte Finanzierungs- und Absicherungsstruktur von NLEX

Abbildung 53: Projektbeteiligte bei NLEX 252

Abbildung 54: Auswirkungen der NLEX auf Wachstum und Armutsreduktion 262

Abbildung 55: Darstellung der Kriterien des Auswahlprozesses 262

Abbildung 56: Einordnung der Themen in die Disziplinen 271 


\section{Tabellenverzeichnis}

Tabelle 1: Zusammenfassung der Infrastrukturdefinitionen

Tabelle 2: Investitionen in die Infrastruktur mit privater Beteiligung, aufgeteilt nach Sektoren, in Milliarden US\$, für den Zeitraum 1996-2005

Tabelle 3: Investitionen in die Infrastruktur mit privater Beteiligung, aufgeteilt nach Regionen, in Milliarden US\$, für den Zeitraum von 1996-2005

Tabelle 4: Zusammenfassung von öffentlich-privaten Finanzierungsmodellen

Tabelle 5: Übersicht über ausgewählte Finanzinstitutionen für private Projekte in Entwicklungsländern

Tabelle 6: Regionen gereiht nach Private Participation in Infrastructur (PPI), in Millionen US\$, für den Zeitraum von 1995-2005

Tabelle 7: Sektoren gereiht nach Private Participation in Infrastructure (PPI), in Millionen US\$, für den Zeitraum von 1995-2005

Tabelle 8: $\quad$ Art der PPI in den Sektoren Energie und Transport in der Region Ostasien und Pazifik, in Millionen US\$, für den Zeitraum 1995-2005

Tabelle 9: Übersicht über die ausgewählten Fallstudien

Tabelle 10: Armutsreduktion in Vietnam im Vergleich zu anderen asiatischen Entwicklungsländern

Tabelle 11: Zusammenfassung der Finanzierung und Absicherung von Phu My 2.2 und Phu My 3

Tabelle 12: Zusammenfassung der Finanzierung und Absicherung von SPI, Phu My 2.2 und Phu My

Tabelle 13: Zusammenfassung der Finanzierung und Absicherung von NLEX

Tabelle 14: Überblick über die Fallstudien 
Bianca Gusenbauer - 978-3-631-75445-0

Downloaded from PubFactory at 01/11/2019 04:47:32AM

via free access 


\section{Abkürzungsverzeichnis}

$\begin{array}{ll}\text { ABS } & \text { Asset Backed Securities } \\ \text { ACP } & \text { African Carribean Pacific } \\ \text { ADA } & \text { Austrian Development Agency } \\ \text { ADB } & \text { Asian Develoment Bank } \\ \text { ADI } & \text { Ausländische Direktinvestition } \\ \text { AFDB } & \text { African Development Bank } \\ \text { AFFG } & \text { Ausfuhrfinanzierungsförderungsgesetz } \\ \text { AFG } & \text { Ausfuhrförderungsgesetz } \\ \text { AKP } & \text { Afrika, Karibik, Pazifik } \\ \text { ASEAN } & \text { Association of Southeast Asian Nations } \\ \text { ASTAE } & \text { Asia Sustainable and Alternative Energy Program } \\ \text { AWS } & \text { Austria Wirtschaftsservice } \\ \text { BIP } & \text { Bruttoinlandsprodukt } \\ \text { BMF } & \text { Bundesministerium für Finanzen } \\ \text { BOOT } & \text { Build Operate Own Transfer } \\ \text { BOT } & \text { Build Operate Transfer } \\ \text { DAC } & \text { Develompent Assistance Committee } \\ \text { DBFO } & \text { Design Build Finance Operate } \\ \text { DFID } & \text { UK Departement for International Development } \\ \text { EAIF } & \text { Emerging Africa Infrastructure Fund } \\ \text { EBRD } & \text { European Bank for Reconstruction and Development } \\ \text { ECA } & \text { Export Credit Agency } \\ \text { EDF } & \text { Electricité de France } \\ \text { EG } & \text { Europäische Gemeinschaft } \\ \text { EIB } & \text { Euroean Investment Bank } \\ \text { EIF } & \text { European Investment Fund } \\ \text { EPC } & \text { Engineering, Procurement, Production } \\ \text { ESAMP } & \text { Energy Sector Management Assistance Program } \\ \text { EVF } & \text { Exportfinanzierungsverfahren } \\ \text { EVN } & \text { Electricity Vietnam } \\ \text { EZA-Gesetz } & \text { Entwicklungszusammenarbeitsgesetz } \\ \text { FC } & \text { Fuel charge } \\ \text { FCC } & \text { Fixed capacity charge } \\ \text { FDI } & \text { Foreign Direct Investment } \\ \text { FIAS } & \text { Foreign Investment Advisory Service } \\ \text { GKA } & \text { Garantie für Kapitalanlagen } \\ \text { GPOBA } & \text { Global Partnership on Output-Based Aid } \\ \text { GUS } & \text { Gemeinschaft Unabhängiger Staaten } \\ \text { HDI } & \text { Human Development Index } \\ \text { HDN } & \text { Human Development Network } \\ & \end{array}$




$\begin{array}{ll}\text { HWWA } & \text { Hamburger Welt-Wirtschafts-Archiv } \\ \text { IADB } & \text { Inter-American Development Bank } \\ \text { IBRD } & \text { International Bank of Reconstruction and Development } \\ \text { IDA } & \text { International Development Agency } \\ \text { ieS } & \text { Im engeren Sinn } \\ \text { IFC } & \text { International Finance Corporation } \\ \text { IFIs } & \text { International Financial Institutions } \\ \text { INEF } & \text { Institut für Entwicklung und Frieden } \\ \text { infoDev } & \text { Information for Development Program } \\ \text { IPP } & \text { Independent Power Producer } \\ \text { IsDB } & \text { Islamic Development Bank } \\ \text { JBG } & \text { Jorong Barutama Greston } \\ \text { JBIC } & \text { Japan Bank for International Cooperation } \\ \text { KfW } & \text { Kreditanstalt für Wiederaufbau } \\ \text { KHI } & \text { Kawasaki Heavy Industries } \\ \text { KPCI } & \text { Kawasaki Plant Construction Inc. } \\ \text { kWh } & \text { Kilowatt per Stunde } \\ \text { MDGs } & \text { Millennium Development Goals } \\ \text { MECO } & \text { Mekong Energy Company Ltd. } \\ \text { MIGA } & \text { Multilateral Investment Guarantee Agency } \\ \text { MNTC } & \text { Manila North Tollways Corporation } \\ \text { MOI } & \text { Ministry of Industry } \\ \text { MTPDP } & \text { Medium-Term Philippine Development Plan } \\ \text { NEDA } & \text { National Economic and Development Authoritiy } \\ \text { NEXI } & \text { Nippon Export and Investment Insurance } \\ \text { NGO } & \text { Non-Governmental Organisation } \\ \text { NLEX } & \text { North Luzon Expressway } \\ \text { NPC } & \text { National Power Corporation } \\ \text { NPM } & \text { New Public Management } \\ \text { O\&M charge } & \text { Operation and Maintenance Charge } \\ \text { OBA } & \text { Output-Based Aid } \\ \text { ODA } & \text { Official Development Assistance } \\ \text { OeKB } & \text { Oesterreichische Kontrollbank } \\ \text { OEZA } & \text { Österreichische Entwicklungszusammenarbeit } \\ \text { PBGs } & \text { Policy Based Guarantees } \\ \text { PCGs } & \text { Partial Credit Guarantees } \\ \text { PHIVIDEC } & \text { Philippine Veterans Industrial Development Corporation } \\ \text { PIDG } & \text { Private Infrastructure Development Group } \\ \text { PM3 } & \text { Phu My 3 BOT Power Company Limited } \\ \text { PNCC } & \text { Philippine National Construction Corporation } \\ \text { PPA } & \text { Purchasing Power Agreement } \\ \text { PPI } & \text { Private Participation in Infrastructure } \\ & \end{array}$




$\begin{array}{ll}\text { PPIAF } & \text { Public-Private Infrastructure Advisory Facility } \\ \text { PPP } & \text { Public Private Partnerships } \\ \text { PRG } & \text { Political Risk Guarantee (ADB) } \\ \text { PRGs } & \text { Partial Risk Guarantees (IBRD und IDA) } \\ \text { PRSPs } & \text { Poverty Reduction Strategy Papers } \\ \text { PSALM } & \text { Power Sector Assets and Liabilities Management Corporation } \\ \text { PV } & \text { Petro Vietnam } \\ \text { ROT } & \text { Rehabilitate Operate Transfer-Concession } \\ \text { SaM } & \text { Service- und Management-Modelle } \\ \text { SAP } & \text { Structural Adjustment Policies (Programs) } \\ \text { SEF } & \text { Stiftung Entwicklung und Frieden } \\ \text { SFF } & \text { Structure Finance Facility } \\ \text { SPI } & \text { STEAG State Power Inc. } \\ \text { SPV } & \text { Special-Purpose-Vehicle - Projektgesellschaft } \\ \text { SSATP } & \text { Sub-Saharan Africa Transport Policy Program (SSATP) } \\ \text { STOA } & \text { Supplemental Toll Agreements } \\ \text { TAF } & \text { Technical Assistance Fund } \\ \text { TOR } & \text { Transfer of Operation Rights-Modelle } \\ \text { UDEC } & \text { Urban Construction Company } \\ \text { UNCTAD } & \text { United Nations Conference on Trade and Development } \\ \text { UNDP } & \text { United Nations Development Program } \\ \text { WSP } & \text { Water and Sanitation Program } \\ \text { WTO } & \text { World Trade Organization }\end{array}$


Bianca Gusenbauer - 978-3-631-75445-0

Downloaded from PubFactory at 01/11/2019 04:47:32AM

via free access 


\section{Einleitung}

\subsection{Positionierung der Arbeit}

Die vorliegende Arbeit ist an der Schnittstelle von Betriebswirtschaftslehre und Entwicklungsökonomie angesiedelt und unternimmt den schwierigen Versuch, beiden Aspekten gerecht zu werden. Die Schwierigkeit dieses Unterfangens lässt sich anhand der verschiedenen Aktionsebenen bzw. Fokusse der Disziplinen erklären. Die Entwicklungsökonomie versucht auf makroökonomischer Ebene die Situation der Entwicklungsländer zu verbessern. Deren Zielgruppe sind daher die Entwicklungsländer, die durch Theorien bzw. strategische Maßnahmen unterstützt werden. Die Betriebswirtschaftslehre hingegen ist auf mikroökonomischer Ebene angesiedelt und versucht effiziente Modelle für einzelne Unternehmen zu entwickeln. Daher kommt es zwischen diesen beiden Disziplinen zu divergierenden Zielen, die in dieser Arbeit jedoch gemeinsam untersucht werden. Da die Verfasserin selbst Betriebswirtin ist, liegt der Schwerpunkt dieser Arbeit auf der Ausarbeitung der betriebswirtschaftlichen Aspekte. Allerdings liefern die Veränderungen in den Ansätzen der Entwicklungsökonomie den Anlass zu dieser Untersuchung.

Die Entwicklungsökonomie unterliegt, wie jede Wissenschaftsdisziplin, Modeerscheinungen, die neue Theorien zu Beginn euphorisch bejubelt und anschließend zu Grabe trägt. Anlass für die vorliegende Arbeit sind die veränderten Theorien der Entwicklungsökonomie bei der Finanzierung von Infrastruktur in den Entwicklungsländern. Beginnend mit dem WashingtonConsensus in den 1980er Jahren und fortgesetzt in seiner überarbeiteten Version, dem Post-Washington-Consensus in den 1990er Jahren, wurden Privatisierungs- und Deregulierungsreformen in den Entwicklungsländern gefordert. Durch diese Reformen wurde der Infrastruktursektor in den Entwicklungsländern für private inländische und ausländische Unternehmen geöffnet.

Mit dem Übergang in das neue Millennium einigten sich fast alle Länder weltweit auf gemeinsame Ziele, nämlich die Millennium Development Goals (MDGs) in der Entwicklungspolitik. Die MDGs umfassen acht Hauptziele, wobei das erste Ziel eine drastische Reduktion der Armut ist. Wesentliche Aufgabe in der Entwicklungspolitik ist es daher, Maßnahmen zu setzen, welche die Armutsreduktion unterstützen. Ein wesentlicher Schwerpunkt liegt dabei auf der Entwicklung des Infrastruktursektors, da diesem ein so genannter ,enabling factor" zugesprochen wird. Infrastruktur ist somit eine wichtige Komponente bei der Erreichung der MDGs.

Eine wesentliche Herausforderung in diesem Zusammenhang stellt die Finanzierung der benötigten Infrastruktur dar, die grundsätzlich aus drei Quellen kommen kann: Dem Staat, der Entwicklungshilfe oder dem privaten Sektor. Zu- 
sätzlich dazu gibt es die Mischform der öffentlich-privaten Modelle, die sowohl in der Entwicklungsökonomie, als auch in der Betriebswirtschaftslehre durch den Begriff „Public Private Partnerships" Ansehen erlangt haben. Die vorliegende Arbeit fokussiert auf die öffentlich-privaten Modelle und diskutiert deren Risiken und Finanzierungsmöglichkeiten durch die Internationalen und nationalen Finanzinstitutionen (IFIs). Durch die Veränderungen bei der Finanzierung von Infrastruktur in der Entwicklungspolitik wird untersucht, ob auch die IFIs ihr Produktangebot bei der Unterstützung von privaten Investitionen geändert haben.

Für die Armutsreduktion und die Erreichung der MDGs spielt Infrastruktur eine Schlüsselrolle, deren Finanzierung vermehrt private Unternehmen übernehmen sollen. Das primäre Ziel eines Unternehmens ist jedoch die Gewinnmaximierung und nicht die Armutsreduktion. Für die Verfasserin dieser Arbeit sind genau diese beiden divergierenden Ziele Anlass für die vorliegende Untersuchung. Die Veränderungen in beiden Disziplinen und die sich daraus ergebenden Fragestellungen werden in Abbildung 1 grafisch dargestellt.

\section{Abbildung 1: Positionierung des Themas}

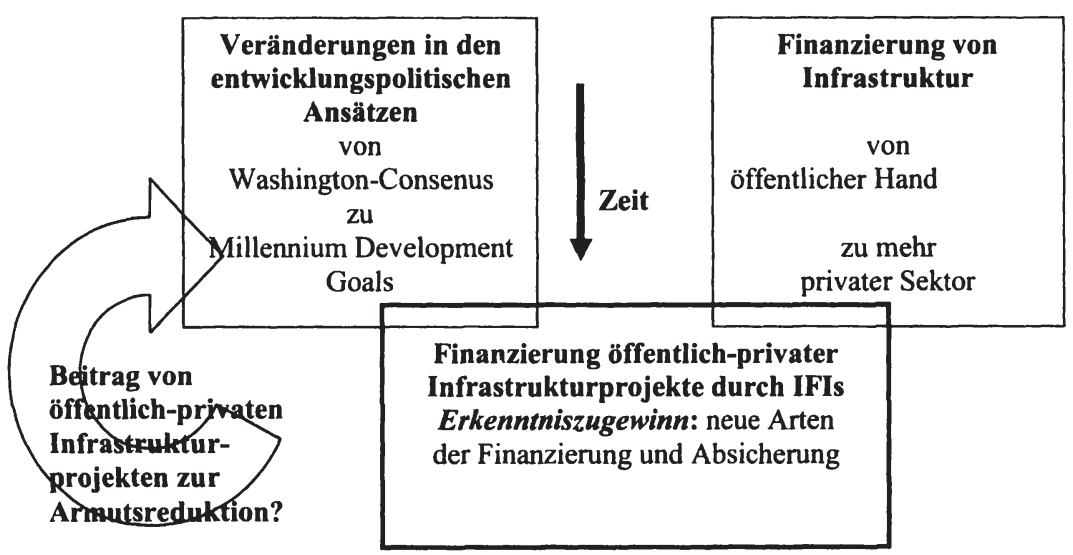

\subsection{Zielsetzung}

Im Rahmen der vorliegenden Dissertation werden, ausgehend von einer theoretischen Darstellung der Rahmenbedingungen, folgende beiden Forschungsfragen $\mathrm{zu}$ beantworten sein: 
1. Welche neuen Formen der Finanzierung und Absicherung sind im Bereich der Förderung von privaten Infrastrukturinvestitionen in den Internationalen Finanzinstitutionen (IFIs) entstanden?

2. Welchen Beitrag leisten öffentlich-privat finanzierte Infrastrukturinvestitionen zur Armutsreduktion?

Abbildung 2 stellt den Zusammenhang der beiden Forschungsfragen nochmals grafisch dar. Die erste Frage beschäftigt sich damit, welche Auswirkungen die Reformen des New Public Management auf das Angebot der Internationalen Finanzinstitutionen (IFIs) haben. Die zweite Forschungsfrage behandelt den positiven Zusammenhang von privater Infrastrukturfinanzierung und Armutsreduktion.

\section{Abbildung 2: Forschungsfragen}

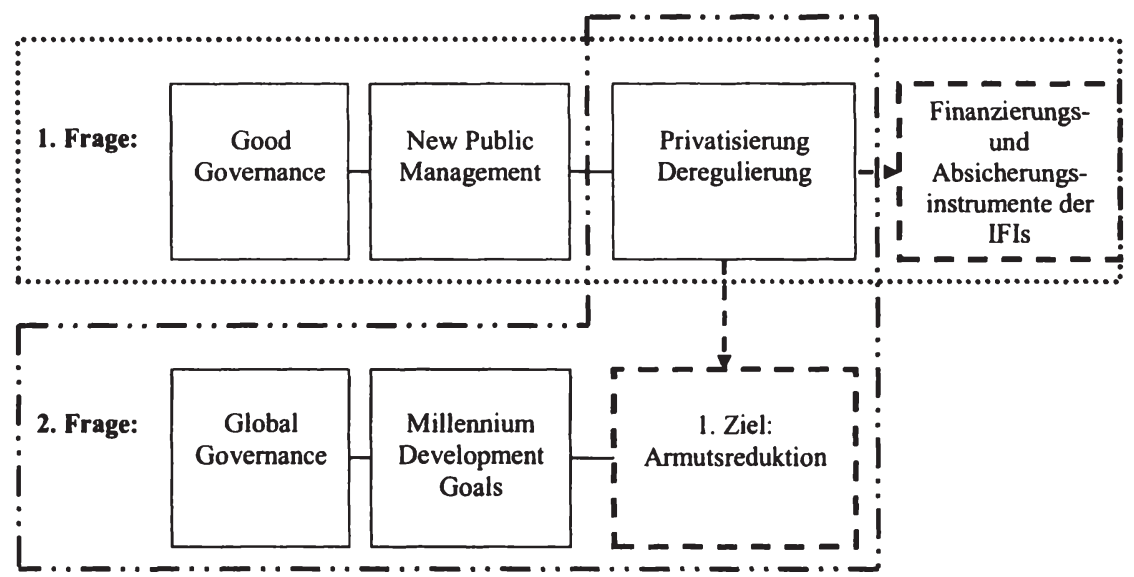

\subsection{Methodik}

„Qualitative data are sexy“ (vgl. Miles/Huberman 1994: 1).

Im folgenden Kapitel wird nach kurzen methodologischen Überlegungen jene empirische Methode vorgestellt, die für die Beantwortung der Forschungsfragen gewählt wurde. Grundsätzlich basiert die Arbeit auf den Annahmen der qualitativen Sozialforschung, die nun abschnittsartig dargestellt werden. 
Werden die quantitativen Untersuchungsmethoden dem kritischen Rationalismus zugeordnet, so können qualitative Designs typischerweise dem Empirismus zugerechnet werden. Laut Francis Bacon, der als der Wegbereiter des Empirismus gilt, kommt man zum wirklichen Abbild der Natur nur über ein Zusammengehen von empirischer Erfahrung und ordnender Tätigkeit des Geistes (vgl. Schülein/Reitze 2002: 64). Für die qualitative Sozialforschung werden als methodologische Grundposition häufig der symbolische Interaktionismus, die Ethnomethodologie und strukturalistische Ansätze genannt (vgl. Wrona 2005: 5). Zentrales Prinzip dieser theoretischen Positionen ist die Interpretation bzw. das Verstehen des untersuchten Gegenstandes.

Den qualitativen Methoden wird typischerweise die Induktion zugeordnet (vgl. Wrona 2005: 4), wobei diese Zuordnung mittlerweile in der empirischen Sozialforschung als sehr naiv angesehen wird (vgl. Strauss 1994: 39). Dabei wird auf Basis einer definierten Anzahl einzelner Fälle auf die Allgemeinheit geschlossen. Diese Methode ist laut John Locke auch in der Wissenschaft legitim, da man aufgrund von beschränkten Mitteln darauf angewiesen ist (vgl. Schülein/Reitze 2002: 73). In der Wissenschaftstheorie wird diese Vorgehensweise dahingehend kritisiert, dass keine Induktion jemals eine Allgemeingültigkeit beweisen könne. Diese Kritik kann jedoch auch bei der quantitativen Sozialforschung angewendet werden, da es nur in den seltensten Fällen zu Vollerhebungen kommt. Resümierend kann aus diesem hier nur kurz angerissenen Paradigmenstreit gesagt werden, dass die Probleme der Induktion und der Deduktion jeweils vom Ausmaß ihrer Verwendung abhängig sind und sich nicht einem qualitativen oder quantitativen Vorgehen zuordnen lassen.

Ein weiteres Merkmal der qualitativen Methodologie ist die Kontextabhängigkeit sozialen Handelns. Dabei wird auf der Grundlage ,ideografischer“ (d.h. das Einmalige beschreibende) Methoden versucht, den Untersuchungsgegenstand in seiner gesamten Vielfalt zu erfassen und dabei insbesondere auch die Beziehungen von Merkmalen des untersuchten Gegenstandes und zu seinem Kontext zu berücksichtigen (vgl. Wrona 2004: 4). Kausaler Verallgemeinerung wird dabei kaum eine Bedeutung beigemessen.

Den qualitativen Methoden liegt im Vergleich zu den quantitativen Methoden, deren Ziel die Konstruktion von Theorien aus dem empirischen Material ist, das „interpretative Paradigma“ zugrunde (vgl. De Geer et al. 2004: 325, Novy 2002, Mayring 1995: 16ff). Diese These basiert auf der Annahme, dass nur der Forscher die Realität über seine Sinne interpretieren kann, da die Realität nicht „direkt" erfahren werden kann (vgl. Novy 2002). Die wesentlichen Stärken der qualitativen Sozialforschung liegen dabei in der Generierung neuer Konzepte, Hypothesen oder gegenstandsbezogener Theorien, indem sie das Aufspüren bisher wenig beachteter Eigenschaften oder Einflussgrößen fördern. Durch den Ansatz der Offenheit können in der qualitativen Sozialforschung auch komplexere Phänomene empirisch erfasst werden, da komplexe soziale Phänomene häufig aus nominalskalierten und mehrdimensionalen Variablen bestehen (vgl. 
Wrona 2004: 10, Baker 2000: 8). In der interpretativen Sozialforschung tritt an Stelle des Entweder-Oder das Sowohl-als-Auch und ist deshalb das ideale Forschungsprogramm interdisziplinärer Entwicklungsforschung (vgl. Novy 2002).

In den Studien, die sich mit den Auswirkungen von Infrastrukturinvestitionen beschäftigen, werden oft qualitative Ansätze verwendet, um die Zusammenhänge zwischen Investitionen und Armutsreduktion besser zu verstehen (vgl. Baker 2000: 7). Dadurch können insbesondere auch neue Zusammenhänge entdeckt werden, die indirekte Auswirkungen haben.

Als Grundlage für die empirische Erhebung wurde in dieser Dissertation ein Multimethodenansatz gewählt, da es für die Beantwortung der Forschungsfragen nicht ausreichen würde, nur eine isolierte Methode zu verwenden. In der Wissenschaft wird die Kombination von qualitativen und quantitativen Ansätzen und Methoden zunehmend akzeptiert. Im Bereich der Messung von direkten Auswirkungen von Maßnahmen auf die Armutsreduktion werden ökonometrische Modelle verwendet, um zu allgemeinen Aussagen zu gelangen. Qualitative Modelle hingegen erlauben eine genauere und kritischere Analyse von einzelnen Fällen, die wiederum die Ergebnisse des ökonometrischen Modells hinterfragen (vgl. Baker 2000: 8).

Im Rahmen dieser Überlegungen wurde ein Fallstudienansatz gewählt, der sich durch eine Kombination von verschiedenen Methoden auszeichnet und es dadurch im Forschungsprojekt ermöglicht, unterschiedliche Aspekte einer Fragestellung $\mathrm{zu}$ beantworten. Es sei allerdings darauf hingewiesen, dass die Einzelfallanalyse nicht automatisch der qualitativen Sozialforschung zuordenbar ist, da auch quantitative Ansätze darin angewendet bzw. verfolgt werden können (vgl. Ghauri 2004: 112). Ein wesentlicher Vorteil der Fallstudienanalyse besteht vor allem auch darin, dass durch den Multimethodenansatz möglichst viele Quellen zur Beantwortung der Forschungsfrage herangezogen werden können (vgl. Baker 2000: 32).

In der englischsprachigen Literatur wird die Verwendung von mehreren Methoden als „Triangulation" diskutiert, welche eine wesentliche Eigenschaft von Fallstudien darstellt (vgl. Ghauri 2004: 115, Hurmerinta-Peltomäki/Nummela 2004: 164, Pauwels/Mattheyssens 2004: 129). Jede Methode weist sowohl Stärken, als auch Schwächen auf. Um diese zu re-duzieren, heben Carvalho/White (1997) die Wichtigkeit der Kombination von qualitativen und quantitativen Ansätzen für die Erhebung von Auswirkungen auf die Armutsreduktion hervor.

Demnach wird unter der Einzelfallanalyse prinzipiell das gesamte Spektrum der sozialwissenschaftlichen Erhebungsmethoden subsumiert (vgl. Lamnek 2005: 298). ' Das Ziel einer Einzelfallanalyse lässt sich demnach als die Dar-

1 Eine weiterführende Diskussion über die Relevanz der Einzelfallanalyse in den Wirtschafts-wissenschaften bieten Piore (2006), Aboyni (2005), Scapens (2004), Chauri 
stellung von interessanten Fällen in möglichst vielen Dimensionen definieren (vgl. Ghauri 2004: 109f, Hurmerinta-Peltomäki/Nummela 2004: 164f, Scapens 2004).

Die folgende Abbildung 3 bietet einen Überblick über die einzelnen Methoden, die in dieser Arbeit verwendet wurden und in Folge kurz dargestellt werden, sollen.

\section{Abbildung 3: Methodische Vorgehensweise}

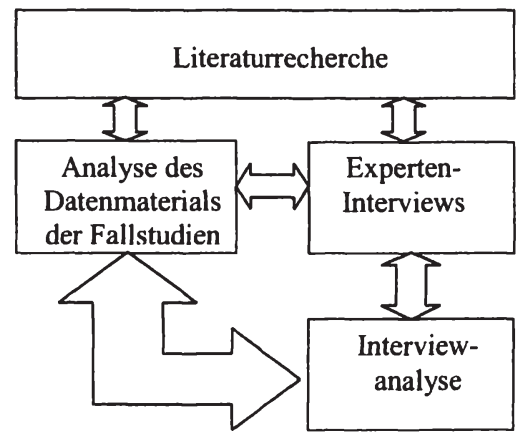

Wie aus Abbildung 3 ersichtlich ist, wurde im Dissertationsprojekt ein zirkuläres Vorgehen verfolgt, wie es in der qualitativen Sozialforschung meist der Fall ist (vgl. Flick 2002: 73). Da Interpretationen immer nur zu vorläufigen und unsicheren Ergebnissen führen, muss man in der Forschung zirkulär vorgehen (vgl. Novy 2002). Dies bedeutet, dass es keine streng lineare Abfolge der einzelnen Schritte gibt, da es im Sinne des Erkennens von neuen Zusammenhängen in der Qualitativen Sozialforschung notwendig ist, immer wieder zur Literatur zurückzukehren.

In der vorliegenden Arbeit wurden die folgenden drei Methoden zur Wissensgenerierung herangezogen:

1. Literaturrecherche,

2. Analyse des Datenmaterials und

3. Experteninterviews.

Die Literaturrecherche stellt den Ausgangspunkt dar und bildet somit die Grundlage für weitere Schritte. Für die spätere empirische Erhebung ist eine Ausarbeitung der zugrunde liegenden theoretischen Konzepte notwendig, da sie

(2004), Pauwels/Mattheyssens (2004), Hurmerinta-Peltomäki/Nummela (2004), De Geer et al. (2004), Wrona (2004), Helper (2000), Yin (1994). 
für die Analyse der Daten und die Entwicklung der Forschungsfrage eine Voraussetzung darstellt. Das damit erlangte Kontextwissen stellt einen wesentlichen Datenfundus dar, der die Sensitivität bei der Theoriebildung erhöht und auch eine Fülle von Möglichkeiten liefert, um Vergleiche anzustellen (vgl. Strauss 1994: 37).

Im Bereich von Infrastruktur in der Entwicklungszusammenarbeit lassen sich im Wesentlichen folgende Quellen zur Literaturrecherche heranziehen:

1. Wissenschaftliche Journale und Publikationen,

2. Dokumente der Internationalen Finanzinstitutionen (IFIs) und

3. das Internet.

Basierend auf den Ergebnissen der Literaturrecherche wurden relevante Fallstudien identifiziert. Die Identifikation der Fallstudien hat sich relativ aufwendig gestaltet, da die Projekte sowohl aktuell sein sollten als auch dem eingeschränkten Fokus der Arbeit entsprechen mussten. Anschließend daran wurden für die ausgewählten Fälle Daten im Internet recherchiert, die im Sinne der Forschungsfragen analysiert wurden.

Aufgrund der Analyse des Datenmaterials konnten dem Stakeholder-Ansatz folgend, relevante Interviewpartner identifiziert werden. Laut diesem Ansatz werden Stakeholder als jene Menschen bezeichnet, die entweder von der Organisation beeinflusst werden oder auch diese selbst beeinflussen können. In dieser Arbeit wurden daher einerseits Personen identifiziert, die in einem konkreten Nahverhältnis zu den Fällen stehen, wie beispielsweise Sponsoren, Lieferanten, Regierung oder Finanzinstitutionen. Andererseits wurden unabhängig von den Fällen auch Experten im Bereich der Entwicklungspolitik interviewt, die entweder in den Bereichen der Infrastruktur oder der Finanzierung von privaten Investitionen tätig sind. Aufgrund von beschränkten Zeitund Finanzmitteln wurde auf eine Befragung der Endabnehmer der Leistung oder der Benutzer der Infrastruktureinrichtungen verzichtet, da sich die Erhebung dieser im Bereich der Infrastruktur als besonders schwierig darstellt.

Beispielsweise können im Bereich der Stromerzeugung die endgültigen Abnehmer der Leistungen nicht mehr identifiziert werden, da die Leistungen in das bestehende Stromnetz gespeist werden. Einfacher würde sich die Identifikation bei so genannten „off-grid“ Projekten gestalten, die eben nicht in das bestehende Netz liefern, sondern direkt an die Endverbraucher. Diese „off-grid“Projekte werden oft in unterentwickelten, ländlichen Regionen durchgeführt, die nur gering besiedelt sind und nicht durch das öffentliche Stromnetz versorgt werden. Solche Projekte müssen wiederum von öffentlicher Hand getragen werden, da sie nicht gewinnbringend und daher für private Investoren uninteressant sind. Im Falle von Mautstraßen könnten zwar die direkten Stakeholder, d.h. die Straßenbenützer, identifiziert werden, aber nicht jene, die aufgrund einer Mauterhöhung die Straße nicht mehr benützen können. 
Die dritte Methode stellen die Experteninterviews oder fokussierten Interviews dar. Solche Interviews können entweder schriftlich oder mündlich (vgl. Mayring 2006:67, Atteslander 1995: 159) und anhand eines Leitfadens teilstrukturiert durchgeführt werden. In der qualitativen Sozialforschung stellen offene Interviews die Regel und geschlossene Interviews die Ausnahme dar (vgl. Carvalho/White 1997: 7). Das Ziel von offenen Konzepten wie dem Experteninterview ist es, Zusammenhänge zu erklären (vgl. Atteslander 1995: 171). Die Interviews haben im vorliegenden Dissertationsprojekt dazu gedient, die Interpretationsleistung bei der Auswertung zu erhöhen. Laut Lamnek (2005: 97) ist es in der qualitativen Sozialforschung möglich, verbesserte Erklärungsmuster für bestimmte Phänomene zu erhalten.

Der Erhebungs- und Analyseprozess kann laut Miles/Huberman (1994: 10) in vier Bestandteile untergliedert werden:

1. Datenerhebung: In diesem Dissertationsprojekt wurden teilstrukturierte Interviews mit Stakeholdern der Fallstudien und Experten in der Entwicklungszusammenarbeit durchgeführt.

2. Datenreduktion: Eine vollständige Transkription der Interviews bildet die Voraussetzung für die Auswertung. Die transkribierten Daten wurden anschließend mit dem Softwareprogramms NVivo7 analysiert. Dieses Programm unterstützt den Analyseprozess, indem zentrale Passagen hervorgehoben und kodiert werden, um Kategorien zu bilden, die zu allgemeineren Erkenntnissen verhelfen (vgl. Strauss 1994: 32). Durch diesen Prozess werden die Daten gefiltert und reduziert.

3. Datendarstellung: Die Ergebnisse des Analyseprozesses wurden grafisch dargestellt, um einen besseren Überblick über die Analyseergebnisse zu erhalten.

4. Ziehung von Schlussfolgerungen: Im letzten Schritt wurden auf Basis der ausgearbeiteten Daten Schlüsse gezogen, die in der Literatur wiederum auf ihre Glaubwürdigkeit und Gültigkeit überprüft wurden.

Abschließend wird in Abbildung 4 das zirkuläre Vorgehen bei der Datenanalyse nochmals grafisch dargestellt. Um zu validen Aussagen zu kommen, müssen die Ergebnisse auf ihre Gültigkeit überprüft werden.

\subsection{Aafbau der Arbeit}

Die vorliegende Dissertation umfasst sieben Kapitel, wobei das erste Kapitel der Einleitung in die Arbeit dient. In diesem Kapitel wird das Thema positioniert und es werden die zugrunde liegenden Forschungsfragen dargestellt. Anschließend daran wird das methodische Vorgehen in der Arbeit erörtert. 


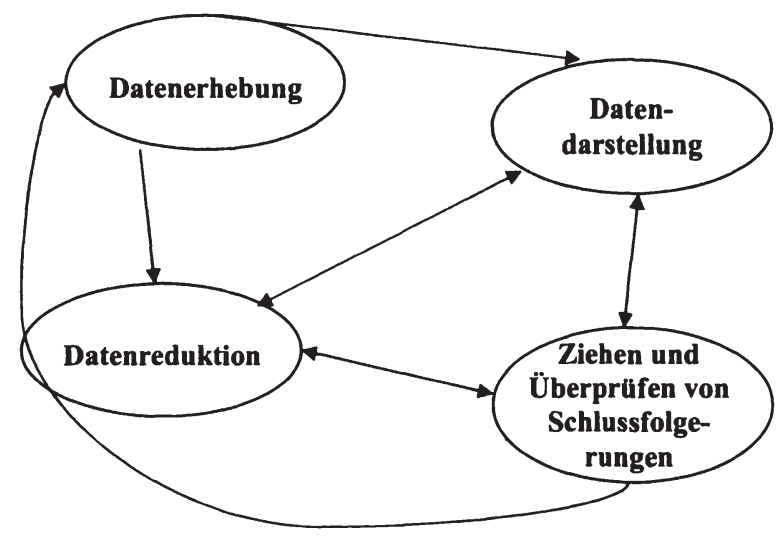

Quelle: Miles/Huberman 1994: 12

Das zweite Kapitel widmet sich den Aspekten der Governance in der Entwicklungspolitik. Nach einer Einführung in das Konzept Governance, werden die beiden Konzepte „Good Governance“ und „Global Governance“ in der Entwicklungspolitik vorgestellt. Beide Konzepte werden in eine historischtheoretische Darstellung eingebettet und anschließend daran einer Kritik unterzogen. Zu Good Governance werden die Ansätze des New Public Management gezählt, von denen die Themen Privatisierung und Dezentralisierung genauer vorgestellt werden.

Im dritten Kapitel wird das Thema der privaten Investitionen in der Infrastruktur in Entwicklungsländern bearbeitet. In einem Einführungskapitel werden zunächst die wichtigsten Begriffe definiert. Im nächsten Abschnitt folgt eine Darstellung der Auswirkungen von ausländischen privaten Investitionen auf die Entwicklungsländer, wobei diese unterteilt werden in den Beitrag zum Wachstum und die möglichen Spillover-Effekte. Der dritte Abschnitt untersucht die direkten und indirekten Auswirkungen von Infrastruktur auf die Armutsreduktion.

Die Darstellung von öffentlich-privaten Infrastrukturmodellen findet im vierten Kapitel statt. Dieses Kapitel beginnt mit einer grundsätzlichen Dis-kussion des Begriffs Partnerschaft. Diese Ausarbeitung dient anschließend zur Abgrenzung des Begriffes „Public Private Partnerships“. Darauf folgen die Ziele und Risiken in diesen Modellen und eine Darstellung des Themas in der Entwicklungspolitik. Im darauf folgenden Abschnitt wird die Entwicklung von privaten Investitionen in der Infrastruktur in den Entwicklungsländern dargestellt. Der dritte Abschnitt beschäftigt sich mit den Länderrisiken von privaten Investitionen in Entwicklungsländern. Aufbauend auf dieser Darstellung werden 
in den darauf folgenden Abschnitten die einzelnen Modelle vorgestellt und die damit verbundenen Risiken diskutiert.

Im fünften Kapitel werden die einzelnen Finanzierungs- und Absicherungsinstrumente der internationalen und nationalen Finanzinstitutionen (IFIs) dargestellt, durch die private Investitionen unterstützt werden können. Der erste Abschnitt stellt prägnant die Relevanz der Projektfinanzierung und die Mindestprojektanforderungen für private Unternehmen dar. Außerdem wird auch Bezug auf die Kofinanzierungsrolle der IFIs genommen. Im zweiten Abschnitt werden auf multilateraler, europäischer und nationaler Ebene die jeweils relevanten Institutionen vorgestellt. Dieser Abschnitt schließt mit einer Zusammenfassung über die verfügbaren Instrumente für den privaten Sektor. Der dritte Abschnitt stellt ausgewählte innovative Instrumente detaillierter dar und unterzieht sie einer krit:schen Würdigung.

Die Fallstudien werden im sechsten Kapitel beschrieben. Die vier Fallstudien werden in den Dimensionen Finanzierung und Absicherung, Vertragsstruktur und deren Beitrag zur Armutsreduktion in Vietnam und auf den Philippinen dargestellt.

Im letzten siebten Kapitel werden die Erkenntnisse der Dissertation nochmals zusammengefasst und es wird ein Ausblick auf weitere Forschungsziele gegeben. 
Die folgende Abbildung 5 gibt den Aufbau der Dissertation nochmals wieder.

Abbildung 5: Aufbau der Arbeit

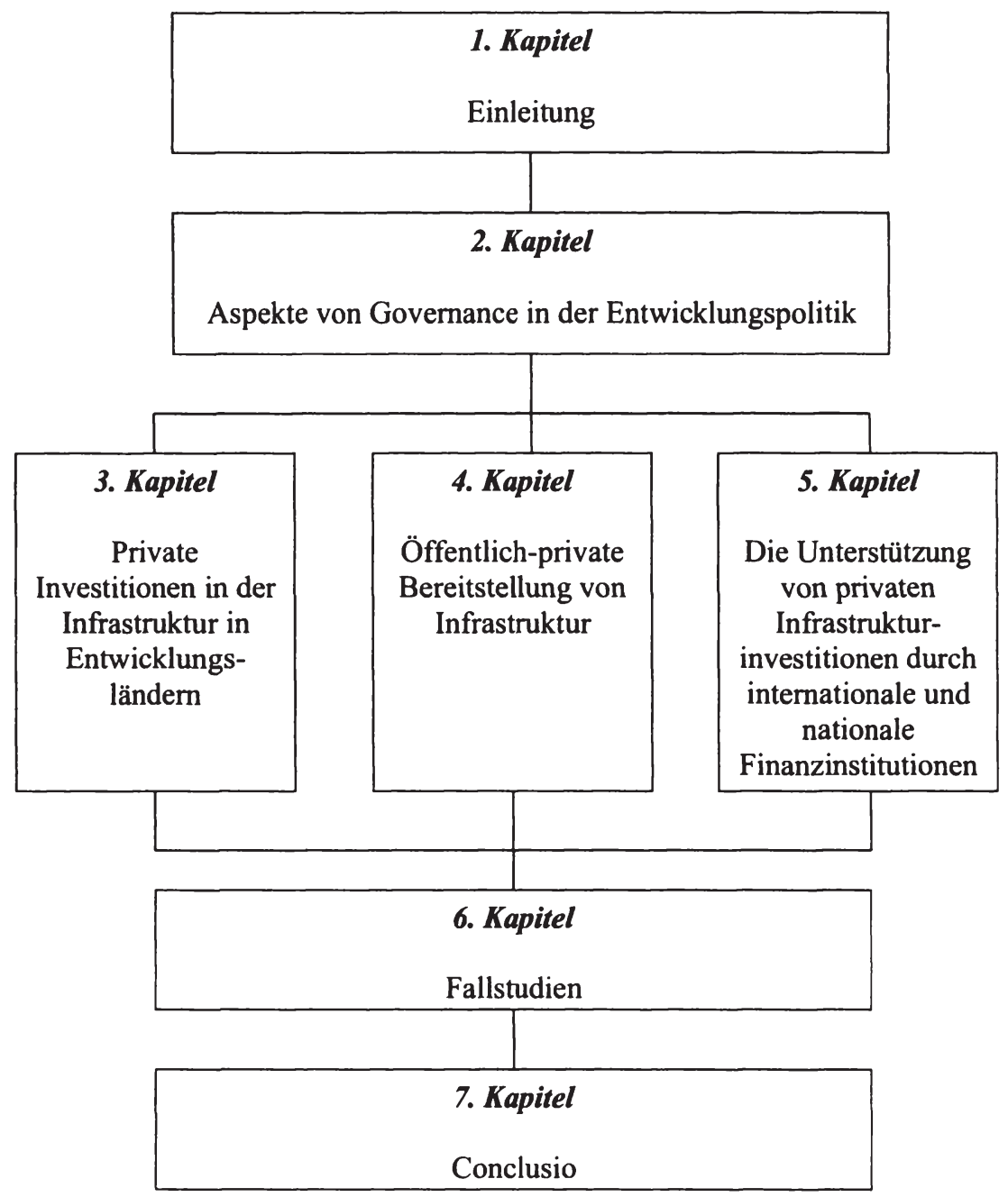


Bianca Gusenbauer - 978-3-631-75445-0

Downloaded from PubFactory at 01/11/2019 04:47:32AM

via free access 


\section{Aspekte von Governance in der Entwicklungspolitik}

Das zweite Kapitel beschäftigt sich mit den Aspekten von Governance in der Entwicklungspolitik. Nach einer Einführung in die Governance-Thematik, werden Good Governance und Global Governance als zwei mögliche Konzepte im Lichte der entwicklungstheoretischen Ansätze dargestellt. Der letzte Abschnitt schließt mit einer Zusammenfassung dieser Thematik das Kapitel ab.

Die folgende Abbildung 6 stellt grafisch nochmals den Aufbau dieses Kapitels vor und dient der besseren Orientierung.

\section{Abbildung 6: Kapitelübersicht Aspekte von Governance in der Entwicklungspolitik}

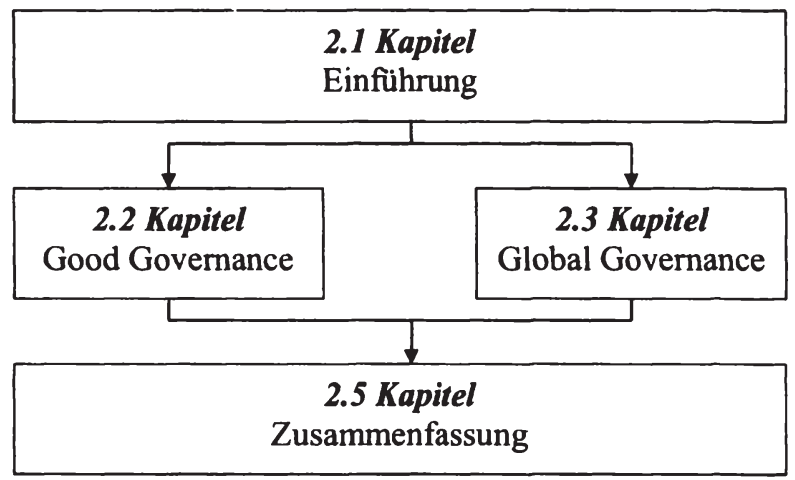

\subsection{Einführung}

Der Begriff „Governance“ stammt ursprünglich aus dem Französischen und wurde bereits im 14. Jahrhundert verwendet (vgl. Pierre/Peters 2000: 1). Das Konzept von Governance erfreut sich seit Ende der 1980er Jahre in verschiedenen Wissenschaftsdisziplinen großer Beliebtheit. Diese Zeit war vor allem geprägt von hoher öffentlicher Verschuldung und sinkendem Staatsbudget. Pierre und Peters (2000: 54) erkennen im Wesentlichen drei Gründe dafür, warum Governance in dieser Zeit zu besonders großer Popularität gelangte:

1. Mit der Einbindung von privaten Akteuren in die Herstellung von öffentlichen Dienstleistungen können trotz Budgetproblemen diese Leistungen weiter angeboten werden. 
2. Die Involvierung von privaten Unternehmen und die Etablierung von Public Private Partnerships signalisiert von öffentlicher Seite die Partizipationsmöglichkeit für den privaten Sektor bei der Mitgestaltung des Staates.

3. Während der wirtschaftlichen Rezession wurde die öffentliche Produktion von Dienstleistungen hinsichtlich ihrer Ineffizienz kritisiert. Daher bedeutete die Übernahme der Managementphilosophie des privaten Sektors eine attraktive Möglichkeit zur Steigerung der Effizienz.

Das Governance-Konzept kann in vielen Fragestellungen eine wesentliche Rolle spielen. Im Zusammenhang mit der vorliegenden Arbeit sind vor allem zwei Governance-Konzepte wesentlich: Good Governance, das ein normatives Konzept der Weltbank darstellt, und Global Governance, das die veränderten internationalen Machtverhältnisse erklärt.

\subsubsection{Definition}

"The concept of governance is notoriously slippery; it is frequently used among both social scientists and practitioners without a definition which all agree on. To be sure, even within each of these groups there are many different definitions and connotations of government" (Pierre/Peters 2000: 7).

In diesem Kapitel wird daher ein Überblick über die in der Literatur am häufigsten verwendeten Definitionen geben. Zusätzlich dazu wird auch die Definition der Weltbank dargestellt, die wesentlich zur Verbreitung des Begriffes in der Entwicklungspolitik beigetragen hat.

Auch wenn der Begriff, wie Pierre und Peters (2000: 7) treffend darstellen, "notoriously slippery" ist und es keine Lehrbuchdefinition für den Begriff Governance gibt, ist das kein Anlass den Begriff abzulehnen (vgl. Benz 2004a: 12). Der Grund für die unterschiedlichen Definitionen liegt in der Vielschichtigkeit des Begriffes, die nur indirekt über einzelne Beobachtungen zu erkennen ist. Daher zählt der Begriff Governance laut Benz (2004a: 15) auch zu den komplexesten Begriffen der Sozialwissenschaften.

", 'Governance' is now fashionable, but the concept is as old as human history" (Weiss 2000). Auch wenn Governance erst in den vergangenen Jahrzehnten wirklich beliebt wurde, entstand, wie Weiss richtig feststellt, der Begriff Governance bereits in den Dreißiger Jahren des vergangenen Jahrhunderts. Der Begriff Governance stammt ursprünglich aus der Ökonomie, wo er von Ronald Coase (1937) erstmals verwendet und anschließend auch von Oliver Williamson (1985) im Rahmen von Unternehmensführung stark geprägt wurde. Willamson (1985: 68ff) konzentriert sich in seinen Ausführungen auf die Governance der verschiedenen vertraglichen Beziehungen von wirtschaftlichen Einrichtungen, die der Verringerung der Transaktionskosten dienen. In der Wirtschaftswissenschaft wird inzwischen von Corporate Governace gesprochen, wenn die 
Organisations- und Leitungsstrukturen oder die Beziehungen zwischen Eigentümern und Management eines Unternehmens gemeint sind (vgl. Benz 2004a: 15).

In der Politikwissenschaft wurde der Begriff zuerst im Teilgebiet der ,Internationalen Beziehungen" verwendet, wo er für die Beschreibung der Interaktionen und Kooperationen zwischen Staaten und anderen Beteiligten herangezogen wird. Laut Benz (2004a: 15) lassen sich internationale Beziehungen durch fehlende hierarchische Über- und Unterordnungsverhältnisse, die Steuerung und Kontrolle durch einseitige Machtausübung, Kommunikation und Verhandlungen sowie die Dominanz von Prozessen über Strukturen und die kontinuierlichen Veränderungen von Strukturen beschreiben. In der Politikforschung wurde auch erkannt, dass gesellschaftliche, komplexe Aufgaben nicht mehr vom souveränen Staat alleine, sondern nur im Zusammenwirken mit anderen Akteuren, seien es solche aus dem öffentlichen oder dem privaten Sektor, erfüllt werden können (vgl. Benz 2004a: 17). Governance kann daher als Begriff beschrieben werden, mit dem die Entscheidungsfindung aufgrund unterschiedlicher Akteure in Institutionen dargestellt wird.

Pierre und Peters (2000: 1) beschreiben diesen Prozess sehr allgemein mit "Governance is the capacity of governments to make and implement policy, in other words, to steer society". Laut Mayntz (1998) sind die Steuerung und Kontrolle jedoch nicht einseitige Tätigkeiten einer zuständigen Institution, sondern Prozesse der Interaktion zwischen kollektiven Akteuren, wobei zwischen Steuerungsobjekt und Steuerungssubjekt nicht mehr unterschieden werden kann. Auch Rhodes (1997: 109) definiert den Begriff ähnlich wie Mayntz, betont aber zusätzlich die beteiligten Netzwerke ,' 'Governance' means there is no one centre but multiple centres; there is no sovereign authority because networks have considerable autonomy".

Die Weltbank (2001a: 48) fokussiert in ihrer Definition hingegen auf funktionierende Institutionen, die für die Versorgung der Bürger essenziel sind "Governance is the institutional capacity of public organizations to provide public and other goods demanded by a country's citizens or their representatives in an effective, transparent, impartial, and accountable manner, subject to resource constraints". Diese Definition erscheint typisch für eine internationale Organisation, die im Rahmen von Reformen einerseits Regierungen beim Steuern bzw. Regieren unterstützen und andererseits die Teilnahme der Bevölkerung am Entscheidungsprozess stärken will (vgl. Kjaer 2004: 11). Stocker (1998: 17) kritisiert jedoch, dass der Begriff Governance im $\mathrm{Zu}$ sammenhang mit der Weltbank und dem Internationalen Währungsfonds in der Vergangenheit auch als "the acceptable face of spending cuts" verwendet wurde. Basierend auf der stärkeren Teilnahme des privaten Sektors und durch die Betonung der daraus resultierenden positiven Effizienzgewinne, kürzen die Regierungen beispielsweise die Budgetausgaben für die Infrastruktur. 
Auch wenn der Begriff Governance nicht einheitlich definiert wird und je nach Besonderheiten des Anwendungsbereichs variiert, lässt sich doch ein konstanter Begriffskern festlegen. Laut Benz (2004a: 25) können folgende vier Bestandteile identifiziert werden:

- Governance bedeutet Steuern und Koordinieren mit dem Ziel des Managements von Interdependenzen zwischen Akteuren.

- Steuerung und Koordination beruhen auf institutionalisierten Regelsystemen, welche das Handeln der Akteure lenken sollen, wobei üblicherweise Kombinationen aus unterschiedlichen Regelsystemen vorliegen.

- Governance umfasst auch Interaktionsmuster und Modi kollektiven Handelns, wie beispielsweise Netzwerke, welche sich im Rahmen von Institutionen ergeben.

- Prozesse des Steuerns bzw. Koordinierens sowie Interaktionsmuster, die der Governance-Begriff erfassen will, überschreiten in aller Regel Organisationsgrenzen, insbesondere aber auch die Grenzen von Staat und Gesellschaft, die in der politischen Praxis fließend geworden sind. Politik in diesem Sinne findet normalerweise im Zusammenwirken staatlicher und nicht-staatlicher Akteure statt.

In der vorliegenden Arbeit wird die allgemeine Definition von Pierre und Peters (2000: 1) durch Bestandteile von Benz (2004a: 25) erweitert. Die verwendete Definition von Governance in dieser Arbeit lautetet daher: Unter Governance versteht man die Fähigkeit einer Regierung, die Interdependenzen unterschiedlicher Akteure zu managen, indem ein institutioneller Rahmen geschaffen wird, der einerseits alle Akteure berïcksichtigt und andererseits auch zur Erreichung der Entwicklungsziele eines Landes beiträgt.

\subsubsection{Entwicklungen des Governance-Konzeptes}

Die Verwendung des Governance-Konzepts bedeutet, dass man die komplexe politische Wirklichkeit in einer bestimmten Weise unter Konzentration auf ausgewählte und unter Vernachlässigung anderer Merkmale betrachten kann. Die Auswahl der Betrachtungsweise ist Bestandteil der Theorie, aber der Begriff Governance ist deswegen nicht mit einer bestimmten Theorie von Politik und Gesellschaft verbunden (vgl. Benz 2004a: 20). Die Governance-Konzepte finden sowohl Anwendung im öffentlichen Bereich als auch auf Unternehmensebene. In Abbildung 7 wird ein grafischer Überblick über die unterschiedliche Verwendung des Governance-Konzeptes gegeben. Eine wesentliche Unterscheidung ist die Trennung in normative und analytische Konzepte, wobei Good Governance auch für die analytischen Konzepte relevant ist. Für diese Arbeit werden zwei Konzepte herangezogen: Good Governance und Global Governance, die in 
der Abbildung blau markiert sind. In den folgenden Kapiteln werden daher nur diese beiden Konzepte weiter behandelt.

\section{Abbildung 7: Übersicht der Governance-Konzepte}

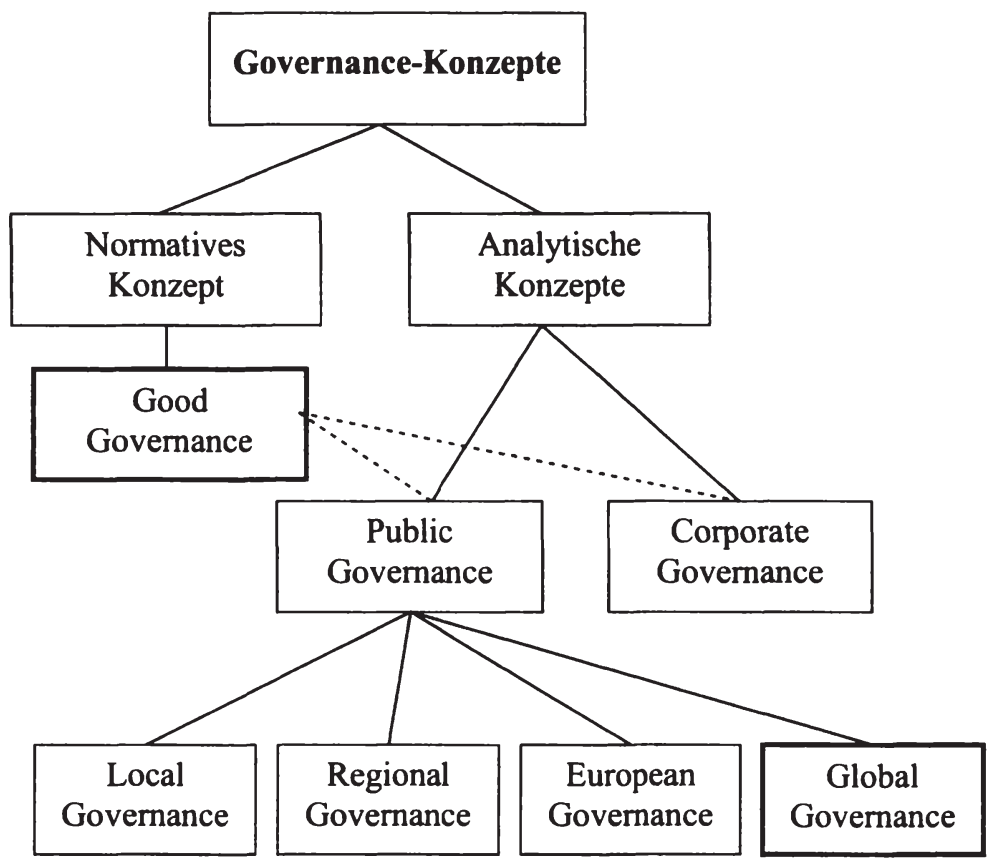

Das Governance-Konzept ersetzt nicht konventionelle Techniken für das Regieren eines Staates, sondern bietet neue, zusätzliche Strategien zur Steuerung eines Landes in einem veränderten Umfeld an. In der herkömmlichen Wahrnehmung sind Regierungen und Staaten sehr mächtig und zentralistisch organisiert. Pierre und Peters (2000: 83ff) erkennen durch die Reformen jedoch eine Verlagerung dieser konzentrierten Macht in folgende drei Richtungen:

1. Verlagerung nach oben $\mathrm{zu}$ den Internationalen Organisationen (siehe Kapitel 2.3),

2. Verlagerung nach unten $\mathrm{zu}$ den regionalen und dezentralen Regierungseinheiten (siehe Kapitel 2.2.4.2) und

3. Verlagerung nach außen zu privaten Unternehmen oder NGOs im Rahmen von Privatisierungen (siehe Kapitel 2.2.4.1). 
Eine wesentliche Veränderung im Zusammenhang mit den Reformen im Rahmen des New Public Managements (siehe Kapitel 2.2.4) ist auch die Entwicklung von Input- zu Output-orientiertem öffentlichem Management (siehe Kapitel 5.3.3). Die Legitimation von öffentlichen Einrichtungen misst sich nicht mehr am Prozess der Entscheidungsfindung, sondern am Output bzw. der Effizienz der politischen Entscheidungen (vgl. Peters/Pierre 2006). Diese starke Output-Orientierung führt zu kurzfristigen Effizienzen und politischer Stimmenmaximierung, die auf Kosten von langfristigen demokratischen Entscheidungen gehen (vgl. Leubolt 2007).

\subsection{Good Governance}

Good Governance ist eines jener beiden Governance-Konzepte, die für die vorliegende Arbeit eine zentrale Rolle spielen. Good Governance ist ein Teilbereich der Governance und stellt eines jener Konzepte dar, die vor allem in der Entwicklungspolitik durch die Weltbank starke Verbreitung gefunden haben. In den folgenden Kapiteln wird nach einer Einführung und der Definition des Begriffes in Kapitel 2.2.1 das Konzept in Kapitel 2.2.2 historisch-theoretisch eingeordnet. Das Konzept von Good Governance blieb in der Vergangenheit jedoch nicht unkritisiert, daher wird in Kapitel 2.2.3 ein Überblick über die Hauptkritikpunkte gegeben. Wesentliche Annahmen von Good Governance werden unter dem Begriff des New Public Management (NPM) zusammengefasst, wobei in der vorliegenden Arbeit vor allem die Privatisierung und Dezentralisierung eine Rolle spielen und in Kapitel 2.2.4 dargestellt werden.

\subsubsection{Einführung und Definition}

Die Weltbank wurde Anfang Ende der 1980er Jahre stark kritisiert, als sie die neoliberalen Ideologien der Geberländer auch auf die Entwicklungsländer übertrug und es aufgrund dieser Maßnahmen zu Krisen in den Entwicklungsländern kam. Daher musste die Weltbank eine Erklärung finden, warum die Entwicklungsländer sich nicht entwickelten, obwohl sie die neoliberalen Strukturanpassungsprogramme durchführten. Die Antwort war „bad governance“. Die Korruption und der Nepotismus in der öffentlichen Hand wurden verantwortlich gemacht für das Scheitern der Reformen (vgl. Kjaer 2004: 138). Daher wurde bei den Reformen, neben den neoliberalen Ansätzen vor allem auch auf eine Stärkung der Regierung und der Governance fokussiert. Dieser Ansatz wurde von der Weltbank als "Good Governance“ eingeführt, der nun auch für die Vergabe von Krediten an die Entwicklungsländer entscheidend ist. Da die Weltbank die größte Institution in der Entwicklungszusammenarbeit darstellt, hat sie auch einen dementsprechend großen Einfluss auf die Verbreitung von neuen entwicklungspolitischen Ansätzen. 
Geprägt von den Veränderungen im öffentlichen Sektor wurde Ende der 1980er Jahre der Begriff Governance auch mit normativen Werten versehen und Good mit den Wertvorstellungen der neoliberalen Politik gefüllt, wobei zwei Varianten identifiziert werden können.

1. Die erste Variante einer normativen Bewertung stellte der Begriff „Good Governance" der Weltbank dar, die damit Kriterien einer effizienten, rechtsstaatlichen und bürgernahen Staats- und Verwaltungspraxis festlegte und diese zur Voraussetzung der Kreditvergabe an Entwicklungsund Transformationsländer machte (vgl. Benz 2004a: 18).

2. Die zweite Variante wurde von den neoliberalen Regierungen festgelegt, die nicht-staatliche Formen der Erfüllung öffentlicher Aufgaben propagierten (vgl. Benz 2004a: 18). Neben der Privatisierung öffentlicher Leistungen und der Idee des „Minimalstaats“ wird auch das New Public Management unter diesem Begriff von Good Governance eingeordnet (vgl. Rhodes 1997: 47ff).

Die Entwicklung des Konzeptes „Good Governance“ wurde sowohl als neues Leitmotiv als und als Paradigmenwechsel beschrieben. Als neues Leitmotiv wurde es deswegen bezeichnet, weil die Vergabe von Krediten der Internationalen Finanzinstitutionen (IFIs) zunehmend an die Erfüllung der Good Governance-Kriterien geknüpft wurde bzw. die Entwicklungshilfe spezifisch auf die Stärkung entsprechender Good Governance-Kriterien ausgerichtet wurde (vgl. Fuster 1997: 1). Der Paradigmenwechsel durch das Good GovernceKonzept kann damit begründet werden, dass internationale Entwicklungshilfe in ihren Ursprungsjahren primär projektorientiert war und sich das Interesse neuerdings zunehmend auf die makroökonomische Ebene (vgl. Kjaer 2004: 174f) verlegt hat.

Good Governance stellt also ein normatives Konzept dar und wird von den meisten wichtigen internationalen Organisationen wie der Weltbank, der OECD, den Vereinten Nationen oder der EU bevorzugt (vgl. Leubolt 2007). Jedoch gibt es keinen klaren Konsens über den konkreten Inhalt von Good Governance (vgl. Fuster 1997: 2, Leftwich 1993) und die Institutionen haben leicht unterschiedliche Konzepte entwickelt, die aber sehr viel Ähnlichkeit aufweisen (vgl. Weiss 2000).

Selbst die Weltbank verwendet unterschiedliche Definitionen und die Verwendung des Begriffs unterscheidet sich je nach Publikation. Im World Development Report von 1992 (World Bank 1992: 3) wird Good Governance als ,the manner in which power is exercised in the management of a country's economic and social resources for development". Dieser Definition folgend stellt Good Governance eine Art des Ressourcenmanagements dar, die zu Entwicklung führt (vgl. Ziai 2006: 71). 
Trotz dieser unterschiedlichen Definitionen ist das Hauptanliegen der Weltbank die Bekämpfung der Korruption und die Stärkung von regelgebundenem Verhalten. Anhand dieser Ziele hat die Weltbank folgende vier Bestandteile für Good Governance definiert, welche die Grundlage für die Politikrichtlinien in den Ländern darstellen (vgl. Kjaer 2004: 173):

1. Die Entwicklung des öffentlichen Sektors beinhaltet die Verbesserung von Bürgerservice und Privatisierungsinitiativen.

2. Die Entwicklung der rechtlichen Rahmenbedingungen soll dafür sorgen, dass es mehr rechtliche Sicherheit, wie beispielsweise gültige, private Eigentumsrechte in einem Land gibt.

3. Die Übernahme von mehr Rechenschaftspflicht (Accountability) soll die Institutionen stärken und sie für getroffene Entscheidungen gegenüber den Bürgern oder dem Parlament verantwortlich machen.

4. Mehr Transparenz und Information sollen für freie Medien und Zugang zu mehr Informationen über makroökonomische Daten sorgen.

Als Arbeitsdefinition wird die Defintion von Ziai (2006: 71) herangezogen, nach der in der entwicklungspolitischen Diskussion Good Governance ein entwicklungspolitisches Konzept bezeichnet, das auf ,gute“ oder „verantwortungsvolle“ Regierungsführung abzielt (vgl. Ziai 2006: 71).

\subsubsection{Historisch-theoretische Darstellung von Good Governance im Lichte der entwicklungstheoretischen Ansätze}

Ziel dieses Kapitels ist es, das Good Governance-Konzept in die entwicklungstheoretischen Ansätze einzuordnen und zu zeigen, dass die Entstehung dieses Konzepts durch die politischen und wirtschaftlichen Rahmenbedingungen erklärt werden kann. Der Fokus dieser Darstellung liegt auf der Entwicklung des Good Governance-Konzepts.

\subsubsection{Von den Anfängen bis zum Washington-Consensus}

Auch wenn es der Sache nach Entwicklungshilfe bereits in der Antike gegeben hat (Altheim 1962 in Kesselring 2004: 203), ist der Begriff neueren Datums. Durch die Auflösung der kolonialen Abhängigkeiten entstand eine kleinere Gruppe von entwickelten Staaten und einer größere Gruppe weniger weit entwickelter Gesellschaften, kurz Nord und Süd. Die Entwicklungspolitik etablierte sich zu einer Nahtstelle zwischen diesen beiden entwicklungspolitischen Hemisphären, mit dem Ziel, den Lebensstandard in den schwächer entwickelten Regionen zu verbessern (vgl. Kesselring 2004: 204). 
Im Jahr 1944, wurden u.a. auch die „Bretton-Woods-Zwillinge“ (Weltbank und Internationaler Währungsfonds) gegründet und mit der Rede des damaligen amerikanischen Präsidenten Truman wurde die erste Dekade der Entwicklungspolitik eingeleitet (vgl. Schicho 2003: 9). Die Entwicklungspolitik jener Zeit verfolgte vor allem auch das politische Ziel, die Entwicklungsländer dem Kapitalismus gegenüber positiv $\mathrm{zu}$ stimmen und somit den Kommunismus zurückzudrängen (vgl. Nuscheler 2004: 122ff).

Die erste ,entwicklungspolitische Dekade“ dauerte von Beginn der 1950er bis Ende der 1970er Jahre und war gekennzeichnet von ,strukturalistischen Ansätzen". Das gemeinsame Merkmal dieser keynesianisch geprägten Modelle bildete die Annahme von strukturellen Rigiditäten in den Wirtschaftssystemen von Entwicklungsländern, wie beispielsweise imperfekte Kapitalmärkte oder das fehlende individuelle Bedürfnis nach Leistung (vgl. Wagner/Kaiser 1995: 32ff). $\mathrm{Zu}$ den strukturalistischen Erklärungsansätzen von Unterentwicklung gehören beispielsweise Dualismus-Modelle, Teufelskreis-Modelle, Bevölkerungstheorien, Dependenztheorien sowie Theorien asymmetrischer Handelsbeziehungen zwischen Industrie- und Entwicklungsländern (vgl. Fuster 1997: 25). In all diesen Theorien hatte der Staat eine stark interventionistische Rolle und griff aktiv in den Wirtschaftsprozess und die Güterproduktion ein.

Mit Beginn der 1980er Jahre begann die nächste Dekade, in der die strukturalistischen Ansätze immer mehr von den neoklassisch orientieren Ökonomen kritisiert wurde. Basierend auf den Ausführungen von Toye (1993) wurden vier wesentliche Kritikpunkte angeführt, die zu einer „Gegenrevolution“ innerhalb der Entwicklungstheorie führten (vgl. Fuster 1997: 28ff):

1. Theoretisch-methodisches Defizit: Die Ad-hoc-Maßnahmen über strukturelle Rigidität wurde von der strukturalistischen Schule nicht hinreichend begründet. Das Resultat war aus neoklassischer Sicht eine hohe „Unschärfe“ und letztendlich eine „Untestbarkeit“ strukturalistischer Ansätze.

2. Geringe empirische Erfolge: Die Erfolge der strukturalistischen Politik waren sehr bescheiden und ließen Zweifel an den stark interventinistischen Maßnahmen wachsen.

3. Überdimensionierter und ineffizienter öffentlicher Sektor: Nach Ansicht der neo-klassischen Ökonomen hat das strukturalistische Dirigismusdogma nicht nur zu einer Vermehrung verzerrender und wohlfahrtsmindender Staatseingriffe im Wirtschaftsprozess geführt, sondern vor allem auch zu einem überdimensionierten und ineffizienten öffentlichen Sektor.

4. Idealistisches Bild des allmächtigen und allwissenden Gemeinwohlmaximierers: Kritisiert wurde die Annahme, dass es auf privater Ebene individuelle Nutzenmaximierer gibt, die aber auf staatlicher Ebene zu Ge- 
meinohlmaximierern werden. Außerdem wurde die Annahme des allwissenden Staates abgelehnt.

Basierend auf diesen Kritikpunkten gewann die neoklassische Theorie als Antithese zu den sturkturalistischen Annahmen wieder an Einfluss. Verfolgt wurde nun der Aufbau einer möglichst unverzerrten Marktwirtschaft ohne staatliche Interventionen. Der Staat sollte sich vielmehr auf die Schaffung einer marktfreundlichen Wirtschaftsordnung auf lange Frist konzentrieren. Die neoklassische Gegenrevolution propagierte daher einen Primat der Ordnungspolitik; mit dem Ideal eines neoliberalen Minimalstaates, der sich darauf beschränkt, die politische Ordnung und die Entscheidungsfreiheit privater Wirtschaftssubjekte zu gewährleisten, die notwendigen Rahmenbedingungen für effiziente Güterund Kapitalmärkte zu etablieren und das Funktionieren des Wettbewerbssystems zu überwachen (vgl. Fuster 1997: 31).

Diese entwicklungspolitische Neuorientierung fand durch den „WashingtonConsensus" ihre Verwirklichung. Das Kernstück des Consensus bilden die Structural Adjustment Policies oder Strukturanpassungsprogramme (SAP) der Bretton-Woods-Institutionen, die auf der Annahme basieren, dass durch Veränderungen von Rahmenbedingungen wie Makro- und Strukturpolitik die Voraussetzungen für eine effiziente Allokation von Ressourcen geschaffen würden. Diese Maßnahmen der Strukturpolitik werden auch unter dem Begriff des New Public Management zusammengefasst und fokussieren auf die Privatisierung und Deregulierung des öffentlichen Sektors.

Ziel dieser Reformen war vor allem auch eine Reduktion der Korruption und des Nepotismus in den öffentlichen Einrichtungen, die in Kapitel 2.2.4 etwas genauer vorgestellt werden. Als die Annahmen des „Washington-Consensus“, die faktisch eine Übertragung der westlichen, liberalen Annahmen auf die Entwicklungsländer bedeuteten, großteils fehlschlugen, entwickelte sich der Consensus zu einem „Post-Washington-Consensus“. Enttäuschend fiel die Bilanz vor allem in Subsahara Afrika aus, wo kaum Fortschritte messbar waren (vgl. Fuster 1997: 33). Das Scheitern der liberalen Reformen war außerdem gekennzeichnet durch die Finanzkrisen zwischen 1997 und 2002 und die ansteigende Armut.

\subsubsection{Post-Washington-Consensus bis zu den Millennium Development Goals}

Das Scheitern des „Washington-Consensus“ leitete Anfang der 1990er Jahre die dritte entwicklungspolitische Dekade ein. Die neoinstitutionelle Ökonomie erklärte die Ergebnisse der Strukturanpassungsprogramme damit, dass in den einzelnen Entwicklungsländern unterschiedliche institutionelle Ausstattung vorhanden war. Es reicht nach den Annahmen der neoinstitutionellen Ökonomen daher für eine Wirtschaft nicht aus, die Ressourcen der ,,invisible hand“, d.h. dem Markt zu übergeben, sondern es bedarf funktionierender Institutionen (vgl. 
Durth et al. 1999: 214ff). Als ein wesentlicher Hauptvertreter dieser Theorie gilt Douglass C. North $(1988,1992)$, der an den neoklassischen Ansätzen zu bedenken gibt, dass die Annahmen eines effizienten Marktes nur dann zutreffen, wenn es dafür auch die institutionellen Gegebenheiten wie eine formale Ordnung bzw. Einrichtungen gibt. Er versteht Institutionen wiederum als Spielregeln, die das Verhalten der Akteure beschränken. ,Wenn wir Institutionen als die Beschränkungen, welche Menschen sich selbst auferlegen, definieren, so ist diese Definition dem wandlungstheoretischen Ansatz der neoklassischen Wirtschaftstheorie komplementär" (North 1992: 6).

Es werden formelle, beispielsweise Eigentumsrechte, und informelle Institutionen, beispielsweise Gebräuche oder Usancen, unterschieden. Diese Institutionen sind aber nur dann wirksam, wenn die Regelsysteme, die das Handeln der Individuen in eine gewünschte Richtung lenken sollen, ein abweichendes, opportunistisches Verhalten sanktionieren und somit kostspielig machen (vgl. Durth et al. 2002: 214).

Die Ökonomen der neoinstitutionellen Theorie warfen den Neoklassikern Kurzsichtigkeit vor, da sie sich alleine auf die Reduktion des Staates konzentriert hatten. Sie hatten vor allem die Existenz von effektiven Instrumenten zur Implementierung des „Washington-Consensus“ stillschweigend angenommen. Wie North (1992: 81) jedoch anführt, fehlt den ,,institutionellen Gegebenheiten der Dritten Welt die formale Ordnung (und Durchsetzbarkeit), auf der effiziente Märkte beruhen ".

Eine wesentliche Bedeutung haben die Transaktionskosten in der neoinstitutionellen Theorie. Als Transaktionskosten bezeichnet man die Kosten der Bereitstellung, Nutzung, Aufrechterhaltung und Änderung von Institutionen, die für ökonomische Transaktionen notwendig sind (vgl. Fuster 1997: 35). Es wird davon ausgegangen, dass durch reduzierte Transaktionskosten auch eine Volkswirtschaft effizienter ist. In der neoklassischen Theorie wurde von einer „NullTransaktionskosten-Welt" ausgegangen, in der die Verfügungsrechte der Individuen eindeutig geregelt waren (vgl. Richter/Bindseil 1995: 133).

Außerdem wurde auch die Existenz der imperfekten Information als wichtig angesehen, da Wirtschaftssubjekte für ein effizientes Handeln auf zuverlässige Informationen angewiesen sind. $\mathrm{Da}$ in der Welt aber ständig unvollständige Informationen vorhanden sind, stellen die Kosten zur Gewinnung von zuverlässigen Informationen wesentliche Transaktionskosten dar. „Die Tatsache, dass Information etwas kostet, ist der entscheidende Aspekt an den Transaktionskosten" (vgl. North 1992: 32). Gleichzeitig werden die Annahmen von North bzgl. Transaktionskosten auch kritisiert, da er nur ein vages Konzept der Transaktionskosten formuliert, die auf Makroebene nicht quantifizierbar sind, und daher $\mathrm{zu}$ einem ,all-purpose tool of explanation" herangezogen werden kann (vgl. Toye 2000: 65). 
North's Versuch, auf Basis der neoklassichen Annahmen eine Theorie für eine langfristige wirtschaftliche und gesellschaftliche Entwicklung zu entwerfen, verhalf ihm $\mathrm{zu}$ großer Anerkennung in der Entwicklungsökonomie. Wie Schwank (2003: 65) jedoch anführt, liefert die Theorie von North nur begrenzt neue Erkenntnis und Toye (2000: 64) bringt diese Kritik auf den Punkt, indem er schreibt ,The main weakness of the New Institutional Economics as a grand theory of socio-economic development is that it is empty. "

Das Good Governance-Konzept, das an der neoinstitutionellen Theorie anknüpft, war für die Bretton-Woods Institutionen eine willkommene Antwort für das Scheitern der Strukturanpassungsprogramme. Dadurch versuchte man den Staat wieder zu stärken, aber gleichzeitig konnte man an den ursprünglichen Annahmen der Strukturanpassungsprogramme festhalten. Mit dem neuen Konzept konnte sich die Weltbank somit teilweise aus der Mitverantwortung für die Dauerkrise entziehen. Das Governance-Konzept entwickelte sich daher gleichzeitig mit der Umwandlung des „Washington-Consensus“ zu einem „PostWashington-Consensus“" (vgl. Leubolt 2007).

Die Weltbank trug wesentlich zur Verbreitung des Good GovernanceKonzeptes bei, indem sie Ende der 1980er Jahre erkannte, dass die schlechte Governance für die Wirtschaftskrisen in den Entwicklungsländern verantwortlich gemacht werden kann. Dadurch entfachte in der Entwicklungspolitik die Diskussion, welche Maßnahmen gesetzt werden können, um „Good Governance“ zu erhöhen. 1989 taucht der Begriff Governance dann erstmals in einer Publikation der Weltbank über ,Sub-Saharan Africa: From Crisis to Sustainable Growth" auf (vgl. Kjaer 2004: 173). Das Ziel dieser Studie war, mittels der aus drei Dekaden afrikanischer Unabhängigkeit gewonnenen Lehren eine wachstumsfördernde Strategie für die 1990er Jahre zu formulieren, die aufgrund der enttäuschenden wirtschaftlichen Rahmenbedingungen in Afrika dringend notwendig waren (vgl. Fuster 1997: 5). Zur Erreichung von nachhaltigem Wachstum wurden von der Weltbank zwei Maßnahmen festgelegt, nämlich (vgl. Weltbank 1989: 4ff):

1. Schaffung eines sogenannten ,enabling environment “ im Bereich ökonomischer Anreize sowie der Infrastruktur. Ein Fokus war auf die Infrastruktur gerichtet, da die Vernachlässigung der physischen Infrastruktur die Geschäftskosten von potenziellen Investoren steigen ließ. Außerdem wurde die Verbesserung der Anreizstruktur für mehr private Investitionen und effizientere Produktion angestrebt.

2. Förderung von ,enhanced capacities “ auf allen Ebenen, von der Dorfstruktur über die Industrie bis hin zur Regierung. Es wurde nicht weniger, sondern eine bessere Regierung gefordert.

Daran anschließend beschäftigte sich 1992 eine eigene Publikation mit dem Thema „Governance and Development “. Dieser Initiative der Weltbank folgten Publikationen von anderen wichtigen internationalen Institutionen wie der 
OECD (1995), den Vereinten Nationen (UNDP 1997) oder der EU (EC2003b) (vgl. Leubolt 2007).

Gekennzeichnet durch den Amtsantritt von Wolfensohn als Präsident der Weltbank 1995, wurde eine klare Wende hin zu einem neuen, gestärkten Staat eingeleitet. Diese veränderte Position spiegelt sich auch im jährlich erscheinenden „World Development Report" der Weltbank wieder, der sich 1997 mit "The State in a Changing World“, 2001 mit ,Attacking Poverty" und 2002 mit „Building Insitutions for the Market “ beschäftigte (vgl. Leubolt 2007).

Good governance wurde zu einem zentralen Konzept in der Weltbank zu einem Zeitpunkt, in der die Weltbank wesentlich für die „verlorene Dekade“ in der Entwicklungspolitik verantwortlich gemacht wurde (vgl. Küblböck/Strickner 1996). Mit diesem Konzept versuchte die Weltbank sich neu und stärker zu positionieren, um ihre Rolle als "the world's leading development agency" wieder aufzubauen (vgl. Leubolt 2007, Weaver/Leiteritz 2005).

Die neue Ära, die mit dem Amtsantritt von Wolfensohn markiert wird, war auch dadurch gekennzeichnet, dass Armutsreduktion wieder zu einem vordergründigen Ziel in der Entwicklungspolitik erklärt wurde. Die Strukturanpassungsprogramme des ,Washington Consensus " basierten im Wesentlichen noch immer auf dem Trickle-Down-Ansatz und erst im Laufe der 1990er Jahre wurde der Zugang zum Armutsproblem innerhalb der Weltbank immer vielschichtiger (vgl. Schwank 2003: 33). Zudem wurde an den bisherigen Maßnahmen der Strukturanpassungsprogramme kritisiert, dass sie zu viele Konditionen enthielten und die Länder diese nicht umsetzen könnten. Zusätzlich dazu stellten die Auflagen eine Diktion aus Washington dar, die daher nur mit geringer Überzeugung und Motivation umgesetzt wurden. Ein neuer Ansatz im "Post Washington Consensus" stellt daher die Selbstbestimmung der Regierungen dar, die mit „Ownership“ bezeichnet wird.

Mit 2000 begann wiederum eine neue Dekade in der Entwicklungspolitik, in der die Millennium Development Goals (MDGs) beschossen wurden. Im September 2000 unterzeichneten unter der Führung der Vereinten Nationen Abgeordnete aus 189 Ländern acht Ziele zur Reduzierung der Armut, die bis 2015 erreicht werden sollen. Diese Ziele stellen die ersten entwicklungspolitischen Vereinbarungen auf globaler Ebene dar und sind daher als ein Ergebnis des Global Governance zu betrachten. Global Governance wird in der vorliegenden Arbeit noch genauer in Kapitel 2.3 behandelt.

Wurde bei den Strukturanpassungsprogrammen der Weltbank vor allem auch der kurze Zeitraum kritisiert, einigte man sich für die Umsetzung der Ziele auf einen längeren Zeithorizont. Erstmals wurden Ziele nicht nur qualitativ formuliert, sondern auch quantifiziert. Die Quantifizierung kommt damit einer bereits seit langem bestehenden Forderung in der Entwicklungspolitik nach, die Erreichung der Ziele überprüfbar zu machen. Kritisch ist jedoch zu sehen, dass ein Verfehlen der Ziele mit keinen Sanktionen geahndet wird. Alle Maßnahmen 
im Bereich der Entwicklungspolitik werden in Hinblick auf die Erreichung der MDGs durchgeführt. In der folgenden Abbildung 8 werden die acht MDGs kurz vorgestellt. Die genaue Auflistung der Ziele ist im Anhang zu finden.

\section{Abbildung 8: Millennium Development Goals}

\section{Ziel 1: Extreme Armut und Hunger beseitigen}

- Die Zahl der Menschen, die von weniger als einem USDollar pro Tag leben, soll um die Hälfte gesenkt werden.

- Der Anteil der Menschen, die unter Hunger leiden, soll um die Hälfte gesenkt werden.

Ziel 2: Grundschulausbildung für alle Kinder gewährleisten

- Alle Jungen und Mädchen sollen eine vollständige Grundausbildung erhalten.

Ziel 3: Gleichstellung und größeren Einfluss der Frauen fördern

- In der Grund- und Mittelschulausbildung soll bis zum Jahr 2005 und auf allen Ausbildungsstufen bis zum Jahr 2015 jede unterschiedliche Behandlung der Geschlechter beseitigt werden.

\section{Ziel 4: Die Kindersterblichkeit senken}

- Die Sterblichkeit von Kindern unter fünf Jahren soll um zwei Drittel gesenkt werden.

\section{Ziel 5: Die Gesundheit der Mütter verbessern}

- Die Müttersterblichkeit soll um drei Viertel gesenkt werden.

\section{Ziel 6: HIV/Aids, Malaria und andere Krankheiten}

\section{bekämpfen}

- Die Ausbreitung von HIV/Aids soll zum Stillstand gebracht und zum Rückzug gezwungen werden.

- Der Ausbruch von Malaria und anderer schwerer Krankheiten soll unterbunden und ihr Auftreten zum Rückzug gezwungen werden.

\section{Ziel 7: Eine nachhaltige Umwelt gewährleisten}

- Die Grundsätze der nachhaltigen Entwicklung sollen in der nationalen Politik übernommen werden; dem Verlust von Umweltressourcen soll Einhalt geboten werden. 
- Die Zahl der Menschen, die über keinen nachhaltigen Zugang zu gesundem Trinkwasser verfügen, soll um die Hälfte gesenkt werden.

Bis zum Jahr 2020 sollen wesentliche Verbesserungen in den Lebensbedingungen von zumindest 1000 Millionen Slumbewohnern erzielt werden.

\section{Ziel 8: Globale Partnerschaft im Dienst der Entwicklung schaffen}

(nur Auszugsweise mit dem Fokus auf Good Governance)

- Ein offenes Handels- und Finanzsystem, das auf festen Regeln beruht, vorhersehbar ist und nicht diskriminierend wirkt, soll weiter ausgebaut werden. Dies schließt eine Verpflichtung zu guter Staatsführung, zur Entwicklung und zur Beseitigung der Armut sowohl auf nationaler wie auf internationaler Ebene ein.

In Zusammenarbeit mit dem Privatsektor sollen die Vorteile der neuen Technologien, insbesondere der Informations- und Kommunikationstechnologien, verfügbar gemacht werden.

Quelle: http://www.unric.org/Millenniums-Entwicklungsziele/, 5. Mai 2007

Wie man an den MDGs sieht, rangiert Governance nur noch als 8. Ziel und wird zusammengefasst mit dem Ziel, die Zusammenarbeit mit dem privaten Sektor zu stärken. Wirtschaftliche und politische Sicherheit stellen wiederum die Voraussetzung für private Investitionen aus dem Ausland dar, die auch gleichzeitig in diesem Ziel berücksichtigt werden. Good Governance wird daher als Bedingung für mehr privates Kapital angesehen.

Im Zusammenhang mit der vorliegenden Arbeit ist aber auch das erste Ziel von besonderer Wichtigkeit. Armutsreduktion steht bei den MDGs an vorderster Stelle und alle Maßnahmen der Entwicklungspolitik sollen direkt oder indirekt dazu beitragen. Eine wesentliche Maßnahme zur Reduzierung der Armut liegt in der Erweiterung und Verbesserung der Infrastruktur. Es wird angenommen, dass Infrastruktur ein sogenannter ,enabling factor" ist und eine gute Infrastrukturversorgung daher am meisten zur Armutsreduktion beitragen kann (vgl. OECD 2006a: 23).

\subsubsection{Zusammenfassung}

Ökonomie kann nicht von Politik getrennt werden und so beeinflussten seit dem Bestehen der Bretton-Woods-Institutionen die politischen Interessen die jeweilige Wirtschaftspolitik. In den beiden vorhergehenden Kapiteln wurde versucht, eine theoretisch-historische Darstellung über die großen Denkschulen 
der Entwicklungsökonomie in den vergangenen Dekaden zu geben und Good Governance darin einzuordnen. In der folgenden Abbildung 9 wird abschließend nochmals grafisch das Kapitel zusammengefasst. Der neoinstitutionellen Theorie ist es zuzuschreiben, dass sie einen Brückenschlag zwischen der strukturalistischen These und der neoklassischen Antithese unternahm. Denn die entwicklungspolitische Erfahrung hatte gezeigt, dass eine effiziente Ressourcenallokation durch eine unsichtbare Hand neoklassischer Prägung ebenso wenig der Realität in Entwicklungsländern zu entsprechen schien wie jene durch einen - faktisch ebenfalls unsichtbaren - Staatsapparat mit alleinigem Gemeinwohlinteresse (vgl. Fuster 1997: 39).

\section{Abbildung 9: Entwicklungstheoretische Einordnung von Good Governance}

\begin{tabular}{|c|c|c|c|}
\hline & $\begin{array}{l}\text { Strukturalistische } \\
\text { These }\end{array}$ & $\begin{array}{l}\text { Neoklassische } \\
\text { These }\end{array}$ & $\begin{array}{l}\text { Neoinstitutionelle } \\
\text { These }\end{array}$ \\
\hline Fokus & $\begin{array}{ll}\text { - } & \text { Strukturelle Rigiditäten } \\
\text { - } & \text { Marktversagen }\end{array}$ & $\begin{array}{l}\text { - Allokationseffizienz } \\
\text { - Staatsversagen }\end{array}$ & $\begin{array}{ll}\text { - Institutioneller } \\
\text { Rahmen } \\
\text { - Transaktionskosten } \\
\text { und Informationen }\end{array}$ \\
\hline Staatsbild & $\begin{array}{l}\text { - Optimistisch } \\
\text { - Gemeinwohlmaximierer }\end{array}$ & $\begin{array}{l}\text { Pessimistisch } \\
\text { - Eigennutzmaximierer }\end{array}$ & $\begin{array}{l}\text { Neutral } \\
\text { durch institu- } \\
\text { tionelle Schranken } \\
\text { kanalisierbarer } \\
\text { Eigennutzen- } \\
\text { maximierer } \\
\end{array}$ \\
\hline $\begin{array}{l}\text { Strategie- } \\
\text { implikation }\end{array}$ & $\begin{array}{l}\text {-Dirigismusdogma“ } \\
\text { Entwicklungsstaat plant } \\
\text { und implementiert ge- } \\
\text { samtwirtschaftliche } \\
\text { Strategien und agiert als } \\
\text { eigentlicher Ent- } \\
\text { wicklungsmotor }\end{array}$ & $\begin{array}{l}\text { "Washington } \\
\text { Consensus“" } \\
\text { Minimalstaat be- } \\
\text { schränkt sich auf die } \\
\text { Schaffung einer } \\
\text { marktfreundlichen } \\
\text { Wirtschaftsordnung } \\
\text { („get the prices right“) }\end{array}$ & $\begin{array}{l}\text { "Good } \\
\text { Governance“ } \\
\text { in Kernfunktionen } \\
\text { starker Staat garan- } \\
\text { tiert effiziente } \\
\text { Insti-tutionen (,get } \\
\text { the institutions } \\
\text { right") }\end{array}$ \\
\hline
\end{tabular}

Quelle: Fuster 1997: 39

\subsubsection{Kritik an Good Governance}

Ziel des vorliegenden Kapitels ist es, die Kritik der Literatur am Konzept von Good Governance prägnant darzustellen, anhand derer ersichtlich wird, wieso das Good Governance-Konzept in den Entwicklungsländern scheiterte.

Wurde das Good Governance-Konzept anfänglich euphorisch als neues Leitmotiv und Paradigmenwechsel dargestellt, folgte dieser anfänglichen Euphorie 
rasch eine gewisse Ernüchterung. Wie Fuster (1997: 348) klar darstellt, kann die breite und rasche Popularisierung dieses Konzepts innerhalb der entwicklungspolitischen Diskussion nur mit der historischen Einmaligkeit des entwicklungspolitischen Umfelds zu Beginn der 1990er Jahre begründet werden. So resümiert er: „Dass ,gute Regierungsführung' für die Entwicklungsaussichten armer Länder des Südens von zentraler Bedeutung ist, dürfte wohl eine an Trivialität nur schwer zu überbietende Binsenwahrheit darstellen." (Fuster 1997: 348).

Dran schließt auch die methodische Kritik an. Erstens wurde keine einheitlich und methodisch strenge Verwendung des Konzepts festgelegt, sondern es wurde „,bisweilen als ein ,Regenschirmkonzept' kritisiert, unter das je nach Gutdünken und Interessen eigene Wunsch- und Wertvorstellungen subsumiert würden " (Fuster 1997: 348). Zweitens bedeutete Governance eine veränderte Fokussierung bei der Unterstützung, da in diesem Konzept vor allem der Aufbau von Institutionen und die politische Ausrichtung unterstützt wurden. Allerdings waren die Ergebnisse aus dieser Unterstützung eher gemischt, da es sich als schwieriger und langwieriger herausstellte Reformen von Institutionen zu unterstützen als ein spezifisches Projekt, wie beispielsweise den Bau einer Straße. "However, the Bank's analysis is naive because it entirely ignores that good governance is not simply available on order " (Leftwich 1993).

Zusätzlich dazu sind die Ergebnisse von Reformen nicht unmittelbar erkennbzw. messbar und können erst mit einer zeitlichen Verspätung wirksam werden. Anfang 2000 verlor das Governance-Konzept daher langsam wieder an Bedeutung in der Weltbank, da es sich als schwierig herausstellte, die gestellten Konditionen von „Good Governance“ zu überwachen (vgl. Kjaer 2004: 174f). Daher wurde bei der Evaluierung der „Good Governance" von einem ex-ante zu einem ex-post-Ansatz gewechselt und „Good Governance“ wird heute als Kriterium für die Vergabe von Unterstützung verwendet (vgl. Doormbos 2001, Fuster 1997: 226). Der Ansatz „Good Governance“ ist daher zwar nicht aus der Weltbank verschwunden, aber die Verwendung des Begriffs hat sich verändert.

Besonders kritisch wird auch die normative Komponente dieses Konzepts gesehen. Die Konditionen für „Good“ werden für die Entwicklungsländer in Washington vordefiniert. Daher werden auch in diesem Konzept vor allem wieder die neoliberalen Wertvorstellungen in die Entwicklungsländer transportiert. Die Weltbank hat dadurch wesentlichen Einfluss auf die wirtschaftspolitische Entwicklung der Länder und beschränkt damit die Freiheit der Entwicklungsländer bei politischen Entscheidungen (vgl. Leubolt 2007). Cooke (2003) betrachtet das vorgegebene Good Governance-Konzept aus historischer Perspektive als , new continuity with colonial administration ".

Außerdem wird die einseitige Ausrichtung des Governance-Konzeptes kritisiert, da die geforderte „Accountability“ bzw. Übernahme von mehr Rechenschaft für das Handeln nur eine nach außen gerichtete ist, welche die Bürger und das Parlament umfasst (vgl. Kjaer 2004: 139). Für Root (1996: 45) greift das zu 
kurz und er kritisiert, dass die Verantwortlichkeit nach innen, wie beispielsweise für die Verwaltung der öffentlichen Ressourcen oder Projekte, nicht berücksichtigt wird. Er kritisiert diese einseitige Orientierung vor allem auch deswegen, weil viele asiatische Länder undemokratische Ansätze verfolgen, aber die interne Verantwortlichkeit sehr gut erfüllen und der öffentliche Sektor zuverlässig ist.

Good Governance stellt Demokratie in den Mittelpunkt von „good“, wie auch Leftwich (1993) feststellt: „Democratic good governance is better understood as an intimate part of the emerging politics of the New World Order". Demokratie und Governance sind zwar eng verbunden, bedeuten jedoch etwas Unterschiedliches (vgl. Kjaer 2004: 163). Tatsächlich liefert die Regierungsform nämlich keine Erklärung, warum manche Länder entwickelt sind und andere nicht (vgl. Root 1996: 170). Daher ist zu hinterfragen, inwieweit diese neoliberalen Ansätze der IFIs wirklich für die Entwicklungsländer von Nutzen sind. Außerdem stellt Root (1996: 147) fest: ,Good governance is not synonymous with good policy". Wichtig erscheint neben der guten Regierungsführung daher, ob eine Regierung auch wirklich in der Lage ist, ihre Versprechen einzuhalten.

Fraglich ist außerdem, inwieweit eine Organisation wie die Weltbank „Accountability“ von Institutionen in Entwicklungsländern fordern kann, wenn sie diese Anforderungen selbst kaum erfüllt. In der Vergangenheit wurden von der Weltbank bereits öfters Projekte finanziert, die negative Auswirkungen auf das Investitionsland und die Bevölkerung hatten und aus reinem wirtschaftspolitischem Kalkül durchgeführt wurden. In diesem Zusammenhang wurde von der Weltbank 1994 das "Inspection Panel“ eingerichtet. In diesem Panel können die Bürger der Entwicklungsländer direkt mit der Bank die negativen Auswirkungen von weltbankfinanzierten Projekten in Bezug auf Umweltschädigung und soziale Belastungen diskutierten (vgl. Kjaer 2004: 184f). Die Mitglieder des Panels sind unabhängig und gehören nicht der Weltbank an. Daher sind sie auch keinem politischen Druck ausgesetzt und können weitere Nachforschungen bei den Projekten vornehmen. Allerdings weist auch dieses Panel Schwächen auf, weil die betroffenen Mitgliedsländer oft weitere Untersuchungen blockieren, da sie wirtschaftspolitische Interessen verfolgen (vgl. Fox 2000). Zusammenfassend kann festgestellt werden, dass die Weltbank aufgrund des externen Drucks der NGOs in den vergangenen Jahren transparenter wurde (vgl. Kjaer 2004: 185).

Wichtig für die Verwendung des Governance-Begriffs ist, dass die Steuerung der Interaktion zwischen Akteuren möglich ist und dass Politik nicht durch wirtschaftliche Zwänge oder Institutionen bestimmt ist (vgl. Benz 2004a: 21). Diese Voraussetzung scheint aber in Hinblick auf die Konditionalität bei der Kreditvergabe durch die IFIs äußerst fraglich.

Rund um die Definition des "Good“ wird auch die Shareholder-Perspektive diskutiert, wobei der einflussreichste Shareholder eben die US sind. ,"There is a 
tendency, then, for Bank rhetoric to be more ideological and 'global', while Bank practice tends to be more in line with neo-liberal ideas." (Kjaer 2004: 183). Die neoliberalen Ansätze sind geprägt durch die Privatisierung von öffentlichen Einrichtungen und die Dezentralisierung der Macht. Diese Reformen werden unter dem Begriff des New Public Managements (NPM) zusammengefasst und im folgenden Kapitel diskutiert.

\subsubsection{New Public Management und Good Governance}

Die veränderte Rolle der öffentlichen Hand wird unter dem Schlagwort des New Public Managements (NPM) zusammengefasst. Das zentrale Credo des NPM ist, dass die klassische bürokratische Steuerung der Verwaltung zunehmend dysfunktionale Folgen zeigt und dass sich Konzepte des modernen betriebswirtschaftlichen Managements mit Erfolg auf die öffentliche Verwaltung übertragen lassen (vgl. Jann/Wegrich 2004: 200). Besonders bekannt wurden die Deregulierungs- und Privatisierungsreformen, die unter den liberalen Regierungen von Thatcher in Großbritannien oder von Reagan in den USA durchgeführt wurden (vgl. Lütz 2004: 154).

Max Weber war der erste Wissenschafter, der sich systematisch mit den Prinzipien einer modernen Bürokratie auseinandersetzte und dabei feststellte, dass öffentliche und private Interessen völlig auseinander liegen (vgl. Weber 1978). Diese Annahme beeinflusst das Handeln der Regierungen in den westlichen Ländern zwar nach wie vor, aber Mitte der 1980er Jahre wurden eine Fülle von Reformen in den westlichen Ländern durchgeführt, welche die Rolle des öffentlichen Sektors stark veränderten. Hatte die Regierung (,government") bisher eine wichtige Rolle bei der Lösung von öffentlichen Problemen, wurde mit der Ära Thatcher und Reagan die Regierung nicht mehr als Lösung, sondern als die Ursache für die Probleme angesehen (vgl. Pierre/Peters 2000: 2). In dieser Zeit wurde die Annahme, dass öffentliche Betriebe eine andere Unternehmenskultur besitzen, abgelehnt und gefordert, dass sie nach den gleichen Prinzipien wie der private Sektor geführt werden sollten (vgl. Pierre/Peters 2000: 64). Die Reformen der neo-liberalen Regierungen wurden daher mit dem Ziel durchgeführt, die Hindernisse für die effiziente Bereitstellung von Dienstleistungen zu entfernen (vgl. Kjaer 2004: 24f).

Die Dominanz des Management-Leitbildes im öffentlichen Sektor hat zwar Ende der 1990er Jahre ihren Zenit überschritten, stellt aber noch immer einen wichtigen Diskussionspunkt dar. Mit dem Umschwung der politischen Debatte vom „schlanken“ zum ,aktivierenden Staat" war der Managementansatz als verwaltungspolitisches Reformkonzept zunehmender Kritik ausgesetzt und immer stärker von Governance als prägendes Leitbild verdrängt (vgl. Jann/Wegrich 2004: 201).

Diese Maßnahmen zur Effizienzgewinnung im öffentlichen Sektor, die ursprünglich in den westlichen Ländern ihren Ursprung hatten und dort 
Anwendung finden sollten, wurden in den 1990er Jahren auch auf die Entwicklungsländer übertragen. Die Internationalen Finanzinstitutionen (IFIs) setzten die Prinzipien des NPM für die Vergabe von Krediten an die Entwicklungsländer fest. Diese Reformen wurden in der Entwicklungspolitik durch die Strukturanpassungsprogramme der Weltbankgruppe bekannt.

In der Literatur werden verschiedene Reformen unter dem Begriff NPM subsumiert, aber es gibt keine einheitliche, exakte Aufzählung darüber (vgl. Kjaer 2004: 25). In den folgenden Unterkapiteln werden daher nur jene Reformen dargestellt, nämlich Privatisierung und Dezentralisierung, die auch für den eingeschränkten Themenbereich der vorliegenden Arbeit relevant sind.

\subsubsection{Privatisierung}

Mit Privatisierung wird der Verkauf oder die Übergabe von bisher öffentlichem Eigentum, wie beispielsweise Infrastruktur, an private Einheiten bezeichnet. Im Rahmen der Privatisierung können verschiedene Organisationsformen die Aufgaben für den Staat erfüllen (vgl. Pierre/Peters 2000: 90). Eine wesentliche Rolle spielen dabei vor allem privatwirtschaftliche Unternehmen oder quasiautonome Organisationen, die von der öffentlichen Hand für einen bestimmten Zweck gegründet werden. Zusätzlich dazu führen Pierre und Peters (2000: 90) auch nichtstaatliche Organisationen an, die in der Infrastruktur auch teilweise Dienstleistungen der öffentlichen Hand übernehmen können.

Im Neo-Institutionalismus basiert die Begründung für eine Privatisierung auf der Annahme, dass privatwirtschaftliche Unternehmen die Leistungen effizienter erzeugen können als die öffentliche Hand. Diese Annahme basiert auf dem Property Rights-Ansatz, der wesentlich von Furubotn/Pejovich (1972) geprägt wurde. Der Ansatz vertritt die Annahme, dass das Recht am Eigentum auch die Nutzung und den Umgang damit verändert (vgl. Feldmann 1995: 47). Der Property Rights-Ansatz untersucht das Verhalten von Individuen in Abhängigkeit von existierenden institutionellen Regelungen und trifft Aussagen über die Effizienz von Property Rights-Strukturen. Außerdem wird auch untersucht wie Property Rights-Strukturen beschaffen sein müssen, um optimale gesamtwirtschaftliche Ergebnisse zu erzielen (vgl. Halin 1995: 12).

Die Vertreter der Privatisierung nehmen an, dass es das originäre Interesse des Eigentümers ist, die Anlage effizient zu betreiben, um Kosten einzusparen bzw. die Gewinne zu erhöhen. Es verändert sich jedoch die Situation, wenn die Property Rights eines Wirtschaftsgutes auf mehrere Personen aufgeteilt werden. Die geteilten Rechte führen dazu, dass das einzelne Individuum die Konsequenzen aus seinem Handeln nicht mehr zur Gänze trägt und sich dadurch die Anreize zum effizienten Umgang verringern (vgl. Strohbach 1999: 205).

Eine weitere Annahme im New Public Management (NPM) ist, dass Wettbewerb zu Effizienzgewinnen führt. Wettbewerb bei öffentlichen Gütern kann wiederum durch Privatisierung erlangt werden, indem staatliche Monopole auf- 
gebrochen werden und sich ein Markt entwickelt. Eine andere Möglichkeit wäre die Einführung von so genannten Quasi-Märkten in den öffentlichen Sektor, beispielsweise im Gesundheitssektor, in dem die Bürger zu Kunden werden und sich frei aussuchen können, von welchem Arzt sie behandelt werden wollen (vgl. Kjaer 2004: 28).

Eine mildere Version der Privatisierung stellt das Contracting-Out dar. Contracting-Out bedeutet, dass der Staat die Produktion der Dienstleistung an ein privates Unternehmen auslagert und von dort zukauft (siehe Kapitel 4.1.2). Großbritannien ist jenes Land, das Dienstleistungen am radikalsten ausgelagert hat. Dabei wurden vormals wesentliche Aufgaben des Staates, wie beispielsweise der Betrieb von Gefängnissen, an privatwirtschaftliche Unternehmen vergeben (vgl. Peters/Wright 1996: 630).

Im Zusammenhang mit Privatisierungen wurden Public Private Partnerships (PPP) zu einen beliebten Instrument, um die Finanzierungsmöglichkeiten für die politischen Institutionen zu verbessern. Insbesondere auf lokaler Ebene wurden PPPs als gute Möglichkeit gesehen, um die Ressourcen zu erhöhen (vgl. Pierre/Peters 2000: 90). Kritikern, die den Weber-Prinzipien folgend keine Kompatibilität von privaten und öffentlichen Interessen sehen, stehen die Befürworter gegenüber, die in einer solchen Partnerschaft neue Möglichkeiten für die Steuerung von Aufgaben sehen (siehe Kapitel 4.1.2).

Die Entwicklungsländer privatisierten Anfang der 1990er Jahre auf Druck des IWF und der Weltbank viele Staatsbetriebe. Neue Kredite waren nur noch jenen Ländern zugänglich, die diese Forderung erfüllten. Ein wesentlicher Kritikpunkt von Seiten des IWF und der Weltbank im Zusammenhang mit öffentlich geführter Infrastruktur in Entwicklungsländer war, dass diese Einrichtung aufgrund von Korruption und Nepotismus ineffizient geführt wurden und die öffentlichen Budgets stark belasteten (vgl. Kjaer 2004: 27). Laut Turner und Hulme (1997: 190) wurden in der Zeit von 1988 bis 1993 mehr als 2.700 öffentliche Unternehmen in mehr als 60 Entwicklungsländern privatisiert.

Im Zusammenhang mit Privatisierung wird angenommen, dass Good Governance eine große Rolle spielt und dass Faktoren wie Korruption, Accountability (Übernahme von mehr Rechenschaft) der Regierung und bestehende Gesetze den Erfolg wesentlich beeinflussen (vgl. Nabli 2001). Es werden in der Literatur verschiedene Gründe diskutiert, die eine Privatisierung befürworten, aber zusammenfassend lässt sich feststellen, dass die vordergründigen Ziele für die Zentralregierung

- die Kostenreduktion und

- die Effizienzsteigerung durch die Einführung von Managementansätzen des privaten Sektors sind (vgl. Pierre/Peters 2000: 91).

Auch wenn das Good Governance-Konzept wieder eine aktivere Rolle des Staates fordert, stellt für die Internationalen Finanzinstitutionen (IFIs) Privatisierung dennoch die Lösung für das Infrastrukturdilemma in den Entwick- 
lungsländern dar. Privatisierung stellt eine wesentliche Strategie von Good Governance dar, da von den IFIs angenommen wird, dass Privatisierung gepaart mit Good Governance die Infrastrukturausstattung in den betreffenden Ländern verbessert.

\subsubsection{Dezentralisierung}

Basierend auf dem Dezentralisierungstheorem von Oates (1972) sollen durch die Dezentralisierung die Effektivität und die Effizienz von Infrastrukturleistungen erhöht werden. Auch neuere und anspruchsvollere Konzepte haben sich mit den Effekten der Dezentralisierung beschäftigt, in denen beispielsweise der asymmetrische Informationsfluss zwischen den Machthabern und den ausführenden Organen berücksichtigt wird (vgl. Laffont/Tirole 1994). Dem Good Governance-Konzept folgend, unterstützt die Dezentralisierung eine verantwortungsvolle Regierungsführung, da eine Aufteilung der Macht vorgenommen wird und sowohl zentralem Machtmissbrauch vorgebeugt als auch Verantwortung an die regionalen Regierungseinheiten bzw. die lokale Bevölkerung weitergegeben wird.

In vielen Entwicklungsländern war die Verwaltung stark zentralisiert und autoritären Präsidenten oder Machthabern unterstellt, da diese Politiker Angst vor Machtverlusten hatten. Daher war die Governance in diesen Ländern dementsprechend schlecht. Unter maßgeblicher Einflussnahme der Geberinstitutionen delegieren aber viele Entwicklungsländer immer mehr ihre politische Entscheidungskraft und finanzielle Verantwortung an regionale Regierungseinheiten. Ziel der Dezentralisierung ist es, die Entscheidungen näher an der Basis anzusiedeln und so die Teilnahme der armen Bevölkerungsschicht bei Entscheidungen zu erhöhen (vgl. Leiderer/Liebig 2006: 4). Dadurch wird auch ein Teil der Verantwortung für Infrastrukturleistungen auf die lokalen Regierungseinheiten übertragen (vgl. Kehew et al. 2005: iv). Es ist auch anzunehmen, dass durch die Dezentralisierung die städtischen Regierungen gestärkt wurden und die Unternehmen nun mehr Anreize sehen, mit den subsouveränen Einheiten zusammenzuarbeiten (vgl. Pierre/Peters 2000: 89).

Neben der Verlagerung der Macht von der zentralen zu dezentralen Einheiten, kann auch innerhalb der lokalen Regierung wiederum Macht weiter nach unten aufgeteilt werden. Die verteilte Macht ist aber eben auch gleichzeitig mit Verpflichtungen verbunden und so hat man, insbesondere während Zeiten von Budgetkürzungen, Interesse, auch die finanziellen Verpflichtungen nach unten auszulagern (vgl. Pierre/Peters 2000: 88).

Außerdem wird angenommen, dass die zentralen Entscheidungseinheiten zu weit von den Verbrauchern entfernt sind und daher nicht mehr wissen, welche Nachfrage wirklich besteht. Wenn hingegen die Aufgaben und die Macht auf untere Ebenen delegiert werden, können die lokalen Regierungen über die Anpassung des Angebots entscheiden. Die zentrale Regierung übernimmt dann nur 
noch die Koordinierungsfunktion und überlässt die Ausübung den dezentralen Regierungseinheiten (vgl. Kjaer 2004: 29).

Eine große Anzahl an Regierungen betrachtet die Produktion von und Versorgung mit Dienstleistungen durch dezentrale Einheiten als effizienter und daher wurden viele Leistungen an die unterstellten Regierungseinheiten ausgelagert. Laut Pierre und Peters (2000: 87) dominierten die Dezentralisierungsbestrebungen die Reformen der Strukturanpassungsprogramme: „...the decentralisation of state authority to regional and local institutions has gained more attention than the strengthening of international structures".

Seit Mitte der 1980er Jahre rückten, wie im Kapitel 2.2.4.1 beschrieben, auch Kooperationsmuster zwischen der öffentlichen Hand und Unternehmen ins Zentrum der Aufmerksamkeit. Außerdem begann man, Chancen wirtschaftlicher Einbettung in nichtmarktförmige Formen der Koordination weniger auf der nationalen Makroebene, sondern auf regionaler und sektoraler Ebene anzusiedeln (vgl. Lütz 2004: 155).

Im Rahmen der Dezentralisierung unterscheidet man zwei mögliche Formen: die Entflechtung (deconcentration) und die Regionalisierung (vgl. Kjaer 2004: 29). Bei der Entflechtung (deconcentration) werden politische Entscheidungen zentral getroffen, aber die regionalen Einheiten setzen die politischen Entscheidungen um, wobei die dezentrale Einheit der zentralen Regierung unterstellt bleibt. Die Regionalisierung ist hingegen jener Zustand, bei dem die gesamte Macht auf die regionalen Einheiten übertragen wird. Die regionale Regierung ist nicht mehr der zentralen Regierung unterstellt und wird direkt vom Volk gewählt (vgl. Turner/Hulme 1997: 154). Bei New Public Management (NPM) handelt es sich meistens um Regionalisierung, wobei es unterschiedliche Auffassungen darüber gibt, wie weit Dezentralisierung gehen soll (vgl. Kjaer 2004: 30).

\subsection{Global Governance}

Wurde im vorangegangenen Kapitel Governance als normatives Konzept bzw. Good Governance dargestellt, so wird im Global Governance-Konzept auf die sich teilweise verändernden Rollen der Staaten im internationalen Rahmen Bezug genommen.

Eine der wesentlichsten Veränderungen seit dem Ende des Zweiten Weltkrieges im Zusammenhang mit der sich ändernden Rolle des Staates stellt die immer größer werdende Wichtigkeit von internationalen Gruppen oder Organisationen dar. Die Staaten haben sich dazu entschieden, gewisse politische Agenden in internationalen Verträgen oder Organisationen zu regeln (vgl. Pierre/Peters 2000: 84). Zweck solcher Zusammenschlüsse ist es, gemeinsam Ziele zu erreichen, auf die jeder einzelne Staat nur wenig Einfluss hat und die 
nur gemeinsam erreicht werden können. Dies können sowohl wirtschaftliche als auch politische Zielsetzungen sein.

Schon mit der Gründung der Bretton-Woods-Institutionen und anderer regionaler Entwicklungsbanken nach dem Ende des Zweiten Weltkrieges wurde Entwicklungspolitik auf eine multilaterale Ebene gestellt. Mit den Millennium Development Goals (MDGs) der Vereinten Nationen ist es aber nun erstmals in der Entwicklungspolitik gelungen, Ziele zu formulieren, die von fast allen Nationen mitgetragen werden.

\subsubsection{Einführung und Definition}

Global Governance ist eng verbunden mit Globalisierung und daher ist es auch notwendig, Globalisierung kurz darzustellen (vgl. Brand et al. 2000: 11). Globalisierung wird in der Literatur oft als die dominanteste und einflussreichste Entwicklung der vergangen Jahrzehnte bezeichnet (vgl. Pierre/Peters 2000: 56).

- Nye (2001) definiert „Globalization can be defined as networks of interdependence at worldwide distances".

- Weiss (2000) beschreibt „Globalisation is neither uniform nor homogeneous, but it is indisputably accelerating the pace and intensity of economic and social interactions at all levels". Globalisierung basiert demnach nicht nur auf wirtschaftlicher Ebene, sondern weist auch politische, kulturelle und soziale Dimensionen auf (vgl. Kjaer 2004: 183).

- Rufera (2001: 11) gibt die folgende sehr umfassende Definition von Globalisierung: Globalisierung ist ein dynamischer Prozess, der durch integrative Kräfte (des Marktes oder von Unternehmen) initiiert und vorangetrieben wird und bewirkt, dass die Verflechtung in den und zwischen den Bereichen Politik, Wirtschaft und Gesellschaft auf eine weltweite Ebene ohne Rücksicht auf nationale Grenzen ausgedehnt wird".

Über die Auswirkungen der Globalisierung ist man sich jedoch uneinig (vgl. Nuscheler 2004: 52ff). Einig ist man sich hingegen über folgendes: "Globalisation has profound consequences for the nature of collective action in both domestic and international politics" (Weiss 2000).

Das Global Governance-Konzept bietet eine Möglichkeit, die verändernden Rahmenbedingungen für Regierungen darzustellen. In der deutschsprachigen Literatur wird das Konzept gelegentlich auch als neue "Weltordnungspolitik" oder ,globale Strukturpolitik" bezeichnet (vgl. Ziai 2006: 72). Das Konzept "Global Governance“ erfreut sich seit Mitte der 1990er Jahre zunehmender Beliebtheit, aber eine einheitliche Definition des Begriffes gibt es bisher nicht. Vielmehr erklärt sich die Attraktivität des Begriffes gerade in seiner Offenheit und Vieldeutigkeit (vgl. Murphy 2000). Das Good Governance-Konzept ist mittlerweile schon in die Jahre gekommen und hinreichend diskutiert worden, „, but the journey to explore global governance has just begun“" (Weiss 2000). 
Der Begriff Global Governance reicht bis in die 1970er Jahre zurück, aber bisher fehlt es an einer einheitlichen Definition (vgl. Lake 1999). Bis Anfang der 1990er Jahre wurde der Begriff empirisch analytisch verwendet und erst durch die Arbeiten am Institut für Entwicklung und Frieden (INEF) und der Stiftung Entwicklung und Frieden (SEF) kam es zu einer normativen Wende (vgl. Behrens 2005a: 11).

Sehr allgemein definiert Rosenau (1995: 13): „Global governance is conceived to include systems of rule at all levels of human activity - from the family to the international organization - in which the pursuit of goals through the exercise of control has transnational repercussions ". Diese Definition von Governance beruht auf der Annahme, dass es keinen zentralen Akteur mehr gibt, dem allein eine Steuerungs- und Kontrollfähigkeit zugesprochen wird, sondern dass Entscheidungsprozesse interaktiv zwischen staatlichen und nichtstaatlichen Akteuren stattfinden. In Global Governance stellt der Nationalstaat daher nicht länger die wesentliche Hauptmacht dar, sondern seine Rolle ist diffuser und die globale Entwicklung wird immer mehr durch multilaterale Institutionen und einige wenige einflussreiche Nationalstaaten, wie beispielsweise die G8, bestimmt (vgl. Lake 1999).

Laut Behrens (2004: 104f) werden bei der Verwendung des Begriffes in der internationalen Politik folgende drei Aspekte betont:

1. Global Governance als Systemmerkmal zur Abgrenzung von nationalem Governance:

Das Systemmerkmal, das nationales von globalem Governance unterscheidet, ist die Tatsache, dass es in der internationalen Politik nie eine Zentralinstanz als Bestandteil eines Weltstaates geben wird, die Entscheidungen autoritär durchsetzen kann.

2. Global Governance als Beschreibung einer neuen Qualität internationaler Politik:

Im Zusammenhang mit internationaler Politik steht Global Governance für Koordinationsleistungen, die nicht alleine von Staaten, sondern von einer Vielzahl gesellschaftlicher Akteure auf verschiedenen Ebenen lokaler, nationaler, regionaler sowie internationaler Politik gleichzeitig erbracht werden.

3. Global Governance als Reformprojekt:

Als Lösung für die Globalisierungsprobleme wird Global Governance auch als normatives Konzept diskutiert. Da die Globalisierung zu einer Aushöhlung der Souveränität staatlichen Handelns geführt hat, sind Einzelstaaten nicht länger in der Lage, die Folgen der Globalisierung alleine zu bewältigen.

Global Governance bedeutet im Zusammenhang mit Entwicklungspolitik die Regierung der Menschheit auf globaler Ebene, durch globale Abkommen 
zwischen staatlichen Regierungen unter Einbeziehung von internationalen Organisationen, Wirtschaftsunternehmen, Nichtregierungsorganisationen sowie regionalen und lokalen Entscheidungsträgern im Interesse der Menschheit und zum Zwecke der Lösung globaler, die Regierungsfähigkeit einzelner Staaten überfordernder Probleme (vgl. Ziai 2006: 73). Diese Definition liegt auch der vorliegenden Arbeit zugrunde.

\subsubsection{Historisch-theoretische Darstellung von Global Governance im Lichte der entwicklungstheoretischen Ansätze}

Ziel dieses Kapitels ist es, das Global Governance-Konzept in die entwicklungstheoretischen Ansätze einzuordnen, um zu zeigen, dass die Entstehung dieses Konzepts durch die sich verändernden politischen und wirtschaftlichen Rahmenbedingungen erklärt werden kann. Der Fokus dieser Darstellung liegt auf der Entwicklung des Global Governance-Konzepts.

Die Entwicklung des Global Governance-Konzeptes wird in der Literatur im Wesentlichen durch die folgenden beiden Ereignisse in der Vergangenheit erklärt.

- Mit der Beendigung des Ost-West-Konfliktes Ende der 1990er Jahre lösten sich politische Grenzen auf, die bisher ausschlaggebend für wirtschaftliche Aktivitäten waren. Gleichzeitig fand ein Globalisierungsprozess statt, durch den andere Probleme sichtbar wurden.

- Mit dem Ende des Zweiten Weltkrieges waren die internationalen Wirtschaftsaktivitäten nach institutionalistischer Auffassung in ein System politischer Regulierung eingebettet (,embedded liberalism"), welches die sozialpolitischen Folgen der Internationalisierung begrenzen sollte (vgl. Behrens 2004: 105).

Mit Ende der 1970er Jahre erfolgte jedoch eine wirtschaftliche Wende hin zum Neoliberalismus. Auf Basis von Deregulierung und Privatisierung wurden die internationalen Wirtschaftsbeziehungen auf Kosten des nationalen Wohlfahrtstaates intensiviert (vgl. Rieger/Leibfried 2001: 57ff). Dadurch löste sich das Regierungsmodell des „embedded liberalism“ in den 1980er Jahren zunehmend auf (vgl. Behrens 2004: 106).

In den 1970er Jahren entwickelte sich als neoliberale Variante des Institutionalismus die Interdependenztheorie, auf die das Global GovernanceKonzept aufbaut. Die Wahrnehmung zunehmender ökonomischer, politischer, sozialer und militärischer Vernetzungen zwischen den Staaten führte in den Theorien der Internationalen Beziehungen seit den 1970er Jahren zur Diskussion über das Konzept der „Interdependenz“ zwischen Staaten (vgl. Messner 2005: 31). Die prominentesten Vertreter dieser Theorie sind Robert O. Keohane und Joseph S. Nye mit ihrem Werk „Power and Interdependence“ (1977). 
Keohane and Nye stellen das internationale System durch ein komplexes Beziehungsgeflecht bzw. eine komplexe Interdependenz zwischen staatlichen wie nichtstaatlichen, nationalen wie transnationalen Akteuren dar, das auf eine zunehmende Internationalisierung von Wirtschaftsaktivitäten zurückgeführt wird (vgl. Rieger/Leibfried 2001: 17). Sie haben mit ihrem Begriff der „komplexen Interdependenz" auf einen qualitativen Wandel im internationalen System hingewiesen (vgl. Messner 2005: 319).

Dies bedeutet, dass ein Staat bei der gemeinsamen Erzeugung einer Dienstleistung oder eines Gutes mit einem anderen Staatswesen, immer eine Beziehung zu der anderen Einheit eingehen muss (vgl. Lake 1999). Außerdem wird angenommen, dass sich alle Akteure opportunistisch oder arglistig verhalten und versuchen, den Partner wenn möglich zu überlisten (vgl. Williamson 1985). Die Staaten sind durch diese komplexen Interdependenzen verwundbarer geworden und es besteht aus Eigeninteresse zur Abwehr möglicher negativer Folgen die Bereitschaft $\mathrm{zu}$ informellen wie formalisierten Kooperationen in Form internationaler Regime und Organisationen (vgl. Behrens 2004: 105).

Gleichzeitig zu den Entwicklungen im Rahmen des New Public Managements (NPM) (siehe Kapitel 2.2.4) stieg in den 1980er Jahren die Anzahl der internationalen Nicht-Regierungsorganisationen (NGOs) stark an, die sich kritisch mit politischen und wirtschaftlichen Fragestellungen auseinandersetzten ( $\mathrm{vgl}$. Meyers 1999 in Behrens 2004: 106). Durch die Privatisierungen nahmen aber auch die privaten Akteure eine immer stärkere Rolle ein und wie Lake (1999) feststellt: "Private actors are central to the phenomenon of globalization". Dadurch nahm die Anzahl an politischen Akteuren zu und die Handlungsfähigkeit auf nationalstaatlicher Ebene ab. Ausgehend von diesen Entwicklungen wird das Konzept Global Governance im angelsächsischen Raum vor allem als empirisch-analytische Kategorie diskutiert und bezeichnet das komplexe System staatlicher wie nichtstaatlicher Koordinationsmechanismen (vgl. Behrens 2004: 106).

Globalisierung schwächt die interne Souveränität der Staaten, zwar nicht im legalen, rechtlichen, jedoch im operationalen Sinne und vergrößert gleichzeitig den Aufgabenbereich der externen Souveränität, d.h. das Management zwischenstaatlicher Beziehungen durch die Außenpolitik und die Herstellung von Sicherheit (vgl. Messner 2005: 32). Die Aufgabenbereiche sind aber im Zeitalter der Globalisierung immer größer und globaler geworden, sodass sie nicht mehr alleine auf nationalstaatlicher Ebene gelöst werden können. Die zentrale Annahme im Global Governance-Konzept ist daher, dass die Nationalstaaten ihre Interessen poolen müssen, da nur so die Handlungsfähigkeit der Politik und deren interne Souveränität wiederhergestellt werden können (vgl. Messner 2005: 34).

Mit der Beendigung des Zweiten Weltkrieges kam es gleichzeitig zur Dekolonialisierung und Staatenbildung in der ehemals so genannten „Dritten 
Welt" (vgl. Behrens 2005a: 16). Aufgrund des Ost-West-Konfliktes spielte es in diesen Ländern jedoch kaum eine Rolle, wer in den Entwicklungsländern regierte, wie z.B. Pol Pot in Kambodscha, solange die Bündnistreue gewährleistet war (vgl. Behrens 2005a: 17). Bis zum schlagartigen Zerfall der Sowjetunion 1989 war die Welt durch eine bipolare Struktur geprägt und die Staaten hatten sich zu entscheiden, welcher Seite sie angehören.

Die Welt hatte zwei Supermächte, die USA und die Sowjetunion, die eine wesentliche stabilisierende Funktion hatten. Durch den Zusammenbruch der Sowjetunion konnten sich die USA jedoch als einzige Supermacht durchsetzen (vgl. Behrens 2005a: 16). Zeitgleich mit der Beendigung des Ost-WestKonfliktes begann auch die Debatte rund um Good Governance in den BrettonWoods-Institutionen, in denen die USA die Mehrheit halten. Unter dem Deckmantel von Good Governance wurde versucht, Demokratie als politisches System in den Entwicklungsländern zu installieren.

\subsubsection{Kritik an Global Governance}

Der Bedarf nach einer globalen Steuerung von Problemen und Konflikten, die aufgrund der Globalisierung entstehen, scheint außer Frage zu stehen und die Herausbildung wird von wissenschaftlicher und politischer Seite gutgeheißen (vgl. Brand/Scherrer 2005: 119). Wie realistisch dies allerdings ist und wie eine globale Strukturpolitik konkret aussehen soll, wird von vielen Seiten unterschiedlich gesehen. An dieser Stelle soll nun prägnant ein Überblick über diese Diskussion gegeben werden.

Die Auflösung der Nationalstaaten müsste einhergehen mit der Auflösung der verbundenen Eigeninteressen der jeweiligen Staaten. Die manifesten Machtstrukturen und die Konzentration auf die nationalstaatlichen Interessen zeigen jedoch, dass globale Lösungen nur beschränkt bzw. nur dann, wenn sie dem eigenen Land dienlich sind, mitgetragen werden. Durch die Machtkonzentration auf einen Staat, die USA, sind multilateral ausgerichtete Politikvorstellungen jedoch tendenziell mit einer abnehmenden Bereitschaft $\mathrm{zu}$ kooperativem Verhalten konfrontiert (vgl. Behrens 2005a: 17).

Auch Entwicklungsländer verweigern sich oft internationalen Lösungsstrategien, weil sie externe Einmischungen starker Akteure in ihre Angelegenheiten fürchten. Für schwache Länder gilt die „nationale Souveränität" oft als die letzte Bastion nationaler Eigenständigkeit (vgl. Messner 2004: 45). Hinter dem Global Governance-Konzept befürchten die Entwicklungsländer, dass dahinter vor allem Versuche der reichen Länder stecken, die Handlungsspielräume der Entwicklungsländer weiter einzuschränken (vgl. Ziai 2006: 88)

Während die EU zunehmend zu einem institutionell verfestigten Regierungssystem wird, sind auf internationaler Ebene lediglich sektor-spezifische Formen des Regierens durch internationale Organisationen oder Regimes zu finden. Um 
diese Organisationen herum oder außerhalb bilden sich durch Koordination und Kooperation Formen von Global Governance zwischen Staaten und privaten Akteuren (vgl. Benz 2004a: 23). Das Konzept des Global Governance bleibt aufgrund fehlender institutioneller Rahmenbedingungen sowie des Schattens der Hierarchie immer relativ instabil und durch Konflikte gefährdet (vgl. Benz 2004a: 23). Aufgrund dieser fehlenden Zentralinstanz bleibt es dem Staat weitgehend selbst überlassen, ob und wie Zielvereinbarungen umgesetzt werden (vgl. Behrens 2004: 104).

Eine sehr ausführliche, kritische Darstellung des Global Governance-Konzeptes wird von Brand et al. geboten, deren Kritikpunkte nun überblicksartig angeführt werden (Brand et al. 2000: 129ff).

- Die Problembeschreibungen bleiben deskriptiv und es fehlt an der Klärung der Problemursachen.

- Die „Weltprobleme“ werden durch technokratische Ansätze, wie beispielsweise durch die Einführung von Besteuerungsmaßnahmen oder die Errichtung neuer internationaler Konventionen, zu lösen versucht. Es soll zu einer „Netzwerkgesellschaft“ kommen, die aber keineswegs hierarchiefrei ist und außerdem $\mathrm{zu}$ komplexeren und intransparenteren Strukturen führen wird.

- Die einzelnen NGOs werden als so genannte „third hand“ mit den anderen Akteuren gleichgestellt. Dies entspricht nicht der Realität, da die NGOs weder eine homogene Gruppe bilden, noch die gleiche Stellung wie Regierungseinheiten haben. Außerdem ist der NGO-Sektor in den Entwicklungsländern noch immer unterentwickelt und daher auch bei globalen Konferenzen unterrepräsentiert.

- Problematisch an allen Governance-Konzepten ist, dass sie die Eigenständigkeit von Ökonomie und Politik verabsolutieren und eine Dichotomie zwischen den beiden konstruieren. Nach diesen Ansätzen ist im Zeitalter der Globalisierung die Handlungsmacht bereits zu Gunsten der Ökonomie entschieden. Sie vergessen dabei, dass der Markt maßgeblich politisch konstruiert ist.

- Ein besonders schwerwiegender Mangel der Governance-Konzepte ist, dass die demokratische Legitimation des Projekts, dem es ja immerhin um weit reichende politische Implikationen geht, kaum reflektiert wird. Die Frage nach globalen, transnationalen Formen von Demokratie wird entweder ausgeklammert oder einer wenig wahrscheinlichen ,globalen Bürgergesellschaft" vorbehalten.

- Der Global Governance-Diskurs erweist sich als geschlechtsblind und übersieht die mit der Globalisierung einhergehende Verschlechterung der Arbeitsverhältnisse für Frauen. 
Brand et al. (2000: 157) resümieren wenig schmeichelhaft „Sie [die Global Governance-Varianten] sind kaum mehr als die allzu versöhnlerische Utopie einer Weltgesellschaft, in der alle widerstreitenden Interessen durch freiwillige Einsicht und durch Verhandlungsprozesse unter Aufsicht der Staaten zu allseitiger Zufriedenheit gelöst werden “.

\subsection{Zusammenfassung}

Ausgehend von einer Einführung in die Thematik, werden in diesem Kapitel zwei wesentliche Governance-Konzepte vorgestellt. Governance wird in der Literatur als „notoriously slippery“ beschrieben (vgl. Pierre/Peters 2000:7). Es lässt sich als die Entscheidungsfindung in Institutionen und die Steuerung bzw. Koordination von Interdependenzen zwischen Akteuren beschreiben. Wurde im Neo-Liberalismus vor allem ein schlanker Staat gefordert, wird die Rolle des Staates im Governance-Konzept neu definiert und es wird zwar ein eher schlanker, aber dennoch aktiver Staat gefordert, der für sein Verhalten zur Rechenschaft herangezogen werden kann.

Good Governance wurde von der Weltbank als ein normatives Konzept entwickelt, das sich mit guter Regierungsführung beschäftigt. Um einen Einblick in die Genese dieses Begriffs zu bekommen, wird das Konzept in eine historisch-theoretische Darstellung eingebettet. Das Good Governance-Konzept wurde in den 1990er Jahren als Antwort auf die unerfreulichen Ergebnisse aus den Reformen des Washington-Consensus verwendet. Mit dem WashingtonConsensus und dem Good Governance-Konzept sind die Theorien des New Public Management (NPM) eng verbunden. Zwei Maßnahmen im Zuge des NPM stellen die Privatisierung und Dezentralisierung dar, die zu einer effizienteren Infrastrukturversorgung führen sollen, um das Wirtschaftswachstum anzukurbeln. Diese Maßnahmen haben die Veränderungen bei der Finanzierung der Infrastruktur nachhaltig beeinflusst.

Nach anfänglicher Euphorie über das Good Governance-Konzept ist nach Ablauf einiger Jahre etwas Ernüchterung eingetreten. Ein wesentlicher Grund dafür ist die Erkenntnis, dass ,gute Staatsführung“ schwer messbar ist. Daher veränderte sich der Ansatz der Weltbank dahingehend, dass Good Governance nun eine Determinante bei der Konditionalisierung für die Kreditvergabe darstellt. Der Vorstellung des Konzepts folgt daher eine kritische Diskussion.

Global Governance beschäftigt sich mit den Auswirkungen der Globalisierung. In diesem Konzept wird versucht eine ,globale Strukturpolitik“ zu gestalten, um globale Probleme, wie beispielsweise Armut, in den Griff zu bekommen. Es beruht auf der Annahme, dass es keinen zentralen Akteur mehr gibt, dem alleine die Steuerungs- und Kontrollfähigkeit zugesprochen wird, sondern dass die Entscheidungsprozesse interaktiv zwischen staatlichen und 
nichtstaatlichen Akteuren stattfinden. Der Bedarf nach einer globalen Steuerung wird zwar gutgeheißen, aber die Wahrscheinlichkeit einer Umsetzung des Konzepts wird sehr unterschiedlich gesehen. Als Hauptgrund für das Scheitern einer Weltpolitik gelten die nationalen Interessen, die immer über dem globalen Wohl stehen werden. Von den Kritikern wird Global Governance daher als Utopie betrachtet, da Nationalstaaten in erster Linie immer für das eigene Wohl handeln. 
Bianca Gusenbauer - 978-3-631-75445-0

Downloaded from PubFactory at 01/11/2019 04:47:32AM

via free access 


\section{Private Infrastrukturinvestitionen in Entwicklungs- ländern}

Das dritte Kapitel beschäftigt sich mit privaten Investitionen in der Infrastruktur, wobei der Arbeitsfokus auf den Entwicklungsländern liegt. Im ersten Kapitel wird eine Begriffsabgrenzung vorgenommen und die Begriffe Ausländische Direktinvestition, Infrastruktur und Armut werden für die Arbeit definiert. Das Kapitel 3.2 gibt einen theoretischen Überblick über die Auswirkungen von Ausländischen Direktinvestitionen (ADI) auf die Entwicklungsländer und geht dabei auf Wachstum und Spillover-Effekte ein. Im Kapitel 3.3 werden die direkten und indirekten Auswirkungen von Infrastruktur allgemein und von Energie- und Transportinfrastruktur im Besonderen auf die Armutsreduktion präsentiert. Die folgende Abbildung 10 stellt grafisch den Aufbau dieses Kapitels vor und dient der besseren Orientierung.

Abbildung 10: Kapitelübersicht Private Investition in die Infrastruktur in Entwicklungsländern

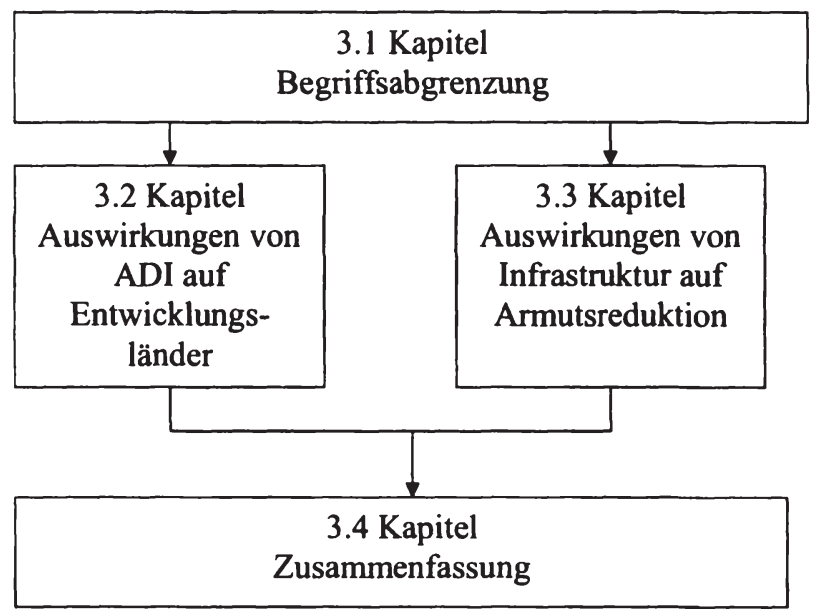

\subsection{Begriffsabgrenzung}

\subsubsection{Definition von Ausländischer Direktinvestition}

Im Infrastruktursektor können inländische und ausländische Investitionen von privater und öffentlicher Hand getätigt werden (vgl. Ebner 1996: 24). Der Begriff der privaten Investitionen (private investment) kommt insbesondere in 
der englischsprachigen Literatur sehr häufig als Überbegriff vor. Im Bereich der privaten Investitionen wird wiederum zwischen zwei möglichen Arten der Investition unterschieden, nämlich Portfolio- und Direktinvestitionen.

Die vorliegende Arbeit konzentriert sich jedoch ausschließlich auf direkte ausländische Privatinvestitionen, die im Deutschen als Ausländische Direktinvestitionen (ADI) oder Direktinvestitionen (DI) bezeichnet werden, wobei bei DI meist implizit angenommen wird, dass es sich um Auslandsinvestitionen handelt und auf den Zusatz ,ausländisch“ verzichtet wird (vgl. Kutschker/Schmid 2004: 82). In Abbildung 11 wird die Einteilung der ausländischen Direktinvestitionen nochmals grafisch dargestellt.

\section{Abbildung 11: Einordnung der ausländischen Direktinvestitionen}

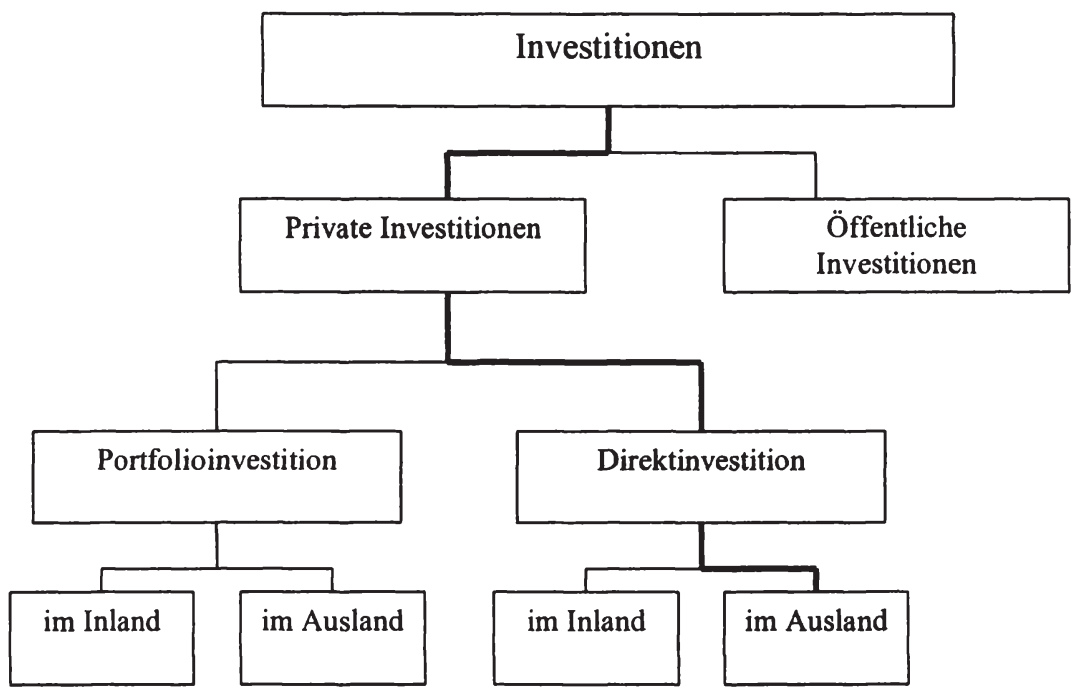

In der Literatur hat sich keine allgemein gültige Definition durchgesetzt (vgl. De Mello 1997), vielmehr wird die Abgrenzung oftmals durch eine Gegenüberstellung der beiden Begriffe vorgenommen. Die Begriffsabgrenzung wird laut Ebner (1996: 24) vor allem anhand von drei verschiedenen Kriterien vorgenommen und zwar

- das Kriterium Managementeinfluss,

- ein quantitatives Kriterium sowie

- das Kriterium der Zeitspanne. 
Der Begriff der Direktinvestitionen oder Ausländischer Direktinvestitionen (ADI) wird in der Literatur nicht einheitlich definiert bzw. werden unterschiedliche Schwerpunkte auf die jeweiligen Ausprägungen gelegt. In vielen Literaturquellen wird die Einflussnahme auf die Geschäftstätigkeit des Unternehmens als wesentliches Merkmal von DI angegeben (vgl. u.a. Bea 1995: 5f, Plum 1995:5; Kutschker/Schmid 2004: 80). Dieses Kontrollmotiv wird in der Literatur überbetont und wie Ebner (1996: 24) herausarbeitet, stellt es nur eines von drei Kriterien dar, die zur Abgrenzung herangezogen werden können.

Beispielsweise berücksichtigt Shapiro (2003: 173) auch eine Quantifizierung der Direktinvestition, die wiederum die Einflussnahme operationalisieren soll: "Direct investments are those where managerial control is exerted ... ownership of at least $10 \%$ of the equity". Diese $10 \%$-Hürde wird auch von der OeNB (2007), der OECD (1999) und dem IWF (1993) als wesentlicher Faktor für die Einflussnahme gesehen. Laut Plum (1995: 7) lässt sich jedoch aufgrund der Beteiligungshöhe nicht zwingend auch das Ausmaß der Kontrolle bestimmen. Auch Bellak (1989: 18) vertritt die Auffassung, dass die Höhe der Beteiligung nicht für den Einfluss entscheidend sein muss. Oftmals kann auch über eine Minderheitsbeteiligung effektiver Einfluss auf die Unternehmensführung genommen werden.

Zeitlich werden Direktinvestitionen den langfristigen Investitionen zugeordnet, da u.a. durch das hohe Investitionsvolumen eine längere Beständigkeit verfolgt wird. Laut IWF wird der Zeitbezug folgendermaßen definiert (1993: 86) „DI ... obtaining a lasting interest in an enterprise resident in another economy". Fast wortgleich liest sich auch die Definition der OECD (1999: 7) ",... objective of obtaining a lasting interest... “.

Basierend auf den vorhergehenden Ausführungen lassen sich folgende Charakteristika für DI festlegen:

- es wird ein Managementeinfluss verfolgt,

- eine Beteiligung von $10 \%$ wird in der Literatur meist als Mindestbeteiligung für DI betrachtet, obwohl eine höhere Quote nicht unbedingt zu einem erhöhten Managementeinfluss führen muss und

- sie werden für einen längerfristigen Zeitraum getätigt.

\subsubsection{Definition von Infrastruktur}

Mit dem Überbegriff Infrastruktur werden in der Literatur verschiedene Infrastruktursektoren zusammengefasst. Es existieren verschiedene Varianten der Untergliederung und daher existiert keine einheitliche Definition von Infrastruktur. In der Mehrzahl der Erklärungsansätze wird für Infrastruktur der öffentlich bereitgestellte Kapitalstock subsumiert (vgl. Kolodziej 1996: 23). 
Im deutschen Sprachraum hat sich vor allem die Untergliederung nach Jochimsen (1966: 103ff) durchgesetzt, der institutionelle, personelle und materielle Infrastruktur unterscheidet. Der Ausbau bzw. die Verbesserung aller drei Aspekte ist für die Entwicklung und das Wachstum eines Landes wichtig.

- Materielle Infrastruktur: umfasst die Verkehrs-, Telekommunikations- und Energieeinrichtungen, aber auch Gebäude und Einrichtungen der staatlichen Verwaltung, des Erziehungs- und Forschungs- sowie des Gesundheits- und Fürsorgewesens. Sie erstellen Vorleistungen, die in die Produktion derjenigen Güter und Dienste eingehen, welche die Endnachfrage aufnimmt. Diese Unterkategorie der Infrastruktur wird in den Wirtschaftswissenschaften auch als Social Overhead Capital bezeichnet.

- Institutionelle Infrastruktur: umschreibt die gesetzlichen und gewachsenen Normen sowie Einrichtungen und Verfahrensweisen der öffentlichen Verwaltung, Polizei und Justiz.

- Personale Infrastruktur: umfasst die geistigen, unternehmerischen, handwerklichen und sonstigen Fähigkeiten der Menschen in einer Gemeinschaft.

Die Organisationen in der Entwicklungspolitik verwenden unterschiedliche Definitionen, die in Folge kurz dargestellt werden.

Die Weltbank und die OECD (vgl. DAC 2006a: 23, World Bank 1994: 17f) unterscheiden zwei Kategorien bei der Infrastruktur:

- Wirtschaftliche Infrastruktur: wird wiederum unterteilt in physische Infrastruktur (Straßen, Energieerzeugung und Wasseranschlüsse) und Dienstleistungen (Transportdienstleistungen, Energie- und Wasserversorgung).

- Soziale Infrastruktur: umfasst alle Investitionen in Gesundheit, Bildung und Kultur.

Die $A D B$ (2007: 43) unterteilt Infrastruktur in

- Physische Infrastruktur: berücksichtigt alle Investitionen im Bereich Transport, Energie, Telekommunikation und Wasser.

- Soziale Infrastruktur: umfasst Maßnahmen, welche in die soziale Entwicklung eines Landes investieren, wie z.B. Governance, Gesundheit oder Bildung.

Bei den Vereinten Nationen (vgl. UN 2006: 5f) wird Infrastruktur in drei Kategorien unterteilt: 
- Infrastrukturbestand: umfasst das gesamte infrastrukturelle Anlagevermögen und kann wiederum unterteilt werden in Verkehr (z.B. Straßen, Zugverbindungen und Häfen), öffentliche Versorgungsbetriebe (z.B. Strom und Wasserversorgung) und Staatsdienstleistungen (z.B. Verteidigung und öffentliche Verwaltung).

- Infrastrukturdienstleistungen: stellen jene Infrastrukturinvestitionen dar, die für die Versorgung zuständig sind.

- Weiche Infrastruktur: bezeichnet die Investitionen in Bildungsund Gesundheitsinfrastruktur.

Die Definitionen sind einander sehr ähnlich, wobei die Verfasserin die Einteilung von OECD und Weltbank als Basis für diese Arbeit am hilfreichsten findet, da unter dem Begriff der wirtschaftlichen Infrastruktur alle Investitionen in die Anlagenerstellung und die Dienstleistungen berücksichtigt werden, diese aber dennoch getrennt behandelt werden. Dies widerspiegelt die Diskussion in Theorie und Praxis, in der die Beteiligung des privaten Sektors vor allem für die Anlagenerstellung diskutiert und umgesetzt wird.

Soziale Infrastruktur stellt hingegen keinen Anwendungsbereich für private Investoren dar und wird in der vorliegenden Arbeit nicht weiter behandelt. In der allgemeinen Diskussion um die Finanzierung von Infrastruktur durch den privaten Sektor wird der Begriff Infrastruktur ausschließlich für wirtschaftliche (auch materielle oder physische) Infrastruktur verwendet. Diese Definitionsbzw. Begriffslogik wird auch in dieser Arbeit beibehalten.

Die folgende Tabelle 1 gibt abschließend noch einen Überblick über die in diesem Kapitel vorgestellten Definitionen und hebt die in der vorliegenden Arbeit verwendete Definition grau hervor.

Tabelle 1: Zusammenfassung der Infrastrukturdefinitionen

\begin{tabular}{|c|c|c|c|c|}
\hline Jochimsen & \multicolumn{2}{|c|}{ Weltbank und OECD } & ADB & $\mathbf{U N}$ \\
\hline \multirow{2}{*}{$\begin{array}{l}\text { Materielle } \\
\text { Infrastruktur }\end{array}$} & \multicolumn{2}{|c|}{ Wirtschaftliche Infrastruktur } & \multirow{2}{*}{$\begin{array}{l}\text { Physische } \\
\text { Infrastruktur }\end{array}$} & Infrastruktur- \\
\hline & $\begin{array}{l}\text { Physische } \\
\text { Infrastruktur }\end{array}$ & $\begin{array}{l}\text { Dienstleist- } \\
\text { ungen }\end{array}$ & & $\begin{array}{l}\text { Infrastruktur- } \\
\text { dienstleistungen }\end{array}$ \\
\hline $\begin{array}{c}\text { Personale } \\
\text { Infrastruktur }\end{array}$ & \multirow{2}{*}{\multicolumn{2}{|c|}{ Soziale Infrastruktur }} & \multirow{2}{*}{ Soziale Infrastruktur } & \multirow{2}{*}{$\begin{array}{c}\text { Weiche } \\
\text { Infrastruktur }\end{array}$} \\
\hline $\begin{array}{l}\text { Institutionelle } \\
\text { Infrastruktur }\end{array}$ & & & & \\
\hline
\end{tabular}




\subsubsection{Definition von Armut}

Armut ist ein schwierig zu definierendes Wort und es gibt unterschiedliche Annahmen darüber, warum Menschen arm sind. Es gibt die radikale Ansicht, dass Armut eine inhärente Eigenschaft des Kapitalismus ist. Am anderen extremen Ende des Kontinuums behaupten die Neoliberalen wiederum, dass Armut nur dadurch entsteht, dass es Unvollkommenheiten am Markt gibt, die den Kapitalismus behindern (vgl. Hulme/Shepherd 2003).

Grundsätzlich unterscheidet man ein- und mehrdimensionale Armutsdefinitionen sowie dynamische Armutskonzepte, die an dieser Stelle überblicksartig vorgestellt werden. Obwohl die Armutsreduktion in den Entwicklungsländern das zentrale Ziel der Inter-nationalen Finanzinstitutionen darstellt, ist es teilweise nicht einfach, die Armutsdefinitionen der jeweiligen Institutionen $\mathrm{zu}$ finden. Meistens werden allgemeine, nicht operationalisierte Definitionen veröffentlicht.

\section{Eindimensionale Armutsdefinition}

Die nach wie vor am häufigsten verwendete Definition ist die eindimensionale und rein quantitativ definierte Armutsgrenze anhand des Pro-Kopf-Einkommens. International haben sich die Vereinten Nationen und die Weltbank auf folgende einheitliche Armutsgrenze geeinigt:

- Armut: weniger als 2 US\$ pro Tag (730 US\$ im Jahr) und

- Extreme Armut: weniger als 1 US\$ (365 US\$ im Jahr).

Regierungen und Ökonomen finden es nach wie vor hilfreich, sich bei der Messung von Armut auf das Einkommen zu konzentrieren, da dieses relativ einfach gemessen werden kann (vgl. ADB 2006d: 93). Auch das erste Ziel der Millennium Development Goals (MDGs) basiert auf dieser Armutsdefinition. Viele Länder setzen aber ihre eigene offizielle Armutsgrenze, auf Basis des ProKopf-Einkommens fest, da sie eine eigene Definition des lebensnotwendigen Minimums haben. Für internationale Vergleiche ist es aber wichtig, dass in allen Ländern die gleiche Definition verwendet wird. Die Messung der Armut ausschließlich auf Basis des Einkommens ist allerdings viel zu eng, da Armut ein mehrdimensionaler Zustand ist, der sich nicht nur auf das Einkommen beschränken lässt (vgl. Hettige 2006: 3, OECD 2001: 25). Deshalb wurden viele, sich großteils sehr ähnliche, multidimensionale Ansätze entwickelt, von denen nun eine kleine Auswahl dargestellt wird.

\section{Multidimensionale Armutsdefinition}

Von den einzelnen Organisationen werden zusätzlich zu der eindimensionalen Armutsdefinition andere Dimensionen für die Armutsdefinition herangezogen. An dieser Stelle wird stellvertretend für alle anderen Institutionen eine Auswahl 
an Institutionen dargestellt. Laut Willoughby (2004b) können die verschiedenen Erweiterungen der einzelnen Institutionen im Wesentlichen in drei Dimensionen erfasst werden:

- das Fehlen von Einkommen und ökonomischer Sicherheit,

- der fehlende Zugang zu Infrastrukturleistungen und

- der fehlende Respekt durch andere und die fehlende politische Macht in der Gesellschaft.

Die Weltbank hat erkannt, dass die Messung der Armut anhand des Pro-KopfEinkommens unzureichend ist und daher wurde im Jahr 2000 eine Untersuchung mit dem Namen "Voices of the Poor" durchgefuihrt, in der die Betroffenen Armut definieren. Basierend auf der Auswertung dieser qualitativen Definitionen hat auch die Weltbank den rein einkommensbasierten Ansatz erweitert, indem bei den Programmen nicht mehr ausschließlich auf eine Erhöhung des Pro-Kopf-Einkommens, sondern auch auf die Förderung von

- „Opportunities“,

- „Security“ und

- „Empowerment" geachtet wird (vgl. World Bank 2001a).

Auch das UNDP definiert ergänzend zu der einkommensorientierten Definition den Human Development Index (HDI), der die durchschnittlichen Lebensbedingungen der Menschen in einem Land ermittelt. Der HDI ist eine $\mathrm{Zu}$ sammensetzung von folgenden drei Indikatoren:

- der Lebensdauer,

- der Alphabetisierung und

- der Kindersterblichkeit.

Das UNDP folgt dem globalen Trend und berücksichtigt nun auch stärker die verbesserte Governance und die Partizipation der Armen als wesentliche Kriterien zur Armutsreduktion (vgl. UNDP 2000). Das Konzept von „Human Development" ist sehr umfangreich und wird natürlich von mehr als drei Indikatoren beeinflusst, jedoch ist eine gesamte Erfassung aller Indikatoren schwierig.

Von der OECD (2001: 25) wird Armut allgemein definiert als ,the inability of people to meet economic, social and other standards of well-being “. Im multidimensionalen Konzept der OECD werden folgende fünf Größen für die Beschreibung von Armut herangezogen, die sich wechselseitig beeinflussen (vgl. OECD 2006c: 16, 2001: 26): 
- Economic capability: beschreibt die Möglichkeit Geld zu verdienen und konsumieren zu können.

- Human capability: basiert auf Gesundheit, Ausbildung, Nahrung, sauberem Wasser und Wohnraum.

- Political capability: beschreibt die Einflussnahme der Bevölkerung auf Entscheidungen und die Menschenrechte in einem Land.

- Socio-cultural capability: beschäftigt sich mit der Möglichkeit, als geschätztes Mitglied in der Gesellschaft wahrgenommen zu werden.

- Protective capability: ermöglicht es Menschen sich vor wirtschaftlichen und anderen externen Schocks zu schützen.

Die folgende Abbildung 12 zeigt den multidimensionalen Ansatz der OECD, beim dem sich die verschiedenen Armutsdimensionen beeinflussen und daher nicht isoliert verändert werden können. Beispielsweise können Haushalte ein geringes Einkommen haben und sind deswegen auch verstärkt Risiken ausgesetzt, da sie nicht die ausreichenden finanziellen Ressourcen haben, sich zu schützen. Durch das geringe Einkommen können sie sich keine Schulbildung leisten und haben nur einen geringen sozialen Status, der ihnen wiederum nur geringe Rechte und Freiheiten zugesteht. Die Tatsache, dass die verschiedenen Armutsdimensionen sehr stark und unterschiedlich zusammenhängen, stellt den Hauptgrund für einen multidimensionalen Ansatz dar.

\section{Abbildung 12: Der multidimensionale Armutsrahmen der OECD}

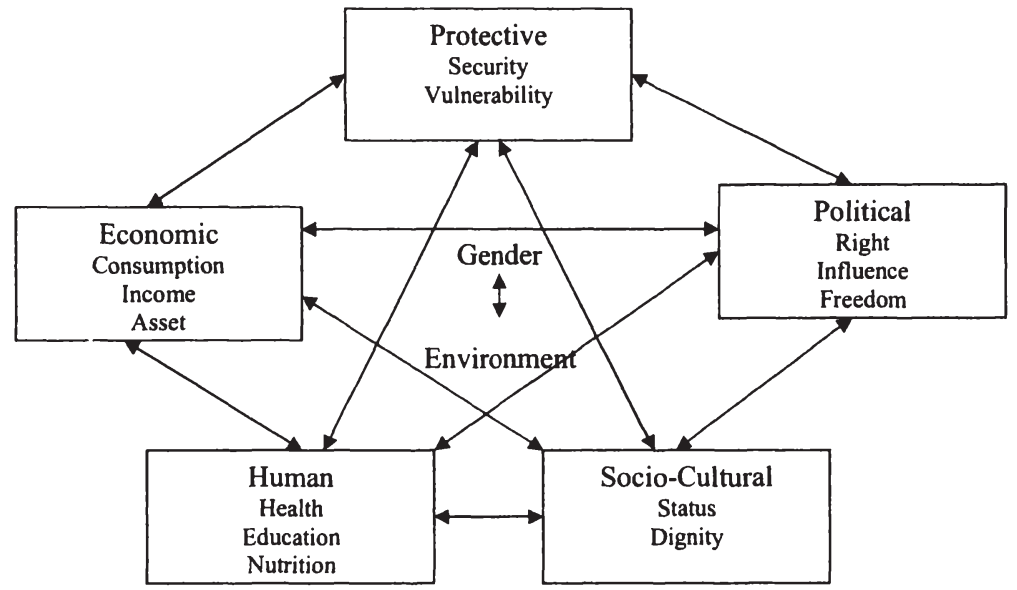

Quelle: OECD 2006: 16 
Die ADB (Asian Development Bank) definiert Armut allgemein als „a deprivation of essential assets and opportunities to which every human is entitled" (ADB 2005a: 7). Jeder soll Zugang zu einer Grundausbildung und zu grundlegender Gesundheitsversorgung haben. Darüber hinaus sollen die Individuen neben einer angemessenen Entlohnung aber auch an Entscheidungsprozessen teilnehmen können, die ihr Leben beeinflussen. Armut sollte laut ADB (2005a) daher umfangreicher und zwar in Form von

- Grundausbildung,

- Gesundheitsversorgung,

- Nahrung,

- Wasserversorgung und

- Einkommen gemessen werden.

Abschließend zu der Vorstellung dieser ein- und multidimensionalen Armutsdefinitionen muss jedoch festgehalten werden, dass Armut, aufgrund der relativ einfachen Durchführbarkeit, nach wie vor hauptsächlich auf Basis des Pro-Kopf-Einkommens gemessen wird, wie beispielsweise bei den Millennium Development Goals (MDGs). Problematisch im Zusammenhang mit dem ersten MDG sehen Hulme/Shepard (2003) die Tatsache, dass Armut undifferenziert in den MDGs behandelt wird und Arme als eine homogene Gruppe gesehen werden. Dadurch werden alle Armen einer Kategorie zugerechnet und nur über eine Ausprägung, nämlich ihr geringes Einkommen, definiert. Die Maßnahmen zur Armutsreduktion bei einer einheitlichen Definition setzen wiederum nur beim zu geringen Einkommen an und daher werden Programme entwickelt, die zu einem höheren Einkommen führen sollen. Dadurch erreichen solche Maßnahmen nur eine beschränkte Gruppe von Armen und es erscheint sinnvoller, die Armut genauer zu analysieren, um die Maßnahmen zielgerichteter einsetzen zu können. Daher unterteilen Hulme/Shepard (2003) Armut in kurzlebige, strukturelle und chronische Armut:

- Die kurzlebige Armut ist häufig das Ergebnis von kurzfristigen Schocks, wie Krieg, Finanz- oder Umweltkrisen, welche die Menschen, die nahe an der Armutsgrenze leben, wieder unter die Armutsgrenze befördern kann (vgl. ADB 2005c: 13).

- Chronische Armut kann durch verschiedene Faktoren entstehen, die das Individuum oder den ganzen Haushalt chronisch in Armut leben lässt. Dies können beispielsweise die ethnische Zugehörigkeit oder auch physische oder geistige Behinderung sein (vgl. ADB 2005c: 13, Jalan/Ravallion 2000).

- Die Strukturelle Armut ist im Gegensatz dazu das Ergebnis von 
fehlenden Möglichkeiten (opportunity) und einer geografischen Abhängigkeit (vgl. Jalan/Ravallion 2002).

Diese Unterteilung signalisiert, dass Armut ein dynamisches Konzept ist und dass Menschen sich von einer Armutskategorie in eine andere bewegen können (vgl. ADB 2005c: 13). In der Studie der ADB (2005a: 194) wird insbesondere auch darauf hingewiesen, dass Armut kein Merkmal eines Dorfes bzw. einer Gemeinschaft ist, sondern das eines einzelnen Haushalts. Dies bedeutet, dass Armut nicht ausreichend auf Basis von aggregierten Daten einer Region oder eines Dorfes erhoben werden kann. Basierend auf dieser Definitionserweiterung, müssen sich effiziente Maßnahmen zur Verringerung der Armut beim jeweiligen Armutskonzept unterscheiden. Infrastruktur kann insbesondere zur Reduktion struktureller Armut beitragen, dá sie, beispielsweise durch bessere Straßen, zu einer Reduktion der geografischen Abhängigkeit beitragen kann.

Zusammenfassend kann daher festgehalten werden, dass Infrastruktur in allen multidimensionalen Armutsdefinitionen enthalten ist und insbesondere positive Auswirkungen auf die Reduktion der strukturellen Armut hat. Wenn in Folge von ,,den Armen " oder der ,armen Bevölkerung “ gesprochen wird, so werden darunter jene Menschen subsumiert, welche laut einkommensbasierter Armutsdefinition der Weltbank und der Vereinten Nationen unter der Armutsgrenze leben. Der Grund dafür ist, dass bei Studien oft Daten herangezogen werden, die auf dem Pro-Kopf-Einkommen basieren, und um Missverständnisse zu vermeiden, wird diese eindimensionale Definition beibehalten. Dennoch ist sich die Autorin der Unzulänglichkeit dieser Definition bewusst und daher werden bei der Diskussion über die Auswirkungen der Infrastrukturinvestitionen auf die Armutsreduktion auch andere qualitative Dimensionen diskutiert.

\subsection{Auswirkungen von ADI auf Entwicklungsländer}

In den folgenden Unterkapiteln werden die Auswirkungen von Ausländischen Direktinvestitionen (ADI) auf Entwicklungsländer diskutiert. Im ersten Unterkapitel werden in der Einleitung die Entwicklung und die Fragestellungen im Zusammenhang mit ADI dargestellt. Daran anschließend werden in den Kapiteln 3.2.2 und 3.2.3 eine Literaturübersicht über die Spillover-Effekte und die Auswirkungen von ADI auf das Wachstum im Gastland gegeben. Im Fokus dieser Literaturübersicht liegt der Schwerpunkt auf den Auswirkungen von ADI in Entwicklungsländern. Dennoch werden auch Publikationen herangezogen die Schwellenländer behandeln, da die verwendeten Daten oftmals weit in die Vergangenheit reichen und die Länder zum damaligen Zeitpunkt noch weniger entwickelt waren. 


\subsubsection{Einführung}

Der weltweite Boom an Ausländischen Direktinvestitionen (ADI) begann in den 1980er Jahren und erreichte die Entwicklungsländer zu Beginn der 1990er Jahre mit zehn Jahren Verspätung. Laut De Mello (1997) kann der Anstieg an ADI durch den Kauf von lokalen Firmen im Zuge des Privatisierungsprozesses, der Globalisierung der Produktionsprozesse und durch die zunehmende Verflechtung im Finanzsektor erklärt werden. 1999 erreichte die Höhe an ADI in Entwicklungsländern ihren bisherigen Höhepunkt. Palmade/Anayiotas (2004) erklären den darauf folgenden, in manchen Sektoren dramatischen Rückgang an ausländischen Investitionen, durch die Anfang der 1990er Jahre im Zuge der durchgeführten Privatisierungen einmalig getätigten, enormen Investitionen in den Bereichen der Infrastruktur, des Finanzsektors und des Erdöls. Dennoch liegen die ADI in den Entwicklungsländern noch weit hinter dem Investitionsvolumen in den entwickelten Ländern.

In der Diskussion über ADI wird in der Theorie ein besonderes Augenmerk auf die Auswirkungen im Gastland gelegt. Nur bei wenigen Fragestellungen in dieser Diskussion kommt man jedoch zu einem einheitlichen Ergebnis. Als gegeben kann allerdings angenommen werden, dass ADI generell Auswirkungen auf das Gastland haben, die sowohl auf makro-, als auch auf mikroökonomischer Ebene auftreten können. Eine geteilte Meinung über die Richtung der Auswirkungen, d.h. ob sie positiv oder negativ für das Gastland sind, spiegelt sich unter anderem in der großen Anzahl an Publikationen zu diesem Thema wider.

Insbesondere Regierungen in Entwicklungsländern messen ADI als Instrument zur Verbesserung der wirtschaftlichen Situation des Gastlandes besondere Bedeutung bei. Als Vorteile werden auf makroökonomischer Ebene das neue Investitionskapital, der Beitrag zum Ausgleich der Zahlungsbilanz und zum zukünftigen wirtschaftlichen Wachstum gesehen (siehe Kapitel 3.2.3). Auf mikroökonomischer Ebene werden der Technologietransfer, eine höhere $\mathrm{Be}$ schäftigungsrate, der Transfer von Managementwissen sowie die Investition in die materielle und personale Infrastruktur genannt (vgl. Moss et al. 2005: 338). Insbesondere als Vehikel zum Technologietransfer in die Entwicklungsländer werden ADI diskutiert, wobei man in der Literatur sehr unterschiedliche Ergebnisse findet, die im Kapitel 3.2.2 noch genauer diskutiert werden. Diese Diskussion ist für die vorliegende Arbeit besonders interessant, da im Infrastruktursektor der Technologietransfer eine wesentliche Rolle spielt.

Beginnend in den 1990er Jahren, verbesserten die Regierungen in den Entwicklungsländern daher ständig die rechtlichen Rahmenbedingungen für ausländische Investitionen. Laut der jährlichen Erhebung von UNCTAD (2003: 21) über die Veränderungen von nationalen Gesetzgebungen für ADI wurden von 1991 bis 2002 über 1.500 Gesetze und Verordnungen zugunsten und nur weniger als 100 zum Nachteil von ADI verändert. Viele Regierungen gehen sogar weiter und gewähren den multinationalen Unternehmen Steuer- oder 
Investitionsvorteile bzw. vorteilhaftere Konditionen als den heimischen Unternehmen. Dies kann unter anderem zu einer Diskriminierung der heimischen Unternehmen führen (vgl. Hanson 2001, Woodward 2001: 162ff). Laut Hanson (2001) lassen sich daher Vergünstigungen nur unter gewissen Voraussetzungen wirtschaftlich rechtfertigen.

Vorwegzunehmen ist, dass bei den meisten Studien, die nun in den folgenden zwei Abschnitten herangezogen werden, nicht auf den Entwicklungsstand des jeweiligen Ziellandes Rücksicht genommen wurde. Dies wird insbesondere in der neueren Literatur zu ADI kritisiert und daher gefordert, dass Aussagen, die in solchen gemischten Untersuchungen getroffen wurden, nicht verallgemeinerbar sind (vgl. Blonigen/Wang 2005: 221, Carkovic/Levine 2005: 196, Nunnenkamp 2004). Agosin/Mayer (2000) warnen vor einer simplifizierten Wahrnehmung von ADI und politischen Maßnahmen, wie beispielsweise der Gewährung von Investitionssubventionen, die höchst wahrscheinlich zu keinen optimalen wirtschaftlichen Ergebnissen führen werden.

\subsubsection{ADI und Spillover-Effekte}

Spillover-Effekte sind jene externen Effekte oder Externalitäten, die von ADI auf das Umfeld und die Produktivität von lokalen Unternehmen einwirken können (vgl. Blomström/Sjöholm 1999). In der Literatur wird darunter u.a. der Technologietransfer verstanden. Spillover-Effekte entstehen dadurch, dass beispielsweise das Wissen von multinationalen Unternehmen nicht vollständig geschützt werden kann und es durch unbeabsichtigten oder beabsichtigten Austausch in die lokale Wirtschaft diffundiert (vgl. Burger 1998: 13f).

\section{Existenz von Spillover-Effekten}

Die Mythen, die sich um die Spillover-Effekte ranken, gleichen denen des Trickle-Down-Ansatzes in der Entwicklungszusammenarbeit - es gibt kaum Beweise für deren Existenz, aber trotzdem werden sie ausgiebig diskutiert und als Entscheidungsgrundlage für die Gewährung von Investitionsanreizen herangezogen (vgl. u.a. Hanson 2001, Aitken/Harrison 1999). Laut Javorcik/Spatareanu (2005: 70): „...there is little conclusive evidence on the existence of spillovers in developing countries. It is a complex issue with no easy answers".

Weiters betonen Javorcik/Spatareanu (2005: 70) auch den Unterschied von Spillover-Effekten bei Entwickelten und Entwicklungsländern „,...we caution researchers about using limited evidence to draw generalized conclusions aboutthe existence of externalities associated with FDI in developing countries". Genau zu diesem Schluss kommt man auch, wenn man die Ergebnisse der Studien von Aitken/Harrison (1999), Chuang/Lin (1999) und Cheng et al. (2004) vergleicht. Aitken/Harrison (1999: 605) stellen als Ergebnis 
in ihrer Unter-suchung fest "We find no evidence supporting the existence of technology „spillovers " from foreign firms to domestically owned firms “. Auch Cheng et. al (2004: 480) befürworten die Spillover-Effekte von ADI und vertreten folgende Meinung: „Multinational Enterprises are the only vehicle of technology transfer between a technologically advanced region, the "North", and a technologically backward region, the "South". Interessanterweise stammen beide Autorengruppen, die in ihren Studien zu positiven Ergebnissen bei den Spillover-Effekten kommen, aus dem asiatischen Raum.

Einen zusammenfassenden Überblick über die unterschiedlichen Auswirkungen von $\mathrm{ADI}$ in Entwicklungsländern geben Agosin/Maỳer (2000). Sie zeigen in ihrer Studie, dass ADI ein starkes Crowding-Out von lokalen Investitionen in Lateinamerika, ein abgeschwächteres Crowding-Out in Afrika und ein starkes Crowding-In in Asien verursacht haben. Auch Kumar/Pradhan (2002) beschäftigen sich mit den Auswirkungen für lokale Unternehmen und kommen in ihrer Untersuchung zu dem Ergebnis, dass es im Allgemeinen eher durch ADI zu Crowding-Out-Effekten kommt, es aber in einigen Ländern auch zu Crowding-In-Effekten gekommen ist. Die Ergebnisse gleichen denen von Agosin/Mayer (2002).

Ob Spillover-Effekte in einem Land realisiert werden können, hängt laut Theorie auch von den unterschiedlichen Entwicklungsniveaus der beiden beteiligten Länder ab. Spillover-Effekte können nicht realisiert werden, wenn die technologische Lücke zwischen den investierenden und den lokalen Unternehmen zu groß ist (vgl. Kumar/Pradhan 2002). Eine Mindestausstattung an personaler Infrastruktur wird als Voraussetzung für die Übertragung von Spillover-Effekten angesehen (vgl. Li et al. 2005, Blomström/Sjöholm 1999, Djankov/Hoekman 1998, Borensztein et al. 1997). Demzufolge können Entwickelte Länder mehr von ADI profitieren als Entwicklungsländer. Sjöholm (1999) findet Spillover-Effekte in Sektoren, die eine sehr hohe Konkurrenz aufweisen und schlussfolgert, dass, je größer die technologische Lücke zwischen lokalen und ausländischen Unternehmen in diesen Sektoren ist, auch die Spillover-Effekte an Größe gewinnen.

Abhängig von der Form der Zusammenarbeit mit lokalen Unternehmen, können die Spillover-Effekte unterschiedlich auftreten. So können die Effekte eine positive Auswirkung auf die direkten lokalen Partnerunternehmen haben, sie können aber nicht von anderen lokalen Unternehmen, die beispielsweise im selben Industriezweig tätig sind, absorbiert werden (vgl. Djankov/Hoekman 1998). Dies wird u.a. auch von Aitkten/Harrision (1999: 605) bestätigt „,The gains from foreign investment appear to be entirely captured by joint ventures ". Des Weiteren sind laut Blomström/Sjöholm (1999) nur jene Unternehmen von Spillover-Effekten betroffen, die ausschließlich für den Heimatmarkt produzieren, da exportorientierte Unternehmen bereits der internationalen Konkurrenz ausgesetzt waren und sich dementsprechend ohnehin anpassen mussten. 


\section{Horizontale- und vertikale Spillover-Effekte}

Die Spillover-Effekte können laut Theorie horizontal oder vertikal auf die heimischen Unternehmen übertragen werden. In den Untersuchungen kommt man u.a. zu dem Ergebnis, dass horizontale Spillover-Effekte sehr fraglich, wenn überhaupt existent, sind (vgl. Konings 2001, Djankov/Hoekman 2000, Aitken/Harrison 1999). Für die vertikalen Effekte werde in der Literatur mehr Beweise gefunden.

Als horizontale Spillover-Effekte bezeichnet man die Auswirkungen von ADI in der gleichen Branche, wobei man in der Literatur zwei entgegengesetzte Ausprägungsformen erkennt (vgl. Javorcik/Spatareanu 2005: 50ff, Aitken/Harrison 1999, Blomström/Sjöholm 1999, Sjöholm 1999):

- Das lokale Unternehmen kann seine Produktion durch Wissenstransfer erhöhen. Dies ist beispielsweise dadurch möglich, dass Arbeiter aufgenommen werden, die zuvor bei der ausländischen Firma tätig waren oder durch Beobachtung der Marketingstrategien des ausländischen Unternehmens.

- Das ausländische Unternehmen übernimmt Marktanteile des lokalen Produzenten. Dieser wird dadurch gezwungen seine Fixkosten auf eine kleinere Produktionsmenge aufzuteilen, er kann somit keine konkurrenzfähigen Preise mehr anbieten und wird langfristig gesehen den Markt verlassen.

Ausländische Unternehmen zahlen im Durchschnitt höhere Löhne als lokale Unternehmen und es wird daher für lokale Unternehmen äußerst schwierig, gut ausgebildetes Personal vom ausländischen Unternehmen abzuwerben. Es entsteht hier eher das Problem, dass die ausländischen Unternehmen das gute Personal vom Markt abziehen und nur noch die Negativselektion für die heimischen Unternehmen übrig bleibt. Weiters werden die ausländischen Unternehmen, insbesondere multinationale Unternehmen, ihr Fachwissen vor Außenstehenden zu schützen wissen. Aus den dargelegten logischen Gründen ist einfach nachzuvollziehen, warum horizontale Spillover-Effekte kaum stattfinden. Selbst in der Studie von Damijan et al. (2001), in der acht Transformationsländer im Zeitraum von 1994-1998 untersucht werden, die laut Theorie eine geringere Technologielücke als Entwicklungsländer aufweisen, kommt es zu keinen positiven horizontalen Spillover-Effekten für die lokalen Unternehmen. Die Studie kam sogar zu dem Ergebnis, dass es in drei Ländern sogar zu signifikanten Crowding-Out-Effekten kam.

Die Spillover-Effekte können also nicht von den lokalen Konkurrenten genützt werden, sondern die multinationalen Unternehmen geben das Wissen an lokale Sublieferanten weiter, um sich vor Ort qualitativ hochwertige Produkte zu günstigen Preisen zu sichern (vgl. Blalock/Gertler 2005: 74). Auch Markusen/ Venables (1997) kommen zu dem Schluss, dass ADI und die Konkurrenz am 
Markt dazu führen, dass die Gewinne der lokalen Unternehmen vermindert werden, es aber bei den Zulieferanten zu Kostenreduktionen und Gewinnsteigerungen kommen kann. In diesem Fall spricht man von einem vertikalen Wissenstransfer.

Im Zusammenhang mit multinationalen Unternehmen, unterscheidet man bei den vertikalen Spillover-Effekten drei mögliche Erscheinungsformen (vgl. Blalock/Gertler 2005: 77ff, Javorcik/Spatareanu 2005: 54ff):

- Es gibt keine Spillover-Effekte, da die multinationalen Unternehmen nur jene lokalen Unternehmen als Sublieferanten nehmen, die ohnehin bereits eine fortgeschrittene Technologie anwenden. Diese Strategie wird in der Literatur auch ,Cherry picking “ genannt.

- Die lokalen Sublieferanten verbessern schon im Vorfeld ihre Produktion. Dies kann einerseits aufgrund von Bestrebungen des Unternehmens basieren oder durch Investitionsanreize durch das multinationale Unternehmen.

- Die Spillover-Effekte treten während der Zusammenarbeit zwischen dem lokalen Unternehmen und dem multinationalen Unternehmen auf.

Zusammenfassend kann festgehalten werden, dass die Existenz von horizontalen Spillover-Effekten in der Theorie stark bezweifelt wird und in den Untersuchungen sogar Crowding-Out-Effekte entdeckt wurden. Die vertikalen Spillover-Effekte werden am ehesten durch den Kontakt zwischen dem multinationalen Unternehmen und dem lokalen Sublieferanten vermutet. Diese Spillover-Effekte können durch direkten Wissenstransfer und durch Qualitätsauflagen erreicht werden. Weiters können für das lokale Unternehmen durch die erhöhte Nachfrage für Halbfertigprodukte die Erlöse steigen und es wird daher dazu motiviert, die Qualität zu steigern, um den Vertrag zu halten (vgl. Javorcik/ Spatareanu 2005: 48).

\subsubsection{ADI und Wirtschaftswachstum}

Wirtschaftswachstum nimmt in der Diskussion über die Auswirkungen von ADI auf die Entwicklungsländer eine zentrale Stellung ein, da in der Literatur angenommen wird, dass Wirtschaftswachstum einen Beitrag zur Armutsreduktion leisten kann. Jenkins/Thomas (2002: 12) stellen in ihrer Arbeit aber klar dar: "While economic growth is not synonymous with economic development, it is at least necessary. Provided that mechanisms exist to facilitate some trickle-down on the benefits of economic growth to the impoverished, economic growth can aid in poverty reduction".

In der Theorie wird oftmals ein sehr klarer Zusammenhang zwischen ADI und Wachstum dargestellt, welcher allerdings auf schwachen empirischen Ergebnissen basiert (vgl. Nunnenkamp 2004, De Mello 1997). Bei empirischen Unter- 
suchungen sind die Ergebnisse nämlich sehr widersprüchlich und, abhängig vom Untersuchungsdesign, kann dieser Zusammenhang bestätigt oder aber auch widerlegt werden (vgl. Carkovic/Levine 2005: 196ff). $\mathrm{Zu}$ unterscheiden sind mikro- und makroökonomische Untersuchungsdesigns sowie der Zeithorizont der Untersuchung und die beteiligten Länder.

Die Problematik der Verfügbarkeit und der Qualität der Daten in Entwicklungsländern führen oftmals zu dem Ergebnis, dass nur zu einem geringen Prozentteil Daten aus diesen Ländern verwendet werden (vgl. Balasumbramanyam et al. 1996). Die Ergebnisse werden aber so dargestellt, dass sie auch für Entwicklungsländer gültig sind (vgl. Bloningen/Wang 2005: 221, Carkovic/Levine 2005: 196). ${ }^{2}$ Des Weiteren stellt De Mello (1997) fest, dass der Zusammenhang von ADI und Wachstum abhängig von länder-spezifischen Faktoren ist, die aber in Zeitreihenanalysen nicht beobachtbar sind.

Im folgenden Abschnitt wird ein Literaturüberblick über die mikro- und makroöknomischen Untersuchungen, über die notwendigen Vorraussetzung für einen positiven Zusammenhang sowie den Zusammenhang von ADI und Wirtschaftswachstum gegeben.

\section{Mikro- und makroökonomische Untersuchungen}

Bei mikroökonomischen Untersuchungen, die auf Firmenebene stattfinden, kommt es häufig zu keinen positiven Ergebnissen. Beispielsweise kommen Aitken/Harrison (1999) in ihrer sehr einflussreichen Studie über venezolanische Unternehmen zu dem Ergebnis, dass ADI einen negativen Einfluss auf lokale Unternehmen haben. Auch Haddad/Harrison (1993) finden in ihrer Untersuchung keine wachstumsfördernden Spillover-Effekte in anderen Ländern. Blomström (1998) hingegen kommt in seiner Studie zu dem Ergebnis, dass mexikanische Sektoren mit einem hohen Anteil an ADI ein höheres Wachstum aufweisen. Fung-Yee Ng/Tuan (2006) können auch für die Region Guangdong in China ein ADI-induziertes Wachstum erkennen.

Die makroökonomischen Studien geben die optimistische Sichtweise der Auswirkungen von $\mathrm{ADI}$ wider und sprechen für einen positiven Zusammenhang zwischen ADI und Wachstum unter bestimmten Bedingungen (vgl. BendeNabende/Ford 1998). Allerdings müssen diese Ergebnisse sehr skeptisch betrachtet werden, da diese Studien oft die länderspezifischen Eigenheiten nicht berücksichtigen. Nair-Reichert/Weinhold (2001: 168) beispielsweise betonen dies in ihrer Untersuchung von 24 Entwicklungsländern ,....we find strong evidence in the data of considerable heterogeneity".

2 Dies wird u.a. auch von De Mello (1997: 30) bestätigt, welcher zwischen den Entwicklungsstufen der Länder unterscheidet : “... the degree of substitutability is higher in technologically advanced than developing recipient economies." 


\section{Voraussetzungen für Wirtschaftswachstum durch ADI}

Einige Studien kommen zu dem Ergebnis, dass ADI wirtschaftliches Wachstum unter bestimmten Voraussetzungen fördert (vgl. Chuang/Lin 1999, Borensztein et al. 1998, Blomström et al. 1994). In den Untersuchungen kommt es zu unterschiedlichen Annahmen, welche Voraussetzungen (,threshold-Hypothese') in einem Land gegeben sein müssen, damit ADI einen Beitrag zum Wachstum leisten kann. Schlussendlich kommen aber alle Untersuchungen zu dem Ergebnis, dass die Entwicklungsländer ein Mindestmaß an wirtschaftlicher Entwicklung bereits erreicht haben müssen, um von den positiven wachstumserhöhenden Auswirkungen von ADI profitieren zu können. (vgl. Nunnenkamp 2004, Bengoa/Sanchez-Roblez 2002).

Die am häufigsten zitierte Annahme ist die von Borensztein et al. (1998), die behauptet, dass es in Ländern mit gut ausgebildeten Arbeitskräften zu einem positiven Wachstum kommen kann. Bende-Nabende/Ford (1998) sehen nur einen indirekten Einfluss von gut ausgebildeten Arbeitskräften auf das Wachstum. Blomström et al. (1994) widerlegen wiederum diese Aussage und können keinen signifikanten Beweis dafür finden, dass Ausbildung für Wachstum wichtig ist. Sie kommen hingegen $\mathrm{zu}$ einem positiven Zusammenhang zwischen ADI und Wachstum, wenn das Gastland ausreichend reich ist.

Alfaro et al. (2004) sehen einen entwickelten Finanzmarkt als Voraussetzung für schnelleres Wachstum. In die gleiche Richtung argumentieren auch Hermes/Lensink (2003), die positive Auswirkungen von ADI in Entwicklungsländern nur dann beobachten konnten, wenn diese das lokale Finanzwesen verbessert haben. Auch Durham (2004: 285) unterstützt die Annahme, dass die Auswirkungen von ADI wesentlich von der Entwicklung des Finanzmarktes eines Landes abhängig ist und spricht von der: „,.., absorptive capacity' of host contries, with particular respect to financial or institutional development".

Balasubramanyam et al. (1996) betonen, dass die Offenheit des Handelssystems eine Voraussetzung für Wachstum darstellt (vgl. auch BendeNabende/Ford 1998). Inwieweit staatliche Regulierungen das Wachstum durch ADI beeinflussen, wird von Busse/Groizard (2006) untersucht. Sie kommen zu dem Ergebnis ,,...that excessive regulations 'restrict growth through FDI only in the most regulated economies ", welches be-deutet, dass gute gesetzliche Regelungen einen indirekten Beitrag zum wirt-schaftlichen Wachstum leisten können (Busse/Groizard 2006: 1).

Zusätzlich zu den bereits genannten Annahmen hängt laut der Studie von De Mello (1997: 29) die Richtung des Zusammenhanges zwischen ADI und Wachstum auch noch von der Faktorausstattung und der Größe eines Landes ab: ,...the direction of causation between FDI and growth may also depend on 
existing factor endowments and scale effects, such that larger economies are more attractive to FDI than smaller ones".

\section{Zusammenhang von ADI und Wirtschaftswachstum}

Aktuellere Studien bezweifeln die positiven Auswirkungen von ADI auf Wachstum. Laut Untersuchungen von Choe (2003) und Chowdhury/Mavrotas (2005) ist ein Zusammenhang zwischen Wachstum und ADI in beide Richtungen möglich, d.h. ADI kann Wachstum schaffen, aber Wachstum kann auch die Voraussetzung für mehr ADI sein. Laut Choe (2003) ist es aber wahrscheinlicher, dass Wachstum eine Voraussetzung für mehr ADI ist. Chowdhury/Mavrotas (2005) stellen beispielsweise in ihrer Studie auch den klaren Zusammenhang dar, dass in Chile wirtschaftliches Wachstum ADI anzieht und nicht umgekehrt. Für Malaysien und Thailand hingegen wird eine Beziehung in beide Richtungen gefunden. Hansen/Rand (2006) weisen außerdem darauf hin, dass lokale Investitionen in der gleichen Weise wachstumsfördernd wirken können wie ADI.

$\mathrm{Li} / \mathrm{Liu}$ (2005) berichten nicht nur von direkten, sondern auch von indirekten positiven Auswirkungen von ADI auf Wachstum, die durch Interaktionen mit dem Humankapital stattfinden. Sie weisen außerdem explizit darauf hin, dass diese Ergebnisse auch für Entwicklungsländer gelten.

Carkovic/Levine (2005: 197) hingegen finden in ihrer aktuellen Studie keinen robusten positiven Zusammenhang zwischen ADI und langfristigem Wachstum „Specifically, there is no reliabel cross-country empirical evidence supporting the claim that FDI per se accelerates economic growth". Nichtsdestotrotz halten sie aber fest, dass ausländisches Kapital für das langfristige Wachstum eines Landes wichtig ist. Lensink/Morrissey (2001) kommen auch zu dem Ergebnis, dass ADI grundsätzlich einen positiven, aber die Volatilität von ADI einen negativen Einfluss auf das Wachstum haben.

Zusammenfassend lässt sich also feststellen, dass eher ADI Wachstum bedingt, als umgekehrt. Insbesondere im Hinblick auf Entwicklungsländer bleibt der Beitrag von ADI zum Wachstum sehr umstritten (vgl. Choe 2003). Abhängig vom Forschungsdesign und den ausgewählten Ländern, kommt es zu sehr konträren Ergebnissen. Wenig überraschend erscheint die Tatsache, dass die Ergebnisse aus dem asiatischen Raum weitestgehend positiv sind, insbesondere dann, wenn China in der Studie vorkommt. Für Afrika und Lateinamerika sind die Ergebnisse aber weit weniger vorteilhaft und Crowding-outEffekte wurden für diese Regionen festgestellt.

Ein wesentlicher Diskussionspunkt, der im engen Zusammenhang mit der umstrittenen Beziehung von ADI und Wachstum steht, ist die Gewährung von Subventionen und Steuervorteilen an ausländische Unternehmen. $\mathrm{Da}$ kein gesicherter positiver Zusammenhang zwischen ADI und Wachstum festgestellt werden kann, werden auch diese Maßnahmen als nicht zielführend angesehen 
(Agosin/Mayer 2004: 14). Deswegen sollten sich die Länder die Kosten, die durch die Gewährung von Investitionsanreizen entstehen, genau überlegen ,...Countries should weigh the cost of policies aimed at attracting FDI versus those that seek to improve local conditions. These two sets of policies need not be incompatible, better local conditions not only attract foreign companies but also they can maximize the benefits of foreign investments" (Alfaro et al. 2004: 23). In die gleiche Richtung argumentieren auch Kumar (2001) und Kumar/Pradhan (2002), die es als zielführender ansehen, dass Entwicklungsländer die Infrastruktur im Land verbessern, um so einerseits für ADI attraktiv zu sein, aber auch um eine Verbesserung der Produktionsbedingungen für die lokalen Unternehmen zu sichern, die wiederum zum Wirtschaftswachstum beitragen.

\subsection{Auswirkungen von Infrastruktur auf Armutsreduktion}

Im vorangegangen Kapitel 3.2 wurden die Auswirkungen von ADI allgemein, d.h. es wurde nicht zwischen ADI im Produktions- und Infrastruktursektor unterschieden, diskutiert. Dieses Kapitel fokussiert nun ausschließlich auf die Auswirkungen von Infrastrukturinvestitionen. In Kapitel 3.3.1 wird eine Einführung in die Thematik und die Problematik bei der Messung der Auswirkungen gegeben. Darauf folgt in Kapitel 3.3.2 die Darstellung des Zusammenhangs zwischen Infrastruktur, wobei auf die direkten und indirekten Auswirkungen auf Wachstum eingegangen wird. In den Kapiteln 3.3.3 und 3.3.4 wird eine Fokussierung vorgenommen, da sich die Fallstudien (siehe Kapitel 6) ausschließlich mit der Energie- und Transportinfrastruktur beschäftigen. In diesen beiden Kapiteln werden daher die Zusammenhänge dieser beiden Sektoren auf die Armutsreduktion diskutiert, die als Basis für die Fallstudien der Arbeit dienen.

\subsubsection{Einführung}

Infrastruktur spielt in der Entwicklungspolitik von Beginn an eine große Rolle, aber die Unterstützungsstrategien haben sich bereits mehrmals geändert. In den 1960er Jahren beschränkten sich von Seiten der internationalen Geber die Investitionen auf große Infrastrukturprojekte, wie beispielsweise Brücken, Häfen und Kraftwerke, um das wirtschaftliche Wachstum in den armen Ländern zu fördern (vgl. ADB 2005a: 1). Infrastrukturinvestitionen wurden mit der Annahme unterstützt, dass diese zu Wachstum beitragen und über den Trickledown-Effekt zu wirtschaftlicher Entwicklung und zur Umverteilung zugunsten der armen Bevölkerung führen würden (vgl. OECD 2006a: 18).

Allerdings stellte man in den 1970er Jahren fest, dass diese Art von Investitionen nicht notwendigerweise den Armen in diesen Ländern zu gute 
kam. Vielmehr förderten sie die Entwicklung von städtischen Gebieten und der Industrie und wendeten sich nicht an die Bedürfnisse der durchwegs armen ländlichen Bevölkerung. Folglich wurde in den 1970er und 1980er Jahren versucht, Infrastrukturinvestitionen zu planen, die sich auf die ländliche Entwicklung konzentrierten. Erst in den 1970er Jahren entdeckte man den Zusammenhang von Armut und ländlicher Bevölkerung und erst Ende der 1990er Jahre wurde damit begonnen, die von der Investition profitierende Bevölkerung in Arme (Poor) und Nicht-Arme (Non-Poor) Gruppen zu unterteilen und auch die Programme Pro-Poor zu gestalten (vgl. ADB 2005a: 1).

Da Infrastrukturleistungen Zwischenprodukte darstellen und zur Produktion von anderen Produkten beitragen, können die Auswirkungen auf die Armutsreduktion nur schwer gemessen werden (vgl. ADB 2005a: 225). Die direkten Auswirkungen einer Infrastrukturinvestition auf die Armutsreduktion können zu einem sehr frühen Stadium des Projektes bereits erhoben werden, während die indirekten Auswirkungen erst zu einem viel späteren Zeitpunkt erfolgen können (vgl. ADB 2005a: 29).

Der Beitrag von Energie- und Verkehrsinfrastruktur zur Armutsreduktion erscheint beispielsweise intuitiv zu bestehen, allerdings sind die empirischen Ergebnisse über diesen Zusammenhang schwach (vgl. ADB 2005a: xv, 2005b: 10). Um einen zuverlässigen Nachweis über den Zusammenhang von Infrastruktur und Armutsreduktion erbringen zu können, müssen ausführliche ex-ante Daten zur Verfügung stehen, um die Auswirkungen der Infrastrukturinvestitionen messen zu können (vgl. Hettige 2006: 35, OECD 2006a: 15, Baker 2000: 56). Bisher wurden in diesem Bereich jedoch kaum ausführliche Daten im Hinblick auf die Auswirkungen zur Armutsreduktion erhoben. Außerdem fehlt den meisten durchgeführten Studien eine verlässliche Methode (vgl. ADB 2005a: xvii). Daher sind die Ergebnisse von vielen Studien in diesem Bereich von fragwürdigem Wahrheitsgehalt (vgl. ADB 2005a: 247). Aber auch die expost Evaluierung muss äußerst kritisch betrachtet werden, da laut ADB (2005a: 23) bisher in keiner im Rahmen der Studie untersuchten Investition eine ex-post Evaluierung der Projekte hinsichtlich ihres Beitrags zur Armutsreduktion durchgeführt wurde.

Eine weitere Problematik bei der Feststellung der Auswirkungen von Infrastruktur auf die Armutsreduktion sind, wie bereits in Kapitel 3.1.2 dargestellt wurde, die unterschiedlichen Definitionen von Infrastruktur. Dadurch können die Ergebnisse der verschiedenen Studien nur beschränkt verglichen werden. Zusätzlich dazu betrachten viele Studien die einzelnen Sektoren isoliert. Jedoch wird in den Studien bestätigt, dass es durch Investitionen in mehrere Sektoren zu Synergieeffekten kommen kann, insbesondere dann, wenn die Infrastrukturinvestitionen im Zusammenhang mit Ausbildungsinitiativen getroffen werden (vgl. Songco 2002). 


\subsubsection{Infrastruktur und Armutsreduktion}

Die Infrastruktur nimmt in der Diskussion über Maßnahmen zur Armutsreduktion eine zentrale Stellung ein. Die Auswirkungen von Infrastruktur auf die Umwelt sind vielfältig und der Zusammenhiang zwischen Infrastruktur und Armutsreduktion kann sowohl direkt, als auch indirekt über Wachstum bestehen (vgl. Hesselbarth 2004: 16).

\subsubsection{Indirekte Auswirkungen von Infrastruktur auf Armutsreduktion}

\section{Wachstum und Armutsreduktion}

Ein wesentlicher Diskussionspunkt im Zusammenhang mit den Auswirkungen von Infrastruktur auf Armutsreduktion basiert auf der Grundsatzdiskussion, inwieweit Wachstum der Armutsreduktion dient. In der Praxis und in vielen Studien wird angenommen, dass Wachstum automatisch zur Armutsreduktion beiträgt (vgl. ADB 2005b: 77, Dollar/Kraay 2002) und allgemeines Wirtschaftswachstum der wesentliche Motor für die Armutsreduktion ist (vgl. Hesselbarth 2004: 16, Krishna 2004, Lopez 2004a: 2, Willoughby 2004b, Klasen 2001). Krishna (2004) gibt aber zu bedenken, dass dieser positive Zusammenhang nicht automatisch für jedes Land oder jede Region zutrifft.

In der Entwicklungsökonomie wurde erkannt, dass nicht nur steigendes Wachstum, sondern vor allem eine Reduktion der ungleichen Verteilung für die Armutsreduktion ausschlaggebend ist (vgl. Lopez 2004a: 18, Dollar/Kraay 2002). Wichtig ist daher, dass Wachstum von Maßnahmen begleitet wird, welche die Umverteilung von Wachstum auf die Armen fördert, da eine hohe Ungleichheit (,, inequality") eine Bremse in der Armutsreduktion darstellt. Ausbildung, Infrastruktur und makroökonomische Stabilität scheinen hingegen positive Auswirkungen auf Wachstum und Einkommensverteilung zu haben (vgl. Lopez 2004b: 15).

Zusammenfassend lässt sich also feststellen, dass es starke Hinweise auf einen positiven Zusammenhang von Wachstum auf Armutsreduktion gibt. In der neueren Entwicklungsökonomie fordert man aber die Fokussierung auf ProPoor-Wachstum, bei dem auch die verteilungspolitischen Aspekte berücksichtigt werden. Pro-Poor-Wachstum erklärt inwieweit Wachstum signifikant zur Armutsreduktion beiträgt und die Situation der Armen verbessert (vgl. Lopez 2004b: 4) und es stellt daher jenes Wachstum dar, bei dem die Armen durch das erhöhte Einkommen durchschnittlich mehr profitieren als die NichtArmen (vgl. Pernia 2003: 2). Diese neue Ausrichtung stellt eine Abkehr des Trickle-Down-Ansatzes in den entwicklungspolitischen Maßnahmen dar. 


\section{Infrastruktur und Wachstum}

In vielen Quellen wird angenommen, dass verlässliche und effiziente Infrastruktur eine wichtige Voraussetzung für Wirtschaftswachstum darstellt (vgl. OECD 2006a: 10, Doh/Ramamurti 2003, Bougheas et al. 2000) und eine Vielzahl an empirischen Studien beschätigt die signifikant positiven Auswirkungen auf den Output (vgl. Willoughby 2004b, Roller/Waverman 2001, Canning 1999, Fernald 1999).

Einige Studien kamen zu dem Schluss, dass öffentliche Ausgaben im Bereich der Transport- und Telekommunikationsinfrastruktur besonders zu Wachstum beitragen (vgl. Teruel/Kuroda 2005, Miller/Tsoukis 2001, Easterly/Rebelo 1993) und andere wiederum, dass es einen negativen Zusammenhang zwischen Wachstum und öffentlichen Ausgaben im Bereich der Infrastruktur gibt (vgl. Devarajan et al. 1996). Unklar sind die Auswirkungen der Teilnahme des Privatsektors und der Regulierungen in der Infrastruktur auf das Wirtschaftswachstum (vgl. Cook/Uchida 2003, Alexander/Estache 2000).

Die positiven Auswirkungen von Infrastruktur auf Wachstum hängen aber nicht nur von der Investition alleine, sondern auch von der Effizienz ab, mit der die Anlage betrieben und erhalten wird. In Studien wurde herausgefunden, dass der effiziente Betrieb von Infrastrukturinvestitionen einen gleichen, wenn nicht sogar einen größeren Einfluss auf Wachstum hat als die Erweiterung von Dienstleistungen (vgl. Willoughby 2004b).

\subsubsection{Direkte Auswirkungen von Infrastruktur auf Armutsreduktion}

Der positive, direkte Zusammenhang von Infrastruktur und Armutsreduktion besteht oft intuitiv, aber nur einzelne Sektoren tragen tatsächlich direkt zur Armutsreduktion bei. Insbesondere im ländlichen Raum kommt der Infrastrukturentwicklung eine wichtige Bedeutung $\mathrm{zu}$, da es dort unter anderem aufgrund der schlechten Versorgung mit Infrastruktur zu hoher Armut kommt (vgl. Estache 2006). Daher werden in der Literatur öffentliche Ausgaben in diesem Bereich als signifikant für die Armutsreduktion angesehen, da es durch diese Investitionen nicht nur zu Verbesserungen im landwirtschaftlichen Sektor, sondern auch in allen anderen Bereichen kommt (vgl. Fan et al. 1999).

Der Beitrag zur Armutsreduktion hängt stark von einem verlässlichen und effizienten Versorgungsnetz ab. Besonders wichtig sind daher nicht nur die Investitionen in die Infrastruktur selbst, sondern auch die zusätzlichen Investitionen in bestehende Versorgungsanlagen, die es auch den Armen er-möglichen, von der besseren Infrastruktur zu profitieren (vgl. ADB 2005a: 258). In der Literatur ist man sich darüber einig, dass Infrastrukturinvestitionen nur dann eine nachhaltige positive Auswirkung auf die Armutsreduktion hat, wenn sie mit anderen Maßnahmen kombiniert werden (vgl. ADB 2005b: 80). 
Estache (2004: 4) hebt in seiner Literaturrecherche hervor, dass die Ergebnisse der Studien über positive Auswirkungen sehr stark von den gewählten Ländern abhängig sind. In Ländern und Regionen mit sehr niedrigem Einkommen haben die Infrastrukturinvestitionen die größten Auswirkungen. Estache (2004) kommt zu dem Schluss, dass die Ergebnisse über die Auswirkungen in den verschiedenen Sektoren großteils konsistent sind, wobei Transportprojekte den größten sozioökonomischen Nutzen aufweisen. Aus den herangezogenen Untersuchungen lässt sich ableiten, dass

- Investitionen in die Infrastruktur (inkl. Transport und Energie), Ausbildung und Landwirtschaft zu Verbesserungen in der ländlichen Produktion und zur ländlichen Armutsreduktion führen (vgl. OECD 2006a: 11, ADB 2005a: 258).

- Straßen als isolierter Infrastruktursektor am meisten zur Armutsreduktion beitragen (vgl. Balisacan 2001, Kwon 2000).

- Investitionen in die Bewässerung und Energie einen positiven Einfluss auf die landwirtschaftliche Produktion haben können, aber wahrscheinlich nur marginal die ländliche Armut beeinflussen (vgl. ADB 2005a: 262). Balisacan und Pernia (2002) entdecken in ihrer Studie über die Philippinen jedoch keinen signifikanten Zusammenhang von Energie und Armutsreduktion, auch nicht in Kombination mit Ausbildung.

- verbesserte Infrastruktur zu einem erhöhten Informationsfluss, zu geringeren Transaktionskosten und erhöhter Mobilität führen, die wiederum einen besseren Zugang zum Arbeitsmarkt fördern (vgl. ADB 2005a: 258).

- Transport und Energieinvestitionen unmittelbar eher den armen Haushalten helfen, die an der Grenze zur extremen Armut leben, aber nicht den extrem Armen, die durch eine chronische Armut von den Infrastrukturleistungen ferngehalten werden (vgl. ADB 2005a: 194).

\subsubsection{Konzeptioneller Rahmen für die Darstellung der Auswirkungen von Infrastruktur auf Armutsreduktion}

Es gibt direkte und indirekte Auswirkungen von Infrastrukturinvestitionen, die sowohl positiv, als auch negativ sein können. Die indirekten Auswirkungen sind schwieriger zu messen als die direkten, da sie sehr umfangreich sein können und die Erhebung wiederum nur erschwert möglich ist (vgl. Hettige 2006: 1). Ein wesentlicher Punkt in dieser Diskussion ist auch die Einbettung von Infrastrukturinvestitionen in das bereits bestehende Netzwerk. Die isolierte Betrachtung von Infrastrukturinvestitionen macht in diesem Zusammenhang wenig Sinn, da es neben den wesentlichen Investitionen auch zu lokalen Investitionen 
kommen muss, die wiederum die erzeugte Dienstleistung auch der armen Bevölkerung zugänglich macht (vgl. Stafford 2005: 26).

Die Auswirkungen von Infrastruktur auf die arme Bevölkerung hängen wesentlich von den sozialen und politischen Rahmenbedingungen in einem Land und im jeweiligen Sektor ab (vgl. ADB 2005a: 193). Gefordert werden daher umfassende und konsistente Strategien für Infrastrukturinvestitionen, die auch andere soziale und ökonomische Sektoren und Pläne berücksichtigen (vgl. OECD 2006a: 11). In der Literatur werden auch die negativen Auswirkungen von Infrastruktur und die ungleiche Verteilung der positiven Effekte diskutiert, da es nicht automatisch zu einer gleichmäßigen Nutzenverteilung kommt und die Zielgruppe oftmals die Nicht-Armen sind (vgl. ADB 2005b: 80). Dies bedeutet, dass Infrastrukturinvestitionen die Armen auch negativ oder nur indirekt positiv beeinflussen können (vgl. ADB 2005b: 11).

Abgesehen von dem gegebenen Kontext in einem Land beeinflussen aber auch situationsbezogene Variablen, wie beispielsweise der Anschluss an die Stromversorgung oder der Zugang zur Straße, die Auswirkungen eines Infrastrukturprojekts auf die Armutsreduktion (vgl. ADB 2005a: 34). Insbesondere die kontextuellen Variablen können die Auswirkungen der Investitionen stark beeinflussen und daher zu unterschiedlichen Ergebnissen in verschiedenen Ländern führen (vgl. ADB 2005b: 80).

In der folgenden Abbildung 13 wird dieser konzeptionelle Rahmen grafisch dargestellt, wobei die Infrastruktur-investitionen indirekte und direkte Auswirkungen auf die Armutsreduktion haben können.

\section{Abbildung 13: Auswirkungen von Infrastruktur auf Wachstum und Armutsreduktion}

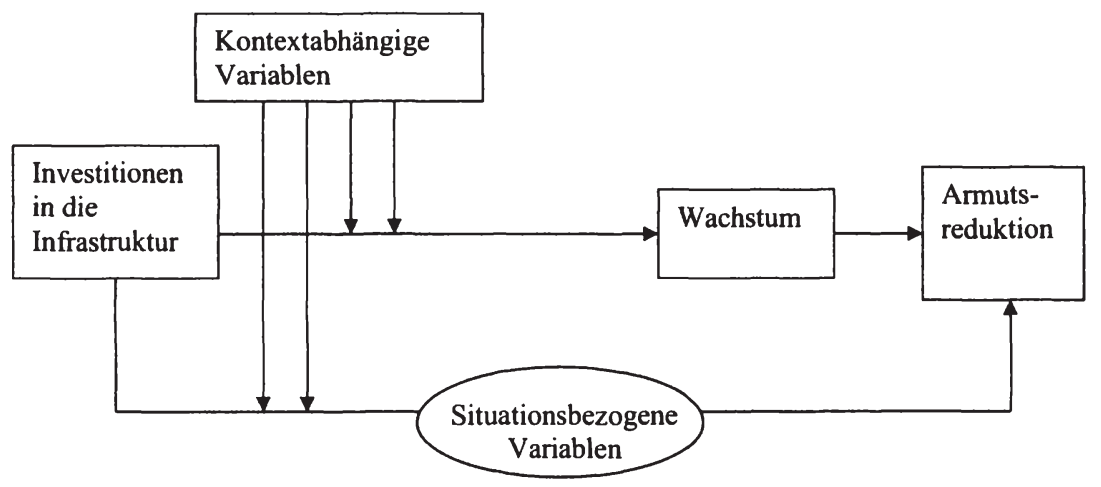

Quelle: in Anlehnung an OECD (2006a: 19), ADB (2005a: 34) 
Basierend auf der Ausarbeitung in Kapitel 3.3.2 über die Auswirkungen von Infrastruktur im Allgemeinen, werden in den folgenden beiden Kapiteln 3.3.3 und 3.3.4 die Auswirkungen von zwei Sektoren der physischen Infrastruktur, Energie- und Transportinfrastruktur, genauer dargestellt.

\subsubsection{Energieinfrastruktur und Armutsreduktion}

Der Beitrag von Energieinfrastruktur zur Armutsreduktion erscheint intuitiv zu bestehen, allerdings sind die empirischen Ergebnisse über diesen Zusammenhang schwach (vgl. ADB 2005a: xv, 2005b: 10). Bisher haben sich auch nur wenige empirische Studien mit den Auswirkungen von Energieinfrastruktur auf die Armutsreduktion beschäftigt und auch wenn der Zusammenhang von Energie und Armutsreduktion behauptet wird, messen tatsächlich nur sehr wenige empirische Studien diesen Effekt (vgl. OECD 2006a: 43). Als Gründe für die positiven Auswirkungen werden die erhöhte wirtschaftliche Aktivität und Wirtschaftswachstum angegeben. Diese Annahmen werden heute als weitgehend unrealistisch betrachtet, da die Bereitstellung von Energie alleine nicht zu erhöhten Wirtschaftsaktivitäten und Armutsreduktion beitragen wird (vgl. ESAMP 2000: 30ff). Allerdings wird in der Literatur die Tatsache anerkannt, dass verfügbare Energie, kombiniert mit anderen verbessernden Faktoren, positive Veränderungen in der wirtschaftlichen Wohlfahrt ermöglicht (vgl. ADB 2005a: 247, Brenneman/Kerf 2002: 12ff).

Eine wesentliche Unterscheidung bei der Diskussion über die Auswirkungen von Investitionen in die Energieinfrastruktur ist zwischen sogannanten „OnGrid" und „Off-Grid"-Projekten zu treffen. „On-Grid“-Projekte stellen Kraftwerke dar, die die produzierte Energie in die bestehende Stromversorgung einspeisen. Typischerweise wird in Entwicklungsländern vor allem in urbanen Gegenden ein Stromnetz aufgebaut. „Off-Grid“-Projekte hingegen liefern den produzierten Strom an ein lokal beschränktes Stromnetz oder direkt an die angeschlossenen Haushalte. Diese Art der Investitionen wird vor allem in den ländlicheren und abgelegenen Regionen der Entwicklungsländer vorgenommen.

Auf dem Energiesektor ist es besonders schwierig, den Wert von Strom für die Armen zu quantifizieren und eine bessere und günstigere Stromversorgung kann sowohl direkte als auch indirekte Auswirkungen auf die Bevölkerung haben. Beispielsweise kann durch die verbesserte Stromversorgung die Arbeitszeit ausgeweitet werden und Arbeitskraft länger in der Erzeugung eingesetzt und dadurch die Produktion pro Haushalt gesteigert werden. Neue Technologie kann die Umweltbelastung senken und dadurch den unmittelbaren Lebensraum verbessern (vgl. ADB 2005a: 248, Brenneman/Kerf 2002: 24ff).

Da der ländliche Raum, der am häufigsten von hoher Armut betroffen ist, nicht durch das bestehende Netz versorgt wird, muss die Bevölkerung andere Quellen als Energielieferanten heranziehen. Diese alternativen Quellen sind jedoch unzuverlässig und verursachen höhere Kosten. Wird aber die Strom- 
versorgung auch auf die ländlichen Regionen erweitert, profitiert die Bevölkerung von einer stabilen Versorgung zu geringern Kosten (vgl. Willoughby 2004a: 3, Brenneman/Kerf 2002: 16).

Strom wird von den Armen meistens für Beleuchtungszwecke verwendet. Daher kann eine stabile und preisgünstige Stromversorgung auch zu einer verbesserten Ausbildung der Kinder führen (vgl. Brenneman/Kerf 2002: 18ff), was allerdings nur beschränkt positive Auswirkungen auf die Armutsreduktion hat (vgl.Willoughby 2004a: 3). Insbesondere für ländliche Regionen, die nicht an das bestehende Stromnetz (off-grid) angeschlossen sind, können kleinere Anlagen mit erneuerbarer Energie die lokalen Bedürfnisse am besten befriedigen (vgl. ADB 2005a: 248).

Die Verfügbarkeit und Verlässlichkeit von moderner Energie ist auch ein wichtiger Faktor für die Standortwahl von Unternehmen (vgl. OECD 2006a: 43). Daher wird davon ausgegangen, dass eine verbesserte Energieversorgung für kommerzielle Zwecke in den ländlichen Regionen zu wirtschaftlichen und sozialen Gewinnen führen kann. Allerdings gibt es auch hier nur geringe Beweise über das Ausmaß, in dem diese positiven Auswirkungen auch wirklich realisiert werden. Die Schlussfolgerung dieser Annahme ist daher, dass eine verbesserte Energieversorgung das wirtschaftliche Wachstum in den ländlichen Regionen unterstützen, aber nicht initiieren kann (vgl. ADB 2005a: 253).

Mögliche positive Auswirkungen von Energieinfrastruktur werden in der folgenden Abbildung 14 kurz zusammengefasst:

\section{Abbildung 14: Mögliche positive Auswirkungen von Energieinfrastrukturleistungen auf die Armen}

\begin{tabular}{|l|l|l|}
\cline { 2 - 3 } \multicolumn{1}{l|}{} & Direkte Auswirkungen & Indirekte Auswirkungen \\
\hline \multirow{2}{*}{ Energie } & $\begin{array}{l}\text { Beleuchtung, TV, Radio (bei niedrigem } \\
\text { Einkommen) }\end{array}$ & $\begin{array}{l}\text { Reduzierte Energiekosten für } \\
\text { Unternehmen können zu } \\
\text { ansteigenden Investitionen } \\
\text { und zu mehr Beschäftigung } \\
\text { führen }\end{array}$ \\
\cline { 2 - 3 } & $\begin{array}{l}\text { Heizung, Kochen, verbesserte Versorgung für } \\
\text { freie Mitarbeiter bzw. Selbständige (bei einem } \\
\text { höheren Einkommensniveau) }\end{array}$ & $\begin{array}{l}\text { Verbesserte } \\
\text { Gesundheitsversorgung }\end{array}$ \\
\cline { 2 - 3 } & $\begin{array}{l}\text { Verbesserter Zugang zu } \\
\text { Informations- und } \\
\text { Kommunikationstechnik }\end{array}$ \\
\hline
\end{tabular}

Quelle: in Anlehnung an ADB (2005b: 81)

Die vorliegende Arbeit konzentriert sich aufgrund der gewählten Fallstudien ausschließlich auf Kraftwerke, die ihre Energieleistung in das bestehende Versorgungsnetz (grid) einspeisen. Solche großen Kraftwerke dienen vor allem der Industrie, der städtischen Bevölkerung und landwirtschaftlichen Benutzern, 
während die ländliche, arme Bevölkerung Biomasse oder andere Energiequellen verwendet (vgl. OECD 2006a: 43).

\subsubsection{Transportinfrastruktur und Armutsreduktion}

Unter Transportinfrastruktur werden Investitionen im Straßenbereich, aber auch in alle anderen Bereichen, wie Häfen oder Schiene, subsumiert. Straßeninfrastruktur wird von öffentlicher Seite als öffentliches Gut gesehen, welches vom Staat kostenlos zur Verfügung gestellt werden soll. Besonders schwierig gestaltet sich in diesem Bereich die Übertragung der Kosten auf die Nutzer oder die Begünstigten (vgl. Curtis 2004).

Die meisten Studien im Bereich des Transports und der Armutsreduktion haben sich bisher mit Straßen im ländlichen Raum beschäftigt. Diese Tendenz erscheint einleuchtend, da die abgelegenen Regionen meist sehr unterentwickelt und sehr arm sind. Im Bereich von städtischem Transport und Autobahnen wurde jedoch bisher noch kaum Forschung betrieben (vgl. ADB 2005a: 234). Viele Studien, die sich mit den Auswirkungen von Infrastruktur im ländlichen Raum beschäftigten, kamen zu dem Ergebnis, dass Investitionen in das Straßennetz am meisten zur Armutsreduktion beitragen (vgl. Estache 2004: 5, Balisacan 2001, Kwon 2000). Allerdings mit der Einschränkung, dass Straßen zwar notwendig für die Armutsreduktion sind, aber keine hinreichende Bedingung sind (vgl. Hettige 2006: 32). Die Transportanforderungen unterscheiden sich außerdem nach den verschiedenen sozioökonomischen Gruppen (vgl. Hettige 2006: 26). Insbesondere die Kombination von Investitionen in die Ausbildung und in Straßeninfrastruktur scheinen positiv $\mathrm{zu}$ korrelieren (vgl. Balisacan/Pernia 2002: 259).

Die Transportkosten stellen für die Armen oft einen überproportional hohen Anteil des Einkommens dar. Der wesentliche und unmittelbare Nutzen von Straßeninvestitionen ist für die Bevölkerung daher oft die Reduktion der Transportkosten (vgl. Brenneman/Kerf 2002: 74ff). Dadurch sollen der Handel und die Produktion vereinfacht und erhöht werden, wodurch wiederum das Einkommen der Bevölkerung steigt (vgl. OECD 2006a: 38, Brenneman/Kerf 2002: 70). Andererseits werden durch den Bau und die Instandhaltung neue Arbeitsplätze geschaffen (vgl. ADB 2005a: 23). Es wird daher angenommen, dass eine bessere Transportinfrastruktur in einem Land zu einem besseren Investitionsklima, zu einer erhöhten Produktivität im Allgemeinen und daher auch zu einem positiven Umfeld für Armutsreduktion beitragen kann (vgl. Brenneman/Kerf 2002: 66ff).

Wie bereits in Kapitel 3.3.2 dargestellt, hängt der Beitrag von Straßeninfrastruktur zur Verbesserung der Lebenssituation der Armen vom strukturellen und institutionellen Kontext ab. Daher sollten Projekte im Zuge von sektorweiten Maßnahmen zur Stärkung der Institutionen realisiert werden (vgl. ADB 2005a: 232, ADB 2005b: 80). 
Eine Zusammenfassung über die möglichen positiven direkten und indirekten Auswirkungen werden in der folgenden Abbildung 15 gegeben.

Abbildung 15: Mögliche positive Auswirkungen von Transportinfrastrukturleistungen auf die Armen

\begin{tabular}{|l|l|l|}
\cline { 2 - 3 } \multicolumn{1}{c|}{$\begin{array}{l}\text { Transport- } \\
\text { infrastruktur }\end{array}$} & Direkte Auswirkungen & Indirekte Auswirkungen \\
\cline { 2 - 3 } & $\begin{array}{l}\text { Zugang zum Arbeitsplatz und zu Märken } \\
\text { Gesung zu Dienstleistungen wie } \\
\text { verbesserter Marktzugang } \\
\text { für Unternehmen und } \\
\text { Dienstleistungsanbieter, } \\
\text { geringere Kosten für die } \\
\text { Versorgung von } \\
\text { abgelegenen Gebieten }\end{array}$ \\
\hline
\end{tabular}

Quelle: in Anlehnung an ADB (2005b: 81), Brenneman/Kerf (2002: 70ff)

Im Bereich der städtischen Infrastruktur ist allerdings weniger klar, wie die Transportinfrastruktur dazu genützt werden kann, die Armutsreduktion zu fördern bzw. den Armen die Infrastruktur konkret zugänglich zu machen. In diesem Bereich werden oft Subventionen eingesetzt, von denen wiederum auch die Nicht-Armen (Non-Poor) profitieren, da sie nicht von den Dienstleistungen ausgeschlossen werden können. Andererseits besteht auch die Möglichkeit, dass für Arme selbst die subventionierten Infrastrukturleistungen noch zu teuer sind und sie daher sowohl von den Leistungen, als auch von den Subventionen ausgeschlossen bleiben. Häufig wirken sich neue Infrastrukturinvestitionen in einer Stadt, wie beispielsweise Schnellstraßen, auch negativ auf die Armen aus. Diese Projekte werden meist in Gebieten geplant, in denen das Land günstig ist und dieses typischerweise von ärmeren Bevölkerungsschichten besiedelt ist. Dies führt zu unfreiwilligen Umsiedelungen oder auch $\mathrm{zu}$ einer erhöhten Umwelt- und Lärmbelastung für die Anrainer (vgl. ADB 2005a: 239, ADB 2005b: 80).

Insbesondere bei großen Infrastrukturinvestitionen, wie beispielsweise Mautstraßen eder Häfen, nimmt man an, dass diese das wirtschaftliche Wachstum einer Region fördern. Ob diese Investitionen allerdings den Armen helfen, ist abhängig davon, inwieweit sie Zugang dazu haben, beispielsweise durch $\mathrm{Zu}-$ bringerstraßen oder ob sie durch den Bau einen Arbeitsplatz bekommen (vgl. ADB 2005a: xxviii).

Bei einer Studie über die Auswirkungen von Instandhaltungs- und Erweiterungsarbeiten an einer Autobahn zwischen Laos und Vietnam wurde entdeckt, dass die indigenen Gruppen entlang der Straße nicht profitierten, sondern negativ beeinträchtigt wurden. Man kam zu dem Schluss, dass die betroffene Bevölkerung nur dann vom Bau profitieren würde, wenn gleichzeitig Zubringerstraßen gebaut würden (vgl. ADB 2005a: 24). 
In einer anderen Studie wurden die Auswirkungen einer Mautstraße in China untersucht. Das Ziel dieses Projektes war in erster Linie die Verbesserung des Zugangs dieser Region zum Hafen, somit die wirtschaftliche Entwicklung und das Wachstum dieser Region. In diesem Fall wurde in der ex-post Analyse festgestellt, dass durch die Verbesserung der Straße der Zugang zu sozialen Leistungen, zum Markt und zum Hafen vereinfacht wurde und dadurch auch die wirtschaftlichen Aktivitäten anstiegen und sich Unternehmen in der Gegend ansiedelten. In dieser chinesischen Region leben jedoch wenig arme Menschen und der mögliche Beitrag dieses Projektes beschränkt sich darauf, dass einerseits arme Menschen aus anderen Regionen zum Bau dieser Straße angestellt wurden und andererseits die Zubringerstraßen zu Gemeinschaften mit niedrigen Einkommen verbessert wurden (vgl. Pan/Shu 2002). Insgesamt haben aber NichtArme mehr von diesem Projekt profitiert. Im Wesentlichen besteht der Hauptnutzen von Autobahnen oder Schnellstraßen in der Reduktion der Transportkosten (vgl. ADB 2005a: 27).

Dieses Straßenprojekt weist viele Parallelen zu der im Rahmen dieser Arbeit gewählten Fallstudie, dem North-Luzon Expressway auf den Philippinen, auf (siehe Kapitel 6.3.3). Im weiteren Verlauf dieser Dissertation beschränkt sich die Autorin aufgrund der gewählten Fallstudie im Transportsektor ausschließlich auf die Auswirkungen von Schnellstraßen auf die Armutsreduktion.

\subsection{Zusammenfassung}

Das dritte Kapitel hat sich mit Investitionen in der Infrastruktur beschäftigt, wobei der Fokus auf den privaten Investitionen liegt. Im einleitenden Kapitel (Kapitel 3.1) werden die Begriffe Ausländische Direktinvestition (ADI), Infrastruktur und Armut definiert.

Als ADI werden private Investitionen bezeichnet, bei denen das private Unternehmen einen Managementeinfluss verfolgt und eine Mindestbeteiligung von $10 \%$ für einen längerfristigen Zeitraum tätigt.

Der Begriff Infrastruktur wird häufig als Überbegriff verwendet und wird, abhängig von der Literaturquelle, in verschiedene Subkategorien unterteilt. Die Verfasserin verwendet für die vorliegende Arbeit die Definition der Weltbank und der OECD, welche die Infrastruktur in wirtschaftliche und soziale Infrastruktur unterteilen. Gegenstand der vorliegenden Untersuchung sind physische Infrastrukturinvestitionen, die eine Untergruppe der wirtschaftlichen Infrastrukturinvestitionen darstellen.

Armut wurde lange Zeit nur auf Basis des Pro-Kopf-Einkommens gemessen. Die Unzulänglichkeit dieser eindimensionalen Definition wurde von vielen Seiten erkannt und sowohl um qualitative, als auch quantitative Dimensionen erweitert. Bei den Maßnahmen zur Armutsreduktion ist insbesondere hervor- 
zuheben, dass Armut ein dynamisches Konzept ist. Dynamisch bedeutet in diesem Zusammenhang, dass es verschiedene Arten bzw. Stadien von Armut gibt und die Betroffenen durch einen externen Schock vorübergehend arm sein können, sie sich aber durch eine Verbesserung der Zustände wieder aus der Armut retten können. Diese verschiedenen Stadien können wiederum nur mit einem Bündel von Maßnahmen bekämpft werden. Infrastruktur trägt am meisten zur Reduktion von struktureller Armut bei.

Im darauf folgenden Kapitel 3.2 wird ein Literaturüberblick über die Auswirkungen von ADI auf Entwicklungsländer gegeben. Nach einer Einleitung, in der auf die sich veränderte Rolle von $\mathrm{ADI}$ in der Entwicklungspolitik und in den Entwicklungsländern eingegangen wird, werden im Kapitel 3.2.2 die SpilloverEffekte von ADI diskutiert.

Fraglich in diesem Zusammenhang ist, ob Spillover-Effekte tatsächlich existieren, wobei man in der Literatur zu unterschiedlichen Ergebnissen kommt. Man unterscheidet dabei horizontale und vertikale Spillover-Effekte. Horizontale Spillover-Effekte sollen laut Theorie zwischen Unternehmen derselben Produktionsstufe stattfinden. Diese Effekte finden aber in den empirischen Untersuchungen nur wenig Beweis. Die vertikalen Spillover-Effekte finden zwischen Unternehmen unterschiedlicher Produktionsstufe statt. Diese Effekte können mit einer höheren Wahr-scheinlichkeit auftreten, wenn lokale Unternehmen an das multinationale Unternehmen zuliefern.

Das Kapitel 3.2.3 beschäftigt sich mit der Fragestellung, ob ADI zum Wirtschaftswachstum beiträgt. In der Wissenschaft gibt es keine Einigkeit darüber und mikroökonomische Untersuchungen sehen diesen Zusammenhang eher negativ. Die Ergebnisse der makroökonomischen Untersuchungen hingegen geben eine optimistischere Sichtweise dieser Beziehung wider.

In vielen Studien wird argumentiert, dass die Voraussetzungen in einem Land, wie beispielsweise ausgebildete Arbeitskräfte oder die Größe des Landes, Wirtschaftswachstum beeinflussen können, aber auch darüber herrscht keine Einigkeit. Eine weitere Frage stellt die Richtung des Zusammenhanges von ADI und Wachstum dar, da sowohl ADI Wachstum, als auch Wachstum ADI bedingen kann. Neuere Untersuchungen kommen allerdings zu dem Ergebnis, dass Wachstum eine Voraussetzung für $\mathrm{ADI}$ ist. Insbesondere im Hinblick auf Entwicklungsländer bleibt der Beitrag von ADI zum Wachstum sehr umstritten und abhängig vom Forschungsdesign und den ausgewählten Ländern, kommt es zu sehr konträren Ergebnissen.

Im Kapitel 3.3 werden die Auswirkungen von Infrastruktur auf Armutsreduktion diskutiert. Nach einer kurzen Einleitung über die Entwicklung von Infrastruktur, beschäftigt sich das Kapitel 3.3.2 mit den Auswirkungen von Infrastruktur auf Armutsreduktion. In der Literatur wird angenommen, dass Wachstum Armutsreduktion indirekt beeinflussen kann und es lässt sich zu-- 
sammenfassend feststellen, dass es starke Hinweise für positive Auswirkungen von Wachstum auf Armutsreduktion gibt.

Über das Ausmaß dieser Auswirkungen wird jedoch heftig diskutiert und es wird Wachstum gefordert, das „Pro-Poor" ist. Der positive, direkte Zusammenhang von Infrastruktur und Armutsreduktion besteht oft intuitiv, aber nur einzelne Sektoren tragen tatsächlich direkt zur Armutsreduktion bei. Vor allem am Land profitieren die Menschen oft direkt von Infrastrukturinvestitionen, da die ländlichen Regionen oft strukturell benachteiligt sind. Zusätzlich dazu hängen die positiven Auswirkungen auch stark von den gewählten Ländern in der Untersuchung und von den Begleitmaßnahmen ab. Im letzten Abschnitt dieses Kapitels wird ein Model vorgestellt, das den konzeptionellen Rahmen wiedergibt und als Basis für die Fallstudien im Kapitel 6 dient.

In dieser Diskussion über Infrastruktur im Allgemeinen werden in den folgenden beiden Unterkapiteln 3.3.3 und 3.3.4 die Auswirkungen von Energieund Transportinfrastruktur auf Armutsreduktion im Speziellen dargestellt. In der Theorie wird oft angenommen, dass Energieinfrastruktur zur Armutsreduktion beiträgt, jedoch sind die empirischen Beweise eher schwach.

Ein wichtiges Kriterium bei der Diskussion über den Beitrag zur Armutsreduktion ist die Unterscheidung von „On- und Off-grid“" Projekten. Typischerweise leben arme Menschen eher im ländlichen Raum, der nicht an eine bestehende Stromversorgung angeschlossen ist. Daher profitieren sie nicht direkt von sogenannten „On-grid“-Projekten, die ihre Energie in ein bestehendes Versorgungsnetz speisen. Straßen tragen laut Literatur am meisten zur Armutsreduktion bei, aber auch nur dann, wenn sie im ländlichen Raum gebaut werden. Direkte positive Auswirkungen durch ein besseres Straßennetz werden in der Reduktion der Transportzeit gesehen, da dadurch Märkte oder auch Arbeitsplätze schneller erreicht werden können. 
Bianca Gusenbauer - 978-3-631-75445-0

Downloaded from PubFactory at 01/11/2019 04:47:32AM

via free access 


\section{4 Öffentlich-private Bereitstellung von Infrastruktur}

Das vierte Kapitel beschäftigt sich mit der öffentlich-privaten Bereitstellung von Infrastruktur. Das erste Kapitel (4.1) dient der Einführung in die Thematik und dient der Festlegung von Arbeitsdefinitionen. Im Kapitel 4.2 werden die private Teilnahme an der Infrastrukturfinanzierung in den Entwicklungsländern anhand von Regionen und Sektoren sowie die historische Entwicklung dargestellt. Das darauf folgende Kapitel (4.3) gibt einen Überblick über die Länderrisiken, die bei privaten Investitionen in die Infrastruktur berücksichtigt werden müssen. Im vierten Abschnitt dieses Kapitels (4.4) werden die einzelnen öffentlich-privaten Finanzierungsmodelle beschrieben. Das abschließende Kapitel (5.4) fasst die Erkenntnisse dieses Kapitels nochmals zusammen.

Die folgende Abbildung 16 stellt grafisch nochmals den Aufbau dieses Kapitels dar und dient der besseren Orientierung.

\section{Abbildung 16: Kapitelübersicht Öffentlich-private Infrastrukturmodelle}

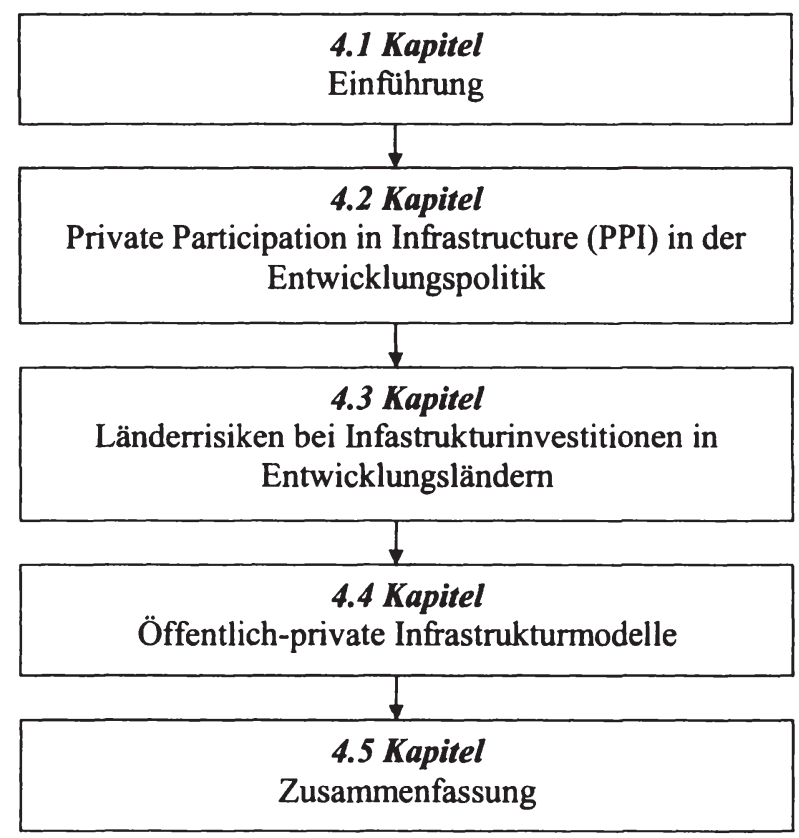




\subsection{Einführung}

Das allgemeine Phänomen von öffentlich-privater Zusammenarbeit in Form von gemeinschaftlichen Betrieben ist grundsätzlich nichts Neues, denn bereits im 17. Jahrhundert wurden erste Verträge für die private Finanzierung von Infrastruktur in Frankreich abgeschlossen (vgl. Grimsey/Lewis 2005: xiii, Scharle 2001). Jedoch haben sich die Partnerschaften, die seit Mitte der 1980er Jahre gegründet worden sind, sowie die Anwendungsfelder stark vermehrt und die Organisationsformen stärker differenziert (vgl. Hauschildt 1997: 11). Zunehmend haben sich aber auch Organisationsformen herausgebildet, die außerhalb der herkömmlichen, ordnungspolitisch definierten Arbeitsteilung zwischen öffentlichen und privaten Sektor angesiedelt sind (vgl. Budäus 1997a: 11). Zusammenfassend kann festgehalten werden, dass das Konzept von Public Private Partnerships (PPP) keine neue Erfindung ist, wie sie teilweise angepriesen wird, sondern nur ein neuer Name für eine bereits länger bekannte Form der $\mathrm{Zu}$ sammenarbeit (vgl. Sass Rubin/Stankiewicz 2001). Auch Wettenthal (2003: 80) stellt in seinem Beitrag über PPP fest ,....we see how the rhetorical power of a new slogan can blind us to the fact that the idea on which it is based is far from new".

Der Begriff Public Private Partnerships (PPP) ist heutzutage weit verbreitet und wird von vielen Quellen als Überbegriff für alle Formen der öffentlichprivaten Zusammenarbeit verwendet. Im Bereich der Literatur zur Finanzierung von Infrastruktur wird der Begriff PPP allerdings nicht einheitlich verwendet. Dies führt in der untersuchten Literatur dazu, dass aufgrund der häufigen und sehr ungenauen Verwendung oft nicht mehr klar ist, was genau unter PPP subsumiert wird. Zusätzlich dazu wird auch der Begriff Private Participation in Infrastructure (PPI) teilweise synonym verwendet. PPI hat aber noch wenig Verbreitung gefunden, sondern wird fast ausschließlich in der Entwicklungspolitik und in Großbritannien verwendet. PPI kann für alle Formen der privaten Teilnahme an der Infrastrukturfinanzierung verwendet werden ohne dabei das oftmals kritisierte falsche Bild einer Partnerschaft zu vermitteln.

In diesem einführenden Kapitel widmet sich der erste Abschnitt der Definition von Partnerschaft in einem kooperativen Modell. Darauf aufbauend werden im folgenden Abschnitt Public Private Partnerships (PPP) definiert. Später folgt eine Darstellung der verfolgten Ziele und Interessen in einer PPP. Dieses Kapitel wird abgerundet von einer Diskussion über die Risiken und Problembereiche bei PPP.

\subsubsection{Definition von Partnerschaft in einem kooperativen Modell}

Partnerschaft, speziell in der Verwendung von PPP, wurde u.a. zu einem Schlüsselbegriff im Public Management, der oftmals nicht mit ausreichender Genauigkeit verwendet wird. McQuaid (2003: 9) hebt hervor, dass trotz der 
großen Anzahl an Publikationen zum Thema Partnerschaft die Definition des Begriffes unzureichend definiert bleibt: „,... the more general theoretical basis for understanding and analysing them remains poorly developed". Auch Faulkner (2004: 65) stellt fest: "In business generally, ,partnership' is an overworked and frequently abused term ". Auch Langford (2002: 69) erkennt den Missbrauch des Begriffs Partnerschaft „...undoubtedly one of the most misused ... in the contemporary administrative lexicon... ".

Der Begriff Partnerschaft umfasst sehr unterschiedliche Konzepte und wird für die Beschreibung einer Vielzahl von Beziehungen in sehr unterschiedlichen Rahmenbedingungen verwendet (vgl. Lewis 2003: 260, McQuaid 2003: 10). Partnerschaft wird typischerweise als eine spezifische Kooperationsform bezeichnet, die von mindestens zwei Partnern eingegangen werden kann, um ein gemeinsames Ziel zu erreichen (vgl. EC 2003a: 34). Die Partner können sowohl aus öffentlichen Instituten als auch der Privatwirtschaft kommen, auch politische Vereinigungen oder Interessensgruppen spielen dabei eine wichtige Rolle (vgl. Eggers 1998: 12ff). Partnerschaften können dabei unterschiedliche rechtliche Formalisierungsgrade aufweisen (vgl. Osei 2004: 253). Als Partnerschaften im engeren Sinne werden Kooperationen zwischen öffentlichen und privaten Akteuren bezeichnet, die formell-rechtlich geregelt sind (vgl. Wettenhall 2002, Rosenau 2000). $\mathrm{Zu}$ den Partnerschaften im weiteren Sinne werden auch informelle Kooperationen gezählt (vgl. Wettenhall 2002, Kouwenhoven 1993: 120).

In einer Partnerschaft werden die daraus erwachsenden Chancen und Risiken geteilt und die jeweils zugedachten Rollen ausgeübt (vgl. Faulkner 2004: 65). Insbesondere die Prozesse des Aushandelns spielen dabei eine zentrale Rolle (vgl. Roggencamp 1999: 56). Lewis (2003: 260) unterscheidet dabei zwischen „aktiven“ und „abhängigen“ Partnerschaften, wobei nur die aktive Partnerschaft auf Verhandlungen basiert und damit auch laufende Änderungen möglich sind. Dem Prinzip folgend, dass die Summe größer ist als die Einzelteile, entsteht bei Partnerschaften ein Synergiepotenzial (vgl. McQuaid 2003: 11). Bei der abhängigen Partnerschaft finden sich Partner zusammen, welche nur die eigenen Interessen verfolgen und in der alle Rollen schon vorgegeben sind. Diese Art der „Partnerschaft" entspricht daher eher dem Contracting-Out.

Partnerschaften haben viele Dimensionen und jede Partnerschaft stellt eine Kombination dieser Ausprägungen dar. McQuaid (2003: 13ff) erklärt Partnerschaften anhand der folgenden Komponenten:

- Ziel: Die wichtigste Dimension bei Partnerschaften ist deren Ziel. Neben dem vorrangigen Ziel gibt es auch unausgesprochene Ziele, wie beispielsweise erhöhter Bekanntheitsgrad in einem neuen Markt oder eine bessere Stromversorgung, die für eine Partnerschaft sehr wichtig sein können.

- Wer: Alle Akteure müssen identifiziert sein und ihr Beitrag festgelegt werden. 
- Wann: Die Zeit ist ein weiterer wichtiger Faktor, der berücksichtigt werden muss. Die Teilnehmer können sich mit der Zeit verändern oder auch in unterschiedlichen Phasen zusammenarbeiten.

- Wo oder Wem: Partnerschaften können auf unterschiedliche geografische Regionen oder auch Kunden beschränkt sein.

- Wie: Diese Dimension beschreibt die Aufgabenteilung zwischen den Partnern.

Als ,Partnerschaft' werden in dieser Arbeit also nur formell-rechtlich geregelte Kooperationen von öffentlichen und privaten Akteuren bezeichnet, die für eine gewisse zeitliche Dauer eingegangen werden sowie auf gemeinsamem Zielen und gemeinsamem Handeln beruhen, wobei alle beteiligten Akteure Ressourcen wie finanzielle Mittel, Zeit, Informationen oder Beziehungen einbringen, die Identität und Verantwortung der Partner intakt bleiben sowie gemeinsam Strukturen und Verfahren für das Management der Partnerschaft festlegen (vgl. Egger 1998: 12)

\subsubsection{Definition von Public Private Partnerships}

Der Begriff von Public Private Partnership (PPP) erfreut sich sowohl in der Wissenschaft auch in der Praxis großer Beliebtheit und ist insbesondere im Public Management auf Konferenzen oder in Publikationen zu einem Muss geworden (vgl. Wettenthall 2003). Außerdem ist der Begriff auch zunehmend im Vokabular von Ökonomen, Juristen oder Wirtschaftspolitikern zu finden; dennoch existiert bis heute keine eindeutige Begriffsdefinition (vgl. Strohbach 2001: 56). Der Begriff PPP ist mit sehr unterschiedlichen Vorstellungen verbunden und auch im internationalen Sprachgebrauch wird er keineswegs einheitlich verwendet (vgl. Weber et al. 2006, Eggers 2004, Carroll/Steane 2003, Zeuchner 2002, Strohbach 2001, Zeiss 2000, Linder 1999, Roggencamp 1999, Kolodziej 1996). Wie auch Sass Rubin/Stankiewicz (2001) feststellen: „... many city officals have begun claiming that all sorts of relationships with priavte and nonprofit actors constitute a ,public-private partnership“. Bestani (2004: 4) sieht die inflationären Verwendung des Begriffs PPP noch drastischer und stellt fest: "This term (PPP) has been overused to the point of becoming a cliche'".

Wesentlicher Grund für die weite Verbreitung des Begriffs PPP ist die Privatisierungswelle in den 1980er Jahren, in der PPP häufig verwendet wurde, um die Kritiker solcher Reformen zu beruhigen, da mit dem Wort Partnerschaft positive Assoziationen transportiert werden (vgl. Carroll/Steane 2003: 43, Budäus 1997a: 16). Wie von Seiten der EU jedoch festgestellt wird, fallen Privatisierungen nicht unter den Begriff PPP, sondern gehören zu der breiter gefassten Kategorie von "Private Participation in Infrastructure (PPI)“ (vgl. EC 2004: 6).

In der Literatur sind folgende zwei unterschiedlichen Strömungen bei der Definition von PPP zu erkennen: 
1. PPP i.w.S. wird für jegliche Zusammenarbeit zwischen privatwirtschaftlichen und öffentlichen Unternehmen gebraucht (vgl. Budäus 2006a: 14, Sass Rubin/Stankiewicz 2001).

Diese PPP-Definition i.w.S umfasst auch einfache Formen des Contracting-Out oder Objektgesellschaften, Betreibermodelle und Leasing (vgl. Grimsey/Lewis 2004: 10, IMF 2004: 6f, Klijn/Teisman 2003a: 85, Hartmann 1994: 37). Der Sinn einer derart weiten Begriffsverwendung ist allerdings gering, denn der Begriff grenzt kaum eine Form der Kooperation aus (vgl. Budäus 1997a: 15). Dennoch gilt eine weiter gefasste Definition von PPP als Mehrheitsmeinung.

2. PPP i.e.S. wird nur für jene Zusammenarbeit zwischen privatwirtschaftlichen und öffentlichen Unternehmen verwendet, die folgende Anforderungen erfüllen (vgl. Peter 1998):

- Jeder Teilnehmer kann für sich selbst verhandeln.

- Die Partnerschaft basiert auf einer lang anhaltenden, formellrechtlich geregelten Beziehung.

- Jeder Partner muss in der Lage sein, entweder materielle oder symbolische Werte in die Partnerschaft einzubringen.

- $\quad$ Alle Teilnehmer haben eine geteilte Verantwortung für das Resultat der PPP.

Diese Definition i.e.S. wird beispielsweise von Klijn/Teisman (2004: 147) vertreten: ,...cooperation between public and private actors with a durable character in which actors develop mutual products and/or services and in which risk, costs and benefits are shared ".

Auch in Deutschland wurde durch das Bundesgutachten „PPP im öffentlichen Hochbau“ 2003 der Versuch eines einheitlichen Verständnisses eingeführt (vgl. Weber et al. 2006: 16f). In diesem werden PPP folgendermaßen definiert: „,...als langfristige, vertraglich geregelte Zusammenarbeit zwischen öffentlicher Hand und Privatwirtschaft, zur wirtschaftlichen Erfüllung öffentlicher Aufgaben, bei der die erforderlichen Ressourcen (z.B. Know-how, Betriebsmittel, Kapital, Personal) in einem gemeinsamen Organisationszusammenhang eingestellt und vorhandene Projektrisiken entsprechend der Risikomanagementkompetenz der Projektpartner angemessen verteilt werden" (vgl. BMVBS 2003: 2f).

In der Literatur gibt es keine weitgehende Einigkeit darüber, welche Kriterien eine PPP konstituieren (vgl. IMF 2004: 6), aber aus der Literaturrecherche lassen sich für PPP i.e.S. folgende fünf Merkmale identifizieren: 
1. Teilnehmer: Es müssen sowohl öffentliche als auch privatrechtliche Leistungsträger an der PPP teilnehmen. Laut Caroll/Steane (2003: 50) werden dadurch verschiedene Partner mit unterschiedlichen Motiven, Werten und Zielen zusammengebracht (siehe Kapitel 4.1.3). Die Rollen der Vertragspartner können in PPP sehr unterschiedlich sein und die Partner können sich unterschiedlich intensiv in die Partnerschaft einbringen (vgl. Gerrard 2001). Die Teilnahme des privaten Sektors bedeutet aber nicht, dass es dabei zu „less government" kommt, sondern dass sich die Rolle des öffentlichen Partners verändert (vgl. Scharle 2001).

Als öffentliche Leistungsträger werden Gebietskörperschaften des Bundes, der Länder und Gemeinden, öffentlich-rechtliche Körperschaften, Anstalten und Stiftungen sowie öffentliche Unternehmen in öffentlichrechtlicher und privatrechtlicher Rechtsform bezeichnet (vgl. Strohbach 2001: 56).

Unter privatrechtlichen Leistungsträgern werden sowohl in privatem Eigentum stehende juristische als auch natürliche Personen bezeichnet (vgl. Strohbach 2001: 56f, Roggencamp 1999: 32).

2. Risiko-, Kosten- und Gewinnteilung: Bei einer PPP muss es zu einer Risikoteilung zwischen den beteiligten Partnern kommen (vgl. Grimsey/Lewis 2005: xiv). Auch für Klijn/Teisman (2003b) und Lewis (2003: 261) ist die Teilung von Risiken, Kosten aber auch der Gewinne eine wesentliche Eigenschaft von PPP. Für Weber et al. (2006: 53) stellt der Lebenszyklusansatz ein weiteres Merkmal dar, d.h. die Integration von Planen, Bauen, Finanzieren und Betreiben. Im Vergleich dazu werden beim Contracting-Out diese Phasen nicht gemeinsam durchgemacht (vgl. Klijn/Teisman 2003a: 85).

3. Vertrauen: Für eine erfolgreiche PPP ist gegenseitige Vertrauen das wesentliche Merkmal (vgl. Budäus 2006a: 19, McQuaid 2003: 14). In den Verträgen einer PPP werden die Rahmenbedingungen für die Zusammenarbeit festgelegt, um den Partnern die notwendige Sicherheit zu bieten, aber zusätzliches Vertrauen der Beteiligten ist notwendig (vgl. Parker/Hartley 2003). Wie auch Parker/Hartley (2003) festhalten: „.... where there is trust there should be less need for detailed and formal contracting". Auch Grimsey/Lewis (2005: xv) stellen fest: „the partnership is a high-trust relationship. ... A PPP has to be built on trust, like all successful partnerships".

4. Abstimmungsbedarf: Ein weiteres wesentliches Merkmal für PPP ist der fallweise oder auch kontinuierliche Abstimmungsbedarf zwischen den 
Partnern (vgl. Roggencamp 1999: 56). Laut Budäus (2006a: 16f) handelt es sich dort, wo alle Leistungen und Gegenleistungen sowie die Risiken des privaten und öffentlichen Partners von vornherein vertraglich klar definiert sind, zwar um eine Kooperationsform, aber nicht um eine PPP. Als Argument gegen Public Private Partnerships werden oftmals die erhöhten Transaktionskosten angeführt, die aufgrund des größeren Verhandlungsaufwand es bei PPP entstehen. Diese sollten jedoch durch das Ergebnis aufgewogen werden und idealerweise sollten PPP zu einem beiderseitigen Mehrwert führen, durch den jene Mehrkosten in Kauf genommen werden können (vgl. Parker/Hartler 2003).

5. Zielkomplementarität: Für eine funktionierende Partnerschaft ist es wichtig, dass die beteiligten Partner komplementäre Ziele aufweisen (vgl. Budäus 2006a: 16, Stiglitz/Wallstein 2000: 42, Budäus 1997a: 16f). Zielkonflikte führen langfristig zu keiner funktionsfähigen PPP, daher muss eine funktionsfähige PPP auf Dauer für beide Partner zu einer Win-WinSituation führen (vgl. Faulkner 2004: 65, McQuaid 2003: 22, Roggencamp 1999: 132ff, Smith 1999: 129).

Zusammenfassend kann aus dieser Diskussion festgehalten werden, dass keine Definition von PPP in der Literatur einer anderen gleicht. Grundsätzlich lassen sich die verschiedenen Definitionen aber in eine Definition i.w.S. und eine Definition i.e.S. unterteilen. Bei den einzelnen Definitionen, die zur Definition i.w.S. gezählt werden können, werden unterschiedliche Schwerpunkte gesetzt und daher auch verschiedene Modelle unter dem Begriff PPP subsumiert. Bei der Definition i.e.S. konnten wesentliche Kriterien in der Literatur entdeckt werden, die zur Definition herangezogen werden. In der vorliegenden Arbeit wird die Definition i.e.S. für PPP verwendet. Als Überbegriff für alle möglichen Kooperationsformen zwischen privater und öffentlicher Hand, die nicht die Kriterien der Definition i.e.S. erfüllen, d.h. PPP i.e.S. und PPP i.w.S., wird der Begriff Private Participation in Infrastructure (PPI) verwendet (vgl. Izagurirre 2004).

\subsubsection{Ziele und Interessen der Partner}

Wie schon eingangs erwähnt wurde, verfolgen die Partner von PPP unterschiedliche Interessen und demzufolge unterscheiden sich auch ihre Ziele. Obwohl die Ziele der Partner grundsätzlich komplementär sein sollten, lässt sich ein gewisses Konfliktpotenzial nicht verhindern (vgl. Eggers 2004:31f). Laut Eggers (2004: 119) können komplementäre Ziele eine notwendige jedoch nicht die ausreichende Bedingung für eine PPP sein. Beide Partner können Ziele formulieren, die sich wiederum in einzelne Subziele oder Interessen unterteilen 
lassen. Laut Literatur sollte es in einer Partnerschaft bzw. in einer PPP zu einer beiderseitigen Win-Win-Situation für die Partner kommen (vgl. Budäus 2006: 16, Faulkner 2004: 65, Smith 1999: 129).

\section{Effizienzsteigerung}

Ein wesentliches Ziel einer PPP für den öffentlichen Bereich ist die Effizienzsteigerung durch den privaten Partner bzw. durch die komplementäre Kombination der Produktionsfaktoren (vgl. Eggers 2004: 32). In der englischen Literatur wird auch vom "collaborative advantage" oder ,added value of synergy" gesprochen, welcher der wesentliche Vorteil einer Partnerschaft ist. In einer Partnerschaft können durch die Kombination oder Integration von unterschiedlichem Wissen und anderen Inputfaktoren Ergebnisse erzielt werden, die ohne diese nicht möglich wären (vgl. Klijn/Teisman 2004: 148, Huxham/ Vangen 2003: 293). Im Infrastrukturbau wird angenommen, dass Effizienzsteigerungen sowohl in der Bau- als auch in der Betriebsphase realisiert werden, die auf der optimalen Risikoteilung zwischen den Partnern und auf der Nützung von Know-How-Vorteilen beruhen (vgl. EC 2004: 8, IMF 2004: 3, Parker/ Hartley 2003, Scharle 2001, Strohbach 2001: 77, Roggencamp 1999).

Scharle (2001: 231) hingegen bezeichnet die Annahme der Effizienzsteigerungen aufgrund geringer Kosten, der Kombination von Ressourcen und der geteilten Risiken als ,good-faith"-Ansatz. Er sieht insbesondere die hohen Transaktionskosten von PPP sehr kritisch. Dies spielt insbesondere dann eine Rolle, wenn bisher wenig Erfahrung bei der Konstruktion von PPP gesammelt worden ist. Im Sinne der Partnerschaft müssen aber viele Details verhandelt werden, die bei einem Alleingang von öffentlicher oder privater Seite nicht notwendig wären.

Viel wahrscheinlicher erscheint hingegen die Annahme, dass private Unternehmen durch ihr Technik- und Management-Know-How Einsparungen erwirtschaften können. Nach Klijn/Teisman (2003b: 137) liegt der Vorteil einer PPP aber nicht ausschließlich in einer einfachen Effizienzsteigerung beim Projekt selbst, sondern aufgrund einer PPP kann ein ,added value of synergy" erzeugt werden. Sie verstehen darunter die Tatsache, dass ohne PPP beide Seiten nicht in der Lage wären, das Produkt zu entwickeln bzw. zu produzieren (vgl. Roggencamp 1999: 147).

Ein weiterer zentraler Aspekt für die Effizienzsteigerung von Infrastrukturprojekten ist die Verlagerung oder Reduzierung von Ungewissheit, da komplexe Projekte durch die Koordination mit dem privaten Partner sichergestellt werden (vgl. EC 2003a: 16). Bei der Errichtung einer Infrastrukturanlage durch einen kompetenten privaten Projektpartner ist auch eine Reduzierung der Projektbauzeit und damit eine Verringerung der Baukosten erzielbar (vgl. Strohbach 2001: 76, Roggencamp 1999: 149). Private verfolgen eine wirtschaftliche Handlungslogik, durch die auch die Einhaltung von Kosten- sowie Finan- 
zierungsplänen aber auch zeitlicher Vorgaben gewährleistet wird (vgl. Roger 1999, Roggencamp 1999: 88). Auch in der Betriebsphase dient das Know-How des privaten Unternehmens einer effizienten Führung.

\section{Zusätzliches Kapital}

Ein weiteres vorrangiges Ziel bzw. Interesse der öffentlichen Hand ist das Kapital der privaten Partner (vgl. EC 2003a: 14, Reijniers 1994). Das Interesse des öffentlichen Sektors liegt vor allem in der Mobilisierung zusätzlicher Finanzmittel, um die angespannte Finanzlage zu verbessern (vgl. IMF 2004: 3, Parker/Hartley 2003, Roger 1999). Basierend auf dieser Budgetknappheit muss der öffentliche Sektor nach alternativen Finanzierungsmöglichkeiten suchen, um weitere Infrastrukturprojekte finanzieren zu können. Dadurch kann teilweise auf Steuererhöhungen verzichtet werden oder es können aufgrund des Erreichens der staatlichen Verschuldungsgrenze ansonsten unfinanzierbare Projekte vorgezogen werden (vgl. Strohbach 2001: 78).

\section{Risikoteilung}

Vor allem kommt es bei einer PPP aber auch zu einer effizienten Aufteilung der Risiken, die von beiden Seiten tragbar sein müssen (vgl. Grohnert 2005, EC 2003a: 15, Scharle 2001, Hall 1998). Für den öffentlichen Bereich kommt es hierbei insbesondere zu einer Teilung des Projektentwicklungs- und Projektdurchführungsrisikos. Laut Roggencamp (1999: 88) bedeutet eine erfolgreiche PPP auch einen Imagegewinn für die öffentliche Hand und dient der Beziehungspflege zur Privatwirtschaft als einflussreicher Interessensgruppe.

\section{Ordnungspolitische Ziele}

Mit der Einbindung von privaten Unternehmen in die Erstellung von Infrastrukturleistungen werden auch ordnungspolitische Ziele verfolgt. Die neoliberale Auffassung von weniger Staat ist global beobachtbar (siehe Kapitel 2.2.4). Dabei wird der Funktionsfähigkeit des Marktmechanismus grundsätzlich Vertrauen geschenkt und durch die Bereitstellung von Gütern und Dienstleistungen über den Markt werden Effizienz- und Wohlstands-steigerungen erwartet (vgl. IMF 2004: 3, Strohbach 2001: 77f). Da aber in manchen Infrastrukturbereichen ein teilweises Marktversagen angenommen wird, wird staatliche Einflussnahme notwendig.

\section{Akquirierung von Aufträgen}

Für private Unternehmen, die insbesondere im Bereich der Erstellung von Infrastruktur tätig sind, ist der öffentliche Sektor ein wesentlicher Auftraggeber. Diese Unternehmen sehen sich aber aufgrund der öffentlichen Budgetknappheit 
mit einem Rückgang bei den Investitionen konfrontiert. Waren sie früher reine Auftragnehmer und Lieferanten für Dienstleistungen, versuchen sie durch innovativere Vertragsformen, wie beispielsweise PPP, an neue Auftäge heranzukommen (vgl. Reijniers 1994). Ein wichtiges Ziel für private Unternehmen ist daher die Akquirierung von neuen Aufträgen durch den öffentlichen Sektor.

\section{Gewinnsteigerung}

Des Weiteren sind private Partner vorrangig daran interessiert, ihr Kapital zu investieren und Gewinn zu realisieren bzw. zu steigern (vgl. Klijn/Teisman 2003b, Roggencamp 1999: 88). Der private Partner wird sein Engagement bei der Verfolgung der Minimalkostenkombination nur dann zur Verfügung stellen, wenn es für ihn rentabel ist (vgl. Eggers 2004: 14). Um Gewinne realisieren zu können, wollen private Akteure auch Verfügungsrechte über öffentliches Eigentum und finanzielle Unterstützung erhalten, etwa durch steuerliche Vergünstigungen oder Fördermittel. Beispielsweise können Regierungen zu günstigeren Konditionen als private Unternehmen Kredite aufnehmen (vgl. Praker/Hartley 2003). Durch ihre Nähe zur Politik und Verwaltung hoffen die privaten Unternehmen außerdem auf eine Absicherung der Investition. Wie bereits erwähnt, sind sie auch an einer Teilung der Projektrisiken interessiert und können durch öffentliches Know-How auch gewisse Risiken reduzieren. Für den privaten Partner ist außerdem der Imagegewinn als kommunaler Partner oder guter Bürger durch die Dokumentation von Verantwortungsbewusstsein und Leistungsfähigkeit von Interesse (vgl. Klijn/Teisman 2003b, Roggencamp 1999: 89).

Aus dieser Darstellung wird ersichtlich, dass sich die Einzelinteressen und ziele in gewisser Weise ergänzen bzw. komplementär oder aber auch entgegengesetzt sind. Dies wird auch in der folgenden Abbildung 17 nochmals übersichtlich dargestellt:

\subsubsection{Risiken und Problembereiche}

Im Bereich der Finanzierung von Infrastruktur und im Speziellen bei der Finanzierung von PPP gibt man sich über gesammelte Erfahrungen eher bedeckt. Die meisten Beiträge zu PPP, die über Erfahrungen in diesem Bereich berichten, befassen sich mit den positiven Auswirkungen. Nur wenige Beiträge befassen sich hingegen mit den Fehlern und Fehlschlägen von PPP, die aber wesentlich zu einer umfassenden Diskussion der Thematik beitragen würden. Die betroffenen Partner haben natürlich kein Interesse daran, Daten über ihre Projekte Preis zu geben, die für eine Analyse herangezogen werden könnten. Aufgrund dieser Tatsache wurde bisher nur wenig über Fehlschläge oder Problembereiche bei PPP berichtet (vgl. Scharle 2002).

Aber auch in der Literatur erkennt man gewisse Problemfelder, die bei PPP auftreten können und Huxham/Vangen (2003: 306) stellen fest: „It seems clear 
that involvement in partnership is challenging“. Laut Grimsey/Lewis (2002) entstehen die meisten Risiken bei einer PPP aus der Komplexität der Vereinbarung selber, wie beispielsweise die aufwendige Dokumentation, die Finanzierung, Versteuerung oder Untervereinbarungen sein, die sich auch im Laufe der Zeit verändern.

\section{Abbildung 17: Ziele und Interessen der Akteure in einer PPP}

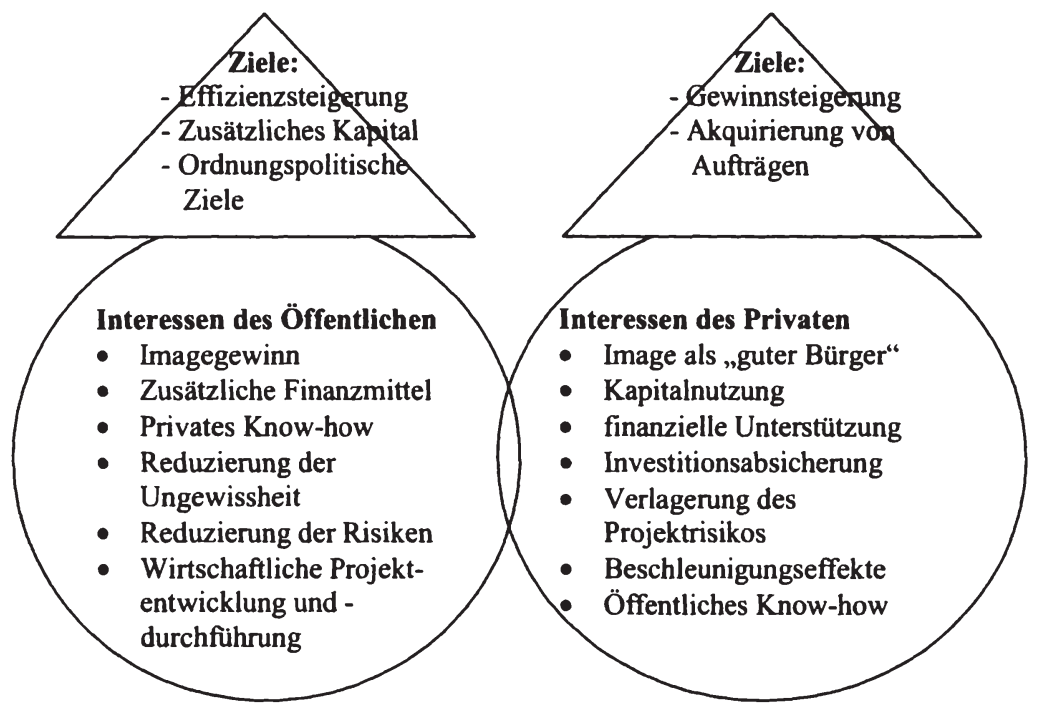

Quelle: in Anlehnung an Roggencamp (1999: 89)

\section{Ursächliches Risiko: Partnerschaft}

Die Partnerschaft selbst wird als Ursache für die Probleme und Risiken, die sich in einer PPP ergeben können, angesehen (vgl. Klijn/Teisman 2004: 148, Scharle 2001). Dies kann u.a. wiederum auf zwei Grundvoraussetzung einer Partnerschaft zurückgeführt werden (vgl. Klijn/Teisman 2003b). Einerseits ergibt sich durch die Zusammenarbeit ein Anstieg der beteiligten Personen am Entscheidungsprozess und andererseits sind die Partner bei einer Partnerschaft auch voneinander abhängig. In sehr ausgeprägten Individualgesellschaften ist es genau diese Abhängigkeit von den Partnern, die Probleme in der Zusammenarbeit verursachen kann. Erst durch das Eingehen der Partnerschaft müssen nämlich Verhandlungen geführt werden, um sowohl die eigenen Interessen zu vertreten, aber auch die der Partner zu berücksichtigen (vgl. Klijn/Teisman 2004: 148).

Grundlegend problematisch erscheint bei PPP der Versuch, von beiden Organisationsformen, d.h. vom privaten und vom öffentlichen Partner, aus- 
schließlich die Vorteile herauszuziehen und dabei die Nachteile zu eliminieren. Dadurch ergibt sich ein neuer Organisationstyp, der in keine der beiden Kategorien passt und deren Handlungsfähigkeit nur schwer einzustufen ist. Vor allem gibt es zwischen öffentlichem und privatem Sektor wesentliche Unterschiede, die bei der Etablierung einer PPP zu wenig berücksichtigt werden. Öffentliche Unternehmen können Verluste tolerieren und sind eher an einer Wiederwahl interessiert. Private Unternehmen hingegen werden ausschließlich anhand ihrer Ergebnisse bewertet (vgl. Klijn/Teisman 2003b). Auch die unterschiedliche Unternehmenskultur von öffentlichen und privaten Unternehmen kann zu Spannungen bei einer PPP beitragen, wie beispielsweise die unterschiedliche Wahrnehmung und Bewertung von Risiken oder auch des Zeitfaktors (vgl. Kouwenhoven 1993: 123).

Für den privaten Partner ist außerdem wichtig, sich vor dem Entstehen einer PPP im Klaren darüber zu sein, dass Regierungen und somit deren politische Ansätze oftmals innerhalb von kurzer Zeit wechseln. Die Zeitspanne, in der eine gewisse Regierung an der Macht ist, stellt vielleicht nur einen kurzen Teil der Projektlaufzeit dar (vgl. Smith 1999: 131). Insbesondere in Entwicklungsländern stellen die politische Instabilität, der häufige Regimewechsel sowie die Korruption in der Politik wesentliche Risiken für PPP dar (vgl. Grimsey/Lewis 2004: 220).

\section{Unterschiedliche Zielsetzungen}

Laut Reijniers (1994) verfolgen privatwirtschaftliche und öffentliche Unternehmen grundsätzlich unterschiedliche Zielsetzungen, die nicht zusammenpassen. Zu Beginn eines Projektes haben solche Spannungen noch keine Auswirkungen, da beide Parteien noch motiviert zusammenzuarbeiten, aber meistens können die Unterschiede nach einer gewissen Zeit zu gravierenden Auswirkungen führen. Klijn/Teisman (2004: 149) bezeichnen PPP als Spiele und sie versuchen anhand der Spieltheorie die Strategien der Beteiligten bzw. deren Verhalten zu erklären. Sie weisen darauf hin, dass alle Beteiligten einer PPP eine unterschiedliche Vergangenheit aufweisen, an unterschiedlichen Netzwerken beteiligt sind und durch Entscheidungen, die in anderen Bereichen (,arena') getroffen werden, ständig beeinflusst werden.

\section{Unterschiedliche Interessen}

Es spielen auch die unterschiedlichen Interessen der Partner sowie die Transaktionskosten für den Erfolg von PPP eine große Rolle. Wie bereits aus der geführten Diskussion hervorgeht (siehe 4.1.3), verfolgen öffentliche und private Partner unterschiedliche Interessen bei der Errichtung einer PPP. Diese Interessen überschneiden sich zwar in gewissen Bereichen, wie beispielsweise bei der Finanzierung oder raschen Fertigstellung der Einrichtung, jedoch kann es auch im Nachhinein zu Interessenskonflikten kommen (vgl. Strohbach 2001: 
79). Je höher die Anzahl der beteiligten Parteien an einer PPP ist, desto unterschiedlicher können auch die Interessen sein und desto höher ist das Konfliktpotenzial. Auch durch die multiplen und unklar definierten Ziele der öffentlichen Hand, können sich zahlreiche Konflikte zwischen den privaten und öffentlichen Aktionären ergeben (vgl. Bös/Schneider 1996).

\section{Erhöhte Kosten}

Werden PPP vor allem aufgrund von möglichen positiven Potenzialen, wie beispielsweise Kosteneinsparung, verwirklicht, können bei PPP aber in den folgenden drei Bereichen erhöhte Kosten entstehen, die wiederum zu Konflikten führen können:

1. Bei PPP steigen aufgrund des erhöhten Verwaltungs- und Kommunikationsaufwandes die Transaktionskosten (vgl. EC 2004: 8). Zusätzlich dazu verursachen der Ausschreibungsprozess und die Auftragsvergabe Kosten, die mit der Komplexität der Modelle und der Anzahl der am Projekt involvierten Parteien steigen (vgl. Strohbach 2001: 79). Diese Kosten kommen vor allem dann verstärkt zum Tragen, wenn bisher noch keine Erfahrungen mit PPP gesammelt worden sind. Insbesondere die komplexen Verträge, die erstellt werden müssen, sowie die Arbeitstreffen, die dafür notwendig sind, können zu erhöhten Kosten führen. Die generellen Kosten der Ausschreibung entstehen aber auch beim Contracting-Out im öffentlichen Bereich.

2. Die privaten Unternehmen werden auch Garantien abschließen, um den wirtschaftlichen Erfolg der Investition zu sichern. Dadurch entstehen auch höhere Kosten, die wiederum von den Konsumenten oder den Steuerzahlern getragen werden müssen.

3. Ein weiterer Diskussionspunkt sind die erhöhten Kosten, die dem privaten Unternehmen aufgrund des schlechteren Ratings bei der Kapitalbeschaffung im Vergleich zur öffentlichen Hand entstehen (vgl. Klein 1997). Generell ist es teurer auf Projektbasis Fremdkapital zu beschaffen, insbesondere bei so langen Laufzeiten wie bei Infrastrukturprojekten erhöhen sich die Kosten für die Finanzierung und Absicherung (vgl. EC 2004: 8).

Eine sehr pessimistische Sichtweise vertreten Klijn/Teisman (2003b, 2004: 159), indem sie anhand von drei Faktoren die Unrealisierbarkeit von PPP erklären. Sie kommen zu dem Schluss, dass ,PPP - the right form at the wrong moment" ist. Als die drei wesentlichen Gründe für das Scheitern von PPP nennen sie die Komplexität der Partnerzusammensetzung, die institutionellen Faktoren sowie das strategische Verhalten der Akteure (vgl. Klijn/Teisman 2003b). Laut Klijn/Teisman passen demnach richtige Partnerschaften nicht in die institut- 
ionellen Regeln, Rollen und Beschaffenheiten des 21. Jahrhunderts, sondern sie bedürfen einer ,network society", um erfolgreich zu sein. Eine solche Gesellschaftsform ist aus heutiger Sicht allerdings sehr unrealistisch.

Partnerschaften in Form von PPP im Infrastruktursektor scheinen tatsächlich schwer realisierbar zu sein, da große Infrastrukturprojekte auch ohne Partnerschaft bereits hoch komplex sind. Dennoch ist ein völliges Scheitern dieser Idee nicht zu erkennen, da vor allem bei kleineren Projekten mit lokalen Partnern die Risiken überschaubarer sind und Synergieeffekte entstehen können.

\subsection{PPI in der Entwicklungspolitik}

Ziel dieses Kapitels ist es, eine Verknüpfung mit den vorgestellten entwicklungstheoretischen Ansätzen im Kapitel 2 und der Teilnahme von privaten Unternehmen in der Infrastrukturfinanzierung herzustellen. Daher wird im ersten Abschnitt ein Überblick über die Auswirkungen der entwicklungspolitischen Veränderungen in der Infrastrukturfinanzierung gegeben. Das daran anschließende Kapitel 4.2.2 zeigt die sich verändernde Rolle der Offical Development Assistance (ODA) in der Infrastrukturfinanzierung. Im Kapitel 4.2.3 wird die Rolle des privaten Sektors bei der Finanzierung von Infrastruktur dargestellt, wobei zuerst auf die öffentlich-privaten Finanzierungsmodelle und daran anschließend auf die PPI-Ströme nach Sektoren und Regionen eingegangen wird und endet mit einer Zusammenfassung der Entwicklung der PPIStröme. Kapitel 4.2.4 befasst sich mit der neuen Rollenaufteilung bei der Infrastrukturfinanzierung.

\subsubsection{Auswirkungen der entwicklungspolitischen Veränderungen in der Infrastrukturfinanzierung}

Als in den späten 1970er und frühen 1980er Jahren viele Länder aufgrund von globaler Rezession, Inflation und gesunkenen Rohstoffpreisen einem erhöhten Finanzdruck ausgesetzt waren, wurde auch der Grundstein für eine ideologische Veränderung zu Gunsten des privaten Sektors in der Infrastrukturfinanzierung gelegt. Diese Entwicklung zeigte sich vor allem in der Verwendung der finanziellen Mittel durch die IFIs. Investierte die Weltbank 1987 noch die Hälfte des Gesamtbudgets in Infrastruktur, waren es 1990 nur noch 30 \% (vgl. World Bank 2006c: xiii). Diese Tendenz reflektiert die Annahme, dass sich der gleichzeitig stattfindende Investitionsboom der privaten Unternehmen der Zukunft fortsetzen würde.

Gleichzeitig wurden die Regierungen der Entwicklungsländer Mitte der 1980er Jahre von Seiten der Gebernationen durch die Strukturanpassungsprogramme (SAP) (siehe Kapitel 2.2.2.1) dazu gezwungen, die Verschuldung abzubauen und sich nicht neu zu verschulden. Die Privatisierung stellte in vielen 
Fällen eine Voraussetzung für Entschuldung und neue Kreditvergabe dar (vgl. Küblböck 2004: 16). Daher wurden die öffentliche Infrastruktur großteils privatisiert und von Seiten der öffentlichen Hand keine oder nur wenige Investitionen in diesem Bereich getätigt (vgl. Nuscheler 2004a: 368). Diese Annahme spiegelt sich im Washington Consensus wider (siehe Kapitel 2.2.2.1), dessen Leitlinien der weitgehende Staatsabbau und die Privatisierung bzw. der Ausverkauf von Staatsbetrieben an Privatfirmen waren (vgl. Stiglitz 2006: 49). Die Argumentationslinien des Washington Consensus blieben jedoch nicht unkritisiert und die Annahmen werden vereinfacht in der folgenden Abbildung 18 dargestellt (vgl. u.a. Stiglitz 2006: 49ff).

Abbildung 18: Vereinfachte Darstellung der Annahmen des Washington Consensus bei der Privatisierung von Infrastruktur

$$
\begin{aligned}
& \text { Annahmen des Washington Consensus: } \\
& \begin{array}{c}
\uparrow \text { Privatisierung }+\uparrow \text { private Investitionen in der Infrastruktur } \\
=\text { Effizienzsteigerung in der Infrastruktur }
\end{array}
\end{aligned}
$$

Afffizienzsteigerung in der Infrastruktur führtzu| Ausländische Direktinvestitionen

$$
=\uparrow \text { Wachstum }=\uparrow_{\text {und Armutsreduktion }}
$$

Durch Privatisierungen sollte es zu mehr privaten Investitionen im Infrastruktursektor kommen, die wiederum aufgrund ihres Managementansatzes zu einer Effizienzsteigerung bei den Infrastrukturleistungen führen sollten. Aufgrund der verbesserten Infrastruktur durch die privaten Unternehmen oder in Kooperation mit dem öffentlichen Sektor wird angenommen, dass die Attraktivität des Landes steigt und mehr ausländische Investitionen in den Produktionssektor getätigt werden. Die Ausländischen Direktinvestitionen (ADI) leisten wiederum einen Beitrag zum Wirtschaftswachstum und dieses soll im Sinne des „TrickleDown"-Ansatzes schließlich auch zur Armutsreduktion beitragen (siehe Kapitel 2.2.2.2).

In den späten 1980er und frühen 1990er Jahren kam es schließlich aufgrund von Budgetproblemen zur bisher größten Privatisierungswelle in den Entwicklungsländern (vgl. Estache 2006, ADB 2005b: 53, Birdsall/Nellis 2005: 3, Osius/Carlson 2004: 5). Laut Nellis (2006) wurden aufgrund von Privatisierungen in den vergangen 25 Jahren mehr als 400 Milliarden US\$ Verkaufserlöse umgesetzt. Grund dafür waren die Ansätze des New Public Managements (siehe Kapitel 2.2.4), die eng mit Good Governance verknüpft sind (siehe Kapitel 2.2). 
Wesentliche Infrastruktureinrichtungen wurden unter dem Druck der „Geberländer" privatisiert, in der Annahme, dass Privatisierung zu einer Steigerung des Wirtschaftswachstums führe (vgl. Nellis/Birdsall 2005).

Theorien, die diese These unterstützen, können sowohl im Bereich der Wirtschaftspolitik, als auch auf der Mikroebene gefunden werden. Auf politischer Ebene wird die Privatisierung als ein neutraler Prozess ökonomischer Veränderung gesehen, der eine politische Antwort auf ein Budgetproblem ist (vgl. Küblböck 2004: 14). Insbesondere aber die Theorien der Mikroökonomie, wie beispielsweise die Property Rights (siehe Kapitel 2.2.4.1), unterstützen die Annahme, dass privat geführte Unternehmen zu einem höheren Wirtschaftswachstum führen (vgl. Cook/Uchida 2003). Die empirischen Ergebnisse über die Auswirkungen von Privatisierungen sind jedoch wenig aussagekräftig, da viele existierende Studien auf Daten basieren, die relativ knapp nach der Privatisierung erhoben wurden und daher nur eingeschränkt über deren langfristigen Auswirkungen berichten können (vgl. Birdsall/Nellis 2005: 25).

\subsubsection{Die Rolle der ODA in der Infrasturkturfinanzierung}

Von öffentlicher Seite kam es gleichzeitig zu den Structural Adjustment Policies (SAP) zu einem starken Rückgang bei den Infrastrukturausgaben. Wurden in den 1970er und 1980er Jahren in den Entwicklungsländern noch durchschnittlich $15 \%$ des Bruttoinlandsproduktes in die Infrastruktur investiert, sind es heute nur noch ca. $3 \%$ (vgl. Hesselbarth 2004: 2). Zusätzlich dazu begannen Anfang der 1990er Jahre auch die „Geberländer“, vermehrt in soziale Aktivitäten $\mathrm{zu}$ investieren, um die negativen Auswirkungen der Structural Adjustment Policies (SAP) abzuschwächen und investierten weniger in die Infrastruktur.

\section{Abbildung 19: Anteil der Bilateralen Entwicklungshilfe für Infrastruktur}

Gesamte bilaterale ODA

$\square$ Gesamte bilaterale ODA für Infrastruktur

- Anteil der Infrastruktur an der gesamten bilateralen ODA in \%

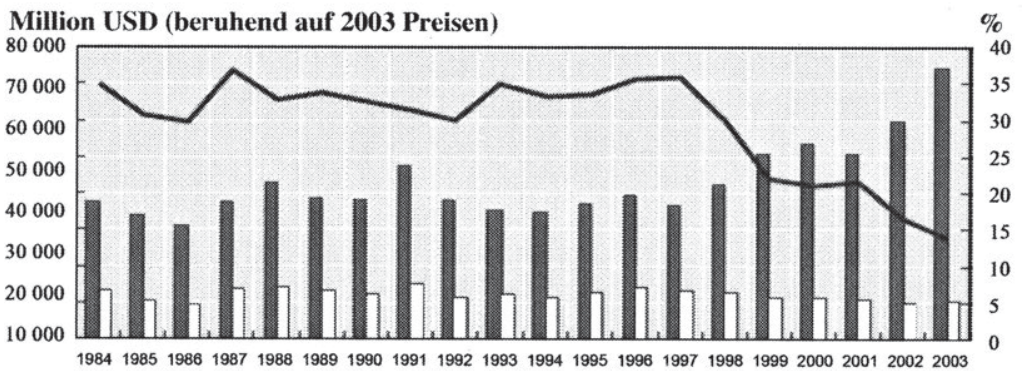

Quelle: OECD Creditor Reporting System nach OECD (2006a: 19) 
Obwohl die Official Development Assistance (ODA) bisher nur einen geringen Anteil am gesamten Infrastrukturvolumen ausmachte, reduzierte sich der Anteil der Entwicklungshilfe in der Infrastruktur nochmals drastisch. In den Jahren von 1996 zu 2002 haben sich die Ausgaben der ODA im Infra-strukturbereich von US\$ 15.175 Millionen auf US\$ 8.174 Millionen beinahe halbiert (vgl. Hesselbarth 2004: 3). Da beide Finanzierungsquellen abnahmen, erhoffte man sich die große Erleichterung durch den privaten Sektor (vgl. OECD 2006a: 21, ADB 2005b: 65).

Der Abwärtstrend der ODA im Infrastruktursektor ist aus Abbildung 19 ersichtlich. Gleichzeitig sind Mitte der 1990er Jahre aber auch die öffentlichen und die privaten Investitionen stark zurückgangen. Daraus resultiert eine große Lücke zwischen der Nachfrage und dem Angebot.

\subsubsection{Die Rolle des privaten Sektors in der Infrastrukturfinanzierung}

Seit dem Paradigmenwechsel hin zum privaten Sektor, ist dieser für die Finanzierung von Infrastruktur in Entwicklungsländern von wachsender Bedeutung. Wurden bisher die Infrastrukturinvestitionen im Wesentlichen aus zwei Quellen finanziert, nämlich der öffentlichen Hand und der ODA, hoffte man ab den 1990er Jahren, dass ein Rückgang dieser beiden Quellen durch den privaten Sektor abgefangen würde (vgl. Estache 2004: 9). Die in den privaten Sektor gesetzte Hoffnung blieb jedoch zum Großteil unerfüllt, da das hohe Investitionsvolumen der 1980er und 1990er Jahre zu optimistisch in die Zukunft prognostiziert wurde (vgl. Willoughby 2004a: 13). Die Investitionen verringerten sich von 1997 bis 1999 beispielsweise um rund 1/5 im Vergleich zu den Prognosen der Weltbank (vgl. Izaguirre 2000). Zusätzlich dazu sind die Investitionen auf einige wenige Sektoren und Länder beschränkt (vgl. Hesselbarth 2004: 12).

\subsubsection{PPI-Ströme nach Sektoren}

Die folgende Tabelle 2 zeigt nach Sektoren aufgeteilt die Investitionsströme von privaten Unternehmen in die Infrastruktur in Entwicklungsländer. Im Zeitraum von 1995 bis 2005 wanderten die meisten Investitionen in den Telekommunikationssektor, der fur private Investoren am lukrativsten erscheint. Auch im Stromsektor hatten 1999 bereits 27 Entwicklungsländer mehr als 700 Projekte mit dem Privatsektor abgeschlossen (vgl. Izaguirre 2000). Die Investitionen im Wassersektor waren eher gering, da dieser Sektor aufgrund seiner Charakteristika für private Investoren zu kompliziert und unrentabel ist. Problematisch im Strom- und Wassersektor ist, dass zuvor nicht die wahren Kosten an die Konsumenten weitergegeben wurden und es durch die privaten Investoren zu einer natürlichen Preiserhöhung kommen musste (vgl. Harris 2003: vii). Im 
Transportsektor können nach einem starken Einbruch ansteigende Investitionen verzeichnet werden. Wie aus der Tabelle 2 ersichtlich ist, kam es nach dem anfänglichen Investitionsboom in den 1980er und 1990er Jahren, der durch die Privatisierungs- und Liberalisierungsbestrebungen der 1980er Jahre ausgelöst worden war, zu einem starken Rückgang der privaten Investitionen in allen Sektoren.

Tabelle 2: Investitionen in die Infrastruktur mit privater Beteiligung, aufgeteilt nach Sektoren in Milliarden US\$, für den Zeitraum 1996-2005

\begin{tabular}{|l|c|c|c|c|c|c|c|c|c|c|c|}
\hline & $\mathbf{1 9 9 6}$ & $\mathbf{1 9 9 7}$ & $\mathbf{1 9 9 8}$ & $\mathbf{1 9 9 9}$ & $\mathbf{2 0 0 0}$ & $\mathbf{2 0 0 1}$ & $\mathbf{2 0 0 2}$ & $\mathbf{2 0 0 3}$ & $\mathbf{2 0 0 4}$ & $\mathbf{2 0 0 5}$ & $\boldsymbol{\Sigma}$ \\
\hline Sektor & & & & & & & & & & & \\
\hline Energie & 30,0 & $\mathbf{4 6 , 3}$ & 29,3 & 21,1 & 27,4 & 15,6 & 19,2 & 17,6 & 12,7 & 17,4 & $\mathbf{2 5 8 , 9}$ \\
\hline Telekommunikation & 24,6 & 39,9 & 51,8 & 36,1 & 48,9 & 45,2 & 33,0 & 33,2 & 45,0 & 59,6 & $\mathbf{4 3 4 , 7}$ \\
\hline Transport & 15,7 & 19,4 & 17,5 & $\mathbf{8 , 2}$ & $\mathbf{9 , 1}$ & $\mathbf{8 , 1}$ & 3,6 & 5,0 & 4,5 & 16,5 & $\mathbf{1 1 5 , 6}$ \\
\hline Wasser/Abwasser & 1,7 & 8,4 & 2,2 & $\mathbf{6 , 5}$ & 4,8 & 2,4 & 2,0 & 1,4 & 1,9 & 1,5 & $\mathbf{3 4 , 2}$ \\
\hline Gesamt & $\mathbf{7 2 , 1}$ & $\mathbf{1 1 4 , 1}$ & $\mathbf{1 0 0 , 9}$ & $\mathbf{7 2 , 0}$ & $\mathbf{9 0 , 2}$ & $\mathbf{7 1 , 3}$ & $\mathbf{5 7 , 8}$ & $\mathbf{5 7 , 2}$ & $\mathbf{6 4 , 1}$ & $\mathbf{9 5 , 0}$ & $\mathbf{8 4 3 , 4}$ \\
\hline
\end{tabular}

Quelle: World Bank (2004a), PPI Project Database

\subsubsection{PPI-Ströme nach Regionen}

Eine weitere Aufteilung der Investitionsströme kann nach Regionen erfolgen, wie aus Tabelle 3 ersichtlich ist. Es ist klar zu erkennen, dass die Investitionen unterschiedlich stark in die jeweiligen Regionen fließen. Die armen Länder Europas und Zentralasiens verzeichnen die meisten ausländischen Investitionen in der Infrastruktur. Subsahara Afrika ist hingegen nur wenig erfolgreich bei privaten Investitionen und nur rund $5 \%$ der gesamten Investitionen fließen in diese Region. Auch die Länder Nordafrikas und des Nahen Ostens bekamen nur $7 \%$ der Gesamtinvestitionen. Lateinamerika und Asien erhielten 2005 annähernd die gleiche Höhe an Investitionen in der Infrastruktur mit privater Beteiligung.

Bei dieser Aufteilung hinkt Afrika allen anderen Regionen dramatisch hinterher. In Asien erreichten die privaten Investitionen 1997 ihren Höhepunkt und nahmen nach der asiatischen Finanzkrise stark ab. Seit wenigen Jahren kann wieder ein leichter Anstieg verzeichnet werden, aber der Höhepunkt von 1997 wurde bisher nicht annähernd erreicht. Die Region Ostasien und Pazifik steht insgesamt an zweiter Stelle bei den privaten Investitionen. Dort begannen in der zweiten Hälfte der 1980er Jahre die ersten Investitionen in Malaysien und auf den Philippinen, wo sie auch in den 1990er Jahren stark anwuchsen. Obwohl alle Länder dieser Region in den 1990er Jahren vermehrt private Investoren in der Infrastruktur anzogen, waren es allen voran China, Indonesien und die Philippinen, die zu den hohen Investitionsvolumen 1996 und 1997 beitrugen (vgl. World Bank 2003c: 23). 
Tabelle 3: Investitionen in die Infrastruktur mit privater Beteiligung, aufgeteilt nach Regionen in USS Milliarden, für den Zeitraum von 1996-2005

\begin{tabular}{|l|c|c|c|c|c|c|c|c|c|c|c|}
\hline & 1996 & 1997 & 1998 & 1999 & 2000 & 2001 & 2002 & 2003 & 2004 & 2005 & $\Sigma$ \\
\hline Regionen & & & & & & & & & & & \\
\hline $\begin{array}{l}\text { Ostasien und } \\
\text { Pazifik }\end{array}$ & 28,0 & 34,9 & 9,7 & 13,1 & 14,3 & 11,0 & 9,7 & 13,0 & 8,7 & 13,5 & 174,7 \\
\hline $\begin{array}{l}\text { Europa und } \\
\text { Zentralasien }\end{array}$ & 10,5 & 14,2 & 12,1 & 9,4 & 25,0 & 12,3 & 16,8 & 12,2 & 12,5 & 34,8 & 167,9 \\
\hline $\begin{array}{l}\text { Lateinamerika } \\
\text { und Karibik }\end{array}$ & 25,8 & 49,3 & 71,2 & 37,3 & 38,7 & 33,7 & 19,6 & 15,8 & 17,4 & 20,5 & 346,4 \\
\hline $\begin{array}{l}\text { Mittlerer } \\
\text { Osten und } \\
\text { Nordafrika }\end{array}$ & 0,3 & 5,1 & 3,1 & 3,0 & 4,1 & 4,4 & 1,6 & 6,2 & 10,9 & 6,7 & 45,5 \\
\hline $\begin{array}{l}\text { Suldasien } \\
\text { Subsahara }\end{array}$ & 5,8 & 6,3 & 2,3 & 4,6 & 4,4 & 4,6 & 6,0 & 3,4 & 9,6 & 13,6 & $\mathbf{6 4 , 4}$ \\
\hline Afrika & $\mathbf{7 2 , 1}$ & 114,1 & 100,9 & $\mathbf{7 2 , 0}$ & $\mathbf{9 0 , 2}$ & $\mathbf{7 1 , 3}$ & $\mathbf{5 7 , 8}$ & $\mathbf{5 7 , 2}$ & $\mathbf{6 4 , 1}$ & $\mathbf{9 5 , 0}$ & $\mathbf{8 4 3 , 4}$ \\
\hline Gesamt & & & & & & & & & & & \\
\hline
\end{tabular}

Quelle: World Bank (2004a), PPI Project Database

\subsubsection{Zusammenfassung der PPI-Entwicklung in den Entwicklungsländern}

Öffentlich-private Modelle haben sich zu einer Alternative für die Herstellung von Infrastrukturleistungen entwickelt, die sowohl in entwickelten Ländern, wie beispielsweise Großbritannien oder Australien, aber auch in der Entwicklungspolitik immer mehr Beachtung findet (vgl. OECD 2006, ADB 2005b, Sagar 2006, Bestani 2004, Osei 2004: 251). Gründe dafür sind sowohl die Privatisierungsbestrebungen der neoliberalen Schule (siehe Kapitel 2.2.4.1), aber auch der enorme Investitionsbedarf zur Verbesserung der Infrastruktur, da der Investitionsbedarf die Möglichkeiten der Entwicklungs- und Schwellenländer und auch der internationalen Gebergemeinschaft bei weitem übersteigt (vgl. IUED 2005: 79, EC 2004: 5).

Ein wesentliches Argument für die Verwendung von öffentlich-privaten Finanzierungsmodellen in der Entwicklungspolitik ist die Annahme, dass es zu Effizienzzugewinnen durch die privaten Unternehmen kommen wird. PPI haben somit das Ziel, privatwirtschaftliches Engagement für entwicklungspolitische Zwecke zu nutzen (vgl. Martens 2004: 51). Theoretisch könnten PPI daher das Infrastrukturdilemma der Entwicklungsländer lösen, aber in Wirklichkeit sind die Ergebnisse von PPI-Finanzierungen der vergangenen Jahre sehr gemischt (vgl. Harris 2003: vii, Farlam 2005: i). Tatsache ist, dass Unternehmen nur dort investieren, wo es für sie am profitabelsten ist - und das ist auch ihr gutes Recht (vgl. Martens 2004: 52). Allerdings gibt es mehr Infrastruktursektoren, wie beispielsweise die Wasser und Abwasserversorgung, die nicht profitabel sind und daher nur von öffentlicher Hand finanziert werden können. 
Der anfänglich große Enthusiasmus gegenüber privaten Investitionen im Infrastruktursektor ist beinahe verschwunden, da aufgrund der asiatischen Finanzkrise viele dieser Projekte nicht überlebt haben. Patterson (1984: 148) bringt die Unvereinbarkeit von privaten Firmen bei der Grundversorgung auf den Punkt: „Business cannot make a profit where potential customers have little or no income". In die gleiche Richtung argumentiert auch Plummer (2002), die eine Übertragung des PPI-Konzepts in die Infrastruktur in Entwicklungsländern sehr kritisch sieht. Vor allem, weil das PPI-Konzept seinen Ursprung in den Ländern des Nordens hat, die normalerweise eine gut entwickelte institutionelle Infrastruktur aufweisen. Dennoch glaubt Patterson (1984: 14), dass private Unternehmen an der Finanzierung von Infrastruktur teilnehmen können, aber nur unter der Voraussetzung, dass die öffentliche Hand das Projekt durch Subventionen oder Garantien unterstützt.

Eine $100 \%$ ige Privatisierung wird daher nicht notwendigerweise in allen Infrastruktursektoren zu Effizienzsteigerungen oder Wachstum führen (vgl. Farlam 2005: 51) und es ist eine Einschränkung für die Verwendung von öffentlichprivaten Finanzierungsmodellen nach Sektoren vorzunehmen (vgl. EC 2003a: 13). In der Entwicklungszusammenarbeit sollen bei der Anwendung von PPI die Auswirkungen auf die Armen im Vordergrund stehen: „It has to be carefully assessed if the benefits of private participation will outweigh the costs and the risks of bringing in the private sector. A very important variable of this assessment is the impact of private sector participation on the poor" (EC 2004: 5). Zusammenfassend kann daher festgehalten werden, dass das Ziel von PPI darin liegt, Chancen zu nützen, die sich aus der Verbindung des verstärkten Engagements von Unternehmen in Entwicklungsländern mit dem spezifischen Know-How der privaten Partner ergeben. PPI soll in den Entwicklungsländern somit einen Entwicklungsbeitrag leisten (vgl. bfai 2004: $11 \mathrm{ff}$ ).

Die unterschiedlichen Interessen der beteiligten Partner dürfen bei PPI in Entwicklungsländern jedoch nicht aus den Augen verloren werden. Für das private Unternehmen ist der wirtschaftliche Erfolg der Investition am wichtigsten und auch die Regierung des investierenden Unternehmens hat ein originäres Interesse an einem guten Projekt, da dies wiederum wichtige Wachstumsimpulse für die Heimatwirtschaft liefert. Außerdem will die öffentliche Hand die Dienstleistung zu einem möglichst günstigen Preis und zu geringen Kosten hergestellt haben. Allerdings sind nicht alle lokalen oder regionalen öffentlichen Einheiten vorbehaltlos enthusiastisch gegenüber der Entwicklung von PPI eingestellt (vgl. Caroll/Steane 2003: 46). Die öffentliche Hand muss auch mit den politischen Auswirkungen von Privatisierungen bzw. PPI-Konzepten umgehen können, da es dabei zu Preiserhöhungen kommen kann.

PPI ist daher kein „Allheilmittel“ für die Finanzierung der fehlenden Infrastruktur in Entwicklungsländern (vgl. Farlam 2005: 55) aber wie Bestani (2004: 5) trotz aller Bedenken enthusiastisch feststellt: „We are now in a position to turn it into an effective and forceful reality". 


\subsubsection{Eine neue Rollenverteilung in der Infrastrukturfinanzierung}

Der große Rückgang bei den Investitionen kam 1997 mit der asiatischen Finanzkrise. Die dadurch gescheiterten Projekte, die zu einer gewissen Resignation führten, sind für den Abschwung mitverantwortlich (vgl. EC 2004: 5). Zusätzlich dazu wurden sowohl von Seiten der Regierungen als auch der Investoren viel $\mathrm{zu}$ hohe Erwartungen in die private Infrastrukturfinanzierung gesetzt (vgl. Harris 2003: vii). Eine weitere Erklärung für den nicht mehr erreichten Höhepunkt von 1997 stellt laut Palmade/Anayiotas (2004) auch die Tatsache dar, dass zu Beginn vor allem sehr große öffentliche Infrastruktureinrichtungen privatisiert wurden.

Die asiatische Finanzkrise löste ein starkes Umdenken sowohl auf öffentlicher als auch auf privater Seite aus (vgl. Izaguirre 2000). Die meisten Infrastrukturinvestitionen waren Dollar-finanziert, da die lokalen Finanzmärkte nicht ausreichend entwickelt waren (vgl. Osius/Carlson 2004: 6). Dies bedeutete aufgrund der dramatischen Entwertung vieler Währungen in den Entwicklungsländern plötzlich eine extreme Steigerung der Verschuldung. Die Unausgeglichenheit zwischen der Dollarverschuldung und den Einnahmen in der heimischen Währung kamen hier verstärkt zum Tragen. Die Folge war ein finanzielles Ungleichgewicht, das in vielen Projekten für Probleme sorgte und manche sogar scheitern ließ (vgl. Bestani 2004).

Aufgrund dieser schwierigen Finanzsituation wurden viele private Infrastrukturabkommen von den betroffen Ländern neu verhandelt oder widerrufen (vgl. ADB 2005b: 54). Viele Regierungen stellten aufgrund der prekären Finanzsituation nämlich plötzlich fest, dass sie zu hohe Unterstützung an die privaten Investoren zahlten und griffen in bestehende Verträge ein oder lösten sie auf. Dadurch wurden die Erwartungen und die Bereitschaft für weitere Investitionen auf Seiten der privaten Investoren großteils nachhaltig gestört (vgl. ADB 2005b: 55). Die verhandelten Verträge stellten sich als nicht krisenresistent heraus und auch die bestehenden rechtlichen Rahmenbedingungen schützten die privaten Investoren nicht. In der Folge waren auch die Banken von den Vertragsbrüchen betroffen, da die investierenden Unternehmen wiederum ihre Schulden bei den Banken nicht beglichen (vgl. Osius/Carlson 2004: 8).

Die Teilnahme des privaten Sektors im Infrastrukturbereich verlief in der Vergangenheit daher vielfach wenig erfolgreich. Auch die Weltbank erkannte, dass die Infrastruktur nicht den privaten Unternehmen alleine überlassen werden kann und es eines starken Staates bedarf (vgl. World Bank 2006c: xv). Aufgrund der Auswirkungen der Finanzkrise erkannten auch die Regierungen die Probleme, die durch die Teilnahme von privaten Unternehmen in einem bestehenden Sektor entstehen können, wenn notwendige begleitende Reformen nicht durchgeführt werden (vgl. World Bank 2004c: 2, Alexander/Estache 2000). Beispielsweise verursachten die zuvor subventionierten Tarife oder Ineffizienzen im Sektor Probleme, welche die privaten Unternehmen durch langfristige Abnahmeverträge mit dem Staat lösen mussten (vgl. Izaguirre 2000). 
Die Probleme, die in der Vergangenheit bei privat finanzierter Infrastruktur aufgetaucht sind, weisen darauf hin, dass die Dienstleistungen von der öffentlichen Hand oftmals nicht kostendeckend abgegeben wurden (vgl. EC 2004: 10). Durch die privaten Unternehmen entstand aber ein erhöhter Druck auf die öffentliche Hand, die Preise für die Dienstleistungen zu erhöhen bzw. ausreichend zu subventionieren.

Resümierend können also zwei Faktoren das Scheitern von vielen internationalen Projekten erklären:

- die fehlenden soliden und verlässlichen Rahmenbedingungen in vielen Sektoren und

- die notwendigen, aber nicht berücksichtigten, neuen Regulierungen und institutionellen Veränderungen im Zuge der Privatisierung, welche die Investoren bei der Krise vor große Probleme stellte (vgl. Cook/Uchida 2003, Alexander/Estache 2000).

Diese Entwicklungen haben zu einer neuen Ausrichtung bei der Infrastrukturfinanzierung in der Entwicklungszusammenarbeit geführt. Ein neuer Ansatz ist die Verwendung von ODA, um private Investitionen anzuziehen bzw. eine Kombination dieser beiden Ressourcen, um ODA-unterstützte öffentlich-privat finanzierte Infrastruktur zu fördern (vgl. OECD 2006b: 12, OECD 2005). ODA soll daher immer mehr als Hebel dienen, privates Kapital zu mobilisieren und in den Infrastrukturbereich der Entwicklungsländer zu kanalisieren. Dies propagieren die Weltbank, die regionalen Entwicklungsbanken, das macht die EU und das fördern die nördlichen Regierungen (vgl. Bär 2004: 87). Seit 2003 steht außerdem die Infrastruktur wieder im Mittelpunkt des Interesses der Weltbank und somit auch bei den meisten anderen IFIs (vgl. World Bank 2006c: xviii).

Die Rolle der privaten Investoren wurde in der Vergangenheit also grundlegend überschätzt und daher wird in den neuen Ansätzen wieder auf einen stärkeren öffentlichen Partner gesetzt, der eine aktive Rolle in der Planung, Finanzierung und Regulierung der Infrastrukturinvestitionen einnehmen soll (vgl. OECD 2006a: 21, ADB 2005b: 21, Meridian Institute 2005). Auch die Annahme, dass Infrastruktur wichtig für die Armutsreduktion ist, hat dazu beigetragen, dass wieder vermehrt öffentliche Investitionen getätigt werden. Darüber hinaus gilt es, die Bedeutung der privaten Investitionen in der Vergangenheit zu relativieren. Die privaten Investitionen sind in den 1990er Jahren zwar stark angestiegen, aber ihr finanzieller Anteil im Vergleich zum Gesamtvolumen blieb auch in der Zeit eher bescheiden (vgl. ADB 2005b: 55f). Dennoch wird angenommen, dass die privaten Investitionen in die Infrastruktur höher sind als die ODA (vgl. Estache 2004: 10).

Zusammenfassend lässt sich daher festhalten, dass

- der Staat im Infrastruktursektor wieder gestärkt werden und eine aktivere Rolle übernehmen soll, 
- die ODA als „Hebel“ für mehr private Investitionen benützt werden soll und

- die privaten Investitionen sich langsam nach der asiatischen Finanzkrise wieder erholen, aber die Investitionen auf wenige Sektoren und Regionen beschränkt sind.

\subsection{Länderrisiken bei Infrastrukturinvestitionen in Entwicklungsländern}

\subsubsection{Einführung}

Grundsätzlich sind alle Investitionen einem gewissen Risikoportefeuille ausgesetzt. Jedoch sind Investitionen im Ausland zusätzlich mit speziellen Risiken behaftet, insbesondere dann, wenn das Zielland ein Entwicklungsland ist (vgl. Nevitt/Fabozzi 2000: 22). In vielen Entwicklungsländern sind die Länderrisiken aufgrund des instabilen politischen oder wirtschaftlichen Umfelds besonders hoch.

Als Risiko werden Ereignisse bezeichnet, die aus unsicheren Erwartungen resultieren oder, enger, nur solche, bei denen eine Verlustgefahr besteht (Büschgen 1997: 283). In der wirtschaftswissenschaftlichen Literatur werden zur Interpretation von Risiko die Entstehungsursachen und die Verlustgefahr herangezogen (vgl. Tytko 1999: 142, Uekermann 1993: 23). Ursachenbezogen ergibt sich das Risiko aus der Unsicherheit zukünftiger Umweltzustände und damit aus unvollständigen Informationen (vgl. Uekermann 1993: 24). Die Entscheidungen stellen sich ex-post als suboptimal und somit als Fehlentscheidungen heraus. Sie führen zu unterwarteten Ergebnissen - und damit zum wirkungsbezogenen Risikobegriff, der sich als Abweichung eines Handelsergebnisses von einer Zielgröße definieren lässt (vgl. Wolf 2003: 45).

Als Länderrisiko kann die Verlustgefahr bezeichnet werden, die aus einem ausländischen Engagement resultiert und nicht auf das einzelne Investitionsobjekt zurückzuführen ist, sondern in den Besonderheiten des Landes selbst begründet ist (vgl. Bäcker 1998: 2, Mrotzek 1988: 77). Das Länderrisiko wird auch mit der Kreditwürdigkeit eines Landes gleichgesetzt, wobei dieses durch den wirtschaftlichen Gesamtzustand und die politische Stabilität bestimmt werden (vgl. Reuter/Wecker 1999: 77, Hinsch/Horn 1985: 216). In der Literatur wird der Begriff Länderrisiko außerdem häufig mit dem Begriff des politischen Risikos gleichgesetzt (vgl. u.a. Madura 2006: 548f, Eiteman et al. 2004: 442f). Auch wenn das Länderrisiko in manchen Publikationen zwischen politischen und wirtschaftlichen Eingriffen getrennt wird, existieren in Wirklichkeit nur wenige Phänomene, die einen rein politischen oder rein wirtschaftlichen Charakter haben (vgl. Przybylski 1993:66). 
Die Anzahl der unterschiedlichen Kategorisierungen der Risiken ist genauso zahlreich wie die Anzahl der Publikationen, in denen sie definiert werden. Die verwendeten Begriffe überschneiden sich teilweise oder es werden gleiche Sachverhalte anders benannt. Eine zu starke Untergliederung ist oftmals weder zielführend noch sinnvoll, da zwischen den Risiken Interdependenzen bestehen (vgl. Mrotzek 1989: 80). Ein weitgehender Konsens in der Literatur herrscht allerdings darüber, dass Länderrisiken in einzelne Kategorien unterteilt werden können.

In der vorliegenden Arbeit wird das Länderrisiko als die Unkenntnis über die Zukunftslage im jeweiligen Gastland definiert. Die unter dem Länderrisiko zusammengefassten Risiken entstehen aus der Unsicherheit über die gesamtwirtschaftliche, politische und soziokulturelle Situation eines Landes und werden nicht durch das investierende Unternehmen bzw. ein Individuum verursacht (vgl. Przybylski 1993: 63). Basierend auf der Einteilung von Häberle (1995: 4) wird in dieser Arbeit das Länderrisiko in wirtschaftliche und politische Risiken sowie Wechselkursrisiken unterteilt. In Folge werden aber nur die politischen Risiken (Kapitel 4.3.3) und die Wechselkursrisiken (Kapitel 4.3.4) genauer erklärt, da diese beiden Risiken insbesondere für Infrastrukturinvestitionen von besonders großer Wichtigkeit sind.

In der folgenden Abbildung 20 wird jene Kategorisierung der Länderrisiken graphisch dargestellt, die in dieser Arbeit verwendet wird. Anschließend werden die einzelnen Kategorien nochmals genauer definiert.

\section{Abbildung 20: Einteilung der Länderrisiken}

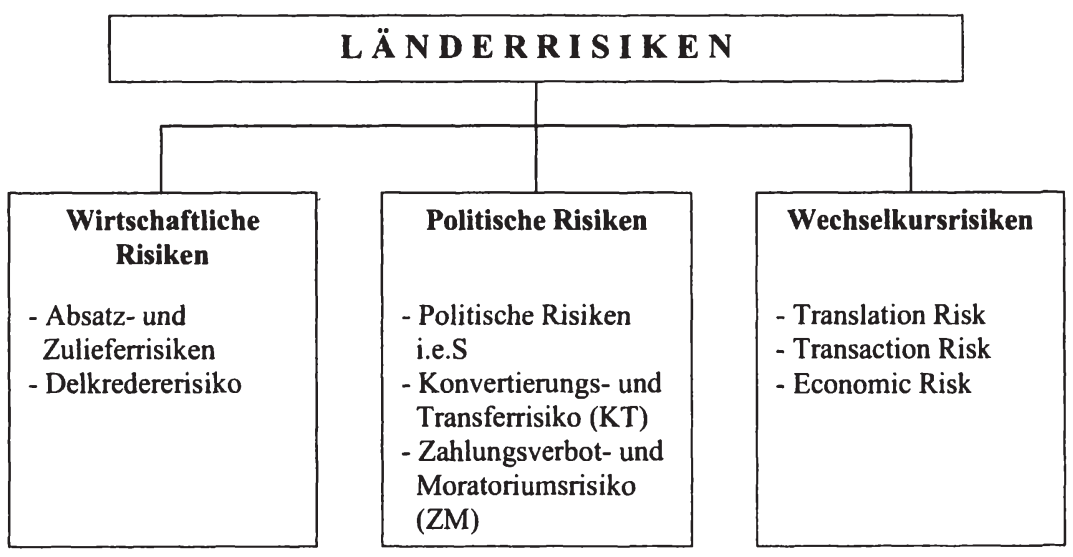

Quelle: in Anlehnung an Häberle (1995: 4) 


\subsubsection{Wirtschaftliche Risiken}

Die wirtschaftlichen Risiken treten hauptsächlich in der Betriebsphase des Projektes auf. Jedoch sind für den Fremdkapitalgeber die wirtschaftlichen Risiken sowohl in der Planungs- und Konstruktionsphase als auch in der Startup-Phase sehr hoch, da erst mit der geplanten Inbetriebnahme Rückflüsse aus dem Projekt zu erwarten sind. In der Betriebsphase fängt das Projektunternehmen an, regelmäßige Einnahmen aus dem Verkauf des Outputs zu erzielen. Dieser erwirtschaftete Cash-Flow soll einerseits die Betriebsausgaben und den Schuldendienst decken und andererseits den Ertragserwartungen der Eigenkapitalgeber genügen (vgl. Wolf 2003: 78).

\subsubsection{Absatz- und Zulieferrisiken}

$\mathrm{Zu}$ den wirtschaftlichen Risiken zählen die Absatz- und Zulieferrisiken. Wenn die tatsächliche Preis- und/oder Mengenentwicklung für den Projektoutput hinter den Erwartungen zurückbleibt und dadurch die tatsächlichen Betriebseinnahmen geringer sind als die prognostizierten, spricht man von Absatzrisiken. (vgl. Wolf 2003: 80). Abhängig davon wie stark der Markt geregelt ist bzw. wieviele Konkurrenzanbieter am Markt aktiv sind, lassen sich unterschiedlich valide Absatzprognosen erstellen (vgl. Nevitt/Fabozzi 2000: 45).

Im Bereich der Infrastruktur versuchen private Unternehmen dieses Risiko durch Absatzverträge abzusichern, wobei man die folgenden zwei Formen unterscheidet: Take-or-Pay und Take-and-Pay Verträge (vgl. Siebel 2001: 54). Der produktionsunabhängige Abnahmevertrag (Take-or-Pay) bietet dem Produzenten die größte Sicherheit, da dieser, unabhängig davon, ob der Kunde konsumiert, für die Produktion bezahlt wird (vgl. Tytko 1999: $61 \mathrm{ff}$ ). Bei produktionsabhängigen Vereinbarungen (Take-and-Pay) verpflichtet sich der Vertragspartner zur Abnahme einer bestimmten Produktionsmenge; die Zahlungsverpflichtung ist jedoch vom tatsächlichen Erhalt der Produkte abhängig (vgl. Tytko 1999: 66f). In Entwicklungsländern sind Abnahmeverträge meistens US dollarindexiert, da die Kredite auch auf US\$ lauten und die Projektgesellschaft eine Abwertung der Währung des Gastlandes befürchtet.

Gleichzeitig versuchen die Projektgesellschaften durch Zulieferverträge die Rohstoffe für die Produktion zu sichern, da es zu unerwarteten Kostensteigerungen oder Produktionsausfällen kommen kann, wenn die notwendigen Rohstoffe nicht in der erwarteten Menge und Qualität und/oder zum erwarteten Preis zur Verfügung stehen (vgl. Siebel 2001: 167, Nevitt/Fabozzi 2000: 45). Die Zulieferverträge können sowohl auf Basis von Take-or-Pay und Take-andPay vereinbart werden. Auch hier werden häufig Mindestabnahmemengen und Dollarindexierungen vereinbart. 


\subsubsection{Delkredererisiko}

Unter Delkredererisiko versteht man im gegebenen Zusammenhang, dass eine oder mehrere Parteien ihren vertraglichen Verpflichtungen nicht mehr nachkommen können und es zu einer Unterfinanzierung des Investitionsvorhabens kommt (vgl. Weber et al. 2006: 117, Siebel 2001: 166). Dieses Risiko betrifft aufgrund der Nachschussverpflichtung vorrangig die Projektgesellschaft und die Sponsoren, aber auch die Banken.

Das Ausfallsrisiko ist von besonderer Bedeutung, da alle Projektbeteiligten und alle Projektphasen davon betroffen sind (vgl. Wolf 2003: 81). Darunter versteht man, dass einzelne Projektbeteiligte in den verschiedenen Phasen nicht ihren Verpflichtungen nachkommen.

Um dieses Risiko bei Infrastrukturprojekten zu minimieren, wird vom Gaststaat meistens eine Staatsgarantie verlangt, in der dem privaten Unternehmen versichert wird, dass die heimischen Unternehmen ihre Verpflichtungen erfüllen werden (vgl. Siebel 2001: 20). Sollte dies nicht der Fall sein, muss der Staat anstelle des Unternehmens den Vertrag erfüllen. Hier sind insbesondere wieder die Abnahme- und Rohstofflieferverträge abgesichert. Die Vergabe von Staatsgarantien wird aber seit der asiatischen Finanzkrise als kritisch gesehen, da sich die Regierungen davor nicht über den Umfang der Garantie bewusst waren. Außerdem hat das private Unternehmen keinen Überblick darüber, wieviele Staatsgarantien die Regierung bereits abgegeben hat und es kann im Fall einer Finanzkrise trotzdem zu einem Zahlungsausfall kommen.

\subsubsection{Politische Risiken}

Das politische Länderrisiko ist durch eine Vielzahl von Definitionsversuchen gekennzeichnet und lässt sich nur schwer operationalisieren (vgl. Büschgen 1997: 290). Laut Mrotzek (1988: 80) lassen sich politische Risiken als Diskontinuitäten definieren, die nur schwer antizipierbar und durch politische Änderungen bedingt sind. Von einem Risiko wird dann gesprochen, wenn mit den Änderungen der politischen Rahmenbedingungen die Möglichkeiten einer positiven oder negativen Beeinflussung der Unternehmenssituation verbunden sind.

\subsubsection{Politische Risiken i.e.S.}

Mit politischen Risiken i.e.S. werden insbesondere Streiks, politische Unruhen, Bürgerkriege oder die Gefahr von Einfuhrsperren bezeichnet, aber auch innenpolitische Entwicklungen können wesentliche Auswirkungen auf die Infrastrukturinvestition haben (vgl. Jonach 1996: 27).

Beispielsweise haben Enteignungen aufgrund von Regimewechseln in der Vergangenheit bereits $\mathrm{zu}$ großen finanziellen Problemen bei ausländischen Investoren und insbesondere bei Infrastrukturinvestitionen geführt (vgl. Meyer 1987: 28). Unter Enteignung versteht man den teilweisen oder vollständigen $\mathrm{Zu}$ - 
griff der Regierung auf Vermögen und Rechte der ausländischen Unternehmung mit oder ohne Entschädigung (vgl. Meyr 1987: 28). Hiermit sind immer zwei Gefahren verbunden: Zum einen der unmittelbare Verlust in Höhe des Wertes der enteigneten Anlagen, zum anderen die Möglichkeit entgangener Gewinne aufgrund von Produktionsausfällen und Versorgungs- bzw. Produktionsschwierigkeiten (Przybylski 1993: 73).

Artikel 2 Absatz 2 der „Charta der wirtschaftlichen Rechte und Pflichten der Staaten" gibt den Mitgliedsstaaten der Vereinten Nationen das Recht zur Regulierung und Überwachung der ausländischen Investitionen und der multinationalen Unternehmen. Insbesondere wird festgestellt, dass jeder Staat das Recht hat, privates Eigentum zu verstaatlichen, zu enteignen oder zu überragen bei Zahlung einer angemessenen Entschädigung (vgl. Büschgen 1997: 285).

Meyer (1987: 28) unterteilt das Risiko der Enteignung in drei Erscheinungsformen:

- Enteignung i.e.S. bezieht sich auf ein einzelnes Unternehmen. Jede souveräne Regierung hat das Recht zu enteignen, wenn sie dabei im öffentlichen Interesse handelt und dem Unternehmen eine angemessene Entschädigung zahlt, wobei die Interpretation des Begriffs ,angemessen“ je nach Sichtweise des Enteignenden und des Enteigneten unterscheiden.

- Nationalisierung bzw. Verstaatlichung betrifft eine ganze Gruppe von Unternehmen oder einen gesamten Wirtschaftssektor. Im Fall der Nationalisierung sind nur ausländische Unternehmungen betroffen, im Fall der Verstaatlichung nur inländische. Das Recht der Nationalisierung ist vielfach in der Verfassung der Regierungen verankert und meistens mit Entschädigungszahlungen verbunden.

- Konfiszierung ist die weitest reichende Form der Enteignung und beinhaltet die Beschlagnahmung von Eigentum ausländischer Unternehmen ohne Entschädigung. Laut Völkerrecht ist eine entschädigungslose Enteignung ausländischen Eigentums verboten und verlangt unverzügliche, angemessene und effektive Entschädigung im Falle der Ent-eignung.

\subsubsection{KT-ZM Risiko}

Es besteht das Risiko, dass ein Staat aufgrund seiner binnen- und außen-wirtschaftlichen Situation nicht über die notwendigen Devisenbestände verfügt, um seinen ausländischen Zahlungsverpflichtungen nachzukommen (vgl. Will 2000: 11, Reuter/Wecker 1999: 77). Die Zahlungsfähigkeit eines Landes ist solange gesichert, wie es über ausreichende Währungsreserven, Deviseneinnahmen aus Exporterlösen und Kreditaufnahmen sowie Zahlungsmöglichkeit in der eigenen Währung verfügt, um die Zahlungsverpflichtungen aus Importen und Schuldverhältnissen zu begleichen (vgl. Büschgen 1997: 285f). 
Als Konvertierungs- und Transferrisiko (KT) bezeichnet man die Gefahr, dass Überweisungen in das Land des ausländischen Investors und die Umwechslung in dessen Landeswährung nicht möglich sind (vgl. Topritzhofer/ Moser 1985: 9). Ein Land, das Probleme im internationalen Zahlungsverkehr hat, weil es nicht genügend Devisen erwirtschaftet, um die finanziellen Verpflichtungen gegenüber dem Ausland zu erfüllen, kann Maßnahmen setzen, um die eigene Währung zu schützen. Diese unterteilen sich in Konvertierungsbeschränkungen (Beschränkung des Devisenverkehrs), die den Umtausch der lokalen Währung in eine harte Auslandswährung nur noch begrenzt oder überhaupt nicht zulassen und in Transferbeschränkungen, die einen Geldexport ins Ausland verhindern (vgl. Häberle 1995: 18, Meyer 1987: 20f). Insbesondere Entwicklungsländer versuchen die aus Projekten erzielten Devisen bei der Zentralbank des Landes zu halten oder dem Land zur Verfügung zu stellen. Die Deviseneinnahmen sollen in der Zentralbank zusammen laufen, um die Zahlungsfähigkeit des Landes aufrechtzuerhalten. Dies ist unter dem Aspekt der Devisenknappheit der meisten Entwicklungsländer nachvollziehbar (vgl. Hinsch/Horn 1985: 217).

Unter Zahlungsverbots- und Moratoriumsrisiko (ZM) wird die Gefahr verstanden, dass ein im Gastland verordnetes Zahlungsverbot bzw. eine Zahlungspause den Zahlungsverkehr an die Muttergesellschaft verhindert bzw. hinausschiebt (vgl. Topritzhofer/Moser 1985: 9). Wenn es sich dabei nur um ein teilweises Verbot der Zahlung oder um einen Zahlungsaufschub handelt, dann liegt ein Moratorium vor (vgl. Häberle 1995: 18). Ein Zahlungsverbot hingegen liegt vor, wenn einem zahlungswilligen und -fähigen Schuldner die Erfüllung seiner Auslandsverbindlichikeiten durch gesetzgeberische Maßnahmen der Regierung untersagt wird (vgl. Meyer 1987: 23).

Die politischen Risiken können durch Staatsgarantien abgesichert werden, die das private Unternehmen vom Gaststaat fordert. Außerdem bieten Finanzinstitutionen, wie beispielsweise die MIGA oder nationale Institutionen wie die OeKB Garantien an, die das politische Risiko absichern. Die einzelnen Institutionen werden im Kapitel 5 dargestellt.

\subsubsection{Wechselkursrisiken}

Unter dem Wechselkursrisiko versteht man die Gefahr, dass sich Wertrelationen zwischen der Heimatwährung des investierenden Unternehmens und der Währung des Gastlandes verändern (vgl. Topritzhofer/Moser 1985: 9). Insbesondere bei Entwicklungsländern ist dieses Risiko tendenziell höher als bei ADI in entwickelten Ländern, da Unternehmen dazu gezwungen sind, den Absatz in einer volatilen Heimatwährung zu fakturieren, die Kredite aber in einer der Weltwährungen getilgt werden müssen. Da die Währungen in Entwicklungsländern oftmals hoch volatil und die zukünftigen Wechselkurse sind nur schwer prognostizierbar sind, entsteht ein Wechselkursrisiko für das Unternehmen. 
Insbesondere bei Infrastrukturprojekten in Entwicklungsländern ist das Wechselkursrisiko besonders hoch, da die Projekte lange Laufzeiten aufweisen und die Kredite großteils in Hartwährung, meistens US Dollar, vergeben werden und die Projekterlöse in heimischer Währung erzielt werden, wie beispielsweise Mautautobahnen oder Kraftwerke (vgl. Tytko 1999: 151). Aber auch auf der Kostenseite können die Rohstoffe und Zulieferprodukte in Fremdwährung fakturiert werden und dem Wechselkursrisiko ausgesetzt sein (vgl. Priermeier 2002). Problematisch ist daher jene Situation, in der es zu einem großen Ungleichgewicht bei den Kosten in Hartwährung und den Erträgen in lokaler Währung kommt (vgl. Haarmeyer/Mody 1997).

Laut der Ergebnisse von Bea (1995: 149) bewerten die an seiner Studie teilnehmenden Unternehmen die Wechselkursrisiken als jenen Faktor, der den größten negativen Einfluss auf das Investitionsvolumen hat. Man unterscheidet die folgenden drei Erscheinungsformen des Wechselkursrisikos.

\subsubsection{Währungsumrechnungsrisiko}

Das Währungsumrechnungsrisiko (translation risk) betrift die buchhalterische Bewertung von auf Fremdwährung lautenden Posten im Jahresabschluss oder die Bewertung anlässlich der Konsolidierung in unterschiedlicher Währung erstellter Jahresabschlüsse zu einer Konzernbilanz (vgl. Madura 2006: 381f, Topritzhofer/Moser 1985: 175). Dieses Risiko ist vergangenheitsorientiert und führt nicht zu realen Auswirkungen aufgrund der Wechselkursveränderungen. In der Theorie ist die Berücksichtigung dieses Risikos umstritten, allerdings gibt es Ansätze, wie beispielsweise den "Stockprice-perspective“, welche die Wichtigkeit dieses Risikos betonen (vgl. Madura 2006: $381 \mathrm{ff}$ ).

Obwohl für Infrastrukturinvestitionen typischerweise Projektgesellschaften gegründet werden, müssen Unternehmen Mehrheitsbeteiligungen und Haftungszusagen bei Projektgesellschaften auch in der eigenen Bilanz anführen. Daher kann das Währungsumrechnungsrisiko auch für Unternehmen wichtig sein, die sich an Projektgesellschaften als Sponsoren mit Eigenkapital beteiligen.

\subsubsection{Währungsumwechslungsrisiko}

Das Währungsumwechslungsrisiko (transaction risk) beschreibt die Unsicherheit betriebswirtschaftlicher Kosten- bzw. Erlösgrößen, die sich aus Wechselkursveränderungen ergeben (vgl. Topritzhofer/Moser 1985: 173). Das Währungsumwechslungsrisiko stellt eines der größten Risiken im Zusammenhang mit Infrastrukturinvestitionen dar, da die Erträge aus den Leistungen in der Heimatwährung des Landes bezahlt werden, in dem die Investition getätigt wurde. Da Einnahmen für die Tilgung der Kredite in Hartwährung, wie beispielsweise US\$ oder EUR, benötigt werden, befürchten die Investoren einen Verlust aus den Erträgen durch eine Abwertung der ausländischen Währung zum US\$ oder EUR. 
Bei Infrastrukturinvestitionen wird das Währungs-umwechslungsrisiko oft dadurch vermieden, dass die Investoren ihre Absatzpreise durch Wertsicherungsklauseln kurssichern. Dabei werden die Einnahmen in lokaler Währung bezahlt, aber deren Höhe ist meistens an den US\$ oder EUR indexiert. Das private Unternehmen erhält die Erträge dabei nach wie vor in der Landeswährung, aber faktisch kann es durch die Indexierung eine Anpassung an Wechselkursveränderungen erreichen und so das Wechselkursrisiko vermeiden. Es können sowohl ausländische als auch inländische Investoren vom Währungsumrechnungsrisiko betroffen sein, da aufgrund der geringen Liquidität der Kapitalmärkte in Entwicklungsländern auch die inländischen Investoren ausländische Kredite aufnehmen müssen. Zusätzlich dazu vergeben auch die Internationalen Finanzinstitutionen (IFIs) ihre Kredite in US\$.

\subsubsection{3 Ökonomische Wechselkursrisiko}

Das ökonomische Wechselkursrisiko (economic risk) geht über das Transaktionsrisiko hinaus und berücksichtig neben den Preis- auch die Mengenauswirkungen aufgrund von zukünftigen Veränderungen des Wechselkurses. Im Mittelpunkt steht dabei die Betrachtung des Gesamtwertes eines Unternehmens. (vgl. Moser: 1985: 112f). Das ökonomische Wechselkursrisiko wird aufgrund seiner Komplexität von Unternehmen nur wenig berücksichtigt (vgl. Glaum 2000: 33, Loderer/Pichler 2000).

Im Zusammenhang mit Infrastrukturinvestitionen in Entwicklungsländern findet es kaum Anwendung, da die Investoren oft Abnahmeverträge zu fixen Preisen mit den öffentlichen Abnehmern vereinbaren und sie daher keinen Einfluss auf die Preisgestaltung haben. Eine Mengenreaktion ist jedoch dann möglich, wenn der private Investor direkt an die Kunden liefert und es aufgrund von erhöhten Preisen zu Mengenveränderungen kommt. Diese Mengenreaktion ist aber wiederum abhängig von der Art der Infrastrukturleistung und dem Wettbewerb am Markt.

\section{4 Öffentlich-private Infrastrukturmodelle}

\subsubsection{Einführung}

Unter dem Begriff der öffentlich-privaten Infrastrukturmodelle werden verschiedene Formen der Zusammenarbeit zwischen dem öffentlichen und dem privaten Sektor subsumiert. Die Formen unterscheiden sich je nach Intensität der jeweiligen Beteiligung der Parnter.

In der folgenden Abbildung 21 werden die Modelle in einem Kontinuum zwischen Staat und Privat dargestellt. Auf der linken Seite der Abbildung 
werden jene Modelle zusammengefasst, bei denen der Staat Eigentümer der Anlage bleibt, aber einzelne Dienstleistungen an private Unternehmen auslagert. Beim staatlichen Eigentum haben Serviceverträge die geringste und Konzessionsmodelle die größte private Beteiligung. Public Private Partnerships weisen sowohl öffentliches als auch privates Eigentum auf. Jene Modelle mit der höchsten privaten Beteiligung werden als Betreiber- oder Build Operate Transfer-Modelle bezeichnet. Abhängig vom gewählten Modell kann die Infrastrukturanlage am Ende der Laufzeit an die öffentliche Hand übergeben werden (BOT-Modell) oder im Eigentum des privaten Unternehmens bleiben (BOO).

In den nun folgenden Abschnitten werden die einzelnen Modelle dargestellt. Die Aufbaulogik folgt dem Staat-Privat Kontinuum und stellt in den Kapiteln 4.4.2 und 4.4.3 die Modelle mit staatlichem Eigentum vor. Bei diesen Formen beschränkt sich die private Beteiligung auf den Betrieb und eventuelle Erweiterungs- und Reparaturinvestitionen. Das private Unternehmen ist aber nicht finanziell bei der origiäneren Errichtung der Anlage beteiligt. Bei diesen Formen wird das private Unternehmen direkt durch den Auftraggeber, z.B. die Regierung des Gastlandes, für die Leistung bezahlt (vgl. World Bank 2004c: 5).

\section{Abbildung 21: Kontinuum Staat - Privat}

Staatliches Eigentum

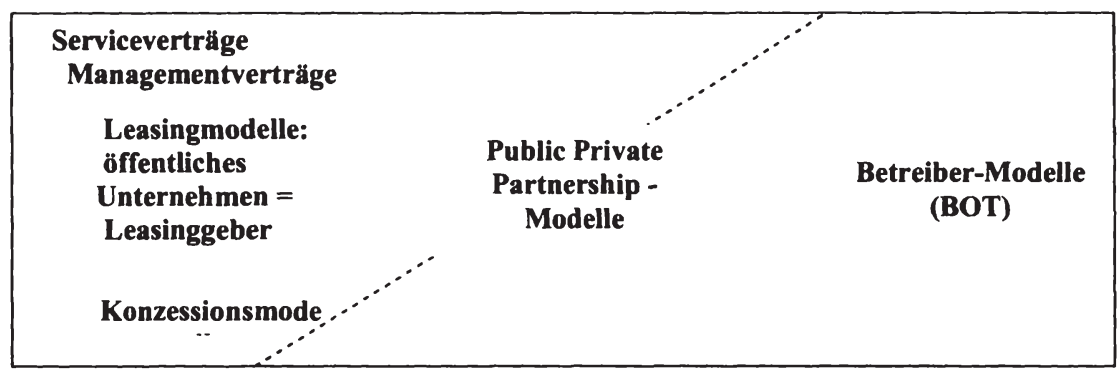

Privates Eigentum

Daran anschließend werden im Kapitel 4.4.4 Public Private Partnership-Modelle diskutiert, die sowohl öffentliches als auch privates Kapital aufweisen. Die beteiligten Partner gründen dafür gemeinsam eine Gesellschaft. Die Modelle mit der höchsten privaten Beteiligung werden im letzten Kapitel 4.4.5 dargestellt. Die Betreibermodelle werden oft synonym auch als BOT-Modelle bezeichnet, obwohl das BOT-Modell nur eine Variante der unterschiedlichen Betreibermodelle darstellt. PPP und Betreibermodelle sehen private Kapitalbeteiligung bereits während der Bauphase vor. Abhängig von der Ausgestaltungsform, 
können bei diesen beiden Modellen die privaten Unternehmen auch direkt Kontakt mit den Kunden haben.

\subsubsection{Service- und Management Modelle}

Bei Service- und Management-Modellen werden Aufträge zur Übernahme des Rechnungswesens, der Datenverarbeitung oder der Personalbeschaffung vergeben (vgl. Zeuchner 2002: 208). Eine Vergabe von solchen Aufgaben kann sowohl an heimische, als auch an Unternehmen aus Industriestaaten vergeben werden. Normalerweise werden in Entwicklungsländern einfache, standardisierte Leistungen an lokale Unternehmen vergeben. Ausländische Partner werden hingegen dann herangezogen, wenn komplexe Leistungen oder Spezialwissen benötigt werden.

Die Risiken für den privaten Unternehmer beschränken sich bei beiden Modellen auf das politische Risiko und das Wechselkursrisiko. Da keine Investitionen getätigt werden, beschränken sich die dem Risiko ausgesetzten Zahlungsströme auf die Zahlung des Entgelts, die auch in der Heimatwährung des Gastlandes vereinbart werden kann. Da Entwicklungsländer häufig hoch volatile Währungen haben und ein durchschnittlich höheres politisches Risiko aufweisen, können durch die Transferierung ins Mutterunternehmen Verluste entstehen. Diese können dadurch vermieden werden, dass die Entgeltszahlung in der Währung des investierenden Unternehmens durchgeführt wird oder sich das ausländische Unternehmen durch eine Versicherungsleistung absichert. Eine Verringerung, allerdings keine Vermeidung, des Wechselkursrisikos kann auch erreicht werden, indem man sich auf eine stabile Drittwährung einigt. Staatlich garantierte Wechselkurse können eine weitere Absicherungsmöglichkeit darstellten. Dabei wird ein Wechselkurs mit der Regierung des Gastlandes vereinbart, zu dem die privaten Vertragspartner ihre Erlöse in eine Fremdwährung tauschen dürfen. In diesem Fall übernimmt die Regierung des Gastlandes das gesamte Wechselkursrisiko

\subsubsection{Serviceverträge}

Wesentliche Merkmale von Serviceverträgen sind das öffentliche Eigentum an der Infrastrukturanlage sowie die öffentliche Finanzierung und Betriebsführung in Verbindung mit der Übertragung bestimmter Serviceleistungen an private Unternehmen (vgl. Zeuchner 2002: 209). Aus dieser Charakterisierung ist erkennbar, dass Serviceverträge die einfachste Form der privaten Teilnahme an einer Infrastrukturleistung darstellen. In der Literatur werden Serviceverträge u.a. auch als Contracting-Out oder Outsourcing bezeichnet (vgl. Savas 2000: 242).

Serviceverträge können vielseitig für genau spezifizierte Leistungen in der Planungs-, Bau- oder Betriebsphase eingesetzt werden (vgl. EC 2003a: 20). Die 
Verantwortung des privaten Bereichs ist somit nur auf die ihm übertragene Leistung begrenzt. Die fixen Zahlungen an den Privaten sind an dessen Leistung gebunden und diese Art der Erfolgsvergütung wird auch als ein wesentlicher Vorteil gegenüber der Leistungserstellung durch den öffentlichen Bereich angesehen (vgl. Zeuchner 2002: 210). Die folgende Abbildung 22 stellt den Ablauf eines Servicevertrages nochmals schematisch dar.

\section{Abbildung 22: Leistungsbeziehungen bei Serviceverträgen}

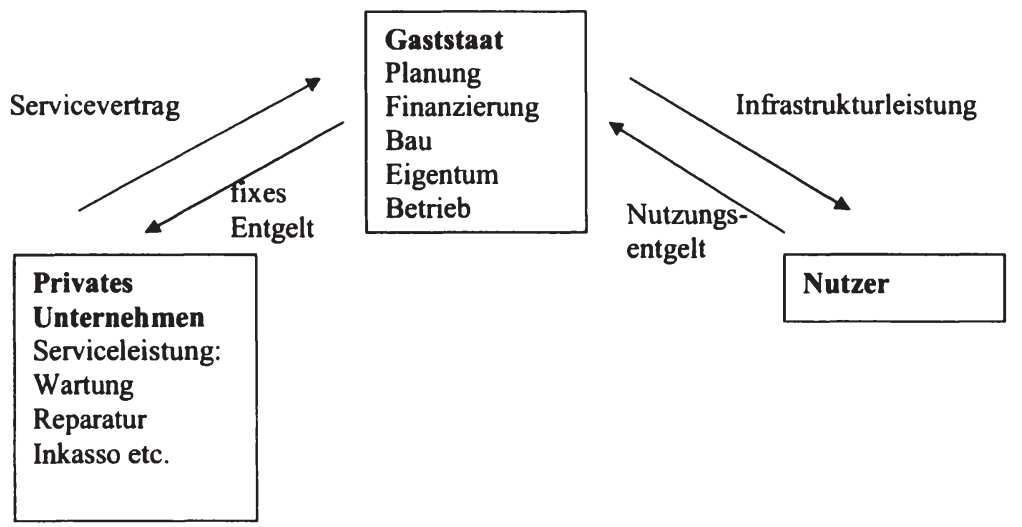

Quelle: Zeuchner (2002: 210)

\subsubsection{Managementverträge}

Managementverträge sind gekennzeichnet durch öffentliches Eigentum einer Infrastrukturanlage, die öffentliche Finanzierung und die private Managementleistung. In dieser Konstruktion überträgt die öffentliche Behörde den Betrieb und die Wartung einer Anlage an ein privates Unternehmen (vgl. World Bank 2006b: 7, EC 2003a: 20, Savas 2000: 242).

Das private Unternehmen ist für die täglichen Managemententscheidungen verantwortlich, ohne aber die Projektrisiken, insbesondere das Eigentumsrisiko, tragen zu müssen (vgl. World Bank 2006b: 7). Der private Betreiber ist nur im Innenverhältnis für die Bereitstellung der Dienstleistung verantwortlich. Die öffentliche Stelle bleibt weiterhin verantwortlich für die Bereitstellung der Leistung und tritt nach außen sowohl bei der Beschaffung als auch beim Absatz als Rechtspersönlichkeit und Vertragspartner auf. Das private Unternehmen erhält für seine Leistung und sein Spezialwissen von der öffentlichen Stelle ein Managemententgelt, das auch eine leistungsbezogene Komponente beinhalten kann (vgl. EC 2003a: 20).

Dadurch soll ein Anreiz zur Produktivitätssteigerung geschaffen werden. Managementverträge haben in der Regel eine mittelfristige Laufzeit von 3 bis 5 
Jahren, deren Zweck darin besteht, die Anlagen rentabel zu machen (vgl. Savas 2000: 242). Managementverträge sind besonders für Sektoren geeignet, die entweder gerade eine Umwandlung von öffentlichem zu privatem Eigentum durchmachen oder für jene Bereiche, die keine weitere Partizipation des privaten Sektors zulassen (vgl. Willoughby 2004a: 14, EC 2003a: 21).

Die folgende Abbildung 23 zeigt wiederum grafisch den Ablauf eines Managementvertrages.

\section{Abbildung 23: Leistungsbeziehungen bei Managementverträgen}

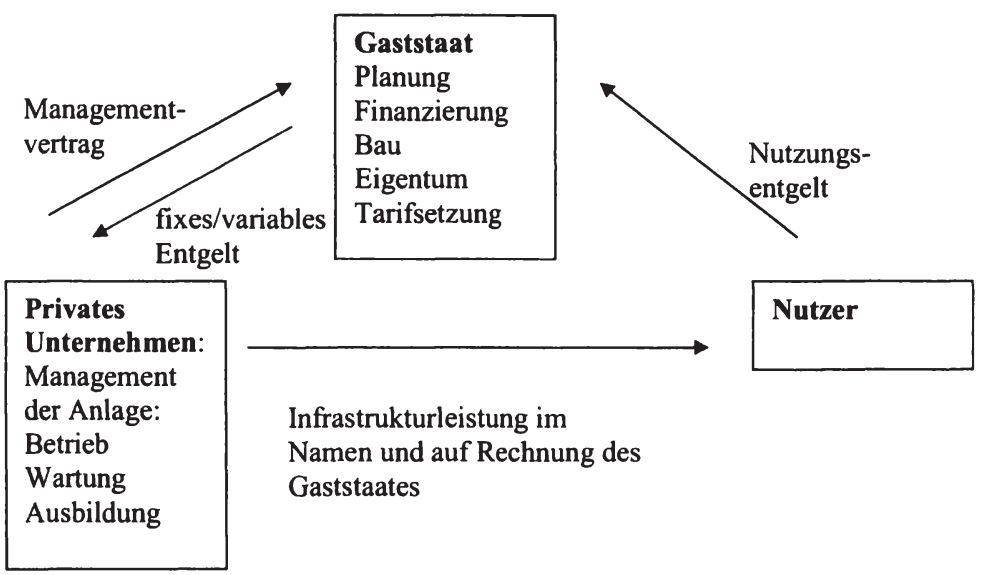

Quelle: Zeuchner (2002: 212)

\subsubsection{Transfer of Operation Rights (TOR) - Modelle}

Unter dem Begriff TOR-Modelle versteht man Leasing- und Konzessionsmodelle, die man im deutschsprachigen Raum auch als Betriebsführungsmodelle bezeichnet (vgl. Zeuchner 2002: 219). Dabei vergibt der Staat eine zeitlich begrenzte Lizenz an ein privates Unternehmen für die Erbringung einer Infrastrukturleistung. Bei diesen Modellen übernimmt das private Unternehmen die volle Verantwortung für den Betrieb der Anlage und muss einen Teil des kommerziellen Risikos für das Leistungsangebot tragen. Das Unternehmen deckt seine Kosten und erwirtschaftet einen Gewinn, in dem es direkt an die Endabnehmer liefert und auch von diesen für die Leistung bezahlt wird (vgl. World Bank 2006b: 7f). Dadurch ist das private Unternehmen auch an einer Tarifdisziplin seitens der Abnehmer interessiert (vgl. World Bank 2004c: 5). Die Regierung überwacht typischerweise die Gebühreneinhebung, indem sie Wettbewerb zulässt und/oder die Preise reguliert. 
Die Risiken, die vom privaten Unternehmen getragen werden müssen, beschränken sich beim Leasing- und Konzessionsmodell einerseits auf das politische und Wechselkursrisiko, andererseits kann es beim Leasingmodell auch zu einer teilweisen Übernahme des wirtschaftlichen Risikos kommen, wenn ein Teil der Entlohnung neben einem festen Sockelbetrag aus der Differenz zwischen Nutzungsentgelt und Leasingrate ermittelt wird. Beim Konzessionsmodell muss das private Unternehmen das Zuliefer- und das Absatzrisiko übernehmen.

Strategien zur Bewältigung der politischen und Wechselkursrisiken wurde bereits im Kapitel 4.4.2 dargelegt. Bei Infrastrukturanlagen ist der Abschluss von Abnahmeverträgen üblich, anhand derer das Absatzrisiko eingeschränkt wird. Hierbei unterscheidet man Take-or-Pay und Take-and-Pay-Verträge (vgl. Siebel 2001: 54). Bei einem Take-or-Pay-Vertrag ist der Abnehmer verpflichtet zu zahlen, unabhängig davon, ob die Dienstleistung geliefert wird bzw. er sie abnimmt (vgl. Tytko 1999: 173). Ziel dabei ist es, zu mindest die Betriebskosten und die Leasingrate bzw. die Abschreibung der Konzession zu decken. Bei einem Take-and-Pay-Vertrag hingegen muss der Abnehmer nur dann für die Leistung zahlen, wenn er diese auch konsumiert.

Eine weitere Möglichkeit sind sogenannte Throughput- und TollingAgreements, die auch das Absatzrisiko begrenzen. Dabei ist der Garantiegeber dazu verpflichtet, eine andere als die aus dem Projekt resultierende Leistung zu erbringen, z.B. eine bestimmte Transportleistung zu bezahlen, unabhängig davon, ob diese auch in Anspruch genommen wird (vgl. Zeuchner 2002: 228). Mit einem Throughput-Vertrag wird das Zulieferrisiko begrenzt. Damit wird das Risiko abgedeckt, dass die für den Betrieb der Anlage benötigten Rohstoffe nicht zu den erwarteten Bedingungen verfügbar sind. Das private Unternehmen kann wiederum Preisanpassungsklauseln mit den Abnehmern vereinbaren oder es können auch besondere Situationen in den Nutzergebühren berücksichtigt werden.

Aus Sicht des Staates ist die adäquate Versorgung der Nutzer mit Infrastrukturleistungen wichtig. Daher wird der Staat nur jene Betreiber auswählen, die das nötige Vorwissen und die Erfahrung aufweisen können. Um sich gegen Nicht- oder Schlechterfüllung zu schützen, können Performance-Bonds vereinbart werden, in denen das private Unternehmen zu einem Schadenersatz verpflichtet wird. Außerdem ist es sinnvoll, die Ausbildung von lokalem Personal vertraglich festzulegen, da die Verträge mit dem privaten Partner zeitlich beschränkt sind.

\subsubsection{Leasingmodelle}

Leasingmodelle können zwei grundlegend verschiedene Ausgestaltungsformen annehmen, da sowohl das private Unternehmen als auch die öffentliche Einrichtung Eigentümer der Infrastrukturanlage sein können. 
Die besonderen Kennzeichen für Leasingmodelle sind das öffentliche Eigen-tum an einer Infrastrukturanlage, die öffentliche Finanzierung und die private Betriebsführung (vgl. Strohbach 2001: 58). Der öffentliche Partner fungiert dabei als Leasinggeber und das private Unternehmen als Leasingnehmer, dem eine nach seinen Anforderungen gebaute Anlage im Rahmen eines Leasingvertrages zur Verfügung gestellt wird (vgl. Grimsey/Lewis 2002: 224). Der Leasingnehmer, d.h. das private Unternehmen, ist alleinig für alle mit dem Betrieb und der Wartung anfälligen Kosten verantwortlich (vgl. World Bank 2006b: 7, Grimsey/Lewis 2004: 224). Dafür hat er im Allgemeinen die ausschließlichen Rechte auf die Einnahmen für einen Zeitraum von fünf bis zehn Jahren (vgl. World Bank 1994: 77). Somit wird auch das wirtschaftliche Risiko auf das private Unternehmen übertragen (vgl. EC 2003a: 21).

Die folgende Abbildung 24 stellt das Verhältnis in einem Leasingmodell dar, in dem der öffentliche Partner als Leasinggeber fungiert.

Abbildung 24: Leistungsbeziehungen in einem Leasingmodell, Staat als Leasinggeber

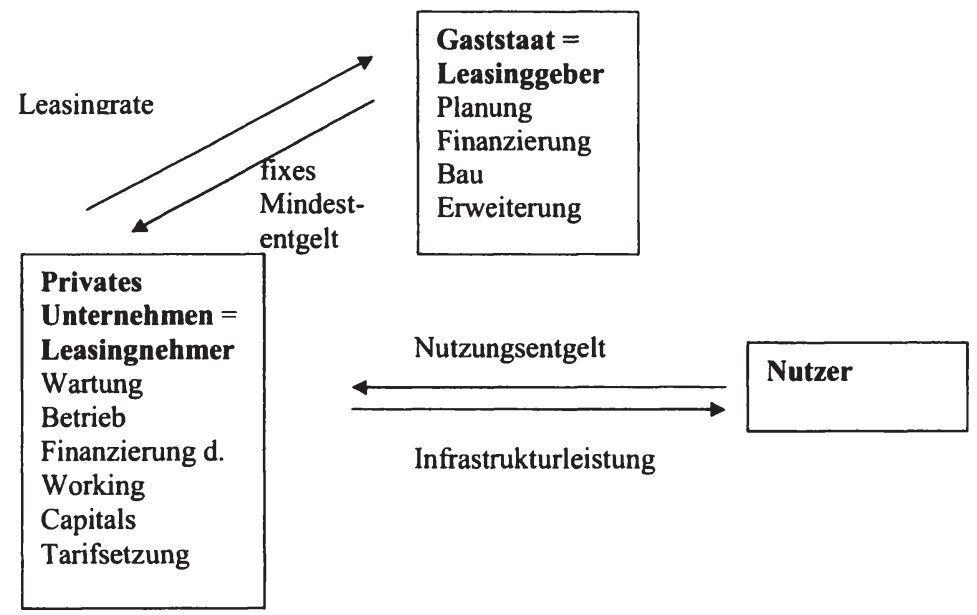

Quelle: Zeuchner (2002: 223)

Der Leasinggeber hingegen, d.h. das öffentliche Unternehmen, bleibt Eigentümer der Anlage und ist nur für die Finanzierung der Investition sowie für Ersatz- und Erweiterungsinvestitionen, Festlegung der Nutzergebühr und die Kostenpolitik zuständig. Im Leasingvertrag werden auch Pönalezahlungen vereinbart, wenn die Dienstleistung des privaten Unternehmens nicht der vereinbarten Qualität entspricht. Der Leasingnehmer trägt die meisten oder alle wirtschaftlichen Risiken für die gesamte Laufzeit des Leasingvertrages, da die 
Leasingrate erfolgsunabhängig zu bezahlen ist (vgl. Tytko 1999: 195f, World Bank 1994: 77). Dabei kann der Leasingnehmer bei sorgfältiger Führung der Anlage eine Wertsteigerung realisieren.

Leasingkonstruktionen werden auch als möglicher Weg für eine intensivere Zusammenarbeit zwischen dem öffentlichen und den privaten Partner in der Zukunft angesehen. Für das private Unternehmen stellt dies außerdem eine Markteintrittsform mit beschränktem Risiko und Investitionsaufwand dar. Da die öffentliche Behörde in vielen Fällen die technischen Projektrisiken übernimmt, muss das private Unternehmen nur noch die wirtschaftlichen Projektrisiken tragen (vgl. Zeuchner 2002: 223).

\section{Abbildung 25: Leistungsbeziehungen im Leasingmodell, Staat als Leasingnehmer}

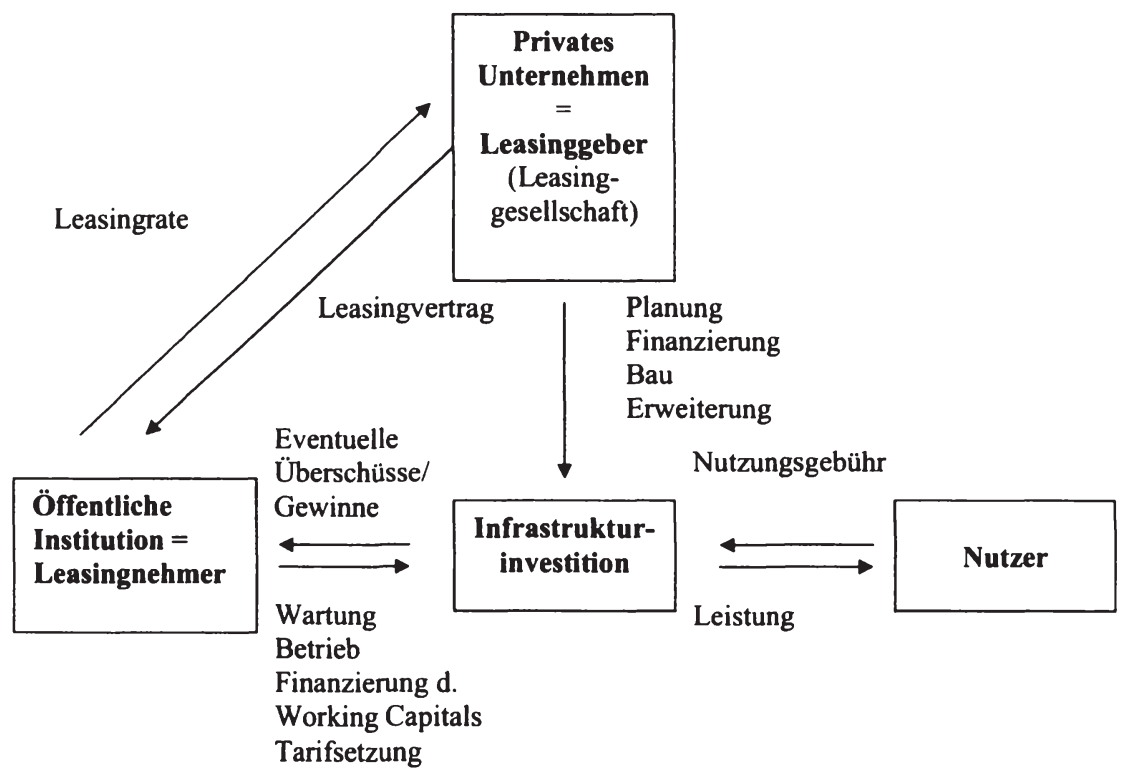

In der Literatur wird aber auch ein anderes Modell beschrieben, bei dem das private Unternehmen als Leasinggeber fungiert (vgl. Backhaus/Werthschulte 2003, Strohbach 2001, Tytko 1999). Häufig wird auch eine weitere Gesellschaft zu Finanzierungszwecken dazwischengeschaltet, die das Leasingobjekt errichtet oder kauft und gegen Bezahlung einer Leasingrate zur Verfügung stellt. Die Leasinggesellschaft wiederum errichtet oder kauft ein Leasingobjekt, welches sie dem Leasingnehmer gegen Bezahlung einer Leasingrate zur Verfügung stellt. 
Die private Gesellschaft ist zivilrechtliche Eigentümerin und Leasinggeberin der Anlage (vgl. Strohbach 2001: 58). Die Verantwortung für den Betrieb der Anlage geht aber in die öffentliche Hand über, die wiederum für die Nutzung der Infrastrukturleistung Gebühren einheben kann (vgl. Backhaus/Werthschulte 2003: 104f).

Wie es bei vielen Leasingverträgen üblich ist, kann auch eine Kaufoption am Ende der Laufzeit festgelegt werden (vgl. Werthschulte 2003: 105, Strohbach 2001: 58f). Außerdem ist es auch möglich, dass die Leasinggesellschaft die Funktion der Planung sowie der Instandhaltung und Wartung übernimmt, da sie zusätzliches Know-How in diesen Bereich einbringen kann. Der Betrieb der Anlage erfolgt schließlich durch den öffentlichen Auftraggeber, der diese gegen fixe Leasingraten von der Leasinggesellschaft mietet (vgl. Strohbach 2001: 59).

Abbildung 25 zeigt wiederum grafisch unterstützt die Leistungs-beziehungen in einem Leasingmodell, wenn der Staat als Leasingnehmer fungiert.

\subsubsection{Konzessionsmodell}

Die wesentlichen Kennzeichen für ein Konzessionsmodell sind das öffentliche Eigentum an der Infrastrukturanlage, die öffentliche Finanzierung der Anfangsinvestition, die private Finanzierung von Erweiterungsinvestitionen und die private Betriebsführung (vgl. World Bank 2006b: 121). Das Konzessionsmodell ist dem Leasingmodell sehr ähnlich, jedoch wird hier auch die teilweise Finanzierung des Anlagenvermögens vom privaten Unternehmen übernommen (vgl. World Bank 1994: 79).

Das Konzessionsmodell ist dadurch gekennzeichnet, dass eine öffentliche Aufgabe auf ein privates Unternehmen, den Konzessionär, übertragen wird (vgl. World Bank 2006b: 10, Eggers 2004: 35, Tytko 1999: 192). Hierbei beschränkt sich die Aufgabe des Konzessionärs, ähnlich dem Leasingnehmer, wieder auf das Management der Infrastrukturanlage. Die Objektgesellschaft erhält von der öffentlichen Hand ein fixes Nutzungsentgelt bis zum Ende der Konzessionsdauer, wobei keine vertragliche Beziehung zwischen der öffentlichen Hand und den Nutzern besteht (vgl. Strohbach 2001: 65f).

Bei international operierenden Kraftwerkslieferanten hat sich die Unterscheidung zwischen Konzessions- und BOT-Modell durchgesetzt (vgl. Zeuchner 2002: 224). Bei einem einfachen Konzessionsmodell wird nur beschränkt Eigenkapital des privaten Unternehmens in die Infrastrukturanlage investiert. Bei den BOT-Modellen (siehe Kapitel 4.4.5) kann das Privatunternehmen als Eigentümer der Anlage fungieren.

Das Konzessionsmodell ist in jenen Ländern von Bedeutung, in denen beispielsweise ausländische Investoren oder private Unternehmen per Gesetz oder Verfassung kein Eigentum an einer Infrastrukturanlage haben dürfen. Im Bereich der Wasserversorgung wurden bereits mehrere Konzessionen in Ent- 
wicklungsländern vergeben. In Manila wurden beispielsweise 1997 gleichzeitig zwei unabhängige Konzessionen vergeben. Ziel war die Ausweitung und Verbesserung des Servicenetzes durch unterschiedliche Technologien (vgl. Harris 2003: 33).

\section{Abbildung 26: Leistungsbeziehungen im Konzessionsmodell}

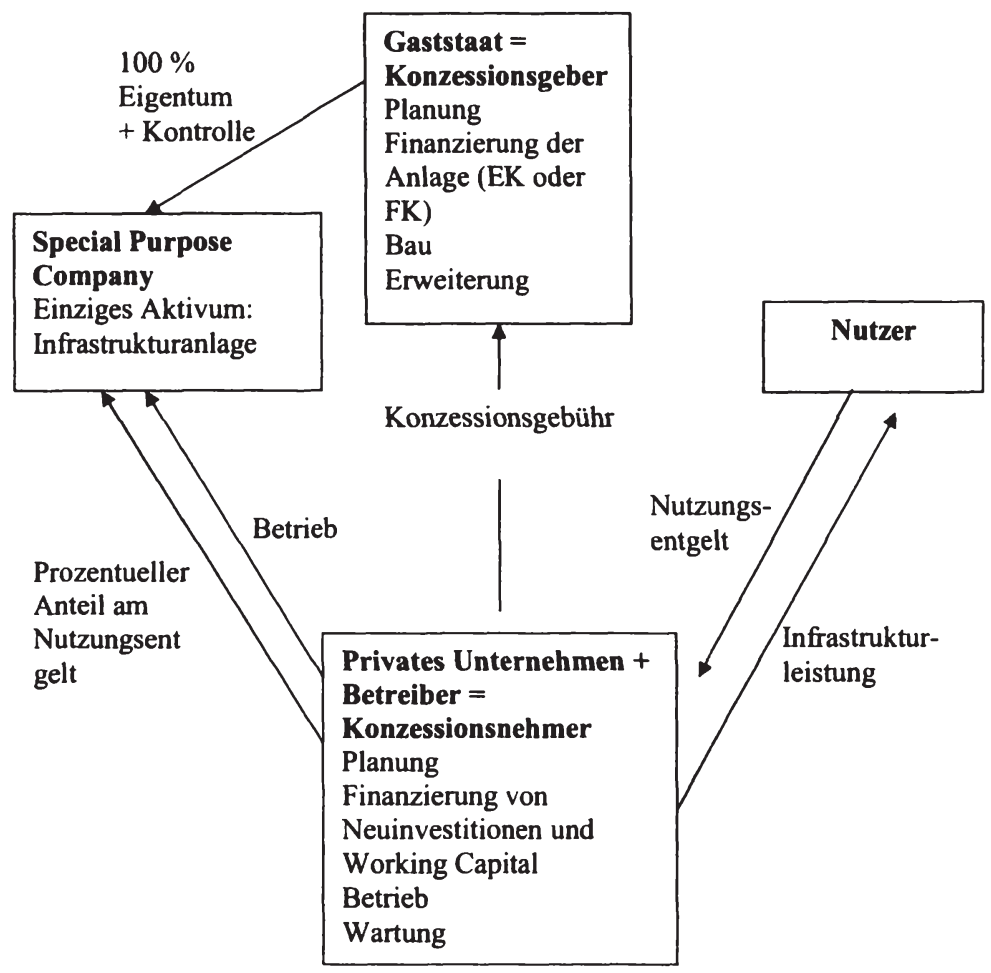

Quelle: Zeuchner (2002: 225)

Für das private Unternehmen ergibt sich der Gewinn wiederum aus der Differenz des Nutzungsentgelts, der Konzessionsgebühr und dem Investitionsaufwand. Aus Sicht des öffentlichen Konzessionsgebers besteht jedoch die Gefahr, dass die Modalitäten der Konzession dem Konzessionsnehmer zu überhöhten Renditen verhelfen und dabei nicht genug Anreize für eine ausreichende Instandhaltung der Anlage und für die Bereitstellung der Leistung geboten werden (vgl. World Bank 1994: 79). Die Abbildung 26 stellt grafisch den Ablauf eines Konzessionsmodells dar. 


\subsubsection{Public Private Partnership - Modelle}

Public Private Partnerships (PPP) i.e.S. werden durch die Gründung von neuen Gesellschaften (Special Purpose Vehicle) gebildet (vgl. IMF 2004: 9, BMVBS 2003: 2f). PPP bedienen sich der Projektfinanzierung, bei der im Mittelpunkt die Gründung einer gemeinsamen Projektgesellschaft (Special Purpose Vehicle) steht. Dadurch können kapitalintensive Projekte so finanziert werden, dass die beteiligten Unternehmen in ihrer Bilanz nur einen Kapitalanteil an der Projektgesellschaft ausweisen und somit nur mit diesem Anteil belastet sind (OffBalance-Sheet-Finanzierung). Außerdem kommt es gleichzeitig auch zu einer Risikoteilung zwischen den involvierten Projektpartnern, wobei die zukünftigen Cash-flows die Voraussetzung für die Investitionsentscheidung darstellen (vgl. Tytko 1999: 9ff).

PPP werden außerdem auch als Joint-Ventures im Infrastrukturbereich bezeichnet, die zur Verfolgung eines gemeinsamen Zieles auf kooperativer Basis zusammenarbeiten (vgl. Tytko 1999: 173). Wesentliche Merkmale von PPP sind das gemeinsame öffentliche und private Eigentum an einer Infrastrukturanlage, die gemeinsame Finanzierung und die private Betriebsführung (vgl. Zeuchner 2002: 250). In einer PPP erfolgt die Infrastrukturbereitstellung durch öffentliche und private Stellen gemeinsam, dabei gründen sie eine privatrechtliche Gesellschaft, in welcher der öffentliche Gesellschafter eine Mehrheitsbeteiligung von $51 \%$ (vgl. Strohbach 2001: 67) und die privaten Investoren eine Minderheitsbeteiligung eingehen (vgl. Tytko 1999: 190). PPP bzw. das Kooperationsmodell finden häufig Anwendung in sicherheits- und qualitätssensiblen Bereichen wie beispielsweise bei der Wasserversorgung, der Altlastensanierung oder der Müllentsorgung (vgl. Strohbach 2001: 67). Die Beteiligung des öffentlichen Partners erfolgt häufig durch die Einbringung von Land oder Entwicklungs- und Baugenehmigungen (vgl. Werthschulte 2003: 107).

In der Regel ist der private Partner mehrheitlich im Vorstand des neu gegründeten Unternehmens vertreten und hat daher die Managementkontrolle im täglichen Geschäftsablauf (vgl. World Bank 2006b: 11). Der Betrieb und die Finanzierung sowie Planung und Bau werden von der Projektgesellschaft übernommen (vgl. Wertschulte 2003: 108). Der Bau der neuen Anlage erfolgt durch einen privaten Anlagenbauer, der mit dem Betreiber identisch sein kann. Eine PPP bietet einen weiten Spielraum für die Gestaltung der Beteiligungsverhältnisse und die Verteilung der erwirtschafteten Gewinne, um das Projekt für den privaten Partner wirtschaftlich attraktiv zu gestalten (vgl. Zeuchner 2002: 247f).

Bei den PPP-Modellen werden die vorhandenen Risiken zwischen den öffentlichen und privaten Partnern geteilt (vgl. BMVBS 2003:2f). Abhängig von der Ausgestaltung des Gewinnabführungsvertrages trägt das private Unternehmen mehr vom Betriebsrisiko als das Gastland. Die Länderrisiken müssen vom privaten Unternehmen getragen werden und umfassen das kommerzielle, politische und das Wechselkursrisiko. 
Die folgende Abbildung 27 stellt die möglichen Leistungsbeziehungen in einer PPP grafisch dar.

\section{Abbildung 27: Leistungsbeziehungen im PPP-Modell}

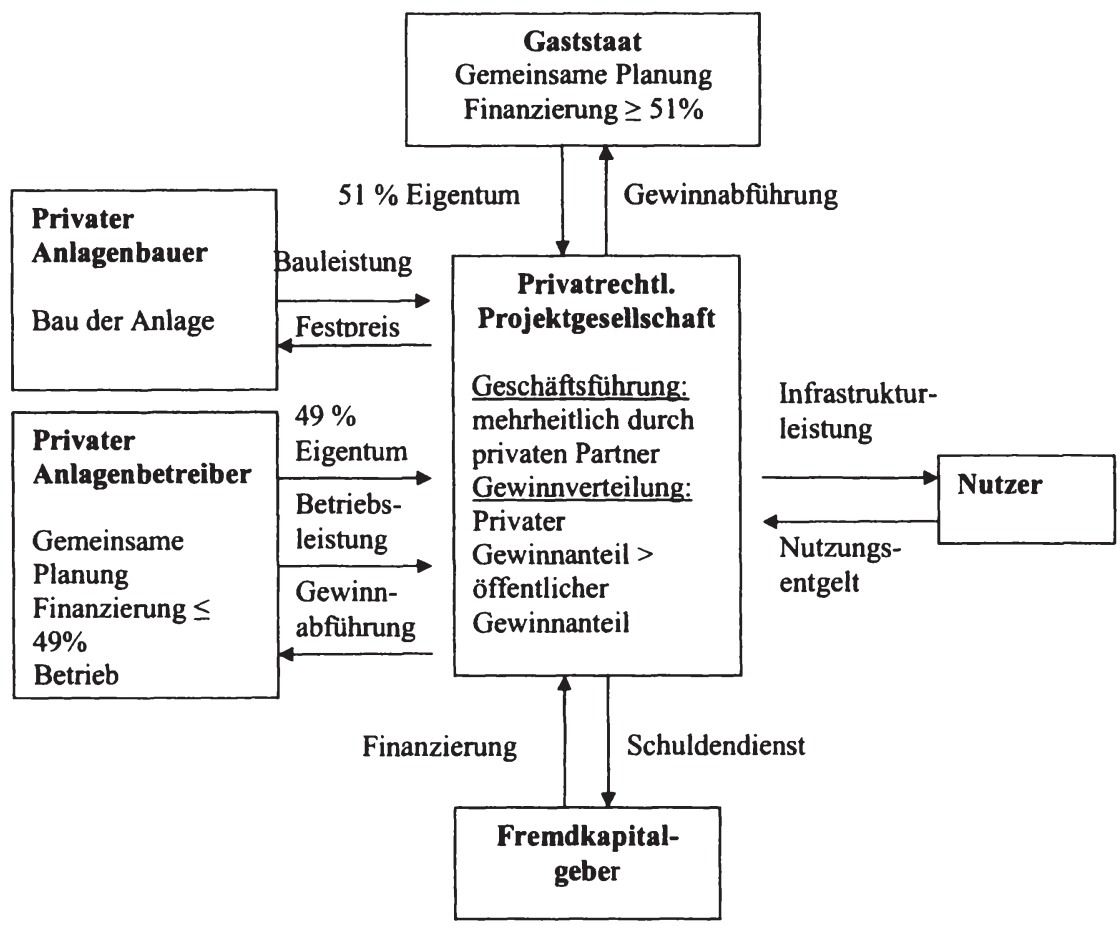

Quelle: Zeuchner 2002 (247)

Um das Zulieferrisiko zu beschränken, können durch langfristige Zulieferverträge (Deliver-or-Pay oder Supply-or-Pay-Verträge) die Risiken auf die Lieferanten übertragen werden. Das Absatzrisiko kann mit Abnahmeverträgen in Form von Take-and-Pay- bzw. Take-or-Pay-Verträgen (vgl. Siebel 2001: 54) reduziert werden. Das Fertigstellungsrisiko hat üblicherweise der Anlagenbauer zu tragen, der dieses durch einen Lump-Sum-Turnkey-Vertrag auf Festpreisbasis auf die Bauunternehmung oder den Anlagenlieferanten abwälzt. In der Praxis werden diese Verträge als EPC-Verträge bezeichnet (vgl. Siebel 2001: 79). Auch Kreditgeber können zu einem geringeren Umfang das Fertigstellungsrisiko übernehmen. Die öffentliche Hand des Gastlandes und die Fremdkapitalgeber können zur Verringerung des Fertigstellungsrisikos Fristen 
setzen und Pönalezahlungen sowie Garantien, wie beispielsweise eine Fertigstellungs-, Erfüllungs- und Gewährleistungsgarantie, vereinbaren.

\subsubsection{Betreibermodelle}

Betreibermodelle, auch Build Operate Transfer (BOT)-Modelle genannt, stellen die Organisationsform mit der höchsten Privatisierungsintensität dar. Die konstituierenden Merkmale für BOT-Modelle sind das private Eigentum, die private Finanzierung, die private Bauleitung und die private Betriebsführung (vgl. Werthschulte 2003: 109, Tytko 1999: 174).

In der Regel wird für BOT eine eigene Projektgesellschaft gegründet, auch Special-Purpose-Vehicle (SPV) genannt, die dann rechtlich für den Bau (Build), für den Betrieb (Operate) und die Übertragung der Anlage an den Staat (Transfer) verantwortlich ist (vgl. Grimsey/Lewis 2004: 225, Werthschulte 2003: 107). Die BOT-Modelle bedienen sich der Projektfinanzierung, die es den beteiligten Unternehmen ermöglicht, off-balance-sheet-Projekte durchzuführen und dadurch ihre eigene Bilanz nur mit der Kapitalbeteiligung an der Projektgesellschaft zu belasten. Außerdem kommt es gleichzeitig auch zu einer Risikoteilung zwischen den Projektpartnern, wobei die zukünftigen Cash-flows die Voraussetzung für die Investitionsentscheidung darstellen (vgl. Tytko 1999: 9ff).

Die öffentliche Hand räumt der Projektgesellschaft im Rahmen einer Konzessionsgewährung die Bau-, Nutzungs- und Gebührenrechte ein (vgl. Strohbach 2001: 70f). Da das Eigentum an der Projektgesellschaft bis zum Transfer überwiegend in privater Hand liegt, werden BOT-Modelle oft auch BOOT (Build Operate Own Transfer)-Modelle genannt. In dieser Arbeit wird aber ausschließlich der Begriff BOT verwendet. Allerdings sind beispielsweise auch BOO (Build Operate Own)-Modelle möglich, bei denen eine Übertragung am Ende der Laufzeit noch nicht im Vorhinein festgelegt wird (vgl. Tytko 1999: 177f). BOT-Modelle sind in allen Infrastrukturbereichen zu finden, in denen grundsätzlich das Kriterium der Ausschließbarkeit von der Nutzung gegeben ist, wobei jedoch Kraftwerke, Straßen- und Schienennetze am häufigsten realisiert werden (vgl. Strohbach 2001: 71).

Der Hersteller einer Infrastrukturanlage geht bei einer BOT-Konstruktion über seine ursprünglichen Kernkompetenzen hinaus und setzt sich dabei höheren Risiken aus (vgl. Tytko 1999: 175). Für die Investoren spielen hier strategische Aspekte eine Rolle, da sie durch das spezielle Angebot Pionier- und Leitkunden oder auch das Verhalten der Wettbewerber beeinflussen wollen. Vor allem Vertriebsüberlegungen spielen dabei eine Rolle, da der Markt für Infrastrukturleistungen von Überkapazitäten und intensivem Preiswettbewerb geprägt ist und durch BOT-Modelle versucht wird, zusätzliche Ertragsmöglichkeiten und Beschäftigungschancen zu gewinnen (vgl. Weber et al. 2006: 32). 
Die folgende Abbildung 28 stellt wiederum grafisch die Leistungsbeziehungen in einem BOT-Modell dar. stellt wiederum grafisch die Leistungsbeziehungen in einem BOT-Modell dar.

Abbildung 28: Leistungsbeziehungen im BOT-Modell

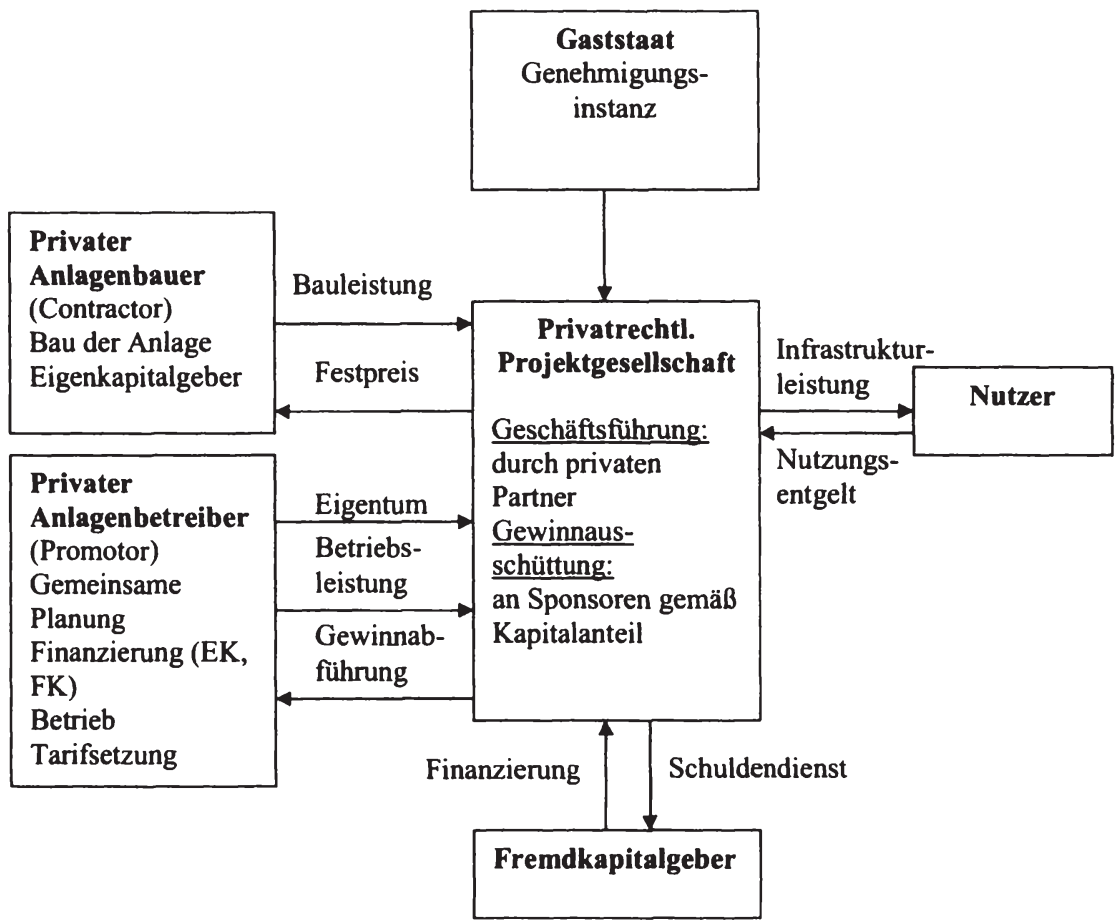

Quelle: Zeuchner (2002: 265)

Am Ende einer im Vorhinein festgelegten Laufzeit geht das Eigentum der Anlage, eventuell gegen Zahlung eines Verkaufspreises, auf den Gaststaat über (vgl. Werthschulte 2003: 109). Diese Übertragung muss auch den Wartungszustand der Anlagen und den Wert nicht kapitalisierten Vermögens berücksichtigen. Der Wartungszustand der Anlagen sollte im Vorfeld genau definiert und kann bei besonders gutem Zustand mit einer Prämie belohnt werden. Die Konzession kann aber auch periodisch neu an Betreiber versteigert oder es können auch Verhandlungen zur Weiterführung vereinbart werden (vgl. Zeuchner 2002: 263).

Sollte die Anlage bis zum Ende der Betriebslaufzeit nicht vollständig amortisiert sein, kann der Betreiber auch eine entsprechende Kompensationszahlung erhalten. Es ist auch möglich, dass die Konzessions- mit der 
Amortisierungszeit nicht übereinstimmt oder sich der Staat das Recht vorbehält, den Vertrag vor der vereinbarten Laufzeit zu beenden. Außerdem erfordert das Infrastrukturangebot manchmal Investitionen, die nicht für die gesamte Laufzeit vorhergesehen werden können.

Es gibt eine Vielzahl von BOT-Varianten, die sich durch die Anzahl der Beteiligten und die Intensität der Beteiligung unterscheiden. Zahlreiche Abkürzungen stehen dabei als Indikatoren für die verschiedenen Ausgestaltungsmöglichkeiten, jedoch werden diese Begriffe nicht einheitlich verwendet. Im Anhang ist eine Aufstellung über mögliche Ausgestaltungsformen von BOT zu finden.

Aufgrund der hohen Involvierung im Projekt, ist das private Unternehmen bei BOT-Modellen Risiken am meisten ausgesetzt. Es ist daher wenig überraschend, dass BOT-Projekte in entwickelten Ländern mehr Erfolg aufweisen als in Entwicklungsländern (vgl. Trujillo et al. 1998).

\subsection{Zusammenfassung}

Das vierte Kapitel beschäftigt sich mit der öffentlich-privaten Bereitstellung von Infrastruktur und beginnt mit einer ausführlichen Auseinandersetzung mit der Thematik in der Einführung. Der erste Abschnitt widmet sich der Definition von Partnerschaft i.e.S. in einem Kooperationsmodell, die wiederum für die Definition von Public Private Partnerships (PPP) herangezogen wird. In der vorliegenden Arbeit wird die Definition von PPP i.e.S. verwendet. Darunter wird jenes Modell verstanden, bei dem es zu einer langfristigen, vertraglich geregelten Zusammenarbeit zwischen öffentlicher Hand und Privatwirtschaft in Hinblick auf wirtschaftliche Erfüllung öffentlicher Aufgaben kommt. Als Überbegriff für alle Formen der privaten Beteiligung an der Infrastrukturbereitstellung wird „Private Participation in Infrastructure" (PPI) verwendet. Darauf folgt die Darstellung der Ziele und Interessen der beteiligten Partner, wobei bei PPP auf eine Zielkomplementarität zu achten ist, da ansonsten die langfristige Zusammenarbeit gefährdet ist. Der abschließende Abschnitt beschäftigt sich mit den Problembereichen bei PPP, wobei die Partnerschaft per se das größte Risiko darstellt.

Das zweite Kapitel widmet sich der PPI in der Entwicklungspolitik. Im ersten Abschnitt werden die Auswirkungen der entwicklungspolitischen Veränderungen in der Infrastruktur dargestellt. Dabei wird Bezug auf die im Kapitel 2.2 dargestellten Reformen wie Privatisierung und Dezentralisierung genommen. Daran anschließend wird die Rolle von Official Development Aid (ODA) in der Infrastrukturfinanzierung beleuchtet, wobei ein stetiger Rückgang von ODA im Infrastruktursektor zu verzeichnen ist. Der folgende Abschnitt beschäftigt sich 
mit der Rolle des privaten Sektors in der Infrastrukturfinanzierung, wobei zu erkennen ist, dass sich die meisten privaten Investitionen auf wenige Sektoren und Regionen beschränken.

Zusätzlich dazu hat Ende der 1990er Jahre die asiatische Finanzkrise viele Projekte gefährdet und die privaten Investoren vom Markt vertrieben. Der starke Rückgang der privaten Investitionen im Infrastruktursektor hatte gravierende, destabilisierende Auswirkungen auf die wirtschaftliche Situation in den Entwicklungsländern und daher wurde die Rolle des Staates und der Entwicklungshilfe (ODA) neu überdacht. Heute wird die Ansicht vertreten, dass der Staat wieder eine aktivere Rolle in der Infrastrukturfinanzierung einnehmen und ODA als „Hebel“ für die Förderung von mehr privaten Investitionen eingesetzt werden soll.

Im Kapitel 4.3 werden die Länderrisiken dargestellt, die in wirtschaftliche, politische und Wechselkurrisiken unterteilt werden. Die wirtschaftlichen Risiken umfassen die Absatz- und Zulieferrisiken und das Delkredererisiko. Die politischen Risiken in Entwicklungsländern umfassen die politischen Risiken im engeren Sinn, Konvertierungs- und Transfer und Zahlungsverbots- und Moratoriumsrisiko. Die dritte Gruppe umfasst die drei Ausprägungsformen des Wechselkursrisikos, wobei das Währungsumwechslungsrisiko aufgrund des starken Ungleichgewichts zwischen US\$-Verschuldung und Einnahmen in lokaler Währung für private Unternehmen sehr groß sein kann.

Im vierten Abschnitt werden die verschiedenen öffentlich-privaten Finanzierungsmodelle vorgestellt. Die Darstellung der einzelnen Modelle folgt der Logik des Staat-Privat-Kontinuums, wobei zuerst jene Formen beschriebenen werden, die meisten staatliches Eigentum aufweisen bis hin zu den Betreibermodellen, bei denen am meisten privates Eigentum zu finden ist.

In der abschließenden Tabelle 4 wird ein Überblick über die Modelle gegeben. Abhängig vom Modell werden die Risiken zwischen den privaten und öffentlichen Beteiligten aufgeteilt. Je weiter man in der Tabelle nach unten rückt, umso stärker nimmt auch der Zeithorizont zu. Wesentlich ist auch, dass bei den letzten beiden Modellen privates Eigentum möglich ist. 
Tabelle 4: Zusammenfassung von öffentlich-privaten Finanzierungsmodellen

\begin{tabular}{|c|c|c|c|c|c|c|c|}
\hline \multirow{6}{*}{$\begin{array}{c}\text { Zunahme } \\
\text { an } \\
\text { Risiko } \\
\text { Dauer } \\
\text { Privatisierung/ } \\
\text { Eigentum }\end{array}$} & Alternativen & Dauer & $\begin{array}{c}\text { Umfang der } \\
\text { Einbindung } \\
\text { Privater }\end{array}$ & Länderrisiken & Projektrisiken & $\begin{array}{l}\text { Eigentum an } \\
\text { Infrastruktur }\end{array}$ & $\begin{array}{c}\text { Investitionen } \\
\text { in } \\
\text { Infrastruktur }\end{array}$ \\
\hline & $\begin{array}{l}\text { Service } \\
\text { Vertrag }\end{array}$ & 1-2 Jahre & $\begin{array}{l}\text { Ausgliederung } \\
\text { genau } \\
\text { spezifizierter } \\
\text { Aufgaben }\end{array}$ & $\begin{array}{l}\text { P: Öffentlich } \\
\text { W: Privat } \\
\text { F: Öffentlich }\end{array}$ & $\begin{array}{l}\text { T: Öffentlich } \\
\text { W: Öffentlich }\end{array}$ & Öffentlich & Öffentlich \\
\hline & $\begin{array}{l}\text { Management } \\
\text { Vertrag }\end{array}$ & 3-5 Jahre & $\begin{array}{l}\text { Ausgliederung } \\
\text { von Betrieb } \\
\text { und Wartung }\end{array}$ & $\begin{array}{l}\text { P: Öffentlich } \\
\text { W: Privat } \\
\text { F: Öffentlich }\end{array}$ & $\begin{array}{l}\text { T: Öffentlich } \\
\text { W: Öffentlich }\end{array}$ & Öffentlich & Öffentlich \\
\hline & Leasing & 8-15 Jahre & $\begin{array}{l}\text { Durchführung } \\
\& \text { Verantwort- } \\
\text { ung für Betrieb, } \\
\text { Wartung u. } \\
\text { Absatz der } \\
\text { Leistung }\end{array}$ & $\begin{array}{l}\text { P: Öffentlich } \\
\text { W: Privat } \\
\text { F: Öffentlich }\end{array}$ & $\begin{array}{l}\text { T: Öffentlich } \\
\text { W: Öffentlich/ } \\
\text { Privat }\end{array}$ & Öffentlich & Öffentlich \\
\hline & Konzessionen & $\begin{array}{l}\text { gewöhnlich: } \\
\text { 5-10 Jahre } \\
\text { möglich: } \\
\text { 20-30 Jahre }\end{array}$ & $\begin{array}{l}\text { Durchführung } \\
\text { \& Verantwort- } \\
\text { ung für Betrieb, } \\
\text { Wartung u. } \\
\text { Absatz der } \\
\text { Leistung, } \\
\text { Ausbau der } \\
\text { Anlage }\end{array}$ & $\begin{array}{l}\text { P: Privat } \\
\text { W: Privat } \\
\text { F: Öffentlich }\end{array}$ & $\begin{array}{l}\text { T: Öffentlich } \\
\text { W: Öffentlich/ } \\
\text { Privat }\end{array}$ & Öffentlich & $\begin{array}{l}\text { Öffentlich/ } \\
\text { Privat }\end{array}$ \\
\hline & $\begin{array}{l}\text { Public Private } \\
\text { Partnerships }\end{array}$ & $\begin{array}{l}\text { unbefristet bis } \\
\text { Beendigung }\end{array}$ & $\begin{array}{l}\text { Planung, Bau } \\
\text { \& Betrieb d. } \\
\text { Anlage, } \\
\text { anteilige } \\
\text { Finanzierung d. } \\
\text { Investition }\end{array}$ & $\begin{array}{l}\text { P: Privat } \\
\text { W: Privat } \\
\text { F: Öffentlich/ } \\
\text { Privat }\end{array}$ & $\begin{array}{l}\text { T: Öffentlich/ } \\
\text { Privat } \\
\text { W: Öffentlich/ } \\
\\
\text { Privat }\end{array}$ & $\begin{array}{l}\text { Öffentlich } \\
(51 \%) \text { und } \\
\text { Privat }(49 \%)\end{array}$ & $\begin{array}{l}\text { Öffentlich und } \\
\text { Private }\end{array}$ \\
\hline
\end{tabular}




\begin{tabular}{|l|l|l|l|l|l|l|l|}
\hline & $\begin{array}{l}\text { Betreibermod } \\
\text { elle } \\
\text { (BOT) }\end{array}$ & 20-30 Jahre & $\begin{array}{l}\text { Planung, } \\
\text { Finanzierung, } \\
\text { Bau, Betrieb \& } \\
\text { Wartung d. } \\
\text { Anlage, } \\
\text { Transfer am } \\
\text { Ende der } \\
\text { Laufzeit }\end{array}$ & $\begin{array}{l}\text { P: Privat } \\
\text { W: Privat }\end{array}$ & W: Privat & $\begin{array}{l}\text { Öfentlich und } \\
\text { Privat }\end{array}$ & Privat \\
& & & & \\
& & & & \\
\hline
\end{tabular}

\section{Anmerkungen:}

Die Länderrisiken werden unterteilt in: Politisches, Wirtschaftliches und Force Majeure Risiko.

Die Projektrisiken werden unterteilt in: Technische und Wirtschaftliche Risiken. 


\section{Die Unterstützung von privaten Infrastruktur- investitionen durch internationalen und nationale Finanzinstitutionen}

Das fünfte Kapitel fokussiert auf die Unterstützung von privaten Infrastrukturfinanzierungen durch internationale und nationale Finanzinstitutionen. Im ersten Kapitel wird eine Einführung in die Thematik gegeben, wobei auf die Rolle der Projektfinanzierung, die Mindestkriterien für eine private Infrastrukturfinanzierung sowie auf die Kofinanzierungsfunktion der internationalen Finanzinstitutionen (IFIs) eingegangen wird. Im zweiten Kapitel (5.2) werden die einzelnen Instrumente der nationalen und internationalen Finanzinstitutionen beschrieben. Anschließend an diese Darstellung werden im Kapitel 5.3 einzelne innovative Ansätze genauer dargestellt und kritisch betrachtet. Im letzten Kapitel (5.4) wird die Thematik abschließend nochmals kurz zusammengefasst. Die folgende Abbildung 29 stellt grafisch den Aufbau dieses Kapitels vor und dient der besseren Orientierung im Kapitel.

Abbildung 29: Kapitelübersicht Unterstützung von privaten Infrastrukturinvestitionen durch internationale und nationale Finanzinstitutionen

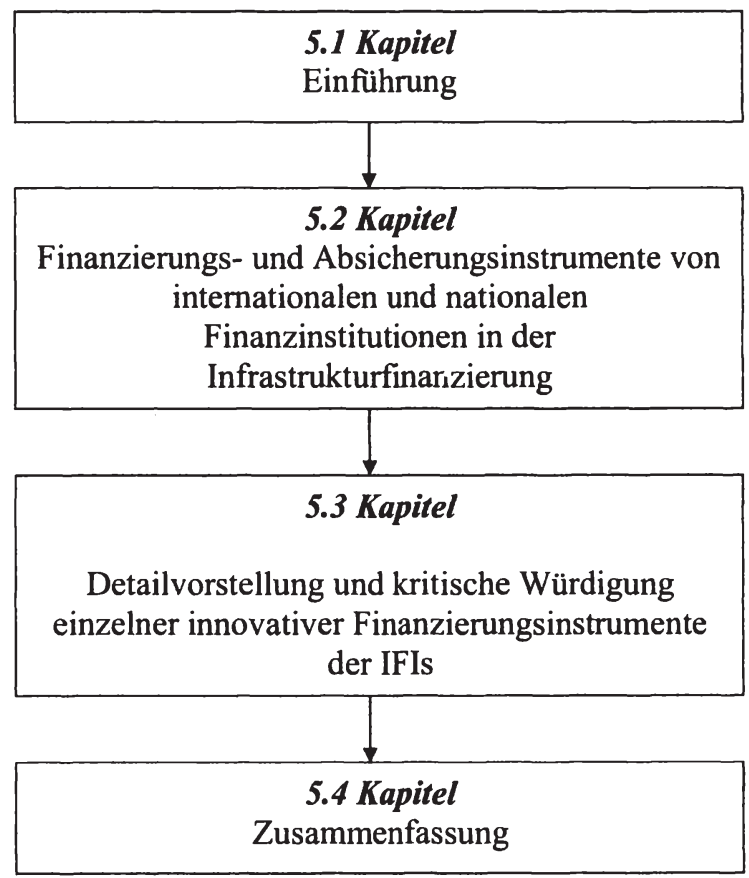




\subsection{Einführung}

Die Projektfinanzierung spielt bei der privaten Finanzierung von Infrastruktur eine wesentliche Rolle, daher wird in der Einführung zunächst darauf Bezug genommen. Daran anschließend werden im zweiten Abschnitt die Mindestkriterien für private Investitionen dargestellt, da nicht alle Infrastrukturprojekte gleichermaßen für den privaten Sektor geeignet sind. Da die Finanzierung der privaten Infrastrukturinvestitionen von den internationalen und nationalen Finanzinstitutionen wesentlich unterstützt wird, gibt der dritte Abschnitt einen Einblick in die Kofinanzierungsfunktion dieser Institutionen.

\subsubsection{Rolle der Projektfinanzierung im Infrastruktursektor}

In den letzten 20 Jahren hat sich die Rolle des öffentlichen Sektors im Bereich der Infrastrukturfinanzierung in allen Ländern stark gewandelt (vgl. Grimsey/Lewis 2004: 81). Wie bereits in den vorangegangen Kapiteln dargestellt wurde, hat sich durch Privatisierungs- und Deregulierungsmaßnahmen in Entwicklungsländern ein neuer Markt für ausländische Investoren aufgetan (vgl. Werthschulte 2005: 47). Dadurch wurde der öffentliche Bereich immer mehr zum „Aufseher" und weniger zum Bereitsteller von Infrastrukturleistungen. Oftmals agiert die öffentliche Hand als Garantiegeber oder gewährt andere Unterstützung (vgl. Wolf 2003: 63, Trujillo et al. 1998).

Sobald der öffentliche Sektor nicht mehr für die Erzeugung der Infrastrukturleistung verantwortlich ist, stellen die zukünftigen Cashflows in der Projektfinanzierung die einzige Finanzierungsquelle dar (vgl. Werthschulte 2005: 34). Im Zuge dieser Verlagerung vom Staat auf das private Unternehmen spricht man bei der Finanzierung von Infrastrukturinvestitionen auch von Financial Engineering. Diese beschäftigt sich mit der Planung und Ausarbeitung von maßgeschneiderten Finanzierungskonzepten, bei denen das gesamte Spektrum der klassischen Finanzierungsinstrumente zum Einsatz kommt (vgl. Werthschulte 2005: 34). Aus diesem Spektrum wird versucht, eine optimale Kombination aller Finanzierungsalternativen zusammenzustellen. Streng genommen handelt es sich hierbei um kein neues Instrument für die Infrastrukturfinanzierung, sondern vielmehr um einen grundsätzlich anderen Denkansatz (vgl. Backhaus/ Werthschulte 2003: 6).

Projektfinanzierung bietet eine Möglichkeit, Eigen- und Fremdkapital für spezielle Infrastrukturprojekte zu mobilisieren, wobei die Risiken und Gewinne nicht alleine vom Sponsor getragen werden, sondern auch die anderen Investoren daran beteiligt sind (vgl. Grimsey/Lewis 2004: 227). Seit mehreren Jahrzehnten wird für die Finanzierung von großvoluminösen, risikoreichen Investitionsvorhaben Projektfinanzierung eingesetzt. Darunter fallen vor allem Projekte im Bereich der Infrastruktur, die aufgrund der hohen Investitions- 
ausgaben und der großen Risiken durch die einzelnen Unternehmen oder Staaten nicht getragen werden können. Die Beurteilung des Projektes basiert vor allem auf den generierten Cash-Flow-Strömen und den mit ihnen verbundenen Risiken (vgl. Wolf 2003: 59). Ein wesentliches Kriterium für Projektfinanzierung ist daher die Tatsache, ob das Projekt wirtschaftlich rentabel ist. Neben risikoreichen Investitionen in Entwicklungs- und Schwellenländer, ist diese Form der Finanzierung insbesondere auch für Projekte geeignet, die im Zuge von Unternehmenskooperationen erstellt und/oder betrieben werden (vgl. Wolf 2003: 64).

\subsubsection{Mindestkriterien für private Infrastrukturinvestitionen}

Wie im Kapitel 4.4 dargestellt wurde, können private Unternehmen mit einer unterschiedlichen Intensität an Infrastrukturinvestitionen beteiligt sein. Damit private Unternehmen in die Infrastruktur investieren, müssen die Projekte aber folgenden Mindestanforderungen entsprechen. Wenn ein Vorhaben nicht alle Mindestanforderungen erfüllt, ist eine rein privatwirtschaftliche Lösung nicht möglich und die Unterstützung durch den Staat oder andere Institutionen erforderlich (vgl. Zeuchner 2002: 242).

1. Das Projekt muss wirtschaftlich tragfähig sein: Das Projekt sollte eine Projektrendite erwirtschaften, die über den marktüblichen Fremdkapitalzinsen liegt. Ein Projekt hingegen, das wirtschaftlich nicht tragfähig ist, aber gesamtwirtschaftlich erwünscht und sinnvoll ist, muss vom Staat oder von Internationalen Finanzinstitutionen (IFIs) unterstützt werden. Dies ist beispielsweise auch möglich, wenn es aufgrund von natürlichen Monopolen nicht zu wettbewerbsfähigen Preisen kommen kann. In diesem Fall muss die öffentliche Hand die Finanzierung alleine übernehmen (vgl. IMF 2004: 10).

2. Das Projekt muss schuldendienstfähig sein: Aus dem Projekt muss ausreichend Cash-Flow generiert werden, aus dem neben den Bau- und Betriebskosten die Zinsen und die Tilgung der erforderlichen Kredite erbracht werden können. Problematisch ist hier insbesondere, dass die Dienstleistungen von der öffentlichen Hand oft zu nicht kostendeckenden Preisen angeboten wurden und es durch die PPI zu Preiserhöhungen und in der Folge zu einem Nachfragerückgang kommen kann. Laut Harris (2003: 31f) sind daher die Finanzierungs- und Preisfestsetzungsentscheidungen für den Erfolg eines Projektes essenziell. Entweder müssen die Steuerzahler oder die Verbraucher für die Kosten der Dienstleistung aufkommen. Um Preiserhöhungen für die Dienstleistung abzuschwächen bzw. zu vermeiden, wurde von der Weltbankgruppe für den Bereich der PPI der „Output Based Aid“-Ansatz (OBA) entwickelt (vgl. Noel/Brzeski 2005: xii). Der OBA-Ansatz wird im Kapitel 5.3.3 genauer dargestellt. 
3. Die Projektrisiken sind unter allen Projektbeteiligten auf eine akzeptable Weise zu verteilen: Die Risikoteilung zwischen den beteiligten Parteien stellt eine Grundvoraussetzung für die Projektfinanzierung dar. Eine adäquate Aufteilung der Risiken ist daher eine Grundvoraussetzung für eine erfolgreiche PPI (vgl. IMF 2004: 15).

4. Die Qualität der Dienstleistung muss vorab festgelegt werden können: Es können nur jene Projekte finanziert werden, deren Leistung gemessen werden kann. Wichtig ist dabei, dass auch die Qualität festgelegt werden kann.

\subsubsection{Die Kofinanzierungsrolle der IFIs bei privaten Investitionen}

Internationale und nationale Finanzinstitutionen (IFIs) erfüllen mit ihren Finanzund Absicherungsinstrumenten, die im folgenden Kapitel 5.2 genauer dargestellt werden, eine wichtige Kofinanzierungsfunktion bei der privaten Finanzierung von Infrastruktur. Von Seiten der ADB wird Kofinanzierung wie folgt definiert „Cofinancing refers to any arrangement under which bank funds or guarantees are associated with funds provided by third parties for a particular project or program" (vgl. ADB Homepage). Als Kofinanzierung wird daher jede Vereinbarung verstanden, aufgrund derer Finanzmittel der Internationalen Finanzinstitutionen mit jenen von weiteren Kapitalgebern außerhalb des kreditnehmenden Landes zur Finanzierung eines Investitionsvorhabens kooperativ eingesetzt werden.

Ziel der Beteiligung von IFIs ist daher die Mobilisierung zusätzlicher Finanzmittel für Projektfinanzierungen. Dies bedeutet, dass kommerzielle Finanzinstitutionen meist erst dann bereit sind in risikoreichen Ländern zu investieren, wenn IFIs beteiligt sind. Durch die Beteiligung von IFIs kann es daher zu einer Hebelwirkung bei der Finanzierung kommen und so wesentlich mehr Projekte finanziert werden, als dies mit den eigenen Mitteln möglich wäre.

Eine wesentliche Sicherheit bietet der „Preferred Creditor Status “ der multilateralen Finanzinstitutionen. Die Schuldner sind dadurch verpflichtet, vorrangig die bestehenden Verpflichtungen dieser Institutionen pünktlich und vollständig zu bedienen (vgl. Deutsche Kommission Justitia et Pax 1999: 19). Dieser Status basiert nicht auf einem rechtlichen Konzept, sondern ist das Ergebnis einer gemeinsamen Absprache zwischen den Internationalen Finanzinstitutionen, den kommerziellen Kreditgebern und den Entwicklungsländern. Dieser Status bedeutet, dass die Kredite der multilateralen Banken bei Konvertierungsrestriktionen vorrangig Zugang zu Devisen haben. Außerdem beinhaltet dieser Status auch Steuerbefreiung und andere Sicherheiten für den Kreditgeber.

Kommerzielle Banken können sich diesen Vorteil der Finanzinstitutionen durch verschiedene Instrumente zu Nutzen machen. Beispielsweise kann durch sogenannte „cross-default"-Klauseln eine Gleichstellung zwischen den be- 
teiligten multilateralen und kommerziellen Finanzinstitutionen hergestellt werden. Wenn ausstehende Kapitalbeträge von den Kreditnehmern an die kommerziellen Banken nicht zurückbezahlt werden, sind auch die Kredite der Entwicklungsbanken umgehend fällig. Aus diesem Grund beteiligen sich Privatinvestoren bereitwilliger an Projektfinanzierungen in Entwicklungsländern. Außerdem bemühen sich die Gastländer die Verträge ordnungsgemäß zu erfüllen, um auch in Zukunft Finanzmittel der jeweiligen Institutionen beantragen zu können.

Zusammenfassend kann als ein wesentlicher Vorteil der Kofinanzierung die Erhöhung der Finanzmittel für jene großen Infrastrukturprojekte genannt werden, die nicht alleine von einer einzigen Finanzinstitution finanziert werden können. Außerdem wird den privaten Investoren durch die Beteiligung der IFIs der Zugang zum internationalen Finanzmarkt und zu längeren Kreditlaufzeiten erleichtert.

\subsection{Finanzierungs- und Absicherungsinstrumente von inter- nationalen und nationalen Finanzinstitutionen in der Infrastrukturfinanzierung}

\subsubsection{Einführung}

Das folgende Kapitel beschäftigt sich mit dem Angebot der multilateralen, europäischen und nationalen Finanzinstitutionen. Dabei werden nur jene Instrumente vorgestellt, die für die Finanzierung von Infrastruktur relevant sind. Der Fokus liegt auf den Instrumenten, die für öffentlich-private Infrastrukturprojekte zur Verfügung stehen. Ausgehend von den internationalen Organisationen, bei denen verschiedenen Nationen beteiligt sind, werden die europäischen und schließlich die österreichischen Institutionen vorgestellt.

Multilaterale

Finanzinstitutionen

[5.2.2]:
Weltbankgruppe:

- IFC

- MIGA

- IBRD und IDA

- Fonds- und Programmbeteiligungen der Weltbankgruppe

Regionale Entwicklungsbank:

- Asian Development Bank (ADB) 
Europäische Institutionen [5.2.3]:

Österreichische

Institutionen [5.2.4]:
- Europäische Investitionsbank (EIB)

- Europäische Kommission

- Austrian Development Agency (ADA)

- Austria Wirtschaftsservice (AWS)

- Oesterreichische Kontrollbank (OeKB)

In Kapitel 5.2.2 werden zuerst die multilateralen Finanzinstitutionen dargestellt. Hier werden die Instrumente der Organisationen der Weltbankgruppe und stellvertretend für alle anderen regionalen Entwicklungsbanken, die Asian Development Bank (ADB) vorgestellt. Im darauf folgenden Kapitel 5.2.3 werden die europäischen und daran anschließend in Kapitel 5.2.4 die österreichischen Organisationen vorgestellt.

In den Institutionen wird klar zwischen den Kundengruppen bzw. zwischen dem öffentlichen und privaten Sektor unterschieden. Abhängig davon werden verschiedene Produkte zu unterschiedlichen Konditionen angeboten. Für öffentlich-privat finanzierte Infrastrukturprojekte sind vor allem direkte und indirekte Kredite und Garantien wesentlich.

\subsubsection{Multilaterale Institutionen}

Wie im Kapitel 5.1.3 dargestellt wurde, leisten die internationalen Finanzinstitutionen im Rahmen von Kofinanzierungen einen wichtigen Beitrag zur Finanzierung von öffentlich-privaten Infrastrukturinvestitionen. Die älteste und daher auch tonangebende IFI stellt mit Sicherheit die Weltbankgruppe mit ihren Untereinheiten dar. Im Bereich der privaten Projektfinanzierung ist vor allem die International Finance Corp. (IFC) aktiv. Auch Multilateral Investment Guarantee Agency (MIGA) bietet Förderprogramme für Direktinvestitionen im Ausland an. Des Weiteren beteiligen sich auch die International Bank of Reconstruction and Development (IBRD) und die International Development Agency (IDA) an der Finanzierung von Infrastrukturinvestitionen, wobei deren Fokus aber am öffentlichen Sektor liegt. Beim International Centre for Settlement of Investment Disputies (ICSID) können Verfahren eingeleitet werden, falls es zu Problemen im Investitionsland kommt. Zusätzlich zu diesen Unterorganisationen gibt es noch eine Unzahl von Fonds- und Programmbeteiligungen, an denen die einzelnen Organisationen investieren können.

Die folgende Abbildung 30 bietet einen grafischen Überblick über die verschiedenen Institutionen der Weltbankgruppe, deren Eingliederung sowie deren 
Beteiligungen. Anschließend an die Vorstellung der relevanten Institutionen für die private Finanzierung von Infrastruktur werden wiederum nur die Fonds- und Programmbeteiligungen in Bereich der Privatsektor und Infrastrukturentwicklung beschrieben.

\section{Abbildung 30: Organisationen der Weltbankgruppe}

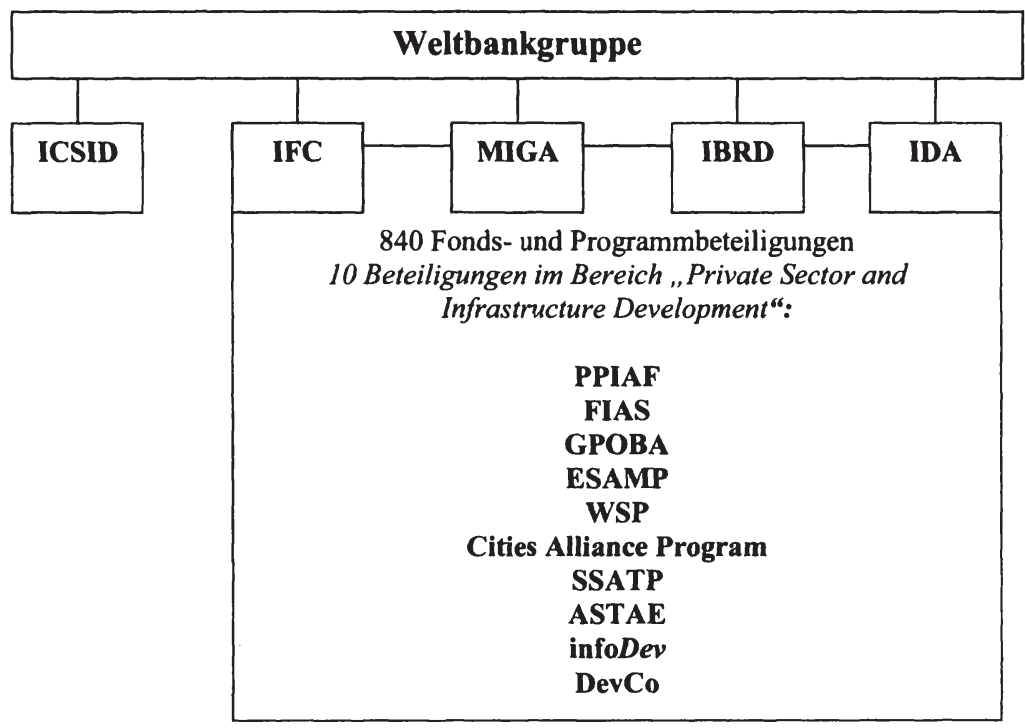

Zusätzlich gibt es multilaterale, regionale Entwicklungsbanken, die jeweils nur auf eine Region spezialisiert sind, wie beispielsweise die Afrikanische, Interamerikanische und Asiatische Entwicklungsbank. Da sich alle Fallstudien (siehe Kapitel 6) in Asien befinden, wird im Zuge der vorliegenden Arbeit nur die Asian Development Bank (ADB) vorgestellt. Die angebotenen Instrumente der Institutionen sind einander jedoch sehr ähnlich.

\subsubsection{IFC}

Im Bereich der Projektfinanzierung ist vor allem die International Finance Corp. (IFC) aktiv. Sie stellt Finanzmittel in Ergänzung zu den privaten Geschäftsbanken zur Verfügung. Außerdem bietet sie technische Unterstützung und Beratungsdienste an. Aufgrund der in den Kreditverträgen vereinbarten Cross-Default-Klauseln sind diese Kredite sehr beliebt. Diese Klauseln legen fest, dass die IFC-Kredite trotz ihres „Preferred creditor status “ gleich wie die kommerziellen Kredite behandelt werden (vgl. Nevitt/Fabozzi 2000: 83). 
Die IFC kann laut Statuten in Projekte investieren,

- die mehrheitlich dem privaten Sektor gehören,

- in Entwicklungsländern liegen,

- die technisch solide sind,

- zur lokalen Entwicklung beitragen und

- welche die IFC Umwelt- und Sozialstandards erfüllen.

Typischerweise stellt die IFC bis zu maximal $25 \%$ der gesamten Projektkosten zur Verfügung und normalerweise beteiligt sie sich mit mindestens 1 Million US\$ (vgl. IFC 2006: 1f).

In Folge werden die einzelnen Instrumente von IFC dargestellt, wobei der Aufbaulogik in Abbildung 31 gefolgt wird. Im Bereich der Infrastrukturfinanzierung stellt die Gewährung von IFC-Krediten die wichtigste Form der Beteiligung dar. IFC investiert aber nicht nur auf Projektbasis, sondern versucht durch die Unterstützung von Investmentfonds, das Geld breiter zu streuen. Hier muss insbesondere auf die Kofinanzierungsfunktion (siehe Kapitel 5.1.3) von IFC hingewiesen, um weitere Finanzmittel anderer Kapitalgeber kooperativ einzusetzen. Eigenkapital wird vor allem für die Entwicklung von Finanzinstitutionen eingesetzt. Zusätzlich zu diesen beiden Hauptinstrumenten hat IFC auch weitere Formen der Beteiligung entwickelt.

Abbildung 31: Instrumente der IFC

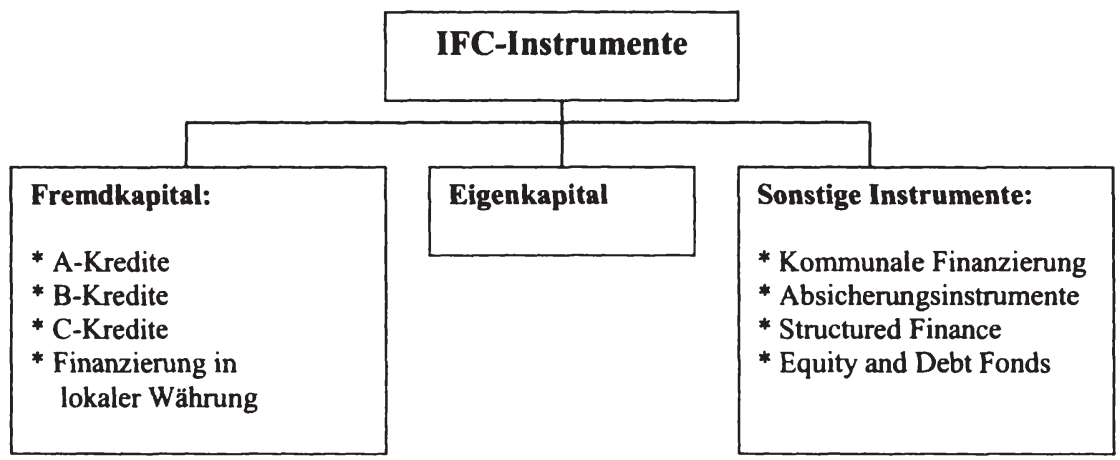

\section{Fremdkapital:}

- A-Kredite: Die IFC gewährt fix- oder variabel verzinste Kredite für den Privatsektor in Entwicklungsländern (A-Kredite). Die Kredite haben typischerweise eine Laufzeit von 7 - 12 Jahren. Wenn die IFC Kredite auf 
kommerzieller Basis vergibt, dann werden nur Projekte mit Gewinnaussicht unterstützt. Dies können Greenfield-Projekte oder aber auch Erweiterungen sein. Die IFC vergibt die Kredite bei einer Projektfinanzierung ohne recourse oder mit limited-recourse auf die Sponsoren.

- B-Kredite: Interessierte Geschäftsbanken werden über B-Kredite an der Finanzierung von Projekten beteiligt. Dies bedeutet, dass die IFC im Außenverhältnis als alleiniger Kreditgeber auftritt und den Geschäftsbanken somit aufgrund ihrer starken politischen Stellung zwar keine juristischen, aber einen faktischen Schutz gegen politische Risiken bietet. Die Syndizierung der Kredite erfolgt nur im Innenverhältnis zwischen der IFC und den Geschäftsbanken (vgl. IFC 2006: 6). Dadurch können außerdem für die privaten Banken Kosten durch Steuereinsparungen reduziert werden (vgl. Osius/Carlson 2004: 20).

- C-Kredite: Die IFC bietet unterschiedliche Quasi-Equity Produkte an, welche Eigenschaften von Eigen- und Fremdkapital aufweisen. Diese Produkte werden C-Kredite genannt und die IFC agiert auf kommerzieller Basis.

- Finanzierung in lokaler Währung: Dem Prinzip folgend, dass die Kosten und die Erlöse in der gleichen Währung entstehen sollen, um das Wechselkurrisiko zu vermeiden, arrangiert die IFC in 23 Ländern Kredite oder Swaps in lokaler Währung (vgl. IFC 2006: 5). Die IFC ermöglicht diese Finanzierung durch vier Konstruktionen:

- Die IFC kann direkt Kredite in lokaler Währung oder

- Währungsswaps zur Verfügung stellen.

- Indirekt kann IFC Instrumente zur Verbesserung der Kreditwürdigkeit gewähren, um in lokaler Währung einen Kredit aufnehmen zu können oder

- durch die Einräumung eines Kreditrahmens bei einer lokalen Finanzinstitution.

\section{Eigenkapital:}

- Beteiligungsfinanzierung: Die IFC kann sich an privatrechtlichen Unternehmen, Finanzinstitutionen oder Investmentfonds mit Eigenkapital beteiligen. Typischerweise wird für eine Laufzeit von $8-15$ Jahren investiert, wobei die IFC nie der größte Teilhaber und höchstens mit $35 \%$ beteiligt sein darf. Die IFC übernimmt keine aktive Rolle im Management des Unternehmens. 


\section{Sonstige Instrumente:}

- Equity and Debt Fonds: Die IFC fördert die Entwicklung von Portfoliofonds, indem sie in viele verschiedene Fonds investieren, um langfristige Finanzierungen in den Entwicklungsländern $\mathrm{zu}$ ermöglichen (vgl. IFC 2006: 6).

- Structured Finance: Im Rahmen der „,Structured Finance“ gewährt die IFC Partial Credit Guarantees (siehe Kapitel 5.2.2.3), die Verbriefung von Krediten und Instrumente zur Teilung der Risiken (vgl. IFC 2006: 7). Die Verbriefung hilft den Kunden Finanzierung am Kapitalmarkt zu erhalten, zu der sie aufgrund des hohen Kreditrisikos keinen Zugang hätten. Die Verbriefung wird typischerweise für Hypotheken oder Vermögenswerte mit gut vorhersehbaren Zahlungsströmen durchgeführt. Die Teilung der Risiken zwischen dem privaten Unternehmen und der IFC wird anhand von Teilgarantien ermöglicht. Der Kunde transferiert die Risiken zur IFC, auch wenn der Vermögensgegenstand in der Bilanz des Kunden bleibt.

- Kommunale Finanzierung: Der „Municipal Fund“ ist eine gemeinsame Initiative zwischen Weltbank und IFC, der Kapital für Gemeinden und lokale Unternehmen zur Verfügung stellt, um die Infrastruktur zu finanzieren (vgl. IFC 2006: 7)

- Absicherungsinstrumente: Die IFC bietet Zugang zu einem langfristigen Derivativmarkt an, z.B. Interest Rate Swaps, um das Wechselkursrisiko, das Zinsrisiko und die Warenpreise abzusichern (vgl. IFC 2006: 7).

\subsubsection{MIGA}

Die Multilateral Investment Guarantee Agency (MIGA) ist jene Tochterorganisation der Weltbank, die Garantien zur Absicherung des Länderrisikos anbietet. Sie bietet Schutz gegen Verluste mittels Garantien (vgl. Homepage MIGA):

- Garantien: Die MIGA bietet in ihrem Garantieprogramm Schutz gegen den Verlust bei folgenden Tatbeständen an: Transferrisiko, Enteignung, Krieg, Unruhen, Terrorismus und Vertragsbruch durch die Gastregierung.

Guarantee: Die Garantien der MIGA werden für private Investoren oder Kreditnehmer angeboten. Die Garantien sollen vor dem politischen Risiko schützen.

Die Beschränkungen der Konvertibilität von Währungen (siehe Kapitel 4.3.4) und die Enteignung durch den Gaststaat kann durch Garantien der MIGA eingeschränkt werden. Schließt der private Investor Verträge mit dem Gaststaat ab, 
so kann er sich mit Hilfe der MIGA zudem gegen Verlust schützen, der durch einen Vertragsbruch des Gaststaates hervorgerufen wurde.

MIGA versichert Investitionen eines ihrer Mitgliedsländer in Entwicklungsländern. Versicherbar sind nicht nur Erstbeteiligungen, sondern auch die Modernisierung, Erweiterung oder Restrukturierung von bereits bestehenden Unternehmungen sowie Unternehmenskäufe im Rahmen der Privatisierung staatlicher Betriebe. Dabei wird nicht nur die Bereitstellung von Eigenkapital oder Gesellschafterdarlehen unterstützt, sondern auch Franchising-, Lizenz- oder Managementverträge sind versicherbar. Es werden nur Projekte unterstützt,

- die finanziell und wirtschaftlich lebensfähig sind sowie

- die Entwicklung des Gastlandes unterstützen und

- umweltverträglich sind. ${ }^{3}$

MIGA sichert explizit vermehrt auch in den Bereichen Private Participation in Infrastructure (PPI) und Infrastruktur ab. Da insbesondere bei Infrastrukturprojekten häufig Verträge mit der lokalen Regierung abgeschlossen werden und die MIGA keine Gegengarantie der Regierungen verlangt, kommt der MIGA in diesem Bereich eine große Bedeutung zu (vgl. Osius/Carlson 2004: 20).

Die MIGA sieht sich nicht als Konkurrenz zu staatlichen oder privaten Versicherungen, sondern als Ergänzung: Sie versucht die Lücken zu schließen, die private oder staatliche Versicherungen nicht ausfüllen. Häufig arbeitet die MIGA dabei auch im Rahmen der Kofinanzierung mit anderen Versicherungen zusammen oder übernimmt die Funktion einer Rückversicherung. Die Höhe der Teilnahme von MIGA ist beschränkt auf 200 Millionen US\$ pro Projekt. Wie im Kapitel 5.1.3 dargestellt, signalisiert sie alleine durch ihre Teilnahme den Projektteilnehmern eine gewisse Rechtssicherheit gegenüber dem Staat (vgl. Osius/Carlson 2004: 20).

\subsubsection{IBRD und IDA}

Die International Bank of Reconstruction and Development (IBRD) und die International Development Agency (IDA) werden weitgehend als Weltbank bezeichnet und bieten unterschiedliche Instrumente zur Finanzierung und Absicherung von Infrastrukturprojekten an. Hauptzielgruppe der beiden Organisationen ist aber der öffentliche Sektor und die Kredite und teilweise auch die Garantien werden nur an den öffentlichen Sektor gewährt. Die IDA konzentriert sich dabei auf die ärmsten Entwicklungsländer, die ein jährliches Pro-KopfEinkommen unter US\$ 896 aufweisen. und die IBRD auf Ent-wicklungsländer mit mittlerem Einkommen. Die IBRD finanziert sich mehrheitlich am Internationalen Finanzmarkt. Die IDA hingegen wird durch die reicheren Mit-

3 http://www.miga.org/sitelevel2/level2.cfin?id=1087, 27.12.2006 
gliedsstaaten finanziert und ist 2005 zum 14. Mal ,aufgefüllt" worden, wobei diese Periode am 30. Juni 2008 enden wird.

Seit 1994 gewährt die Weltbank auch politische Garantien im Infrastruktursektor, um die steigende Nachfrage von privaten Unternehmen und kommerziellen Kreditgebern zu unterstützen. Daher bieten die IBRD und die IDA auf den Infrastruktursektor beschränkt auch für den Privatsektor Garantien an. Alle anderen Produkte, wie beispielsweise zinsgünstige Kredite oder $\mathrm{Zu}$ schüsse, werden nur an den öffentlichen Sektor vergeben.

- Garantien: Die IBRD und die IDA gewähren drei Arten von Garantien, wobei nur die Partial Risk Guarantees (PRGs) dem privaten Investor zur Verfügung stehen. Hingegen können alle drei Garantien bei der Finanzierung von öffentlich-privaten Infrastrukturinvestitionen eine Rolle spielen.

Partial Risk Die Partial Risk Guarantees bieten die IBRD und die Guarantees IDA für private Investoren und Kreditgeber an. Da in (PRGs): der Infrastrukturfinanzierung politische Risiken eine besondere Rolle spielen, soll diese Garantie vor dem Risiko der Nichterfüllung des Vertrages durch die öffentliche Hand schützen. Die wesentlichen Risikokategorien, die durch diese Garantie abgesichert werden, umfassen Vertragsbruch durch die Regierung, Konvertierungs- und Transferbeschränkungen durch Regierungsmaßnahmen, Politische Force MajeureRisiken und Hemmnissen von Schlichtungsverfahren.PRGs garantieren Zahlungen im Falle einer Vertragsverletzung durch die Regierung und schützen die kommerziellen Kreditinstitute vor einem Ausfall der Zins- und Kreditrückzahlungen.

\subsubsection{Fonds- und Programmbeteiligungen der Weltbankgruppe}

Zusätzlich zu den Hauptinstitutionen existieren noch eine Vielzahl an Fazilitäten bzw. Treuhandfonds (, trust fonds"), die von der Weltbank bzw. ihren Teilorganisationen oder von nationalen Institutionen gegründet wurden oder in welche die Organisationen nur investieren. Diese Fonds investieren wiederum in unterschiedlichen Sektoren, die den internationalen Entwicklungszielen und der Erreichung der MDGs dienen.

Laut Weltbank-Bericht (World Bank 2005c: 2) waren dies 2005 rund 840 Beteiligungen, wobei 705 Fonds von der IBRD und IDA, 124 vom IFC und 11 von der MIGA verwaltet wurden. Im Fokus dieser Arbeit liegen vor allem die Bereiche der Infrastrukturfinanzierung und der ,private participation in infra- 
structure finance". Für diese Arbeit sind deshalb vor allem jene Fonds von Bedeutung, die von der Weltbankgruppe (vgl. World Bank 2005c: 57ff) in der Gruppe "Private Sector and Infrastructure Development" zusammengefasst werden. Das Hauptaugenmerk dieser Fonds liegt auf der Verbesserung des Investitionsklimas für private Investoren und einer Stärkung der privaten Investitionen im Bereich der Infrastrukturfinanzierung. Die in der Folge präsentierten zehn Fonds gehöre zu der Gruppe "Private Sector and Infrastructure Development".

\section{Asia Sustainable and Alternative Energy (ASTAE) Program}

ASTAE wurde 1992 gegründet und unterstützt die Weltbankgruppe bei den steigenden Investitionen im Energiesektor in Asien. Das Programm unterstützt außerdem auch Investitionen im Bereich von erneuerbarer Energie und Energieeffizienz (vgl. World Bank 2005c: 62).

\section{Cities Alliance Program}

Cities Alliance wurde 1999 gegründet und unterstützt die Entwicklung der Städte und eine Reduktion der Slums. Aufgrund des rapiden Bevölkerungswachstums und einer Verstädterung wird in diesem Programm verstärkt auf die Infrastrukturentwicklung in den Städten fokussiert (vgl. World Bank 2005c: 61).

\section{Public-Private Infrastructure Advisory Facility (PPIAF)}

PPIAF ist eine Fazilität, die den Entwicklungsländern technische Unterstützung anbietet, um die lokale Infrastruktur durch die Privatwirtschaft zu verbessern. Dieser ,multi-donor" Fonds wurde 1999 eingeführt und wird von 14 Geberinstitutionen bzw. -ländern unterstützt. Diese Fazilität kanalisiert die technische Unterstützung für die Regierungen in den Entwicklungsländern, um private Investitionen im Bereich der Infrastruktur zu bekommen. Außerdem versucht sie Erfahrungen und „best practices“ zu identifizieren und bekannt zu machen (vgl. World Bank 2005c: 57).

\section{Global Partnership on Output-Based Aid (GPOBA)}

GPOBA ist ein "multi-donor" Fonds, der 2003 von der Weltbank und dem britischen Departement for International Development (DFID) gegründet wurde. Das Ziel dieser Fazilität ist die Verbesserung von Infrastrukturdienstleistungen durch eine Ausweitung des ,output-based aid“-Ansatzes. Output-based aid (OBA) beinhaltet die Vergabe von leistungsabhängigen Subventionen, um eine Leistungserstellung durch Dritte zu ermüglichen. Diese Dritten können sowohl private Unternehmen, NGOs oder staatseigene Unternehmen sein, die dann leistungsbezogen für die wirklich gelieferten Dienstleistungen öffentliche Unter- 
stützung erhalten. GPOBA unterstützt sowohl Maßnahmen, die zur Verbreitung des Ansatzes führen, als auch die Subventionen an Dritte (vgl. World Bank 2005c: 58). Dieser Out-put orientierte Ansatz wird im Kapitel 5.3.3 nochmals genauer diskutiert.

\section{Foreign Investment Advisory Service (FIAS)}

FIAS ist eine gemeinsame Einheit der Weltbank und der IFC, welche den Regierungen in den Entwicklungsländern helfen soll, ausländische Direktinvestitionen anzulocken und dabei den Beitrag zur Armutsreduktion zu maximieren. FIAS besteht bereits seit 1985 und hat bereits mehr als 130 Länder bei 600 Projekten beraten (vgl. World Bank 2005c: 58).

\section{Energy Sector Management Assistance Program (ESMAP)}

Diese Fazilität besteht bereits seit 1983 und ist eine globale Partnerschaft für technische Unterstützung. ESAMP berät Regierungen, bietet Zugang zu technischer Unterstützung und unterstützt Pilotprojekte zur Verwendung nachhaltiger Energie zur Armutsreduktion in Entwicklungs- und Transformationsländern (vgl. World Bank 2005c: 59).

\section{Water and Sanitation Program (WSP)}

WSP besteht bereits seit 1979 und wird von 14 Ländern unterstützt. Diese Fazilität fördert die Verbesserung der Wasser- und Sanitärsituation in Entwicklungsländern. Das Hauptaugenmerk liegt dabei auf der Erreichung der MDGs durch Sektorreformen und Strategien, die einen maximalen Beitrag zur Armutsreduktion leisten (vgl. World Bank 2005c: 60).

\section{Sub-Saharan Africa Transport Policy Program (SSATP)}

Diese Fazilität wurde 2004 als internationale Partnerschaft gegründet und versucht die Strategieentwicklung im Transportsektor in Sub-Sahara Afrika zu vereinfachen. Durch die Unterstützung von öffentlichen Strategien, die zu einem verlässlicheren und kostengünstigeren Transportnetz führen, soll ein wesentlicher Beitrag zur Armutsreduktion geleistet werden (vgl. World Bank 2005c: 61).

\section{Information for Development (infoDev) Program}

Dieses Program ist ein ,multi-donor'-Programm, das sich insbesondere mit den Chancen und Herausforderungen von Informationstechnologie für Entwicklung beschäftigt. Es wurde 1995 gegründet und unterstützt seither die Verwendung 
von Informationstechnologie zur Reduktion von Armut (vgl. World Bank 2005c: $63)$.

\section{DevCo}

Diese „mulit-donor“ Fazilität unterstützt die Beratungsarbeit der IFC bei der Privatisierung von Infrastruktur. DevCo konzentriert sich dabei auf Projekte in den ärmsten Entwicklungsländern, wobei sie zusätzlich auch noch die Beratungskosten für die Vorbereitung von Infrastrukturprojekten trägt. ${ }^{4}$

Diese zehn Fonds unterstützen sehr unterschiedliche Aspekte der Infrastrukturentwicklung, wobei hierbei folgende Schwerpunkte zu erkennen sind:

1. Die Unterstützung von privaten Investoren in der Infrastruktur (PPIAF, FIAS, DevCo und GPOBA),

2. Fokus auf einzelne Infrastruktursektoren (ESAMP, SSATP, ASTAE, infoDev) und

3. Fokus auf Regionen (SSATP, ASTAE).

Die wenigsten Fonds investieren direkt in einzelne private Infrastrukturinvestitionen, sondern versuchen bessere Rahmenbedingungen dafür zu schaffen. Jene Fonds, die sich auf einzelne Sektoren konzentrieren, versuchen insbesondere im öffentlichen Bereich durch Sektorreformen die Infrastrukturversorgung $\mathrm{zu}$ verbessern.

\subsubsection{Asian Development Bank (ADB)}

Die regionalen Entwicklungsbanken verfolgen das Prinzip der Subsidiarität und sind daher in jene Projekte involviert, in denen eine eigenständige Finanzierung für Geschäftsbanken wegen der politischen oder wirtschaftlichen Projektrisiken nicht realisierbar ist. In diesen Fällen entwickelt die Entwicklungsbank Finanzierungsstrukturen, bei denen sie nicht nur eigene Kredite, Eigenkapital oder Garantien zur Verfügung stellt, sondern auch Gemeinschaftsfinanzierungen mit anderen multilateralen Organisationen (wie etwa der IFC), mit Exportkreditversicherungen sowie mit Geschäftsbanken arrangiert. Insbesondere durch die geografische Nähe und die Expertise über diese Länder unterscheidet sie sich von den zentralen Institutionen.

Da sich die in dieser Arbeit dargestellten Fallbeispiele alle in Asien befinden, werden die Finanzierungs- und Absicherungsinstrumente der ADB beispielhaft für die anderen Entwicklungsbanken genauer dargestellt. Da in fast allen Fallstudien die ADB an der Finanzierung und Absicherung beteiligt ist, werden die

4 http://www.ifc.org/ifcext/psa.nsf/content/Devco, 26.12.2006 
Instrumente an dieser Stelle etwas genauer und, wenn sinnvoll, grafisch dargestellt.

Die ADB konzentriert sich ausschließlich auf die Unterstützung von Infrastrukturprojekten. Projekte im Infrastruktursektor werden bei mehrheitlich privater Beteiligung gefördert, die eine effiziente Privatisierung der Einrichtungen fördern. Insgesamt beteiligt sich die ADB mit maximal $25 \%$ der Gesamtkosten, unabhängig davon, welche Instrumente zum Zuge kommen. Bei gemeinsamen Projekten von öffentlicher und privater Seite muss der private Partner mehrheitlich am Projekt beteiligt sein. Sollte die öffentliche Hand mehrheitlich am Projekt beteiligt sein, wird die ADB Kapital investieren, wenn die Umstände eine solche Intervention rechtfertigen (vgl. ADB 2006a: 7). Die ADB hilft auch bei der Ausarbeitung und der Überprüfung der Realisierbarkeit des Projektes (vgl. ADB 2006a: 10). In Abbildung 32 wird ein Überblick über die Instrumente der Abteilung „Private Sector Development and Finance“ der ADB gegeben. Im Rahmen der Kofinanzierung (siehe Kapitel 5.1.3) bietet die ADB das "Complementary Financing Scheme" und zwei Garantien an, bei denen sie auch als "Guarantor of record" fungieren kann. Voraussetzung für die Vergabe dieser Instrumente in einem Projekt stellt eine Beteiligung der ADB durch einen direkten Kredit dar.

\section{Abbildung 32: Instrumente der $A D B$}

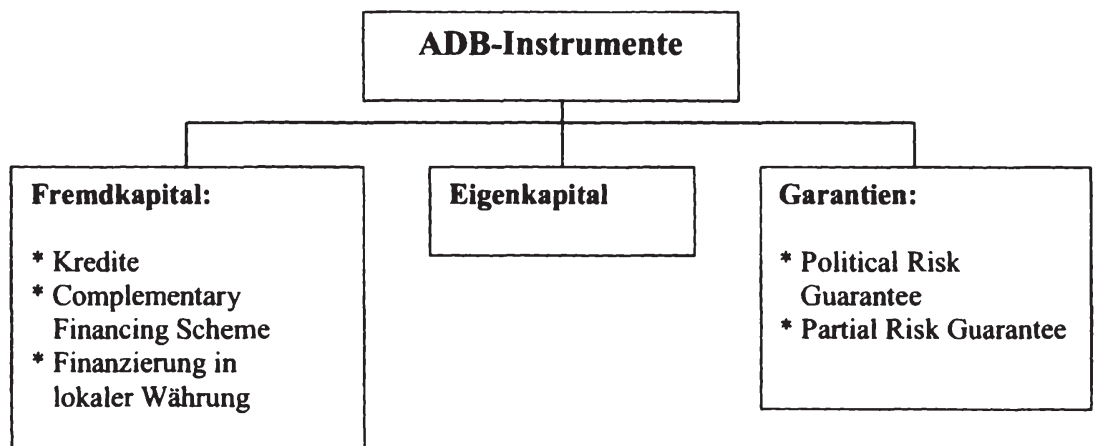

\section{Fremdkapital}

- Kredite: Die Kredite werden an die Laufzeit der Projekte angepasst, allerdings mit einer maximalen Laufzeit von 15 Jahren. Auch die Zinsen werden auf Basis der involvierten Risiken und Ansprüche bestimmt. Zusätzlich dazu werden auch Gebühren (Vorabgebühr, Bereitstellungsprovision und eventuell eine Bewertungsprovision) verrechnet (vgl. ADB 2006a: 10ff). Die Vergabe von direkten Krediten an ein Projekt stellt die Voraussetzung für die Vergabe von Garantien und dem „Complementary Financing Scheme" dar. 
- Complementary Financing Scheme (CFS): Die ADB bietet das "Complementary Financing Scheme" an (ähnlich dem IFC-B-Darlehen), bei dem die ADB als "lender of record" auftritt, aber kommerzielle Kreditgeber das Kapital zur Verfügung stellen. ${ }^{5}$ CFS ist nur für jene privaten Infrastrukturprojekte möglich, in denen die ADB bereits ein direkter Partner ist. Durch die Rolle der ADB als ,,lender of record" entstehen aber keine Rückgriffsrechte der kommerziellen Banken auf die ADB für etwaige Probleme beim Zinsendienst. Dennoch genießen die kommerziellen Banken durch die Beteiligung der ADB im CFS einige Privilegien aufgrund des ,preferred creditor status " der ADB (vgl. ADB 2006a: 15f). Wegen dieses Statuses werden die Forderungen bei Konvertierungsproblemen gegenüber kommerziellen Forderungen vorrangig bedient (siehe dazu Kapitel 5.1.3). Die folgende Abbildung 33 stellt grafisch den Ablauf des CFS dar.

\section{Abbildung 33: Ablauf des Complementary Financing Schemes}

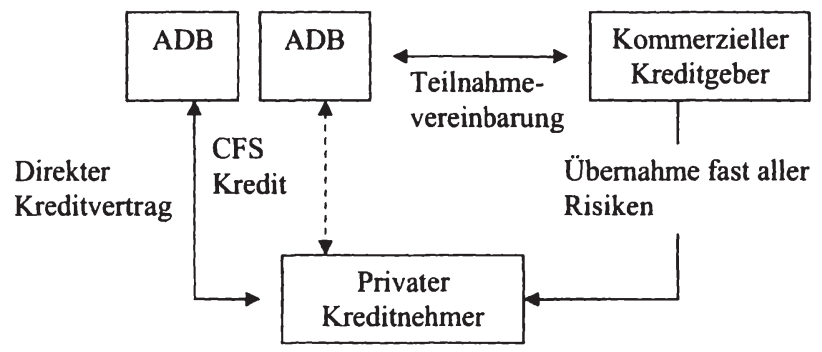

Quelle: in Anlehnung an http://www.adb.org/Cofinancing/com financing.asp, 3.10.2007

- Finanzierung in lokaler Währung: Das Wechselkursrisiko spielt insbesondere bei Infrastrukturfinanzierungen eine besonders große Rolle für alle Beteiligte. Daher bietet die ADB Finanzierungen in der lokalen Währung an, um die Kosten und die Erlöse abzustimmen. Bis jetzt gibt es diese Möglichkeit auf den Philippinen, in Indien und in der Volksrepublik China (vgl. ADB 2006a: 3f). Die Finanzierung kann durch die Vergabe von Krediten oder Cross-Currency Swaps erfolgen (vgl. Sagar 2006: 6). Eine weiterführende Diskussion dazu findet sich in Kapitel 5.3 statt.

\section{Eigenkapital}

- Eigenkapital: Kapitalinvestitionen werden von der ADB bis zu einer Höhe von $25 \%$ der Gesamtkosten getätigt. Es wird Eigenkapital sowohl direkt als auch indirekt über Fonds in private Projekte investiert. Die

5 http://www.adb.org/Cofinancing/com financing.asp, 2.10 .2007 
ADB übernimmt bei beiden Möglichkeiten keine Managementfunktionen. Eigenkapital wird nur in den seltensten Fällen für klassische Infrastrukturinvestitionen zur Verfügung gestellt. Das Eigenkapital wird vorrangig in Finanzinstitutionen investiert und dient der Unterstützung der Kapitalmarktentwicklung.

\section{Garantien}

- Garantien: Durch Garantien können durch ihren Schutzumfang die Kreditwürdigkeit (,, credit enhancement") der privaten Investoren verbessern, da insbesondere bei Infrastrukturprojekten der private Investor die Risiken nicht alleine tragen kann. Dadurch erhalten sie eine vorteilhaftere Finanzierung von den kommerziellen Banken und können die Projektkosten senken. Garantien werden nur für jene Projekte vergeben, an denen die ADB bereits mit einem direkten Kredit oder Eigenkapital beteiligt ist. Die Vergabe von Garantien spielt auch eine wesentliche Rolle bei der Kofinanzierung (siehe Kapitel 5.1.3). Außerdem kann die ADB auch als "Guarantor of record" bei der Vergabe von Garantien fungieren. Dabei vergibt die ADB eine Garantie, behält aber nur einen Teil der Garantie für sich und syndiziert das verbleibende Risiko an private Exportkreditversicherungsunternehmen. Die ADB bietet folgende zwei Garantiearten an (vgl. ADB 2006a: 16f):

\section{Abbildung 34: Ablauf einer Political Risk Guarantee}

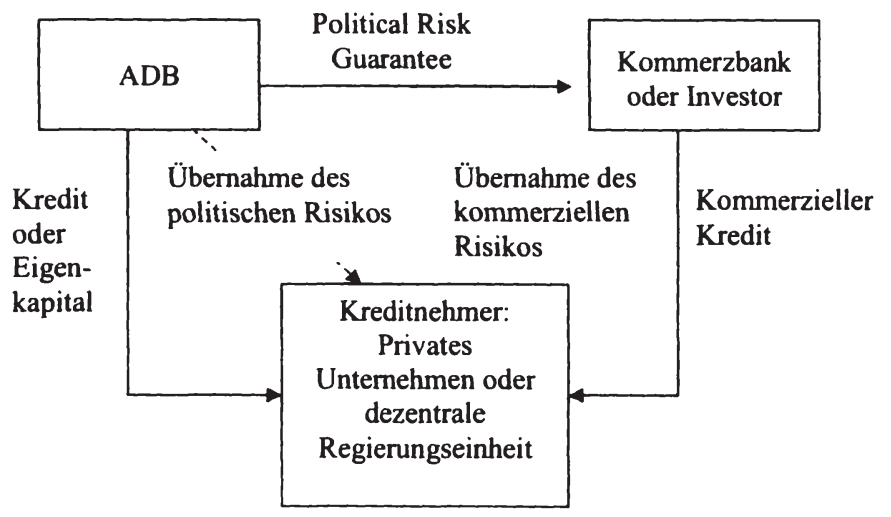

Political Die PRG schützt vor politischen Risiken und lässt die Risk kommerziellen Risiken beim privaten Investor. Es Guarantee werden bis zu max. 150 Mio. US\$ oder $50 \%$ der 
(PRG): gesamten Projektkosten abgesichert. Durch die Absicherung der politischen Risiken sollen die privaten Investoren einfacher Mitfinanzierer finden (vgl. ADB 2000: 1).

In Abbildung 34 wird der Ablauf einer Political Risk Guarantee (PRG) grafisch dargestellt. Dabei vergibt die ADB eine PRG an die Kommerzbank oder den Investor, um ihn gegen politische Risiken zu schützen.

Partial Diese Partial Credit Guarantees schützen sowohl vor Credit kommerziellen als auch vor politischen Risiken.

Guarantees Diese Garantien werden insbesondere für Projekte mit (PCGs): langer Laufzeit vergeben und umfassen einen definierten Teil des kommerziellen Risikos. PCGs schützen daher vor allen Vorkommnissen, die eine Nichterfüllung der versicherten Verpflichtung hervorrufen würden. Es werden bis zu 75 Mio. US\$ oder $25 \%$ der gesamten Projektkosten abgesichert. Im Allgemeinen versichert diese Garantie Kredite inkl. Zinsen für Laufzeiten, die ohne eine Garantie von kommerziellen Banken nicht angeboten werden würden. Diese Garantien wurden entwickelt, um sowohl Kredite in US\$ als auch in lokaler Währung zu ermöglichen.

\section{Abbildung 35: Ablauf einer Partial Risk Guarantee}

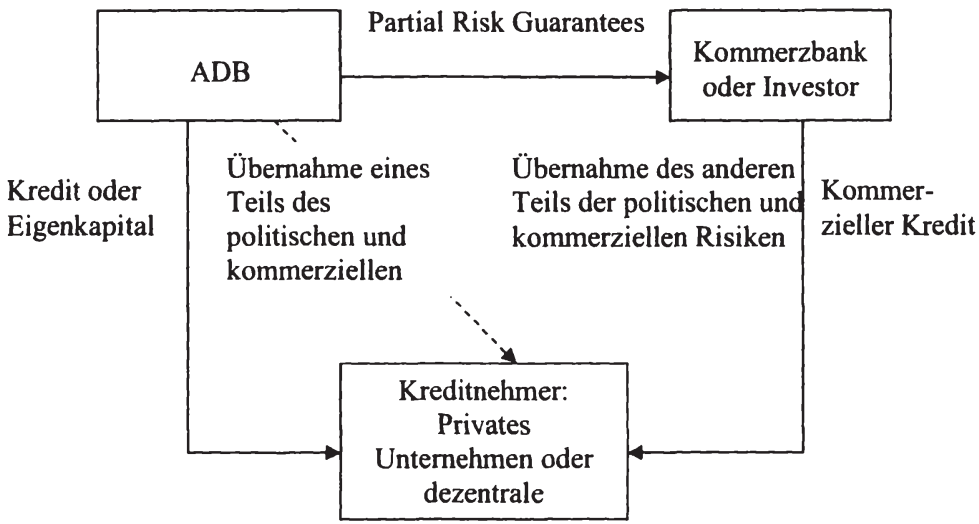


Die Partial Credit Guarantee (PCG) wird in Abbildung 35 dargestellt. Bei dieser Garantie übernimmt die ADB einen Teil der politischen und kommerziellen Risiken und überlässt den anderen Teil dieser Risiken dem Kreditgeber oder Investor. Allerdings versichert die ADB nur bis zu $25 \%$ der gesamten Projektkosten, bei der einer Political Risk Guarantee hingegen bis zu $50 \%$.

\subsubsection{Europäische Institutionen}

Auf europäischer Ebene gibt es von Seiten der EU Unterstützung für Infrastrukturprojekte in Entwicklungsländern. Einerseits gibt es die Europäische Investitionsbank (EIB) mit dem Europäischen Entwicklungsfonds (EEF) und andererseits die Europäische Kommission.

\subsubsection{Europäische Investitionsbank (EIB)}

Die primäre Aufgabe der Europäischen Investitionsbank $(E I B)$ ist es, jene Projekte durch langfristige Kredite zu fördern, die den Zielen der EU entsprechen und $\mathrm{zu}$ einer ausgeglichen Entwicklung in den Mitgliedsstaaten beitragen sollen. Zusätzlich zu diesem Europafokus werden aber auch Länder in Asien, Lateinamerika und Afrika unterstützt, die eine Kooperationsvereinbarung mit der EU unterzeichnet haben. Es werden u.a. existenzfähige (,viable ") öffentliche und private Projekte in der Infrastruktur finanziert, wobei der Schwerpunkt im Umweltschutz liegt.

Als Kreditnehmer kommen sowohl öffentliche und private Körperschaften, als auch private Unternehmen in Frage. Es werden ausschließlich Kredite von der EIB vergeben, die sich wiederum aufgrund ihres eigenen AAA-Ratings günstig am Kapitalmarkt refinanzieren können. Diese günstigen Konditionen werden an die Kreditnehmer weitergegeben, indem sie Kredite zum „Selbstkostenpreis" vergibt (vgl. Weber et al. 2006: 251).

- Einzelkredite: Diese Kredite werden für jene Projekte vergeben, die ein Investitionsvolumen von 25 Millionen $€$ übersteigen. Im Infrastrukturbereich werden sie für eine Dauer von 20 Jahren gewährt.

- Globalkredite: Wenn Projekte ein geringeres Investitionsvolumen als $€$ 25 Millionen aufweisen, können lokale Körperschaften für Infrastrukturprojekte einen Globalkredit beantragen. Es werden max. $50 \%$ der Investitionskosten bis zu einer Höhe von max. 12,5 Millionen $€$ gewährt.

- Structure Finance Facility: Die EIB hat die „Structure Finance Facility“ (SFF) gegründet, durch die verschiedene Finanzierungsprodukte angeboten werden, wie beispielsweise Senior und Nachrangige Darlehen, Mezzaninekapital sowie projektbezogene Derivate. Diese Instrumente sollen zwar hauptsächlich in den EU-Mitgliedsländern zur Anwendung 
kommen, aber es wird darauf hingewiesen, dass sie auch für NichtMitgliedsländer möglich sind. ${ }^{6}$

Der Europäische Entwicklungsfonds (EEF) stellt das wichtigste Hilfsinstrument der Europäischen Union für die Zusammenarbeit mit den AKPStaaten und den überseeischen Ländern und Gebieten dar. Der EEF vergibt Kredite an den Privatsektor, Risikokapital und nicht-zurückzahlbare Hilfe, wobei die EIB die Kredite und das Risikokapital verwaltet. ${ }^{7}$

\subsubsection{Europäische Kommission}

Die Europäische Kommission gewährt finanzielle Hilfe für Projekte und Maßnahmen, die mit der Politik der Europäischen Union zusammenhängen. Die Empfänger dieser Finanzhilfen sind vorwiegend private und öffentliche Einrichtungen, in Ausnahmefällen auch Privatpersonen, die von der Kommission ausgewählt werden.

Die Finanzhilfen stellen eine komplementäre Finanzierung dar. Das bedeutet, dass die EU in der Regel nicht die Gesamtkosten eines Projektes übernimmt. Die Möglichkeit einer Vollfinanzierung besteht ausschließlich für Projekte, die außerhalb der EU durchgeführt werden. Sie dienen der Kostendeckung, also dürfen dadurch keine Gewinne erzielt werden. Sie dürfen außerdem nicht rückwirkend und nur ein Mal pro Projekt gewährt werden.

Teil der Europäischen Kommission ist Europe Aid, das Programme für Infrastrukturmaßnahmen in den ACP-Ländern ("African, Carribean, Pacific“) und in Asien (,ASEAN") anbietet:

- ACP-EU Water Facility: Durch diese Fazilität werden Investitionen im Bereich der Wasser- und Abwasserinfrastruktur durch Darlehen oder Garantien gefördert. ${ }^{8}$ Die EU stellt insgesamt 500 Millionen $€$ für die Unterstützung afrikanischer Initiativen sowie für Projekte in den AKPLändern im Bereich Wasser und Siedlungshygiene bereit. ${ }^{9}$

- ACP-EU Energy Facility: Mit dieser Fazilität werden Infrastrukturinvestitionen im Energiesektor durch Darlehen gefördert. Zusätzlich zur „Energy Facility“ wurden speziell für die afrikanischen Länder im Rahmen der "EU-Africa Partnership on Infrastructure“ 10 Millionen $€$ Unterstützung 2006 zur Verfügung gestellt. ${ }^{10}$

\footnotetext{
6 http://www.eib.org/projects/dynamic. asp?cat $=209,19.12 .2006$

7 http://europa.eu/scadaplus/leg/de/lvb/r12102.htm, 28.12.2006

8 http://ec.europa.eu/europeaid/projects/water/index en.htm, 19.12.2006

9 http://www.ada.gv.at/view.php3?f id =1786\&LNG=de\&version $=, 26.12 .2006$

10 http://ec.europa.eu/europeaid/projects/energy/index en.htm, 19.12.2006
} 
- EC-Asean Energy Facility: Diese Fazilität stellt eine Kooperation zwischen der Europäischen Gemeinschaft (EG) und der „Association of Southeast Asian Nations (ASEAN) dar, um Partnerschaften im Energiesektor zu vereinfachen und die regionale Nachfrage nach Energieprojekten anzuregen. Es werden Beihilfen zu unterschiedlichen Maßnahmen, wie beispielsweise Erstellung einer Feasibilty Studie, projektabhängig von bis zu 50 \% gewährt. ${ }^{11}$

- EC-Asean Cogen: Dieses Programm unterstützt die Identifizierung und Demonstration von adäquaten Kraft-Wärme-Koppelung-Technologien in Europa, die natürlich erneuerbare Ressourcen verwenden. Es wird sowohl Unterstützung bei der Projektentwicklung als auch bei Finanzierung gewährt. $^{12}$

- Politische Garantien: Es werden Garantien für EIB-Kredite gewährt, die das politische Risiko außerhalb der EU absichern (vgl. EC 2004: 24). Zu den politischen Risiken zählen der Vertragsbruch durch die Regierung, Konvertierungs- und Transferbeschränkungen durch Regierungsmaßnahmen, Politische Force Majeure-Risiken und Hemmnisse bei Schlichtungsverfahren.

\subsection{4 Österreichische Beteiligungen an Internationalen Finanz- institutionen}

Die Republik Österreich beteiligt sich an wesentlichen internationalen und regionalen Finanzinstitutionen, wie aus der nachfolgenden Abbildung $36 \mathrm{zu}$ entnehmen ist. Sie wird in den Institutionen durch den Bundesminister für Finanzen in den Gouverneursräten vertreten. Die Abbildung gibt außerdem einen guten Überblick über die Einordnung der einzelnen Institutionen.

\subsection{5 Österreichische Institutionen}

Auf nationaler Ebene werden private Investitionen in Entwicklungsländern sowohl in der Risikokapitalaufbringung und -absicherung, als auch im Rahmen der österreichischen Entwicklungszusammenarbeit (OEZA) unterstützt. Nationale Institutionen unterstützen typischerweise Projekte, an denen nationale Unternehmen beteiligt sind.

2004 wurde in Österreich die Austrian Development Agency (ADA) gegründet, die im Auftrag des Außenministeriums in der Entwicklungs-

11 http://www.aseanenergy.org/eaef/index.php?view=4 facilities 5 subsectors, 19.12.2006

12 http://www.cogen3.net/, 19.12.2006 
zusammenarbeit tätig ist. Im Bereich der Förderung von ausländischen Direktinvestitionen in Entwicklungsländern bieten auch das Österreichische Wirtschaftsservice (AWS) und die Oesterreichische Kontrollbank (OeKB) finanzielle Unterstützung an.

Abbildung 36: Österreichische Beteiligungen an Internationalen Finanzinstitutionen

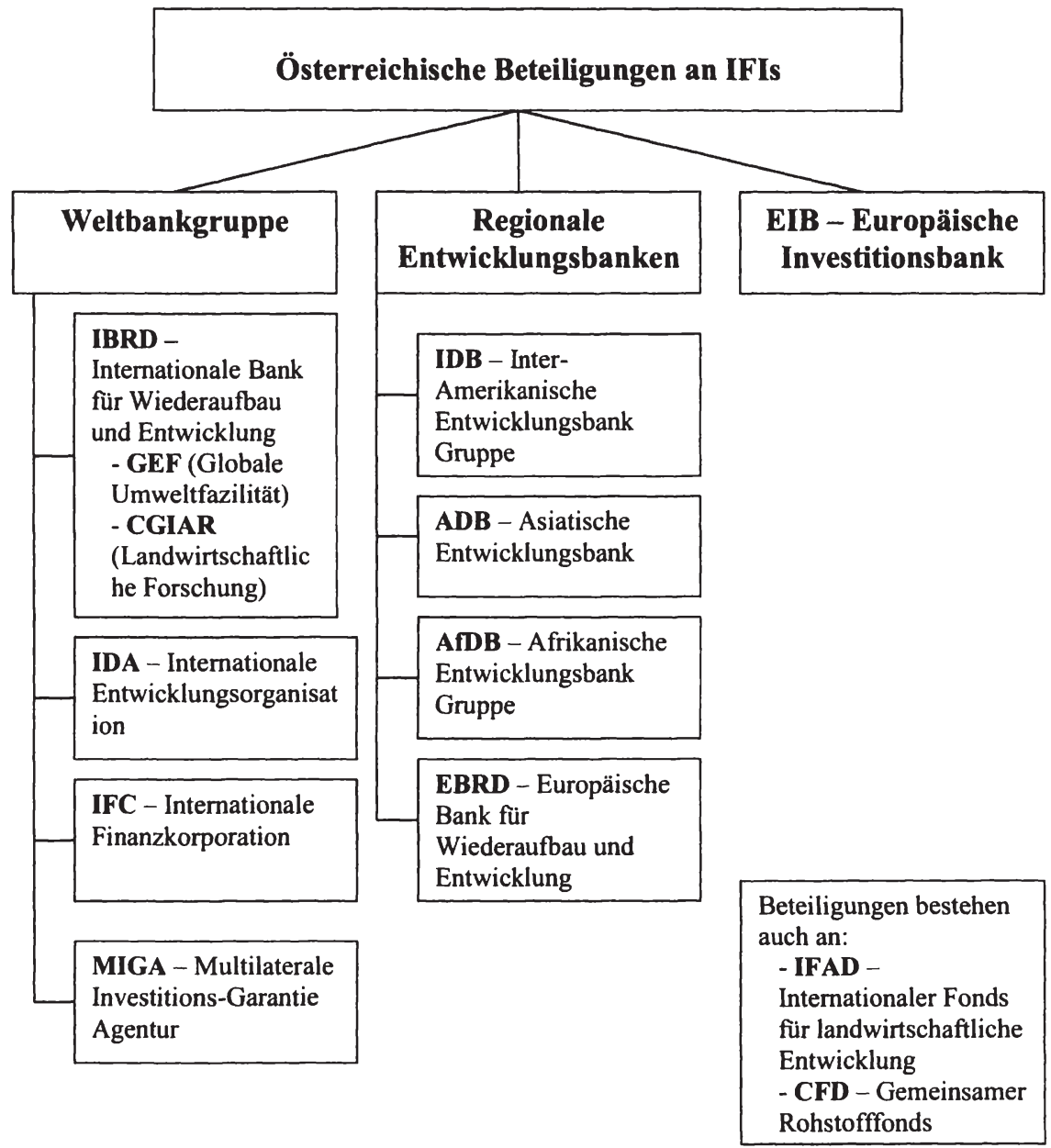

Quelle: laut Information des Bundesministeriums für Finanzen, 28.11.2006

\subsubsection{Austrian Development Agency (ADA)}

Die Austrian Development Agency (ADA) ist eine $\mathrm{GmbH}$, deren alleiniger Gründer der Bund ist; als Eigentümervertreter gegenüber der ADA fungiert das 
Außenministerium. Die Arbeitsgrundlage der ADA ist das Dreijahresprogramm der Österreichischen Entwicklungspolitik, das vom Außenministerium erstellt wird und die zentralen entwicklungspolitischen Positionen und strategischen Rahmenbedingungen der Österreichischen Entwicklungs- und Ostzusammenarbeit definiert. ${ }^{13}$ Die Gesellschaft nahm mit 1.1.2004 ihre Tätigkeit auf und ist für den operativen Teil der Entwicklungszusammenarbeit zuständig. ${ }^{14}$

Die Ziele und Prinzipien der Österreichischen Entwicklungszusammenarbeit (OEZA) sind im Entwicklungszusammenarbeitsgesetz (EZA-Gesetz) festgeschrieben, welches 2002 beschlossen und 2003 novelliert wurde. Das EZAGesetz bildet die Grundlage für eine einheitliche österreichische Entwicklungspolitik und enthält einen konkreten Zielkatalog, der für die gesamte Bundesverwaltung entwicklungspolitische Kriterien vorschreibt. ${ }^{15}$ Die thematischen Schwerpunkte der OEZA im Bereich der materiellen Infrastruktur sind die Wasser- und Siedlungshygiene und die Energie. ${ }^{16}$ Konkret unterstützt die ADA private oder öffentlich-private Projekte durch die Gewährung von $\mathbf{Z u}$ schüssen für die Erstellung von Machbarkeitsstudien und Know-How.

Seit Juli 2006 ist die ADA Mitglied des PIDG-Fonds, welche eine „,...multidonor, member-managed organisation..." ist, deren Ziel die finanzielle, praktische und strategische Unterstützung von privaten Infrastrukturinvestitionen in den Entwicklungsländern darstellt. ${ }^{17}$ Die Mitglieder ${ }^{18}$ können $^{-}$ sich wiederum wahlweise an folgenden Fazilitäten des PIDG-Fonds beteiligen:

- Emerging Africa Infrastructure Fund (EAIF): Dieser Fonds ist ein Rentenfonds, der insgesamt 305 Millionen US\$ für die Finanzierung von privaten Infrastrukturinvestitionen in Subsahara Afrika zur Verfügung stellt. ${ }^{19}$

- GuarantCo: Diese Fazilität unterstützt private Infrastrukturinvestitionen, deren Erlöse teilweise oder gänzlich in der Währung des jeweiligen Gastlandes anfallen und somit dem Wechselkursrisiko und dem KT-ZMRisiken ausgesetzt sind. ${ }^{20}$

13 http://www.ada.gv.at/view.php3?f id =8102\&LNG =de\&version=, 26.12.2006

14 http://www.bmaa.gv.at/view.php3?f id =82648\&LNG=de\&version=, 26.12.2006

15 http://www.bmaa.gv.at/view.php3?f id=82648\&LNG=de\&version $=, 26.12 .2006$

16 http://www.ada.gv.at/view.php3?r id $=100 \& L N G=$ de\&version $=, 26.12 .2006$

17 http://www.pidg.org, 26.12.2006

18 Die Mitglieder des PIDG-Fonds sind: UK Department for International Development (DFID), the Swiss State Secretariat for Economic Affairs (SECO), the Netherlands Ministry of Foreign Affairs (DGIS), the Swedish International Development Cooperation Agency (SIDA), the World Bank.

19 http://www.emergingafricafund.com, 26.12.2006

20 http://www.guarantco.com, 26.12.2006 
- InfraCo: Diese Einrichtung ist ein Projektentwicklungsunternehmen, welches privat finanzierte Infrastrukturprojekte in Afrika und Asien entwickelt und anschließend an private Unternehmen verkauft. ${ }^{21}$

- Technical Assistance Fund (TAF): Das Hauptziel dieses Fonds ist die Unterstützung von privat finanzierten Infrastrukturprojekten bei der Evaluierung, Strukturierung und Entwicklung der Projektrisiken und bei der Kapitalbeschaffung. TAF unterstützt wiederum Projekte der EAIF, GuarantCo und InfraCo. ${ }^{22}$

Tochtergesellschaften des PIDG-Fonds sind

- DevCo und

- GPOBA.

Beide Fazilitäten wurden bereits in Kapitel 5.2.2.4 kurz vorgestellt. Sie stellen wiederum ein Bindeglied zur Weltbankgruppe dar, da diese auch am PIDGFonds beteiligt ist.

\subsubsection{Austria Wirtschafisservice (AWS)}

Langfristig fixierte Kredite zu vorteilhaften Konditionen können für ausgewählte Projekte sowohl von nationalen als auch von internationalen Entwicklungsbanken gewährt werden. Als Förderbank des Bundes ist die AWS die zentrale Abwicklungsstelle für die unternehmensbezogene Wirtschaftsförderung. ${ }^{23}$ Die AWS ist spezialisiert auf die Förderung von kleinen und mittleren Betrieben und bietet folgende Produkte für ausländische Investitionen in der Infrastruktur an. ${ }^{24}$

- Kredite: Die zinsgünstigen erp-Kredite ${ }^{25}$ mit langer Laufzeit werden von der AWS vergeben. Die erp-Kredite sind fokussiert auf Direktinvestitionen in Mittel- und Osteuropa, aber auch in China und Indien. ${ }^{26}$

- Garantien: Die AWS vergibt Garantien für Direktinvestitionen im Rahmen des Ost-West-Fonds für Beteiligungen und Internationalisierungen. Im Auslandsengagement übernimmt die AWS die Absicherung des Projekt- und Finanzierungsrisikos (d.h. des wirtschaftlichen Risikos). Durch die Übernahme von Haftungen werden fehlende dingliche Sicherheiten umgewandelt und ermöglichen so günstigere Finanzierungs-

21 http://www.infracolimited.com, 26.12.2006

22 http://www.pidg.org, 26.12.2006

23 http://www.awsg.at, 29.12.2006

24 http://www.awsg.at/portal/index.php? $x=199 \& n=197,29.12 .2006$

25 Die Mittel dafür stammen aus dem Vermögen des erp-Fonds.

26 Albanien, Bosnien-Herzegowina, Kroatien, Mazedonien, Montenegro, Serbien, Russische Förderation, Ukraine, Türkei http://www.awsg.at/portal/index.php?n=373, 20.10.2007 
konditionen bei den privaten Banken. Darüber hinaus sichert die AWS private Investitionen in viel versprechende Unternehmen mit der Einzelkapitalgarantie ab.

- Zuschüsse: Die AWS vergibt Zuschüsse an Leitprojekte in regionalen Fördergebieten sowie zu verschiedenen Maßnahmen, wie beispielsweise Machbarkeitsstudien oder Marktrecherchen.

\subsubsection{Oesterreichische Kontrollbank (OeKB)}

Staatliche Exportkreditversicherungen existieren ausdrücklich dafür, die heimischen Exporte im Ausland zu fördern (vgl. Osius/Carlson 2004: 23). In Österreich ist dies die Oesterreichische Kontrollbank (OeKB). Sie ist eine Spezialbank für die österreichische Exportwirtschaft und ist Bevollmächtigte der Republik im Rahmen des Exportfinanzierungs- und -garantiesystems. Die OeKB kann aufgrund ihres Status günstige Finanzierungen für einen langen Zeitraum zur Verfügung stellen. Aufgrund ihres guten Ratings als Bevollmächtigte der Republik Österreich, kann sie sich auf dem Finanzmarkt vorteilhaft refinanzieren. Die OeKB bietet im Rahmen des Exportförderungsverfahrens (EFV) folgende Instrumente für die Finanzierung von Infrastrukturinvestitonen an (vgl. OeKB 2004: 2ff):

- Kredite: Die OeKB bietet im Rahmen eines Refinanzierungsverfahrens die Möglichkeit der Vergabe eines Kredites zur Finanzierung von Beteiligungen im Ausland an. Die Kreditlaufzeit kann bis zu 25 Jahre betragen und es können Zusagen für Einzelgeschäfte oder Kreditrahmen erteilt werden. Die Vergabe eines Kredites ist gebunden an die gleichzeitige Existenz einer G4, einer Wechselbürgschaft nach dem AFG, der AWS, einer internationalen Organisation gemäß AFFG oder durch einen Kreditversicherer (vgl. OeKB 2004: 5).

- Garantien: Die Oesterreichische Kontrollbank (OeKB) vergibt im Auftrag des Bundes im Rahmen des AFG eine Garantie (G4), die das politische Risiko bei Auslandsengagements absichert. ${ }^{27}$ Diese Garantie schützt den Garantienehmer vor Zerstörung von Vermögensteilen im Fall eines Krieges oder einer Revolution, Beschränkung der freien Verfügbarkeit oder des Transfers von Dividenden, Beteiligungserträgen, Kapitalund Zinstilgungen aus einem beteiligungsähnlichen Darlehen und Erlösen aus dem Verkauf von Beteiligungsrechten.

Eine beliebte Finanzierungsmöglichkeit von Infrastruktur durch die öffentliche Hand stellen sogennante Soft Loans dar. Diese Rahmen II-Kredite sind jedoch keine Kredite, die für Projektfinanzierungen verwendet werden können. Da

27 http://www.oekb.at/control/index.html?id=69241, 29.12.2006 
diese Kredite aber oft im Zusammenhang mit Infrastrukturfinanzierung genannt werden, werden sie an dieser Stelle kurz angeführt.

- Soft Loans (Rahmen II-Kredite): Soft Loans werden ausländischen Regierungen für Infrastrukturprojekte gewährt, die nicht wirtschaftlich lebensfähig sind. Die Kredite müssen einen Schenkungsanteil von $35 \%$ aufweisen. Dieser wird in Österreich durch unter dem Marktzinssatz liegende Zinsen, tilgungsfreie Zeitspannen und lange Laufzeiten charakterisiert. Soft Loans können nur für Entwicklungsländer mit einem BNP-Einkommen unter 3.465 US\$ pro Kopf gewährt werden. Bei diesen Krediten handelt es sich um Lieferkredite, die im Rahmen einer Projektfinanzierung aber nicht in Frage kommen.

\subsubsection{Zusammenfassung der Instrumente für PPI}

In der folgenden Tabelle 5 werden die Instrumente der nationalen und internationalen Finanzinstitutionen nochmals zusammengefasst. Der Fokus dieser Ausarbeitung liegt auf der Unterstützung des privaten Sektors und daher werden keine Zuschüsse und Kredite mit Schenkungsanteil an den öffentlichen Sektor angeführt. Diese Instrumente können natürlich auch unterschiedlich positive Auswirkungen auf die privaten Unternehmen haben.

Wie aus Tabelle 5 ersichtlich, gleicht sich das Angebot an Instrumenten von den IFIs stark. Einschränkungen bei der Unterstützung basieren oftmals auf den Statuten der jeweiligen Organisationen und regionalen Beschränkungen. So unterstützt die Weltbankgruppe Projekte weltweit, die ADB und die europäischen Institutionen weisen hingegen einen regionalen Fokus auf.

Aufgrund ihrer Statuten kann die Weltbank keine bzw. nur begrenzt direkte Unterstützung an private Unternehmen vergeben. Die Tochterorganisation IFC kann wiederum den öffentlichen Sektor nicht unterstützen. Innerhalb der ADB gibt es wiederum eine strikte Trennung zwischen dem ,Public Sector" und dem „Private Sector“. Der Public Sector kann nur Kredite an Regierungen vergeben. Der Private Sector bietet auf kommerzieller Basis Kredite und Absicherung für private Unternehmen an. Ein wesentlicher Unterschied zwischen der ADB und der IFC ist die Einschränkung der ADB auf die Infrastruktur und der starke Fokus der IFC auf ADI im Produktionsbereich.

Im Rahmen der Fallstudien in Kapitel 6 werden einige der vorgestellten Instrumente angewendet und in diesem Zusammenhang nochmals dargestellt. 
Tabelle 5: Übersicht über ausgewählte Finanzierungspartner für private Projekte in Entwicklungsländern

\begin{tabular}{|c|c|c|c|c|c|c|c|c|c|c|}
\hline $\begin{array}{l}\text { Finanzierungs- bzw. } \\
\text { Absicherungsinstrumente }\end{array}$ & IFC & MIGA & $\begin{array}{l}\text { IBRD } \\
\text { und } \\
\text { IDA }\end{array}$ & ADB & EIB & EEF & $\begin{array}{l}\text { Europ. } \\
\text { Komm. }\end{array}$ & ADA & AWS & OeKB \\
\hline Kredite & $\mathrm{X}$ & & & $\mathrm{X}$ & $\mathrm{X}$ & $\mathrm{X}$ & $\mathrm{X}$ & & $\mathrm{X}$ & $\mathrm{X}$ \\
\hline Syndizierte Kredite & $\mathrm{X}$ & & & $\mathrm{X}$ & & & & & & \\
\hline $\begin{array}{l}\text { Finanzierung in lokaler } \\
\text { Währung }\end{array}$ & $\mathrm{X}$ & & & $\mathrm{X}$ & & & & & & \\
\hline Eigenkapital & $\mathrm{X}$ & & & $\mathrm{X}$ & & & & & & \\
\hline Quasi-Equity & $\mathrm{X}$ & & & & & & & & & \\
\hline Structured Finance & $\mathrm{X}$ & & & & $\mathrm{X}$ & & & & & \\
\hline $\begin{array}{l}\text { Garantien für politisches } \\
\text { Risiko }\end{array}$ & & $\mathrm{X}$ & $\mathrm{X}$ & $\mathrm{X}$ & & & $\mathrm{X}$ & & & $\mathrm{X}$ \\
\hline $\begin{array}{l}\text { Garantien für } \\
\text { wirtschaftliches und } \\
\text { politisches Risiko }\end{array}$ & & & & $\mathrm{X}$ & & & & & $\mathrm{X}$ & \\
\hline Absicherungsinstrumente & $\mathrm{X}$ & & & & & & & & & \\
\hline Zuschüsse & & & & & & $\mathrm{X}$ & $\mathbf{X}$ & $\mathrm{X}$ & $\mathrm{X}$ & \\
\hline Beratung / Unterstützung & $\mathrm{X}$ & $\mathrm{X}$ & $\mathrm{X}$ & $\mathrm{X}$ & & & & $\mathrm{X}$ & $\mathrm{X}$ & \\
\hline \multicolumn{11}{|l|}{ Reichweite } \\
\hline Weltweit & $\mathrm{X}$ & $\mathrm{X}$ & $\mathrm{X}$ & & & & & & & $\mathrm{X}$ \\
\hline Regionale Beschränkung & & & & $\mathrm{X}$ & $\mathrm{X}$ & $\mathrm{X}$ & $\mathrm{X}$ & $\mathrm{X}$ & $\mathrm{X}$ & \\
\hline
\end{tabular}




\subsection{Detailvorstellung und kritische Würdigung einzelner innovativer Finanzierungsinstrumente der IFIs}

Die Maßnahmen der Structural Adjustment Policies (SAP) im Rahmen des Washington-Consensus und die asiatische Finanzkrise 1997 veränderten die Rahmenbedingungen der Entwicklungspolitik und auch die Wahrnehmung der Risiken bzw. Herausforderungen bei der Infrastrukturfinanzierung. Auch von Seiten der IFIs wurde auf diese veränderten Rahmenbedingungen reagiert und es wurden neue, innovative Instrumente zur Risikovermeidung entwickelt. Im Wesentlichen konnten durch die Interviews und Recherchearbeiten vier innovative Ansätze in der Finanzierung von Infrastrukturprojekten identifiziert werden, wobei

1. die Garantien und

2. die Finanzierung in lokaler Währung

in erster Linie eine Rolle für den privaten Sektor und sub-souveräne Regierungseinheiten spielen.

Im Zusammenhang mit der Herstellung der Infrastrukturleistung durch den privaten Sektor kann es zu Preiserhöhungen kommen, da die Preise oft von öffentlicher Seite subventioniert werden. Dafür gibt es einen neuen Ansatz, von dem sowohl der private Produzent, als auch die Regierung profitieren können, nämlich

\section{Output-Based Aid.}

Ein weiterer Trend in den Entwicklungsländern stellt die Dezentralisierung und die Stärkung der regionalen Regierungseinheiten dar. Dadurch werden Aufgaben, wie die Finanzierung und Instandhaltung der Infrastruktur auch auf die dezentralen Regierungseinheiten ausgelagert. Daraus ist neben dem Staat und dem privaten Sektor eine neue Kundegruppe mit spezifischen Charakteristika entstanden. Die Fragestellungen, die sich bei diesen Kunden ergeben, werden unter der

\section{Sub-souveräne Finanzierung}

diskutiert.

\subsubsection{Garantien}

Die Vergabe von Garantien zur Absicherung des politischen Risikos hat eine lange Tradition in den IFIs, allerdings hat sich die Kundengruppe erweitert. ${ }^{28}$ Die neuen Kunden stellen vermehrt private Unternehmen oder sub-souveräne Regierungseinheiten dar, welche Garantien zur Erhöhung der Kreditwürdigkeit

28 Eine detaillierte Vorstellung der Funktionsweise von Garantien erfolgte bereits im Kapitel 5.2.2.5. 
benötigen, um an zusätzliche Finanzierung am Kapitalmarkt zu gelangen. Die wesentliche Besonderheit stellt für die IFIs dabei das erhöhte Risiko dar, da private Unternehmen und dezentrale Regierungseinheiten außerhalb der starken Einflusssphäre der IFIs liegen und zusätzlich dazu nicht die gleiche Finanzstärke wie ein Staat aufweisen. Bei einer Vergabe von Garantien an den öffentlichen Sektor können IFIs durch politische Intervention beispielsweise die Erfüllung von offenen Krediten herbeiführen. Daher stellte die Vergabe von Garantien an Staaten bisher ein geringes Risikopotenzial dar, da Garantien kaum jemals wirklich in Anspruch genommen wurden.

Die teilweise Absicherung von politischen und wirtschaftlichen Risiken durch Garantien stellt ein wesentliches Instrument zur Verbesserung der Kreditwürdigkeit (,credit enhancement ") der Investoren dar. Private Investoren benötigen diese Garantien für die kommerzielle Kapitalaufbringung, um einerseits die Finanzierung zu bekommen und andererseits, um durch die erhöhte Kreditwürdigkeit die Finanzierungskosten zu senken. Dabei vergeben die IFIs auch Garantien an die kommerziellen Banken, um die privaten Unternehmen bei der Kapitalaufbringung zu unterstützen.

Dezentrale Regierungseinheiten haben, wie in Kapitel 5.3.4 noch genauer besprochen werden wird, nur eine schwache Einnahmenstruktur. Sollte eine dezentrale Regierungseinheit daher einen Kredit nicht zurückzahlen können, kann der Kreditgeber wiederum nur auf die geringe Sicherheit, nämlich die spärlichen, nicht beeinflussbaren Einnahmen zurückgreifen. Die Einflusssphäre von IFIs auf die dezentralen Regierungseinheiten ist in diesem Fall stark beschränkt und so könnte es im Falle eines Defaults einer Regionalregierung zu einem völligen Zahlungsausfall kommen.

Die IFIs übernehmen durch die Vergabe von Garantien an diese beiden Kundengruppen eine wesentliche Rolle in der Finanzierung von Infrastruktur. Dies ist auch in Kapitel 6 zu sehen, da in jeder Fallstudie Garantien vergeben wurden.

\subsubsection{Finanzierung in lokaler Währung}

Im Zusammenhang mit Infrastrukturfinanzierung in Entwicklungsländern stellt das Wechselkursrisiko eines der wesentlichen Risiken für die Projektsponsoren dar. Dies wurde den Sponsoren und Regierungen vor allem bei der asiatischen Finanzkrise vor Augen geführt. Viele Projekte scheiterten am Ungleichgewicht zwischen Verschuldung in US\$ und den Einnahmen in der lokalen Währung. Gleichzeitig waren viele Regierungen, die ohnehin bereits stark verschuldet waren erstmals an Abnahmeverträge mit privaten Unternehmen gebunden, die dollarindexiert waren. Dies führte wiederum dazu, dass Regierungen ihre Verbindlichkeiten nicht oder nur teilweise begleichen konnten. 
Infrastrukturinvestitionen haben eine lange Laufzeit und benötigen daher Kredite mit einer entsprechenden Dauer. Typischerweise stehen Kredite mit langen Laufzeiten aber nur auf gut entwickelten Kapitalmärkten bzw. in stabiler Währung zur Verfügung. Da die Kapitalmärkte in den Entwicklungsländern meistens unterentwickelt sind, besteht nicht automatisch die Möglichkeit, Kredite in lokaler Währung mit langer Laufzeit aufzunehmen. Bisher konnten Kredite in lokaler Währung abhängig vom jeweiligen Land nur für kleinere und mittlere Projekte oder für Teilfinanzierungen bei großen Projekten aufgenommen werden.

Eine weitere wesentliche Beschränkung des lokalen Kapitalmarktes im Zusammenhang mit Projektfinanzierung stellen die personalen Ressourcen dar. In den Entwicklungsländern gibt es noch nicht das notwendige Know-How, Projektfinanzierungen selbständig durchzuführen. Es mangelt vor allem an ausgebildetem Personal in der Risikoeinschätzung und im Risikomanagement.

Die Finanzierung in lokaler Währung ist für die Infrastrukturfinanzierung sehr wichtig und die Beteiligung von lokalen Banken im Infrastruktursektor verspricht viel Erfolg. Aus diesem Grund unterstützen viele IFIs auch die Entwicklung der Kapitalmärkte und Institutionen in den Entwicklungsländern (vgl. Estache 2006, Bestani/Sagar 2004). Eine große Herausforderung besteht vor allem in der Entwicklung der Bankvorschriften und des Know-How-Transfers, da diese in der Vergangenheit relativ schwach waren (vgl. Osius/Carlson 2004: 13).

Solange die Kapitalmärkte aber noch unterentwickelt und nicht in der Lage sind, selbständig langfristige Kredite in lokaler Währung zu vergeben, können IFIs diese Rolle übernehmen. Grundsätzlich existieren für IFIs zwei Möglichkeiten, um langfristige Finanzierungen in lokaler Währung zur Verfügung zu stellen. Dies ist einerseits die

- Emission von Anleihen in lokaler Währung und

- andererseits Cross-Currency Swaps.

\section{Emission von Anleihen in lokaler Währung durch die IFIs}

Die Emission von Anleihen in lokaler Währung ist mit sehr hohem Planungsaufwand verbunden. Idealerweise würden Pensions- oder Versicherungsfonds in Anleihen investieren, die die gleiche Laufzeit wie der Kredit aufweisen. Jedoch ist der Anleihenmarkt unterentwickelt und Vorschriften verhindern oftmals sogar die Beteiligung von institutionellen Anlegern, wie beispielsweise Pensionsfonds oder Versicherungsunternehmen (vgl. Osius/Carlson 2004: 14).

Die Emission von Anleihen ist außerdem mit einem hohen administrativen Aufwand verbunden. Sobald die Emission erfolgreich abgeschlossen ist, muss das Kapital in lokaler Währung wiederum von der IFI durch Kredite vergeben werden. Diese beiden Zeitpunkte, d.h. die erfolgreiche Emission und die 
Vergabe des Kredites, sind aber in den seltensten Fällen ident. Fallen diese beiden Zeitpunkte also nicht zusammen, muss die IFI das Geld bis zur Kreditvergabe zusätzlich managen, da jeder ungenützte Kapitallagertag mit Kosten verbunden ist.

Infrastrukturprojekte haben typischerweise auch aufgrund ihres Projektvolumens einen langen Planungszeitraum, bei dem es viele Unsicherheiten gibt und daher der genaue Zeitpunkt für die Inanspruchnahme des Kredites nicht taggenau geplant werden kann. Aus diesen Gründen wurde die Finanzierung in lokaler Währung durch die Emission von Anleihen noch nie erfolgreich umgesetzt.

\section{Bereitstellung von Cross-Currency Swaps}

Bisher gibt es noch keine mittel- oder langfristigen Cross-Currency bzw. Währungsswaps in Entwicklungsländern, die zu einem geringeren Wechselkursrisiko bei den Infrastrukturprojekten beitragen würden (vgl. Bestani 2004, Osius/Carlson 2004: 14). Von Seiten der ADB gibt es aber Bestrebungen, durch Cross-Currency-Fazilitäten zusätzliche Ressourcen in lokaler Währung bereitzustellen.

Um zusätzliche Kredite in lokaler Währung zu schaffen, tauscht die ADB mit der lokalen Regierung ausländische, konvertible Währungen gegen einheimische Währung und gibt die einheimische Währung mit fixer Verzinsung wiederum an kommerzielle Banken, die wiederum Kredite an private Unternehmen vergeben können. Internationale Banken, die mindestens ein BBB-Rating aufweisen, können äquivalent in lokaler Währung bis zu 75 Millionen US\$ und lokale Banken bis zu 10 Millionen US\$ ausleihen (vgl. Osius/Carlson 2004: 21). Diese Weitergabe der Kredite ist wiederum beschränkt auf gewisse Sektoren, wie beispielsweise die Infrastruktur. Zusätzlich dazu müssen es auch Projekte sein, die sich positiv auf die Entwicklung des Landes auswirken (vgl. Bestani/Sagar 2004). Diese Einschränkung ist vor allem aufgrund der Statuten der ADB wichtig, laut derer sie nur Projekte fördern, die auch einen positiven Beitrag zur Entwicklung leisten. Nicht nur neue Projekte können von diesem Instrument profitieren, sondern auch Restrukturierungsfinanzierungen sind möglich. In der folgenden Abbildung 37 wird der Ablauf eines Währungsswaps zwischen der ADB und einer lokalen Bank beispielhaft dargestellt.

Bei einem Cross-Currency Swap durch die ADB werden nicht wie bei einem klassischen Währungsswap variable gegen fixe Zinszahlungen, sondern US\$ gegen heimische Währung getauscht. Gleichzeitig ist nicht das private Unternehmen der Initiator des Swaps, sondern die ADB. 


\section{Erster Haupttausch}

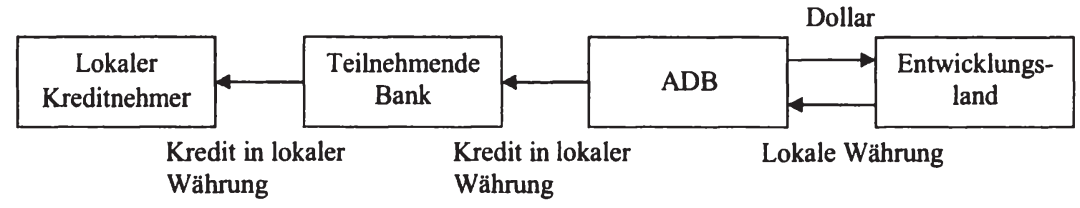

2. Swap/Zinszahlung

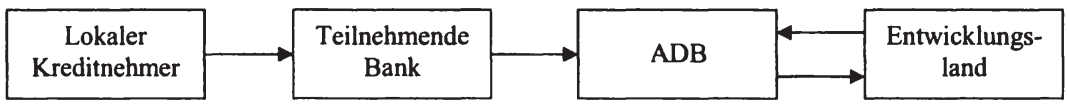

Lokale Währung

Lokale Währung

Lokale Währung

3. Letzter Haupttausch

Dollar

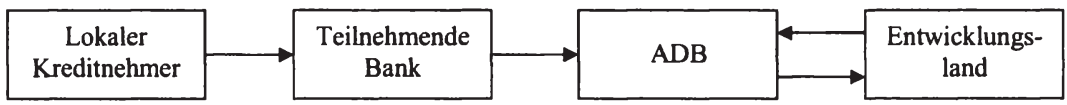

Lokale Währung

Lokale Währung

Lokale Währung

Quelle: in Anlehnung an Bestani/Sagar (2004)

Die Emission von Anleihen beansprucht viel Planungsaufwand und ist daher nicht einfach zu bewerkstelligen. Währungsswaps sind dagegen viel kurzfristiger angelegt, können den Kunden relativ rasch zur Verfügung gestellt werden und sind somit erfolgsversprechender. Generell ist aber der Erfolg von Krediten in lokaler Währung momentan noch relativ gering. Man ist sich darüber einig, dass durch dieses Instrument die Risiken für beide, den privaten und den öffentlichen Partner, gesenkt werden könnten, aber noch scheiden sich die Geister über die Machbarkeit.

Vor allem würde die Vergabe von Krediten in lokaler Währung auch positive Auswirkungen auf die Entwicklungsländer haben, da im Infrastruktursektor in den Abnahmeverträgen keine oder nur noch sehr geringe Dollarindexierungen notwendig wären. Dadurch würden sich für die Entwicklungsländer die Wechselkursrisiken und die daraus resultierenden Kosten verringern. Dies ist auch in den philippinischen Fallstudien im Kapitel $6 \mathrm{zu}$ sehen, die eine partielle Finanzierung in lokaler Währung und eine geringer Dollarindexierung aufweisen.

Bisher hat sich vor allem die ADB mit dieser Thematik beschäftigt. Wesentlicher Grund dafür ist, dass die Weltbankgruppe aufgrund ihrer Statuten keine Cross-Currency-Swaps durchführen kann, da die Weltbank keine Transaktion 
mit dem Privatsektor und IFC keine mit dem öffentlichen Sektor durchführen darf.

\subsubsection{Output-based Aid}

Die Subventionierung von Infrastrukturleistungen hat in den Entwicklungsländern eine lange Tradition. In der nahen Vergangenheit wurden Subventionen in diesem Zusammenhang oft kritisiert, da sie nicht ausreichend zielgerichtet waren. Es wurden generelle Beihilfen für Projekte gewährt, die dann oftmals nicht transparent verwendet wurden und dazu führten, dass die bereits besser gestellten Haushalte und nicht die Armen davon profitierten (vgl. CEC 2004: 19). Außerdem wurden Zuschüsse auch dahingehend kritisiert, dass sie keine Anreize für die Organisationen schafften, effizienter zu arbeiten (vgl. Smith 2001: 91).

Daher wurde in den vergangenen Jahren an neuen Ansätzen gearbeitet, um Beihilfen nur auf Basis von vorher definierten Zielen zu vergeben. Zusätzlich dazu werden in der Entwicklungszusammenarbeit wieder verstärkt Subventionen bei der privaten Finanzierung von Infrastruktur gefordert und stellen heutzutage wieder eine legitime Unterstützung des privaten oder öffentlichen Sektors in der Herstellung von Infrastrukturleistungen dar.

Im Zusammenhang mit öffentlich-privaten Infrastrukturmodellen erscheint insbesondere Output-based Aid (OBA) geeignet. OBA stellt eine neue Strategie zur Beschaffung von Infrastruktur dar, indem versucht wird, auf Basis des Outputs die Effizienz der Leistungen und der Subventionen zu erhöhen (GPOBA 2005). Diese Output-Orientierung gewährleistet, dass nur dann an das private Unternehmen bezahlt wird, wenn die Leistungen in der vordefinierten Qualität geliefert werden. Dieser Ansatz steht im starken Gegensatz zur bisherigen Vergabe von Subventionen, da bisher die Inputs subventioniert wurden. OBA wird explizit für eine gewisse Leistung vereinbart und diese wiederum abhängig von der wirklichen Leistung ausbezahlt. Beispielsweise kann der Energiesektor durch OBA unterstützt werden, indem der Stromproduzent nur auf Basis der produzierten kWh Subvention erhält. Bei einer Input-orientierten Subvention wird hingegen nicht der wahre Output gemessen, sondern die Unterstützung anhand der geplanten Produktionsleistung gewährt.

Durch den OBA-Ansatz ergeben sich wesentliche Vorteile (vgl. GPOBA 2005):

1. Die Output-Fokussierung verbessert die Qualität und die Quantität der Leistungen.

2. Durch die Konzentration auf den Output kann die Unterstützung besser verfolgt und die Verantwortlichkeit der Geber und der Regierungen erhöht werden. 
3. Durch die explizite Gewährung der Subventionen kann die Transparenz der Zahlungen erhöht werden.

4. Durch die Subventionierung kommt es zu einer Erhöhung der Tarife und dadurch können mehr private Investoren angelockt werden.

5. OBA ermöglicht es, die Unterstützung zielgenauer armen Haushalten zukommen zu lassen.

OBA stellt daher keine Subventionierung dar, welche private Unternehmen anzulocken versucht, sondern eine Ausgleichsfinanzierung, um die geringen Ausgaben der armen Haushalte auszugleichen. Jedoch wird OBA als Instrument betrachtet, das dazu führen soll mehr PPI zu unterstützen, die zu einer verbesserten Infrastrukturleistung führen. OBA kann in unterschiedlichen Bereichen der Infrastruktur eingesetzt werden und stellt beispielsweise in der Strom- und Straßenversorgung eine gute Möglichkeit zur zielsicheren Vergabe von Subventionen dar.

Grafisch wird in der folgenden Abbildung 38 die Gestaltung der OBASubvention dargestellt. Sie stellt eine zeitlich beschränkte Subventionierung der Tarife dar und wird nach einer im Voraus bestimmten Zeit wieder eingestellt. Durch ein öffentliches Ausschreibungsverfahren soll jenes Unternehmen für die Produktion ausgewählt werden, das die Infrastrukturleistung, wie beispielsweise Strom, am effizientesten erzeugen kann. Die Verbraucherpreise werden kontinuierlich den wahren Kosten angepasst, damit am Ende der OBA-Laufzeit die Kunden die gesamten Kosten selber tragen können.

\section{Abbildung 38: Gestaltung der Übergangssubvention}

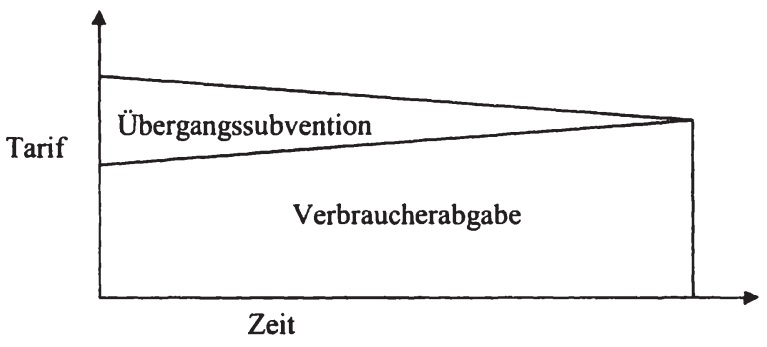

Quelle: in Anlehnung an GPOBA (2005: 15)

Im Vergleich zur üblichen Input-Subventionierung entsteht bei OBA ein zusätzlicher Überwachungsaufwand (vgl. CEC 2004: 19). Die größte Herausforderung stellt für OBA die Festlegung und Messung des Outputs dar, vor allem dann, wenn die Dienstleistung weiter verarbeitet und nicht sofort konsumiert wird, wie dies beispielsweise im Energiesektor der Fall ist (vgl. GPOBA 
2005). Grundsätzlich sollten Outputs definiert werden, die so nahe wie möglich am gewünschten Output liegen.

Bisher gibt es noch keine ausreichenden Belege für die Wirksamkeit von OBA (vgl. GPOBA 2005). Jedoch wird auf Basis der bereits vorliegenden positiven Ergebnisse davon ausgegangen, dass OBA ein effizientes Unterstützungsinstrument darstellt, das die Infrastrukturleistungen verbessert und damit auch zur Armutsreduktion und zur Erreichung der MDGs beitragen wird.

Kritisch zu sehen ist der reine Fokus auf den Output, da dies kurzfristig zu einer Verbesserung der Dienstleistungen führen und auch der Stimmenmaximierung dienen kann. Daher sollte auch der Input bei diesem Ansatz berücksichtigt werden, da die strategischen Infrastrukturmaßnahmen über die politische Periode hinaus der Entwicklung des Landes dienen sollen.

\subsubsection{Sub-souveräne Finanzierung}

Im Englischen werden unter "Sub-sovereign financing“"29,30 alle Kredite an dezentrale Regierungseinheiten zusammengefasst. Die Dezentralisierung stellt weltweit und besonders in den Entwicklungsländern eine entscheidende politische Entwicklung dar (vgl. OECD 2004), jedoch waren die Erfolge von Dezentralisierungen in der Vergangenheit eher gemischt (vgl. World Bank 2004). Diese auf dem Dezentralisierungstheorem (siehe Kapitel 2.2.4.2) basierende Entwicklung stößt in Entwicklungsländern an ihre Grenzen. Die Anforderungen bzw. Hindernisse für eine erfolgreiche Dezentralisierung in Entwicklungsländern werden an dieser Stelle angeführt. Auf Basis dieser Hindernisse werden die Herausforderungen an eine sub-souveräne Finanzierung hergeleitet.

Der Zielkonflikt zwischen Skaleneffekten bei der Ressourcenbeschaffung auf zentraler Ebene und der besseren Information auf dezentraler Ebene wird im Dezentralisierungstheorem nicht berücksichtigt. Außerdem gibt es eine Vielzahl von Infrastrukturleistungen, deren Externalitäten sich nicht auf einen bestimmten Raum beschränken lassen. Daher ist es im Falle von auftretenden Externalitäten sinnvoll, die Planung nicht zu dezentralisieren (vgl. Leiderer/ Liebig 2006: 7).

Des Weiteren erfüllen nicht alle Entwicklungsländer die Voraussetzungen für eine sub-souveräne Finanzierung, da es oft sowohl an Transparenz als auch an

29 Sub-souveräne Einrichtungen sind normalerweise nur eingeschränkt autonom und können daher auch keine Garantien abgeben. In den Ländern gibt es meistens mehrere Ebenen von sub-souveränen Strukturen.

30 Selbst nach intensiver Recherche konnte keine ideale deutsche Übersetzung für diesen Begriff gefunden werden, da sich in der deutschen Literatur bislang noch keine deutsche Übersetzung dieses Begriffes durchgesetzt hat und auch in der Entwicklungspolitik der englische Begriff verwendet wird. 
gut ausgebildetem Personal mangelt (vgl. OECD 2004). Die Dezentralisierung bedeutet auch einen Machtverlust für die zentrale Regierung und kann darin resultieren, dass sie zwar formal aber nicht faktisch unterstützt wird. Eine Dezentralisierung kann im Falle von Zahlungsschwierigkeiten auf lokaler Ebene auch zu makroökonomischen Krisen führen. Insbesondere dann, wenn Gemeinden auf eine implizite Garantie von der zentralen Regierung hoffen und es dadurch zu einem riskanten Verhalten bei den Kreditgebern und -nehmern kommt (vgl. Leiderer/Liebig 2006: 10).

Die Dezentralisierung birgt aufgrund der Vielzahl von Untereinheiten erhöhte Koordinationsanforderungen in sich, sowohl vertikal (zwischen der zentralen und den lokalen Regierungen) als auch horizontal (zwischen den einzelnen lokalen Regierungen) (vgl. ADB 2005b: 168). Die Thematik der Dezentralisierung steht somit auch im engen Zusammenhang mit dem Finanzmanagement und der Informationspolitik von öffentlichen Einrichtungen (vgl. OECD 2004). Lokale Regierungseinheiten müssen aber ein gutes Finanzmanagment aufweisen, um kreditwürdig zu sein. Daher erfordert dieser Dezentralisierungsprozess eben auch gut ausgebildetes Personal auf lokaler Ebene, um den Anforderungen der Kreditgeber gerecht zu werden.

Den dezentralen Einheiten stehen im Wesentlichen drei Finanzierungsmöglichkeiten zur Verfügung, nämlich

- die traditionelle Form durch Mittel der zentralen Regierung,

- durch öffentliche Finanzintermediäre, wie beispielsweise durch kommunale Fonds, und

- durch die direkte Mittelaufbringung bei kommerziellen Banken (vgl. Leiderer/Liebig 2006: 9, OECD 2004).

Eine wesentliche Herausforderung im Rahmen der Dezentralisierung stellt die Ressourcenakquirierung dar, da diese häufig nicht gleichzeitig mit der Aufgabenverteilung dezentralisiert wird (vgl. ADB 2006c: 219, Petersen/Freire 2004: 12). Durch die verspätete oder nur teilweise durchgeführte Dezentralisierung der Mitteleinnahmen durch die zentrale Regierung kommt es in vielen Ländern zu einer Lücke bei den übertragenen Aufgaben und den finanziellen Ressourcen der lokalen Regierungen. Durch die Ausweitung der übertragenen Obliegenheiten bei gleichzeitig gleichbleibenden Einnahmen von der zentralen Regierung, kommt es zu einem Engpass bei der Finanzierung. Dies ist beispielsweise auf den Philippinen zu sehen, wo bereits 1991 unter dem Local Government Code damit begonnen wurde, die öffentlichen Aufgaben stärker zu dezentralisieren und bis heute keine adäquate Anpassung der Einnahmen stattgefunden hat (vgl. ADB 2006c: 219).

Durch die Möglichkeit der unabhängigen Kapitalaufnahme durch die lokalen Regierungseinheiten kann die Abhängigkeit von der Zentralregierung verringert werden (vgl. Leiderer/Liebig 2006: 1). Zusätzlich wird seit geraumer Zeit insbesondere der lokale Finanzmarkt als Kapitalgeber diskutiert, der in manchen 
Ländern auch schon für mittelfristige Finanzierungen zur Verfügung steht. Damit aber die sub-souveräne Finanzierung in Entwicklungsländern erfolgreich sein kann, müssen sich sowohl der lokale Kapitalmarkt als auch die Institutionen bzw. die Regierungen weiterentwickeln.

Insbesondere muss sich aber die Kreditwürdigkeit der dezentralen $\mathrm{Re}$ gierungen erhöhen, da die Vergabe von Krediten an dezentrale Regierungseinheiten mit besonderen Risiken verbunden ist. Das wesentliche Risiko stellt dabei die Finanzkraft bzw. die Zahlungsfähigkeit der jeweiligen Einheit dar, da die sehr eingeschränkten Einnahmen der dezentralen Regierungen aus den limitierten lokalen und der Umverteilung von zentral verwalteten Steuereinnahmen bestehen (vgl. Kehew et al. 2004: 1, OECD 2004). Außerdem verschuldet sich die souveräne Regierung unabhängig von den dezentralen Einheiten und belastet dadurch auch die Kreditwürdigkeit der dezentralen Einheiten. Ein weiteres Risiko stellt auch das erhöhte politische Risiko bei dezentralen Einheiten dar, da IFIs normalerweise eng mit den zentralen Regierungen zusammenarbeiten und diese bei Zahlungsschwierigkeiten beeinflussen können, auf dezentrale Regierungen aber nur beschränkt oder keinen Einfluss haben.

Um die Kreditwürdigkeit der dezentralen Regierungseinheiten zu erhöhen, können IFIs nicht nur den geforderten langfristigen Prozess zur Erhöhung der Kreditwürdigkeit der dezentralen Einheiten unterstützen, sondern auch operative Instrumente zur Verfügung stellen (vgl. OECD 2004). Garantien stellen in diesem Zusammenhang das am häufigsten verwendete Instrument dar, wobei man globale und Teilversicherungen unterscheidet. Die globale Versicherung sichert sowohl die Kredit-, als auch Zinszahlungen aufgrund von Zahlungsschwierigkeiten ab. Bei der Teilgarantie teilt sich der Versicherer das Risiko mit dem Kreditgeber auf Basis von vorher festgelegten Vereinbarungen (vgl. Kehew et al. 2004: 3). Weiters könnten die IFIs die dezentralen Einheiten dabei unterstützen, weitere Kreditgeber zu finden oder auch als Finanzintermediäre auftreten (vgl. OECD 2004). Die Garantien werden nicht ausreichen, die Finanzierungslücke zu reduzieren (vgl. PwC 2003: 10).

Von den IFIs wird die direkte Vergabe von Krediten an jene dezentralen Regierungseinheiten gefordert, die beispielsweise durch staatliche Gegengarantien abgesichert werden können (vgl. PwC 2003: 44). Hinderlich sind hierbei oftmals die Statuten und die politische Ausrichtung der verschiedenen Institutionen, die entweder den öffentlichen oder den privaten Sektor finanzieren. In der ADB gibt es allerdings verstärkt Bestrebungen, den Bereich der privaten Finanzierungen im öffentlichen Sektor auszubauen. Dies bedeutet, dass in Zukunft auch ausgegliederte Unterorganisationen durch die ADB unterstützt werden können.

Die folgenden Abbildungen 39 und 40 zeigen den Unterschied zwischen einer indirekten Vergabe eines Kredites durch den Staat und der direkten Kreditgewährung an eine dezentrale Regierungseinheit. Bei der indirekten Vergabe eines Kredites an die dezentrale Regierungseinheit bleibt der souveräne Staat der 
Kreditnehmer und sichert diesen durch eine staatliche Garantie ab. Wird hingegen ein direkter Kredit an die sub-souveräne Regierungseinheit gegeben, dann kann zusätzlich zur Risikominierung eine Garantie vom Staat oder von den IFIs vergeben werden.

\section{Abbildung 39: Indirekter Kredit durch den souveränen Staat an die dezentrale Regierungseinheit}

\begin{tabular}{|c|l|}
\hline $\begin{array}{c}\text { ADB } \\
\text { oder IFC }\end{array}$ & $\begin{array}{l}\text { Direkter Kredit in } \\
\text { ausländischer } \\
\text { Währung }\end{array}$ \\
\hline
\end{tabular}

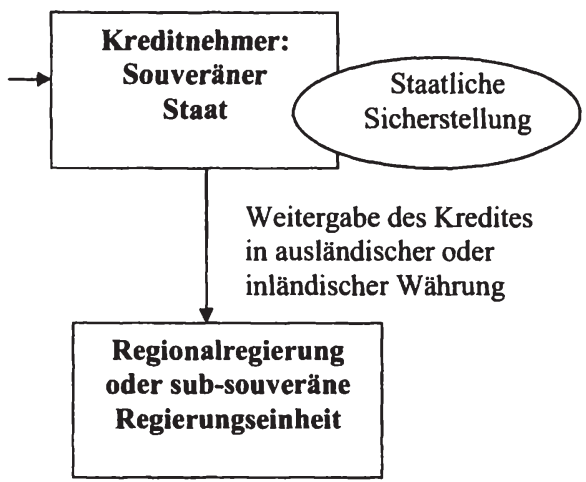

Abbildung 40: Direkter Kredit an die sub-souveräne Regierungseinheit

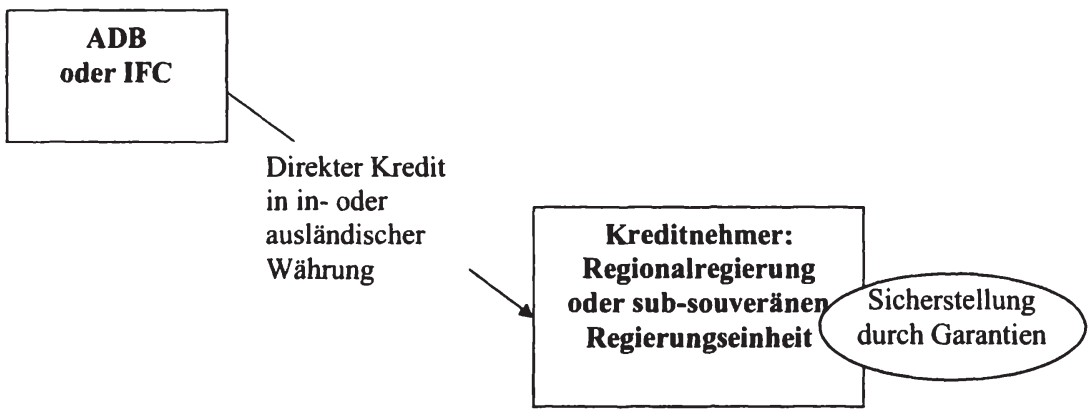

Die Finanzierung von sub-souveränen Regierungseinheiten steht bereits seit längerem im Mittelpunkt der Diskussionen in der Entwicklungspolitik. Da die ländlichen Regionen am meisten von Armut betroffen sind, befürchtet man, dass es aufgrund der geringen Finanzkraft der sub-souveränen Einheiten zu keinen oder geringen Infrastrukturinvestitionen kommt und sich die Armut dadurch vergrößert. Unter diesem Aspekt erscheint es sehr kritisch, Infrastrukturkompetenz großteils an die sub-souveränen Regierungseinheiten zu vergeben. Kurzfristig, im Sinne der politischen Stimmenmaximierung, bedeutet eine Ausgliederung 
der Kompetenzen für die zentralen Regierungen eine Verbesserung des Budgets. Bedenkt man allerdings, dass fehlende Investitionen in neue Infrastruktur oder in den Bestand nicht sofort, sondern erst nach gewisser Zeit erkennbar sind, kann davon ausgegangen werden, dass es langfristig gesehen zu einer Armutserhöhung kommt. Ziel der IFIs ist es daher, die Kreditwürdigkeit der subsouveränen Regierungseinheiten zu erhöhen, um Investitionen in den Infrastruktursektor von öffentlicher Seite zu ermöglichen.

\subsection{Zusammenfassung}

Das fünfte Kapitel widmete sich der Rolle der internationalen und nationalen Finanzinstitutionen bei privaten Infrastrukturinvestitionen und unterteilt sich in vier Unterkapitel.

Das erste Kapitel gibt eine Einführung in die Rolle der Projektfinanzierung im Infrastruktursektor und die Mindestkriterien für private Investitionen, da die Projekte wirtschaftlich tragfähig sein müssen. Daran anschließend wird auf die Kofinanzierungsrolle der Finanzinstitutionen eingegangen, aufgrund deren Beteiligung zusätzliches Kapital mobilisiert werden kann.

Im zweiten Kapitel werden die Finanzierungs- und Absicherungsinstrumente von einzelnen nationalen und internationalen Finanzinstitutionen in der Infrastrukturfinanzierung dargestellt. Beginnend bei der multilateralen Ebene, werden zunächst die Institutionen der Weltbankgruppe vorgestellt. Für die private Finanzierung des Infrastruktursektors sind vor allem die IFC und die MIGA wesentlich, da sie ausschließlich Instrumente für den privaten Sektor anbieten. Die Hauptprodukte der IFC stellen direkte oder indirekte Kredite an private Unternehmen dar. Sie bietet aber auch Eigenkapital und investiert in Fonds. Die MIGA ist wiederum spezialisiert auf die Absicherung von politischen Risiken durch Garantien. Auch die Weltbank vergibt eine Garantie an den privaten Sektor, die das politische Risiko absichert. Die Organisationen der Weltbankgruppe investieren zusätzlich in Fonds und Programme, die wiederum auf die Infrastrukturentwicklung fokussieren.

Daran anschließend wird die Asian Development Bank (ADB) vorgestellt. Da sich alle Fallstudien im 6. Kapitel in Asien befinden und die ADB wesentlich an der Finanzierung der Infrastrukturprojekte in dieser Region beteiligt ist, werden ihre Instrumente stellvertretend für die restlichen regionalen Entwicklungsbanken dargestellt. Die ADB verfügt über ein ,Private Sector Operation Department", das ausschließlich private Unternehmen unterstützt. Von diesem Department werden direkte und indirekte Kredite und Garantien vergeben. Zusätzlich wird auch Eigenkapital zur Verfügung gestellt, das nur in den seltensten Fällen in physische Infrastruktur, sondern meist in den Ausbau des Finanzsektors investiert wird. Zusammenfassend kann festgestellt werden, 
dass sich die Produkte, abhängig von der Kompetenz der einzelnen Institutionen, stark ähneln.

Im dritten Abschnitt werden die Institutionen auf europäischer Ebene vorgestellt, welche die Finanzierung von Infrastruktur unterstützen. Die Europäische Investitionsbank (EIB) beschränkt sich auf die Vergabe von Krediten an private und öffentliche Sponsoren. Die Europäische Kommission konzentriert sich auf die Infrastrukturentwicklung in den AKP-Ländern und in Asien durch Beihilfen. Außerdem bietet sie auch eine politische Garantie für EIB-Kredite an.

Der vierte Abschnitt zeigt in einer Übersicht die österreichischen Beteiligungen bei multilateralen und europäischen Institutionen, wobei Österreich an allen wesentlichen Institutionen beteiligt ist und vom Bundesministerium für Finanzen vertreten wird.

Im fünften Abschnitt werden die österreichischen Institutionen vorgestellt, die eine Rolle bei der privaten Finanzierung von Infrastruktur spielen, wobei es bisher nur beschränkt österreichische Investitionen im Infrastruktursektor gegeben hat. Die Austrian Development Agency (ADA) unterstützt Unternehmen vor allem mit Zuschüssen und Know-How, da sie in den österreichischen Schwerpunktländern Vertretungen hat. Seit Juli 2006 ist die ADA auch am PIDG-Fonds beteiligt, dessen Ziel die umfassende Unterstützung von privaten Investoren im Infrastruktursektor ist. Das Austria Wirtschaftsservice (AWS) bietet Garantien und Kredite an, wobei es einen Mittel- und Osteuropaschwerpunkt hat. Die Oesterreichische Kontrollbank (OeKB) kann private österreichische Investitionen durch die Vergabe von politischen Garantien unterstützen.

Im abschließenden sechsten Abschnitt werden die Instrumente grafisch in einer Tabelle zusammenfasst. Aus dieser Zusammenstellung ist ersichtlich, dass sich das Angebot an Instrumenten der IFIs sehr stark gleicht. Einschränkungen bei der Unterstützung basieren oftmals auf den Statuten der jeweiligen Organisationen und auf regionalen Beschränkungen.

Im dritten Kapitel werden vier ausgewählte, innovative Instrumente genauer vorgestellt und einer kritischen Würdigung unterzogen. Die Vergabe von Garantien stellt per se kein innovatives Instrument dar, jedoch hat sich der Kundenkreis für die Institutionen und die damit verbundenen Risiken stark verändert. Durch die Privatisierung und Deregulierung benötigen nun private Unternehmen und sub-souveräne Regierungseinheiten vermehrt Schutz vor politischen Risiken. Die Garantien stellen ein sehr beliebtes Instrument dar.

Ein wesentliches Problem bei der Infrastrukturfinanzierung stellt das Ungleichgewicht bei der Verschuldung in US Dollar und den Einnahmen in lokaler Währung dar. Daher wird von IFC und ADB versucht, Kredite in lokaler Währung anzubieten. Allerdings hat dieses Instrument noch kaum Verbreitung gefunden, da sich die Durchführung als relativ kompliziert herausgestellt hat. 
Ziel dieser Kredite in lokaler Währung wäre die Reduktion der Verschuldung in US Dollar und in Folge eine Reduktion der Dollarindexierung in den Abnahmeverträgen, die für Regierungen bei Wechselkurschwankungen ein hohes Risiko bedeuten.

Infrastrukturleistungen werden oftmals subventioniert, damit die Leistungen auch für die arme Bevölkerung leistbar werden. Bisher wurde der Input subventioniert. Da dieser Input-Ansatz aber teilweise nicht zu den Effizienzgewinnen geführt hat, versucht man nun mit Output-based Aid einen Anreiz für ein besseres Ergebnis zu schaffen. Bisher wurden nur wenige Projekte auf Basis dieses Ansatzes subventioniert und die Erfahrungen daraus sind nur beschränkt zu verallgemeinern.

Eine weitere wesentliche Entwicklung im Infrastrukturbereich stellt aufgrund der Dezentralisierung die Finanzierung von sub-souveränen Regierungseinheiten dar. Die dezentralen Regierungseinheiten sind maßgeblich für die Infrastrukturentwicklung in der Region verantwortlich und benötigen daher auch Finanzmittel. Diese sind aber mit einem erhöhten Risikoprofil ausgestattet und benötigen daher die Unterstützung der internationalen Finanzinstitutionen, um sich am Kapitalmarkt finanzieren zu können. Die Unterstützung der dezentralen Regierungseinheiten ist von hoher Priorität für die Armutsreduktion und man setzt sich intensiv mit diesem Thema auseinander. Momentan werden vor allem Garantien vergeben, um die Kreditwürdigkeit zu erhöhen und die Finanzierungskosten zu senken.

Zusammenfassend lässt sich daher feststellen, dass sich die Instrumente der multilateralen Institutionen sehr stark ähneln und die privaten Investoren vor allem durch direkte und indirekte Kredite sowie Garantien unterstützen. Auch auf europäischer und nationaler Ebene werden ähnliche Instrumente angeboten. Aufgrund der Privatisierung und Dezentralisierung von Infrastruktur konnten vier neue Ansätze identifiziert werden, die eine unterschiedliche Rolle bei der privaten Infrastrukturfinanzierung spielen. 
Bianca Gusenbauer - 978-3-631-75445-0

Downloaded from PubFactory at 01/11/2019 04:47:32AM

via free access 


\section{Fallstudien}

Im sechsten Kapitel werden insgesamt vier Fallstudien vorgestellt. Im ersten Teil werden in einer Einführung das Ziel, der Auswahlprozess und der Aufbau der Fallstudien dargestellt. Im Kapitel 6.2 werden zwei Fallstudien in Vietnam und im Kapitel 6.3 zwei Fallstudien auf den Philippinen dargestellt. Die folgende Abbildung 41 stellt nochmals grafisch den Aufbau dieses Kapitels vor und dient der besseren Orientierung im Kapitel.

Abbildung 41: Kapitelübersicht Fallstudien

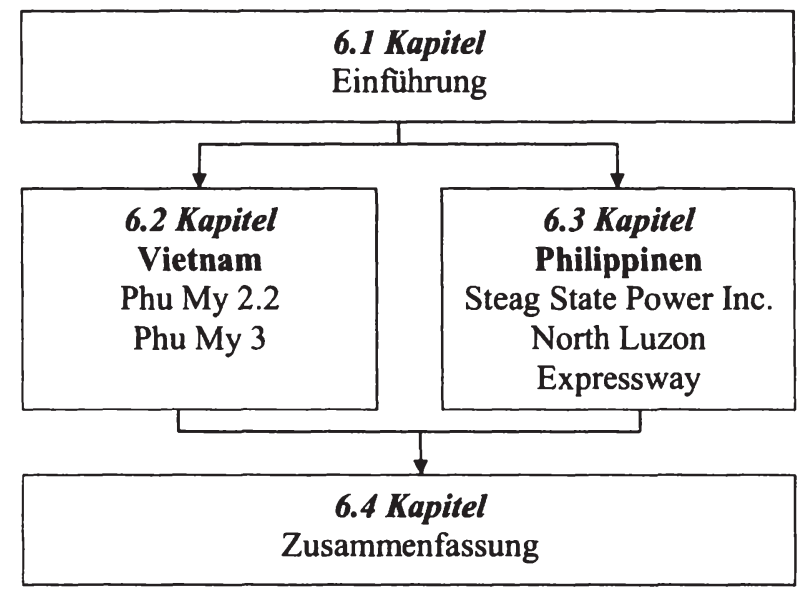

\subsection{Einführung}

Wie bereits im Kapitel 1.3 dargestellt wurde, basiert diese Dissertation auf dem Multimethodenansatz und Teil dieses Ansatzes ist auch die Darstellung von vier Fallstudien. In der vorliegenden Arbeit werden Infrastrukturinvestitionen dargestellt, die durch den privaten Sektor finanziert worden sind. Ziel der Fallstudien ist es zu zeigen, auf welche Weise bzw. durch welche Instrumente diese Projekte von nationalen und internationalen Finanzinstitutionen unterstützt wurden und die Diskussion über den Beitrag der Investitionen zur Armutsreduktion darzustellen. 


\subsubsection{Kriterien für die Auswahl der Fallstudien}

An dieser Stelle wird der Auswahlprozess der Fallstudien anhand von fünf Kriterien dargestellt.

\subsubsection{Region}

Die Weltbank unterteilt die Entwicklungsländer in sechs Regionen ein, diese Einteilung wurde auch für die vorliegende Arbeit herangezogen. In der folgenden Tabelle 6 werden die Regionen anhand der PPI in US\$ Millionen gereiht.

Tabelle 6: Regionen gereiht nach Private Participation in Infrastructur (PPI), in Millionen US\$, für den Zeitraum von 1990-2005

\begin{tabular}{|c|c|}
\hline \multicolumn{2}{|c|}{ Regionen gereiht nach PPI: in Millionen US\$ } \\
\hline Lateinamerika und Karibik & 407.341 \\
\hline Ostasien und Pazifik & 224.548 \\
\hline Europa und Zentralasien & 182.455 \\
\hline Südasien & 70.435 \\
\hline Mittlerer Osten und Nordafrika & 41.163 \\
\hline Subsahara Afrika & 36.510 \\
\hline
\end{tabular}

Quelle: PPI Database, http://ppi.worldbank.org/explore/ppi exploreRankings.aspx, 23.8.2007

Die meisten Investitionen gingen in der Vergangenheit nach Lateinamerika und in die Karibik. In den meisten Ländern dieser Region wird Spanisch gesprochen. Da die Autorin diese Sprache nicht ausreichend gut beherrscht, wurde diese Region für Fallstudien ausgeschlossen, da auch viele Dokumente nur auf Spanisch zur Verfügung stehen.

Wie aus der Tabelle 6 ersichtlich wird, wurden die zweitmeisten Investitionen in der Vergangenheit in der Region Ostasien und Pazifik ${ }^{31}$ getätigt. Daher wurden die Länder dieser Region in die engere Auswahl gezogen.

31 Zu den Ländern der Region East Asia and Pacific zählen: Kambodscha, China, Fiji, Indonesien, Kiribati, Korea, Laos, Malaysien, die Marshall Inseln, Mikronesien, Mongolei, Palau, Papua Neuguinea, Philippinen, Samoa, die Solomon Inseln, Thailand, Ost-Timor, Tonga, Vanuatu und Vietnam. 


\subsubsection{Sektor}

Wie bereits im Kapitel 3.1.2 dieser Arbeit festgelegt wurde, liegt der Fokus dieser Arbeit auf der wirtschaftlichen Infrastruktur. Innerhalb dieser Einteilung konzentriert sich die Autorin wiederum auf die physische Infrastruktur.

Die wirtschaftliche Infrastruktur kann laut Weltbank in vier Kategorien unterteilt werden und die folgende Tabelle 7 gibt einen Überblick über Höhe der PPI im Zeitraum von 1990-2005 in der Region Ostasien und Pazifik in diese Kategorien.

Tabelle 7: Sektoren gereiht nach Private Participation in Infrastructur (PPI), in Millionen US\$, für den Zeitraum von 1990-2005

\begin{tabular}{|l|r|}
\hline PPI im Zeitraum von 1990-2005: in US\$ Millionen \\
\hline Energie & 86.841 \\
\hline Telekommunikation & 64.757 \\
\hline Transport & 49.613 \\
\hline Wasser und Abwasser & 23.969 \\
\hline
\end{tabular}

Quelle: PPI Database, http://ppi.worldbank.org/explore/ppi exploreRankings.aspx, 23.8.2007

Wie aus der Tabelle 7 ersichtlich ist, liegt der Engergiesektor in der Region Ostasien und Pazifik an erster Stelle bei PPI, wobei private Investoren vor allem in die Produktion, aber weniger in die Versorgung investieren. Da der Fokus der vorliegenden Arbeit auch auf der physischen Infrastruktur, d.h. auf der Herstellung von Energie liegt, wurde in diesem Sektor nach Fallstudien gesucht.

Weltweit war das Investitionsvolumen im Telekommunikationssektor in der Vergangenheit am höchsten (siehe Kapitel 4.2.3.1). Wie aus der Tabelle 7 ersichtlich ist, nimmt dieser Sektor in dieser Region nur Platz zwei ein. In der Vergangenheit hat sich jedoch gezeigt, dass private Unternehmen einen Großteil der Versorgung in diesem Sektor erfolgreich übernehmen können. Daher werden in den Finanzinstitutionen Projekte in diesen Sektor nur noch beschränkt und nur dann gefördert, wenn das einzelne Projekt positive Auswirkung auf die regionale Entwicklung aufweist. Dies ist vor allem dann der Fall, wenn in Regionen mit einer hohen Armutsrate investiert wird. Alle anderen Projekte werden auf Anfrage nur noch mit Instrumenten zu kommerziellen Konditionen unterstützt. Basierend auf dieser Entwicklung wurde dieser Sektor für eine Evaluierung ausgeschlossen.

An dritter Stelle bei PPI liegt der Transportsektor. Private Investoren finanzieren in diesem Sektor bevorzugt nur in jene Projekte, bei denen die Kundengruppe identifiziert und damit der Cashflow kalkulierbar ist. Als Projekte für private Investoren kommen daher vor allem Projekte in Frage, die räumlich be- 
schränkt sind, wie beispielsweise Mautstraßen oder Häfen. Dieser eingeschränkte Bereich im Transportsektor wurde daher auch für die Fallstudien herangezogen.

In den Wassersektor investieren private Unternehmen hingegen aufgrund des hohen Risikos und der gleichzeitig niedrigen Rendite nur sehr beschränkt. Da die Beteiligung privater Unternehmen in diesen Sektor sich vor allem auf die Versorgung und nicht auf die physische Infrastruktur beschränkt, wurde dieser Sektor nicht für Fallstudien herangezogen.

Zusammenfassend lässt sich daher festhalten, dass der Energie- und der Transportsektor für die Suche nach Fallstudien herangezogen wurden.

\subsubsection{Art der PPI}

In der Region Ostasien und Pazifik in den Bereichen Infrastruktur- und Transportsektor wurde die Art der Investitionen genauer betrachtet. Als Quelle wurde die PPI Datenbank der Weltbank herangezogen, die eine Einteilung in vier Kategorien vornimmt. ${ }^{32}$ Die meisten PPI-Projekte können einer dieser Kategorien zugeteilt werden, aber deren Grenzen sind nicht immer klar und einige Projekte können unterschiedliche Eigenschaften aufweisen.

Bei dieser Analyse stellte sich heraus, wie aus der folgenden Tabelle 8 ersichtlich ist, dass Greenfield-Investitionen am häufigsten und Konzessionen am zweit häufigsten getätigt werden. Unter Greenfield-Investitionen fallen laut Weltbank BOT-Modelle, die in eine neue Infrastruktur investieren.

Tabelle 8: Art der PPI in den Sektoren Energie und Transport in der Region Ostasien und Pazifik, in Millionen US\$, für den Zeitraum von 1990-2005

\begin{tabular}{|l|r|r|r|r|r|}
\hline & Konzession & $\begin{array}{l}\text { Veräußerung } \\
\text { (Brownfield) }\end{array}$ & $\begin{array}{l}\text { Greenfield } \\
\text { Projekte }\end{array}$ & $\begin{array}{l}\text { Management- } \\
\text { und Leasing- } \\
\text { verträge }\end{array}$ & Gesamt \\
\hline Energie & 1.610 & 15.137 & 70.093 & 0 & 86.841 \\
\hline Transport & 11.288 & 6.161 & 31.531 & 1 & 48.981 \\
\hline Alle Sektoren & 39.154 & 45.099 & 140.273 & 22 & 224.548 \\
\hline
\end{tabular}

Quelle: PPI Database, http://ppi.worldbank.org/explore/ppi exploreRegion.aspx?regionID=2, 23.8.2007

In beiden Sektoren wurden BOT-Modelle am häufigsten angewendet, aber im Transportsektor spielen auch Konzessionen eine wichtige Rolle. Basierend auf diesen Daten wurde in beiden Sektoren nach BOT-Projekten gesucht.

32 Eine genauere Erklärung der Kategorisierung ist im Anhang zu finden.

33 Die Gesamtsumme stimmt nicht überein mit der Gesamtsumme der Tabelle 7. Diese Differenz basiert jedoch auf den zur Verfügung stehenden Daten der Weltbank und kann von der Autorin daher nicht korrigiert werden. 


\subsubsection{Aktualität der Fallstudien}

Die asiatische Finanzkrise Ende der 1990er Jahre hat das Investitionsklima in Asien nachhaltig beeinträchtigt. Durch die prekäre Finanzsituation waren private Infrastrukturinvestitionen Anfang des neuen Millenniums mit Finanzierungsengpässen, erhöhten Risiken und somit erhöhten Finanzierungskosten konfrontiert. Die Autorin war daher bemüht, aktuelle Fallstudien zu bekommen, die genau dieses wirtschaftliche Umfeld wiedergeben.

\subsubsection{Zugang zu den Daten}

Ein weiteres wesentliches Kriterium für die Auswahl der Fallstudien war der Zugang zu Daten bzw. die Gesprächsbereitschaft. Insbesondere im Bereich der Finanzienung sind Firmen oftmals nicht bereit, Daten preis zu geben. Für die vorliegende Arbeit wurde durch verschiedene Quellen versucht, Kontakte zu den Unternehmen und zu Angestellten der verschiedenen Finanzinstitutionen herzustellen. Dieses Kriterium war schlussendlich ausschlaggebend für die engere Auswahl der Projekte.

\subsubsection{Ergebnis des Auswahlprozesses}

Am Ende des Auswahlprozesses wurden insgesamt vier Fallstudien für die vorliegende Arbeit identifiziert. Es wurden jeweils zwei Fallstudien in Vietnam und auf den Philippinen gewählt. In Vietnam handelt es sich um zwei Gaskraftwerke und auf den Philippinen jeweils um ein Kohlekraftwerk und eine Schnellstraße. Da Vietnam und die Philippinen derzeit ein sehr konträres wirtschaftliches Umfeld ausweisen, wurden Projekte in diesen beiden Ländern gewählt. Die folgende Tabelle 9 gibt einen Überblick über die ausgewählten Projekte im jeweiligen Land.

Für den Umfang der Arbeit wäre es ausreichend gewesen, nur einen Sektor zu bearbeiten, nämlich den Energiesektor. Da aber das Straßenprojekt (North Luzon Expressway) insbesondere aus Finanzierungssicht interessant ist, wurde es zusätzlich ausgewählt.

In die Fallstudien fließen einerseits die durch Recherchearbeiten erhobenen Daten und andererseits die ausgewerteten Interviews ein. 
Tabelle 9: Übersicht über die ausgewählten Fallstudien

\begin{tabular}{|l|l|}
\hline Land: & Projekt: \\
\hline $\begin{array}{l}\text { Sozialistische Republik } \\
\text { Vietnam }\end{array}$ & Phu My 2.2 - Kraftwerk \\
\hline & Phu My 3 - Kraftwerk \\
\hline Republik der Philippinen & Steag State Power Inc. - Kraftwerk \\
\hline & Northern Luzon Expressway \\
\hline
\end{tabular}

\subsubsection{Aufbau der Fallstudien}

Die Fallstudien dienen dazu, die in den vorangegangen Kapiteln erarbeitete Literatur darzustellen. Es folgen nun zwei Kapitel (6.2 und 6.3) in denen das wirtschaftliche und politische Umfeld von Vietnam und den Philippinen prägnant vorgestellt wird. Daran anschließend werden die Fallstudien einzeln vorgestellt, welche die folgenden Punkte umfassen:

1. die Eckdaten des Projekts,

2. die Entstehung des Projektes,

3. die verwendeten Finanzierungs- und Absicherungsinstrumente,

4. die Darstellung der wesentlichen Verträge,

5. zusammenfassende Gegenüberstellung der Instrumente und der Vertragsstruktur und

6. die Auswirkungen auf die Armutsreduktion.

In den Gesprächen mit Angestellten der Internationalen Finanzinstitutionen (IFIs) und auch in der Literatur wurde immer wieder darauf hingewiesen, dass ein wesentlicher Schwachpunkt in der Diskussion des Zusammenhanges zwischen Infrastruktur und Armutsreduktion die fehlenden Baselines sind, die eine Voraussetzung für eine ex-post Evaluierung der Projekte darstellen. Zusätzlich dazu können die Auswirkungen von Kraftwerken, die in ein bestehendes Versorgungsnetz ihre Energie einspeisen, nicht direkt gemessen werden. In der vorliegenden Arbeit wird die Diskussion über die Auswirkungen der Infrastrukturinvestitionen auf Basis des in Kapitel 3.3.2.3 vorgestellten konzeptuellen Rahmens geführt. 


\subsection{Vietnam}

Das folgende Kapitel widmet sich der Darstellung von zwei Fallstudien in Vietnam. Beginnend mit einer Einführung wird Vietnam anhand von Eckdaten und der wirtschaftlichen Entwicklung dargestellt. Daran anschließend folgen die Beschreibungen der Fallstudien Phu My 2.2 und Phu My 3. Die vietnamesischen Fallstudien ähneln einander sehr stark, da sie im Rahmen eines großen Projektes verwirklicht worden sind, jedoch unterscheiden sie sich aufgrund der Eigentümerstruktur bei der Finanzierung und Absicherung. In einer Gegenüberstellung werden abschließend sowohl die Finanzierungs- und Absicherungsinstrumente als auch die Vertragsstruktur zusammengefasst. Aufgrund der großen Ähnlichkeit schließt dieses Kapitel mit einer gemeinsamen Diskussion, inwieweit die beiden Kraftwerke zur Armutsreduktion beitragen.

\subsubsection{Einführung}

\subsubsection{Eckdaten}

Fläche:

Einwohner:

Hauptstadt:

Staat:

Währung:

$331.114 \mathrm{~km}^{2}$

84.100 .000

Hanoi

Sozialistischer Staat seit 1980

1 Dong $(D)=100 \mathrm{Xu}$

Quelle: Von Baratta 2001: 857

Wirtschaft:

Wachstum des BIP: $\quad 2001: 6,9 \%$ 2003: $7,3 \%$ 2005: $8,4 \%$

Inflation: $\quad 2001:-0,4 \%$ 2003: $3,1 \%$ 2005: $8,3 \%$

Arbeitslosenrate: $\quad 2001: 6,3 \%$ 2003: $5,8 \%$ 2005: $5,1 \%$

Anstieg der $\quad 2001: 6,5 \%$

Warenexporte: $\quad 2003: 20,4 \%$

ADI Zufluss: $\quad 2001: 273$

in Millionen US\$ 2003: 1.222

2005: 1.914

Quelle: ADB 2006c 
Armut:

Bruttoinlandsprodukt pro Kopf:

HDI:

MDG 1:

$\%$ der Bevölkerung unter

$1 \$$ pro Tag

Quelle: UNDP 2006

Infrastrukturausstattung:

Haushalte mit 1998: 63

Stromanschluss in \% 2003: 81
2002: 430 US\$

2005: 620 US\$

2006: 0,709 (an 109. Stelle von 177

Ländern)

2003: $9,7 \%$

Quelle: ADB 2007b

\subsubsection{Wirtschaftliche Rahmenbedingungen}

\section{Wirtschaftspolitische Entwicklung}

In den vergangen zwei Jahrzehnten war Vietnam eines der am schnellsten wachsenden Länder in Asien und wird im gleichen Atemzug mit China genannt. Dieser Erfolg wird einer Serie von Wirtschaftsreformen zugerechnet, die Ende der 1980er Jahre durchgeführt wurden und die als „doi moi“ (Erneuerung) bekannt sind (vgl. Leproux/Brooks 2004). Begonnen wurde mit Reformen im Agrarsektor bis hin zu einer Liberalisierung des Außenhandels und der Investitionen (vgl. Balisacan et al. 2003). Diese Reformen haben in den vergangen Jahren zu einem durchschnittlichen Wirtschaftswachstum von 7 - $8 \%$ geführt. Durch die weit angelegten Reformen konnte auch die Armutsgrenze stark gesenkt werden und die Anzahl der unter der Armutsgrenze lebenden Vietnamesen hat sich drastisch reduziert, nämlich von 1993 bis 2004 von rund $58 \%$ auf 19,5 $\%$ (vgl. ADB 2006c: 232).

Der Übergang von einer zentralstaatlichen Planung hin zu einem offeneren, sozialistisch-orientierten Wirtschaftssystem hat auch die Liberalisierung der Märkte, die Anerkennung von privatem Eigentum und die schrittweise Öffnung des Marktes für ausländische Investoren mit sich gebracht (vgl. Leproux/Brooks 2004). Lange Zeit waren private Investitionen, insbesondere von ausländischen Unternehmen, unerwünscht. Da der private Sektor aber seit Anfang der 1990er Jahre wesentlich zum Wirtschaftswachstum beigetragen hat, werden seit geraumer Zeit auch die privaten Investitionen in den Infrastruktursektor durch die notwendigen rechtlichen Rahmenbedingungen gefördert (vgl. ADB 2006c: 232). 
Seit Jänner 2007 ist Vietnam nun auch Mitglied der WTO; dadurch wird die vietnamesische Wirtschaft immer stärker in die globale Wirtschaft integriert. Im Rahmen der Vorbereitungen für den WTO-Beitritt hat Vietnam bereits im Vorfeld viele Voraussetzungen für ein dauerhaftes Wirtschaftswachstum geschaffen und bietet für ausländische Investoren viele Anreize (vgl. Massmann 2007: xv). Vietnam gilt nach China als eines der attraktivsten Länder für ausländische Investoren. Gründe dafür sind die günstige geographische Lage, die reichhaltigen Rohstoffvorkommen, die stabile politische Lage sowie die trotz des stetig ansteigenden Lebensstandards weiterhin sehr niedrigen Lohnkosten (vgl. Massmann 2007: xv).

\section{Armutsreduktion}

In der folgenden Tabelle 10 werden Armutsreduktion und Wirtschaftswachstum gleichzeitig dargestellt. Es ist erkennbar, dass das Wirtschaftswachstum von Vietnam über dem durchschnittlichen Wachstum in der Region liegt, China jedoch das stärkste Wirtschaftswachstum aufweist. In Bezug auf Armutsreduktion ist allerdings Vietnam das erfolgreichste Land in dieser Region.

Tabelle 10: Armutsreduktion in Vietnam im Vergleich zu anderen asiatischen Entwicklungsländern

\begin{tabular}{|l|c|c|c|}
\hline Land & Zeitraum & $\begin{array}{c}\% \text { der } \\
\text { Armutsreduktion }\end{array}$ & $\begin{array}{c}\text { O Wirtschaftswachstum pro } \\
\text { Kopf in \% }\end{array}$ \\
\hline $\begin{array}{l}\text { Gesamt Ostasien } \\
\text { und Pazifik }\end{array}$ & $1993-98$ & $-2,0 \%$ & $5,6 \%$ \\
\hline Vietnam & $1993-98$ & $-\mathbf{4 , 1} \%$ & $\mathbf{6 , 8} \%$ \\
\hline Philippinen & $1994-97$ & $-1,3 \%$ & $\mathbf{1 , 9} \%$ \\
\hline Bangladesch & $1992-96$ & $-1,7 \%$ & $2,8 \%$ \\
\hline Kambodscha & $1994-97$ & $-1,0 \%$ & $2,6 \%$ \\
\hline China & $1993-98$ & $-2,5 \%$ & $10,4 \%$ \\
\hline Indien & $1992-97$ & $-1,4 \%$ & $3,8 \%$ \\
\hline Indonesien & $1990-96$ & $-2,1 \%$ & $6,4 \%$ \\
\hline Thailand & $1992-96$ & $-1,0 \%$ & $7,2 \%$ \\
\hline
\end{tabular}

Quelle: Balisacan et al. 2003: 1

Vietnam gilt heute als das Vorzeigeland für erfolgreiche Armutsreduktion und das Wirtschaftswachstum scheint wesentlich dazu beigetragen zu haben. Es ist davon auszugehen, dass die Wirtschaftsreformen (,doi moi') stark Pro-Poor ausgerichtet waren, da in der durchgeführten Studie von Balisacan et al. (2003: 14) das Einkommen der Armen durch das Wirtschaftswachstum stärker anstieg als jenes der wohlhabenden Bevölkerung. Die Ergebnisse von Vietnam deuten daher darauf hin, dass ein schnelles Wachstum zu einer geringeren Bedeutung von Umverteilungsmaßnahmen führt und die arme Bevölkerung direkt davon 
profitiert. Dennoch gibt es immer noch eine beträchtliche Menge an Menschen, die in Armut leben. Vor allem die Gruppe der extrem Armen hat bisher noch nicht ausreichend von den Reformen und dem Wirtschaftswachstum profitiert (vgl. Bhushan et al. 2002).

\section{Stromsektor}

Der vietnamesische Stromsektor ist extremen Herausforderungen ausgesetzt, da aufgrund des stetigen Wirtschaftswachstums auch die Energienachfrage steigt. Mit dem jährlichen Ansteigen der Nachfrage um ca. 15\% wird auch ein massiver Ausbau der Energieinfrastruktur benötigt. Laut World Bank (2006d: 3) muss sich die Energieversorgung im Zeitraum von 2006-2010 verdoppeln, um der steigenden Nachfrage nachzukommen. Die steigende Energieproduktion wird in der folgenden Abbildung 42 grafisch dargestellt, wobei mit einem weiteren Anstieg zu rechnen ist.

\section{Abbildung 42: Energieproduktion in Vietnam}

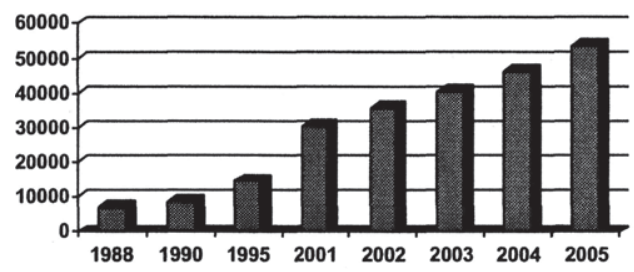

Quelle: ADB (2006b: 386)

Eine große Herausforderung stellt in diesem Zusammenhang die Finanzierung der benötigten Investitionen dar, da nur eine beschränkte Projektanzahl durch den öffentlichen Sektor finanziert werden kann. Lokale und internationale Unternehmen können in kleinere Kraftwerke mit BOO-Modellen investieren (vgl. ADB 2007c). Die einzigen bisher durchgeführten großen ausländischen BOT-Modelle stellen Phu My 2.2 und Phu My 3 dar. Diese großen Projekte werden als Independent Power Producers (IPPs) bezeichnet, wobei die Projektgesellschaft einen Vertrag mit der Regierung eingeht. Gleichzeitig zu den Veränderungen in der Finanzierung macht auch der Energiesektor momentan eine wesentliche Reform durch, bei der es zu einer Restrukturierung der Institutionen und zu einer Erhöhung des Wettbewerbs kommen soll (vgl. World Bank 2006d: 3). Gleichzeitig mit der erhöhten Energieproduktion muss es auch $\mathrm{zu}$ einer Ausweitung der Stromversorgung kommen, die aber in Vietnam in rein öffentlicher Hand liegt. Eine wesentliche Errungenschaft stellt in diesem Zusammenhang die Ausweitung der Stromversorgung im ländlichen Raum dar, 
wobei 2004 rund $88 \%$ der Haushalte mit Strom versorgt wurden (vgl. World Bank 2006d: 3).

Da die Energieversorgung eine wesentliche Voraussetzung für die Entwicklung des Industriesektors und des Wachstums darstellt, entschied die vietnamesische Regierung Mitte der 1990er Jahre, der Energieentwicklung hohe Aufmerksamkeit zukommen zu lassen. Im Rahmen des 5. „Power Master Development Plan“ wurde das „Phu My Power Generation Center" geplant, das fünf kombinierte Kraftwerke umfasst (vgl. ADB 2002: 1). Zu diesem Kraftwerkszentrum gehören auch die beiden Fallstudien (siehe Kapitel 6.2.2 und Kapitel 0). Da es Anfang 2005 während der Trockenzeit zu großen Energieengpässen und somit zu Stromausfällen kam, wurde eine stärke Diversifizierung der Stromproduktion diskutiert, da ein Großteil des Stromes in Vietnam von Wasserkraftwerken erzeugt wird (vgl. World Bank 2006d: 3). Im neuen Gesamtplan, dem mittlerweile sechsten „Power Master Development Plan“, wird diese neue Ausrichtung berücksichtigt und der Bau von neuen Kraftwerken für den Zeitraum von 2006-2015 geplant, wobei das jährliche Investitionsvolumen auf über 3 Milliarden US\$ geschätzt wird (vgl. World Bank 2006d: 5). In diesem Plan wurde wieder der private Sektor für die Finanzierung der Kraftwerke berücksichtigt.

\subsubsection{Phu My 2.2}

\subsubsection{Eckdaten}

Land:

Sozialistische Republik Vietnam

Projektname:

Phu My 2.2

Art der Investition:

Gaskraftwerk

Lage:

Region Vung Tau (BR-VT)

$70 \mathrm{~km}$ südöstlich von Ho Chi Minh City

Finanzierungsmodell: BOT-Modell

Laufzeit:

20 Jahre

Projektgesellschaft: Mekong Energy Company Ltd. (MECO)

Projektsponsoren: $\quad 56,25 \%$ EDF International (EDFI) (Frankreich)

$28,125 \%$ Sumitomo Corporation (Japan)

$15,625 \%$ Tokyo Electric Power Company (TEPCO)

Installierte

$715 \mathrm{MW}$ (Japan)

Antriebsleitung:

Phu My 2.2 stellt das erste BOT in Vietnam dar, das durch eine öffentliche Ausschreibung zustande gekommen ist. Es stellt ein sogenanntes DownstreamProjekt dar, da die Projektgesellschaft keinerlei Verbindung zu den Gaslieferanten hat. Die Ausschreibung wurde durch die Weltbank unterstützt, um die vietnamesische Regierung mit dem Prozess der Ausschreibung vetraut zu 
machen und um ein Fallbeispiel mit Vorbildcharakter zu realisieren. Heute dienen der Ausschreibungs- und Projektablauf von Phu My 2.2 als Mustervorlage für andere Kraftwerksprojekte. Ein wesentliches Ziel der öffentlichen Ausschreibung war auch das Erreichen eines möglichst günstigen Tarifes, durch den der Marktpreis für weitere Projekte festlegt werden kann.

Phu My 2.2 ist eines von fünf Kraftwerken im Rahmen des „Phu My Power Generation“- Komplexes, das 715 Mega Watt produziert. Das Kraftwerk ist für eine Betriebsdauer von 30 Jahren konzipiert und soll davon 20 Jahre durch die Projektgesellschaft MECO betrieben und anschließend an das vietnamesische Industrieministerium übergeben werden.

\subsubsection{Entstehung}

Ende 1997

Anfang 1999

Ende 2001

Ende 2002
Internationale Ausschreibung mit 5 Anbietern

Es wird das EDFI-angeführte Konsortium ausgewählt. BOT-Vertrag und Investmentlizenz werden unterzeichnet Finanzierungsdokumente werden unterzeichnet

Die Ausschreibung für das Phu My 2.2-Kraftwerk hat zeitgleich mit dem Beginn der asiatischen Finanzkrise stattgefunden und dadurch hat der Ausschreibungsprozess beinahe zwei Jahre gedauert. Insgesamt haben fün Anbieter bei der Ausschreibung teilgenommen und schließlich wurde das EDFI-angeführte Konsortium ausgewählt.

Wie bereits erwähnt, stellt Phu My 2.2 das erste BOT-Projekt mit internationaler Ausschreibung in Vietnam dar. Da in Vietnam bisher noch keine Erfahrungen im Bereich von privaten Investitionen in den Stromsektor gesammelt wurden, unterstützte die Weltbank den Verhandlungs- und Entscheidungsprozess. Die angespannte Finanzsituation im asiatischem Raum und die vietnamesische Unerfahrenheit bei BOT-Projekten haben dazu geführt, dass die Verhandlungen beinahe fünf Jahre gedauert haben. Insbesondere die Tarifverhandlungen haben viel Zeit in Anspruch genommen, da bisher nur öffentliche Unternehmen den Strom erzeugt haben und der Tarif daher nicht notwendigerweise kostendeckend war. In den Verhandlungen mussten Tarifkomponenten festgelegt werden, die im Rahmen von Abnahmeverträgen den Produzenten zugesichert wurden. Ziel war die Festlegung eines Tarifes, der sowohl von vietnamesischer Seite leistbar, aber auch gewinn- und kostendeckend ist. Erst Ende 2002, nach rund fünf Jahren Verhandlungen, wurden die Finanzierungsdokumente unterzeichnet und mit dem Bau des Kraftwerkes begonnen.

\subsubsection{Finanzierungs- und Absicherungsinstrumente}

MECO sicherte sich ein Gesamtkapital in der Höhe von 480 Mio. US\$, wobei rund 80 Mio. US\$ als Stand-by Finanzierung geplant und eine Nachschuss- 
verpflichtung auf einem 50:50 Verhältnis vereinbart wurde. Die Basiskosten belaufen 400 Mio. US\$ und weisen einen Verschuldungsgrad von 75:25 auf. Die gesamte Finanzierung inklusive der Stand-by Finanzierung hat einen Verschuldungsgrad von 70,8:29,2. Rund 20,8 \% des gesamten Fremdkapitals stammen von kommerziellen Kreditgebern und rund $50 \%$ von internationalen und nationalen Finanzinstitutionen. Das Fremdkapital besteht unter anderem aus zwei kommerziellen Banktranchen, die durch ADB und IDA abgesichert werden. Außerdem wurden Kredite von ADB, JBIC und Proparco gewährt.

\section{Finanzierung:}

Gesamtes FK: 70,8 \% Fremdkapital:

Basis FK: $75 \%$

Stand-by Fremdkapital

1. Tranche der kommerziellen

Kredite

(IDA PRG)

2. Tranche der kommerziellen 25 Mio. US\$

Kredite (ADB PRG)

ADB Kredit

JBIC Kredit

Proparco Kredit

Gesamtes EK: 29,2 \% Eigenkapital:

Basis EK: $25 \%$
50 Mio. US\$

150 Mio. US\$

40 Mio. US\$

\begin{tabular}{lc} 
Eigenkapitalbasis & 100 Mio. US\$ \\
Stand-by Eigenkapital & 40 Mio. US $\$$ \\
\hline Gesamtkapital (inkl. Stand-by & 480 Mio. US\$ \\
Kapital): & 80 Mio. US\$ \\
Stand-by Kapital & \\
\hline
\end{tabular}

40 Mio. US\$

75 Mio. US\$

Die Finanzierungs- und Absicherungsstruktur stellt eine typische Form einer parallelen Kofinanzierung dar (siehe Kapitel 5.1.3), wie es bei Infrastrukturprojekten aufgrund des großen Projektvolumens häufig vorkommt. Die beteiligten Institutionen verfolgen dabei folgende Interessen:

- Die Institutionen stammen aus dem Herkunftsland der Investoren (Proparco, JBIC) und wollen die heimischen Unternehmen bei Auslandsinvestitionen unter-stützen.

- Die Institutionen verfolgen entwicklungspolitische Interessen (IDA) und weisen einen regionalen Bezug $(A D B)$ auf.

Die einzelnen Finanzierungs- und Absicherungsinstrumente der beteiligten Institutionen sollen nun in alphabetischer Reihenfolge genauer vorgestellt. 


\section{Kredit: $\quad 50$ Mio. US\$}

Der Private Sector der ADB gewährt der Projektgesellschaft (MECO) einen Kredit. Da von der vietnamesischen Regierung keine Rückgarantie für die Finanzierung gewährt wurde und dadurch erhöhte Risiken für die Bank bestehen, sind die Kosten für diese privaten Kredite höher als für öffentliche Kredite.

Political Risk Guarantee (PRG):

\section{Mio. US\$}

Diese Garantie schützt die kommerziellen Kreditgeber vor einem Ausfall der Zins- und Kreditrückzahlungen aufgrund von poli-tischen Risiken. Diese Risiken können eine Kombination folgender Tatbestände sein: Vertragsbruch durch die Regierung, Konvertierungs- und Transferbeschränkungen durch Regierungsmaßnahmen, Konfiszierung von Anlagen und politische Unruhen. Die Vergabe von Garantien ist in der ADB an die gleichzeitige Vergabe von Krediten gebunden.

In diesem Fall agierte die ADB zum ersten Mal bei einer PRG als „Guarantor of record". Als solcher vergibt die $\mathrm{ADB}$ eine Garantie für den gesamten Betrag im Namen eines privaten Versicherungsunternehmens. Die PRG wurde ohne Rückgarantie der vietnamesischen Regierung vergeben.

Garantievereinbarung: Diese Vereinbarung regelt zwischen ANZ, als Stellvertreter für die beteiligten kommerziellen Banken, und der $\mathrm{ADB}$ die abgesicherten Risiken.

Garantiebeteiligungsvereinbarung:
Diese Vereinbarung regelt zwischen * ADB, * dem privaten Versicherer

Sovereign Risk Insurance Limited und

* der ANZ als Stellvertreter für die beteiligten kommerziellen Banken die Beteiligung der privaten Kreditversicherung. 
Partial Risk 75 Mio. US\$ für kommerziellen Kredit

Guarantee Diese Garantie schützt die kommerziellen Kreditinstitute vor (PRG): einem Ausfall der Zins- und Kreditrückzahlungen.

Die wesentlichen Risikokategorien, die durch diese Garantie abgesichert werden, umfassen Vertragsbruch durch die Regierung, Konvertierungs- und Transferbeschränkungen durch Regierungsmaßnahmen, Politische Force MajeureRisiken und Hemmnissen von Schlichtungsverfahren.

Garantievereinbarung: Diese Vereinbarung regelt zwischen dem Kreditgeber und der IDA die abge-sicherten Risiken.

Projektvereinbarung: MECO verpflichtet sich darin, den Umweltrichtlinien der Weltbank zu ent-sprechen.

Entschädigungsvereinbarung:
IDA

Unterstützung

(Technical

Assistance):
- unterstützte die 1. Phase von Phu My 2 als ein öffentliches Projekt

- unterstützte die technische Entwicklung in der 2. Phase von Phu My 2 als BOT-Modell (Phu My 2.2)

\section{JBIC Instrument:}

Kredit: $\quad 150$ Mio. US\$

JBIC stellt den größten Kreditgeber im Projekt dar.

\section{Proparco Instrument:}

Kredit: $\quad 40$ Mio. US\$

Die französische Proparco (Promotion et Participation pour la coopération économique) gewährt einen Kredit in der Höhe von 40 Mio. US\$ an MECO. 
Die Vergabe von Garantien an den privaten Sektor stellt einen relativ neuen Bereich für die IDA dar, da bisher nur an den öffentlichen Sektor Kredite und Garantien vergeben wurden. Da die IDA bei Phu My 2.2 ihre erste PRG vergeben hat, nimmt dieses Projekt in der Weltbank eine besondere Stellung ein. Auch für die ADB stellt Phu My 2.2 ein besonderes Projekt dar, da sie zum ersten Mal bei einer PRG als , Guarantor of record " aufgetreten ist.

Kommerzielle Kredite für Projekte in Entwicklungsländern sind aufgrund der involvierten Risken sehr teuer. Durch die gewährten Garantien und Kapitalengagement der Finanzinstitutionen wurde es der Projektgesellschaft ermöglicht, günstigere Kredite zu mobilisieren. Die gewährten Garantien stellen jedoch vielmehr einen „Deckmantel“ für die Beteiligung der IFIs dar, da sie nur selten eingelöst werden. Dies resultiert aus der Eigentümerstruktur der IFIs und deren Bedeutung für die Entwicklungsländer. Die Mehrzahl der Entwicklungsländer sind Miteigentümer der einzelnen Finanzinstitutionen, die für die Finanzierung der jeweiligen Projekte zur Verfügung stehen. Daher werden die Regierungen versuchen, ihre Verpflichtungen zu erfüllen.

Die folgende Abbildung 43 zeigt nochmals grafisch den Zusammenhang der verschiedenen Finanzierungs- und Absicherungsinstrumente bzw. die involvierten Institutionen im Phu My 2.2 Projekt.

\section{Abbildung 43: Finanzierungs- und Absicherungsstruktur von Phu My 2.2}

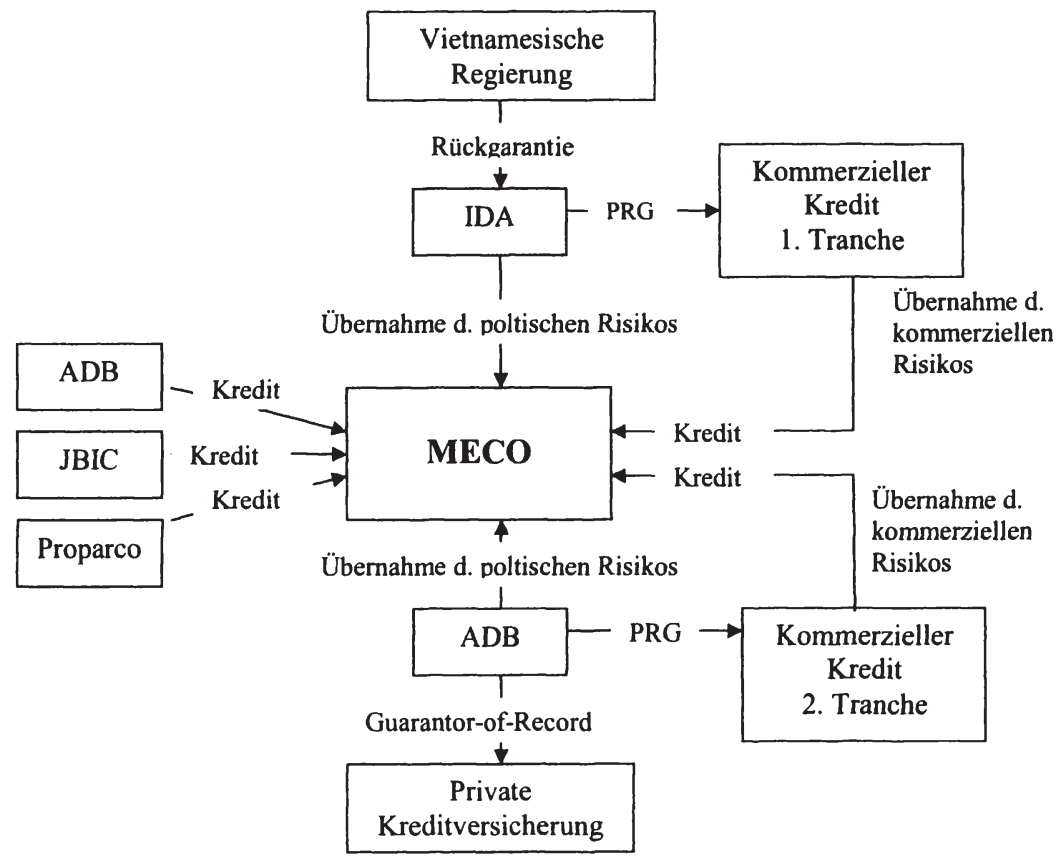




\subsubsection{Vertragsstruktur}

Die Vertragsstruktur bei Projektfinanzierungen ist sehr umfangreich. Daher wird in der folgenden Abbildung 44 zunächst das Vertragsnetz grafisch dargestellt. In dieser Übersicht werden nun zusätzlich zu den Verträgen mit den Finanzinstitutionen auch die Verträge mit den vietnamesischen und anderen privaten Unternehmen berücksichtigt. Im Anschluss daran werden die wichtigsten Verträge mit vietnamesischen und privaten Partnern etwas genauer vorgestellt. 


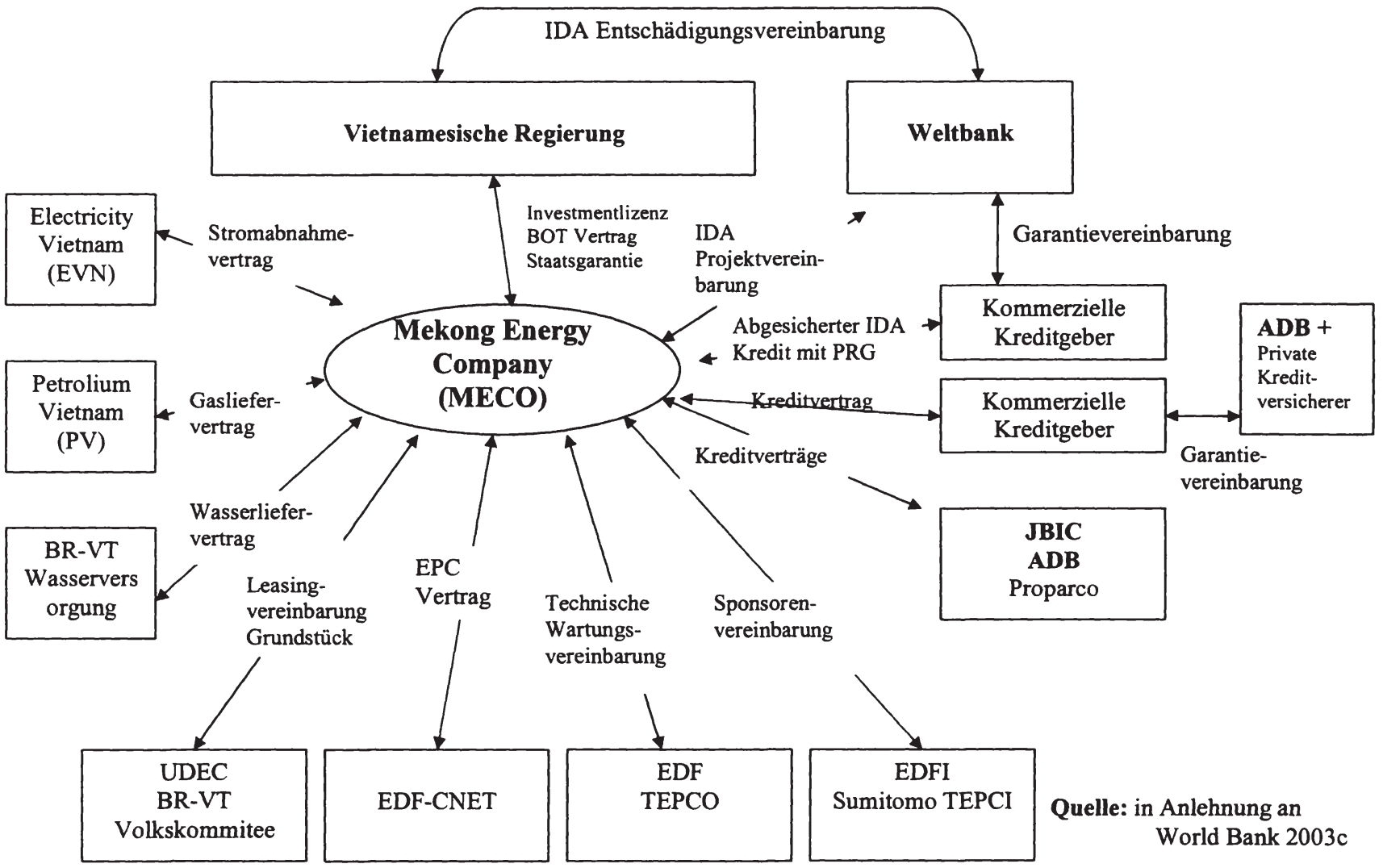


Investmentlizenz:

BOT-Vertrag:
Vertragspartner: Ministerium für Planung und Investition und MECO

Inhalt: Bedingungen für den Bau der Anlage und der Entstehung der Projektgesellschaft (MECO) werden festgelegt.

Vertragspartner: Industrieministerium und MECO

Inhalt: Rechte und Pflichten von MECO und dem Industrieministerium werden festgelegt.

$\rightarrow$ Das Industrieministerium garantiert MECO das exklusive Recht das Kraftwerk zu bauen, zu besitzen und zu betreiben.

$\rightarrow$ MECO wird nach Ablauf von 20 Betriebsjahren die Anlage kostenlos an das Industrieministerium übertragen.

Stromabnahmevertrag: Vertragspartner: EVN (Electricity of Vietnam) und MECO

Inhalt: Stromtarif und Strommenge werden festgelegt.

$\rightarrow$ Auf Basis eines Take-or-pay Vertrages garantiert EVN die Abnahme des Stroms zu einem fixierten Preis (US\$ 4,1 Cent/kWh).

$\rightarrow$ Der Tarif ist zweigeteilt und wird in Dong bezahlt.

$\rightarrow$ Ein Teil des Tarifs ist Dollarindexiert und der andere Teil berücksichtigt die Inflation.

Gasliefervertrag: Vertragspartner: PV (Petro Vietnam) und MECO

Inhalt: Gastarif und Gasmenge sind festgelegt.

$\rightarrow$ PV liefert sämtliches Gas für das Kraftwerk.

$\rightarrow$ MECO ist verpflichtet einen Minimum an Gas zu kaufen.

$\rightarrow$ Der Gaspreis ist Dollarindexiert und steigt jährlich um fixierte $2 \%$ p.a.

Wasserliefervertrag: Vertragspartner: lokale Wasserlieferant (BR-VT WSC) und MECO

Inhalt: Versorgung und Preis von Trink- und Industriewasser sind festgelegt. 
Leasingvereinbarung

für das Grundstück:

Staatsgarantie:
Vertragspartner: Urban Development Construction Company (UDEC) und MECO

Inhalt: Das Grundstück für den Bau des Kraftwerkes wird MECO kostenlos zur Verfügung gestellt.

Vertragspartner: Vietnamesische Regierung und MECO

Inhalt: Vietnamesische Regierung sichert MECO die Einhaltung aller Rechte und Verpflichtungen aus den Verträgen mit den vietnamesischen Partnern. Weiters schützt sie auch vor dem Konvertierungs- und Transferrisiko.

Wesentliche Verträge mit anderen privaten Unternehmen:

EPC Vertrag:

Vertrag über den

Betrieb und die

technische Wartung:
Vertragspartner: MECO und EDF-CNET

Inhalt: MECO beauftragt darin EDF-CNET mit der Erstellung eins schlüsselfertigen Kraftwerkes $\mathrm{zu}$ einem fixierten Preis und einem fixierten Termin.

Vertragspartner: MECO mit EDF und TEPCO Inhalt: MECO vereinbart mit den beiden Firmen die Unterstützung beim Betrieb und der Erhaltung der Anlage.

Vertragspartner: MECO und GE International

Langfristige

Inhalt: GE International ist der Hauptlieferant der Anlage und verpflichtet sich im Vertrag, MECO bis zur ersten Gesamtüberholung nach sechs Jahren technisch zu unterstützten.

\subsubsection{Phu My 3}

\subsubsection{Eckdaten}

Land:

Projektname:

Sozialistische Republik Vietnam

Art der Investition:

Lage:

Phu My 3

Gaskraftwerk

Region Vung Tau (BR-VT)

$70 \mathrm{~km}$ südöstlich von Ho Chi Minh City 
Finanzierungsmodell: BOT-Modell

BOT-Laufzeit:

Betriebslaufzeit:

Projektgesellschaft:
23 Jahre

20 Jahre

Phu My 3 BOT Power Company Limited. (PM3)

PM3 besteht aus 3 Anlagen:

1. dem Kraftwerk,

2. der Anlage, die zum frühzeitigen Transfer von der Projektgesellschaft gebaut wurde und

3. Infrastruktureinrichtungen in Vietnam.

Projektsponsoren: $\quad 331 / 3 \%$ BP Holding BV (Niederlande)

$331 / 3 \%$ SembCorp Industries (Singapur)

33 1/3 \% Kyuden International Corporation (Japan) und Sojitz Corporation (Japan)

Installierte

$716,8 \mathrm{MW}$

Antriebsleistung:

Das Phu My 3 Projekt ist ungefähr zeitgleich mit Phu My 2.2 entstanden und ist auch Teil des „Phu My Power Generation“-Komplexes. Phu My 3 ist ein Gaskraftwerk, das $716,8 \mathrm{MW}$ produziert. Der BOT-Vertrag ist auf 23 Jahre beschränkt und berücksichtigt auch den Bau des Kraftwerkes (vgl. ADB 2007c: 16). Die Betriebslaufszeit von Phu My 3 ist jedoch auf 20 Jahre begrenzt und nach Ablauf wird die Projektgesellschaft PM3 das Kraftwerk an die vietnamesische Regierung übergeben.

Phu My 3 ist ein sogenanntes Upstream Projekt, da BP die Gasvorkommen Nam Con Son fördert, die rund $40 \%$ der gesamten bekannten Gasvorkommen in Vietnam darstellen. Nach Abschluß des Gasfördervertrages begannen BP und die vietnamesische Regierung mit den Verhandlungen für das vereinbarte Kraftwerk, das BP zugesichert wurde. Durch die Förderung des Gases hat BP ein originäres Interesse am Bau von Phu My 3, nämlich die sichergestellte Abnahme des geförderten Gases. Ende 1998 akzeptierte das Industrieministerium die vorgelegte Durchführbarkeitsstudie von BP.

Da Phu My 3 durch ein privates Unternehmen, nämlich BP, initiiert wurde, obliegen viele Daten der Geheimhaltung. Da die Projektgesellschaft zusätzlich noch japanisch geführt wird, war PM3 nur äußerst beschränkt bereit Daten Preis zu geben. Dadurch mussten andere Beteiligte, wie beispielsweise Sponsoren, Lieferanten und IFIs, befragt werden oder Dokumente für die Beschreibung herangezogen werden. Insbesondere der Tarif obliegt strenger Geheimhaltungspflicht. Laut Massmann (2003: 11) wurden allerdings zwei Tarife für unterschiedliche Laufzeiten vereinbart, nämlich ein Tarif in der Höhe von 7,59 UScent/kWh für die ersten vier Jahre, und ein Tarif in der Höhe von 4,59 UScent/kWh für die restlichen 16 Jahre. Daraus ergibt sich ein Durchschnitts- 
tarif von 5 UScent $/ \mathrm{kWh}$, der um 0,9 UScent $/ \mathrm{kWh}$ über den Tarif von Phu My 2.2 liegt. Dieser Tarif wurde aber weder von der Projektgesellschaft noch von öffentlicher Seite bestätigt.

Die Ausschreibung von Phu My 2.2 erfolgte zeitlich erst später und die Projekte wurden anschließend parallel entwickelt. Durch die Auschreibung wurde sichergestellt, dass für Phu My 2.2 der möglichst geringste Tarif angesetzt wurde. Auf Basis dieses Benchmarks wurden auch die Verhandlungen mit Phu My 3 geführt.

\subsubsection{Entstehung}

In den frühen $1990 \mathrm{er}$ Jahren

1997

Dezember 1998

Große natürliche Gasvorkommen (Nam Con Son) wurden in Vietnam entdeckt. Diese stellen $40 \%$ der gesamten Gasreserven in Vietnam dar $\mathrm{BP}$ wurde als exklusiver Projektsponsor für Phu My 3 nominiert

Das Ministerium für Industrie (MOI) akzeptiert die Durchführbarkeitsstudie für PM3 auf Basis eines BOT-Konzepts

Januar 2001 Vietnamesische Regierung akzeptiert die Tarife Mai 2001

November 2001

März 2004 BOT Verträge und Investitionslizenz werden unterzeichnet

Baustart

Start des kommerziellen Betriebes

Phu My 3 ist gleichzeitig zum Phu My 2.2 von der vietnamesischen Regierung verhandelt worden, jedoch wurde der Bau dieses Kraftwerkes BP bereits 1997 zugesichert. Durch den Wegfall des Ausschreibungsprozesses beanspruchte die Verhandlung der Verträge weniger Zeit als bei Phu My 2.2. Insbesondere für die Tarifverhandlungen war der Entstehungsprozess von Phu My 2.2 auch ausschlaggebend für Phu My 3, da durch den Ausschreibungsprozess der Marktpreis für Strom von Gaskraftwerken festgelegt wurde.

BP, ein mutinationales und finanzkräftiges Unternehmen, hat die Führung in der Strukturierung des Projektes übernommen. Aufgrund der eigenen Finanzstärke von BP, konnte sofort mit der Planung und dem Bau des Projektes begonnen werden. Erst zu einem späteren Zeitpunkt wurde mit potenziellen Projektpartnern verhandelt und die Finanzierung durch kommerzielle Banken und IFIs geregelt. Dies hat zu einer verkürzten Projektdauer geführt.

\subsubsection{Finanzierung- und Absicherungsinstrumente}

Phu My 3 benötigte insgesamt ein Gesamtkapital von 385,9 Mio. US\$, bei einem Verschuldungsgrad von 75:25. Rund 47,2 \% des Fremdkapitals wurden von internationalen Finanzinstitutionen zur Verfügung gestellt. Die kommer- 
ziellen Kredite werden zu einem Großteil durch Garantien der ADB und MIGA vor politschem Risiko gesichert. Auch das Privatkapital wird beinahe zur Hälfte durch eine politische Garantie der MIGA geschützt.

Finanzierung:

$75 \%$

Fremdkapital:

ADB Kredit

37,5 Mio. US\$

JBIC Kredit

99,0 Mio. US\$

Bankenkonsortium

152,9 Mio. US\$

$25 \%$

(ADB + MIGA PRG)

Eigenkapital:

(MIGA PRG)

96,5 Mio. US\$

Gesamtkapital:

385,9 Mio. US\$

Die Finanzierungs- und Absicherungsstruktur weist auch wie bei Phu My 2.2 (siehe Kapitel 6.2.2.3) eine parallele Kofinanzierung auf. Jedoch beteiligte sich keine europäische Finanzinstitution an der Finanzierung des Projektes. Lediglich JBIC weist einen nationalen Bezug zu einem der drei Sponsoren auf. Die einzelnen Finanzierungs- und Absicherungsinstrumente der beteiligten Institutionen werden nun in alphabetischer Reihenfolge genauer vorgestellt.

\section{ADB Instrumente:}

Kredit:

37,5 Mio. US\$

Der "Private Sector" der ADB gewährt der Projektgesellschaft (PM3) einen Kredit ohne dafür eine Regierungsgarantie zu verlangen. Durch den Wegfall der Regierungsgarantie entstehen erhöhte Risiken für die Bank, die daher diese privaten Kredite zu ungünstigeren Konditionen vergibt als öffentliche Kredite.

Political Risk

Guarantee (PRG):
30 Mio. US\$

Diese Garantie wurde zu Gunsten von folgenden fünf kommerziellen Kreditgebern ausgestellt: Credit Agricole Indosuez, Crédit Lyonnais S.A., Fortis Bank S.A./N.V., Mizuho Corporate Bank Ltd., Bank of Tokyo-Mitsubishi Ltd.

Es wird ein Kredit in der Höhe von 30 Mio. US\$ gegen eine Kombination folgender Tatbestände abgesichert: Vertragsbruch durch die Regierung, Konvertierungs- und Transferbeschränkungen durch Regierungsmaßnahmen, Konfiszierung von Anlagen und politische Unruhen. Die Vergabe 
von Garantien ist in der ADB an die gleichzeitige Vergabe von Krediten gebunden.

Garantievereinbarung: Diese Vereinbarung regelt zwischen ADB und den kommerziellen Banken die abgesicherten Riken.

JBIC Instrument:

Kredit: $\quad 99$ Mio. US\$

JBIC hat der Projektgesellschaft einen Kredit gewährt und stellt den größten Kreditgeber dar.

MIGA Instrumente:

Political Risk

Guarartee:
43,2 Mio. US\$ für Eigenkapital

75 Mio. US\$ für Fremdkapital

15 Mio. US\$ zur Abdeckung eines Finanzierungsswaps, der von Calyon durchgeführt wurde

Die Garantie von MIGA sichert sowohl Teile des Eigen- als auch des Fremdkapitals ab und schützt vor folgenden Tatbeständen: Transferrisiko, Enteignung, Krieg, Unruhen, Terrorismus und Vertragsbruch durch die Gastregierung.

Garantievereinbarung: Diese Vereinbarung regelt zwischen den jeweiligen Parteien und MIGA die abgesicherten Risiken.

NEXI Instrument:

Garantie:

Information aufgrund von Datenschutz nicht verfügbar.

Bei Phu My 3 wurden von den Finanzinstitutionen ausschließlich klassische Kredite und Garantien vergeben. In diesem Projekt ist die ADB wieder mit einem Kredit und einer Garantie involviert. Eine Besonderheit der ADB stellt bei der Vergabe von Garantien die gleichzeitige Koppelung an die Vergabe von Krediten dar. Dies bedeutet, dass nur dann Garantien an ein Projekt vergeben werden, wenn die ADB auch gleichzeitig einen Kredit für dieses Projekt vergibt und eine sogenannte Ankerinvestition aufweist.

Bei Phu My 3 ist nicht direkt die Weltbank, sondern die MIGA als Teil der Weltbankgruppe beteiligt. Sie vergibt in diesem Projekt sowohl Garantien für das Fremd- als auch für das Eigenkapital. Eine Besonderheit stellt für die MIGA in diesem Projekt die Vergabe einer Garantie für die Absicherung des Zins- 
änderungsrisikos dar. Da die kommerziellen Kredite der Projektgesellschaft auf Basis einer variablen Verzinsung gewährt wurden, sieht sich das Unternehmen mit einem potenziellen Finanzrisiko konfrontiert. Insbesondere im Stromsektor stellen die sich verändernden Kapitalkosten aufgrund von Zinsschwankungen ein wesentliches Risiko dar, da das Unternehmen die Erlöse im Rahmen des Abnahmevertrages bereits fixiert hat und dadurch verändernde Zinsschwankungen im Verkaufspreis nicht berücksichtigen kann. Aus diesem Grund hat die Projektgesellschaft PM3 einen Interest Rate Swap gewählt, mit dem sie das Zinsänderungsrisiko absichern kann. Die MIGA schützt den Anbieter des Swaps (Calyon) vor Verlusten im Swapgeschäft, die aus den abgesicherten politischen Risiken resultieren. Durch diese Garantie wurde die Verwendung eines Instrumentes zur Absicherung der Zinsänderungen ermöglicht, das in Entwicklungsländern aber noch nicht so häufig Anwendung findet.

NEXI steht für „Nippon Export and Investment Insurance“ und ist die staatliche japanische Kreditversicherung. NEXI erscheint in Dokumenten der Weltbank und anderen Finanzinstitutionen mehrmals als Garantiegeber für Phu My 3 , jedoch wollte die Projektgesellschaft aufgrund von Datenschutzgründen die Höhe und den Garantieumfang nicht bekannt geben. In den folgenden Abbildungen 45 und 46 wird NEXI daher berücksichtigt, aber die fehlende Information ist durch die gestrichelte Linie gekennzeichnet.

Die nun folgende Abbildung 45 zeigt nochmals grafisch den Zusammenhang der verschiedenen Finanzierungs- und Absicherungsinstrumente bzw. die involvierten Institutionen im Phu My 3-Projekt.

\section{Abbildung 45: Finanzierungs- und Absicherungsstruktur von Phu My 3}

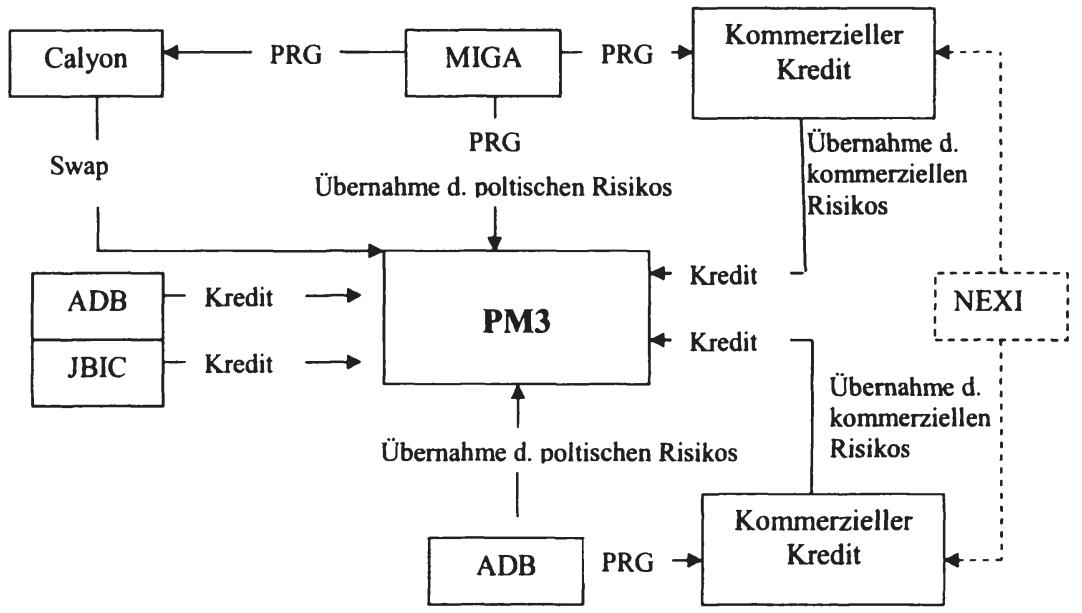




\subsubsection{Vertragsstruktur}

Aufgrund der umfangreichen Vertragsstruktur wird zunächst in Abbildung 46 das Vertragsnetz grafisch und im Anschluss daran die wichtigsten Verträge etwas genauer dargestellt. In dieser Übersicht werden nun zusätzlich zu den Veträgen mit den Finanzinstitutionen auch die Verträge mit den vietnamesischen und anderen privaten Unternehmen berücksichtigt. 
Abbildung 46: Projektbeteiligte Phu My3

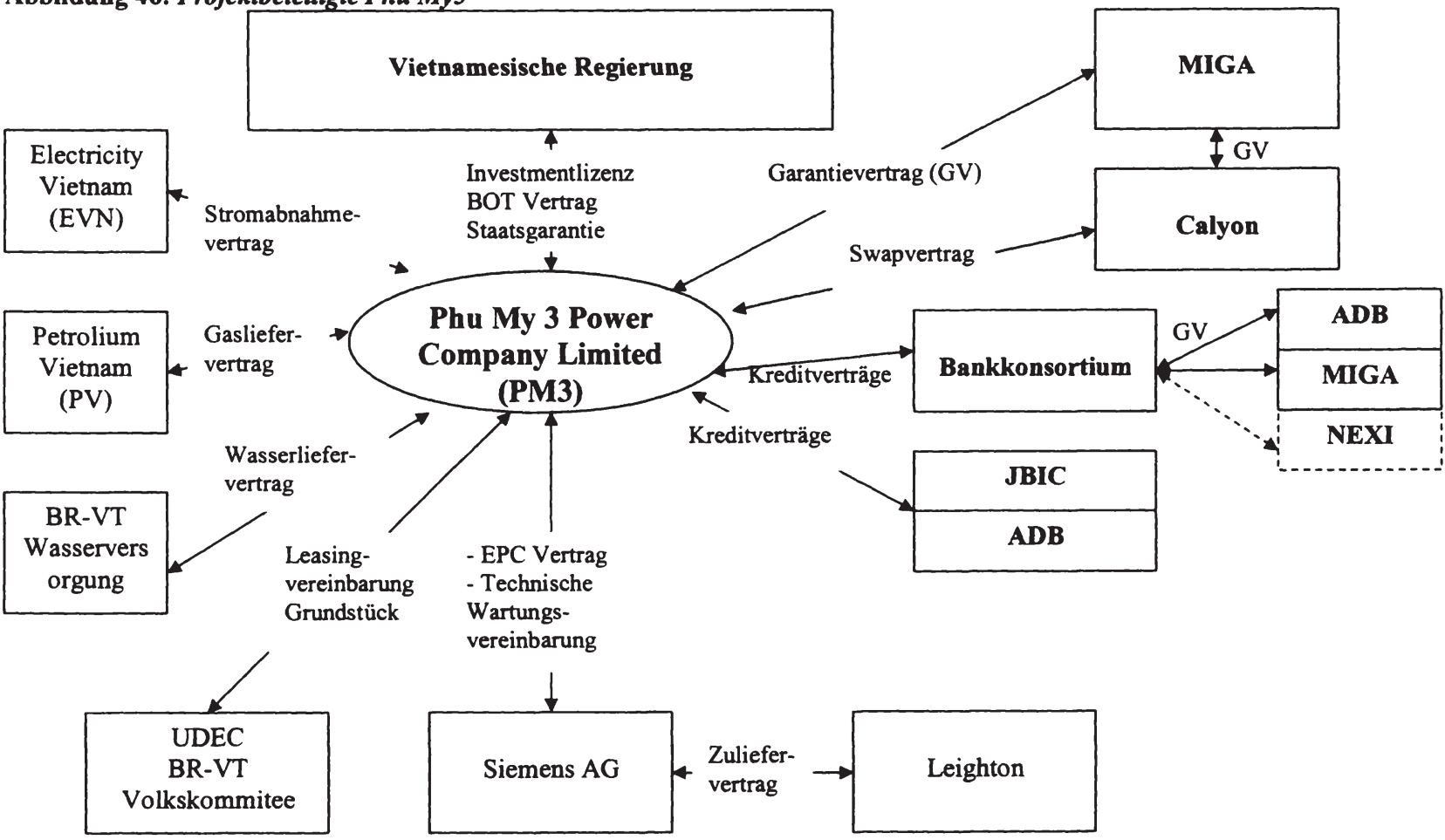


Investitionslizenz:

BOT-Vertrag:

Stromabnahmevertrag: Vertragspartner: EVN (Electricity of Vietnam) und PM3

Inhalt: Stromtarif und Strommenge werden festgelegt.

$\rightarrow$ Auf Basis eines Take-or-pay Vertrages garantiert EVN die Abnahme des Stromes zu einem fixierten Preis.

Die Tarifhöhe ist aufgrund von Datenschutz nicht verfügbar.

$\rightarrow$ Der Tarif ist zweigeteilt und wird in Dong bezahlt.

$\rightarrow$ Ein Teil des Tarifs ist Dollarindexiert und der andere Teil berücksichtigt die Inflation.

Gasliefervertrag: $\quad$ Vertragspartner: PV (Petro Vietnam) und PM 3 Inhalt: Gastarif und Gasmenge sind festgelegt.

$\rightarrow$ PV liefert das gesamte Gas für das Kraftwerk. Das Gas stammt vom Nam Con Son Gas Basin Block 6.1, das PV gemeinsam mit BP und Oil and Natural Gas Corporation Ltd. gehört.

$\rightarrow$ Die Gaslieferung basiert auf einem 20jährigen Gas-liefervertrag auf Basis eines take-or-pay Vertrages.

$\rightarrow$ Der Gaspreis ist ein reiner Durchlaufposten unter dem Stromabnahmevertrag, wobei EVN 
(Electricity of Vietnam) einen Tarif zahlt, der den Gaspreis berücksichtigt.

Wasserliefervertrag: Vertragspartner: lokale Wasserlieferant (BR-VT WSC) und PM3

Inhalt: Versorgung und Preis von Trink- und Industrie-wasser sind festgelegt.

$\rightarrow$ Der Preis ist nicht indexiert und es wird der allgemeine Tarif verrechnet.

Leasingvereinbarung für das Grundstück:

Staatsgarantie:
Vertragspartner: Urban Development Construction Company (UDEC) und PM3

Inhalt: Das Grundstück für den Bau des Kraftwerkes wird PM3 kostenlos zur Verfügung gestellt.

Vertragspartner: Vietnamesische Regierung und PM3

Inhalt: Das Ministerium für Planung und Investition (MPI) garantiert im Namen der Regierung die Erfüllung aller Pflichten und Verbindlichkeiten der vietnamesischen Partner von PM3.

$\rightarrow$ Diese Garantie beinhaltet den BOT-Vertrag, den Stromabnahmevertrag, den Gasliefervertrag, den Wasserliefervertrag und die Leasingvereinbarung.

$\rightarrow$ Weiters schützt die Garantie auch vor Konvertierungs- und Transferrisiko.

$\rightarrow$ Die Garantie genehmigt off-shore Konten für die Projektfinanzierung und garantiert, dass die rechtlichen und steuerlichen Rahmenbedingungen, auf die man sich geeinigt hat, während der PM3-Laufzeit gültig und stabil bleiben.

Hauptverträge mit anderen privaten Unternehmen:

EPC Vertrag:

Vertragspartner: Siemens AG und PM3

Inhalt: PM3 beauftragt darin die Siemens AG mit der Erstellung eines schlüsselfertigen Kraftwerkes zu einem fixierten Preis und einem fixierten Termin. 
Zuliefervertrag:

Langfristige

Wartungsvereinbarung:
Vertragspartner: Siemens AG und Leighton

Inhalt: Zwischen den Vertragspartnern besteht ein Zuliefervertrag über die Durchführung der Bauarbeiten. Der Bau startete im November 2001 und das Projekt wurde im November 2003 fertig gestellt.

Vertragspartner: Siemens AG und PM3

Inhalt: Die Siemens AG bietet auch die Wartungs-dienstleistungen an.

\subsubsection{Zusammenfassende Gegenüberstellung von Phu My 2.2 und Phu My 3}

In dieser nun folgenden zusammenfassenden Gegenüberstellung werden zuerst die Finanzierungs- und Absicherungsinstrumente und daran anschließend die Vertragsstruktur diskutiert.

\section{Finanzierungs- und Absicherungsinstrumente}

Phu My 2.2 und Phu My 3 stellen die ersten privat finanzierten Kraftwerke in Vietnam dar. Beide Projekte befinden sich im selben Kraftwerkskomplex und wurden ungefähr zeitgleich entwickelt. Ein wesentlicher Unterschied zwischen diesen beiden Projekten stellt die Berechtigung zum Bau des Kraftwerkes dar. Phu My 2.2 ist ein reines Down Stream-Projekt, das keinen Bezug zur Rohstoffquelle besitzt und die Projektgesellschaft durch einen Ausschreibungsprozess ausgewählt wurde. Phu My 3 ist hingegen ein Up Stream-Projekt, da die Kernkompetenz von BP in der Förderung von Gas liegt. Im Rahmen des Vertrages zwischen der vietnamesischen Regierung und BP zur Förderung von Gas wurde auch vereinbart, dass BP Investitionen tätigen kann, welche BP die Abnahme des geförderten Gases sichern. Aufgrund der unterschiedlichen Bauberechtigung und Motivation der Investoren dauerte der Entstehungsprozess der Projekte unterschiedlich lange und es wurden verschiedene Institutionen für die Unterstützung herangezogen. Die folgende Tabelle 11 soll die Finanzierung und Absicherung der beiden Projekte nochmals zusammenfassen und daran anschließend werden die Gemeinsamkeiten und Unterschiede in der Finanzierung und Absicherung diskutiert.

Unter institutionellen Krediten wird jenes Fremdkapital subsumiert, das von Entwicklungsbanken oder Exportagenturen der Projektgesellschaft zur Verfügung gestellt wird. Diese Institutionen unterstützen oftmals sowohl den öffentlichen als auch den privaten Sektor. Die Vergabe von Krediten an den öffentlichen Sektor in Entwicklungsländern erfolgt unter den Marktkosten, da sie den Regierungen im Sinne der Entwicklungshilfe eine günstige Finanzierung an- 
bieten. Diese Form des Kredites wird auch als ODA-Kredit ${ }^{34}$ bezeichnet. An den Privatsektor werden hingegen Kredite zu kommerziellen Konditionen gewährt. Der Vorteil eines Kredites von einer multilateralen Bank liegt daher nicht notwendigerweise in den reduzierten Finanzierungskosten, sondern in der automatischen Sicherstellung durch deren Beteiligung aufgrund ihres sogenannten "preferred creditor status ".

Tabelle 11: Zusammenfassung der Finanzierung und Absicherung von Phu My 2.2 und Phu My 3

\begin{tabular}{|c|c|c|c|}
\hline \multicolumn{2}{|c|}{ 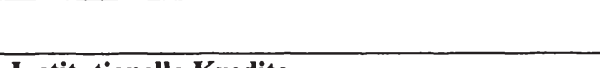 } & Phu My 2.2 & Phu My 3 \\
\hline \multicolumn{4}{|c|}{ Institutionelle Kredite } \\
\hline \multicolumn{2}{|l|}{ ADB } & 50,0 Mio. US\$ & 37,5 Mio. US\$ \\
\hline \multicolumn{2}{|l|}{ JBIC } & 150,0 Mio. US\$ & 99,0 Mio. US\$ \\
\hline \multicolumn{2}{|l|}{ Proparco } & 40,0 Mio. US $\$$ & \\
\hline \multicolumn{2}{|c|}{\begin{tabular}{l|l} 
& Gesamtsumme Institutionelle Kredite \\
\end{tabular}} & 240,0 Mio. USS & 136,5 Mio. USS \\
\hline \multicolumn{2}{|c|}{ Kommerzielle Kredite } & 100,0 Mio. US\$ & 152,9 Mio. US\$ \\
\hline \multicolumn{2}{|c|}{ Gesamtes Fremdkapital (FK) } & 340,0 Mio. USS & 289,4 Mio. USS \\
\hline \multicolumn{2}{|c|}{ Kommerzielles : Institutionelles FK } & $29,4: 70,6$ & $52,8: 47,2$ \\
\hline \multicolumn{2}{|c|}{$\%$-Anteil von JBIC am Institutionellen FK } & $62,5 \%$ & $72,5 \%$ \\
\hline \multicolumn{2}{|l|}{ Garantien } & & \\
\hline$\overline{\mathrm{ADB}}$ & $\begin{array}{l}\text { Political Risk Garantie } \\
\text { Guarantor of record }\end{array}$ & 25,0 Mio. US\$ & 30,0 Mio. US\$ \\
\hline & Partial Risk Garantie & 75,0 Mio. US\$ & \\
\hline MIGA & $\begin{array}{l}\text { Political Risk Garantie } \\
\text { für Eigenkapital } \\
\text { für Fremdkapital } \\
\text { für Abdeckung eines Swaps }\end{array}$ & & $\begin{array}{l}\text { 43,2 Mio. US\$ } \\
75,0 \text { Mio. US\$ } \\
15,0 \text { Mio. US\$ }\end{array}$ \\
\hline NEXI & $\begin{array}{l}\text { Overseas United Loan } \\
\text { Insurance }\end{array}$ & & $\begin{array}{l}\text { Summe ist nicht } \\
\text { bekannt }\end{array}$ \\
\hline $\begin{array}{l}\text { Bundesrepublik } \\
\text { Deutschland }\end{array}$ & DiA & & \\
\hline \multicolumn{2}{|c|}{ Gesamtsumme Garantien } & 100,0 Mio. USS & 163,2 Mio. USS \\
\hline \multirow{2}{*}{\multicolumn{2}{|c|}{$\begin{array}{l}\text { Gesamtsumme Eigenkapital (EK) } \\
\text { \%-Deckung des kommerziellen FK }\end{array}$}} & 140,0 Mio. US4 & 96,5 Mio. US\$ \\
\hline & & $100 \%$ & $59 \%$ \\
\hline \multicolumn{2}{|c|}{ \%-Deckung des institutionellen FK } & $0 \%$ & $0 \%$ \\
\hline \multirow{2}{*}{\multicolumn{2}{|c|}{$\begin{array}{l}\text { \%-Deckung des gesamten FKs } \\
\text { \%-Deckung des Eigenkapitals }\end{array}$}} & $29,4 \%$ & $56,4 \%$ \\
\hline & & $0 \%$ & $77,7 \%$ \\
\hline
\end{tabular}

JBIC ist in beiden Projekten der dominanteste institutionelle Geldgeber. Bei Phu My 2.2 stammen rund 62,5\% und bei Phu My 3 rund 72,5\% der institutionellen Kredite von JBIC. In beiden Projekten sind japanische Firmen an der Projekt-

34 Official Development Assistance, siehe dazu Kapitel 4.2.2 
gesellschaft oder am Bau beteiligt. Japan verfolgt daher bei der Unterstützung ein wirtschaftliches Interesse.

Die ADB ist in beiden Projekten mit Krediten und Garantien involviert, wobei sie bei Phu My 2.2 erstmals als Guarantor of record fungierte. Insgesamt übernehmen daher ADB und JBIC in beiden Projekten die wichtigste Finanzierungsrolle. Aufgrund der französischen Beteiligung in Phu My 2.2 wurde das Projekt auch von französischer Seite durch Proparco unterstützt.

Eine Besonderheit bei Phu My 2.2 stellt die Unterstützung der Weltbank dar, da diese bisher nur den öffentlichen Sektor unterstützt hat. Da die Weltbank aber ein originäres Interesse hat, das sozialistische Regime in Vietnam zu öffnen und private Investitionen in die Infrastruktur zu ermöglichen, unterstützte die IDA Phu My 2.2 beim Ausschreibungsverfahren und vergab zusätzlich eine Garantie gegen politische Risiken, die aber in der IDA als Partial Risk Guarantee bezeichnet wird. Wichtig im Rahmen der Unterstützung des Ausschreibungsverfahrens war auch die Erarbeitung der notwendigen Vertragsstruktur, die für Folgeprojekte herangezogen werden kann. Wesentliches Ziel der öffentlichen Ausschreibung war aber auch die Festlegung eines Marktpreises für den Strom. Auf Basis dessen konnte auch der Preis für Phu My 3 und weiterer Krafwerke diskutiert werden.

Phu My 3 wurde von BP ohne Ausschreibungsverfahren durchgeführt, da die vietnamesische Regierung BP bereits das Baurecht für das Kraftwerk im Rahmen der Förderung von Gasvorkommen zugesprochen hatte. Die Weltbankgruppe vergab, vertreten durch die MIGA, an Phu My 3 Garantien für drei verschiedene Bereiche. Eine Besonderheit bzw. Novum stellt dabei die Vergabe einer Garantie für die Deckung eines Zinsenswaps dar. Auch NEXI hat Phu My 3 mit Garantien unterstützt, jedoch sind aufgrund von Datenschutz Haftungsund Deckungsumfang nicht bekannt.

Es kann daher festgehalten werden, dass sich die Finanzierungsstruktur dieser beiden Projekte stark ähnelt, die Instrumente aber unterschiedlich sind. Von den Finanzinstitutionen wurden Phu My 2.2 insgesamt ca. 100 Mio. US\$ mehr Fremdkapital gewährt als Phu My 3, wobei 40 Mio. US\$ als Stand-by Kapital eingeräumt wurden. Wesentliche Gründe für diesen Unterschied könnten die starke Finanzkraft von BP und deren führende Rolle bei der Strukturierung der Projektgesellschaft und der Finanzierung des Projektes sein. Die Strukturierung der Finanzierung hat im Fall von Phu My 3 gleichzeitig mit der Planung und Beginn der Bauphase begonnen. Wohingegen für Phu My 2.2 die Finanzierung des Projektes ausschlaggebend für die Realisierung war und diese erst nach fünf Verhandlungsjahren feststand. Zusätzlich zur Pionierrolle von Phu My 2.2 wurde die Strukturierung der Finanzierung durch die asiatische Finanzkrise erschwert und eine hohe institutionelle Unterstützung notwendig.

Durch das bereits hohe direkte Kapitalengagement der IFIs und einem vergleichsweise geringem Engagement der kommerziellen Banken wurde bei Phu 
My 2.2 auch eine geringere Summe an Garantien benötigt. Jedoch werden die kommerziellen Kredite bei Phu My 2.2 zu $100 \%$ von Garantien gedeckt. Für das Eigenkapital wurden bei Phu My 2.2 hingegen keine Garantien vergeben.

Bei Phu My 3 werden rund 59 \% der kommerziellen Kredite mit Garantien abgedeckt. Jedoch ist die Höhe der NEXI-Garantie nicht bekannt und daher kann kein Rückschluß darauf gezogen werden, wie hoch die wahre Deckung der kommerziellen Kredite ist. Dazu wird von der MIGA-Garantie auch der Zinsswap mit Calyon abgesichert. Zusätzlich zur Deckung des Fremdkapitals werden $77,7 \%$ des Eigenkapitals mit einer Garantie gegen politisches Risiko abgesichert.

Auch wenn die Finanzierungs- und Absicherungsstruktur in beiden Projekten sehr ähnlich ist, wurden dennoch von jeweils unterschiedlichen Institutionen neue Instrumente angeboten. In Phu My 2.2 stellt die Vergabe einer Garantie zur Absicherung des politischen Risikos von der IDA eine Besonderheit dar, da der Kernfokus dieser Institution im öffentlichen Bereich liegt und sie auch laut Statuten nur äußerst beschränkt den privaten Sektor unterstützen darf. Auch die ADB hat durch die Rolle als Guarantor of record im Rahmen einer politischen Garantie eine neue Form der Unterstützung gewählt. Bei Phu My 3 stellt die Vergabe einer politischen Garantie für einen Zinsswap eine bisher noch nicht gewählte Form der Unterstützung für die MIGA dar.

\section{Vertragsstruktur}

Die Vertragsstruktur der beiden Kraftwerke gleicht sich stark, was darauf zurückgeführt werden kann, dass sie parallel verhandelt worden ist. Im Wesentlichen wird nun auf die folgenden vier Punkte eingegangen:

\section{Die Vereinbarung von Take-or-pay Abnahmeverträgen.}

Eine ausschlaggebende Komponente für die Finanzierung von Kraftwerken durch den privaten Sektor und durch kommerzielle Banken stellen Abnahmeverträge dar, da bei Projektfinanzierungen die zukünftigen CashFlows für die Bewertung des Projektes herangezogen werden. Darin wird in den vorliegenden beiden Fallstudien der jeweiligen Projektgesellschaft die fixe Abnahme von Strom zu einem im Vorhinein fixierten Betrag vom Industrieministerium zugesichert. Besonders bedeutend für die privaten Investoren ist in diesen Verträgen die Dollarindexierung, die ihnen unabhängig von Wechselkursveränderungen einen stabilen Abnahmepreis garantiert. Kritisch werden jedoch die Auswirkungen dieser Abnahmeverträge für die Entwicklungsländer gesehen, wenn es zu einer starken Inflation bzw. zu einer Finanzkrise kommt. Genau vor solchen Situationen werden die privaten Investoren durch die Abnahmeverträge jedoch geschützt. Die Bindung an Abnahmeverträge kann aber auf öffentlicher Seite 
zu einem destabilisierenden Teufelskreis beitragen, da Infrastrukturleistungen einen wesentlichen Beitrag zur Stabilität leisten, aber deren Fehlen oder Ausbleiben aufgrund von Zahlungsschwierigkeiten gravierende wirtschaftliche Auswirkungen haben. Auch die Zusicherung von Staatsgarantien für die Einhaltung der Abnahmeverträge bietet bei Finanzkrisen keinen Schutz, da zahlungsunfähige Regierungen, wie bei den Finanzkrisen in Lateinamerika und Asien, ihre Schulden nicht mehr begleichen können.

\section{Die Rolle des Liefervertrages:}

Die Lieferung von Gas ist in Vietnam an das staatseigene Unternehmen Petro Vietnam (PV) gebunden. Bei Phu My 2.2 wurde im Liefervertrag die Abnahme einer Mindestmenge zu einem fixierten Betrag vereinbart. Bei Phu My 3 wurde hingegen ein Take-or-Pay-Vertrag zwischen der Projektgesellschaft (PM3) und PV vereinbart. Für PM3 stellen die Gaskosten jedoch einen reinen Durchlaufposten dar, da der Stromabnahmevertrag auch auf einer Take-or-Pay-Basis vereinbart wurde und der Stromtarif wiederum die Gaskosten berücksichtigt.

\section{Zusammensetzung der Projektgesellschaften.}

Die beiden Projektgesellschaften weisen eine unterschiedliche Eigentümerstruktur auf. Bei Phu My 2.2 hält EDFI die Mehrheitsbeteiligung, die auch als Konsortialführerin fungierte. Das originäre Interesse von BP am Kraftwerksprojekt war ausschlaggebend für die Gründung von PM3, wobei die Projektgesellschaft dann schließlich gleichmäßig auf drei Investoren aufgeteilt wurde.

\section{Die Rolle der Projektsponsoren im EPC-Vertrag.}

Die unterschiedlichen Interessen der Projektsponsoren beim Bau des Kraftwerkes sind durch die Unterscheidung des down-stream (Phu My 2.2) und up-stream-Ansatzes (Phu My 3) erkennbar. Bei Phu My 2.2 hat das französische Unternehmen EDFI Interesse an der Konstruktion des Kraftwerkes und im Rahmen des EPC-Vertrages wurde ein Tochterunternehmen damit beauftragt. Bei Phu My 3 liegt hingegen die Kernkompetenz des ursprünglichen Projektsponsors BP in der Gasförderung und BP verfolgte mit der Erstellung des Kraftwerkes primär die Sicherstellung des Gasverbrauches. In der Projektgesellschaft sind insgesamt neben BP zwei weitere Unternehmen beteiligt, wobei keines davon am Bau beteiligt ist und der EPC-Vertrag extern an Siemens vergeben wurde. 


\subsubsection{Auswirkungen von Phu My 2.2 und Phu My 3 auf die Armuts- reduktion}

In diesem Kapitel werden die Auswirkungen der beiden Kraftwerke (Phu My 2.2 und Phu My 3) auf die Armutsreduktion diskutiert. Die Erörterung basiert auf der Literaturausarbeitung im Kapitel 3.3. Da die beiden Kraftwerke im selben Komplex untergebracht sind und dieselbe Technologie anwenden, werden die Auswirkungen gemeinsam dargestellt. Auf Projektebene kann es aufgrund der unterschiedlichen Aktivitäten der Projektgesellschaften zu leichten Unterschieden kommen, auf die im Text gesondert hingewiesen wird.

Phu My 2.2 und Phu My 3, die im Rahmen des Power Generation Komplexes gebaut worden sind, fallen in die Kategorie der On-Grid Projekte. Der produzierte Strom wird daher in das bestehende Stromnetz gespeist und dient zur Stabilisierung der bestehenden Stromversorgung, wobei Vietnam zurzeit eine ca. 80 \%ige flächendeckende Stromversorgung aufweist (vgl. World Bank 2006d: 3 ). Von der erhöhten Stromproduktion durch Phu My 2.2 und Phu My 3 profitieren daher jene Haushalte, die ans Stromnetz angeschlossen sind. Vorallem die ländlichen Gebiete, die zu den ärmsten Gegenden zählen, sind jedoch meistens nicht an die Stromversorgung angeschlossen. Da die Stromversorgung der öffentlichen EVN obliegt, kann nicht automatisch davon ausgegangen werden, dass parallel zur erhöhten Stromproduktion die Versorgung ausgebaut wurde. Die Auswirkungen von Phu My 2.2 und 3 beschränken sich somit auf jenen Teil der Bevölkerung, der bereits an das bestehende Stromnetz angeschlossen ist. Gleichzeitig ist ein wesentlicher Vorteil privater Investitionen, dass das öffentliche Budget weitgehend unbelastet bleibt und anderweitig eingesetzt werden kann, wie beispielsweise in der Ausweitung der Stromversorgung.

Vietnam weist seit Jahren ein stabiles Wirtschaftswachstum auf, das auch auf das Ansteigen von inländischen und ausländischen Direktinvestitionen in personalintensiven Branchen zurückzuführen ist. Eine Ausweitung der Produktion unterstützt das Wirtschaftswachstum, bedingt aber auch eine höhere Stromnachfrage. Vor der Errichtung von Phu My 2.2 und Phu My 3 stellte für Vietnam die große Abhängigkeit von Hydrokraftwerken ein großes Problem dar, da in extremen Trockenzeiten nicht die notwendige Menge an Strom, sondern nur $40-50 \%$ der maximalen Kapazität produziert werden konnten. Durch die beiden Kraftwerke konnte diese Abhängigkeit reduziert werden und so werden heute nur noch rund $37 \%$ des Stromes durch Wasserkraftwerke erzeugt (vgl. ADB 2007c: 19). Für den Wirtschaftsstandort Vietnam ist es essenziell, die industrielle Stromversorgung gewährleisten zu können. Die beiden Kraftwerke tragen wesentlich zur Stabilisierung des bestehenden Stromnetzes bei und können daher positive Auswirkung auf das Wirtschaftswachstum haben.

Gleichzeitig ist durch den steigenden Wohlstand auch die private Stromnachfrage gestiegen, da sich die Menschen mehr elektronische Geräte, wie beispielsweise Kühlschränke oder Fernsehgeräte, leisten können. Ist das Einkommen eines Haushaltes aber bereits so hoch, dass elektronische Geräte ge- 
kauft werden können, dann scheint die Armutsgrenze von 1 US\$ pro Tag bereits überschritten zu sein. In diesem Fall tragen Phu My 2.2 und Phu My 3 nicht zur Armutsreduktion, sondern zu einer Steigerung der Lebensqualität jenes Teils der Bevölkerung bei, der nicht mehr zu den Armen gerechnet wird.

Für jene Armen, die bereits an das bestehende Versorgungsnetz angeschlossen sind, ist vor allem die Tarifhöhe wichtig, da die Stromausgaben einen Teil des Einkommens aufbrauchen. EVN kauft auf Basis von Take-or-Pay Verträgen den Strom von den Kraftwerken und verrechnet den Kundengruppen, abhängig von der abgenommen Menge, unterschiedliche Tarife. Dadurch werden die Tarife quersubveniontiert und die Tarife der Kundengruppe angepasst. MECO und PM3 haben keinen Einfluss auf die Tarife und beeinflussen die Tarifgestaltung nur sehr indirekt, nämlich über den vereinbarten Tarif im Abnahmevertrag. Je günstiger sie den Tarif im Abnahmevertrag vereinbaren, desto mehr Spielraum bleibt für EVN. Die Tarifgestaltung basiert jedoch nicht nur auf den Kosten, sondern der Tarif wird auch als regualtives Instrument eingesetzt. Aufgrund dieser Einschränkung erscheinen die direkten positiven Auswirkungen auf die am Netz angeschlossen Armen nochmals geringer. Indirekt könnten sie dadurch profitieren, dass der Staat eventuell das öffentliche Budget für andere Maßnahmen investiert, die von direktem Nutzen für sie sind.

Direkte positive Auswirkungen haben Phu My 2.2 und 3 auf jene arme Bevölkerung, die durch den Bau der Kraftwerke Arbeit gefunden hat. Der Bau der beiden Kraftwerke hat mehr als drei Jahre gedauert und es wurden sowohl Schulungen der Arbeiter als auch verschiedene Entwicklungsprogramme in der Region durchgeführt. Die Auswirkungen der Bautätigkeit haben aber nur kurzfristige positive Auswirkungen, da der Großteil der Arbeiter nach Fertigstellung der Bauarbeiten wieder gekündigt wurde. Die Schulungs- und Sozialprogramme haben hingegen langfristige postive Auswirkungen auf die betroffenen Menschen.

MECO und PM3 tragen im Gebiet, Ba Ria-Vung Tau, in dem das Kraftwerk gebaut wurde, außerdem wesentlich zu einer Erhöhung der Steuereinnahmen bei. Das Bruttoinlandsprodukt stieg im Zeitraum von 2002 bis 2005 um $23 \%$, das ist ein signifikant stärkerer Anstieg als in Gesamtvietnam (vgl. ADB 2007c: 7). Abhängig davon, wie die Steuereinnahmen verwendet werden, können sie direkte positive Auswirkungen auf die Armutsreduktion haben, wenn diese für Investitionen verwendet werden, von denen auch die Armen profitieren.

Eine weitere indirekte positive Auswirkung auf die Bevölkerung ohne Stromanschluss könnte durch die stabilere Stromversorgung eine verbesserte Gesundheitsversorgung sein. Dies betrifft vor allem die Gesundheitsversorgung in den Trockenzeiten, die von Stromausfällen betroffen war.

Eine wesentliche direkte negative Auswirkung der Kraftwerke auf die Bevölkerung in Ba Ria-Vung Tau besteht durch die höhere Umweltbelastung. Die Kraftwerke haben die Umweltauflagen der IFIs und Vietnams zu erfüllen, aller- 
dings können die Maßnahmen nur die Umweltbelastungen beschränken und nicht vollständig vermeiden. Eine weitere direkte negative Auswirkung entstand für die ehemaligen Bewohner der Baufläche, die vom Staat umgesiedelt worden sind. Die Umsiedelung wurde im Namen der Regierung von der Urban Development and Construction Company durchgefuihrt, von der wiederum die Projektgesellschaften das Land pachten. Die Projektgesellschaften hatten keinen Einfluss auf den Standort des Kraftwerkes, sondern dieser wurde von der Regierung zugewiesen. Der Kraftwerkskomplex umfasst mehrere Kraftwerke, daher ist anzunehmen, dass die Regierung eine gering besiedelte Fläche dafür ausgewählt hat.

Zusammenfassend lässt sich feststellen, dass diese beiden Kraftwerke nur einen beschränkten direkten Beitrag zur Armutsreduktion leisten. Ein wesentlicher direkter Beitrag ist die Arbeitsplatzbeschaffung während der Bauphase. Allerdings ist die Arbeitslosenrate in Vietnam ohnehin bereits sehr niedrig. Ein weiterer wesentlicher direkter Beitrag besteht in der Stabilisierung des Stromnetzes, der indirekt zum Wirtschaftswachstum beitragen kann. Indirekt können das stabile Wirtschaftswachstum mit gleichzeitig stattfindenden Pro-Poor Reformen seitens der Regierung, wie beispielsweise die Doi Moi-Reformen, wiederum positive Auswirkungen auf die langfristige Armutsreduktion haben. In der Literatur werden auch mögliche Technologie-Spillover-Effekte diskutiert, die in diesem Projekt auf der Mitarbeiterebene stattfinden könnten. Eine Beschränkung der Spillover-Effekte stellt jedoch die Tatsache dar, dass beide Projekte ausschließlich von ausländischen Unternehmen durchgeführt werden.

Die folgende Abbildung 47 gibt die Diskussion über die Auswirkungen von Phu My 2.2 und Phu My 3 auf Wachstum und Armutsreduktion grafisch wieder. In dieser Abbildung signalisiert die Stärke der Striche jeweils die Intensität des Einflusses.

\section{Abbildung 47: Auswirkungen von Phu My 2.2 und Phu My 3 auf Wachstum und} Armutsreduktion

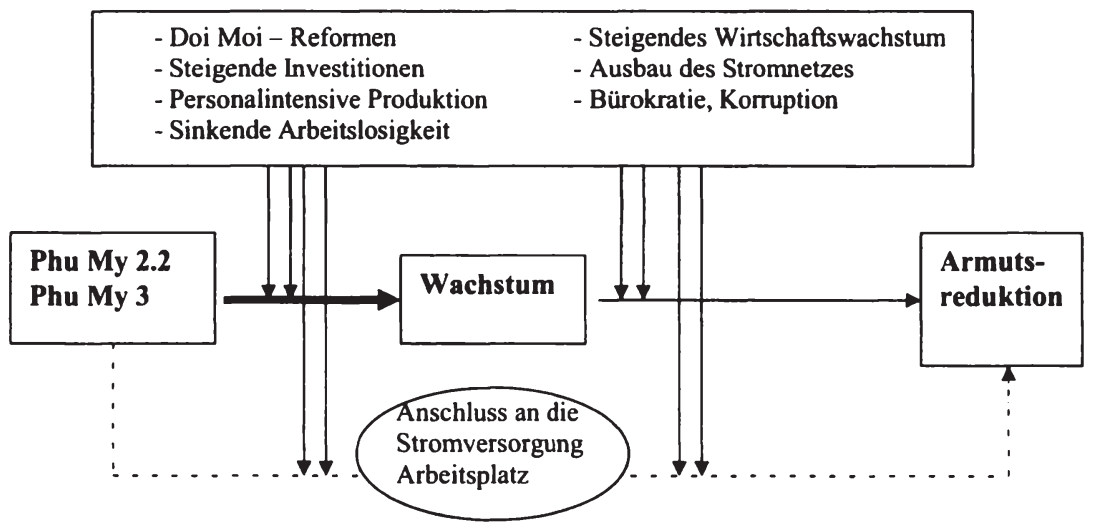




\subsection{Philippinen}

Das folgende Kapitel widmet sich der Darstellung von zwei Fallstudien auf den Philippinen. Beginnend mit einer Einführung werden die Philippinen anhand von Eckdaten und der wirtschaftlichen Entwicklung dargestellt. Daran anschließend folgen die Beschreibungen der Fallstudien SPI Mindanao und NLEX, wobei das SPI-Projekt ein Kraftwerk und NLEX eine Schnellstraße ist. Die Finanzierungs- und Absicherungsinstrumente und die Vertragsstruktur werden in einer Gegenüberstellung zusammengefasst. Außerdem schließt jede Fallstudie mit einer Diskussion über deren Beitrag zur Armutsreduktion.

\subsubsection{Einführung}

\subsubsection{Eckdaten}

Fläche:

$300.000 \mathrm{~km}^{2}$

Einwohner:

85.200 .000

Hauptstadt:

Manila

Staat:

Währung:

Präsidialrepublik seit 1987

1 Peso $=100$ Centavos

Quelle: Von Baratta 2001: 635

Wirtschaft:

Wachstum des BIP: $\quad 2001: 1,8 \%$

2003: $4,5 \%$

2005: $5,1 \%$

Inflation:

2001: $6,1 \%$

2003: $3,5 \%$

2005: $7,6 \%$

Arbeitslosenrate:

2001: $11,1 \%$

2003: $11,3 \%$

2005: $11,4 \%$

Anstieg der

2001: $-16,2 \%$

Warenexporte:

2003: $2,7 \%$

2005: $3,7 \%$

ADI Zufluss:

2001: 335

in Millionen US\$

2003: 188

2005: 970

Quelle: ADB 2006c 
Armut:

Bruttoinlandsprodukt

je Einwohner:

HDI:

MDG 1:

$\%$ der Bevölkerung unter

1 \$ pro Tag
1999: 1.045 US\$

2001: 970 US\$

2003: 1.038 US\$

2006: 0,763 (an 84. Stelle von 177 Ländern)

1990: $19,1 \%$

2002: $11,5 \%$

Quelle: UNDP 2006, ADB 2005c

Infrastrukturausstattung:

Haushalte mit

1998: 72

Stromanschluss in \%

2003: 79

Kilometer an asphaltieren

1998: 16.029

Autobahnen, Schnell-

2003: 19.228

straßen oder nationalen

Straßen

Quelle: ADB 2005c

\subsubsection{Wirtschaftliche Rahmenbedingungen}

\section{Wirtschaftspolitische Entwicklung}

Bevor die Bevölkerung 1986 den Diktator Ferdinand Marcos durch eine Revolution stürzte, wurde das Land mehr als 30 Jahre autokratisch geführt. Marcos hat während der langen Jahre seiner Herrschaft eine politische Elite herangezogen, die bedingungslos seine Vorhaben unterstützte. "Instead of the creation of new institutions, the personalization of authority and the role of patronage in politics attained new peaks " (Root 1996: 111). Mit dem Umsturz der Marcos-Regierung wurde 1986 die Präsidialrepublik als neue Staatsform festgelegt. Auch wenn die Philippinen in den vergangenen 20 Jahren der neuen Staatsform viel zur Demokratisierung beigetragen haben, kämpfen sie noch immer mit den Nachwehen der Marcos Regierung und sind daher eine schwache Demokratie geblieben. Deshalb sind die politische Vergangenheit und Gegenwart noch immer geprägt von Nepotismus, Korruption und Personenwahlkampf anstelle von starken Parteien (vgl. Reiterer 1997: 182). Die ADB (2005c: 100) beschreibt die politische Situation treffend „In the Philippines, political parties are seen as personal tools of self-serving politicians rather than as social vehicles of collective interest ". 
Das moderate Wirtschaftwachstum der vergangenen Jahre war sehr be-scheiden und viel zu gering, um neue Arbeitsplätze zu schaffen (vgl. ADB 2004: 8). Die Arbeitslosenrate ist seit Jahren extrem hoch und liegt immer über $10 \%$, wobei die Dunkelziffer wahrscheinlich weitaus höher ist, da die Philippinen eine starke Verstädterung aufweisen und die Zählung in den städtischen Slums nur beschränkt möglich ist (vgl. ADB 2006c: 214). Auf den Philippinen stellt der private Konsum den größten Beitrag zum Wirtschaftswachstum dar, 2005 waren. dies rund 3,9 \%. Diese Kaufkraft basiert wiederum auf den hohen Geldüberweisungen von philippinischen Überseearbeitern. Der öffentliche Konsum hingegen hat im Jahr 2005 nur 0,2 \% zum Wirtschaftswachstum beigetragen, da die Regierung hohe Defizite hat (vgl. ADB 2006c: 214). Während Budgetprobleme öffentliche Investitionen verhindern, trägt gleichzeitig ein unsicheres Investitionsklima aufgrund von schlechter Governance und schwachen Finanzinstitutionen zu einem Rückgang bei den privaten Investitionen bei (vgl. ADB 2004: 8).

\section{Infrastruktur}

Das Hauptproblem bei der Planung von Infrastruktur auf den Philippinen ist die Tatsache, dass langfristige Visionen und Entwicklungspläne von kurzfristigen, politischen Maßnahmen durchkreuzt werden. Zwischen den Zielen, die in den Plänen gesetzt werden, und der Realität gibt es große Lücken und die Auswirkungen dieser schwachen Koordination sind in allen philippinischen Infrastruktursektoren zu sehen (vgl. ADB 2005b: 15).

Die National Economic and Development Authoritiy (NEDA), die für langfristige Pläne in der Infrastruktur zuständig ist, hat tatsächlich nur eine sehr schwache Position und ist in erster Linie ein ausführendes Organ für die Regierung. Nicht Entwicklung und Planung, sondern Krisenmanagement scheint daher NEDAs Haupttätigkeit zu sein. Problematisch in diesem Zusammenhang ist eine Politik, in der die Politiker ihre Position stärken, indem sie einerseits unqualifizierte Familienangehörige oder Verwandte in politischen Ämtern einstellen und andererseits politisch berechnete Geldzuwendungen aus dem Infrastrukturbudget zur Stimmenmaximierung einsetzen (vgl. ADB 2005b: 107f). Mit diesen Rahmenbedingungen im Hinterkopf müssen auch die Entwicklungspläne der Regierung betrachtet werden.

Die philippinische Regierung hat den „Medium-Term Philippine Development Plan (MTPDP)“ für den Zeitraum von 2005 bis 2010 ausgearbeitet, der sich an den MDGs orientiert (vgl. ADB 2004: 8). Die Ziele, beispielsweise im Hinblick auf das Wirtschaftswachstum, sind sehr ambitioniert und 2009 soll dieses rund 7-8 \% betragen. Das wäre jedoch eine Wachstumsrate, die zuletzt in den 1970er Jahren auf den Philippinen erreicht wurde und wirklich notwendig wäre, um einen wesentlichen Beitrag zur Armutsreduktion leisten zu können (vgl. ADB 2004: 8). 


\section{Stromsektor}

Die Reduzierung des öffentlichen Defizits stellt eines der wesentlichen politischen Ziele dar, wobei vor allem die Privatisierungsreformen im Infrastruktursektor im Mittelpunkt stehen. Der Stromsektor stellt bei diesem Vorhaben eine der wesentlichen Reformherausforderungen auf den Philippinen dar, da er in den vergangenen Jahren einer der Hauptgründe für die steuerlichen Probleme und somit auch ausschlaggebend für das schwache Investitionsklima war. Die Sanierung des öffentlichen Budgets soll daher vor allem durch die Einhebung von neuen Steuern und eine Erhöhung der Strompreise erreicht werden (vgl. ADB 2004: 8).

Problemkind im Stromsektor ist die staatliche National Power Corporation (NPC), die in den vergangenen Jahren immer größere Verluste erwirtschaftet hat. Wurden 1997 noch Gewinne geschrieben, musste 2003 ein Verlust von 117 Milliarden Peso verzeichnet werden, der mehr als die Hälfte des nationalen Budgets aufbrauchte (vgl. ADB 2004: 12). Eine rasche Reformierung des Stromsektors wäre daher für die Sanierung von NPC notwendig gewesen, allerdings führte der Präsidentschaftswahlkampf im Mai 2004 zu verzögerten Reformen im Stromsektor. Um die Wählerschaft nicht zu verärgern, wurde mit Preiserhöhungen bis kurz nach den Wahlen gewartet. Gleichzeitig musste NPC finanziell für den Wahlkampf saniert werden und daher wurde viel öffentliches Budget investiert. Deswegen waren in dieser Zeit kaum andere Investitionen möglich, da die finanzielle Unterstützung von NPC alle anderen Investitionen verdrängte.

Im Juni 2001 wurde allerdings mit dem Electric Power Industry Reform Act ein Gesetz verabschiedet, das neue Rahmenbedingungen in Bezug auf Restrukturierung und Privatisierung darstellt (vgl. ADB 2006c: 219). Aus dem Verkauf der öffentlichen Strominfrastruktur erwartet die Regierung in den nächsten Jahren rund 4-5 Milliarden US\$ (vgl. ADB 2004: 12). Bisher sind der Verkauf bzw. die Auslagerung der Anlagen und der Versorgung jedoch weder vollständig noch erfolgreich durchgeführt worden. Nichtsdestotrotz konnte aber die Strompreiserhöhung in den Jahren 2004 und 2005 die Verluste der NPC reduzieren (vgl. ADB 2006c: 215).

Zusammenfassend lässt sich feststellen, dass in allen Infrastruktursektoren auf den Philippinen großes Investitions- und Verbesserungspotenzial vorhanden ist. Wesentliches Hindernis zur Verbesserung der Infrastruktur stellt die schwache politische Struktur dar, da durch politische Berechnung oder Bereicherung Investitionen verschleppt oder verhindert werden. 


\subsubsection{Kraftwerk Steag State Power Inc. Mindanao}

\subsubsection{Eckdaten}

Land:

Art der Investition:

Lage:

Finanzierungsmodell:

Laufzeit:

Projektgesellschaft:

Projektsponsoren:
Republik der Philippinen

Kohlekraftwerk

Philippine Veterans Industrial Development

Corporation (PHIVIDEC) Estate, Villanueva, Misamis Oriental

BOT

25 Jahre

STEAG State Power Inc. SPI (ehemals State Power Development Corporation)

$89 \%$ STEAG GmbH (Deutschland)

$11 \%$ State Investment Trust, Inc.

(Philippinen)

Installierte Antriebsleistung: $200 \mathrm{MW}$

Dieses privat finanzierte Kraftwerk stellt eines der wenigen BOT-Projekte auf den Philippinen dar, die kürzlich realisiert wurden. Das Kraftwerk wurde in einem Industriegebiet auf der südlichen insel Mindanao gebaut. Die BOTLaufzeit beträgt 25 Jahre und beinhaltet die Erstellung und den Betrieb eines Kohlekraftwerkes mit $200 \mathrm{MW}$ installierter Antriebsleistung. Nach Ablauf der 25 Jahre wird das Kraftwerk an die philippinische Regierung übergeben.

Die Projektgesellschaft SPI besteht aus zwei Sponsoren, wobei die deutsche Beteiligung durch STEAG GmbH eine Mehrheitsbeteiligung von $89 \%$ hält. Der ursprüngliche Name der Projektgesellschaft war State Power Development Corporation und bestand $\mathrm{zu} 100 \%$ aus philippinischer Beteiligung durch die State Investment Trust, Inc. Durch die Beteilgung der STEAG GmbH wurde auch der Name der Projektgesellschaft in STEAG State Power Inc. (SPI) umgeändert.

\subsubsection{Entstehung}

April 1995

Internationale Ausschreibung für ein BOT-Modell

Dezember 1995

Gesellschaftsgründung von State Power Development Corporation (SPDC) durch State Investment Trust Inc. (SITI)

State Investment Trust Inc. (SITI) wird im Zuge der öffentlichen Ausschreibung für das Projekt ausgewählt.

1997

Das Projekt wird von der National and Economic Development Authority (NEDA) überprüft und genehmigt. 
März 2001

(SITI) wird unterzeichnet und von NEDA genehmigt.

Dezember 2001

Der Stromabnahmevertrag wird wirksam.

2003

November 2003

Dezember 2003

Die Feasibilty Studie wird eingereicht.

STEAG AG beteiligt sich am Projekt

November 2006

Financial Closing

Baubeginn

Beginn des kommerziellen Betriebes

Der ursprüngliche Start für dieses Kraftwerk hat bereits 1995 unter der Präsidentschaft von Fidel Ramos begonnen und stellt das letzte genehmigte Infrastrukturprojekt unter dessen Präsidentschaft (1992 - 1998) dar. Ramos versuchte anhand von sogenannten ,Independent Power Producers " die Stromknappheit auf den Philippinen zu überwinden, indem er die Stromproduktion für den privaten Sektor öffnete. 1996 wurde das philippinische Unternehmen State Investment Trust Inc. (SITI) im Rahmen einer internationalen Ausschreibung für das Kraftwerk in Mindanao ausgewählt. Die Genehmigung und die Verhandlungen für den Stromabnahmevertrag wurden auch bereits 1998 durchgeführt.

Dieser Entstehungs- und Genehmigungsprozess fiel jedoch genau in die asiatische Finanzkrise und das Kraftwerk konnte von der dafür gegründeten Projektgesellschaft State Power Development Corporation (SPDC) nicht mehr durchgeführt werden. Die State Investment Trust Inc. wurde schwer von der Finanzkrise getroffen und war daher nicht mehr in der Lage, das Projekt abzuwickeln. 2003 beteiligte sich schließlich die STEAG AG an dem Projekt und trat in die Projektgesellschaft ein, die aufgrund des Beteiligungswechsels in STEAG State Power Inc. (SPI) umbenannt wurde. Ende 2003 wurde die Finanzierung des Kraftwerks unterzeichnet und mit dem Bau begonnen. Im November 2006, rund drei Jahre später, konnte das Kraftwerk den Betrieb aufnehmen.

Da das Projekt schon in den 1990er Jahren genehmigt wurde, war die ursprüngliche Projektgesellschaft bereits mit einem langfristen take-and-pay Abnahmevertrag ausgestattet. Seit der Reformierung des Stromsektors im Jahre 2001 dürfen keine langfristigen Abnahmeverträge mehr von Regierungseinheiten unterzeichnt werden und dieses Projekt stellt diesbezüglich eine Besonderheit dar.

\subsubsection{Finanzierungs- und Absicherungsinstrumente}

SPI benötigte insgesamt ein Gesamtkapital in der Höhe von 305 Mio. US\$, bei einem Verschuldungsgrad von 71:29. Rund $93 \%$ des Fremdkapitals stellen eine vorrangige Verbindlichkeit (d.h. Senior Debt) dar, wobei diese Fazilitäten in US\$ sind. Die restlichen $7 \%$ resultieren aus einem Kredit in lokaler Währung, der in erster Linie für die Bezahlung der lokalen Gehälter benötigt wurde. SPI wäre bei der Strukturierung der Finanzierung daran interessiert gewesen, höhere Kredite in lokaler Währung aufzunehmen, jedoch gab es auf dem philippi- 
nischen Kapitalmarkt wenig Interesse dafür. Die philippinische Banco de Oro gewährte schließlich SPI einen Kredit in philippinischen Peso in der Höhe von umgerechnet 15 Mio. US\$.

\section{Finanzierung:}

$71 \%$

Fremdkapital:

Kommerzielle Kredite (DiA) 100,0 Mio. US\$

JBIC Kredit Tranche A 60,6 Mio. US\$

JBIC Kredit Tranche B 40,4 Mio. US\$

(NEXI PRG)

Kommerzieller Kredit - Banco de 15,0 Mio. US\$

Oro (Lokale Währung)

$29 \% \quad$ Eigenkapital:

STEAG GmbH 79,2 Mio. US\$

State Investment Trust, Inc. 9,8 Mio. US\$

Gesamtkapital 305,0 Mio. US\$

Die Finanzierungs- und Absicherungsstruktur weist eine parallele Kofinanzierung auf (siehe Kapitel 6.2.2.3), jedoch beteiligen sich keine multilateralen Finanzinstitutionen an der Finanzierung. Zum Zeitpunkt der Unterzeichnung der Finanzierung wurde die DiA-Fazilität von der Bundesrepublik Deutschland noch unter dem Namen "Garantie für Kapitalanlagen“ (GKA) vergeben und erst zu einem späteren Zeitpunkt umbenannt. Durch die hohe Beteiligung von japanischen Unternehmen beim Bau des Kraftwerkes kommt es auch zu einer starken Beteiligung von japanischen Institutionen. Die einzelnen Finanzierungs- und Absicherungsinstrumente der beteiligten Institutionen werden nun in alphabetischer Reihenfolge genauer vorgestellt.

\section{Bundesrepublik Deutschland:}

Bundesgarantie 95 Mio. US\$

für

Die Investitionsgarantie des Bundes bietet deutschen

Direktinvestitionen Unternehmen Schutz für deutsche Direktinvestitionen in im Ausland (DiA): Form von Beteiligungen, beteiligungsähnlichen Darlehen, Dotationskapital und anderen vermögenswerten Rechten gegen politische Risiken in Schwellen- und Entwicklungsländern. Diese Garantie wurde im vorliegenden Fall für die kommerziellen Kreditgeber, wie z.B. HVB und $\mathrm{KfW}$, ausgestellt. Die Kredite wurden fast zu $100 \%$ syndiziert.

Insgesamt werden $95 \%$ der gesamten Kreditsumme gegen folgende Tatbestände abgesichert: Verstaatlichung, 
Enteignung, enteignungsgleiche Eingriffe, Bruch von Zusagen staatlicher oder staatlich kontrollierter bzw. gelenkter Stellen, Störungen der inneren Sicherheit, Aufruhr, Krieg, terroristische Akte sowie Konvertierungsund Transferschwierigkeiten.

Die Garantien werden im Auftrag der Bundesrepublik durch die PwC AG abgewickelt und die Anträge vom Interministeriellen Ausschuss (IMA) entschieden.

Garantievereinbarung: Diese Vereinbarung regelt zwischen der Bundesrepublik Deutschland und den kommerziellen Kreditgebern die abgesicherten Risiken.

JBIC Instrument:

Kredit: 101 Mio. US\$

JBIC hat an das Projekt einen Kredit in zwei Tranchen vergeben, wobei eine Tranche nicht und die andere

Tranche durch die NEXI-Garantie abgesichert sind.

\section{NEXI Instrument:}

Overseas Untied

Loan Insurance (OULI):
40,4 Mio. US\$

Diese Garantie sichert eine Tranche des JBIC-Kredites gegen folgende Tatbestände ab: Krieg, Revolution, Konvertierungsverbot, Moratoriumsrisiko, Force Majeure und Insolvenz eines Kreditnehmers.

Garantievereinbarung: In dieser Vereinbarung wird der Haftungsumfang zwischen NEXI und JBIC geregelt.

Für SPI war es aufgrund der schlechten wirtschaftlichen Marktlage nicht einfach, die Strukturierung der Finanzierung für das Kraftwerk durchzuführen. Besonders problematisch für die Finanzierung des Projektes war die kritischere Einstufung der politischen Risiken durch die Banken und Versicherungen auf den Philippinen nach den Anschlägen von New York 2001. Das entsprechende Interesse, Risiken zu übernehmen, war dadurch deutlich zurückgegangen. Daher hat beispielsweise die DiA-Garantie eine wesentliche Rolle für die SPI bei der Mobilisierung der kommerziellen Kredite gespielt, da diese Garantie beinahe zu $100 \%$ das politische Risiko übernimmt. 
Interessant bei der Finanzierung dieses Kraftwerks erscheint die Tatsache, dass keine Instrumente zur Finanzierung oder Absicherung von den internationalen Finanzinstitutionen in Anspruch genommen wurden. Laut Interviewpartnern liegt der Grund dafür in den hohen veranschlagten Kosten von MIGA und ADB, die mit den Konditionen der nationalen Institutionen nicht konkurrieren konnten. Zusätzlich dazu können die STEAG GmbH und die deutschen Institutionen bereits auf eine gemeinsame Zusammenarbeit in der Vergangenheit zurückblicken. Dadurch bestehen bereits gute Kontakte, welche die Vorbereitung eines neuen Projektes erleichtern. Die hohe japanische Beteiligung, sowohl in der Finanzierung als auch in der Absicherung, ist durch die hohe Mitwirkung von Kawasaki beim Bau des Kraftwerkes zu erklären.

Es wurden in diesem Projekt keine innovativen oder neuen Formen der Unterstützung gewählt, da ausschließlich Kredite und Garantien vergeben wurden. Von finanzwirtschaftlicher Seite erscheint die, wenn auch geringe, Finanzierung in lokaler Währung interessant und so wurden rund $7,5 \%$ des Fremdkapitals in philippinischen Pesos aufgenommen.

Die nun folgende Abbildung 48 zeigt nochmals grafisch den Zusammenhang der verschiedenen Finanzierungs- und Absicherungsinstrumente bzw. die involvierten Institutionen im SPI-Projekt.

\section{Abbildung 48: Finanzierungs- und Absicherungsstruktur von SPI Mindanao}

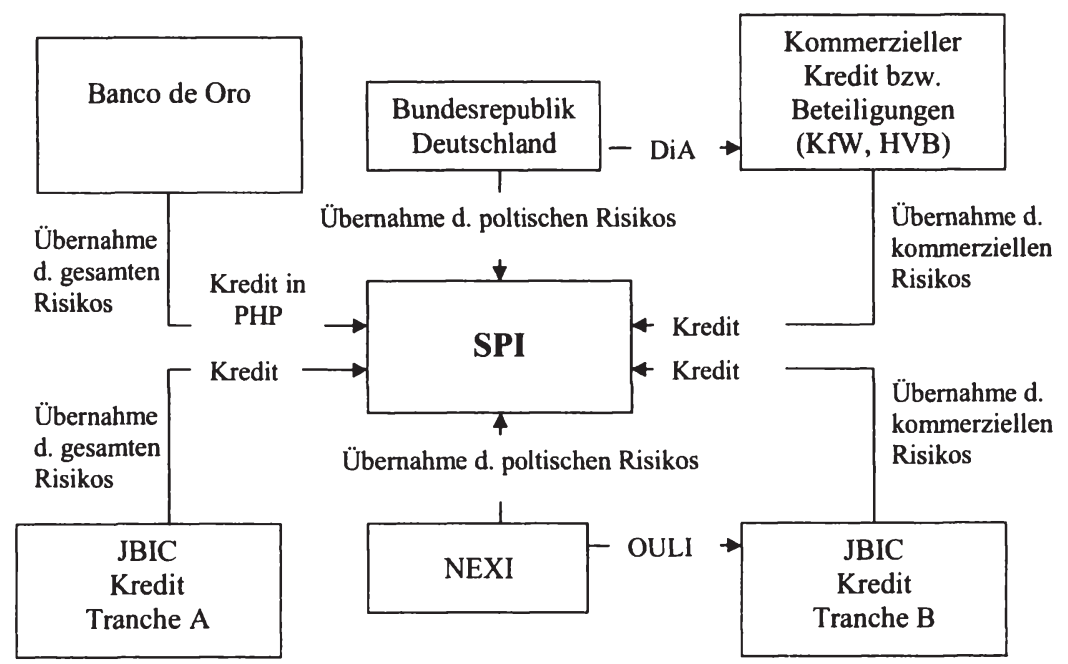




\subsubsection{Vertragsstruktur}

Die umfangreiche Vertragsstruktur des SPI Kraftwerkes wird in Abbildung 49 vorerst grafisch und im Anschluss daran die wichtigsten Verträge etwas genauer dargestllt. In dieser Übersicht werden nun zusätzlich zu den Verträgen mit Finanzinstitutionen auch die Verträge mit den philippinschen und anderen privaten Unternehmen berücksichtigt. 


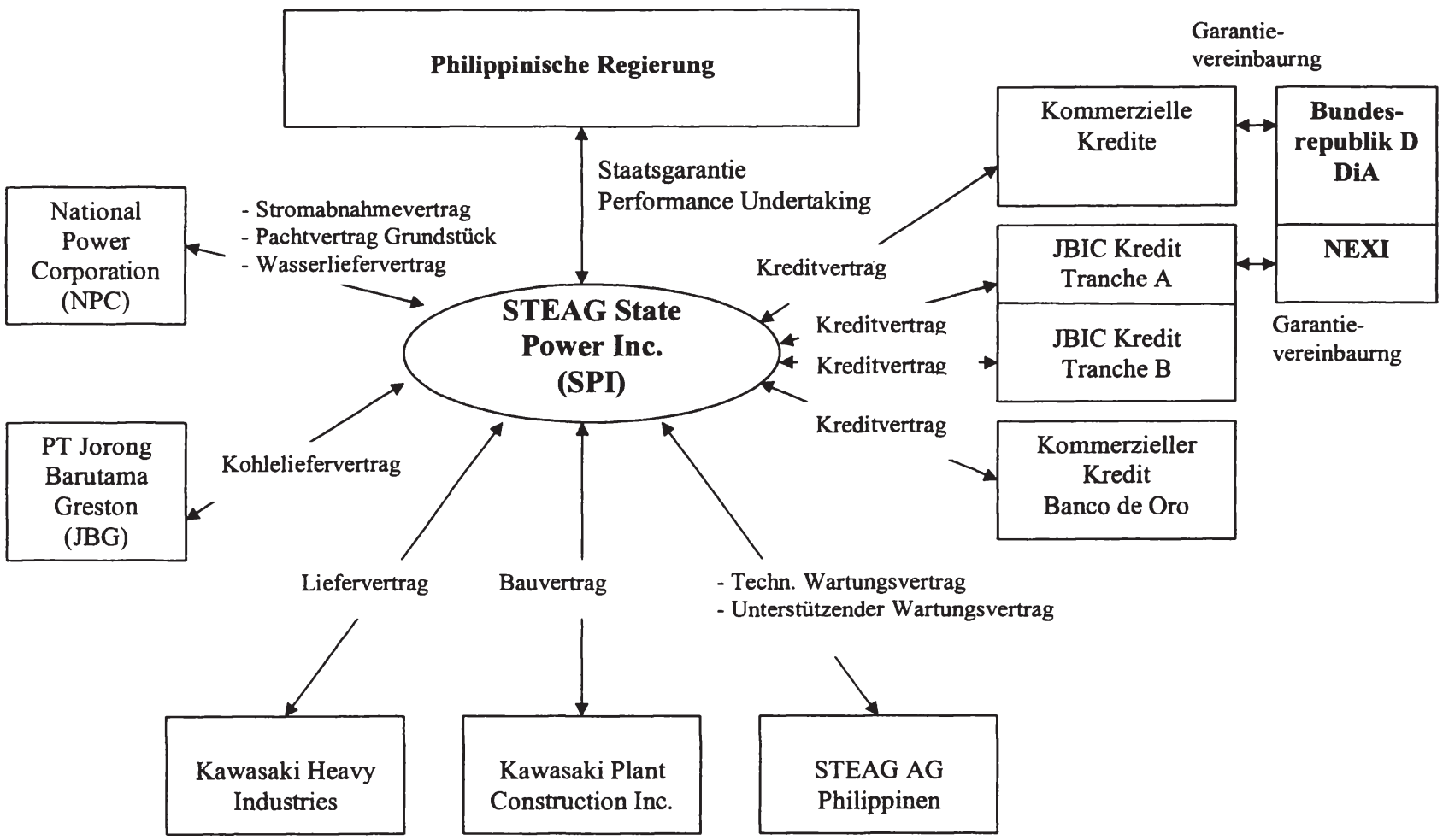


Stromabnahmevertrag: Vertragspartner: Steag State Power Inc. (SPI), National Power Corporation (NPC) und Power Sector Assets and Liabilities Management Corporation (PSALM)

Inhalt: Im Vertrag werden die Finanzierung, das Design, der Bau, der Betrieb und die Erhaltung eines 200 MW Kohlekraftwerkes festgelegt.

$\rightarrow$ Nach Ablauf der BOT-Laufzeit wird SPI das Kohlekraftwerk an NPC kostenlos übergeben.

$\rightarrow$ NPC sichert die Abnahme des Stroms für die nächsten 25 Jahre ab Fertigstellung des Kraftwerks zu einem fixierten Preis.

$\rightarrow$ Der fixierte Abnahmepreis basiert auf einer Tarifformel, die zu $85 \%$ an den Dollar indexiert ist und zu $15 \%$ dem Wechselkurs ausgesetzt ist.

Performance

Undertaking:

Vertragspartner: Philippinische Regierung und Steag State Power Inc. (SPI)

Inhalt: Die philippinische Regierung versichert, dass sie für die Kosten aus dem Stromabnahmevertrag aufkommen wird, falls NPC nicht bezahlt.

Wasserliefervertrag: Vertragspartner: Steag State Power Inc. (SPI) und National Power Corporation (NPC)

Inhalt: NPC versichert SPI in den 25 Jahren Laufzeit ímmer die Erlaubnis für die Wassergewinnung.

Pachtvertrag für das

Grundstück:
Vertragspartner: Steag State Power Inc. (SPI) und National Power Corporation (NPC)

Inhalt: NPC stellt das Land für 25 Jahre SPI zur Verfügung.

Staatsgarantie:

Vertragspartner: Philippinische Regierung und 
Steag State Power Inc. (SPI)

Inhalt: Die Einhaltung aller Verpflichtungen von NPC im Stromabnahmevertrag werden durch die philippinische Regierung zugesichert.

Wesentliche Verträge mit anderen privaten Unternehmen:

Kohleliefervertrag:

Liefervertrag:

Bauvertrag:

Technischer

Wartungsvertrag für den Betrieb und die Instandhaltung:
Vertragspartner: PT Jorong Barutama Greston (JBG) und Steag State Power Inc. (SPI)

Inhalt: In diesem Liefervertrag sichert JBG die Lieferung einer definierten Kohlenmenge bis 31 . Dezember 2019. Insgesamt werden ca. 800.000 Tonnen Kohle pro Jahr benötigt.

Vertragspartner: Kawasaki Heavy Industries (KHI) und Steag State Power Inc. (SPI)

Inhalt: KHI verpflichtet sich darin, die Gestaltung, die Konstruktion und die Arbeitskräftebeschaffung außerhalb der Philippinen für den Kraftwerksbau zu übernehmen.

Vertragspartner: Kawasaki Plant Construction Inc (KPCI) und Steag State Power Inc. (SPI)

Inhalt: KPCI verpflichtet sich darin für die Gestaltung, Konstruktion, die Auftragsvergabe, die Überprüfung, Fertigstellung und Erstinbetriebnahme des Kraftwerks und die Arbeitskräftebeschaffung innerhalb der Philippinen.

Vertragspartner: Regionalleitung der Steag AG auf den Philippinen und Steag State Power Inc. (SPI)

Inhalt: In diesem Vertrag wird die technische Unterstützung von STEAG AG für den Bau, Testlauf, Inbetriebnahme, Auftragsverteilung und Instandhaltung festgelegt.

$\rightarrow$ Abhängig davon welcher Zeitpunkt am 
frühesten eintritt, läuft der Vertrag entweder bis

a) zum 1. Dezember 2018 oder

b) bis zur Rückzahlung aller Schulden an die Gläubiger von SPI.

Unterstützender Wartungsvertrag:
Vertragspartner: Regionalleitung der Steag AG auf den Philippinen und Steag State Power Inc. (SPI)

Inhalt: In diesem Vertrag werden die Beratungsdienste von STEAG AG für SPI im Zusammenhang mit Bau, Testlauf, der Inbetriebnahme, Auftragsverteilung sowie dem Betrieb und der Instandhaltung des Kraftwerkes außerhalb der Philippinen festgelegt.

$\rightarrow$ Abhängig davon welcher Zeitpunkt am frühesten eintritt, läuft der Vertrag entweder bis

a) zum 1. Dezember 2018 oder

b) bis zur Rückzahlung aller Schulden an die Gläubiger von SPI.

\subsubsection{Zusammenfassende Gegenüberstellung von SPI}

In dieser nun folgenden zusammenfassenden Gegenüberstellung werden zuerst die Finanzierungs- und Absicherungsinstrumente und daran anschließend die Vertragsstruktur diskutiert.

\section{Finanzierung- und Absicherungsinstrumente}

Die folgende Tabelle 12 soll die Finanzierung des SPI-Kraftwerkes nochmals zusammenfassen und gleichzeitig den vietnamesischen Kraftwerken Phu My 2.2 und Phu My 3 gegenüberstellen. Daran anschließend werden die Gemeinsamkeiten und Unterschiede diskutiert.

Das SPI-Kraftwerk auf Mindanao stellt das aktuelleste BOT-Kraftwerk auf den Philippinen dar. Aufgrund der asiatischen Finanzkrise wurde der Bau des Kraftwerkes um einige Jahre verzögert, die Finanzierung erschwert und die Projektsponsoren verändert. Im Vergleich zu Vietnam waren die Philippinen lange Vorreiter auf dem Gebiet der BOT-Projekte im asiatischen Raum. Jedoch haben sowohl außen- als auch innenpolitische Unruhen wesentlich dazu beigetragen, dass es zu einem gewissen Stillstand in diesem Bereich gekommen ist. 
Tabelle 12: Zusammenfassung der Finanzierung und Absicherung von SPI, Phu My 2.2 und Phu My 3

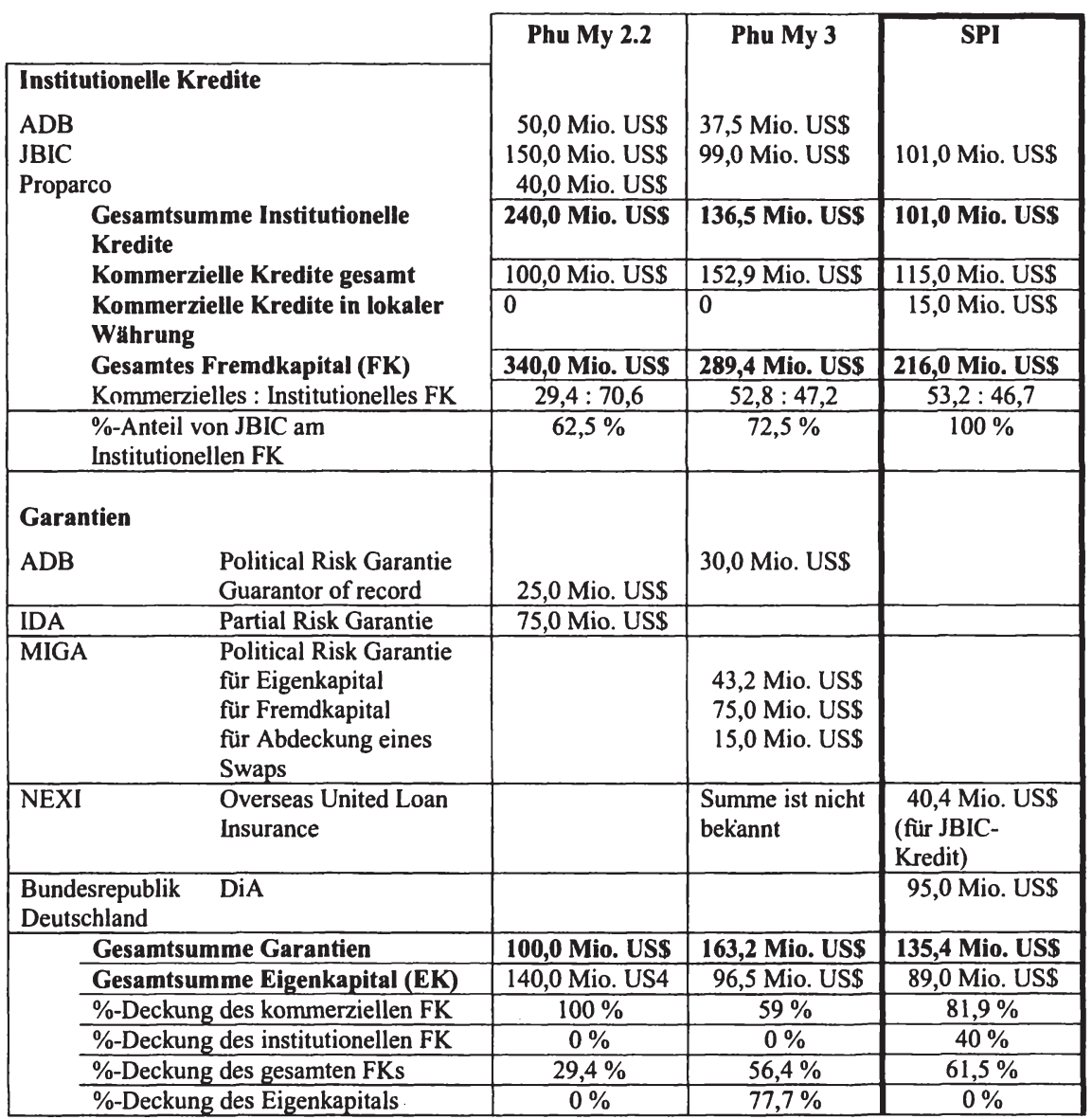

Knapp die Hälfte $46,7 \%$ des gesamten Fremdkapitals stammt von einem institiutionellen Geldgeber, nämlich JBIC. Auch in den beiden Projekten in Vietnam hat JBIC eine wichtige Rolle als institutioneller Geldgeber übernommen, aber nur im SPI-Projekt stellt sie die einzige Finanzinstitution dar. Außerdem wurden Garantien vergeben, die insgesamt $61,5 \%$ des Fremdkapitals absichern. Dies stellt eine relativ hohe Abdeckung dar, die daher rührt, dass die Deutsche Bundesregierung eine sehr hohe Garantie für die kommerziellen Kredite aus Deutschland vergeben hat. Darin spiegelt sich auch die schlechte Marktsituation zum Zeitpunkt der Finanzierung wider, die eine hohe Absicherung des Fremdkapitals verlangte. Die Vergabe der DiA-Garantie er- 
möglichte es außerdem den beteiligten Banken, ihre Forderungen an andere Banken zu syndizieren. Eine Ausnahme im Vergleich zu den beiden anderen Projekten stellt die Garantie von NEXI dar, die einen Teil des JBIC-Kredites deckt.

Eine Besonderheit stellt die Vergabe eines PHP-Kredites an die Projektgesellschaft dar, da bei den anderen beiden Projekten ausschließlich Kredite in US\$ gewährt wurden. Im Falle des SPI-Projektes wurde der PHP-Kredit für die Bezahlung der lokalen Arbeitnehmer genützt. Gleichzeitig ist die Projektgesellschaft aber auch einem höheren Wechselkursrisiko ausgesetzt, da im Abnahmevertrag die Dollarindexierung auf $85 \%$ beschränkt ist und somit $15 \%$ der Einnahmen dem Wechselkursrisiko ausgesetzt sind.

Zusammenfassend lässt sich daher festhalten, dass die Finanzierung des SPIProjekts einen stark nationalen Bezug aufweist, da ausschließlich deutsche und japanische Institutionen, aber keine einzige multilaterale Organisation an der Finanzierung und Absicherung beteiligt sind. Von den Institutionen wurden keine innovativen Instrumente verwendet, sondern ausschließlich Kredite und Garantien im klassischen Sinne vergeben.

\section{Vertragsstruktur}

Die Vertragstruktur ist in der Projektfinanzierung großteils stark standardisiert, jedoch gibt es auf Projektebene Unterschiede. Daher wird die Vertragsstruktur des SPI-Kraftwerkes nun im Vergleich zu jenen von Phu My 2.2 und Phu My 3 dargestellt, wobei im Wesentlichen nun auf die folgenden sechs Punkte eingegangen wird:

1. Es gibt keinen separaten BOT-Vertrag.

Die BOT-Vereinbarungen über den Bau, den Betrieb und den Transfer des Kraftwerkes durch SPI wurden im Rahmen des Stromabnahmevertrages vereinbart.

3. Die Rolle der Projektsponsoren beim Bau des Kraftwerkes.

Die Kernkompetenz der STEAG GmbH liegt in der Planung und im Betrieb, aber nicht im Bau von Kraftwerken und daher hat die Projektgesellschaft den Bau an Kawasaki augelagert. Die STEAG GmbH hat aber ein originäres Interesse am Betrieb des Kraftwerkes und ist daher durch Wartungsverträge für den Betrieb und die Instandhaltung des Kraftwerks verantwortlich.

4. Es gibt einen Take-AND-Pay und keinen Take-OR-Pay-Vertrag.

Ein Take-or-Pay-Vertrag bietet die größte Sicherheit für den privaten Investor. Die Projektgesellschaft wird unabhängig davon bezahlt, ob der öffentliche Abnehmer die Leistung konsumieren kann. Laut SPI ist aber ein 
Take-and-Pay-Vertrag für dieses Kraftwerk ausreichend, da die Region ohnehin an einer Unterversorgung von Strom leidet und daher das Kraftwerk immer auf maximaler Leistung läuft. Es wurde daher auch keine Vereinbarung für die Abnahme einer Mindestmenge abgeschlossen.

Seit der Reformierung des Stromsektors im Jahre 2001 dürfen überhaupt keine langfristigen Abnahmeverträge mehr durch NPC abgeschlossen werden, sondern es müssen die Abnahmerisiken zu $100 \%$ von den privaten Unternehmen übernommen werden.

5. Zusätzlich zur Staatsgarantie gibt es für den Abnahmevertrag eine separate Absicherung durch die Vereinbarung „Performance Undertaking".

Die Vereinbarung „Performance Undertaking“ beschränkt sich ausschließlich auf die Einhaltung der Zahlungsverpflichtungen der NPC die aufgrund des Abnahmevertrages entstehen.

6. Die Zulieferverträge unterscheiden sich.

Aufgrund der großen Gasvorkommen in Vietnam ist die vietnamesische Regierung auch an der Lieferung des Rohstoffes für das Kraftwerk involviert. Der Gaspreis stellt im Tarif daher einen Durchlaufposten dar, dessen Höhe im Abnahmevertrag berücksichtigt wird. Auf Basis eines Take-or-Pay Liefervertrages bzw. Verpflichtung zu einer Mindestabnahme sind die Projektgesellschaften an die Abnahme des Gases gebunden.

Beim SPI-Kraftwerk auf den Philippinen wurde der Kohleliefervertrag mit einem externen indonesischen Lieferanten abgeschlossen. Die Kohlekosten werden nicht als Durchlaufposten im Abnahmevertrag behandelt.

\subsubsection{Auswirkungen des Kraftwerks Steag State Power Inc. Mindanao auf die Armutsreduktion}

In diesem Kapitel werden die Auswirkungen des Kraftwerks Steag State Power Inc. Mindanao auf die Armutsreduktion diskutiert. Die Erörterung basiert auf der Literaturausarbeitung im Kapitel 3.3.

Die Philippinen bestehen aus insgesamt 7197 Inseln, wobei $94 \%$ der Bevölkerung auf den elf größten Inseln leben (vgl. von Baratta 2001: 635). Die Insel Mindanao zählt zu den drei größten Inseln und stellt eine unterwickelte Region auf den Philippinen dar (vgl. ADB 2005c: xiii). Auf der Insel leben mehr als $50 \%$ der Bevölkerung unter der Armutsgrenze. Ein Großteil der dort lebenden Bevölkerung gehört der muslimischen Glaubensgemeinschaft an, die auf den Philippinen eine Minderheit von ca. 4,6\% darstellt (vgl. von Baratta 2001: 635). Da die Philippinen aber mehrheitlich katholisch sind und die muslimische Minderheit auf Mindanao wirtschaftlich benachteiligt ist, gibt es zwischen Mindanao und der Regierung in Manila seit Jahrzehnten ein ange- 
spanntes Verhältnis. Besondere Berühmtheit hat dieser Konflikt durch die bewaffneten Aktionen von Abu Sayaf erlangt (vgl. HDN 2005: 7ff).

Das Kohlekraftwerk auf der Insel Mindanao fällt in die Kategorie der OnGrid-Projekte. Der produzierte Strom wird in die bestehende Stromversorgung gespeist und dient zur Stabilisierung des bestehenden Stromnetzes. Das Kraftwerk produziert insgesamt $15 \%$ der gesamten Energienachfrage auf Mindanao. Die Philippinen weisen insgesamt eine $79 \%$ ig flächendeckende Stromversorgung auf. Aufgrund der hohen Armut und der generellen infrastrukturellen Unterentwicklung auf Mindanao ist anzunehmen, dass auch die Stromversorgung verhältnismäßig geringer ist als im besser entwickelten Norden der Philippinen. Es sind jedoch keine Daten darüber verfügbar, ob gleichzeitig mit dem Bau des Kraftwerkes das Versorgungsnetz ausgebaut wurde. Es ist allerdings anzunehmen, dass keine diesbezüglichen Investitionen getätigt wurden, da NPC einerseits mit Budgetproblemen zu kämpfen hat und andererseits seit längerem erfolglos versucht, die Versorgung des Stromnetzes zu privatisieren. Daher beschränken sich die Auswirkungen dieses Kraftwerks nur auf jenen Teil der Bevölkerung, der bereits an das bestehende Stromnetz angeschlossen ist.

Auch auf Mindanao ist die Stromnachfrage in den vergangen Jahren gestiegen. Bedenkt man, dass dieses Kraftwerk bereits 1996 genehmigt wurde, so ist anzunehmen, dass bereits zu diesem Zeitpunkt Bedarf vorhanden gewesen wäre. Ein Großteil des Stromes wird auf Mindanao von Wasserkraftwerken produziert, die insbesondere in Trockenzeiten nur eine geringe Kapazität haben. Der Bau eines Kohlekraftwerkes diversifiziert daher die Stromproduktion und verringert die Engpässe in der Trockenzeit.

Für jene Armen, die bereits an das bestehende Versorgungsnetz angeschlossen sind, ist vor allem die Tarifhöhe wichtig, auf die SPI jedoch keinen Einfluss hat. Je günstiger der vereinbarte Tarif im Abnahmevertrag ist, desto mehr Spielraum bleibt allerdings für NPC. Die Preissetzung auf den Philippinen basiert auf einer politischen Entscheidung, da NPC 2003 große Verluste hatte und es 2004 und 2005 zu Strompreiserhöhungen kam. Die effiziente Produktion zu günstigen Preisen wird daher keine direkten Auswirkungen auf den Strompreis haben.

Die Philippinen weisen nur ein geringes Wirtschaftswachstum, eine hohe Arbeitslosigkeit und einen hohen Bevölkerungszuwachs auf. Der Bau des Kraftwerkes hat direkte, kurzfristig positive Auswirkungen auf die lokale Bevölkerung, die im Zuge des Baus Arbeit gefunden haben, da zu Bauhochzeiten rund 4.000 Menschen beschäftigt waren. Seit Fertigstellung des Kraftwerkes sind weniger als 180 Menschen für den Betrieb beschäftigt. Zusätzlich dazu hat SPI ein Entwicklungsprogramm für die lokale Bevölkerung gestartet, im Zuge dessen soziale Projekte finanziell unterstützt werden, wie beispielsweise Zugang zu medizinischer Vorsorgung, aber auch neue Projekte ins Leben gerufen wurden, bei denen die Menschen eine Anstellung fanden. Welche Auswirkungen diese Maßnahmen auf die Armutsredution haben, wurde bisher aber noch 
nicht gemessen. Insgesamt werden die Effekte auf die Teilnehmer positiv eingeschätzt, jedoch ist deren Anzahl sehr beschränkt. Außerdem geht aus den Unterlagen nicht hervor, ob die Teilnehmer unter der Armutsgrenze leben.

Eine indirekte positive Auswirkung einer stabileren Stromversorgung könnte für Mindanao die Erhöhung der Standortattraktivität sein, um so neue Investitionen anzulocken. Es ist anzunehmen, dass durch die geringe Entwicklung die Lohnkosten sehr gering sind und daher personalintensive Produktionen preisgünstig möglich wären.

Eine wesentliche direkte negative Auswirkung des Kraftwerkes auf die Bevölkerung in Villanueva besteht durch die höhere Umweltbelastung, die auch durch state-of-the-art-Technologie nicht vermieden werden kann. Eine weitere direkte negative Auswirkung ist für die ehemaligen Bewohner der Baufläche entstanden, die umgesiedelt werden mussten. Da jedoch eine Fläche in einer Industriezone ausgewählt wurde, waren nur wenige Familien davon betroffen. Die Auswahl des Standorts und die Umsiedelung wurden von NPC vorgenommen.

Zusammenfassend kann festgestellt werden, dass dieses Kraftwerk nur einen beschränkten direkten Beitrag zur Armutsreduktion leistet, da der Strom nur jenen Menschen zu Gute kommt, die bereits an das bestehende Stromnetz angeschlossen sind. Beachtet man aber das politische und wirtschaftliche Umfeld auf den Philippinen, so hat das Projekt vor allem andere positive Auswirkungen. In Anbetracht der Tatsache, dass nur eine sehr geringe Anzahl an Infrastrukturprojekten auf den Philippinen und beinahe keine auf Mindanao in der Vergangenheit umgesetzt wurden, ist jede Infrastrukturinvestition ein Zugewinn für die Region. Das Kraftwerk auf Mindanao, der unterentwickeltsten Region auf den Philippinen, stabilisiert daher nicht nur das bestehende Stromnetz, sondern hat auch eine wichtige Signalwirkung nach außen, da Mindanao aufgrund der politischen Spannungen für ausländische Investitionen als unattraktiv gilt. Das Wirtschaftswachstum auf den Philippinen ist gering und ein Kraftwerk alleine wird im gegebenen politischen und wirtschaftlichen Umfeld nicht oder wenn ja, nur sehr beschränkt zu mehr Wirtschaftswachstum beitragen können.

Zusätzlich wäre es möglich, dass es zu Know-How Transfer in der Projektgesellschaft kommt, da mit einem geringen Anteil die philippinische State Investment Trust Inc. an der Projektgesellschaft beteiligt ist. Es wäre daher möglich, dass es sowohl in der Planungsphase als auch in der Bau- und Betriebsphase zu positiven Spillover-Effekten kommt.

Die folgende Abbildung 50 gibt die Diskussion über die Auswirkungen des SPI Kraftwerkes auf Wachstum und Armutsreduktion wieder. In dieser Abbildung signalisiert die Stärke der Striche die Intensität des Einflusses. Da das geringe Wachstum auf den Philippinen nicht Pro-Poor ist (siehe Kapitel 3.3.2), 
sind auch die indirekten Auswirkungen auf die Armutsreduktion sehr gering. Dies wird in dieser Grafik durch die strichlierte Linie dargestellt.

Abbildung 50: Auswirkungen des SPI Kraftwerkes auf Wachstum und Armutsreduktion

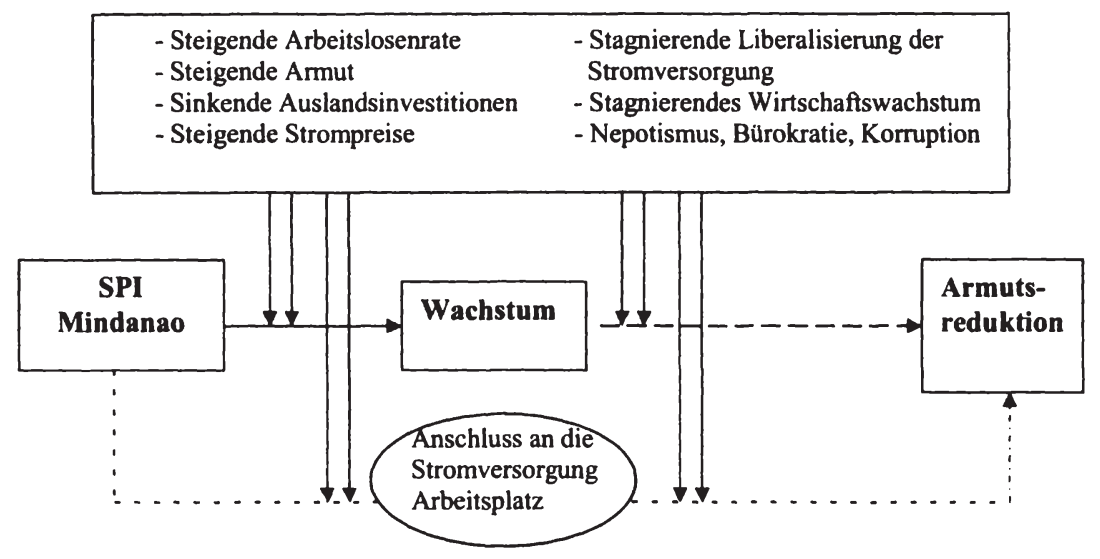

\subsubsection{North Luzon Expressway (NLEX)}

\subsubsection{Eckdaten}

Land:

Projektname:

Sektor:

Lage:

Finanzienungsmodell: Rehabilitate Operate Transfer-Modell (ROT)

ROT Laufzeit:

Projektgesellschaft: Projektsponsoren:

Republik der Philippinen

Transport

North Luzon welches Ereignis früher eintritt

Manila North Tollways Corporation (MNTC)
North Luzon Expressway Project, North Luzon

Expressway Rehabilitation and Expansion (NLEX)

30 Jahre oder bis 31. Dezember 2030, abhängig davon

$67,1 \%$ First Philippine Infrastructure Development Corporation (Lopez Gruppe)

$16,5 \%$ Leighton Asia (Southern) Limited

$13,9 \%$ Egis Projects, S.A.

$2,5 \%$ Philippine National Construction Corporation (PNCC) 

North Luzon Expressways, einschließlich des Baus und der Sanierung von 14 Autobahnkreuzen, 24 Brücken und 31 Straßenüberführungen von Manila (Balintawak exit) bis Clark Special Economic Zone (Sta. Ines exit), und den Betrieb von $8 \mathrm{~km}$ Autobahn in der Subic Special Economic Zone, deren Bau 1996 abgeschlossen wurde.

Das vorliegende Straßenprojekt wurde als Rehabilitate Operate Transfer-Projekt (ROT) vergeben, da bereits Teile der Straße existieren. ROT ist dem BOTModell sehr ähnlich, da der Konzessionär einen Vertrag mit der Regierung des Gastlandes eingeht, in dem er die Renovierung einer bereits existierenden Straße und der Mautinfrastruktur übernimmt und am Ende der Laufzeit die Straße der Regierung wieder überlässt (vgl. Amoatey 2007: 78).

Die Projektgesellschaft besteht aus mehreren philippinischen und ausländischen Sponsoren, wobei die Lopez Gruppe eine Mehrheitsbeteiligung hält. Die geringste Beteiligung wird von der philippinischen PNCC gehalten, die bereits die existierende Straße vor mehr als 30 Jahren gebaut hat. Die beiden ausländischen Unternehmen stammen aus Frankreich und Australien; Egis Projects, S.A. und Leighton Asia. Egis Projects, S.A., eine Tochtergesellschaft des französischen Konzern Egis, ist für die Telekommunikation und das System der Mauteinhebung zuständig. Leighton Asia ist Teil einer der größten australischen Baufirmen, die auf Infrastrukturprojekte spezialisiert ist. Leighton Asia wurde schließlich auch mit den Bauarbeiten beauftragt.

Im Zusammenhang mit der Konzession ist die Tatsache wichtig, dass an MNTC keine neue Konzession vergeben wurde, sondern stattdessen PNCC die Konzessionsrechte an MNTC übertrug und selbst Konzessionsnehmer blieb. Bisher wurde im Projekt die erste Phase, d.h. die Sanierung der bestehenden Schnellstraße durchgeführt. In der zweiten Phase kommt es zum Ausbau der Straße durch neue Autobahnkreuze, Brücken und Überführungen.

\subsubsection{Entstehung}

1970er Jahre

1990er Jahre

Februar 1997

April 1998

Juli 2001

Februar 2003

Februar 2003

Februar 2005
Bau der ursprünglichen Straße

Regierung tritt an die Lopez Gruppe heran

Errichtung der Projektgesellschaft

Unterzeichnung des Supplemental Toll Operation

Agreements

Unterzeichnung der Finanzierungsdokumente

Financial Closing der ursprünglichen

Projektfinanzierung

Baustart

Betriebsaufnahme 
NLEX stellte eine Brownfield-Investition dar, da bereits in den 1970er Jahren die ursprüngliche Straße von der Philippine National Construction Corporation (PNCC) gebaut wurde. PNCC, vormals ein privates Unternehmen, geriet aber in finanzielle Schwierigkeiten und führte daher auch keine Reparaturarbeiten durch. Das Unternehmen wurde daraufhin verstaatlicht, aber auch der Staat konnte die Sanierung der Straße nicht übernehmen. Da sich die Straßenqualität in den letzten 20 Jahren stark verschlechtert hatte, musste die Regierung nach neuen Kapitalgebern suchen, weil sie selber die Sanierung nicht übernehmen wollte. Daher wurden Gespräche mit verschiedenen philippinischen Unternehmen über die Sanierung geführt. Schließlich wurde die philippinische Lopez Gruppe ausgewählt, die zu der Gruppe der finanzstärksten Unternehmen auf den Philippinen zählt.

Anfang 1997, als die Projektgesellschaft errichtet wurde, gab es jedoch bereits die ersten Anzeichen der asiatischen Finanzkrise. Die ursprünglich geplante rein kommerzielle Finanzierung des Projektes wurde aufgrund der Finanzkrise unmöglich. 1999 kontaktierten die Sponsoren verschiedene IFIs, um von ihnen Unterstützung zu bekommen. ADB und IFC übernahmen daraufhin die Führung bei der Strukturierung der Finanzierung und im Juli 2001 wurde nach mehr als vier Jahren die Finanzierung unterzeichnet. 2003 wurde die Finanzierung abgeschlossen und mit der ersten Bauphase begonnen. Zwei Jahre später, 2005, konnte die erste Teilstrecke in Betrieb genommen werden.

Im April 2006 wurde begonnen, über eine mögliche Refinanzierung nachzudenken. Grund dafür waren die Erholung des Finanzmarktes, eine Aufwertung des philippinischen Pesos und die drohende Reduzierung der Dollarindexierung im Rahmen der Tarifvereinbarungen. Ziel dieser Refinanzierung war es, die Dollarschulden zu minimieren, um das Wechselkursrisiko zu senken. Dies sollte gleichzeitig auch zu einer Reduzierung der Darlehensverpflichtungen führen, um für die geplante zweite Bauphase neue Kredite aufnehmen zu können. Im November 2006 konnte schließlich die Refinanzierung erfolgreich abgeschlossen werden.

\subsubsection{Finanzierungs- und Absicherungsinstrumente}

MNTC benötigte ein Gesamtkapital für die ursprüngliche Finanzierung in der Höhe von 371 Mio. US\$, bei einem Verschuldungsgrad von 68,3:29. Alle Kredite wurden in US\$ gewährt, wobei $58 \%$ des gesamten Fremdkapitals von Finanzinstitutionen stammen. In Folge werden nun zuerst die ursprüngliche Finanzierung und daran anschließend die Restrukturierung der Finanzierung dargestellt. 


\section{Ursprüngliche Projektfinanzierung}

\begin{tabular}{llc}
$\mathbf{6 8 , 3} \%$ & Fremdkapital: & \\
& ADB Complementary Financing & 25,0 Mio. US\$ \\
Scheme & \\
ADB Kredit & 45,0 Mio. US\$ \\
& IFC Kredit & 46,0 Mio. US\$ \\
& Australia's EFIC & 55,0 Mio. US\$ \\
& Kommerzielle Kredite & 82,5 Mio. US\$ \\
& (Coface und MIGA Garantien) & \\
& Kommerzieller Kredit in lokaler & 7,5 Mio. US\$ \\
& Währung & 117,5 Mio. US\$ \\
\hline $\mathbf{3 1 , 7} \%$ & Eigenkapital: & \\
\cline { 2 - 2 } & Gesamtkapital der ursprünglichen & $\mathbf{3 7 1 , 0}$ Mio. US\$ \\
\hline
\end{tabular}

Die Finanzierung- und Absicherungsstruktur weist eine parallele Kofinanzierung auf (siehe Kapitel 6.2.2.3) und es sind sowohl nationale als auch multinationale Institutionen beteiligt. ADB und MIGA haben die Strukturierung der Finanzierung übernommen, da die Projektgesellschaft (MNTC) aufgrund der Finanzlage in Asien nicht in der Lage war, die Projektfinanzierung alleine durchzuführen. Aufgrund der französichen Projektbeteiligung hat die Coface eine Garantie für einen kommerziellen Kredit vergeben. Die australische Export Finance and Insurance Corporation (EFIC) hat aufgrund des australischen Sponsors Leighton der Projektgesellschaft einen Kredit gewährt.

In einzelnen Finanzierungs- und Absicherungsinstrumente der beteiligten Institutionen werden nun in alphabetischer Reihenfolge genauer vorgestellt.

\section{ADB Instrumente:}

Kredit:

Complementary financing scheme:
45 Mio. US\$

Kredit mit 10 Jahren Laufzeit und gleichbleibender Tilgung zu gängigen Konditionen. Der Kredit ist abgesichert durch die wesentlichen Projektverträge, wie beispielsweise durch die Einnahmen des Betriebs oder abgeschlossene Versicherungen, und Hypotheken über existente bewegliche Güter und Liegenschaften von MNTC.

\section{Mio. US\$}

Die ADB tritt als Kreditgeber auf und syndiziert das Darlehen an kommerzielle Banken. Dadurch entstehen keine Rückgriffsrechte auf die ADB, dennoch aber genießen die beteiligten kommerziellen Banken einen 
besseren Schutz vor dem Länderrisiko, da die ADB den "preferred creditor status " aufweist. Dadurch wird dieser Kredit im Falle des Eintretens von politischen Risiken, wie beispielsweise Konvertierungsbeschränkungen, vorrangig behandelt. Die Geschäftsbanken übernehmen das wirschaftliche Risiko, ohne jedoch mit dem Kreditnehmer ein eigenes Rechtsverhältnis zu begründen.

Kreditvereinbarung: ADB und die Projektgesellschaft schließen einen Kreditvertrag ab.

Ergänzende Kreditvereinbarung:

ADB und die Projektgesellschaft vereinbaren die Syndizierung.

TeilnahmeADB und die teilnehmenden vereinbarung: Kreditgeber schließen eine Vereinbarung $a b$.

\section{Australia's Export Finance and Security Corporation (EFIC) Instrument:}

Kredit:

Coface Instrument:

EFIC hat der Projektgesellschaft einen Kredit gewährt und stellt den größten Kreditgeber dar.

Garantie:

35 Mio. US\$

Mit der Garantie von Coface werden kommerzielle Kredite in der Höhe von 35 Mio. US\$ teilweise gegen politische und wirtschaftliche Risiken geschützt. Die genaue Deckung konnte nicht in Erfahrung gebracht werden.

Garantievereinbarung: Diese Vereinbarung regelt zwischen den kommerziellen Banken und Coface den Haftungsumfang.

\section{IFC Instrument:}

Kredit:

46,0 Mio. US\$

Die IFC gewährt einen direkten Kredit an die Projektgesellschaft. 
Political Risk

Guarantee:
47,5 US\$ Mio.

Mit dieser Garantie sichert die MIGA Teile des Fremdkapitals gegen folgende Tatbestände ab: Transferrisiko, Enteignung, Krieg, Unruhen, Terrorismus und Vertragsbruch durch die Gastregierung.

Garantievereinbarung: Diese Verinbarung regelt zwischen den jeweiligen Partnern und MIGA die abgesicherten Risiken.

An der Finanzierung der NLEX beteiligten sich insgesamt drei multinationale und zwei nationale Institutionen, wobei die ADB und die MIGA die Strukturierung übernahmen.

Abbildung 51 zeigt grafisch den Zusammenhang der verschiedenen Finanzierungs- und Absicherungsinstrumente bzw. die involvierten Institutionen im NLEX-Projekt.

\section{Abbildung 51: Ursprüngliche Finanzierungs- und Absicherungsstruktur von NLEX}

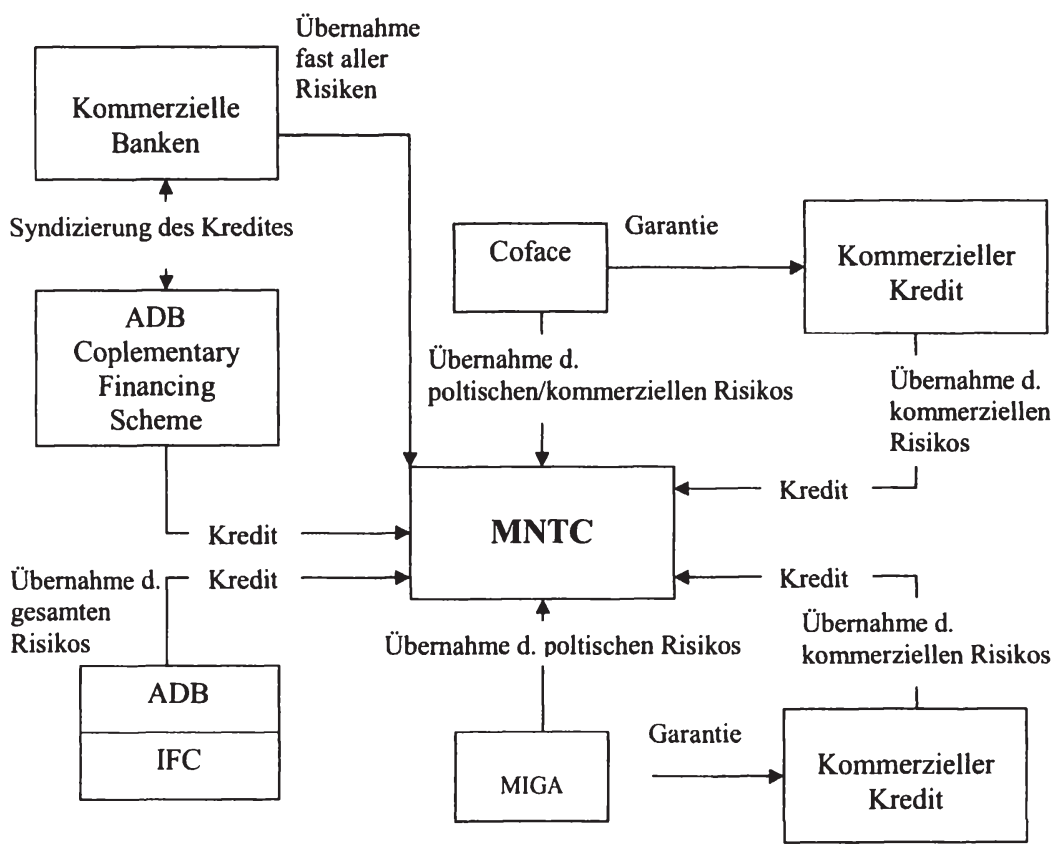


In diesem Projekt stellt die australische EFIC den größten direkten Kredit zur Verfügung. Die ADB weist aber insgesamt die größte Beteiligung auf, da sie sowohl über einen direkten als auch über einen indirekten Kredit das Projekt unterstützt. Beim indirekten Kredit, der als „Complementary financing scheme“ bezeichnet wird tritt die ADB als "lendor of record" auf und syndiziert die Kreditforderung an kommerzielle Banken. Dadurch übernehmen die kommerziellen Banken beinahe alle involvierten Risiken, aber sie profitieren von den gleichen Privilegien und Sicherheiten wie ein direkter ADB-Kredit (siehe Kapitel 5.1.3) Die Weltbankgruppe hat klassische Produkte an das Projekt vergeben. Die IFC gewährte MNTC einen direkten Kredit und die MIGA eine politische Garantie.

Abgesehen vom direkten Kredit der EFIC hat sich auch die französiche Exportkreditversicherung Coface mit einer Garantie am Projekt beteiligt. Diese Garantie deckt Teile des kommerziellen und politischen Risikos ab.

\section{Restrukturierung der Projektfinanzierung}

Durch den ungünstigen Zeitpunkt bei der Strukturierung der ursprünglichen Finanzierung war die Projektgesellschaft mit hohen Finanzierungskosten und Wechselkursrisiken aufgrund der US\$-Kredite belastet. Die ursprüngliche Finanzierung beruhte ausschließlich auf US\$-Krediten und es wurde eine Mautgebühr vereinbart, der nur in den ersten Jahren zu $100 \%$ an den US\$ indexiert war. Im Rahmen des Supplemental Toll Agreements (STOA) wurde für das Jahr 2007 eine Reduzierung der Dollarindexierung von 100 auf $50 \%$ vereinbart. Daher wollte MNTC gleichzeitig auch seine Dollarkredite im Jahr 2007 senken, um die Höhe der Einnahmen an die der Ausgaben etwas anzupassen.

Da sich der Kapitalmarkt nach der Finanzkrise wieder langsam zu erholen begann, interessierte sich MNTC für eine Restrukturierung der Finanzierung, um das Wechselkursrisiko zu senken. MNTC wollte durch die Restrukturierung zusätzlich auch die Kreditverpflichtungen senken, um den Bau der 2. Phase einfacher finanzieren zu können.

Im April 2006 begann man daher über eine Restrukturierung der Finanzierung mit den beteiligten Banken zu diskutieren. MNTC wollte durch die Restrukturierung vordergründig das Wechselkursrisiko senken und versuchte daher, möglichst viel an US\$-Schulden frühzeitig mit einer gleichzeitigen Verschuldung in Peso zurückzubezahlen. Dadurch verringerte MNTC das Ungleichgewicht zwischen US\$-Schulden und Peso-Einnahmen soweit, dass von der ursprünglichen Finanzierung nur noch 100 Mio. US\$ Schulden übrig blieben und die restlichen Schulden auf Peso lauteten.

MNTC erkundigte sich bei den Kreditgebern der ursprünglichen Finanzierung, welche Schulden frühzeitig zu begleichen seien. Da ein wirtschaftlich stabiles Unternehmen auch im Interesse der beteiligten Banken ist, willigten sie in eine 
vorzeitige Rückzahlung der US\$ ein. Einige Kredite, wie beispielsweise jener von IFC, wurden vollständig zurückbezahlt, andere nur teilweise. Durch die Reduktion des Fremdkapitals kam es auch zu einer frühzeitigen Auflösung oder Reduzierung von Garantien. Die vorzeitige Rückzahlung wurde durch die Emission von Unternehmensanleihen ermöglicht.

\section{Restrukturierung der Finanzierung: Fremdkapital \\ ADB Complementary Loan Financing ADB Kredit \\ Australia's Export Finance and Insurance Corporation \\ Kommerzieller Kredit \\ Kommerzieller Kredit (Coface) \\ Unternehmensanleihen \\ Verbleibendes Fremdkapital nach der \\ Restrukturierung \\ Eigenkapital \\ Gesamtkapital}

20,0 Mio. US\$

18,0 Mio. US\$

15,0 Mio. US\$

32,05 Mio. US\$

14,95 Mio. US\$

5,5 Mrd. Peso

Ca.210,0 Mio. US\$

117,5 Mio. US\$

327,5 Mio. US\$

Durch die Restrukturierung blieb von den multinationalen Institutionen nur noch ADB am Projekt beteiligt. Sowohl die Höhe des direkten, als auch des indirekten Kredites wurde gesenkt. Der Kredit der EFIC und die Garantie von Coface wurden auch wesentlich reduziert.

Die Emission von kurzfristigen, fix-verzinsten Unternehmensanleihen in philippinischen Peso stellte eine private Platzierung an ausgewählte institutionelle Kreditgeber dar. Die Anleihen wurden vom Kapitalmarkt sehr positiv aufgenommen und es wurde mehr Kapital geboten als MNTC Anleihen aufnehmen wollte. Schließlich einigte man sich auf 5,5 Milliarden Peso mit einer Laufzeit von sieben Jahren, die mit einem fixen jährlichen Zins von 9,75\% bedient werden. Gleichzeitig wurde mit der Ausgabe der Anleihen eine Brückenfinanzierung in der Höhe von 96 Millionen US\$ mit der japanischen Bank Mizuho Corporate Bank Ltd. organisiert. Diese Brückenfinanzierung war vor allem deswegen notwendig, weil die Konvertierung des philippinischen Pesos in eine ausländische Währung durch die philippinische Zentralbank reguliert ist und MNTC daher nicht sofort die gesamten 5,5 Milliarden Peso in US\$ umtauschen konnte. Die Brückenfinanzierung ermöglichte MNTC die geplante Rückzahlung der US\$-Kredite unabhängig von der endgültigen Umwechslung der Pesosumme in US\$ durch die philippinische Zentralbank. Im Gegenzug dazu, wurde die Brückenfinanzierung wiederum durch die US\$ aus der Anleihe befriedigt. In der nun folgenden Abbildung 52 wird abschließend noch grafisch die Finanzierungs- und Absicherungsstruktur nach der Restrukturierung dargestellt. 
Abbildung 52: Restrukturierte Finanzierungs- und Absicherungsstruktur von NLEX

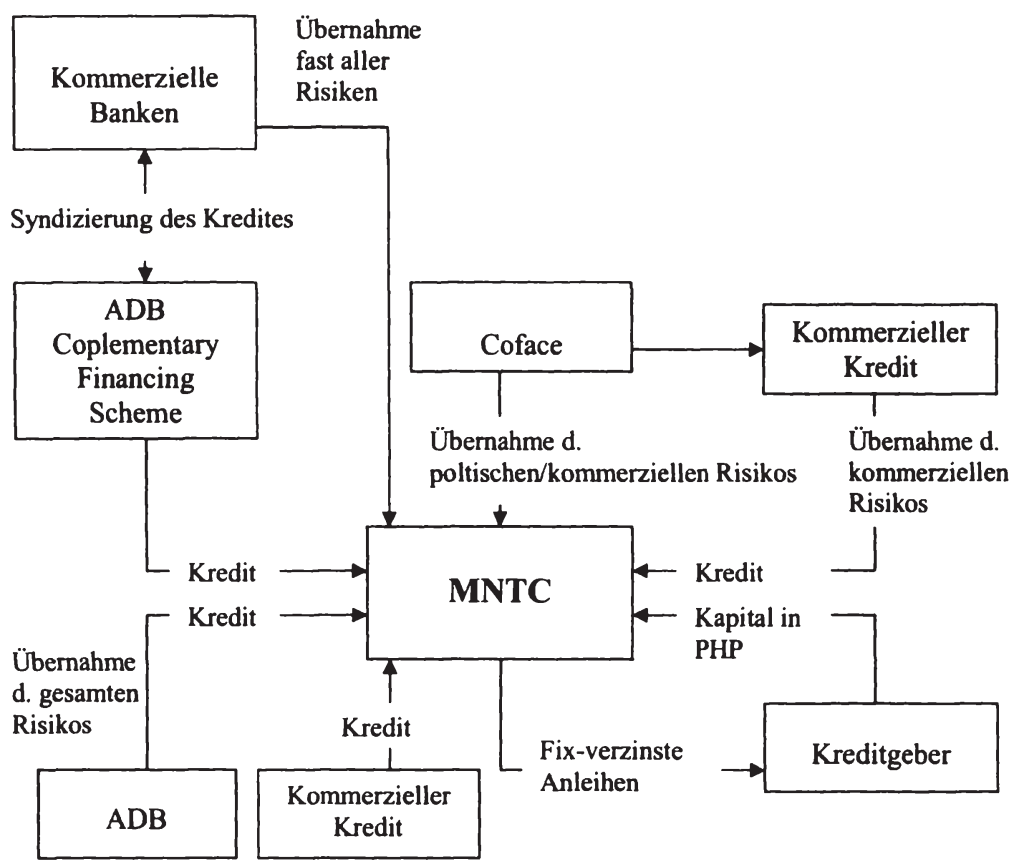

\subsubsection{Vertragsstruktur}

Aufgrund der umfangreichen Vertragsstruktur wird zunächst in der folgenden Abbildung 53 das Vertragsnetz grafisch dargestellt. Im Anschluss daran werden die wichtigsten Verträge etwas genauer dargestellt. In dieser Übersicht werden nun zusätzlich zu den Verträgen mit den Finanzinstitutionen auch die Verträge mit philippinischen und anderen privaten Unternehmen berücksichtigt. 


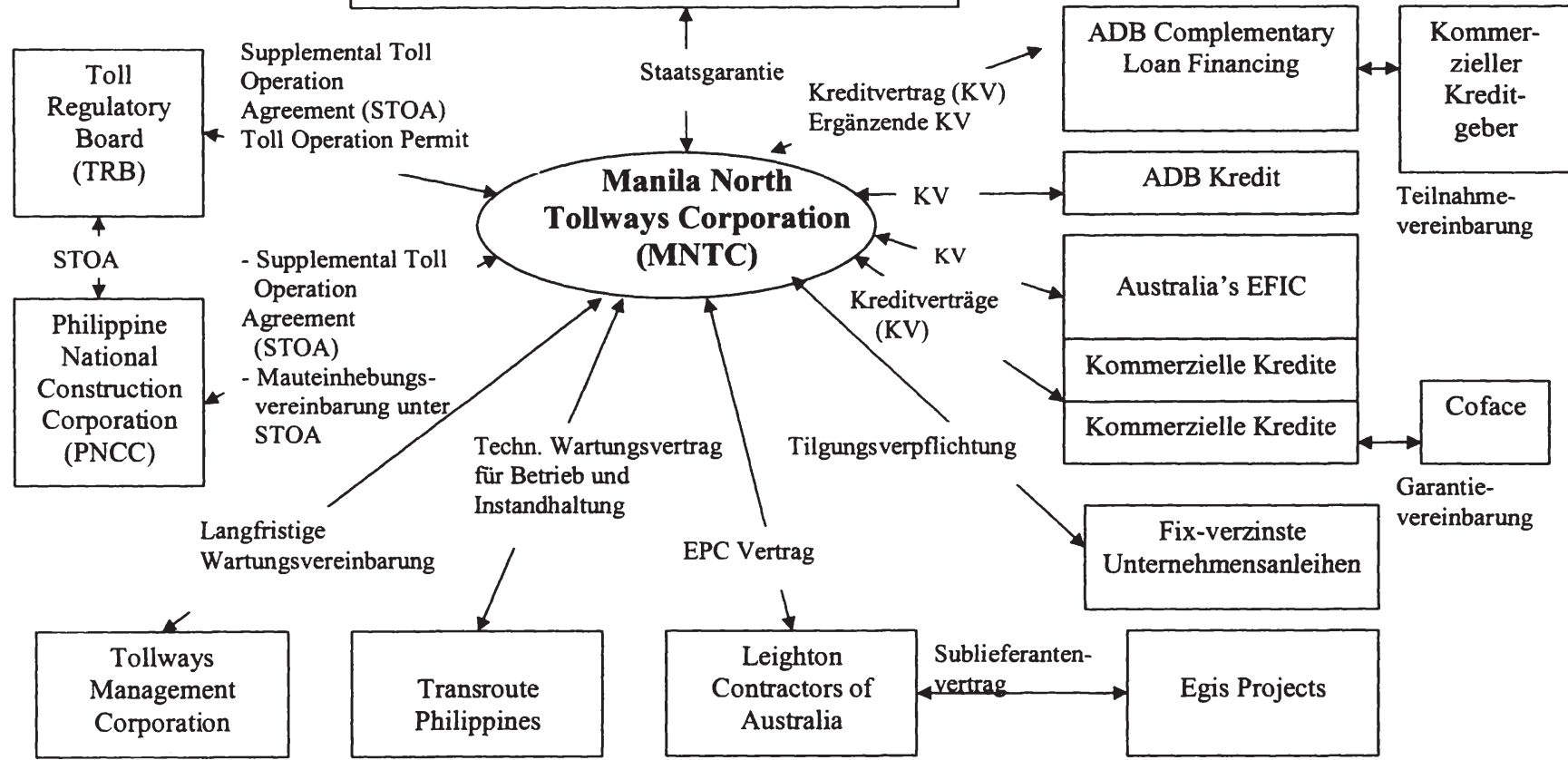


Supplemental Toll

Operation Agreement (STOA):

Mauteinhebungsvereinbarung unter STOA:
Vertragspartner: Toll Regulatory Board, Philippine National Construction Corporation (PNCC) und die Projektgesellschaft (MNTC)

Inhalt: MNTC wird nach Ablauf der Konzessionslaufzeit das Projekt kostenlos an die philippinische Regierung zurückgeben.

$\rightarrow$ Die Konzessionsrechte von MNTC basieren auf dem STOA.

Vertragspartner: PNCC und MNTC

Inhalt: Es wird ein zweiteiliger Tarif in PHP, eine Dollarindexierung und Inflationsanpassung vereinbart.

$\rightarrow$ Pauschaltarif: Alle Fahrzeuge, die durch den offenen Abschnitt fahren, müssen unabhängig von der Fahrtdauer, eine Pauschale bezahlen.

$\rightarrow$ Individueller Tarif: Für den geschlossenen Abschnitt wird eine Maut für die Länge der Fahrt eingehoben, die bei mehreren Mauthaltestellen bezahlt werden kann.

$\rightarrow$ Die Maut wird periodisch überprüft und erlaubt MNTC, die Höhe der Maut an die vereinbarten Indizes anzupassen.

$\rightarrow$ MNTC darf das Wechselkursrisiko aus dem Fremdkapital im Tarif berücksichtigen. Die Indexienung von $100 \%$ für die ersten zwei Betriebsjahre wird ab 2007 auf $50 \%$ reduziert werden.

$\rightarrow$ Die Tarife werden auch an die Inflation angepasst und es wird eine jährliche Mindestanpassung von $1,25 \%$ vereinbart.

Zollbetriebsgenehmigung: Vertragspartner: Toll Regulatory Board (Toll Operation Permit) (TRB) und MNTC

Inhalt: Die Mauteinhebung für die Phase 1 von MNTC wird geregelt.

$\rightarrow$ Nach 2 Jahren Bauzeit begann MNTC ab 8. Februar 2005 mit der Einhebung der Maut.

Staatsgarantie:

Vertragspartner: Philippinische Regierung und MNTC

Inhalt: Sie garantiert MNTC die Verfügbarkeit, 
Konvertierbarkeit und Transferierbarkeit von ausländischer Währung und erlaubt Off-shore Konten für die Projektfinanzierung.

\section{Wesentliche Verträge mit anderen privaten Unternehmen}

EPC Vertrag:

Wartungsvertrag für

den Betrieb und die Instandhaltung:

Langfristige Wartungsvereinbarung:
Technischer

Vertragspartner: Leighton Contractors of Australia und MNTC

Inhalt: MNTC beauftragt darin Leighton Contractors of Australia mit dem Bau und der Renovierung der Straße.

Sublieferantenvertrag: Vertragspartner: Egis Projects und Leighton Contractors of Australia

Inhalt: Egis Projects wird beauftragt die Telekommunikationssysteme und Verkehrsmanagementsysteme für die Mauteinhebung zu liefern.

Vertragspartner: Transroute Philippines und MNTC

Inhalt: Die technische Wartung für den Betrieb und die Instandhaltung wird an Transroute Philippines vergeben.

Vertragspartner: Tollways Management Corporation und MNTC

Inhalt: Die langfristige Wartungsvereinbarung für Betrieb und Instandhaltung wird an Tollways Management Corporation vergeben.

\subsubsection{Zusammenfassende Gegenüberstellung von NLEX}

In dieser nun folgenden zusammenfassenden Gegenüberstellung werden zuerst die Finanzierungs- und Absicherungsinstrumente und daran anschließend die Vertragsstruktur diskutiert. Einschränkend muss an dieser Stelle jedoch festgehalten werden, dass NLEX das einzige Straßenprojekt in der vorliegenden Arbeit darstellt und daher teilweise andere Verträge und Finanzierungscharakteristika aufweist.

\section{Finanzierungs- und Absicherungsinstrumente}

Das NLEX-Projekt stellt eines der wenigen Mautstraßenprojekte auf den Philippinen dar, das erfolgreich an private Unternehmen vergeben wurde. 
Besonders dabei hervorzuheben ist die hohe philippinische Beteiligung an der Projektgesellschaft. Bei der Strukturierung der Finanzierung Ende der 1990er Jahre war die Projektgesellschaft stark von den Auswirkungen der asiatischen Finanzkrise betroffen.

Die ursprünglich geplante rein kommerzielle Finanzierung konnte nicht einmal Ansatzweise durchgeführt werden und MNTC benötigte die Unterstützung der multilateralen Institutionen, um eine Finanzierung für das Projekt aufstellen zu können. ADB und IFC übernahmen bei der Strukturierung schließlich die Führerschaft.

Aufgrund der schlechten Finanzlage wurden MNTC ausschließlich Dollarkredite gewährt, die ein wesentliches Wechselkursrisiko darstellen. Die Einnahmen von MNTC basieren auf den Tarifvereinbarungen mit der philippinischen Regierung, wobei eine Dollarindexierung vorgesehen ist, die aber 2007 reduziert wurde. Gleichzeitig erholte sich der Finanzmarkt und MNTC restrukturierte die ursprüngliche Projektfinanzierung, als sie einen Teil der Dollarkredite frühzeitig zurückbezahlte, indem sie Unternehmsanleihen emittierte.

Eine Zusammenfassung der ursprünglichen, wie auch der restrukturierten Finanzierung wird in der folgenden Tabelle 13 gegeben. Diese soll nochmals die Finanzierung und Absicherung der beiden Projekte zusammenfassen und daran anschließend werden die Gemeinsamkeiten und Unterschiede diskutiert.

NLEX weist mit 32,5\% eine relativ niedrige Summe an kommerziellen Krediten im Vergleich zum Gesamtkapital auf, die ferner zu $100 \%$ mit Garantien abgedeckt ist. Die institutionellen Kredite blieben hingegen unbesichert. Die geringe kommerzielle Beteiligung lässt sich auf die schwierige Finanzinstitution zum Zeitpunkt der Strukturierung der Finanzierung zurückführen. Auch bei Phu My 2.2 kam es zu einer sehr geringen Beteiligung der kommerziellen Banken und beide Projekte wurden bei der Strukturierung der Finanzierung von multilateralen Institutionen stark unterstützt.

Auch an diesem Projekt widerspiegelt sich die Herkunft der Projektsponsoren in der Finanzierung. Der größte institutionelle Kreditgeber ist die australische EFIC, die einen direkten Kredit an das Projekt vergibt. Die französische Beteiligung ist anhand der Garantie der Coface zu erkennen, die einen Teil des kommerziellen Fremdkapitals absichert.

NLEX ist das einzige dieser vier Projekte, dem ein indirekter Kredit im Rahmen des "Complementary Financing Schemes" durch die ADB gewährt wurde. Durch diese Konstellation können sich kommerzielle Kreditgeber an der Finanzierung eines Projektes beteiligen, welche die gleichen Privilegien wie die ADB genießen. Hier sind insbesondere der „preferred creditor status “ der multilateralen Banken und deren Steuerbefreiung von Vorteil. Gleichzeitig überlassen die Kreditgeber der $\mathrm{ADB}$, die als ,lendor-of-record" agiert, auch die Administration des Kredites. 
Tabelle 13: Zusammenfassung der Finanzierung und Absicherung von NLEX

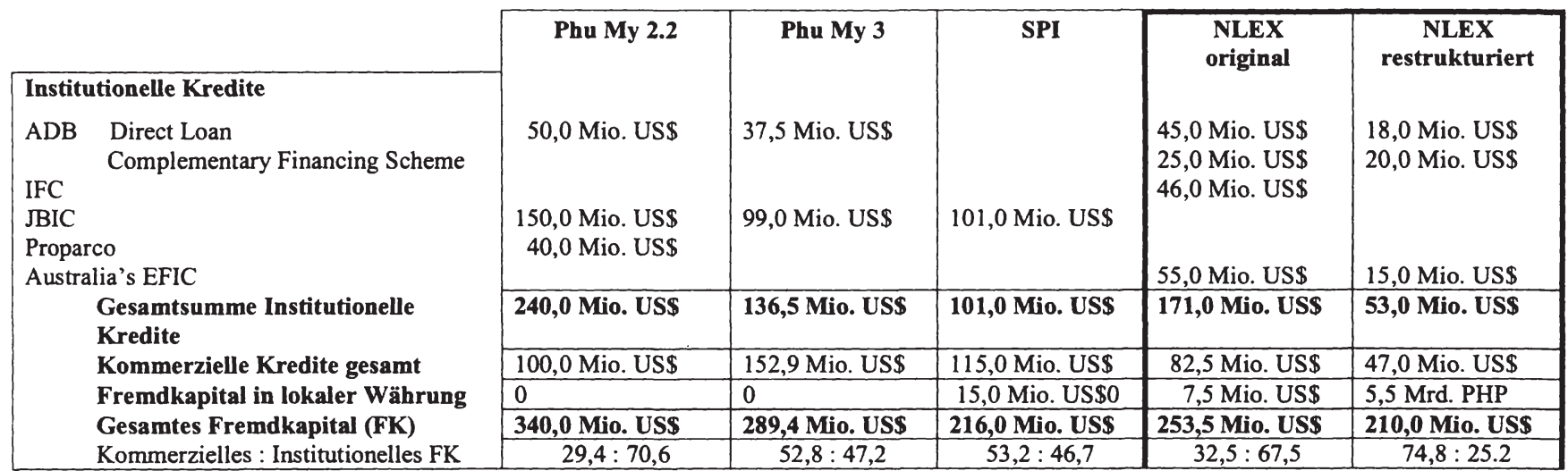




\begin{tabular}{|c|c|c|c|c|c|c|c|}
\hline & & & Phu My 2.2 & Phu My 3 & SPI & $\begin{array}{c}\text { NLEX } \\
\text { original }\end{array}$ & $\begin{array}{c}\text { NLEX } \\
\text { restrukturiert }\end{array}$ \\
\hline \multirow{11}{*}{ 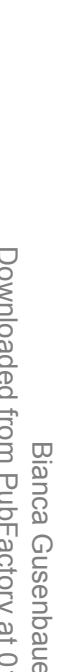 } & ADB & $\begin{array}{l}\text { Political Risk Garantie } \\
\text { Guarantor of record }\end{array}$ & 25,0 Mio. US\$ & 30,0 Mio. US\$ & & & \\
\hline & BR Deutschland & $\mathrm{DiA}$ & & & 95,0 Mio. US\$ & & \\
\hline & Coface & & & & & 35,0 Mio. US\$ & 14,95 Mio. US\$ \\
\hline & IDA & Partial Risk Garantie & 75,0 Mio. US\$ & & & & \\
\hline & MIGA & $\begin{array}{l}\text { Political Risk Garantie } \\
\text { für Eigenkapital } \\
\text { für Fremdkapital } \\
\text { für Abdeckung eines } \\
\text { Swaps }\end{array}$ & & $\begin{array}{l}\text { 43,2 Mio. US\$ } \\
75,0 \text { Mio. US\$ } \\
15,0 \text { Mio. US\$ }\end{array}$ & & 47,5 Mio US\$ & \\
\hline & NEXI & $\begin{array}{l}\text { Overseas United Loan } \\
\text { Insurance }\end{array}$ & & $\begin{array}{l}\text { Summe ist nicht } \\
\text { bekannt }\end{array}$ & $\begin{array}{l}\text { 40,4 Mio. US\$ } \\
\text { (JBIC Kredit) }\end{array}$ & & \\
\hline & \multirow{2}{*}{\multicolumn{2}{|c|}{$\begin{array}{l}\text { Gesamtsumme Garantien } \\
\text { Gesamtsumme Eigenkapital (EK) } \\
\% \text {-Deckung des kommerziellen FK }\end{array}$}} & 100,0 Mio. USS & 163,2 Mio. USS & 135,4 Mio. US\$ & 82,5 Mio. USS & 14,95 Mio. US\$ \\
\hline & & & $\begin{array}{c}140,0 \text { Mio. US4 } \\
100 \%\end{array}$ & $\begin{array}{l}96,5 \text { Mio. US\$ } \\
59 \%\end{array}$ & $\begin{array}{c}89,0 \text { Mio. US } \$ \\
81,9 \%\end{array}$ & $\begin{array}{c}117,5 \text { Mio. US\$ } \\
100 \%\end{array}$ & $\begin{array}{c}117,5 \text { Mio. US } \$ \\
9,5 \%\end{array}$ \\
\hline & \multicolumn{2}{|c|}{ \%-Deckung des institutionellen FK } & $0 \%$ & $0 \%$ & $40 \%$ & $0 \%$ & $0 \%$ \\
\hline & \multicolumn{2}{|c|}{$\%$-Deckung des gesamten FKs } & $29,4 \%$ & $56,4 \%$ & $61,5 \%$ & $32,5 \%$ & $7,1 \%$ \\
\hline & \multicolumn{2}{|c|}{$\%$-Deckung des Eigenkapitals } & $0 \%$ & $77,7 \%$ & $0 \%$ & $0 \%$ & $0 \%$ \\
\hline
\end{tabular}


Aufgrund der Restrukturierung der Finanzierung haben sich die Kredit- und Garantiegeber am Projekt verändert und NLEX stellt die erste limited-recourse Projektfinanzierung auf den Philippinen dar, die erfolgreich die Finanzierung restrukturiert hat. Da zum Zeitpunkt der ursprünglichen Finanzierung nur eine beschränkte Finanzierung in lokaler Währung möglich war, die Projektgesellschaft aber gleichzeitig durch die abnehmende Dollarindexierung im Tarifvertrag vermehrt dem Wechselkursrisiko ausgesetzt war, wurde die Finanzierung restrukturiert. Durch die Emission von Unternehmensanleihen wurde mehr als die Hälfte der Kredite frühzeitig zurückbezahlt. Momentan sind auf den Philippinen Finanzierungen in Peso bis maximal sieben Jahre möglich. Diese zeitliche Einschränkung verhindert jedoch eine völlige Finanzierung in heimischer Währung, da Projektfinanzierungen typischerweise längere Laufzeiten aufweisen. Dennoch wurde anhand von NLEX und SPI Mindanao gezeigt, dass auf den Philippinen bereits beschränkt die direkte Finanzierung in lokaler Währung besteht.

Im Zuge der Restrukturierung wurden alle verbleibenden kommerziellen und institutionellen Kredite stark reduziert und die Weltbankgruppe ist völlig aus dem Projekt ausgeschieden. Dadurch konnte das Verhältnis zwischen kommerziellem und institutionellem Fremdkapital umgedreht werden und in der neuen Finanzierung stammen $74,8 \%$ des gesamten Fremdkapitals von kommerzieller Seite.

Die ADB blieb als einzige multilaterale Institution mit beiden Krediten im Projekt. Nach der Restrukturierung sind nur noch 9,5\% des kommerziellen Fremdkapitals (inkl. Unternehmensanleihen) mit Garantien abgesichert. Die Deckung des gesamten Fremdkapitals ist sogar noch niedriger und liegt bei 7,1 $\%$. Das Projekt weist im Vergleich zu den anderen Projekten daher bei weitem die geringste Garantiesumme auf und dies deutet auf ein relativ hohes Vertrauen der Kreditgeber in das Projekt.

\section{Vertragsstruktur}

Die Vertragsstruktur ist in der Projektfinanzierung großteils stark standardisiert, doch stellt NLEX das einzige Straßenprojekt in der vorliegenden Arbeit dar. Daher können die Verträge nicht direkt miteinander verglichen werden. In Folge werden daher Besonderheiten des NLEX-Projektes vorgestellt und es wird auf wesentliche Unterschiede zu den anderen Projekten hingewiesen.

\section{Hohe philippinische Beteiligung bei der Projektgesellschaft.}

MNTC ist das einzige der vier Projekte, das eine einheimische Mehrheitsbeteiligung aufweist. Die beiden ausländischen Sponsoren sind gemeinsam nur mit knapp $30 \%$ beteiligt. 
2. Es wurde keine neue Konzession vergeben.

PNCC hat bereits eine Konzession zum Bau der ursprünglichen Straße von der philippinischen Regierung erhalten. Da PNCC wiederum Sponsor der neuen Projektgesellschaft ist, wurde die Konzession von PNCC an die neue Projektgesellschaft geleast. Dadurch bestand für die neue Projektgesellschaft kein Bedarf an einer neuen Konzession.

\section{Reduzierung der Dollarindexierung in der Mauteinhebungsver- einbarung.}

Bei Mautautobahnen gibt es im Gegensatz zu Kraftwerken keinen Abnahmevertrag, sondern eine Mauteinhebungsvereinbarung. Sowohl die Abnahmeverträge als auch die Mauteinhebungsvereinbarung sind Dollarindexiert. NLEX stellt das einzige Projekt dar, bei dem eine Reduktion der Dollarindexierung vereinbart wurde. Dies bedeutet für die Unternehmen ein erhöhtes Wechselkurrisiko und daher auch einen Handlungsbedarf für die Projektgesellschaft bei der Reduktion der Dollarverschuldung.

\section{Eine aktive Rolle der ausländischen Sponsoren.}

Beide ausländischen Investoren, EGIS Projects und Leighton Asia, übernehmen ihrer Kernkompetenz entsprechend eine führende Rolle beim Bau und bei der Sanierung der NLEX.

\subsubsection{Auswirkungen der NLEX auf die Armutsreduktion}

NLEX ist eine Schnellstraße, die den Großraum Manila mit der Region Northern Luzon verbindet, die eine der reichsten Regionen auf den Philippinen ist. Dieses Projekt stellt eine Sanierung der ursprünglichen Straße aus den 1970er Jahren dar und beeinflusst positiv Transportzeit und -qualität. Im Norden von Manila befinden sich Wirtschaftszentren, wie beispielsweise Subic Bay, welche die philippinische Regierung als wesentliche Wirtschaftsstandorte entwickeln möchte.

Die wesentlichen direkten positiven Auswirkungen der Sanierung der Straße bestehen daher in der Verkürzung der Transportzeit und dadurch in der Reduktion der Transportkosten durch Einsparungen bei Treibstoff und Zeit. Eine bessere Verbindung nach Manila unterstützt die wirtschaftliche Entwicklung in dieser Region und trägt zu einer höhern Standortattraktivität bei. Auf den Philippinen konzentriert sich das wirtschaftliche Geschehen auf den Großraum Manila und durch die bessere Straße wird auch der Zugang zu Arbeitsplätzen, Ausbildungsstätten und Märkten erleichtert. Diese positiven Auswirkungen betref- 
fen aber vor allem jene Menschengruppe auf den Philippinen, die nicht $\mathrm{zu}$ den Armen oder extrem Armen gezählt werden kann. Die Armen werden diese Straße nur unregelmäßig nützen, da ihr Leben und ihre Aktivitäten in ihrem nahen Lebensraum stattfinden.

Wie im Kapitel 3.3.4 dargestellt wurde, profitieren arme Menschen nur sehr beschränkt von Schnellstraßen; vor allem die ländlichen Armen können nur dann von Schnellstraßen profitieren, wenn gleichzeitig Zubringerstraßen zu ihren Siedlungen gebaut werden. Im Fall von NLEX werden zusätzlich zur Sanierung neue Brücken und Straßenüberführungen gebaut, die aber vor allem der besseren Verbindung der existierenden Schnellstraßen in Manila und der Vermeidung von Umwegen dienen soll. Dies kann wiederum zu verkürzten Transportzeiten und aufgrund des geringeren Treibstoffverbrauches auch zu einer geringeren Umweltbelastung im Großraum Manila führen. Jedoch sind in diesem Projekt konkret keine Zubringerstraßen für entlegene Region entlang der Mautstraße geplant.

Die arme Bevölkerung kann direkt durch den Bau und der Sanierung der Straße profitieren, da neue Arbeitsplätze geschaffen werden. Auf den Philippinen ist die Arbeitslosigkeit seit Jahren extrem hoch, insbesondere in Manila aufgrund der Landflucht. Zusätzlich dazu sind durch die Bautätigkeit neue Mikrounternehmen entlang der Straße entstanden, die beispielsweise die Bauarbeiter mit Essen versorgen.

Durch die Verbesserung der Straßenqualität wurde auch die Anzahl der Verkehrsunfälle drastisch reduziert. In Anbetracht der Tatsache, dass in Entwicklungsländern Arme meistens Verkehrsmittel benützen müssen, die sich in einem sehr schlechten Zustand befinden, können sie überproportional von dieser Entwicklung profitieren.

Direkte negative Auswirkungen hat der Bau auf jene Armen, die aufgrund von Umsiedelungsmaßnahmen von der Regierung ihren Lebensraum verloren haben. Im städtischen Raum sind von Baumaßnahmen vor allem Lebensräume von Armen betroffen, da diese meistens legal oder illegal an den schlechtesten Orten der Stadt, wie beispielsweise unter Autobahnen, leben. Durch die Umsiedlungsmaßnahmen der Regierung wurde auch der Baustart von NLEX verzögert. Genaue Zahlen über die Anzahl der Umgesiedelten sind nicht verfügbar. Auch für die Phase 2 von NLEX müssen wieder Menschen umgesiedelt werden, da es in Manila kaum unbebaute Flächen gibt. Eine weitere direkte negative Auswirkung entsteht für jene Armen, die durch die Erweiterungen der bestehenden NLEX von mehr Verkehrslärm und Umweltbelastung betroffen sind.

Eine indirekte negative Auswirkung auf die Armen entstand durch die Erhöhung der Tarife. Die Transportkosten stellen für die Armen oft einen überproportional hohen Anteil des Einkommens dar und eine geringe Preiserhöhung kann gravierende Auswirkungen haben. Vor allem im städtischen Raum hat sich 
die Tarifgestaltung verändert. Es wird für die ersten Kilometer ein Pauschalpreis unabhängig von der Fahrdistanz eingehoben.

Zusammenfassend lässt sich feststellen, dass NLEX nur einen beschränkten direkten positiven Beitrag zur Armutsreduktion leistet, da die Sanierung und der Ausbau der Straße vor allem der wirtschaftlichen Entwicklung der Region dient. Die Armen können direkt über die Schaffung der Arbeitsplätze in der Bauphase, die Verkürzung der Transportzeit, die allerdings aufgrund der Mauterhöhung, mit erhöhten Kosten verbunden ist, und die Reduktion der Verkehrsunfälle profitieren. Da die Transportkosten oft einen wesentlichen Teil des Einkommens armer Menschen darstellen, kann die Erhöhung der Maut dazu führen, dass sie die Straße nicht mehr benützen oder mehr Einkommen für den Transport aufwenden müssen. Indirekt können die Armen von der Straße dann profitieren, wenn sich die Region Northern Luzon wirtschaftlich weiterentwickelt und es zu einem höheren Wachstum kommt, von dem auch die arme Bevölkerung profitiert. Die geringen direkten positiven Auswirkungen sind vor allem auch auf die Tatsache zurückzuführen, dass Arme kaum Kraftfahrzeuge besitzen, die auf Mautstraßen erlaubt sind und nur dann die Straße benützen werden, wenn sie öffentliche Verkehrsmittel verwenden.

Berücksichtigt man aber wiederum die geringen öffentlichen Ausgaben im Infrastruktursektor, die hohe Arbeitslosigkeit und das geringe Wirtschaftswachstum, dann hat die Sanierung und der Ausbau vor allem positive Auswirkungen auf den Wirtschaftsstandort Northern Luzon. Das Wirtschaftswachstum auf den Philippinen ist gering und eine sanierte bzw. ausgebaute Schnellstraße alleine wird im gegebenen politischen und wirtschaftlichen Umfeld nicht oder wenn ja, nur sehr beschränkt zu mehr Wirtschaftswachstum beitragen können.

Im Zusammenhang mit dem 8. Ziel bei den MDGs (siehe Kapitel 2.2.2.2) ist jedoch festzuhalten, dass dieses Projekt wesentlich zur Erreichung dieses Zieles beitragen kann. Durch die Bildung einer Projektgesellschaft mit hoher in- und ausländischer Beteiligung kann es zu einem Know-How Transfer kommen, da die philippinischen Partner bisher noch wenig Erfahrung im Betreiben von Mautstraßen hatten. Es wurde von beiden, also sowohl von philippinischer als auch ausländischer Seite darauf hingewiesen, dass der jeweils andere Partner sehr wichtig für das Projekt sei.

Die folgende Abbildung 54 gibt die Diskussion über die Auswirkungen der NLEX auf Wachstum und Armutsreduktion wieder. In dieser Abbildung signalisiert die Stärke der Striche die Intensität des Einflusses. Auch in diesem Beispiel sind aufgrund des geringen Pro-Poor Wachstums die indirekten Auswirkungen auf die Armutsreduktion sehr gering. Dies wird in dieser Grafik durch die strichlierte Linie dargestellt. 


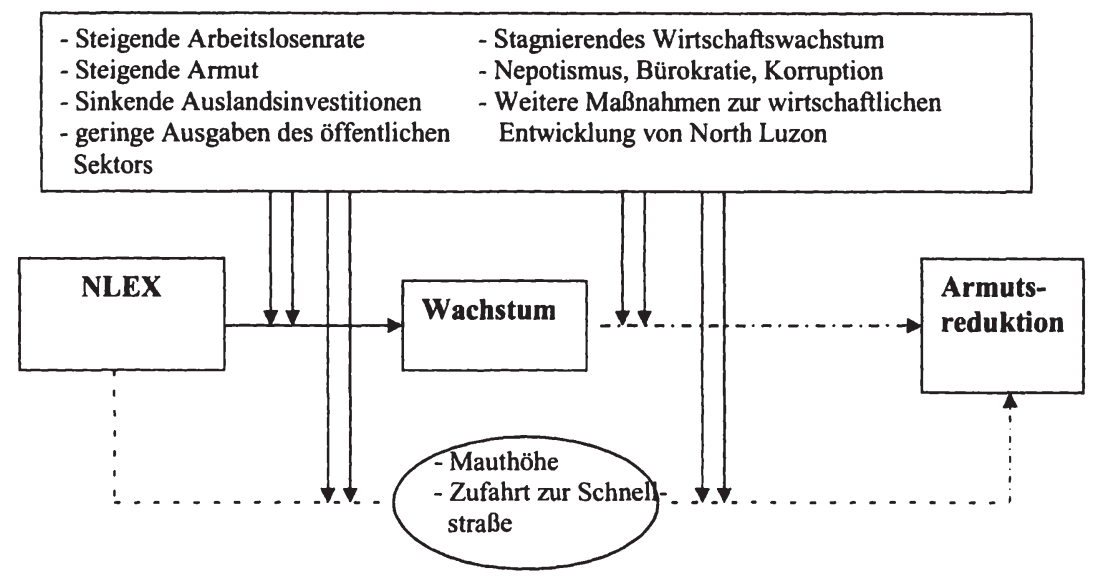

\subsection{Zusammenfassung}

In der vorliegenden Arbeit wurden anhand von fünf Kriterien vier Fallstudien identifiziert. Die Kriterien werden in der folgenden Abbildung 55 grafisch dargestellt werden.

\section{Abbildung 55: Darstellung der Kriterien des Auswahlprozesses}

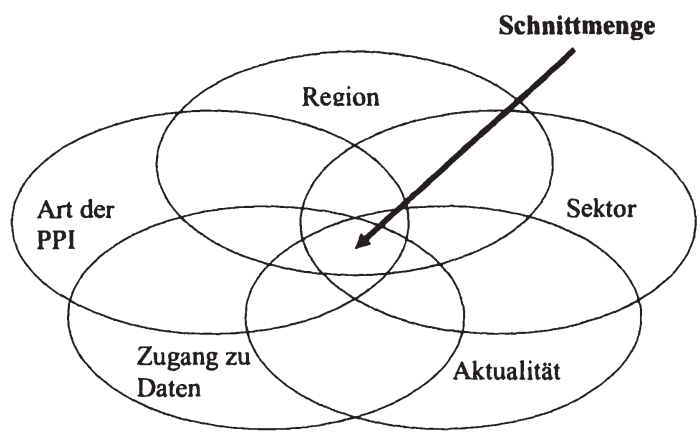


Basierend auf diesen Kriterien wurden die folgenden vier Fallstudien in zwei Ländern identifiziert:

- zwei Kraftwerke in Vietnam (Phu My 2.2 und Phu My 3) und

- ein Kraftwerk und eine Schnellstraße auf den Philippinen (SPI Mindanao und NLEX).

Von jeder Fallstudie wurden folgende Aspekte dargestellt:

- Eckdaten und die Entstehung,

- die Finanzierungs- und Absicherungsinstrumente,

- die Vertragsstruktur und

- deren Beitrag zur Armutsreduktion.

In der folgenden Zusammenfassung werden die Ergebnisse der vier Fallstudien nochmals anhand dieser Struktur prägnant zusammengefasst.

\section{Eckdaten und Entstehung}

Alle vier Projekte wurden als BOT-Modelle durchgeführt, wobei für die Mautstraße eine modifizierte Form, nämlich ein ROT-Modell, verwendet wurde. Dies ist darauf zurückzuführen, dass die Kraftwerke Greenfield Investitionen und das Straßenprojekt eine Brownfield Investition darstellen.

Phu My 2.2 und Phu My 3 sind die ersten BOT-Projekte in Vietnam und die Projektgesellschaften bestehen zu $100 \%$ aus ausländischer Beteiligung. Auf den Philippinen hingegen existiert bereits eine längere Tradition bei der Verwendung des BOT-Modelles und beide Projekte weisen philippinische Beteiligung auf. Zusätzlich dazu sind auf den Philippinen rein rechtlich keine $100 \%$ igen Auslandsinvestitionen in die Infrastruktur erlaubt. Das Kraftwerk SPI weist eine philippinische Minderheitsbeteiligung von $11 \%$ und das Straßenprojekt (NLEX) eine philippinische Mehrheitsbeteiligung von 69,6\% auf. Rund um die Diskussion über Partnerschaften bei der Finanzierung von Infrastruktur ist festzustellen, dass nur die Projektgesellschaft für den North Luzon Expressway aus entwicklungspolitischer Perspektive den Kriterien einer Partnerschaft entspricht. Dadurch sind sowohl positive horizontale als auch vertikale Spillover-Effekte möglich und es kann durch die Zusammenarbeit zu einem Know-How Transfer kommen.

Die Projektgesellschaft von Phu My 2.2 wurde als einzige durch eine internationale Ausschreibung ausgewählt. Ursprünglich war auch die Projektgesellschaft des philippinischen SPI-Projektes durch eine Ausschreibung ausgewählt worden, aber aufgrund finanzieller Schwierigkeiten wurde das Projekt still gelegt, bis die Steag GmbH als Hauptsponsor in das Projekt aufgenommen wurde. Das ursprüngliche Unternehmen hält nur noch eine geringe Beteiligung. 
Phu My 3 stellt ein sogenanntes Downstream-Projekt dar, bei dem das gasfördernde Unternehmen BP bereits den Zuschlag für das Projekt im Rahmen des Förderabkommens bekommen hat. Die ursprüngliche Projektgesellschaft von NLEX wurde in den 1970er Jahren für den Bau der Straße ausgewählt und der neue Hauptsponsor im Rahmen von Gesprächen ausgewählt. Die von multilateraler Seite oft geförderte Transparenz durch Ausschreibungs-prozesse wurde daher nur bei Phu My 2.2 verwirklicht.

Wesentlich für die private Beteiligung bei der Finanzierung von Infrastruktur ist die Identifizierung der Kunden bzw. Nutzer und somit die Planbarkeit der Einnahmen. Im Falle des Kraftwerkes stellt der öffentliche Energieversorger den einzigen Kunden dar. Beim Mautstraßenprojekt können die Kunden auch identifiziert, aber das genaue Ausmaß der Nutzung nicht genau prognostiziert werden.

Alle vier Projekte sind ungefähr zeitgleich entstanden und waren bei der Strukturierung wesentlich von den Auswirkungen der asiatischen Finanzkrise betroffen. Aufgrund der prekären wirtschaftlichen Lage war es für alle Projekte schwierig, die Finanzierung zu sichern. Insbesondere bei Phu My 2.2 und NLEX wurden multilaterale Institutionen zur Unterstützung herangezogen.

\section{Finanzierungs- und Absicherungsinstrumente}

Alle Projekte weisen eine parallele Kofinanzierung auf. Drei der vier Projekte weisen Unterstützung von multilateralen Institutionen, wie beispielweise der ADB oder Institutionen der Weltbank auf. An allen Projekten sind zusätzlich auch nationale Institutionen beteiligt. JBIC spielt bei drei Projekten eine wesentliche Rolle, aber abhängig von der Projektbeteiligung kam es auch zu australischer, deutscher und französischer Unterstützung. Das SPI-Projekt stellt in diesem Zusammenhang eine Besonderheit dar, da keine multilateralen Institutionen am Projekt beteiligt sind.

Bei den Fallstudien wurde zwischen kommerziellem und institutionellem Fremdkapital unterschieden. Als kommerzielles Fremdkapital werden jene Kredite bezeichnet, die durch Geschäftsbanken gewährt werden. Institutionelle Kredite hingegen werden von mulitlateralen und nationalen Institutionen vergeben. Die institutionellen Kredite an die privaten Unternehmen werden auch zu gängigen Marktkonditionen angeboten. Die Beteiligung einer multilateralen Bank führt daher nicht notwendigerweise zu einer Reduktion der Finanzierungskosten, sondern zu einer erhöhten Sicherheit im Projekt aufgrund dessen „preferred creditor status “ (siehe Kapitel 5.1.3).

Die Entwicklung von Phu My 2.2 wurde von Anfang an, also auch bereits in der Ausschreibungsphase, stark von der Weltbank unterstützt. Bei Phu My 3 spielten die IFIs eine geringere Rolle und wurden erst im Rahmen der Finanzierungsstrukturierung benötigt. NLEX konnte aufgrund der schwierigen Finanzlage nicht selbstständig die Finanzierung über den Kapitalmarkt bereitstellen und benötigte die Unterstützung der IFIs, um auch kommerzielle 
Finanzierung für das Projekt zu bekommen. Einzig das SPI-Kraftwerk verzichtete auf die Beteiligung von multilateralen Institutionen und finanzierte das gesamte Projekt über nationale Finanzinstitutionen.

Aus den Fallstudien geht hervor, dass auf den Philippinen bereits, wenn auch nur beschränkt, die Finanzierung in lokaler Währung möglich ist. Hinderlich in Hinblick auf die Projektfinanzierung ist die zu kurze Laufzeit der Kredite. Im Fall des SPI-Projekts wurde der Kredit in lokaler Währung vor allem für die Bezahlung der Personalkosten herangezogen. NLEX hat auch in der ursprünglichen Finanzierung einen vergleichsweise kleinen Kredit in lokaler Währung. Die Projektgesellschaft hat aber sehr erfolgreich eine Restrukturierung der Projektfinanzierung geschafft und durch die Emission von Unternehmensanleihen die Hälfte des gesamten Fremdkapitals in heimische Währung umgewandelt. Insbesondere für diese beiden philippinischen Projekte ist eine Reduktion des Wechselkursrisikos von Bedeutung, da die Tarifvereinbarungen nicht zu $100 \%$ an den Dollar indexiert sind. Bei den vietnamesischen Projekten kam es zu keiner lokalen Finanzierung. Dies kann daher rühren, dass der vietnamesische Kapitalmarkt noch nicht ausreichend entwickelt ist, und dass gleichzeitig aufgrund der hohen Dollarindexierung der Tarife für die privaten Unternehmen kein Handlungsbedarf besteht.

Bei der Finanzierung und Absicherung durch die multilateralen Institutionen wurden wenige neue Instrumente verwendet. Im Bereich der Finanzierung könnte das „Complementary Finance Scheme“ der ADB genannt werden. Die effektiven Kreditgeber sind dabei kommerzielle Banken, die aufgrund des „preferred creditor status" besondere Sicherheit genießen. Das gleiche System wurde auch auf Garantieseite angewendet, indem die ADB als ,guarantor of record" fungiert, die wahren Garantiegeber aber private Versicherungsunternehmen sind. Bei beiden Instrumenten nahm die ADB ihre Kofinanzierungsfunktion wahr (siehe Kapitel 5.1.3).

Eine wesentliche politische Entscheidung der IDA war die Vergabe von politischen Garantien an den privaten Sektor bei Phu My 2.2. Das Produkt selbst ist kein Novum, jedoch stellt es eine Kompetenzausweitung der IDA in den privaten Sektor dar. Bis zu diesem Zeitpunkt war die IDA ausschließlich für die Unterstützung des öffentlichen Sektors zuständig. Durch die Vergabe dieser Garantie kann man einen politischen wie auch institutionellen Wandel hin zur Förderung des privaten Sektors in der Infrastruktur erkennen. Auch die MIGA, die eigentlich in der Weltbankgruppe für Absicherungen zuständig ist, hat erstmals bei Phu My 3 eine Garantie für einen Zinsswap vergeben. Durch diese Garantie wurde die Verwendung eines Instruments zur Absicherung der Zinsänderungen ermöglicht, das in Entwicklungsländern noch nicht so häufig verwendet wird. Auch in diesem Fall stellt die Form der Absicherung keine Innovation dar, jedoch wurde der Anwendungsbereich erweitert.

Von den nationalen Institionen wurden ausschließlich Kredite und Garantien gewährt, die keinerlei Innovation aufweisen. JBIC spielt eine entscheidende 
Rolle bei der Finanzierung von Infrastruktur in Südost-Asien. Dies ist auch durch die hohe Beteiligung von JBIC in allen drei Projekten zu sehen und insgesamt stammt die Mehrheit des institutionellen Kapitals von JBIC. Die Tabelle 14 gibt einen abschießenden Überblick über die Finanzierung der einzelnen Projekte.

\section{Vertragsstruktur}

Die Fallstudien weisen großteils die gleichen Verträge auf, wobei sich Phu My 2.2 und Phu My 3 aufgrund der zeitgleichen Entwicklung besonders ähnlich sind. Alle, außer dem SPI-Projekt, haben einen EPC-Vertrag mit einem Unternehmen abgeschlossen und somit die Baurisiken auf dieses Generalunternehmen übergeben. Die ausländischen Sponsoren der jeweiligen Projekte übernehmen großteils eine wesentliche Rolle beim Bau der Infrastruktur-projekte.

Im Zusammenhang mit Infrastrukturinvestitionen in Entwicklungsländern sind vor allem die Tarif-, Abnahme- und Lieferverträge für private Unternehmen von Wichtigkeit. Abnahmeverträge stellen im Kraftwerksbau standisierte Verträge dar, mit denen sich die privaten Sponsoren gegen das hohe Risiko der Infrastrukturinvestition absichern wollen. Auch bei den Mautstraßen werden Dollarindexierte Verträge vereinbart, wobei jedoch keine Menge fixiert wird. Da die Kapitalmärkte in den Entwicklungsländern nicht ausreichend entwickelt sind, um Infrastrukturfinanzierungen in der lokalen Währung zu ermöglichen, müssen sich die Unternehmen meistens zu $100 \%$ in US\$ verschulden. Um dieses Ungleichgewicht zwischen den Ausgaben in US\$ und den Einnahmen in heimischer Währung zu verringern, vereinbaren die Sponsoren mit den Regierungen in den Abnahme- oder Tarifverträgen US\$-Indexierungen.

Alle drei Kraftwerksprojekte weisen eine US\$-Indexierung in ihren Verträgen auf, aber in keinem der Verträge gibt es eine 100\%ige Anpassung. In Vietnam wurden take-or-pay Abnahmeverträge in Dong vereinbart, wobei ein Teil des Tarifes eine Dollarindexierung und der andere Teil die Inflation berücksichtigen. Dies bedeutet für die privaten Unternehmen, dass sie das Wechselkursrisiko durch diese Wertsicherungsklausel weitestgehend auf die vietnamesische Regierung abgewälzt haben. Zusätzlich dazu sind alle Verträge mit vietnamesischen Partnern durch eine Staatsgarantie abgesichert. Sollte die vietnamesische Versorgungsgesellschaft EVN daher den Abnahmevertrag nicht erfüllen, muss der Staat für dessen Erfüllung aufkommen. Auch die Zulieferverträge bei den Kraftwerken beruhen teilweise auf dollarindexierten take-or-pay Verträgen.

Das SPI-Projekt auf den Philippinen hat einen take-and-pay-Vertrag, in dem ein Tarif mit einer 80 \%igen Dollarindexierung vereinbart wurde. Die Projektgesellschaft argumentiert, dass in ihrem Fall aufgrund der schlechten Stromversorgung auf Mindanao kein take-or-pay-Vertrag notwendig sei und sie immer auf voller Kapazität Strom produzieren und liefern können. Auf den Philippinen 
werden aber von der Regierung keine neuen Abnahmeverträge mehr vergeben, da sie das Abnahmerisiko auf das private Unternehmen abwälzen wollen.

Im Fall der NLEX gibt es zwar keinen Abnahmevertrag, da die Gebühr direkt von den Benutzern eingehoben wird, aber die Projektgesellschaft vereinbarte mit der philippinischen Regierung einen Tarif. In dieser Tarifvereinbarung wurde für die ersten zwei Jahre eine 100 \%ige Dollarindexierung vereinbart, da sich auch die Projektgesellschaft (MNTC) zu $100 \%$ in US\$ finanzieren musste. Es wurde jedoch vereinbart, dass die Dollarindexierung 2007 auf $50 \%$ reduziert wird und deshalb sah sich die Projektgesellschaft stark dem Wechselkursrisiko ausgesetzt. Daher restrukturierte MNTC 2006 die Finanzierung und reduzierte dabei die US\$-Kredite um mehr als die Hälfte, indem sie gleichzeitig Unternehmensanleihen in Peso emittierte, um damit die Kredite frühzeitig zu begleichen.

In beiden philippinischen Projekten wurde deutlich sichtbar, dass am philippinischen Markt bereits eine beschränkte direkte Finanzierung in Peso möglich ist. Für eine Projektfinanzierung mangelt es aber noch immer an ausreichend langen Laufzeiten. Im entwicklungspolitischen Kontext werden oftmals die Abnahmeverträge zwischen Staat und dem privaten Unternehmen kritisiert, da diese zwar eine große Sicherheit für das private Unternehmen bedeuten, aber gleichzeitig das ganze Risiko auf den Staat abwälzen. Solange aber das Wechselkursrisiko aufgrund der verschiedenen Währungen bei den Einnahmen und Ausgaben so stark auseinanderfallen, werden private Unternehmen auf dollarindexierten Tarifen bestehen.

\section{Beitrag zur Armutsreduktion}

Der Beiträge von Infrastruktur zur Armutsreduktion können direkt oder indirekt stattfinden. Indirekt kann das Wirtschaftswachstum zur Armutsreduktion beitragen, wobei die wirtschaftlichen Rahmenbedingungen wesentlich das Ausmaß beeinflussen. In der vorliegenden Arbeit wurden daher bewusst zwei Länder gewählt, die ein sehr konträres wirtschaftliches Umfeld bieten.

Keines der vier Projekte leistet einen wesentlichen direkten Beitrag zur langfristigen Armutsreduktion, aber kurzfristig werden durch den Bau neue Arbeitsplätze geschaffen. Zusätzlich dazu wurde Personal geschult und teilweise in kleine soziale Projekte investiert. Auf den Philippinen liegt die Arbeitslosigkeit im zweistelligen Bereich und der personalintensive Bau von Infrastruktur kann mittelfristig, lokal beschränkt die Arbeitslosigkeit senken. In Vietnam ist die Arbeitslosenrate zwar viel geringer, aber auch hier hat der Bau positive Auswirkungen auf die lokale Reduktion der Arbeitslosigkeit. Nach Beendigung der Bautätigkeit bieten die Unternehmen immer noch, aber weitaus weniger Arbeitsplätze an.

Das vordergründige Ziel dieser Investitionen ist nicht Armutsreduktion, sondern Wirtschaftswachstum. Inwieweit aber Wirtschaftswachstum zur Ar- 
mutsreduktion beiträgt hängt davon ab, ob das Wachstum Pro-Poor ist und das wirtschaftspolitische Umfeld Armutsreduktion unterstützt.

Die Philippinen sind gekennzeichnet durch politische Unruhen und schwache Governance, die dazu geführt hat, dass sich das Investitionsklima verschlechtert hat und Reformen im Bereich der Infrastruktur und der Armutsbekämpfung verschleppt werden. Zusätzlich dazu haben die Philippinen seit Jahren kein ausreichend hohes Wirtschaftswachstum für die Armutsreduktion. Die geringen Erfolge bei der Armutsbekämpfung weisen auch darauf hin, dass die gesetzten Maßnahmen für das Wirtschaftswachstum nicht ausreichend Pro-Poor waren.

Vietnam hingegen wird in einem Atemzug mit China genannt, da das Land wirtschaftlich prosperiert. Gleichzeitig ist es Liebkind der Weltbankgruppe, da die Armut drastisch reduziert werden konnte. Auch Vietnam hat mit Korruption und Bürokratie zu kämpfen, aber die stabile politische Lage wurde von allen Gesprächspartnern als großer Vorteil angesehen und spiegelt sich auch in den ansteigenden ausländischen Investitionen wider. Gleichzeitig dazu verfolgt die vietnamesische Politik in ihren "Doi Moi“ Wirtschaftsreformen einen sehr umfassenden Ansatz bei der Armutsbekämpfung, indem sie das „Geld regnen“ lässt und alle Bevölkerungsgruppen davon profitieren.

Die Annahme, dass der private Sektor die notwendigen Infrastruktureinrichtungen zur Armutsreduktion besser als der Staat erbringen kann, stimmt daher nur sehr eingeschränkt. In der Praxis kommen nachhaltige Maßnahmen zur Armutsreduktion fast ausschließlich vom Staat und nicht vom Privatsektor. Der Privatsektor will vordergründig Gewinne realisieren, was durchaus legitim ist, aber dadurch wird der Investitionsradius stark eingeschränkt. Die Versorgung von armen Menschen ist hingegen meist defizitär, da diese oft in entlegenen Gebieten auf dem Land leben, weniger konsumieren und eine geringe Kaufkraft aufweisen. Für private Unternehmen kommen daher nur jene Projekte in Frage, in denen sie den Kundenkreis identifizieren und die Einnahmen planen können. Abgesehen vom Staat als Abnehmer sind vor allem jene Bevölkerungsgruppen profitabel, die im urbanen Raum leben und ein gewisses Einkommensniveau aufweisen.

Zusammenfassend muss daher festgestellt werden, dass der private Sektor nur sehr beschränkt nachhaltig zur direkten Armutsreduktion beitragen kann. Primär unterstützen die privatfinanzierten Investitionen das Wirtschaftswachstum, das aber bei einem ausreichenden Wachstum und unterstützenden Rahmenbedingungen zur Armutsreduktion beitragen kann. Die öffentliche Hand muss daher umfangreich in die physische Infrastruktur in abgelegene, arme Regionen investieren, um strukturelle Armut nachhaltig zu bekämpfen. 
Tabelle 14: Überblick über die Fallstudien

\begin{tabular}{|c|c|c|c|c|c|c|c|c|c|}
\hline & Projekt & $\begin{array}{c}\text { PPI- } \\
\text { Modell }\end{array}$ & Sponsoren & ADB & Weltbank & IFC & MIGA & JBIC & $\begin{array}{c}\text { nationale } \\
\text { Institutionen }\end{array}$ \\
\hline & Phu My 2.2 & BOT & $\begin{array}{cl}56,25 \% & \text { EDF International } \\
28,125 \% & \text { Sumitomo } \\
& \text { Corporation } \\
15,625 \% & \text { Tokyo Electric } \\
& \text { Power Company } \\
& \end{array}$ & $\begin{array}{l}\text { Political Risk } \\
\text { Guarantee } \\
\text { Kredit }\end{array}$ & $\begin{array}{l}\text { Partial Risk } \\
\text { Guarantee } \\
\text { Technical } \\
\text { Assistance }\end{array}$ & & & Kredit & $\begin{array}{l}\text { Proparco: } \\
\text { Kredit }\end{array}$ \\
\hline 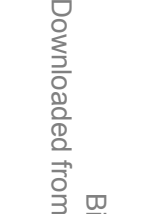 & Phu My 3 & BOT & $\begin{array}{c}331 / 3 \% \text { BP Holding BV } \\
331 / 3 \% \text { SembCorp } \\
\text { Industries } \\
331 / 3 \% \text { Kyuden } \\
\text { International } \\
\text { Corporation und } \\
\text { Sojitz Corporation } \\
\end{array}$ & $\begin{array}{l}\text { Political Risk } \\
\text { Guarantee } \\
\text { Kredit }\end{array}$ & & & $\begin{array}{l}\text { Political Risk } \\
\text { Guarantee }\end{array}$ & Kredit & $\begin{array}{l}\text { NEXI: } \\
\text { Political Risk } \\
\text { Guarantee }\end{array}$ \\
\hline 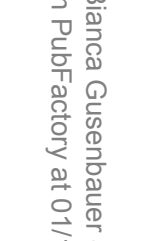 & $\begin{array}{l}\text { SPI } \\
\text { Mindanao }\end{array}$ & BOT & $\begin{array}{ll}89 \% & \text { STEAG GmbH } \\
11 \% & \text { State Investment } \\
& \text { Trust, Inc. }\end{array}$ & & & & & Kredit & $\begin{array}{l}\text { NEXI: } \\
\text { Political Risk } \\
\text { Guarantee } \\
\text { Bundes- } \\
\text { republik } \\
\text { Deutschland } \\
\text { DiA Garantie }\end{array}$ \\
\hline 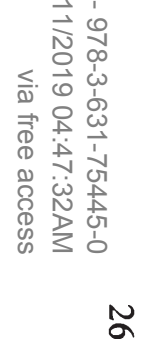 & NLEX & ROT & $\begin{aligned} 67.1 \% & \begin{array}{l}\text { First Philippine } \\
\text { Infrastructure } \\
\text { Development } \\
\text { Corporation }\end{array} \\
16.5 \% & \begin{array}{l}\text { Leighton Asia } \\
\text { (Southern) Limited }\end{array} \\
13.9 \% & \begin{array}{l}\text { Egis Projects, } \\
\text { S.A. }\end{array} \\
2.5 \% & \begin{array}{l}\text { Philippine National } \\
\text { Construction } \\
\text { Corporation (PNCC) }\end{array} \\
& \end{aligned}$ & $\begin{array}{l}\text { Kredit } \\
\text { Complement- } \\
\text { ary financing } \\
\text { scheme }\end{array}$ & & $\begin{array}{l}\text { Kredit } \\
\text { (ursprüng- } \\
\text { lich) }\end{array}$ & $\begin{array}{l}\text { Political Risk } \\
\text { Guarantee } \\
\text { (ursprüng- } \\
\text { lich) }\end{array}$ & & $\begin{array}{l}\text { Australia's } \\
\text { Finance and } \\
\text { Security } \\
\text { Corporation } \\
\text { Kredit } \\
\text { Coface: } \\
\text { Garantie }\end{array}$ \\
\hline
\end{tabular}


Bianca Gusenbauer - 978-3-631-75445-0

Downloaded from PubFactory at 01/11/2019 04:47:32AM

via free access 


\section{Conclusio}

Im abschließenden siebenten Kapitel wird im ersten Abschnitt im Resümee nochmals eine Zusammenfassung der gesamten Arbeit gegeben. Daran anschließend wird im zweiten Ausschnitt ein Fazit gezogen und aufbauend darauf ein Forschungsausblick gegeben.

\subsection{Resümee}

In der vorliegenden Arbeit wurde versucht, zwei Forschungsfragen zu beantworten. In der ersten Frage beschäftigte sich die Autorin damit, ob es aufgrund der veränderten Ausrichtung der Entwicklungspolitik auch zu Veränderungen bei Finanzierungs- und Absicherungsinstrumenten in den IFIs gekommen ist. Die zweite Frage untersucht, inwieweit die öffentlich-privat finanzierten Infrastrukturinvestitionen zur Armutsreduktion beitragen.

Aus diesen beiden Fragestellungen geht hervor, dass die Arbeit an der Schnittstelle zwischen Betriebswirtschaftslehre und Entwicklungsökonomie angesiedelt ist. Die folgende Abbildung 56 zeigt grafisch die thematische Zuordnung der Themen zu den Wissenschaftsdisziplinen. In den Fallstudien werden diese beiden Aspekte zusammenfassend diskutiert.

Abbildung 56: Einordnung der Themen in die Disziplinen

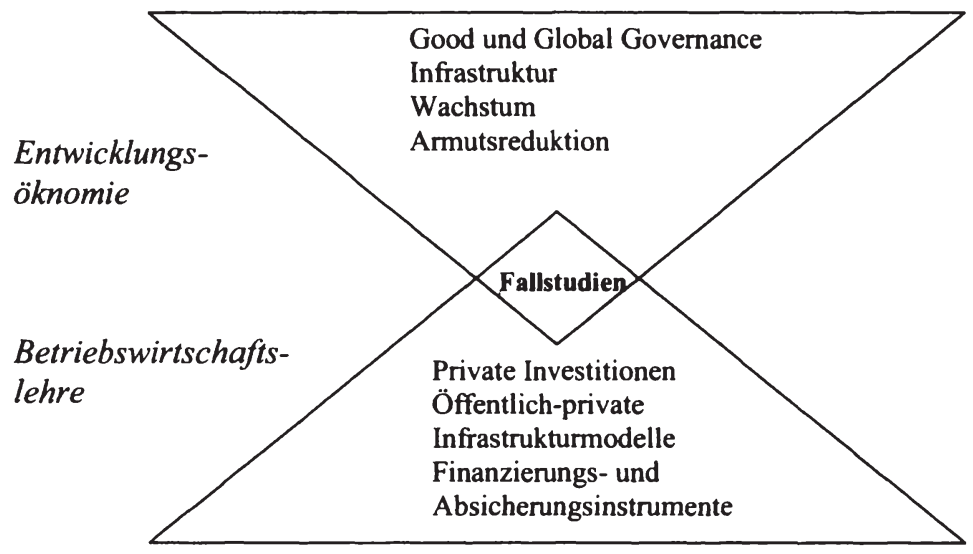

Für die vorliegende Arbeit wurde ein Multimethodenansatz verfolgt und für die Darstellung Fallstudien gewählt wurden. Das Ziel der Einzelfallanalyse ist die Darstellung von interessanten Fällen in möglichst vielen Dimensionen, wobei 
die Methoden kombiniert werden können. Durch Interviews und Internetrecherche wurden zunächst interessante Fälle identifiziert. Daran anschließend wurden teilstrukturierte Interviews mit Experten der einzelnen Fallstudien und Mitarbeitern der internationalen Finanzinstitutionen geführt. Die Interviews wurden basierend auf der zirkulären Vorgehensweise im Analyseprozess von Miles und Hubermann (1994) analysiert und die Ergebnisse zur Beantwortung der Forschungsfragen herangezogen.

Die Arbeit untergliedert sich in sieben Kapitel, wobei nach einer Einführung im ersten Kapitel, sich das zweite Kapitel mit dem Konzept des Governance in der Entwicklungspolitik beschäftigte. Darin wurden das Good Governance und das Global Governance-Konzept dargestellt und in das historisch-theoretische Umfeld eingeordnet. Die Entwicklung und die Auswirkungen dieser beiden Konzepte wurden kritisch dargestellt, wobei insbesondere auf die Privatisierung und die Dezentralisierung in der Infrastruktur Bezug genommen wurde, da diese zu wesentlichen Veränderungen in der Finanzierung geführt haben.

Im dritten Kapitel wurden die Auswirkungen von privaten Investitionen in Entwicklungsländern und die Auswirkungen von Infrastruktur auf die Armutsreduktion diskutiert. Beginnend mit einer Darstellung der privaten Investitionen wurde ein State-of-the-Field über den Zusammenhang von Ausländischen Direktinvestitionen (ADI), Wirtschaftswachstum und Spillover-Effekten erarbeitet. Aus dieser Darstellung geht klar hervor, dass es große Uneinigkeit über die Auswirkungen auf diesem Gebiet gibt. Zusammenfassend lässt sich festhalten, dass die positiven Auswirkungen auf das Gastland oftmals zu hoch eingeschätzt werden, die Ergebnisse stark vom Untersuchungsdesign abhängen und keine allgemeinen Schlüsse gezogen werden können.

Daran anschließend wurde der direkte und indirekte Zusammenhang zwischen Infrastruktur und Armutsreduktion dargestellt. Der indirekte Zusammenhang besteht zwischen Wachstum und Armutsreduktion, wobei das Ausmaß ein wesentlicher Diskussionspunkt der Entwicklungsökonomie ist. Der neuere Ansatz der Entwicklungsökonomie besagt, dass Wirtschaftswachstum „Pro-Poor“ sein muss, um positive Auswirkungen auf die Armutsreduktion zu haben. In Folge wurden die Auswirkungen von Energie- und Transportinfrastruktur auf die Armutsreduktion diskutiert. Hier kann zusammenfassend festgehalten werden, dass Infrastruktur nur beschränkt, abhängig von der Zielgruppe und dem Sektor, positive Auswirkungen auf die Armutsreduktion haben kann. Wobei Energieinfrastruktur nur wenig bis keinen direkten positiven Einfluss auf die Armutsreduktion hat und Investitionen in die Transportinfrastruktur nur dann, wenn sie auf eine arme Zielgruppe ausgerichtet ist. Kritisiert wurde in dieser Diskussion die isolierte Betrachtung von Investitionen, da man auch die kontext- und situationsabhängige Variablen berücksichtigen muss, um Schlüsse über die Auswirkungen ziehen zu können. 
Das vierte Kapitel widmete sich den öffentlich-privaten Infrastrukturmodellen, wobei in der Einführung festgestellt wurde, dass der Begriff "Partnerschaft“ einer der am häufigsten „missbrauchten“" Wörter in den Wirtschaftswissenschaften ist. Da Partnerschaft im Zusammenhang mit Entwicklungspolitik ein ohnehin sensibles und inflationär verwendetes Wort ist, vertritt die Autorin die Ansicht, dass darunter nur jene Formen der Zusammenarbeit subsumiert werden sollen, die echte partnerschaftliche Züge aufweisen. Basierend auf dieser Definition werden Public Private Partnerships (PPP) abgrenzt, wobei Definitionen im engeren und weiteren Sinn existieren. Von der Autorin wird aufgrund der engeren Partnerschaftsdefinition auch PPP i.e.S. gebraucht. Als Überbegriff für alle anderen möglichen Kooperationsformen zwischen privater und öffentlicher Hand, die nicht die Kriterien der Definition i.e.S. erfüllen, wird der Begriffe Private Participation in Infrastructure (PPI) verwendet.

Der zweite Abschnitt des vierten Kapitels widmete sich den PPI in der Entwicklungspolitik. Dabei wurden die Auswirkungen der entwicklungspolitischen Veränderungen anhand des Infrastruktursektors dargestellt und es wurde erkennbar, dass aufgrund der extern auferlegten Restriktionen die Infrastrukturfinanzierung von öffentlicher Seite in den vergangenen Jahrzehnten stark zurückgegangen ist. Gleichzeitig nahmen auch die Entwicklungshilfeflüsse (ODA) in den Infrastruktursektor ab. Zu Beginn der Privatisierungen nahmen die privaten Finanzierungen in ausgewählten Infrastruktursektoren zu, die aber aufgrund der asiatischen und argentinischen Finanzkrisen in große finanzielle Probleme gerieten und abschreckend für weitere Investitionen waren. Aus dieser Darstellung kann festgehalten werden, dass aufgrund des jahrelangen Rückgangs aller drei Finanzströme eine große Finanzierungslücke im Infrastruktursektor entstanden ist und dieser nun wieder im Mittelpunkt der Entwicklungspolitik steht und unter anderem durch mehr private Investitionen geschlossen werden soll. Dabei soll der Staat wieder gestärkt werden und die Official Development Assistance (ODA) als Hebel für die Förderung von privaten Investitionen eingesetzt werden.

Im dritten Abschnitt wurden die Länderrisiken bei privaten Infrastrukturinvestitionen dargestellt, die in kommerzielle und politische Risiken sowie Wechselkursrisiken unterteilt werden. Im vierten Abschnitt wurden die öffentlich-privaten Infrastrukturmodelle vorgestellt, wobei die Modelle nach der Beteiligungsintensität des privaten Sektors unterschieden werden können.

Das fünfte Kapitel beschäftigte sich mit den Finanzierungs- und Absicherungsinstrumenten der internationalen und nationalen Finanzorganisationen für private Investitionen in die Infrastruktur. Ziel dieser Ausarbeitung war es, einen Überblick über das Angebot und neue Instrumente genauer darzustellen und kritisch zu würdigen. Zusammenfassend ist festzuhalten, dass sich das Angebot der Institutionen stark ähnelt und die privaten Investitionen meistens durch Garantien und Kredite unterstützt werden. Von den neueren Ansätzen stellen 
insbesondere die Finanzierung in lokaler Währung und die Unterstützung von sub-souveränen Regierungseinheiten eine Herausforderung für die IFIs dar.

Im sechsten Kapitel wurden folgende Aspekte von vier Fallstudien dargestellt:

- Eckdaten und die Entstehung,

- die Finanzierungs- und Absicherungsinstrumente,

- die Vertragsstruktur und

- deren Beitrag zur Armutsreduktion.

Die Finanzierungs- und Absicherungsstruktur der einzelnen Fallstudien weisen Parallelen auf, sie sind aber aufgrund ihrer Entstehungsgeschichte dennoch unterschiedlich. Die Projekte in Vietnam gehören zu $100 \%$ ausländischen Unternehmen. Auf den Philippinen ist hingegen in einem Projekt ein philippinisches Unternehmen Hauptsponsor und es bestehen in beiden Projekten öffentliche Beteiligungen, die per Gesetz vorgesehen sind.

Die IFIs übernehmen in allen Projekten eine wichtige Rolle durch die Vergabe von Krediten und Garantien. Die Projekte weisen außerdem klaren nationalen Bezug auf, da abhängig von den beteiligten Unternehmen auch die nationalen Institutionen eine wesentliche Rolle gespielt haben. Insbesondere die japanische Bank ,JBIC" ist in drei der vier Projekte ein dominanter Kreditgeber. Im Vergleich zu Vietnam, war auf den Philippinen in beiden Projekten auch eine teilweise Finanzierung in heimischer Währung möglich.

Ferner gleicht sich die Vertragsstruktur der Projekte stark, was darauf zurück $\mathrm{zu}$ führen ist, dass in der Internationalen Projektfinanzierung standardisierte Verträge vorgesehen sind. Das Kraftwerksprojekt auf den Philippinen weist jedoch Abweichungen auf. Die Abnahmeverträge bzw. Tarifvereinbarungen spielen eine wesentliche Rolle bei privaten Infrastrukturprojekten. In allen Projekten wurden Dollarindexierungen vereinbart, jedoch unterscheidet sich das Ausmaß der Indexierung. Die Philippinen weisen generell eine geringere prozentuelle Indexierung auf.

Abschließend wurden noch die Auswirkungen der Projekte auf die Armutsreduktion diskutiert. Zusammenfassend kann festgestellt werden, dass der private Sektor nur sehr beschränkt nachhaltig zur direkten Armutsreduktion beitragen kann. Primär unterstützen die privaten Infrastrukturinvestitionen aufgrund ihrer Projektcharakteristika das Wirtschaftswachstum, das aber bei ausreichendem Wachstum und unterstützenden Rahmenbedingungen zur Armutsreduktion beitragen kann. Die öffentliche Hand muss daher umfangreich in die physische Infrastruktur in abgelegene, arme Regionen investieren, um strukturelle Armut nachhaltig zu bekämpfen. 


\subsection{Fazit und Ausblick}

Ziel dieser Arbeit war es, zwei Tendenzen in der Entwicklungspolitik gemeinsam darzustellen, nämlich die Armutsreduktion im Rahmen der MDGs und die erhöhte Teilnahme von privaten Investoren in der Infrastrukturfinanzierung.

Die Veränderungen in den Ansätzen der Entwicklungsökonomie haben dazu geführt, dass Privatisierung und Deregulierung in den Entwicklungsländern stark gefördert werden, um die Effizienz der Anlagen zu erhöhen. Für ausländische Investoren stellen die Entwicklungsländer einen zusätzlichen Absatzmarkt dar, auf dem sie gewinnbringend ihre Güter verkaufen können. Die oftmals von NGOs kritisierte Gewinnorientierung von privaten Unternehmen ist durchaus legitim, wenn die privaten Unternehmen die wirtschaftsethischen Auflagen erfüllen, da sie den marktwirtschaftlichen Gesetzen unterliegen. Die Gewinnmöglichkeit stellt jedoch genau jenes Kriterium dar, anhand dessen die privaten Unternehmen entscheiden, ob sie Projekte finanzieren.

Wenn man allerdings die Charakteristika der Infrastruktur bedenkt, dann werden nur in jenen Infrastrukturbereichen Gewinne erzielt, in denen auch die Kunden identifizierbar sind und vom Konsum ausgeschlossen werden können. Der Einsatzbereich für private Unternehmen beschränkt sich somit auf jene Infrastrukturbereiche, in denen das private Unternehmen entweder direkt vom identifizierten Kunden und vom Staat bezahlt wird. Konkret geeignet ist etwa die Energieproduktion, da sich die Anlage auf einen Ort beschränkt und der Staat einen Abnahmevertrag mit dem Produzenten eingeht. Die Energieversorgung ist hingegen bereits kritischer und bleibt meistens in öffentlicher Hand, da sie zu viele Risiken und zu geringe Gewinne in sich birgt. Auch wurde in der Vergangenheit bereits mehrmals versucht die Wasserversorgung mittels Konzessionen an private Unternehmen auszulagern, allerdings mit gemischtem Erfolg. Bei der Transportinfrastruktur ist das Ausschlussprinzip ein wichtiger Faktor. Beispielsweise können Häfen aufgrund der geringen Ausbreitung der Anlage und des klar definierten Kundenkreises, gut von privaten Unternehmen betrieben werden. Straßen hingegen werden großteils von der öffentlichen Hand betrieben und nur bei Mautstraßen kann es zu einem Ausschluss kommen.

Wenn man also zusammenfassend festhält, dass private Infrastrukturfinanzierung im Wesentlichen nur für die Stromerzeugung, Häfen, Mautstraßen und in sehr eingeschränktem Maße für die Energieversorgung in Frage kommt, so ist der tatsächliche Investitionsbereich relativ klein. Dieser Tatsache müssen die Erkenntnisse aus den Untersuchungen über die Auswirkungen auf die Armutsreduktion gegenüber gestellt werden. Aus diesen geht hervor, dass Straßen in ländlichen Gegenden den größten und Energieversorgung nur in manchen Fällen einen Beitrag zur Armutsreduktion leisten können. Bringt man diese beiden Erkenntnisse zusammen, so ergeben sich keine Überschneidungen. 
Zusätzlich zu den sektoralen Einschränkungen investieren private Unternehmen nicht in jene Entwicklungsländer, die am meisten unter Armut leiden, wie beispielsweise in Länder in Subsahara Afrika. Fazit ist daher, dass privat finanzierte Infrastrukturinvestitionen keinen direkten Beitrag zur Armutsreduktion, sondern nur einen Beitrag zum Wirtschaftswachstum leisten können. Wichtig in diesem Zusammenhang ist, dass die Infrastrukturinvestitionen von öffentlichen Reformen begleitet werden, die positive, indirekte Auswirkungen fördern und das Wirtschaftswachstum Pro-Poor gestalten.

Privatisierung und Dezentralisierung haben aber nicht nur auf Länderebene, sondern auch in den Internationalen Finanzinstitutionen zu Veränderungen geführt. Der private Sektor spielte als Kunde bis zum Zeitpunkt dieser Reformen nur eine sehr untergeordnete Rolle bei der Finanzierung von Infrastruktur. Da der private Sektor aber an Bedeutung zunahm und eine Kundengruppe darstellt, deren Charakteristika sich wesentlich von denen des öffentlichen Sektors unterscheiden, mussten auch die Instrumente an diese neue Kundengruppe angepasst werden. Insbesondere die Auswirkungen der asiatischen Finanzkrise zeigten, dass die privaten Unternehmen stark dem Wechselkurs- und dem politischen Risiko ausgesetzt sind.

Seither haben sich die Finanzmärkte in den Entwicklungsländern wieder erholt und an Liquidität gewonnen. Dennoch werden internationale Projekte kaum von lokalen Banken finanziert, sondern spielen die IFIs dabei nach wie vor eine große Rolle. Die IFIs können die privaten Unternehmen sowohl durch Beratung und Know-How, aber auch durch effektive Instrumente unterstützen. Da die privaten Unternehmen ein anderes Risikoprofil als öffentliche Kreditnehmer aufweisen, mussten auch der Abwicklungsprozess und die Instrumente adaptiert werden.

Gleichzeitig sollen IFIs nicht in Konkurrenz mit kommerziellen Insti-tutionen stehen, sondern eine Kofinanzierungsfunktion übernehmen. Das Fazit ist, dass die IFIs die Instrumente an den privaten Sektor $\mathrm{zu}$,kommerziellen“ Konditionen vergeben, welche aber unter denen der kommerziellen Banken liegen können. Grund dafür ist das bessere Rating der IFIs und deren günstigere Refinanzierung am Kapitalmarkt.

Die asiatische Finanzkrise zeigte klar, dass die Entwicklungsländer aufgrund von dollarindexierten Abnahmeverträgen mit hohen Forderungen von privaten Unternehmen konfrontiert waren. Um dieses Ungleichgewicht $\mathrm{zu}$ senken, versuchten die IFIs Kredite in lokaler Währung zur Verfügung zu stellen, da diese das Wechselkursrisiko für die privaten Unternehmen reduzieren würden. Bisher jedoch nur mit beschränktem Erfolg, da sich die Abwicklung aufwendiger gestaltet als für einen gewöhnlichen US\$-Kredit. Fazit ist, dass die teilweise Finanzierung in lokaler Währung auch zu einer Reduktion der Dollarindexierung führen würde und dies das Staatsbudget entlasten würde. Dadurch könnten die Einsparungen vom Staat für andere Investitionen, beispielsweise in abgelegenen Regionen, genützt werden. Ziel der IFIs muss daher sein, nicht nur 
direkt selbst einen Kredit in lokaler Währung anzubieten, sondern in die Entwicklung des Finanzmarktes zu investieren, da für die Vergabe von Krediten in lokaler Währung weiterhin die IFIs als Intermediäre benötigt werden. Durch Stärkung des lokalen Finanzmarktes können die lokalen Finanzinstitutionen langfristig soweit entwickelt werden, dass ohne Zutun der IFIs längerfristige Kredite an private Unternehmen vergeben werden können.

Aufgrund der regionalen Einschränkung der Fallstudien wurden die meisten Interviews mit Mitarbeitern der Asian Development Bank (ADB) geführt. Daher kann an dieser Stelle nun ausschließlich auf die ADB Bezug genommen werden. In der ADB gibt es die Unterteilung zwischen ,public sector" und „private sector“, die jeweils eine andere Zielgruppe unterstützen. Das „Private Sector Operations Department" unterstützt private Investoren und hat in den vergangen Jahren kontinuierlich den Umsatz erhöht. Eine grundlegende Voraussetzung für die Unterstützung von privaten Infrastrukturprojekten durch die ADB ist, dass das Projekt einen ,"clear development impact “ aufweisen muss.

Jedoch konnte in den Interviews weder eine ADB-gültige Definition für Entwicklung erarbeitet werden, noch bestehen bankintern die Möglichkeiten bzw. die Methoden diese ,klare Auswirkung“ zu messen. Wie sinnvoll es daher ist, ein Kriterium für die Unterstützung festzulegen, das weder operationalisierbar ist, noch einheitlich erhoben wird, ist fraglich. Vielmehr erscheint in diesem Zusammenhang die Verschwommenheit des Begriffes „Entwicklung“ für die Bank sehr dienlich. Fazit ist, dass auf institutioneller Ebene ein großer Forschungsbedarf bei den Methoden zur Erhebung der Auswirkungen und ein Handlungsbedarf bei der Erhebung von ex-ante Daten für eine methodisch reine Messung der Aus-wirkungen für Infrastrukturprojekte bestehen.

Für zukünftige Forschungsthemen im Zusammenhang mit den Ergebnissen dieser Arbeit ergeben sich - im Rahmen eines Ausblicks - weitere Ansatzpunkte.

Ein erster Ansatzpunkt besteht darin, die Beziehung zwischen Finanzierung in lokaler Währung und Dollarindexierung in den Abnahmeverträgen genauer zu untersuchen. Der Zusammenhang könnte auf Basis von teilstrukturierten Interviews erforscht werden. Die Effekte auf das Staatsbudget aufgrund einer Reduktion der Indexierung könnten hingegen anhand von makroökonomischen Untersuchungen erhoben werden.

Des Weiteren könnten der Know-how-Transfer und die Spillover-Effekte der Kooperation von lokalen und ausländischen Unternehmen im Rahmen eines BOT-Projektes untersucht werden. Dieser Ansatz könnte anhand von qualitativen Methoden erforscht werden.

Wie bereits im Fazit festgestellt wurde, fehlt es auf institutioneller Ebene an einem effizienten Verfahren, das für die Evaluierung der einzelnen Investitionen 
herangezogen werden kann. Daher wäre ein sinnvoller Beitrag, ein quantitatives Modell zu entwickeln, das versucht, den Beitrag der Infrastrukturinvestition auf die Armutsreduktion zu messen. 


\section{Anhang}

Anhang 1: Millennium Development Goals (MDGs) 291

Anhang 2: Varianten von BOT-Modellen

293

Anhang 3: Kategorisierung der Investitionen laut PPI-Databases

294 


\section{Millennium Development Goals}

\section{Ziel: Extreme Armut und Hunger beseitigen}

- Die Zahl der Menschen, die von weniger als einem US-Dollar pro Tag leben, soll um die Hälfte gesenkt werden.

- Der Anteil der Menschen, die unter Hunger leiden, soll um die Hälfte gesenkt werden.

\section{Ziel: Grundschulausbildung für alle Kinder gewährleisten}

- Alle Jungen und Mädchen sollen eine vollständige Grundschulausbildung erhalten.

\section{Ziel: Gleichstellung und größeren Einfluss der Frauen fördern}

- In der Grund- und Mittelschulausbildung soll bis zum Jahr 2005 und auf allen Ausbildungsstufen bis zum Jahr 2015 jede unterschiedliche Behandlung der Geschlechter beseitigt werden.

\section{Ziel: Die Kindersterblichkeit senken}

- Die Sterblichkeit von Kindern unter fünf Jahren soll um zwei Drittel gesenkt werden.

\section{Ziel: Die Gesundheit der Mütter verbessern}

- Die Müttersterblichkeit soll um drei Viertel gesenkt werden.

\section{Ziel: HIV/Aids, Malaria und andere Krankheiten bekämpfen}

- Die Ausbreitung von HIV/Aids soll zum Stillstand gebracht und zum Rückzug gezwungen werden.

- Der Ausbruch von Malaria und anderer schwerer Krankheiten soll unterbunden und ihr Auftreten zum Rückzug gezwungen werden.

\section{Ziel: Eine nachhaltige Umwelt gewährleisten}

- Die Grundsätze der nachhaltigen Entwicklung sollen in der nationalen Politik übernommen werden; dem Verlust von Umweltressourcen soll Einhalt geboten werden. 
- Die Zahl der Menschen, die über keinen nachhaltigen Zugang zu gesundem Trinkwasser verfügen, soll um die Hälfte gesenkt werden

- Bis zum Jahr 2020 sollen wesentliche Verbesserungen in den Lebensbedingungen von zumindest 100 Millionen Slumbewohnern erzielt werden.

\section{Ziel: Eine nachhaltige Umwelt gewährleisten}

- Ein offenes Handels- und Finanzsystem, das auf festen Regeln beruht, vorhersehbar ist und nicht diskriminierend wirkt, soll weiter ausgebaut werden. Dies schließt eine Verpflichtung zu guter Staatsführung, zur Entwicklung und zur Beseitigung der Armut sowohl auf nationaler wie auf internationaler Ebene ein.

- Auf die besonderen Bedürfnisse der am wenigsten entwickelten Länder muss entsprechend eingegangen werden. Dazu gehören der zoll- und quotenfreie Marktzugang für die Exporte dieser Länder; die verstärkte Schuldenerleichterung für die hochverschuldeten armen Länder; die Streichung aller bilateralen öffentlichen Schulden dieser Länder; sowie eine großzügigere Entwicklungshilfe für Länder, die wirkliche Anstrengungen zur Senkung der Armut unternehmen.

- Auf die besonderen Bedürfnisse der Binnenstaaten und der kleinen Inselentwicklungsländer muss entsprechend eingegangen werden.

- Die Schuldenprobleme der Entwicklungsländer mit niedrigen und mittleren Einkommen müssen durch Maßnahmen auf nationaler und internationaler Ebene umfassend und wirksam angegangen werden, damit ihre Schulden auf lange Sicht tragbar werden.

- In Zusammenarbeit mit den Entwicklungsländern soll für die Schaffung menschenwürdiger und produktiver Arbeitsplätze für junge Menschen gesorgt werden.

- In Zusammenarbeit mit der pharmazeutischen Industrie sollen lebenswichtige Medikamente in den Entwicklungsländern zu erschwinglichen Preisen verfügbar gemacht werden.

- In Zusammenarbeit mit dem Privatsektor sollen die Vorteile der neuen Technologien, insbesondere der Informations- und Kommunikationstechnologien, verfügbar gemacht werden.

Quelle: http://www.unric.org/html/german/millennium/ziele/index.htm, 12. Mai 2007 


\begin{tabular}{|c|c|}
\hline BLOT/BOLT & $\begin{array}{l}\text { Build Lease Operate Transfer: Leasinggeber ist eine Leasing- } \\
\text { gesellschaft, die die Projektanlage erstellen lässt und an- } \\
\text { schließend als ihr Eigentümer auftritt. Verantwortlich für die } \\
\text { Bereitstellung der Leistung ist eine private Projektgesellschaft } \\
\text { (Leasingnehmer). Die Leistung umfasst den Betrieb und die } \\
\text { Wartung der Anlage gegen Zahlung eines Entgeltes, der } \\
\text { Leasinggebühr. }\end{array}$ \\
\hline BLTM & Build Lease Transfer Maintain \\
\hline BOD & Build, Operate, Deliver \\
\hline BOT/BOOT & $\begin{array}{l}\text { Build Operate (Own) Transfer: Bau und Betrieb, privates } \\
\text { Eigentum während der Betriebsphase, Transfer der Anlage an } \\
\text { den Gaststaat am Ende der Laufzeit. }\end{array}$ \\
\hline BOO & $\begin{array}{l}\text { Build Own Operate: Die Betriebsphase wird für die } \\
\text { Projektgesellschaft auf die Lebensdauer der Anlage ausgedehnt, } \\
\text { privates Eigentum über die gesamte Lebensdauer. }\end{array}$ \\
\hline BOOR & Build, Own, Operate, Remove \\
\hline BOOST & $\begin{array}{l}\text { Build Own Operate Subsidise Transfer: Projekte, die während } \\
\text { der Betriebsphase vom Staat subventioniert werden. }\end{array}$ \\
\hline BOOT & Build, Own, Operate, Transfer \\
\hline $\begin{array}{l}\text { Reverse } \\
\text { BOOT }\end{array}$ & $\begin{array}{l}\text { Finanzierung und Bau durch den öffentlichen Bereich und } \\
\text { Vermietung an private Betreiber mit Transfer an den privaten } \\
\text { Bereich am Ende der Laufzeit. }\end{array}$ \\
\hline BOTT & $\begin{array}{l}\text { Build Operate Train Transfer: Wie BOT, bezieht aber auch die } \\
\text { Ausbildung der Mitarbeiter oder eine Einarbeitung der zu- } \\
\text { künftigen neuen Betreibers ein. }\end{array}$ \\
\hline BROT & Build, Rent, Operate, Transfer \\
\hline BRT & Build, Rent, Transfer \\
\hline BTO & Build, Transfer, Operate \\
\hline DBFO & $\begin{array}{l}\text { Design Build Finance Operate: Für die Abnehmer des } \\
\text { Projektoutputs fallen keine unmittelbaren Benutzungsgebühren } \\
\text { an, sondern die Bezahlung erfolgt im Rahmen eines Shadow } \\
\text { Tolling. }\end{array}$ \\
\hline DBFOM & Design, Build, Finance, Operate, Manage \\
\hline DBO & $\begin{array}{l}\text { Develop Build Operate: Der Betreiber plant und entwickelt, } \\
\text { baut und betreibt die Anlage. Dabei trägt er zunächst kein } \\
\text { Projektrisiko, ist aber verantwortlich für die Finanzierung und } \\
\text { den Betrieb nach bestimmten Leistungskriterien. }\end{array}$ \\
\hline DBOM & Design, Build, Operate, Maintain \\
\hline DCMF & Design, Construct, Manage, Finance \\
\hline FBOOT & Finance, Build, Own, Operate, Transfer \\
\hline ROT & Rehabilitate, Operate, Transfer \\
\hline
\end{tabular}


Sub-Type of Private Participation in Infrastructure: The database identifies sub-categories for each of the four types of projects:

Management and Lease Contracts - A private entity takes over the management of a state-owned enterprise for a fixed period while ownership and investment decisions remain with the state. There are two subclasses of management and lease contracts:

- management contract - The government pays a private operator to manage the facility. The operational risk remains with the government.

- lease contract - The government leases the assets to a private operator for a fee. The private operator takes on the operational risk.

Concessions - A private entity takes over the management of a state-owned enterprise for a given period during which it also assumes significant investment risk. The database classifies concessions according to the following categories:

- Rehabilitate, operate, and transfer: A private sponsor rehabilitates an existing facility, then operates and maintains the facility at its own risk for the contract period.

- Rehabilitate, lease or rent, and transfer: A private sponsor rehabilitates an existing facility at its own risk, leases or rents the facility from the government owner, then operates and maintains the facility at its own risk for the contract period.

Build, rehabilitate, operate, and transfer: A private developer builds an add-on to an existing facility or completes a partially built facility and rehabilitates existing assets, then operates and maintains the facility at its own risk for the contract period.

Greenfield Projects - A private entity or a public-private joint venture builds and operates a new facility for the period specified in the project contract. The facility may return to the public sector at the end of the concession period. The database classifies greenfield projects in four categories:

- Build, lease, and own: A private sponsor builds a new facility largely at its own risk, transfers ownership to the government, leases the facility from the government and operates it at its own risk, then receives full ownership of the facility at the end of the concession period. The government usually provides revenue guarantees through long-term takeor-pay contracts for bulk supply facilities or minimum traffic revenue guarantees.

- Build, own, transfer, or build, own, operate, transfer: A private sponsor builds a new facility at its own risk, owns and operates the facility at its 
own risk, then transfers ownership of the facility to the government at the end of the concession period. The government usually provides revenue guarantees through long-term take-or-pay contracts for bulk supply facilities or minimum traffic revenue guarantees.

- Build, own, and operate: A private sponsor builds a new facility at its own risk, then owns and operates the facility at its own risk. The government usually provides revenue guarantees through long-term take-or-pay contracts for bulk supply facilities or minimum traffic revenue guarantees.

- Merchant: A private sponsor builds a new facility in a liberalized market in which the government provides no revenue guarantees. The private developer assumes construction, operating, and market risk for the project (for example, a merchant power plant).

Divestitures - A private entity buys an equity stake in a state-owned enterprise through an asset sale, public offering, or mass privatization program. The database classifies divestitures in two categories:

- Full: The government transfers $100 \%$ of the equity in the state-owned company to private entities (operator, institutional investors, and the like).

Partial: The government transfers part of the equity in the state-owned company to private entities (operator, institutional investors, and the like). The private stake may or may not imply private management of the facility.

Quelle: http://ppi.worldbank.org/index.aspx, 23. September 2007 


\section{Literaturverzeichnis}

ADB (2000): Review of the Partial Guarantee of the Asian Development Bank, Manila

ADB (2002): Summary Environmental Impact Assessment, Phu My 2.2 Power Project in the Socialist Republic of Viet Nam, Manila

ADB (2004): Country Economic Review Philippines, Manila

ADB (2005a): Assessing the Impact of Transport and Energy Infrastructure on Poverty Reductions, Manila

ADB (2005b): Connecting East Asia, A New Framework for Infrastructure, Manila

ADB (2005c): Poverty in the Philippines: Income, Assets, and Access, Manila

ADB (2006a): Private Sector Operations, Catalyzing Private Investments Across Asia and the Pacific, Manila

ADB (2006b): Key Indicators of Developing Asian and Pacific Countries

ADB (2006c): Asian Development Outlook 2006, Manila

ADB (2006d): Poverty and Development Indicators: Statistics Glossary, Manila

ADB (2007a): ADB's Infrastructure Operations, Responding to Client Needs, Manila

ADB (2007b): ADB \& Viet Nam 2007, A Fact Sheet, Manila

ADB (2007c): Viet Nam: Loan and Political Risk Guarantee to the Phu My 3 Power Project, Manila

Agosin, M.R.; Mayer, R. (2000): Foreign Investment in Developing Countries: Does it Crowd in Domestic Investment?, UNCTAD, Discussion Paper No. 146

Aitken, B.J.; Harrison, A.E. (1999): Do Domestic Firms Benefit from Direct Foreign Investment? Evidence from Venezuela, in The American Economic Review, S. 605-618

Alexander, I.; Estache, A. (2000): Infrastructure Restructuring and Regulation, Building a Base for Sustainable Growth, Policy Research Working Paper 2415, World Bank

Alfaro, L.; Chanda, A.; Kalemli-Ozcan, S.; Sayek, S. (2004): FDI and Economic Growth: The Role of Local Financial Markets, in Journal of International Economics, Vol. 64/1, S. 89-112

Amoatey, C.T. (2007): User Financed Road Infrastructure in Ghana: Opportunities for Road Concessioning, Dissertation, Stuttgart 
Atteslander, P. (1995): Methoden der empirischen Sozialforschung, Walter de Gruyter Verlag, Berlin

Backhaus, K.; Werthschulte, H. (2003): Die Projektfinanzierung im Kontext der internationalen Auftragsfinanzierung, in Backhaus, K.; Werthschulte, H. (Hrsg.) (2003): Projektfinanzierung, Schäffer Poeschel, Stuttgart

Backhaus, K.; Werthschulte, H. (Hrsg.) (2003): Projektfinanzierung, Schäffer Poeschel, Stuttgart

Baker, J. (2000): Evaluating the Impact of Development Projects on Poverty: A Handbook for Practitioners, World Bank, Washington

Balasubramanyam, V.N.; Salisu, M.; Sapsford, D. (1996): Foreign Direct Investment and Growth in EP and is Countries, in The Economic Journal, Vol. 106/434 1996, S. 92-105

Balisacan, A.M. (2001): Pathways of Poverty Reduction: Rural Development and Transmission Mechanisms in the Philippines, in: Edwards, C.M. (Hrsg.) (2003): Reducing poverty in Asia: emerging issues in growth, targeting, and measurement, Elgar, Cheltenham

Balisacan, A.M.; Pernia, E.M. (2002): Probing Beneath Cross-National Averages: Poverty, Inequality, and Growth in the Philippines, ERD Working Paper Series No. 7, ADB, Manila

Balisacan, A.M.; Pernia, E.M. (2002): What Else Besides Growth Matters to Poverty Reduction? Philippines, ADB ERD Policy Brief Series 5, Manila

Balisacan, A.M.; Pernia, E.M.; Estrada, G.E.B. (2003): Economic Growth and Poverty Reduction in Viet Nam, ERD Working Paper Series No. 42, ADB, Manila

Ball, D. A.; McCulloch, W.H., Frantz, P.L.; Geringer, M.J.; Minor, M.S. (2006): International Business: The Challenge of Global Competition, McGrawHill/Irwin, New York

Ball, R.; Heafey, M.; King, D. (2004): Risk Management and the Private Finance Initiative, in Ghobadian, A.; Gallear, D.; O'Regan, N.; Viney, H. (Hrsg.) (2004): Public-Private Partnerships, Policy and Experience, palgrave Macmillan, New York

Bär, R. (2004): Public-Private Partnerships - Analyse und Kritik der Schweizer Position, in ÖFSE (2004): Armutsminderung durch den Privatsektor?, ÖFSE Edition 12, Südwind-Verlag Wien

Batten, D. F.; Kalsson, C. (Hrsg.) (1996): Infrastructure and the Complexity of Economic Development, Springer-Verlag, Berlin

Bea, S. (1995): Direktinvestitionen in Entwicklungsländern: Auswirkungen von Stabilisierungsmaßnahmen und Strukturreformen in Mexiko, Peter Lang, Frankfurt am Main 
Behrens, M. (2004): Global Governance, in Benz, A. (Hrsg.) (2004b): Governance - Regieren in komplexen Regelsystemen, VS Verlag für Sozialwissenschaften, Wiesbaden

Behrens, M. (2005a): Global Governance - Eine Einführung, in Behrens, M. (Hrsg.) (2005b): Globalisierung als politische Herausforderung: Global Governance zwischen Utopie und Realität, VS Verlag für Sozialwissenschaften, Wiesbaden

Behrens, M. (Hrsg.) (2005b): Globalisierung als politische Herausforderung: Global Governance zwischen Utopie und Realität, VS Verlag für Sozialwissenschaften, Wiesbaden

Bellak, C. (2000): Ausländische Direktinvestitionen - die nachhaltige Variante?, in ÖFSE 10 (2000): Private Kapitalflüsse - Weg aus der Krise?, Südwind-Verlag, Wien

Bellak, C. (2004): The Impact of Enlargement on the Race for FDI, Working Paper, 2004

Bellak, C.; Fischer, O.; Oettl, M.; Schönhofer, P. (1989): Internationalisierung, Band 1: Die Internationalisierung der österreichischen Industrie: Die erste Standortbestimmung, Reichard-Martini Verlag, Wien

Bende-Nabende, A.; Ford, J.L. (1998): FDI, Policy Adjustment and Endogenous Growth: Multiplier Effects from a Small Dynamic Model for Taiwan, 1959-1995, in World Development, Vol. 26/7, S. 1315-1330

Bendick, M. Jr. (1984): Privatization of Public Services: Recent Experience, in Brooks, H.; Liebman, L.; Schelling, C. (Hrsg.) (1984): Public-Private Partnership: New Opportunities for Meeting Social Needs, Ballinger Publishing Company, Cambridge

Bengoa, M.; Sanchez-Robles, B. (2003): Foreign direct investment, economic freedom and growth: new evidence from Latin America, in European Journal of Political Economy, Vol. 19, S. 529-545

Benz, A. (2004a): Governance - Modebegriff oder nützliches sozialwissenschaftliches Konzept?, in Benz, A. (Hrsg.) (2004b): Governance - Regieren in komplexen Regelsystemen, VS Verlag für Sozialwissenschaften, Wiesbaden

Benz, A. (Hrsg.) (2004b): Governance - Regieren in komplexen Regelsystemen, VS Verlag für Sozialwissenschaften, Wiesbaden

Benz, A.; Papadopoulos, Y. (Hrsg.) (2006): Governance and Democracy, Comparing national, European and international experiences, Routledge, London

Berg, S.V.; Pollitt, M.G.; Tsuji, M. (2002): Private Initiatives in Infrastructure: Priorities, Incentives and Performance, Edward Elgar, Cheltenham 
Bestani, R. (2004): A new key to Asian financing today, in: Euromoney Yearsbook's Project Finance

Bestani, R.; Sagar, A. (2004): The Local Currency Financing Revolution, ADB, Manila

Bewley, T. (2002): Interviews as a valid empirical tool in economics, in Journal of Socio-Economics 31, S. 343-353

bfai (2004): Mit Public Private Partnerships nach Asien, Neue Chancen für erfolgreiche Investitionen im Ausland, DIHK, Köln

Bhushan, I.; Bloom, E.; Thang, N.M. (2002): Unequal Benefits of Growth in Viet Nam, ERD Policy Brief Series No. 3, ADB, Manila

Birdsall, N.; Nellis, J. (2003): Winners and Losers: Assessing the Distributional Impact of Privatization, in World Development. Vol. 31/10, S. 1617-1633

Birdsall, N.; Nellis, J. (2005): Privatization Reality Check: Distributional Effects on Developing Countries, in Nellis, J.; Birdsall, N. (Hrsg.) (2005): Reality Check, The Distributional Impact of Privatization in Developing countries, Center for Global Development, Washington

Blalock, G.; Gertler, P.J. (2005): Foreign Direct Investment and Externalities: The Case for Public Intervention, in Moran T.H. et al. (2005) (Hrsg.): Does Foreign Direct Investment Promote Development?, Center for Global Development, Washington

Block, S. A.; Valer, P.M. (2004): The price of democracy: sovereign risk ratings, bond spread and political business cycles in developing countries, in Journal of International Money and Finance, Vol.23 2004, S. 917-846

Blomström, M. (1986): Foreign Investment and Productive Efficiency: The Case of Mexico, in The Journal of Industrial Economics, Vol. 35/1, S. 97-110

Blomström, M.; Lipsey, R.E.; Zejan, M. (1994): What Explains Developing Country Growth? in Baumol, W. et al. (Hrsg.): Convergence and Productivity: Gross-National Studies and Historical Evidence, Oxford University Press, Oxford

Blomström, M.; Sjöholm, F. (1999): Technology transfer and spillovers: Does local participation with multinationals matter?, in European Economic Review, Vol. 43, S. 915-923

Blonigen, B.A.; Wang, M.G. (2005): Inappropriate Pooling of Wealthy and Poor Countries in Empirical FDI Studies, in Moran T.H. et al. (2005) (Hrsg.): Does Foreign Direct Investment Promote Development?, Center for Global Development, Washington

BMF (2005): Strategischer Leitfaden des BMF für die Internationalen Finanzinstitutionen, Wien 
Borensztein, E.; De Gregorio, J.; Lee, J-W. (1998): How does foregin direct investment affect economic growth, in Journal of International Economics, Vol. 45, S. 115-135

Bös, D.; Schneider, F. (1996): Private-public partnership: Gemeinschaftsunternehmen zwischen Privaten und der öffentlichen Hand, Working Paper

Bougheas, S.; Demetriades, P.O.; Mamuneas, T.P. (2000): Infrastructure, Specialization, and Economic Growth, in The Canadian Journal of Economics, Vol. 33/2, S.506-522

Brand, U.; Scherrer, C. (2005): Contested Global Governance: Konkurrierende Formen und Inhalte globaler Regulierung, in Behrens, M. (Hrsg.) (2005b): Globalisierung als politische Herausforderung: Global Governance zwischen Utopie und Realität, VS Verlag für Sozialwissenschaften, Wiesbaden

Brand, U.; Brunnegräber, A.; Schrader, L.; Stock, C.; Wahl, P. (2000): Global governance: Alternative zur neoliberalen Globalisierung?, Westfälisches Dampfboot, Münster

Brenneman, A.; Kerf, M. (2002): Infrastructure \& Poverty Linkages, World Bank, Washington

Brockman, R. A. C.; Williams, A. (Hrsg.) (1996): Urban Infrastructure Finance, Asian Development Bank, Manila

Brook, P.J.; Irwin, T.C. (2003): Infrastructure for Poor People: Public Policy for Private Provision, World Bank, Washington D.C.

Brook, P.J.; Smith, S. M. (Hrsg.) (2001): Contracting for public services: Output-based aid and its applications, World Bank, Washington D.C.

Brooks, H.; Liebman, L.; Schelling, C. (Hrsg.) (1984): Public-Private Partnership: New Opportunities for Meeting Social Needs, Ballinger Publishing Company, Cambridge

Budäus, D. (2006a): Public Private Partnership - Kooperationsbedarfe, Grundkategorien und Entwicklungsperspektiven, in Budäus, D. (Hrsg.) (2006b): Kooperationsformen zwischen Staat und Markt, Nomos, Baden-Baden

Budäus, D. (Hrsg.) (1997a): Public Private Partnership I - State of the Art , Diskussionsbeiträge Nr. 32, Hamburg

Budäus, D. (Hrsg.) (1997b): Public Private Partnership II - Methodische Grundlagen und Elemente einer Theorie der Public Private Partnership, Diskussionsbeiträge Nr. 33, Hamburg

Budäus, D. (Hrsg.) (1998): Public Private Partnership III - Theorie der Public Private Partnership, Diskkusionsbeiträge Nr. 34, Hamburg

Budäus, D. (Hrsg.) (2006b): Kooperationsformen zwischen Staat und Markt, Nomos, Baden-Baden 
Bundesministerium für Verkehr, Bau und Stadtentwicklung (BMVBS) (2003): PPP im öffentlichen Hochbau, Band I: Leitfaden

Burger, B. (1998): Ausländische Direktinvestitionen, technologische SpilloverEffekte und industrielle Entwicklung, dargestellt am Beispiel Mexiko, Nomos Verlagsgesellschaft, Baden-Baden

Büschgen, H.E. (1997): Internationales Finanzmanagement, Fritz Knapp Verlag, Frankfurt a. M.

Busse, M.; Groizard, J.L. (2006): Foreign Direct Investment, Regulations, and Growth, World Bank Policy Research Working Paper 3882

Canning, D. (1999): The Contribution of Infrastructure to Aggregate Output, World Bank Infrastructure Working Paper, No. 2246, Washington

Canning, D.; Bennathan, E. (2000): The Social Rate of Return on Infrastructure Investment, World Bank Policy Research Working Paper 2390, Washington

Carkovic, M.; Levine, R. (2002): Does Foreign Direct Investment Accelerate Economic Growth?

Caroll, P.; Steane, P. (2003). Public-private partnerships, Sectoral perspectives, in Osborne, S.P. (Hrsg.) (2003): Public-Private Partnerships, Theory and practice in international perspectives, Routledge, New York

Carvalho, S.; White, H. (1997): Combining the Quantitative and Qualitative Approaches to Poverty Measurement and Analysis, World Bank Technical Paper 266, Washington

Cheng, L.K.; Qiu, L.D.; Tan, G. (2005): Foreign direct investment and international trade in a continuum Ricardian trade model, in Journal of Development Economics, Vol. 77, S. 477-501

Choe, J.I. (2003): Do Foreign Direct Investment and Gross Domestic Investment Promote Economic Growth?, in Review of Development Economics, Vol. 7/1, S. 44-57

Chowdhury, A.; Mavrotas, G. (2005): FDI and Growth: A Causal Relationship, WIDER

Chuang, Y-C.; Lin, C-M. (1999): Foreign Direct Investment, R\&D and Spillover Efficiency: Evidence from Taiwan's Manufacturing Firms, in The Journal of Development Studies, Vol. 4, S. 117-137

Coase, R.H. (1937): The Nature of the Firm, in Economica 1937, S. 386-405

Commission of the European Communities (CEC) (2004): Public-Private Partnerships in Developing and Transition countries - A Critical Review of Existing Experiences and Analysis of Possibilities of Donor Intervention, Brüssel 
Common, R. (2003): The East Asian region, Do public-private partnerships make sense? in Osborne, S.P. (Hrsg.) (2003): Public-Private Partnerships, Theory and practice in international perspectives, Routledge, New York

Cooke, B.; Kuthari, U. (2000): Participation: A New Tyranny?, Zed Books, London

Cook, P.; Uchida, Y. (2003): Privatisation and Economic Growth in Developing Countries, in The Journal of Development Studies, Vol. 19/6, S. 121-154

Curtis, L. (2004): POVNET: Infrastructure Financing, Current Initiatives, New Opportunities

Damijan, J.P.; Majcen, B.; Rojec, M.; Knell, M. (2001): The Role of FDI, R\&D Accumulation and Trade in Transferring Technology to Transition Countries: Evidence from Firm Panel Data for eight Transition Countries, Working Paper No. 10, Institute for Economic Research

Daniels, R.J.; Trebilcock, M.J. (1996): Private Provision of Public Infrastructure: An Organizational Analysis of the next Privatization Frontier, in University of Toronto Law Journal, Vol. XLVI/3, S. 375-426

Daniels, R.J.; Trebilcock, M.J. (2000): An Organizational Analysis of the Public-Private Partnership in the Provision of Public Infrastructure, in Rosenau, P.V. (Hrsg.) (2000): Public-Private Policy Partnerships, Massachusetts Institute of Technology Press, Cambridge

De Geer, H.; Borglund, T.; Frostenson, M. (2004): Interpreting the International Firm: Going Beyond Interviews, in: Marschan-Piekkari, R.; Welch, C. (Hrsg.) (2004): Handbook of Qualitative Research Methods for International Business, Edward Elgar, Cheltenham

De Mello, L.R. Jr. (1997): Foreign Direct Investment in Developing Countries and Growth: A Selective Summary, in The Journal of Development Studies, Vol. 34/1, S.1-34

De Mello, L.R. Jr. (1999): Foreign direct-investment-led growth: Evidence from time series and panel data, in Oxford Economic Papers, Vol. 51/1, S. 133151

Deutsche Kommission Justitia et Pax (1999): Neue Wege zur Lösung der internationalen Schuldenfrage, Bonn

Devarajan, S.; Swaroop, V.; Zhou, H-F. (1996): The Composition of Public Expenditure and Economic Growth, in Journal of Monetary Economics, Vol. 37/2-3, S. 313-344

DFID (2002): Making Connections: Infrastructure for Poverty Reduction, London 
Djankov, S.; Hoekman, B. (1998): Foreign Investment and Productivity Growth in Czech Enterprises, World Bank Economic Review

Doh, P.J.; Ramamurti, R. (2003): Reassessing Risk in Developing Country Infrastructure, in Long Range Planning Journal, Vol. 36, S. 337-353

Dollar, D.; Kraay, A. (2002): Growth Is Good for the Poor, World Bank, Development Research Group

Doornbos, M. (2001): 'Good Governance': The Rise and Decline of a Policy Metaphor? In The Journal of Development Studies, Vol. 37/6, S. 93-108

Durth, R.; Körner, H.; Michaelowa, K. (2002): Neue Entwicklungsökonomik, Lucius \& Lucius, Stuttgart

Easterly, W.; Rebelo, S. (1993): Fiscal Policy and Economic Growth: An Empirical Investigation, in Journal of Monetary Economics, Vol. 32, S. 417-458

Ebner, C. (1997): Determinanten österreichischer Direktinvestitionen in den USA, Dissertation, WU Wien

EC (European Commission) (2003a): Guidelines for Successful Public-Private Partnerships, Brussels

EC (European Commission) (2003b): Report from the Commission on European Governance, Luxembourg

EC (European Commission) (2004): Public-Private Partnerships in Developing and Transition countries - A critical review of existing experiences and analysis of possibilities of donor intervention, Brussels

Edwards, C.M. (Hrsg.) (2003): Reducing poverty in Asia: emerging issues in growth, targeting, and measurement, Elgar, Cheltenham

Egger, U. (1998): Öffentlich-private Partnerschaften zur regionalen Entwicklung: Theoretische Grundlagen und Fallstudien in der Schweiz, Disertation, Difo-Druck, Bamberg

Eggers, M. (2004): Public Private Partnership: Eine strukturierte Analyse auf der Grundlage von ökonomischen und politischen Potentialen, Dissertation, Peter Lang, Frankfurt a. M.

Eiteman, D.K.; Stonehill, A.I.; Moffet, M.H. (2004): Multinational Business Finance, Pearson - Addison Wesley, Boston

ESMAP (2000): Energy and Development Report 2000: Energy Services for the World's Poor, World Bank, Washington

Estache, A. (2004): A Selected Survey of Recent Economic Literature on Emerging Infrastructure Policy Issues in Developing Countries, INFVP, World Bank

Estache, A. (2006): Infrastructure: A survey of recent and upcoming issues, World Bank 
Fan, S.; Hazell, P.; Thorat, S. (1999): Linkages between Government Spending, Growth and Poverty in Rural India, IFPRI Research Report no. 110, Washington

Farlam, P. (2005): Working Together: Assessing Public-Private Partnerships in Africa, NEPAD Policy Focus Report No. 2

Faschingeder, G.; Ornig, N. (Hrsg.) (2005): Globalisierung Ent-Wickeln, Mandelbaum Verlag, Wien

Faulkner, K. (2004): Public-Private Partnerships, in Ghobadian, A.; Gallear, D.; O’Regan, N.; Viney, H. (Hrsg.) (2004): Public-Private Partnerships, Policy and Experience, palgrave Macmillan, New York

Feldmann, H. (1995): Eine institutionalistische Revolution? Zur dogmengeschichtlichen Bedeutung der modernen Institutionenökonomik, Volkswirtschaftliche Schriften, Berlin

Fernald, J.G. (1999): Roads to Prosperity? Assessing the Link between Public Capital and Productivity, in The American Economic Review, Vol. 89/3, S. 619638

Fischbacher, M.; Beaumont, P.B. (2003): PFI, Public Private Partnerships and the Neglected Importance of Process: Stakeholders and the Employment Dimension, in Public Money \& Management, Vol. 23, S. 171-176

Fischer, K.; Hanak, I.; Parnreiter, Ch. (Hrsg.) (2003): Internationale Entwicklung: Eine Einführung in Probleme, Mechanismen und Theorien, 3. Auflage, Brandes \& Apsel Verlag GmbH, Frankfurt a. M.

Fischer, K.; Hödl, G.; Maral-Hanak, I.; Parnreiter, Ch., (Hrsg.) (2004): Entwicklung und Unterentwicklung: Eine Einführung in Probleme, Theorien und Strategien, Mandelbaum Verlag, Wien

Flick, U. (2002): Qualitative Sozialforschung, Rohwolt Taschenbuch Verlag, Reinbek bei Hamburg

Flick, U.; von Kardorff, E.; Steinke, I. (Hrsg.) (2003): Qualitative Forschung, Ein Handbuch, Rohwohlt Taschenbuch Verlag, Reinbek bei Hamburg

Fox, J.A. (2000): The World Bank Inspection Panel: Lessons from the First Five Years, in Global Governance, Vol. 6/3, S. 279-319

Freiere, M.; Petersen (Hrsg.) (2004): Subnational Capital Markets in Developing Countries: From Theory to Practice, World Bank, Washington

Fritzen, S. (2002): Growth, Inequality and the Future of Poverty Reduction in Vietnam, in: Journal of Asian Economics, Vol. 13/5, S. 635-657

Fung-Yee Ng, L.; Tuan, C. (2006): Spatial agglomeration, FDI, and regional growth in China: Locality of local and foreign manufacturing investments, in Journal of Asian Economics 2006, doi:10.1016/j.asieco.2006.06.008 
Furubotn, E.G.; Pejovich, S. (1972): Property Rights and Economic Theory: A Survey of Recent Literature, in Journal of Economic Literature, S. 1137-1162

Furubotn, E.G.; Richter, R. (Hrsg.) (1991): The New Institutional Economics, Mohr, Tübingen

Fuster, T. (1997): Die „Good Governance“ Diskussion der Jahre 1989 bis 1994, Ein Beitrag zur jüngeren Geschichte der Entwicklungspolitik unter spezieller Berücksichtigung der Weltbank und des DAC, Dissertation, Verlag Paul Haupt, Bern

Gadamer, H.-G. (1986): Gesammelte Werke, Hermeneutik II, J.C.B. Mohr, Tübingen

Garz, D. (Hrsg.) (1983): Brauchen wir neue Forschungsmethoden?: Beiträge zum Verfahren interpretativer Verfahren, Scriptor-Verlag, Frankfurt a. M.

Gerrard, M.B. (2001): Public-Private Partnerships: What are public-private partnerships, and how do they differ from privatization?, in Finance \& Development, Vol. 38/3, S.48-51

Ghauri, P. (2004): Designing and Conduction Case Studies in International Business Research, in: Marschan-Piekkari, R.; Welch, C. (Hrsg.) (2004): Handbook of Qualitative Research Methods for International Business, Edward Elgar, Cheltenham

Ghobadian, A.; Gallear, D.; O'Regan, N.; Viney, H. (Hrsg.) (2004): PublicPrivate Partnerships, Policy and Experience, palgrave Macmillan, New York

Gholami, R.; Lee, S-Y. T.; Heshmati, A. (2005): The Causal Relationship between ICT and FDI, WIDER

Glaum, M. (2000): Finanzwirtschaftliches Risikomanagement deutscher Industrie- und Handelsunternehmen

Godin, R.E.; Klingemann, H.-D. (Hrsg.) (1996): A New Handbook of Political Sciences, Oxford University Press, Oxford

GPOBA (2005): Output-based Aid: Supporting Infrastructure Delivery Through Explicit and Performance-based Subsidies, OBA Working Paper Series, Paper No. 4 ,

Grimsey, D.; Lewis, M.K. (2002): Evaluating the risks of public private partnerships for infrastructure projects, in International Journal of Project Management, Vol. 20, S. $107-118$

Grimsey, D.; Lewis, M.K. (2004): Public Private Partnerships, The Worldwide Revolution in Infrastructure Provision and Project Finance, Edward Elgar, Cheltenham

Grimsey, D.; Lewis, M.K. (Hrsg.) (2005): The Economics of Public Private Partnerships, An Elgar Reference Collection, Cheltenham 
Grohnert, A.-C. (2005): Öffentlich-Private-Partnerschaft - Allheilmittel oder Mogelpackung?, in Immobilien \& Finanzierung, Vol. 18, S. 636-638

Gruber, W.; Rothenberger, D. (2005): Öffentlich-private Partnerschaften - ein Ansatz zur Verbesserung der Infrastrukturversorgung, in: Schweizerisches Jahrbuch für Entwicklungspolitik

Gstöhl, S. (Hrsg.) (2003): Global Governance und die G8: Gipfelimpulse für die Weltwirtschaft und Weltpolitik, LIT Verlag, Münster

Guislain, P.; Kerf, M. (1995): Concessions - the way to privatize infrastructure in sector monopolies, in Public Policy for the Private Sector Note Nr. 59, Weltbank

Haarmeyer, D.; Mody, A. (1997): Private Capital in Water and Sanitation, in Finance \& Development, Vol. 34/1, S. 34-37

Haddad, M.; Harrison, A. (1993): Are There Positive Spillovers from Direct Foreign Investment? Evidence from Panel Data for Morocco, in Journal of Development Economics, Vol. 42, S. 51-74

Halin, A. (1995): Vertikale Innovationskooperation. Eine transaktionskostentheoretische Analyse, Dissertation, Frankfurt a. M.

Hall, J. (1998): Private Opportunity, Public Benefit?, in Fiscal Studies, Vol. 19/2, S. 121-140

Hansen, H.; Rand, J. (2006): On the Causal Links Between FDI and Growth in Developing Countries, United Nations University

Hanson, G.H. (2001): Should Countries Promote Foreign Direct Investment, UNCTAD, Discussion Paper No. 9

Harris, C. (2003): Private Participation in Infrastructure in Developing Countries, World Bank Working Paper No. 5

Harris, J.; Hunter, J.; Lewis, C.M. (Hrsg.) (2000): The New Institutional Economics and Third World Development, Routledge, London

Hartmann, P. (1994): Beziehungen zwischen Staat und Wirtschaft - unter besonderer Berücksichtigung neuartiger Kooperationsformen im Bereich der regionalen und kommunalen Wirtschaftspolitik, Nomos, Baden-Baden

Hauschildt, J. (1997): Innovationsmanagement, Vahlen, München

Herdzina, K.; Nolte, B. (1995): Die Entwicklung einer zieladäquaten Infrastruktur für die Raumkategorien des ländlichen Raumes, Europäische Forschungsstelle für den Ländlichen Raum, Universität Hohenheim, Stuttgart

Hermes, N.; Lensink, R. (2003): Foreign Direct Investment, Financial Development and Economic Growth, in The Journal of Development Studies, Vol. 40/1, S. 142-163 
Hesselbarth, S. (2004): Donors Practices and the Development of Bilateral Donor's Infrastructure Portfolio, OECD Room Document 3, Paris

Hettige, H. (2006): When do rural roads benefit the poor and how?, ADB, Manila

Hinsch, L.C.; Horn, N. (1985): Das Vertragsrecht der internationalen Konsortialkredite und Projektfinanzeierungen, Walter de Gruyter, Berlin

Holz-Klemmer, F. (2001): Die Determinanten des Investitionsklimas im Neuen Südafrika und ihre Auswirkungen auf ausländische Direktinvestitionen, Peter Lang, Frankfurt am Main

Hopf, C. (2003): Qualitative Interviews - Ein Überblick, in Flick, U.; von Kardorff, E.; Steinke, I. (Hrsg.), Qualitative Forschung, Ein Handbuch, Rohwohlt Taschenbuch Verlag, Reinbek bei Hamburg

Hsiao, C.; Shen, Y. (2003): Foreign Direct Investment and Economic Growth: The Importance of Institutions and Urbanization, in Economic Development and Cultural Change, Vol 51/4, S. 883-896

Hulme, D.; Shepherd, A. (2003): Conceptualizing Chronic Poverty, in World Development, Vol. 33/1, S. 403-423

Hurmerinta-Peltomäki, L.; Nummela, N. (2004): First the sugar, Then the Eggs. Or the Other Way Round? Mixing Methods in International Business Research, in: Marschan-Piekkari, R.; Welch, C. (Hrsg.) (2004): Handbook of Qualitative Research Methods for International Business, Edward Elgar, Cheltenham

Huxham, C.; Vangen, S. (2003): What makes partnerships work?, in Osborne, S.P. (Hrsg.) (2003): Public-Private Partnerships, Theory and practice in international perspectives, Routledge, New York

IDA (2004): Strengthening the Private Sector in IDA Countries: Status of World Bank Group Collaboration, Washington

IFC (2006): IFC's Products and Services, Washington

IMF (1993): Balance of Payments Manual, Washington

IMF (2004): Public-Private Partnerships, Washington

IUED (2005): Schweizerisches Jahrbuch für Entwicklungspolitik, Band 24 Nr. 2

Izaguirre, A. K. (2000): Private participation in energy, Private Sector Note No. 208, World Bank

Izaguirre, A. K. (2005): Private Infrastructure: Emerging Market Sponsors Dominate Private Flows, in; Public Policy for the Private Sector, World Bank Group

Jalan, J.; Ravallion, M. (2000): Is Transient Poverty Different? Evidence for Rural China, in Journal of Development Studies, Vol. 36, S. 82-99 
Jalan, J.; Ravallion, M. (2002): Geographic Poverty Traps? A Micro Model of Consumption Growth in Rural China, in Journal of Applied Econometrics, Vol. $17 / 4$, S. 329-346

Jann, W.; Wegrich, K. (2004): Governance und Verwaltungspolitik, in Benz, A. (Hrsg.) (2004b): Governance - Regieren in komplexen Regelsystemen, VS Verlag für Sozialwissenschaften, Wiesbaden

Javorcik, B.S.; Spatareanu, M. (2005): Disentangling FDI Spillover Effects: What Do Firm Perception Tell Us?, in Moran T.H. et al. (2005) (Hrsg.): Does Foreign Direct Investment Promote Development?, Center for Global Development, Washington

Jenkins, C.; Thomas, L. (2002): Foreign Direct Investment in Southern Africa: Determinants, Characteristics and Implications for Economic Growth and Poverty Alleviation, CSAE, CREFSA

Jochimsen, R. (1966): Theorie der Infrastruktur, J. C. B. Mohr, Tübingen

Jochimsen, R.; Gustafsson, K. (1977): Infrastruktur, Grundlage der marktwirtschaftlichen Entwicklung, in Simonis, U.E. (Hrsg.) (1977): Infrastruktur, Theorie und Praxis, Kiepenheuer \& Witsch

Jochimsen, R.; Simonis, U. E. (Hrsg.) (1970): Theorie und Praxis der Infrastrukturpolitik, Duncker \& Humblot, Berlin

Kaiser, M. (Hrsg.) (1986): Entwicklungspolitik: Grundlagen - Probleme - Aufgaben, Schriftenreihe der Bundeszentrale für Politische Bildung, Bonn

Kehew, R.; Matsukawa, T.; Petersen, J. (2005): Local Financing for SubSovereign Infrastructure in Developing Countries: Case Studies of Innovative Domestic Credit Enhancement Entities and Techniques, World Bank Discussion Paper No. 1, Washington

Kjaer, A.M. (2004): Governance, Polity Press, Cambridge

Klasen, S. (2001): In Search of the Holy Grail: How to Achieve Pro-Poor Growth?, GTZ

Klaus, J. (Hrsg.) (1984): Entscheidungshilfen für die Infrastrukturplanung, Nomos Verlagsgesellschaft, Baden-Baden

Klein, M. (1997): The Risk Premium for Evaluating Public Projects, in Oxford Review of Economic Policy, Vol. 13/4, S. 29-42

Klijn, E.-H.; Teisman, G.R. (2003a): Governing public-private partnerships, Analysing and managing the processes and institutional characteristics of public-private partnerships, in Osborne, S.P. (Hrsg.) (2003): Public-Private Partnerships, Theory and practice in international perspectives, Routledge, New York 
Klijn, E.-H.; Teisman, G.R. (2003b): Institutional and Strategic Barriers to Public-Private Partnership: An Analysis of Dutch Cases, in Public Money \& Management, Vol. 23, S. 137-146

Klijn, E.-H.; Teisman, G.R. (2004): Public-Private Partnerships: The Right Form at the Wrong Moment? An Analysis of Institutional and Strategic Obstacles, in Ghobadian, A.; Gallear, D.; O'Regan, N.; Viney, H. (Hrsg.) (2004): Public-Private Partnerships, Policy and Experience, Palgrave Macmillan, New York

Kolland, F. (2003): Entwicklungstheorien bis in die 1980er-Jahre und ihre politischen Implikationen, in Fischer, K. et al. (Hrsg.) (2003): Internationale Entwicklung, Brandes \& Apsel Verlag, Frankfurt a. M.

Kolodziej, M. (1996): Die private Finanzierung von Infrastruktur, Dissertation, Peter Lang, Europäische Hochschulschriften, Frankfurt a. M.

Konings, J. (2000): The Effects of Direct Foreign Investment on Domestic Firms: Evidence from Firm Level Panel Data in Emerging Economies, William Davidson Institute, Working Paper No. 344

Kooiman, J. (Hrsg.) (1993): Modern Governance - New Government-Society Interactions, SAGE, Lodon

Kouwenhoven, V. (1993): Public Private Partnership: A Model for the Management of Public-Private Cooperation, in: Kooiman, J. (Hrsg.) (1993): Modern Governance - New Government-Society Interactions, SAGE, London

Krayenbuehl, T. E. (1988): Country Risk: Assessment and monitoring, Woodhead-Faulkner Ltd, Cambridge

Krishna, A. (2004): Escaping Poverty and Becoming Poor: Who gains, Who loses and Why?, in World Development, Vol. 32/1, S. 121-136

Küblböck, K.; Strickner, A. (1996): Neo-Cepalismo: eine Alternative jenseits des Neoliberalismus, in Journal für Entwicklungspolitik, Vol. 1, S. 39-48

Küblböck, K. (2004): Privatisierung von öffentlichen Dienstleistungen in Entwicklungsländern, in ÖFSE 12 (2004): Armutsminderung durch den Privatsektor?, Südwind-Verlag, Wien

Kumar, N. (2001): Infrastructure Availability, Foreign Direct Investment Inflows and Their Export-orientation: A Cross-Country Exploration, Research and Information System for Developing countries

Kumar, N.; Pradhan, J.P. (2002): Foreign Direct Investment, Externalities and Economic Growth in Developing Countries: Some Empirical Explorations and Implications for WTO Negotiations on Investment, RIS Discussion Paper

Kutschker, M.; Schmid, S. (2004): Internationales Management, Oldenbourg Verlag, München 
Kwon, E. (2000): A Link Between Infrastructure, Growth, and Poverty in Indonesia: Stage 1 report, $\mathrm{ADB}$, Manila

Laffont, J.-J.; Tirole, J. (1994): A theory of incentives in procurement and regulation, MIT Press, Cambridge

Lake, D.A. (1999): Global governance, A relational contracting approach, in Prakash, A.; Hart, J.A. (Hrsg.) (1999): Globalization and Governance, Routledge, London

Lamnek, S. (1995): Qualitative Sozialforschung, Band 1: Methodologien, Band 2: Methoden und Techniken, Psychologie-Verlags-Union, Weinheim

Langford, R. (2002): Managing Public-Private Partnerships in Canada, in Meredith, E.; Langford, R. (Hrsg.) (2002): New Players, Partners and Processes: A Public Sector Without Boundaries, National Institute for Governance, University of Canberra, Center for Public Studies, University of Victoria, British Colombia

Leftwich, A. (1993): Governance, democracy and development in the Third World, in Third World Quarterly, Vol. 14/3, S. 605-624

Leiderer, S.; Liebig, K. (2006): Public Financial Management and SubSovereign Lending for Infrastructure Investment in Africa: When Second Best is not Enough, Workshop on Public Expenditure and Service Delivery in Africa, Zambia, United Nations Economic Commission for Africa

Lensink, R.; Morrissey, O. (2001): Foreign Direct Investment: Flows, Volatility and Growth, DESG

Leproux, V.; Brooks, D.H. (2004): Viet Nam: Foreign Direct Investment and Postcrisis Regional Integration, ERD Working Paper Series No. 56, ADB, Manila

Leubolt, B. (2007): On the historical and current developments of the governance concept, in Journal für Entwicklungspolitik, Vol. 2007/1

Lewis, D. (2003): Building 'active' partnerships in aid-recipient countries, Lessons from a rural development project in Bangladesh, in Osborne, S.P. (Hrsg.) (2003): Public-Private Partnerships, Theory and practice in international perspectives, Routledge, New York

Li, X.; Liu, X. (2005): Foreign Direct Investment and Economic Growth: An Increasingly Endogenous Relationship, in World Development, Vol. 33, S. 393407

Lim, E.-G. (2001): Determinants of, and the Relation Between, Foreign Direct Investment and Growth: A Summary of the Recent Literature, IMF Working Paper 175 
Linder, S.H. (1999): Coming to Terms With Public-Private Partnership: A Grammar of Multiple Meaning, in American Behavioral Scientist, Vol. 43/1, S. $35-51$

Lipsey, R.E. (1999): The Role of Foreign Direct Investment in International Capital Flows, NBER Working Paper 7094

Lipsey, R.E.; Sjöholm, F. (2005): The Impact of Inward FDI on Host Countries: Why Such Different Answers? in Moran T.H. et al. (2005) (Hrsg.): Does Foreign Direct Investment Promote Development?, Center for Global Development, Washington

Liu, Z. (2006): Foreign direct investment and technology spillovers: Theory and evidence, in Journal of Development Economics 2006

Loderer, C.; Pichler, K. (2000): Firms, do you know your currency risk exposure? Survey results, in Journal of Empirical Finance, Vol. 7, S. 317-344

Lopez, J.H. (2004a): Pro-growth, pro-poor: Is there a tradeoff?, World Bank Policy Research Working Paper 3378

Lopez, J.H. (2004b): Pro-poor growth: a review of what we know (and of what we don't), World Bank (PRMPR)

Lütz, S. (2004): Governance in der politischen Ökonomie, in Benz, A. (Hrsg.) (2004b): Governance - Regieren in komplexen Regelsystemen, VS Verlag für Sozialwissenschaften, Wiesbaden

Madura, J. (2006): International Corporate Finance, Thomson South-Western, Toronto

Markusen, J.R.; Venables, A.J. (1999): Foreign direct investment as a catalyst for industrial development, in European Economic Review, Vol. 43, S. 335-356

Marschan-Piekkari, R.; Welch, C. (Hrsg.) (2004): Handbook of Qualitative Research Methods for International Business, Edward Elgar, Cheltenham

Martens, J. (2004): Private Akteure und Partnerschaftsinitiativen in der internationalen Politik, in ÖFSE (2004): Armutsminderung durch den Privatsektor?, ÖFSE Edition 12, Südwind-Verlag Wien

Massmann, O. (2003): Vietnam Investitionsführer und Leitfaden für Lieferfirmen, 7. Buch, Hanoi

Massmann, O. (2007): Vietnam Investitionsführer und Leitfaden für ausländische Lieferfirmen, 1. Buch, Hanoi

Mayntz, R. (1998): New Challenges to Governance Theory, Jean Monnet Chair Paper 50, Robert Schuman Centre of the European University Institute, Florenze

Mayring, P. (1995): Qualitative Inhaltsanalyse: Grundlagen und Techniken, Dt. Studien-Verlag, Wiesbaden 
Mayring, P. (2002): Einführung in die qualitative Sozialforschung: Eine Anleitung zu qualitativen Denken, Psychologie-Verlags-Union, Weinheim

McQuaid, R.W. (2003): The theory of partnership, Why have partnerships?, in Osborne, S.P. (Hrsg.) (2003): Public-Private Partnerships, Theory and practice in international perspectives, Routledge, New York

Meier, G.M.; Stiglitz, J.E. (Hrsg.) (2001): Frontiers of Development Economics, Oxford University Press, New York

Menzel, U. (1992): Das Ende der Dritten Welt und das Scheitern der Großen Theorie, Suhrkamp, Frankfurt a. M.

Meredith, E.; Langford, R. (Hrsg.) (2002): New Players, Partners and Processes: A Public Sector Without Boundaries, National Institute for Governance, University of Canberra, Center for Public Studies, University of Victoria, British Colombia

Meridian Institute (2005): The Provision of Infrastructure Services in Developing Countries

Messner, D. (2005): Global Governance: Globalisierung im 21. Jahrhundert gestalten, in Behrens, M. (Hrsg.) (2005b): Globalisierung als politische Herausforderung: Global Governance zwischen Utopie und Realität, VS Verlag für Sozialwissenschaften, Wiesbaden

Meyer, M. (1987): Die Beurteilung von Länderrisiken der internationalen Unternehmung, Duckner \& Humblot, Berlin

Miles, M.B.; Huberman, A. M. (1995): Qualitative data analysis: an expand sourcebook, Sage Publicatiosn, London

Miller, N.J.; Tsoukis, C. (2001): On the Optimality of Public Capital for LongRun Economic Growth: Evidence from Panel Data, in Applied Economics, Vol. $3 / 9$, S. $1117-1129$

Momberg, R. (2000): Theorie und Politik der Infrastruktur unter Berücksichtigung institutionen- und polit-ökonomischer Einflussfaktoren, Peter Lang Verlag, Frankfurt am Main

Moore, D.B.; Schmitz, G.J. (Hrsg.) (1995): Debating Development Discourse, St. Martin's Press, New York

Moran, T.H.; Graham, E.M., Blomström M. (Hrsg.) (2005): Does Foreign Direct Investment Promote Development?, Center for Global Development, Washington

Moser, R. (1985): Preis- und Finanzierungsentscheidungen im Ausland, Verlag der Österreichischen Akademie der Wissenschaft, Wien

Moss, T,; Ramachandran, V.; Shah, M.K. (2005): Is Africa's Skepticism of Foreign Capital Justified? Evidence from East African Firm Survey Data, in 
Moran et al. (2005) (Hrsg.): Does Foreign Direct Investment promote Development?, Center for Global Development, Washington

Moss, T.; Ramachandran, V. (2005): Foreign Investment and Economic Development: Evidence from Private Firms in East Africa, Center for Global Development

Mrotzek, R. (1989): Bewertung direkter Auslandsinvestitionen mit Hilfe betrieblicher Investitionskalküle, Gabler Verlag, Wiesbaden

Murphy, C.N. (2000): Global Governance: poorly done and poorly understood, in International Affairs, Vol. 76, S. 780-803

Nabli, M.K. (2001): Privatization and Institutions in Developing and Transition Economies, in Stiglitz, J.E.; Muet, P.-A. (Hrsg.) (2001): Governance, Equity and Global Markets, University Press, Oxford

Nair-Reichert, U.; Weinhold, D. (2001): Causality tests for cross-country panels: a new look at FDI and economic growth in developing countries, in Oxford Bulletin of Economics and Statistics, Vol. 63/2, S.153-171

Nellis, J. (2006): Privatization - A Summary Assessment, Working Paper Number 87, Center for Global Development

Nellis, J.; Birdsall, N. (Hrsg.) (2005): Reality Check, The Distributional Impact of Privatization in Developing countries, Center for Global Development, Washington

Nevitt, P.K.; Fabozzi, F.J. (2000): Project Finance, Euromoney Institutional Investor, London

Noel, M.; Brzeski, W.J. (2005): Mobilizing Private Finance for Local Infrastructure in Europe and Central Asia, An Alternative Public Private Partnership Framework, World Bank Working Paper No. 46

North, D.C. (1988): Theorie des institutionellen Wandels, Mohr, Tübingen

North, D.C. (1991): Institutions, in: Journal of Economic Perspectives, Bd. 5, Nr. 1, S. 97-112

North, D.C. (1992): Institutionen, institutioneller Wandel und Wirtschaftsleistung, Mohr, Tübingen

Novy, A. (2002): Die Methodologie interpretativer Sozialforschung, SRE Discussion Paper, 2002/01

Novy, A. (2004): Entwicklung gestalten: Gesellschaftsveränderung in der Einen Welt, Brandes \& Apsel / Südwind, Frankfurt a. M.

Nunnenkamp, P. (2002): Determinants of FDI in Developing Countries: Has Globalization Changed the Rules of the Game?, Kiel Working Paper No. 1122

Nunnenkamp, P. (2004): To What Extent Can Foreign Direct Investment Help Achieve International Development Goals?, Kiel Institute for World Economics 
Nuscheler, F. (2004a): Entwicklungspolitik, Verlag J. H. W. Dietz Nachf. $\mathrm{GmbH}$, Bonn

Nuscheler, F. (2004b): Welchen Beitrag kann der Privatsektor zum Erreichen der Millenniumziele leisten? in ÖFSE (2004): Armutsminderung durch den Privatsektor?, ÖFSE Edition 12, Südwind-Verlag Wien

Nye, J.S. (2001): Globalization's Democratic Deficit: How to make International Institutions More Accountable, in Foreign Affairs, Vol. 80/4, S. 26

Oates, W.E. (1972): Fiscal Federalism, Harcourt Brace Jovanovich, New York

OECD (1999): Benchmark Definition of Foreign Direct Investment

OECD (2001): DAC Guidelines on Poverty Reduction, Paris

OECD (2003): Improving Water Management, IWA Publishing, Paris

OECD (2004): Domestic Finance Mobilization for Pro-Poor Infrastructure: An Exploration of Sub-Sovereign Finance Issues and Policy Guidance, Paris

OECD (2005): Mobilising Private Investment for Development: Policy Lessons on the role of ODA

OECD (2006a): Promoting Pro-Poor Growth: Infrastructure, Paris

OECD (2006b): Promoting Private Investment for Development, The Role of ODA, Paris

OECD (2006c): Promoting Pro-Poor Growth: Key Policy Issues, Paris

OeKB (2004): Auslandsinvestitionen: Bewährte Instrumente für Ihre Internationalisierung, Wien

ÖFSE (2000): Private Kapitalflüsse - Weg aus der Krise, ÖFSE Edition 10, Südwind-Verlag Wien

ÖFSE (2004): Armutsminderung durch den Privatsektor?, ÖFSE Edition 12, Südwind-Verlag Wien

Osborne, S.P. (Hrsg.) (2003): Public-Private Partnerships, Theory and practice in international perspectives, Routledge, New York

Osei, P.D. (2004): Public-Private Partnerships in Service Delivery in Developing Countries: Jamaican Examples, in Ghobadian, A.; Gallear, D.; O’Regan, N.; Viney, H. (Hrsg.) (2004): Public-Private Partnerships, Policy and Experience, palgrave Macmillan, New York

Osius, M. E.; Carlson, C. (2004): International Financing Sources in Support of "Pro-Poor/Pro-Growth" Infrastructure Development, Povnet Conference, Berlin

o.V. (2007): Die Entwicklungsbank steht, in der Standard, Printausgabe 18.10.2007 
Owen, G.; Merna, A. (1997): The Private Finance Initiative, in Engineering, Construction and Architectural Management, Vol. 4/3, S. 163-177

Palmade, V.; Anayiotas, A. (2004): FDI Trends, in Public Policy for the private sector, Note number 273, The World Bank Group

Pan, Y.; Shu, M. (2002): Road Sector Impact Study, Draft Final Report, ADB Technical Assistance to the Beijing-Tongjiang Transport Corridor in China, Manila

Parker, D.; Hartley, K. (2003): Transaction Costs, relational contracting and public private partnerships: a case study of UK defence, in Journal of Purchasing and Supply Management, Vol. 9/3, S. 97-108

Patterson, O. (1984): The Realities of Intervention in Alienated Cultures: A Jamaican Case Study, in Brooks, H.; Liebman, L.; Schelling, C. (Hrsg.) (1984): Public-Private Partnership: New Opportunities for Meeting Social Needs, Ballinger Publishing Company, Cambridge

Pausenberger, E. (1980): Internationale Unternehmungen in Entwicklungsländern: Ihre Strategien und Erfahrungen, Düsseldorf

Pausenberger, E. (Hrsg.) (1994): Internationalisierung von Unternehmen, Schäffer-Poeschel Verlag, Stuttgart

Pauwels, P.; Mattheyssens, P. (2004): The Architecture of Multiple Case Study Research in International Business, in: Marschan-Piekkari, R.; Welch, C. (Hrsg.) (2004): Handbook of Qualitative Research Methods for International Business, Edward Elgar, Cheltenham

Pernia, E.M. (2003): Pro-poor Growth: What is it and How is it important?, ADB ERD Policy Brief No. 17, Manila

Peters, B.G.; Pierre, J. (2006): Governance, accountability and democratic legitimacy, in Benz, A.; Papadopoulos, Y. (Hrsg.) (2006): Governance and Democracy, Comparing national, European and international experiences, Routledge, London

Peters, G.B. (1998): With a little help from our friends: Public-private partnerships as institutions and instruments, in Pierre, J. (Hrsg.) (1998): Partnerships in urban governance, St. Martin's Press, New York

Peters, G.B.; Wright, V. (1996): Public Policy and Administration, Old and New, in Godin, R.E.; Klingemann, H.-D. (Hrsg.) (1996): A New Handbook of Political Sciences, Oxford University Press, Oxford

Petersen, J.; Freire, M. (2004): Political, Legal and Financial Framework, in Freiere, M.; Petersen (Hrsg.) (2004): Subnational Capital Markets in Developing Countries: From Theory to Practice, World Bank, Washington

Pierre, J. (Hrsg.) (1998): Partnerships in urban governance, St. Martin's Press, New York 
Pierre, J.; Peters, G.B. (2000): Governance, Politics and the State, St. Martin's Press, New York

Plane, P. (1997): Privatisation and Economic Growth: An Empirical Investigation from a Sample of Developing Market Economies, in Applied Economics, Vol. 29/2, S. 161-178

Plum, M. (1994): Auswirkungen von Direktinvestitionen in Empfängerländer, Verlag Josef Eul, Bergisch Gladbach

Plummer, J. (2002): Developing Inclusive Public-Private Partnerships: The role of small-scale independent providers in the delivery of water and sanitation services, WDR Workshop 2003/2004

Prakash, A.; Hart, J.A. (Hrsg.) (1999): Globalization and Governance, Routledge, London

PriceWaterhouseCoopers Securities (2003): Comparative Review of Risk Mitigation Instruments and Direct Sub-Sovereign Lending, World Bank, Washington

Priermeier, T. (2002): Steuerung von Finanzrisiken in der Projektfinanzierung, in Die Bank, Vol. 6, S. 392-396

Przybylski, R. (1993): Neuere Aspekte der Länderrisikobeurteilung internationaler Unternehmen, Verlag Dr. Kovač, Hamburg

Puls, U. (2003): Spezifität, Kapitalstruktur und Projektfinanzierung, Dissertation, Peter Lang, Frankfurt a. M.

Ramamurti, R.; Doh, J.P. (2004): Rethinking foreign infrastructure investment in developing countries, in Journal of World Business, Vol. 39, S. 151-167

Redwood, H.J. (2004): Public-Private Partnerships and Private Finance, in Ghobadian, A.; Gallear, D.; O'Regan, N.; Viney, H. (Hrsg.) (2004): PublicPrivate Partnerships, Policy and Experience, palgrave Macmillan, New York

Reijniers, J.J.A.M. (1994): Organization of public-private partnership projects, in International Journal of Project Management, Vol. 12/3, S. 137-142

Reiterer, G.M. (1997): Die Philippinen, Kontinuität und Wandel, Sonderzahl Verlagsgesllschaft m. b. H., Wien

Reuter, A., Wecker, C. (1999): Projektfinanzierung, Schäffer Poeschel, Stuttgart

Richter, R.; Bindseil, U. (1995): Neue Institutionenökonomik, in Wirtschaftswissenschaftliches Studium (WiSt), Vol. 3, S. 132-140

Roeskau, M. (2005): Synergies between private investment and foreign aid, OECD

Roger, N. (1999): Recent trends in private participation in infrastructure, Private Sector, World Bank 
Roggencamp, S. (1999): Public Private Partnership: Entstehung und Funktionsweise kooperative Arrangements zwischen öffentlichen Sektor und Privatwirtschaft, Dissertation, Peter Lang, Frankfurt a. M.

Rhodes, R.A.W. (1997): Understanding Governance, Policy Networks, Governance, Reflexivity and Accountability, Open University Press, Buckingham

Rieger, E.; Leibfried, S. (2001): Grundlagen der Globalisierung, Perspektiven des Wohlstandes, Edition Suhrkamp, Frankfurt a. M.

Roller, L-H.; Waverman, L. (2001): Telecommunications Infrastructure and Economic Development, in American Economic Review, Vol. 91, S. 909-923

Root, H.L. (1996): Small Countries, Big Lessons, Governance and the Rise of East Asia, Oxford University Press, ADB und Oxford

Rosenau, J.N. (1995): Governance in the Twenty-First Century, in Global Governance, Vol. 7/1, S. 25-50

Rosenau, P.V. (Hrsg.) (2000): Public-Private Policy Partnerships, Massachusetts Institute of Technology Press, Cambridge

Sagar, A. (2006): Infrastructure and Project Financing Asia Pacific Scenario, Asian Development Bank, Manila

Samii, R.; Van Wassenhove, L.N.; Bhattacharya, S. (2002): An Innovative Public-Private Partnership: New Approach to Development, in World Development, Vol. 30/6, S. 991-1008

Sass Rubin, J.: Stankiewicz, G.M. (2001): The Los Angeles Community Development Bank: The Possible Pitfalls of Public-Private Partnerships, in Journal of Urban Affairs, Vol. 23/2, S. 133-153

Sass, M. (2003): Competitiveness and Economic Policies Related to Foreign Direct Investment, Working Paper, Ministry of Finance Hungary

Savas, E.S. (2000): Privatization and Public-Private Partnerships, Seven Bridges Press, New York

Scapens, R.W. (2004): The many Skills of the Case Researcher, in: MarschanPiekkari, R.; Welch, C. (Hrsg.) (2004): Handbook of Qualitative Research Methods for International Business, Edward Elgar, Cheltenham

Scharle, P. (2002): Public-Private Partnerships (PPP) as a Social Game, in Innovation, Vol. 15/3, S. 227-252

Schicho, W. (2003): Entwicklung als gesellschaftlicher Auftrag, in Fischer, K.; Hanak, I.; Parnreiter, Ch. (Hrsg.) (2003): Internationale Entwicklung: Eine Einführung in Probleme, Mechanismen und Theorien, 3. Auflage, Brandes \& Apsel Verlag GmbH, Frankfurt a. M. 
Schmidt, C. (2003): Analyse von Leitfadeninterviews, in Flick, U.; von Kardorff, E.; Steinke, I. (Hrsg.), Qualitative Forschung, Ein Handbuch, Rohwohlt Taschenbuch Verlag, Reinbek bei Hamburg

Schröer, N. (1994): Interpretative Sozialforschung, Westdeutscher Verlag, Opladen

Schülein, A.J.; Reitze, S. (2002): Wissenschaftstheorie für Anfänger, Facultas Verlags- und Buchhandlungs AG, Wien

Schwank, O. (2003): Neuorientierung der Weltbank in den 90er Jahren, ÖFSE, Wien

Shapiro, A.C. (2003): Multinational Financial Management, John Wiley \& Son, New York

Siebel, U.R. (Hrsg.) (2001): Handbuch Projekte und Projektfinanzierung, Verlag C.H.Beck, München

Simonis, U.E. (Hrsg.) (1977): Infrastruktur, Theorie und Praxis, Kiepenheuer \& Witsch

Sjöholm, F. (1999): Technology Gap, Competition and Spillovers from Direct Foreign Investment: Evidence from Establishment Data, in The Journal of Development Studies, Vol. 36, S. 53-73

Smith, A.J. (1999): Philosophical Aspects of the Public/Private Sector Partnerships, in Grimsey D.; Lewis, M.K. (Hrsg.) (2005): The Economics of Public Private Partnerships, An Elgar Reference Collection, Cheltenham

Smith, W. (2001): Designing output-based aid schemes: a checklist in Brook, P.J.; Smith, S. M. (Hrsg.) (2001): Contracting for public services: Output-based aid and its applications, World Bank, Washington D.C.

Songco, J.A. (2002): Do Rural Infrastructure Investments Benefit the Poor?, World Bank Working Paper No. 2796, Washington D.C.

Stafford, D. (2005): Regional and Cross-border Infrastructure and its Role in Trade, Pro Poor Economic Growth and Poverty Reduction, OECD Room Document 3, Tokyo

Stiglitz, J. E. (1998): Towards a New Paradigm for Development: Strategies, Policies, and Process, Prebisch Lecture at UNCTAD, Geneva

Stiglitz, J. E. (2006): Die Chancen der Globalisierung, Siedler Verlag, München

Stiglitz, J.E.; Muet, P.-A. (Hrsg.) (2001): Governance, Equity and Global Markets, University Press, Oxford

Stiglitz, J.E.; Wallstein, S.J. (2000): Public-Private Technology Partnerships, Promises and Pitfalls, in Rosenau, P.V. (Hrsg.) (2000): Public-Private Policy Partnerships, Massachusetts Institute of Technology Press, Cambridge 
Stocker, F. (1999): Spaß mit Mikro, Einführung in die Mikroökonomie, R. Oldenbourg Verlag, München

Stocker, G. (1998): Governance as Theory: Five Propositions, in Governance International Social Science Journal, Vol. March/155, S.17-29

Störig, H. J. (1999): Kleine Weltgeschichte der Philosophie, Kohlhammer, Stuttgart

Stosberg, J. (2005): Political Risk and the Institutional Environment for Foreign Direct Investment in Latin America, Peter Lang, Frankfurt a. M.

Strauss, A. L. (1994): Grundlagen qualitativer Sozialforschung, Wilhelm Fink Verlag, München

Strohbach, H. (1999): Build Operate Transfer-Modelle zur Finanzierung von Infrastrukturinvestitionen, Dissertation, Peter Lang, Europäische Hochschulschriften, Frankfurt a. M.

Strübing. J. (2004): Methodologie Interpretativer Sozialforschung, UVKVerlagsgesellschaft, Konstanz

Teruel, R.G.; Kuroda, Y. (2005): Public infrastructure and productive growth in Philippine agriculture, 1974-2000, in Journal of Asian Economics, Vol. 16, S. 555-567

Topritzhofer, E.; Moser, R. (1985): Das Exportgeschäft, Seine Abwicklung und Absicherung, Service Fachverlag, Wien

Toye, J. (1993): Dilemmas of Development, Reflections on the CounterRevolution in Development Economics, Blackwell, Oxford

Toye, J. (2000): The New Institutional Economics and its Implication for Development Theory, in Harris, J.; Hunter, J.; Lewis, C.M. (Hrsg.) (2000): The New Institutional Economics and Third World Development, Routledge, London

Toye, J. (Hrsg.) (2003): Trade and Development, Directions for the $21^{\text {st }}$ century, Edward Elgar Publishing Ltd., Cheltenham

Trujillo, J.A.M Cohen, R.; Freixas, X.; Sheehy, R. (1998): Infrastructure Financing with Unbundled Mechanisms, in The Financier, Vol. 5/4, S. 10-27

Turner, M, Hulme, D. (1997): Governance, Administration and Development, Making the State Work, Macmillan, London

Tytko, D. (1999): Grundlagen der Projektfinanzierung, Schäfer Poeschel, Stuttgart

Tytko, D. (2003): Grundlagen der Projektfinanzierung, in Backhaus, K.; Werthschulte, H. (Hrsg.) (2003): Projektfinanzierung, Schäffer Poeschel, Stuttgart 
Uekermann, H. (1993): Risikopolitik bei Projektfinanzierungen: Maßnahmen und ihre Ausgestaltung, Dissertation, DUV, Münster

UN (2006): Enhancing Regional Cooperation in Infrastructure Development including that related to disaster management, ESCAP

UNCTAD (1995): Foreign Direct Investment in Africa, Geneva

UNCTAD (2003): World Investment Report, United Nations, New York

UNCTAD (2005a): Economic Development in Africa, Rethinking the Role of Foreign Direct Investment, Geneva

UNCTAD (2005b): World Investment Report, United Nations, New York

UNCTAD (2005c): Report of the Expert Meeting on the Impact of FDI on Development, Held in Geneva from 24 to 26 January 2005

UNDP (1997): Governance for sustainable human development, New York

UNDP (2000): Overcoming Human Poverty: UNDP Human Poverty Report, New York

UNDP (2006): Human Development Report 2006, Country Fact Sheets, Online Publication, New York

Von Baratta, M. (Hrsg.) (2001): Der Fischer Weltalmanach 2002, Fischer Taschenbuch Verlag, Frankfurt a. M.

Wagner, N.; Kaiser, M. (1995): Ökonomie der Entwicklungsländer: eine Einführung, Gustav Fischer Verlag, Jena

Walldorf, E.G. (1992): Die Wahl zwischen unterschiedlichen Formen der internationalen Unternehmer-Aktivität, in: Handbuch der Internationalen Unternehmenstätigkeit, C.H.Beck'sche Verlagsbuchhandlung, München

Weaver, C.; Leiteritz, R.J. (2005): Our Poverty Is a World Full of Dreams, Reforming the World Bank, in Global Governance, Vol. 11/3, S.369-388

Weber, B.; Alfen, H.W.; Maser, S. (2006): Projektfinanzierung und PPP, Praktische Anleitung für PPP und andere Projektfinanzierungen, bank-verlag, Köln

Weber, M. (1978): Economy and Society, An Outline of Interpretive Sociology, University of California Press, Berkely

Wei, Y.A.; Balasubramanyam, V.N. (Hrsg.) (2004): Foreign Direct Investment, Edward Elgar Publishing Limited, Cheltenham

Weiss, T. G. (2000): Governance, Good Governance and Global Governance: conceptual and actual challenges, in Third World Quarterly, Vol. 21/5, S. 795814 
Werthschulte, H. (2003): Projektfinanzierungsstrukturen zur privaten Finanzierung von Infrastrukturinvestitionen, in Backhaus, K.; Werthschulte, $\mathrm{H}$. (Hrsg.) (2003): Projektfinanzierung, Schäffer Poeschel, Stuttgart

Werthschulte, H. (2005): Kreditrisikomessung bei Projektfinanzierungen durch Risikosimulation, Dissertation, Deutscher Universitäts-Verlag, Wiesbaden

Wettenhall, R. (2003): The Rhetoric and Reality of Pubic-Private Partnerships, in Public Organization Review: A Global Journal, Vol. 3, S. 77-107

Will, F. (2001): Länder- und Hoheitsrisiken, Verlag Dr. Kovač, Hamburg

Williamson, O.E. (1985): The Economic Institutions of Capitalism, Free Press, New York

Willms, M. (1998): Private Finanzierung von Infrastrukturinvestitionen, Nomos Verlagsgesellschaft, Baden-Baden

Willoughby, C. (2004a): Infrastructure and the Millennium Development Goals, Session on Complementarity of Infrastrucutre for Achieving the MDGs, Berlin

Willoughby, C. (2004b): How important is infrastructure for achieving pro-poor growth?, OECD, Paris

Wolf, B.; Hill, M.; Pfaue, M. (2003): Strukturierte Finanzierung, Schäffer Poeschel, Stuttgart

Woodward, D. (2001): The next crisis?: Direct and Equity Investment in Developing Countries, Zed Books Ltd.

World Bank (1989): Sub-Saharan Africa: From Crisis to Sustainable Growth, Washington

World Bank (1992): Governance and Development, Washington

World Bank (1994): Weltentwicklungsbericht 1994: Infrastruktur und Entwicklung, Washington

World Bank (1997): The State in a Changing World, World Development Report, Oxford

World Bank (2001a): Attacking Poverty: Opportunity, Empowerment, and Security, World Development Report, Oxford

World Bank (200lb): Global Economic Prospects and the Developing Countries, Washington

World Bank (2002): Building Institutions for Markets, World Development Report, Oxford

World Bank (2003a): Making Service Work for Poor People, World Development Report, 2004Washington 
World Bank (2003b): IDA Guarantee supports first competitively-bid private power project in Vietnam, Washington

World Bank (2003c): Private Participation in Infrastructure: Trends in Developing Countries in 1990 - 2001, Washington

World Bank (2004a): World Development Indicators, Washington

World Bank (2004b): Global Development Finance, Harnessing Cyclical Gains for Development, Analysis and Summary Tables, New York

World Bank (2004c): Public and Private Sector Roles in the Supply of Electricity Services, Washington

World Bank (2004d): World Development Report, Washington

World Bank (2005a): Global Development Finance 2005: Mobilizing Finance and Managing Vulnerability, Washington

World Bank (2005b): Global Economic Prospects: Trade, Regionalism and Development, Washington

World Bank (2005c): The World Bank Group, 2005 Trust Funds Annual Report, Washington

World Bank (2006a): Peru Rethinking Private Sector Participation in Infrastructure

World Bank (2006b): Approaches to Private Participation in Water Services, Washington

World Bank (2006c): Infrastructure at the Crossroads, Lessons from 20 Years of World Bank Experience, Washington

World Bank (2006d): Power Strategy: Managing Growth and reform, Vietnam

Wrona, T. (2005): Die Fallstudienanalyse als wissenschaftliche Forschungsmethode, ESCP-EAP Working Paper, Berlin

Yao, X. (2003): Infrastructure and Poverty Reduction Making Markets Work for the Poor, Asian Development Bank, ERD Policy Brief, Manila

Zeiss, Ch. (2000): Privatfinanzierung staatlicher Infrastruktur, Dissertation, Bonn

Zeuchner, S. (2002): Privatwirtschaftliche Organisationsformen der Bereitstellung und Finanzierung von Infrastrukturleistungen in Entwicklungsländern: Eine ökonomische Effizienzanalyse unter Berücksichtigung von Projekt- und Länderrisiken, Dissertation, Verlag für Entwicklungspolitik, Saarbrücken

Ziai, A. (2006): Zwischen Global Governance und Post-Development, Entwicklungspolitik aus diskursanalytischer Perspektive, Westfälisches Dampfboot, Münster 


\section{Internetquellen:}

Bei der Recherche der Instiutionen und Institute wurden die jeweiligen Homepages maßgeblich herangezogen. Daher wurde bei der Beschreibung im Text nicht einzeln auf diese verwiesen.

Austrian Development Agency (ADA):

www.ada.gv.at

Asiatische Entwicklungsbank (ADB):

www.adb.org

Austria Wirtschaftsservice (aws):

www.awsg.at

EC-Asean Cogen:

www.cogen3.net

EC-Asean Energy Facility:

www.aseanenergy.com

Emerging Africa Infrastructure Fund (EAIF):

www.emergingafricafund.com

Europäische Investitionsbank (EIB):

www.eib.org

Europäische Kommission (EC):

http://ec.europa.eu

Finanzministerium:

www.bmf.gv.at

GuarantCo:

www.guarantco.com

IFC:

www.ifc.org

InfraCo:

www.infracolimited.com

MIGA:

www.miga.org

Oesterreichische Kontrollbank: 
www.oekb.at

PIDG Fonds:

www.pidg.org

PPI Project Database:

http://ppi.worldbank.org

Public-Private Infrastructure Advisory Facility (PPIAF):

www.ppiaf.org

World Bank:

http://www.worldbank.org 


\section{Forschungsergebnisse der Wirtschaftsuniversität Wien}

Herausgeber: Wirtschaftsuniversität Wien vertreten durch a.o. Univ. Prof. Dr. Barbara Sporn

Band 1 Stefan Felder: Frequenzallokation in der Telekommunikation. Ökonomische Analyse der Vergabe von Frequenzen unter besonderer Berücksichtigung der UMTS-Auktionen. 2004.

Band 2 Thomas Haller: Marketing im liberalisierten Strommarkt. Kommunikation und Produktplanung im Privatkundenmarkt. 2005.

Band 3 Alexander Stremitzer: Agency Theory: Methodology, Analysis. A Structured Approach to Writing Contracts. 2005.

Band 4 Günther Sedlacek: Analyse der Studiendauer und des Studienabbruch-Risikos. Unter Verwendung der statistischen Methoden der Ereignisanalyse. 2004.

Band 5 Monika Knassmüller: Unternehmensleitbilder im Vergleich. Sinn- und Bedeutungsrahmen deutschsprachiger Unternehmensleitbilder - Versuch einer empirischen (Re-)Konstruktion. 2005.

Band 6 Matthias Fink: Erfolgsfaktor Selbstverpflichtung bei vertrauensbasierten Kooperationen. Mit einem empirischen Befund. 2005.

Band 7 Michael Gerhard Kraft: Ökonomie zwischen Wissenschaft und Ethik. Eine dogmenhistorische Untersuchung von Léon M.E. Walras bis Milton Friedman. 2005.

Band 8 Ingrid Zechmeister: Mental Health Care Financing in the Process of Change. Challenges and Approaches for Austria. 2005.

Band 9 Sarah Meisenberger: Strukturierte Organisationen und Wissen. 2005.

Band 10 Anne-Katrin Neyer: Multinational teams in the European Commission and the European Parliament. 2005.

Band 11 Birgit Trukeschitz: Im Dienst Sozialer Dienste. Ökonomische Analyse der Beschäftigung in sozialen Dienstleistungseinrichtungen des Nonprofit Sektors. 2006

Band 12 Marcus Kölling: Interkulturelles Wissensmanagement. Deutschland Ost und West. 2006.

Band 13 Ulrich Berger: The Economics of Two-way Interconnection. 2006.

Band 14 Susanne Guth: Interoperability of DRM Systems. Exchanging and Processing XML-based Rights Expressions. 2006.

Band 15 Bernhard Klement: Ökonomische Kriterien und Anreizmechanismen für eine effiziente Förderung von industrieller Forschung und Innovation. Mit einer empirischen Quantifizierung der Hebeleffekte von F\&E-Förderinstrumenten in Österreich. 2006.

Band 16 Markus Imgrund: Wege aus der Insolvenz. Eine Analyse der Fortführung und Sanierung insolventer Klein- und Mittelbetriebe unter besonderer Berücksichtigung des Konfigurationsansatzes. 2007.

Band 17 Nicolas Knotzer: Product Recommendations in E-Commerce Retailing Applications. 2008.

Band 18 Astrid Dickinger: Perceived Quality of Mobile Services. A Segment-Specific Analysis. 2007.

Band 19 Nadine Wiedermann-Ondrej: Hybride Finanzierungsinstrumente in der nationalen und internationalen Besteuerung der USA. 2008.

Band 20 Helmut Sorger: Entscheidungsorientiertes Risikomanagement in der Industrieunternehmung. 2008.

Band 21 Martin Rietsch: Messung und Analyse des ökonomischen Wechselkursrisikos aus Unternehmenssicht: Ein stochastischer Simulationsansatz. 2008. 
Band 22 Hans Christian Mantler: Makroökonomische Effizienz des Finanzsektors. Herleitung eines theoretischen Modells und Schätzung der Wachstumsimplikationen für die Marktwirtschaften und Transformationsökonomien Europas. 2008.

Band 23 Youri Tacoun: La théorie de la valeur de Christian von Ehrenfels. 2008.

Band 24 Monika Koller: Longitudinale Betrachtung der Kognitiven Dissonanz. Eine Tagebuchstudie zur Reiseentscheidung. 2008.

Band 25 Marcus Scheiblecker: The Austrian Business Cycle in the European Context. 2008.

Band 26 Aida Numic: Multinational Teams in European and American Companies. 2008.

Band 27 Ulrike Bauernfeind: User Satisfaction with Personalised Internet Applications. 2008.

Band 28 Reinhold Schodl: Systematische Analyse und Bewertung komplexer Supply Chain Prozesse bei dynamischer Festlegung des Auftragsentkopplungspunkts. 2008.

Band 29 Bianca Gusenbauer: Öffentlich-private Finanzierung von Infrastruktur in Entwicklungsländern und deren Beitrag zur Armutsreduktion. Fallstudien in Vietnam und auf den Philippinen. 2009.

www.peterlang.de 


\section{Systematische Analyse} und Bewertung komplexer Supply Chain Prozesse bei dynamischer Festlegung des Auftragsentkopplungspunkts

Frankfurt am Main, Berlin, Bern, Bruxelles, New York, Oxford, Wien, 2008. 154 S., 16 Abb., 20 Tab.

Forschungsergebnisse der Wirtschaftsuniversität Wien. Herausgegeben von der Wirtschaftsuniversität Wien, vertreten durch Barbara Sporn. Bd. 28 ISBN 978-3-631-57961-9 • br. € 34.-*

Ein entscheidender Erfolgsfaktor jeder Supply Chain ist das adäquate Design ihrer Leistungserstellungsprozesse. Da diese Prozesse regelmäßig durch einen hohen Grad an Komplexität gekennzeichnet sind, stellt deren Gestaltung eine große Herausforderung dar. In dieser Forschungsarbeit wird deshalb die Frage behandelt, wie komplexe Leitungserstellungsprozesse einer Supply Chain effektiv und effizient verbessert werden können. Dazu wird eine systematische Vorgehensweise für die Analyse und Bewertung von Supply Chain Prozessen vorgestellt und im Rahmen einer Fallstudie auf eine reale Logistikkette in der Elektronikindustrie angewandt. Bei der Prozessanalyse wird eine Reduktion der Komplexität durch eine Kombination von analytischen Methoden und Simulation erzielt, sodass nicht nur die Validität der Ergebnisse, sondern auch die praktische Umsetzbarkeit gewährleistet ist. Die Bewertung von alternativen Prozessdesigns erfolgt multikriteriell und strategiebasierend, damit sowohl Zielkonflikte als auch die verfolgte Strategie der Logistikkette Berücksichtigung finden. Generell spielt die Wahl des marktbezogenen Produktionstyps (z. B. Make-to-Order, Make-to-Stock) bei der Verbesserung von Supply Chain Prozessen eine entscheidende Rolle. Daher wird auf diesen Aspekt besonders eingegangen, wobei der hybride Produktionstyp Make-to-Forecast in einem Supply Chain-Kontext in der Fallstudie implementiert wird.

Frankfurt am Main - Berlin - Bern - Bruxelles - New York · Oxford - Wien

Auslieferung: Verlag Peter Lang AG

Moosstr. 1, $\mathrm{CH}-2542$ Pieterlen

Telefax 0041 (0) $32 / 3761727$

*inklusive der in Deutschland gültigen Mehrwertsteuer

Preisänderungen vorbehalten

Homepage http://www.peterlang.de 